\title{
Ni(II)-Catalyzed Asymmetric Nitration of Oxindoles: Construction of
}

\section{Cipargamin Analogues}

Mingjun Lv, Xiaoxun Li*

Department of Medicinal Chemistry, Key Laboratory of Chemical Biology (Ministry of Education),

School of Pharmaceutical Sciences, Cheeloo College of Medicine, Shandong University, Jinan, Shandong, 250012, China.

*Correspondence to: xli@sdu.edu.cn. 


\section{Table of Contents}

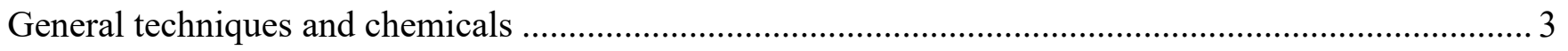

Procedures for the preparation of 3 -alkyl- $N$-protected oxindoles ...................................................... 4

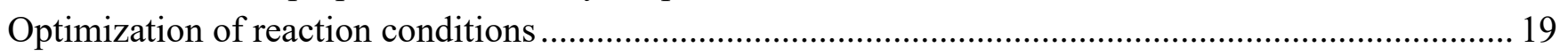

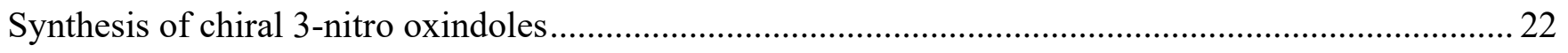

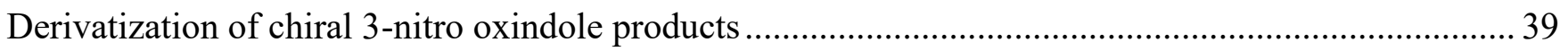

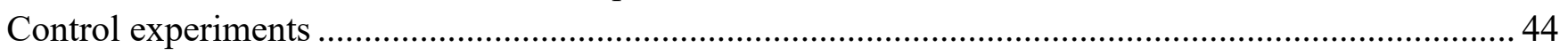

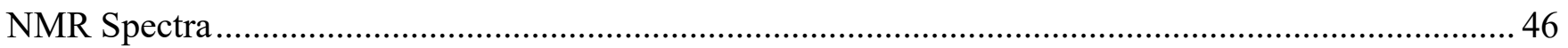

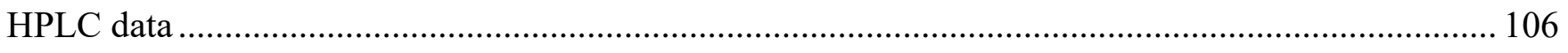

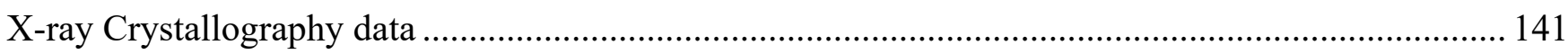

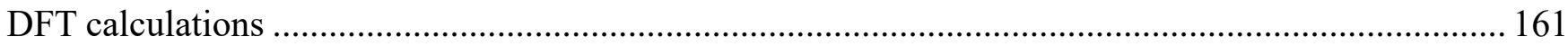

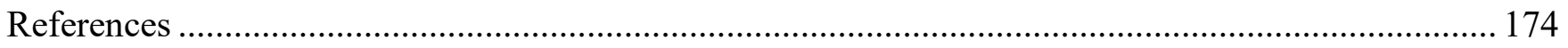




\section{General techniques and chemicals}

Unless otherwise noted, all the reactions for the preparation of the substrates were performed in ovendried glassware under oxygen atmosphere with freshly distilled solvents. The solvents were purified by distillation from calcium hydride unless otherwise noted. All other commercial reagents were used without further purification unless otherwise indicated. ${ }^{1} \mathrm{H}$ NMR and ${ }^{13} \mathrm{C}$ NMR spectra were recorded on Bruker $400 \mathrm{MHz}$ spectrometer (ADVNCE III) using chloroform- $d\left(\mathrm{CDCl}_{3}\right)$ and dimethyl sulphoxide- $d 6$ (DMSO-d6) as the internal standard. Chemical shifts were reported in parts per million (ppm), and the residual solvent peak was used as an internal reference: proton (chloroform $\delta 7.26$ ), carbon (chloroform $\delta 77.1$ ) or tetramethylsilane (TMS $\delta 0.00$ ) was used as a reference. Data are reported as follows: chemical shift, multiplicity $(\mathrm{s}=$ singlet, $\mathrm{d}=$ doublet, $\mathrm{t}=$ triplet, $\mathrm{q}=$ quartet, $\mathrm{m}=$ multiplet, $\mathrm{br}=$ broad $)$, coupling constants $(\mathrm{Hz})$ and integration. The enantiomeric excesses (ee) was determined by HPLC analysis on Agilent 1260 Infinity II Prime using Daicel CHIRALPAK® column IA-U, IB-U, IC-U and ID-U. X-ray diffraction analyses were carried out on a microcrystalline powder using a Rigaku Oxford Diffraction XtaLAB Synergy-S diffractometer using Cu radiation $(\lambda=1.54184 \AA)$. If not specially mentioned, flash column chromatography was performed using 200-300 silica gel purchased from Yaitai Chemicals (China). High-resolution mass spectra (HRMS) were recorded on a Bruker Apex IV FTMS mass spectrometer using ESI (electrospray ionization) as ionization method. IR spectra were analyzed on Thermo NICOLET iN10 spectrometer. Optical rotations were recorded on an AUTOPOL II digital polarimeter at $589 \mathrm{~nm}$ and are recorded as $[\alpha]_{\mathrm{D}}^{\mathrm{T}}$ (concentration in grams $/ 100 \mathrm{~mL}$ solvent). 


\section{Procedures for the preparation of 3-alkyl- $N$-protected oxindoles}

All 3-alkyl oxindoles were prepared directly from commercially available oxindoles except 1g, 1u-1w. After alkylation, the 3-alkyl- $N$-protected oxindoles was achieved through modified literature procedures. ${ }^{[1]}$ Oxindoles 1za-1zc were prepared according to the literature procedures. ${ }^{[2]}$

\section{General procedure for oxindole GP1}

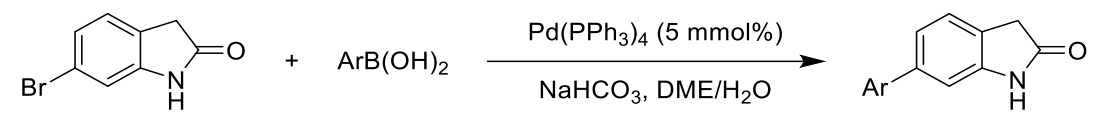

To a solution of 6-Bromo-2-oxindole $\left(10 \mathrm{mmol}, 1\right.$ equiv.) in DME (100 ml) and saturated $\mathrm{NaHCO}_{3}$ aqueous (50 ml) under nitrogen, arylboronic acid (11 mmol, 1 equiv.) and $\mathrm{Pd}\left(\mathrm{PPh}_{3}\right)_{4}(0.5 \mathrm{mmol}, 5 \mathrm{~mol} \%)$ was added. The reaction mixture was refluxed until the starting oxindole was consumed, the solvent was removed under reduced pressure. The residue was extracted with EtOAc $(3 \times 40 \mathrm{ml})$, the combined organic phase washed with brine and dried over $\mathrm{Na}_{2} \mathrm{SO}_{4}$, filtered and solvent was evaporated under reduced pressure. The crude products was recrystallization by $\mathrm{MeOH}$ for next step without further purification.

General procedure for $N$-protection of alkylated oxindole GP2
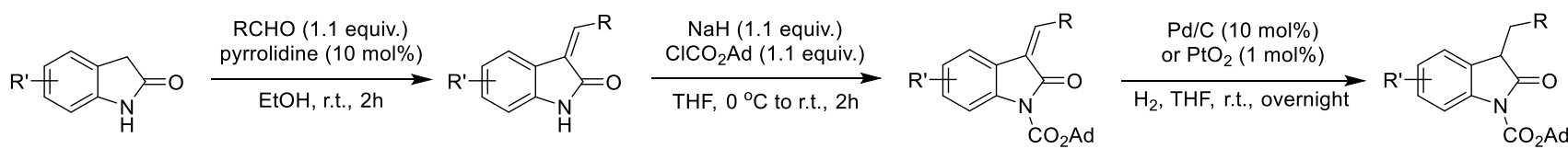

To a solution of oxindole (10 mmol, 1 equiv.) dissolved in EtOH (40 ml), RCHO (11 mmol, 1.1 equiv.) and pyrrolidine ( $1 \mathrm{mmol}, 10 \mathrm{~mol} \%$ ) were added and the resulting mixture were stirred at room temperature for 2 hours. The reaction mixture was concentrated under reduced pressure and filtrated-off to give the desired product. To a solution of the product obtained above ( $5 \mathrm{mmol}, 1$ equiv.) dissolved in anhydrous THF (20 ml), NaH (5.5 mmol, 1 equiv.) was added portion at $0{ }^{\circ} \mathrm{C}$ and the acyl chloride $(5.5 \mathrm{mmol}, 1$ equiv.) dissolved in THF was added dropwise, then the mixture was warmed to room temperature and continued stirred for 2 hours. The reaction was quenched by $\mathrm{H}_{2} \mathrm{O}(50 \mathrm{ml})$ and extracted by EtOAc $(2 \times 30$ $\mathrm{ml}$ ), the combined organic phase washed with brine and dried over $\mathrm{Na}_{2} \mathrm{SO}_{4}$, filtered and solvent was evaporated under reduced pressure. The crude products were purified by column chromatography on silica-gel to give the desired product. To a solution of the product obtained above ( $3 \mathrm{mmol}, 1$ equiv.) in THF (15 ml), $\mathrm{Pd} / \mathrm{C}$ (0.1 g, compound containing bromide or chlorine used $\mathrm{PtO}_{2}$ instead) was added, and resulting mixture was stirred under hydrogen atmosphere over night at room temperature. The reaction mixture was filtered through celite to remove $\mathrm{Pd} / \mathrm{C}$ and removed the solvent under reduced pressure. The crude product was purified by column chromatography to give target product. 

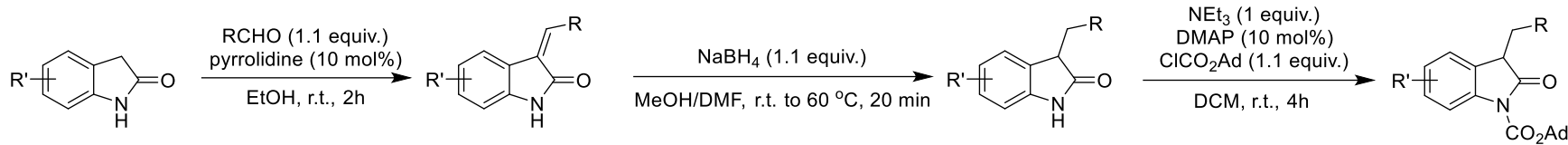

To a solution of oxindole (10 mmol, 1 equiv.) dissolved in EtOH (40 ml), RCHO (11 mmol, 1.1 equiv.) and pyrrolidine ( $1 \mathrm{mmol}, 10 \mathrm{~mol} \%)$ were added and the resulting mixture were stirred at room temperature for 2 hours. The reaction mixture was concentrated under reduced pressure and filtrated-off to give the desired product. To a solution of the product obtained above ( $5 \mathrm{mmol}, 1$ equiv.) dissolved in a mixture of DMF (15 ml) and methanol $(25 \mathrm{ml})$, sodium borohydride $(5.5 \mathrm{mmol}, 1.1$ equiv.) was added at room temperature, and heated at $60{ }^{\circ} \mathrm{C}$ for $20 \mathrm{~min}$. After the mixture was cooled to room temperature, saturated aqueous solution of $\mathrm{NH}_{4} \mathrm{Cl}(60 \mathrm{ml})$ was added for quench and extracted with EtOAc $(2 \times 30 \mathrm{ml})$. The organic phase was dried over $\mathrm{Na}_{2} \mathrm{SO}_{4}$, filtered and solvent was evaporated under reduced pressure. The crude products were purified by column chromatography on silica-gel to give the desired product. To a mixture of the product obtained above ( 3 mmol, 1 equiv.), $\mathrm{NEt}_{3}$ ( 3 mmol, 1 equiv.) and DMAP ( 0.3 mmol, $10 \mathrm{~mol} \%$ ) in DCM (20 ml), the acyl chloride (3.3 mmol, 1 equiv.) dissolved in DCM (5 ml) was added dropwise at $0{ }^{\circ} \mathrm{C}$, then the mixture was warmed to room temperature and continued stirred for 4 hours. The solvent was removed under reduced pressure and purified by column chromatography to give target product.

\section{General procedure for $N$-protection of alkylated oxindole GP4}
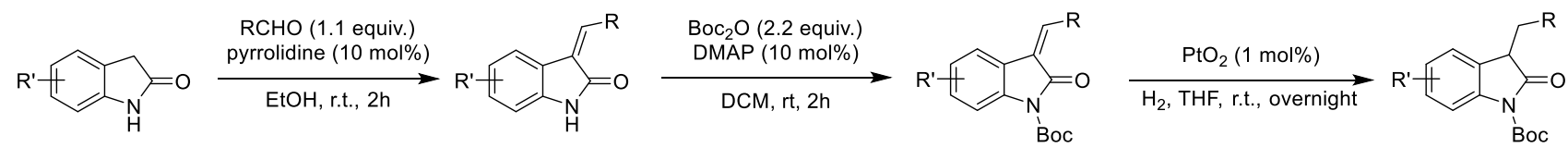

To a solution of oxindole (10 mmol, 1 equiv.) dissolved in EtOH (40 ml), RCHO (11 mmol, 1.1 equiv.) and pyrrolidine ( $1 \mathrm{mmol}, 10 \mathrm{~mol} \%)$ were added and the resulting mixture were stirred at room temperature for 2 hours. The reaction mixture was concentrated under reduced pressure and filtrated-off to give the desired product. 1 equiv. To a solution of the product obtained above (5.0 mmol, 1 equiv.) in DCM (20 $\mathrm{mL})$ was added DMAP $(0.5 \mathrm{mmol}, 10 \mathrm{~mol} \%)$ and (Boc $)_{2} \mathrm{O}(11 \mathrm{mmol}, 2.2$ equiv. $)$ at room temperature, and then the mixture was stirred for 2 hours. Then the solvent was removed under reduced pressure, and purified by column chromatography on silica-gel to provide the desired product. To a solution of the product obtained above $(3.0 \mathrm{mmol})$ in THF $(15 \mathrm{~mL}), \mathrm{PtO}_{2}(0.03 \mathrm{mmol}, 1 \mathrm{~mol} \%)$ was added, and resulting mixture was stirred under hydrogen atmosphere over night at room temperature. The reaction mixture was filtered through celite to remove $\mathrm{PtO}_{2}$, and purified by column chromatography on silica-gel to give target. 


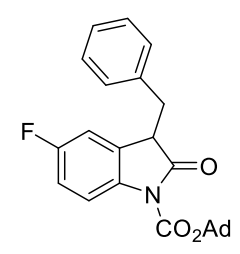

Prepared according to the GP2 to provide the title compound as white solid.

m.p. $134-135{ }^{\circ} \mathrm{C}$.

${ }^{1}$ H NMR $\left(400 \mathrm{MHz}, \mathrm{CDCl}_{3}\right) \delta 7.72(\mathrm{dd}, J=8.9,4.6 \mathrm{~Hz}, 1 \mathrm{H}), 7.27(\mathrm{~m}, 3 \mathrm{H}), 7.20-7.09(\mathrm{~m}, 2 \mathrm{H}), 6.92(\mathrm{~m}$, $1 \mathrm{H}), 6.42(\mathrm{dd}, J=8.0,2.8 \mathrm{~Hz}, 1 \mathrm{H}), 3.79(\mathrm{dd}, J=9.3,4.5 \mathrm{~Hz}, 1 \mathrm{H}), 3.49(\mathrm{dd}, J=13.7,4.6 \mathrm{~Hz}, 1 \mathrm{H}), 2.93$ (dd, $J=13.7,9.3 \mathrm{~Hz}, 1 \mathrm{H}), 2.40-2.22(\mathrm{~m}, 9 \mathrm{H}), 1.71(\mathrm{~m}, 6 \mathrm{H})$.

${ }^{13}$ C NMR $\left(100 \mathrm{MHz}, \mathrm{CDCl}_{3}\right) \delta 175.0,159.4\left(\mathrm{~d}, \mathrm{C}-\mathrm{F},{ }^{1} J_{\mathrm{C}-\mathrm{F}}=242.9 \mathrm{~Hz}\right), 148.5,136.9,136.0\left(\mathrm{~d}, \mathrm{C}-\mathrm{F},{ }^{4} J_{C-}\right.$ $F=2.5 \mathrm{~Hz}), 129.4,129.0\left(\mathrm{~d}, \mathrm{C}-\mathrm{F},{ }^{3} J_{C-F}=8.6 \mathrm{~Hz}\right), 128.6,127.1,116.1\left(\mathrm{~d}, \mathrm{C}-\mathrm{F},{ }^{3} J_{C-F}=8.0 \mathrm{~Hz}\right), 114.6(\mathrm{~d}$, $\left.\mathrm{C}-\mathrm{F},{ }^{2} J_{C-F}=22.7 \mathrm{~Hz}\right), 111.9\left(\mathrm{~d}, \mathrm{C}-\mathrm{F},{ }^{2} J_{C-F}=24.8 \mathrm{~Hz}\right), 84.5,47.7\left(\mathrm{~d}, \mathrm{C}-\mathrm{F},{ }^{4} J_{C-F}=1.9 \mathrm{~Hz}\right), 41.4,37.4,36.1$, 31.0.

IR (ATR, neat): 2923, 2849, 1784, 1704, 1603, 1482, 1454, 1315, 1293, 1235, 1155.

HRMS (ESI) calcd $(\mathrm{m} / \mathrm{z})$ for $\mathrm{C}_{26} \mathrm{H}_{26} \mathrm{FNO}_{3}$ : $[\mathrm{M}+\mathrm{Na}]^{+} 442.1789$, found 442.1790.

Adamantyl 6-fluoro-3-benzyl-2-oxoindoline-1-carboxylate (1b)

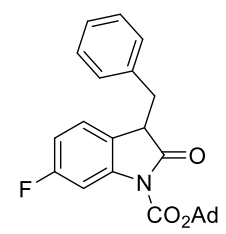

Prepared according to the GP2 to provide the title compound as colorless oil.

${ }^{1} \mathbf{H}$ NMR $\left(400 \mathrm{MHz}, \mathrm{CDCl}_{3}\right) \delta 7.52(\mathrm{dd}, J=10.4,2.4 \mathrm{~Hz}, 1 \mathrm{H}), 7.24(\mathrm{~m}, 3 \mathrm{H}), 7.18-7.10(\mathrm{~m}, 2 \mathrm{H}), 6.73-$ $6.60(\mathrm{~m}, 2 \mathrm{H}), 3.77(\mathrm{dd}, J=9.3,4.5 \mathrm{~Hz}, 1 \mathrm{H}), 3.47$ (dd, $J=13.7,4.6 \mathrm{~Hz}, 1 \mathrm{H}), 2.90$ (dd, $J=13.7,9.2 \mathrm{~Hz}$, $1 \mathrm{H}), 2.36-2.18(\mathrm{~m}, 9 \mathrm{H}), 1.71(\mathrm{~m}, 6 \mathrm{H})$.

${ }^{13} \mathrm{C}$ NMR $\left(100 \mathrm{MHz}, \mathrm{CDCl}_{3}\right) \delta 175.3,162.5\left(\mathrm{~d}, \mathrm{C}-\mathrm{F},{ }^{1} J_{\mathrm{C}-F}=244.0 \mathrm{~Hz}\right), 148.2,141.1\left(\mathrm{~d}, \mathrm{C}-\mathrm{F},{ }^{3} J_{C-F}=12.4\right.$ $\mathrm{Hz}), 137.1,129.4,128.5,126.9,125.3$ (d, C-F, $\left.{ }^{3} J_{C-F}=9.3 \mathrm{~Hz}\right), 122.5$ (d, C-F, $\left.{ }^{4} J_{C-F}=3.0 \mathrm{~Hz}\right), 110.4(\mathrm{~d}$, C-F, $\left.{ }^{2} J_{C-F}=22.4 \mathrm{~Hz}\right), 103.7\left(\mathrm{~d}, \mathrm{C}-\mathrm{F},{ }^{2} J_{C-F}=29.5 \mathrm{~Hz}\right), 84.7,47.0,41.3,37.7,36.0,31.0$.

IR (ATR, neat): 2912, 2853, 1768, 1729, 1604, 1489, 1351, 1251, 1145.

HRMS (ESI) calcd $(\mathrm{m} / z)$ for $\mathrm{C}_{26} \mathrm{H}_{26} \mathrm{FNO}_{3}:[\mathrm{M}+\mathrm{Na}]^{+} 442.1789$, found 442.1788 .

Adamantyl 5-chloro-3-benzyl-2-oxoindoline-1-carboxylate (1c) 


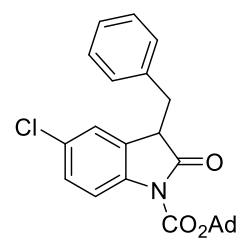

Prepared according to the GP2 to provide the title compound as white solid.

m.p. $94-96{ }^{\circ} \mathrm{C}$.

${ }^{1}$ H NMR $\left(400 \mathrm{MHz}, \mathrm{CDCl}_{3}\right) \delta 7.68(\mathrm{~d}, J=8.7 \mathrm{~Hz}, 1 \mathrm{H}), 7.31-7.23(\mathrm{~m}, 3 \mathrm{H}), 7.19(\mathrm{dd}, J=8.7,2.3 \mathrm{~Hz}, 1 \mathrm{H})$, 7.17-7.09 (m, 2H), 6.69 (d, $J=2.2 \mathrm{~Hz}, 1 \mathrm{H}), 3.78$ (dd, $J=9.0,4.6 \mathrm{~Hz}, 1 \mathrm{H}), 3.47$ (dd, $J=13.7,4.6 \mathrm{~Hz}$, 1H), 2.95 (dd, $J=13.8,9.0 \mathrm{~Hz}, 1 \mathrm{H}), 2.27$ (m, 9H), $1.71(\mathrm{~m}, 6 \mathrm{H})$.

${ }^{13} \mathbf{C}$ NMR $\left(100 \mathrm{MHz}, \mathrm{CDCl}_{3}\right) \delta 174.6,148.4,138.6,136.8,129.4,129.3,128.9,128.6,128.1,127.1,124.6$, $116.1,84.6,47.5,41.4,37.4,36.1,31.0$.

IR (ATR, neat): 2908, 2850, 1791, 1473, 1454, 1313, 1291, 1230, 1206, 1161.

HRMS (ESI) calcd $(m / z)$ for $\mathrm{C}_{26} \mathrm{H}_{26} \mathrm{ClNO}_{3}$ : $[\mathrm{M}+\mathrm{Na}]^{+}$458.1493, found 442.1493.

Adamantyl 6-chloro-3-benzyl-2-oxoindoline-1-carboxylate (1d)

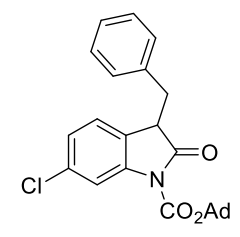

Prepared according to the GP2 to provide the title compound as white solid.

m.p. $100-102{ }^{\circ} \mathrm{C}$.

${ }^{1} \mathbf{H}$ NMR $\left(400 \mathrm{MHz}, \mathrm{CDCl}_{3}\right) \delta 7.81(\mathrm{~d}, J=1.9 \mathrm{~Hz}, 1 \mathrm{H}), 7.39-7.20(\mathrm{~m}, 4 \mathrm{H}), 7.21-7.12(\mathrm{~m}, 2 \mathrm{H}), 6.97(\mathrm{dd}$, $J=8.0,2.0 \mathrm{~Hz}, 1 \mathrm{H}), 6.60(\mathrm{~d}, J=8.0 \mathrm{~Hz}, 1 \mathrm{H}), 3.77(\mathrm{dd}, J=9.3,4.6 \mathrm{~Hz}, 1 \mathrm{H}), 3.49(\mathrm{dd}, J=13.7,4.6 \mathrm{~Hz}$, 1H), 2.91 (dd, $J=13.7,9.3 \mathrm{~Hz}, 1 \mathrm{H}), 2.40-2.25(\mathrm{~m}, 9 \mathrm{H}), 1.71(\mathrm{~m}, 6 \mathrm{H})$.

${ }^{13}$ C NMR $\left(100 \mathrm{MHz}, \mathrm{CDCl}_{3}\right) \delta 175.0,148.3,141.0,137.0,134.0,129.4,128.6,127.1,125.6,125.2,123.9$, $115.7,84.9,47.2,41.3,37.6,36.1,31.1$.

IR (ATR, neat): 2910, 2849, 1774, 1718, 1598, 1477, 1451, 1342, 1262, 1233, 1157.

HRMS (ESI) calcd $(m / z)$ for $\mathrm{C}_{26} \mathrm{H}_{26} \mathrm{ClNO}_{3}$ : $[\mathrm{M}+\mathrm{Na}]^{+}$458.1493, found 458.1492.

Adamantyl 6-bromo-3-benzyl-2-oxoindoline-1-carboxylate (1e)

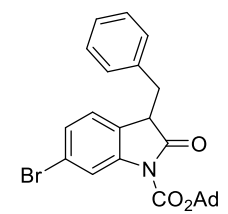

Prepared according to the GP2 to provide the title compound as white solid.

m.p. $114-115^{\circ} \mathrm{C}$. 
${ }^{1}$ H NMR $\left(400 \mathrm{MHz}, \mathrm{CDCl}_{3}\right) \delta 7.97(\mathrm{~d}, J=1.8 \mathrm{~Hz}, 1 \mathrm{H}), 7.32-7.19(\mathrm{~m}, 3 \mathrm{H}), 7.12(\mathrm{~m}, 3 \mathrm{H}), 6.54(\mathrm{~d}, J=8.0$ $\mathrm{Hz}, 1 \mathrm{H}), 3.74(\mathrm{dd}, J=9.2,4.5 \mathrm{~Hz}, 1 \mathrm{H}), 3.48(\mathrm{dd}, J=13.7,4.6 \mathrm{~Hz}, 1 \mathrm{H}), 2.90(\mathrm{dd}, J=13.7,9.3 \mathrm{~Hz}, 1 \mathrm{H})$, 2.36-2.20 (m, 9H), $1.71(\mathrm{~m}, 6 \mathrm{H})$.

${ }^{13} \mathrm{C} \mathrm{NMR}\left(100 \mathrm{MHz}, \mathrm{CDCl}_{3}\right) \delta 174.7,148.3,141.1,137.0,129.4,128.5,127.0,126.8,126.0,125.6,121.8$, $118.4,84.8,47.2,41.3,37.5,36.0,31.0$.

IR (ATR, neat): 2908, 2847, 1775, 1719, 1601, 1581, 1475, 1453, 1419, 1346, 1310, 1291, 1260, 1234, 1203, 1156, 1102.

HRMS (ESI) calcd $(m / z)$ for $\mathrm{C}_{26} \mathrm{H}_{26} \mathrm{BrNO}_{3}:[\mathrm{M}+\mathrm{Na}]^{+}$502.0988, found 502.0992.

Adamantyl 6-methoxy-3-benzyl-2-oxoindoline-1-carboxylate (1f)

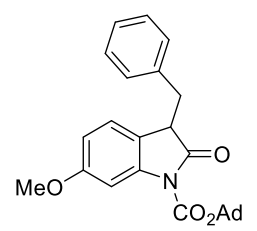

Prepared according to the GP2 to provide the title compound as white solid.

m.p. $130-131{ }^{\circ} \mathrm{C}$.

${ }^{1} \mathbf{H}$ NMR $\left(400 \mathrm{MHz}, \mathrm{CDCl}_{3}\right) \delta 7.39(\mathrm{~d}, J=2.3 \mathrm{~Hz}, 1 \mathrm{H}), 7.30-7.21(\mathrm{~m}, 3 \mathrm{H}), 7.19-7.11(\mathrm{~m}, 2 \mathrm{H}), 6.63-6.49$ $(\mathrm{m}, 2 \mathrm{H}), 3.78(\mathrm{~s}, 3 \mathrm{H}), 3.77-3.73(\mathrm{dd}, J=9.4,4.5 \mathrm{~Hz}, 1 \mathrm{H}), 3.47(\mathrm{dd}, J=13.6,4.5 \mathrm{~Hz}, 1 \mathrm{H}), 2.88(\mathrm{dd}, J=$ 13.6, 9.4 Hz, 1H), $2.27(\mathrm{~m}, 9 \mathrm{H}), 1.71(\mathrm{~m}, 6 \mathrm{H})$.

${ }^{13}$ C NMR $\left(100 \mathrm{MHz}, \mathrm{CDCl}_{3}\right) \delta 176.1,159.7,148.6,141.0,137.6,129.5,128.5,126.8,125.0,119.1,109.5$, 101.8, 84.3, 55.5, 47.1, 41.4, 37.9, 36.1, 31.1.

IR (ATR, neat): 2907, 2851, 1766, 1719, 1622, 1598, 1483, 1445, 1357, 1313, 1286, 1254, 1204, 1165, 1112.

HRMS (ESI) calcd $(m / z)$ for $\mathrm{C}_{27} \mathrm{H}_{29} \mathrm{NO}_{4}:[\mathrm{M}+\mathrm{Na}]^{+} 454.1989$, found 454.1994.

Adamantyl 6-phenyl-3-benzyl-2-oxoindoline-1-carboxylate (1g)

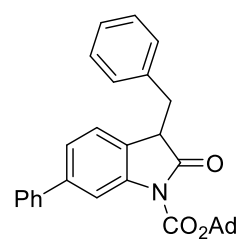

Prepared according to the GP1 and GP2 to provide the title compound as yellow oil.

${ }^{1}$ H NMR (400 MHz, $\left.\mathrm{CDCl}_{3}\right) \delta 8.05(\mathrm{~d}, J=1.5 \mathrm{~Hz}, 1 \mathrm{H}), 7.55(\mathrm{~d}, J=7.3 \mathrm{~Hz}, 2 \mathrm{H}), 7.40(\mathrm{t}, J=7.5 \mathrm{~Hz}, 2 \mathrm{H})$, 7.35-7.15 (m, 7H), $6.74(\mathrm{~d}, J=7.8 \mathrm{~Hz}, 1 \mathrm{H}), 3.83(\mathrm{dd}, J=9.3,4.5 \mathrm{~Hz}, 1 \mathrm{H}), 3.53(\mathrm{dd}, J=13.7,4.5 \mathrm{~Hz}$, 1H), 2.94 (dd, $J=13.7,9.4 \mathrm{~Hz}, 1 \mathrm{H}), 2.27$ (m, 9H), 1.76-1.68 (m, 6H). 
${ }^{13}$ C NMR $\left(101 \mathrm{MHz}, \mathrm{CDCl}_{3}\right) \delta 175.5,148.6,141.4,137.5,129.4,128.8,128.5,127.5,127.1,126.8,126.2$, 124.6, 122.7, 113.9, 84.3, 47.4, 41.4, 37.7, 36.1, 31.0.

IR (ATR, neat): 2910, 2852, 1764, 1723, 1616, 1480, 1454, 1422, 1351, 1315, 1289, 1257, 1220, 1159, 1107.

HRMS (ESI) calcd $(m / z)$ for $\mathrm{C}_{32} \mathrm{H}_{31} \mathrm{NO}_{3}:[\mathrm{M}+\mathrm{Na}]^{+}$500.2196, found 500.2193.

Adamantyl 3-benzyl-2-oxoindoline-1-carboxylate (1h)

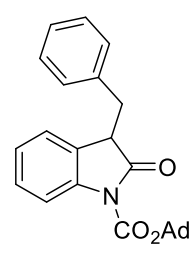

Prepared according to the GP2 to provide the title compound as colorless oil.

${ }^{1} \mathbf{H}$ NMR $\left(400 \mathrm{MHz}, \mathrm{CDCl}_{3}\right) \delta 7.73(\mathrm{~d}, J=8.1 \mathrm{~Hz}, 1 \mathrm{H}), 7.32-7.19(\mathrm{~m}, 4 \mathrm{H}), 7.19-7.11(\mathrm{~m}, 2 \mathrm{H}), 6.98(\mathrm{t}, J$ $=7.5 \mathrm{~Hz}, 1 \mathrm{H}), 6.72(\mathrm{~d}, J=7.5 \mathrm{~Hz}, 1 \mathrm{H}), 3.80(\mathrm{dd}, J=9.2,4.5 \mathrm{~Hz}, 1 \mathrm{H}), 3.49(\mathrm{dd}, J=13.7,4.5 \mathrm{~Hz}, 1 \mathrm{H})$, $2.93(\mathrm{dd}, J=13.7,9.2 \mathrm{~Hz}, 1 \mathrm{H}), 2.27(\mathrm{~m}, 9 \mathrm{H}), 1.71(\mathrm{~m}, 6 \mathrm{H})$.

${ }^{13}$ C NMR $\left(100 \mathrm{MHz} \mathrm{CDCl}_{3}\right) \delta 175.5,148.5,140.0,137.4,129.4,128.4,128.1,127.2,126.8,124.3,123.8$, $114.9,84.2,47.5,41.3,37.6,36.1,31.0$.

IR (ATR, neat): 2910, 2851, 1783, 1703, 1607, 1497, 1478, 1469, 1345, 1317, 1296, 1239, 1156.

HRMS (ESI) calcd $(m / z)$ for $\mathrm{C}_{26} \mathrm{H}_{27} \mathrm{NO}_{3}:[\mathrm{M}+\mathrm{Na}]^{+}$424.1883, found 424.1882.

Adamantyl 5-fluoro-3-(4-methylbenzyl)-2-oxoindoline-1-carboxylate (1i)

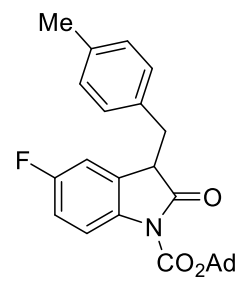

Prepared according to the GP2 to provide the title compound as white solid.

m.p. $155-157^{\circ} \mathrm{C}$.

${ }^{1}$ H NMR $\left(400 \mathrm{MHz}, \mathrm{CDCl}_{3}\right) \delta 7.72(\mathrm{dd}, J=9.0,4.6 \mathrm{~Hz}, 1 \mathrm{H}), 7.07(\mathrm{~d}, J=7.8 \mathrm{~Hz}, 2 \mathrm{H}), 7.02(\mathrm{~d}, J=8.0$ $\mathrm{Hz}, 2 \mathrm{H}), 6.91(\mathrm{~m}, 1 \mathrm{H}), 6.45(\mathrm{dd}, J=8.0,2.7 \mathrm{~Hz}, 1 \mathrm{H}), 3.75(\mathrm{dd}, J=9.3,4.5 \mathrm{~Hz}, 1 \mathrm{H}), 3.44(\mathrm{dd}, J=13.8$, 4.6 Hz, 1H), $2.88(\mathrm{dd}, J=13.8,9.2 \mathrm{~Hz}, 1 \mathrm{H}), 2.27(\mathrm{~m}, 12 \mathrm{H}), 1.71(\mathrm{~m}, 6 \mathrm{H})$.

${ }^{13}$ C NMR $\left(100 \mathrm{MHz}, \mathrm{CDCl}_{3}\right) \delta 174.9,159.4\left(\mathrm{~d}, \mathrm{C}-\mathrm{F},{ }^{1} J_{C-F}=242.9 \mathrm{~Hz}\right), 148.5,136.5,136.0\left(\mathrm{~d}, \mathrm{C}-\mathrm{F},{ }^{4} J_{C-}\right.$ $F=2.5 \mathrm{~Hz}), 133.8,129.2,129.2,129.1,116.1\left(\mathrm{~d}, \mathrm{C}-\mathrm{F},{ }^{3} J_{C-F}=8.0 \mathrm{~Hz}\right), 114.5\left(\mathrm{~d}, \mathrm{C}-\mathrm{F},{ }^{2} J_{C-F}=22.8 \mathrm{~Hz}\right)$, $111.9\left(\mathrm{~d}, \mathrm{C}-\mathrm{F},{ }^{2} J_{\mathrm{C}-F}=24.6 \mathrm{~Hz}\right), 84.4,47.7\left(\mathrm{~d}, \mathrm{C}-\mathrm{F},{ }^{4} J_{C-F}=1.9 \mathrm{~Hz}\right), 41.4,37.0,36.1,31.0,21.1$.

IR (ATR, neat): 2910, 2849, 1787, 1709, 1481, 1454, 1312, 1290, 1233, 1154. 
HRMS (ESI) calcd $(m / z)$ for $\mathrm{C}_{27} \mathrm{H}_{28} \mathrm{FNO}_{3}$ : $[\mathrm{M}+\mathrm{Na}]^{+}$456.1945, found 456.1946.

Adamantyl 5-fluoro-3-(3-methylbenzyl)-2-oxoindoline-1-carboxylate (1j)

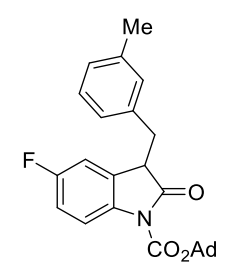

Prepared according to the GP2 to provide the title compound as white solid.

m.p. $143-145^{\circ} \mathrm{C}$.

${ }^{1} \mathbf{H}$ NMR $\left(400 \mathrm{MHz}, \mathrm{CDCl}_{3}\right) \delta 7.72(\mathrm{dd}, J=9.0,4.6 \mathrm{~Hz}, 1 \mathrm{H}), 7.16(\mathrm{~m}, 1 \mathrm{H}), 7.05(\mathrm{~m}, 1 \mathrm{H}), 7.01-6.87(\mathrm{~m}$, 3H), $6.41(\mathrm{dd}, J=8.1,2.8 \mathrm{~Hz}, 1 \mathrm{H}), 3.77(\mathrm{dd}, J=9.5,4.5 \mathrm{~Hz}, 1 \mathrm{H}), 3.46(\mathrm{dd}, J=13.7,4.6 \mathrm{~Hz}, 1 \mathrm{H}), 2.86$ $(\mathrm{dd}, J=13.7,9.4 \mathrm{~Hz}, 1 \mathrm{H}), 2.28(\mathrm{~m}, 12 \mathrm{H}), 1.71(\mathrm{~m}, 6 \mathrm{H})$.

${ }^{13} \mathrm{C}$ NMR $\left(100 \mathrm{MHz}, \mathrm{CDCl}_{3}\right) \delta 175.0,159.4\left(\mathrm{~d}, \mathrm{C}-\mathrm{F},{ }^{1} J_{C-F}=242.9 \mathrm{~Hz}\right), 148.5,138.2,136.9,136.0(\mathrm{~d}, \mathrm{C}-$ $\left.\mathrm{F},{ }^{4} J_{C-F}=2.5 \mathrm{~Hz}\right), 130.0,129.1\left(\mathrm{~d}, \mathrm{C}-\mathrm{F},{ }^{3} J_{C-F}=8.6 \mathrm{~Hz}\right), 128.4,127.8,126.3,116.1\left(\mathrm{~d}, \mathrm{C}-\mathrm{F},{ }^{3} J_{C-F}=7.9 \mathrm{~Hz}\right)$, $114.5\left(\mathrm{~d}, \mathrm{C}-\mathrm{F},{ }^{2} J_{C-F}=23.0 \mathrm{~Hz}\right), 112.0\left(\mathrm{~d}, \mathrm{C}-\mathrm{F},{ }^{2} J_{C-F}=24.6 \mathrm{~Hz}\right), 84.4,47.6\left(\mathrm{~d}, \mathrm{C}-\mathrm{F},{ }^{4} J_{C-F}=1.9 \mathrm{~Hz}\right), 41.3$, $37.4,36.1,31.0,21.4$.

IR (ATR, neat): 2912, 2862, 1764, 1716, 1606, 1479, 1455, 1342, 1286, 1260, 1230, 1133.

HRMS (ESI) calcd $(\mathrm{m} / \mathrm{z})$ for $\mathrm{C}_{27} \mathrm{H}_{28} \mathrm{FNO}_{3}$ : $[\mathrm{M}+\mathrm{Na}]^{+}$456.1945, found 456.1950.

Adamantyl 5-fluoro-3-(2-methylbenzyl)-2-oxoindoline-1-carboxylate (1k)

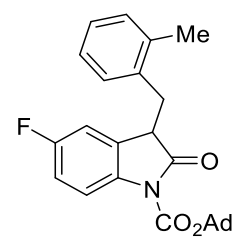

Prepared according to the GP2 to provide the title compound as white solid.

m.p. $140-141^{\circ} \mathrm{C}$.

${ }^{1}$ H NMR $\left(400 \mathrm{MHz}, \mathrm{CDCl}_{3}\right) \delta 7.80(\mathrm{dd}, J=9.0,4.6 \mathrm{~Hz}, 1 \mathrm{H}), 7.25-7.09$ (m, 4H), 6.94 (td, $J=9.0,2.8$ $\mathrm{Hz}, 1 \mathrm{H}), 6.22(\mathrm{dd}, J=8.1,2.8 \mathrm{~Hz}, 1 \mathrm{H}), 3.73(\mathrm{dd}, J=10.9,4.5 \mathrm{~Hz}, 1 \mathrm{H}), 3.54(\mathrm{dd}, J=14.0,4.5 \mathrm{~Hz}, 1 \mathrm{H})$, $2.80(\mathrm{dd}, J=14.0,10.9 \mathrm{~Hz}, 1 \mathrm{H}), 2.40-2.22(\mathrm{~m}, 12 \mathrm{H}), 1.72(\mathrm{~m}, 6 \mathrm{H})$.

${ }^{13} \mathrm{C}$ NMR $\left(100 \mathrm{MHz}, \mathrm{CDCl}_{3}\right) \delta 175.0,159.4\left(\mathrm{~d}, \mathrm{C}-\mathrm{F},{ }^{1} J_{C-F}=243.0 \mathrm{~Hz}\right), 148.6,136.6,135.9\left(\mathrm{~d}, \mathrm{C}-\mathrm{F},{ }^{4} J_{C-}\right.$ $F=2.5 \mathrm{~Hz}), 135.6,130.7,130.0,129.2\left(\mathrm{~d}, \mathrm{C}-\mathrm{F},{ }^{3} J_{C-F}=8.7 \mathrm{~Hz}\right), 127.3,126.1,116.1\left(\mathrm{~d}, \mathrm{C}-\mathrm{F},{ }^{3} J_{C-F}=7.8\right.$ $\mathrm{Hz}), 114.6\left(\mathrm{~d}, \mathrm{C}-\mathrm{F},{ }^{2} J_{C-F}=22.7 \mathrm{~Hz}\right), 112.1\left(\mathrm{~d}, \mathrm{C}-\mathrm{F},{ }^{2} J_{C-F}=24.8 \mathrm{~Hz}\right), 84.5,46.2\left(\mathrm{~d}, \mathrm{C}-\mathrm{F},{ }^{4} J_{C-F}=1.9 \mathrm{~Hz}\right)$, $41.4,36.0,35.2,31.0,19.5$.

IR (ATR, neat): 2915, 2863, 1765, 1715, 1605, 1478, 1344, 1311, 1286, 1259, 1237, 1145, 1106. 
HRMS (ESI) calcd $(m / z)$ for $\mathrm{C}_{27} \mathrm{H}_{28} \mathrm{FNO}_{3}:[\mathrm{M}+\mathrm{Na}]^{+}$456.1945, found 456.1947.

Adamantyl 5-fluoro-3-(4-methoxybenzyl)-2-oxoindoline-1-carboxylate (11)

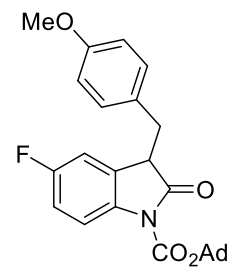

Prepared according to the GP2 to provide the title compound as white solid.

m.p. $136-138^{\circ} \mathrm{C}$.

${ }^{1} \mathbf{H}$ NMR $\left(400 \mathrm{MHz}, \mathrm{CDCl}_{3}\right) \delta 7.71(\mathrm{dd}, J=8.9,4.6 \mathrm{~Hz}, 1 \mathrm{H}), 7.04(\mathrm{~d}, J=8.6 \mathrm{~Hz}, 2 \mathrm{H}), 6.92(\mathrm{td}, J=9.0$, $2.8 \mathrm{~Hz}, 1 \mathrm{H}), 6.79(\mathrm{~d}, J=8.6 \mathrm{~Hz}, 2 \mathrm{H}), 6.48(\mathrm{dd}, J=7.9,2.2 \mathrm{~Hz}, 1 \mathrm{H}), 3.77$ (s, 3H), $3.74(\mathrm{dd}, J=8.9,4.4$ $\mathrm{Hz}, 1 \mathrm{H}), 3.41(\mathrm{dd}, J=13.8,4.6 \mathrm{~Hz}, 1 \mathrm{H}), 2.90(\mathrm{dd}, J=13.9,9.0 \mathrm{~Hz}, 1 \mathrm{H}), 2.26(\mathrm{~m}, 9 \mathrm{H}), 1.71(\mathrm{~m}, 6 \mathrm{H})$. ${ }^{13} \mathrm{C}$ NMR $\left(100 \mathrm{MHz}, \mathrm{CDCl}_{3}\right) \delta 175.0,159.1\left(\mathrm{~d}, \mathrm{C}-\mathrm{F},{ }^{1} J_{C-F}=242.9 \mathrm{~Hz}\right), 158.2,148.4,136.0\left(\mathrm{~d}, \mathrm{C}-\mathrm{F},{ }^{4} J_{C-}\right.$ $F=2.4 \mathrm{~Hz}), 130.3,129.1\left(\mathrm{~d}, \mathrm{C}-\mathrm{F},{ }^{3} J_{C-F}=8.5 \mathrm{~Hz}\right), 128.7,116.1\left(\mathrm{~d}, \mathrm{C}-\mathrm{F},{ }^{3} J_{C-F}=8.1 \mathrm{~Hz}\right), 114.5(\mathrm{~d}, \mathrm{C}-\mathrm{F}$, $\left.{ }^{2} J_{C-F}=22.7 \mathrm{~Hz}\right), 113.9,111.9\left(\mathrm{~d}, \mathrm{C}-\mathrm{F},{ }^{2} J_{C-F}=24.6 \mathrm{~Hz}\right), 84.4,55.2,47.8\left(\mathrm{~d}, \mathrm{C}-\mathrm{F},{ }^{4} J_{C-F}=2.0 \mathrm{~Hz}\right), 41.3$, $36.6,36.0,31.0$.

IR (ATR, neat): 2908, 2848, 1788, 1712, 1510, 1480, 1454, 1313, 1283, 1230, 1151.

HRMS (ESI) calcd $(\mathrm{m} / z)$ for $\mathrm{C}_{27} \mathrm{H}_{28} \mathrm{FNO}_{4}:[\mathrm{M}+\mathrm{Na}]^{+}$472.1895, found 472.1891.

Adamantyl 5-fluoro-3-(3,5-dimethylbenzyl)-2-oxoindoline-1-carboxylate (1m)

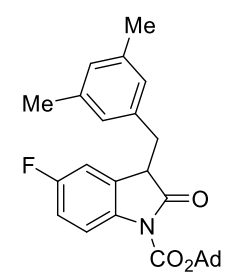

Prepared according to the GP2 to provide the title compound as white solid.

m.p. $149-151{ }^{\circ} \mathrm{C}$.

${ }^{1}$ H NMR $\left(400 \mathrm{MHz}, \mathrm{CDCl}_{3}\right) \delta 7.73(\mathrm{dd}, J=9.0,4.6 \mathrm{~Hz}, 1 \mathrm{H}), 6.98-6.89(\mathrm{~m}, 1 \mathrm{H}), 6.86(\mathrm{~m}, 1 \mathrm{H}), 6.77$ (s, 2H), $6.42(\mathrm{dd}, J=8.1,2.7 \mathrm{~Hz}, 1 \mathrm{H}), 3.75(\mathrm{dd}, J=9.5,4.5 \mathrm{~Hz}, 1 \mathrm{H}), 3.43(\mathrm{dd}, J=13.7,4.5 \mathrm{~Hz}, 1 \mathrm{H}), 2.79$ (dd, $J=13.7,9.5 \mathrm{~Hz}, 1 \mathrm{H}), 2.39-2.20(\mathrm{~m}, 15 \mathrm{H}), 1.71(\mathrm{~m}, 6 \mathrm{H})$.

${ }^{13} \mathrm{C}$ NMR $\left(100 \mathrm{MHz}, \mathrm{CDCl}_{3}\right) \delta 175.0,159.4\left(\mathrm{~d}, \mathrm{C}-\mathrm{F},{ }^{1} J_{\mathrm{C}-F}=242.8 \mathrm{~Hz}\right), 148.5,138.0,136.8,135.9$ (d, C$\left.\mathrm{F},{ }^{4} J_{C-F}=2.3 \mathrm{~Hz}\right), 129.2\left(\mathrm{~d}, \mathrm{C}-\mathrm{F},{ }^{3} J_{C-F}=8.6 \mathrm{~Hz}\right), 128.6,127.1,116.0\left(\mathrm{~d}, \mathrm{C}-\mathrm{F},{ }^{3} J_{C-F}=7.9 \mathrm{~Hz}\right), 114.5(\mathrm{~d}, \mathrm{C}-$ $\left.\mathrm{F},{ }^{2} J_{C-F}=22.9 \mathrm{~Hz}\right), 112.0\left(\mathrm{~d}, \mathrm{C}-\mathrm{F},{ }^{2} J_{C-F}=24.7 \mathrm{~Hz}\right), 84.3,47.6\left(\mathrm{~d}, \mathrm{C}-\mathrm{F},{ }^{4} J_{C-F}=1.8 \mathrm{~Hz}\right), 41.3,37.4,36.0$, 31.0, 21.2.

IR (ATR, neat): 2910, 2857, 1764, 1719, 1604, 1479, 1451, 1344, 1289, 1254, 1233, 1142. 
HRMS (ESI) calcd $(m / z)$ for $\mathrm{C}_{28} \mathrm{H}_{30} \mathrm{FNO}_{3}:[\mathrm{M}+\mathrm{Na}]^{+}$470.2102, found 470.2104.

Adamantyl 5-fluoro-3-(3,5-dimethoxybenzyl)-2-oxoindoline-1-carboxylate (1n)

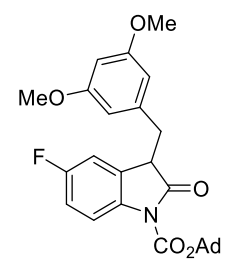

Prepared according to the GP2 to provide the title compound as white solid.

m.p. $109-111^{\circ} \mathrm{C}$.

${ }^{1}$ H NMR $\left(400 \mathrm{MHz}, \mathrm{CDCl}_{3}\right) \delta 7.73(\mathrm{dd}, J=8.9,4.6 \mathrm{~Hz}, 1 \mathrm{H}), 6.93(\mathrm{td}, J=9.0,2.8 \mathrm{~Hz}, 1 \mathrm{H}), 6.55(\mathrm{dd}, J$ $=8.0,2.8 \mathrm{~Hz}, 1 \mathrm{H}), 6.33(\mathrm{t}, J=2.3 \mathrm{~Hz}, 1 \mathrm{H}), 6.30(\mathrm{~d}, J=2.3 \mathrm{~Hz}, 2 \mathrm{H}), 3.78(\mathrm{dd}, J=9.2,4.5 \mathrm{~Hz}, 1 \mathrm{H}), 3.73$ (s, 6H), $3.42(\mathrm{dd}, J=13.7,4.4 \mathrm{~Hz}, 1 \mathrm{H}), 2.87(\mathrm{dd}, J=13.7,9.2 \mathrm{~Hz}, 1 \mathrm{H}), 2.26(\mathrm{~m}, 9 \mathrm{H}), 1.71(\mathrm{~m}, 6 \mathrm{H})$.

${ }^{13}$ C NMR $\left(100 \mathrm{MHz}, \mathrm{CDCl}_{3}\right) \delta 174.9,160.8,159.4\left(\mathrm{~d}, \mathrm{C}-\mathrm{F},{ }^{1} J_{C-F}=243.0 \mathrm{~Hz}\right), 148.5,139.2,136.0(\mathrm{~d}, \mathrm{C}-$ $\left.\mathrm{F},{ }^{4} J_{C-F}=2.5 \mathrm{~Hz}\right), 129.0\left(\mathrm{~d}, \mathrm{C}-\mathrm{F},{ }^{3} J_{C-F}=8.6 \mathrm{~Hz}\right), 116.1\left(\mathrm{~d}, \mathrm{C}-\mathrm{F},{ }^{3} J_{C-F}=7.7 \mathrm{~Hz}\right), 114.6\left(\mathrm{~d}, \mathrm{C}-\mathrm{F},{ }^{2} J_{C-F}=22.8\right.$ $\mathrm{Hz}), 112.0\left(\mathrm{~d}, \mathrm{C}-\mathrm{F},{ }^{2} J_{C-F}=24.6 \mathrm{~Hz}\right), 107.2,99.2,84.4,55.3,47.4\left(\mathrm{~d}, \mathrm{C}-\mathrm{F},{ }^{4} J_{C-F}=1.9 \mathrm{~Hz}\right), 41.3,37.7,36.0$, 31.0 .

IR (ATR, neat): 2912, 2849, 1779, 1595, 1471, 1313, 1283, 1227, 1203, 1148.

HRMS (ESI) calcd $(\mathrm{m} / z)$ for $\mathrm{C}_{28} \mathrm{H}_{30} \mathrm{FNO}_{5}:[\mathrm{M}+\mathrm{Na}]^{+}$502.2000, found 502.2001.

Adamantyl 5-fluoro-3-([1,1'-biphenyl]-4-ylmethyl)-2-oxoindoline-1-carboxylate (10)

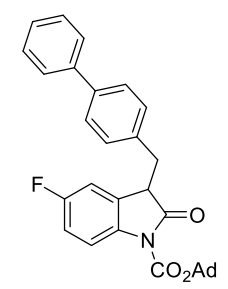

Prepared according to the GP2 to provide the title compound as white solid.

m.p. $123-124{ }^{\circ} \mathrm{C}$.

${ }^{1} \mathbf{H}$ NMR $\left(400 \mathrm{MHz}, \mathrm{CDCl}_{3}\right) \delta 7.72(\mathrm{dd}, J=9.0,4.6 \mathrm{~Hz}, 1 \mathrm{H}), 7.62-7.54(\mathrm{~m}, 2 \mathrm{H}), 7.50(\mathrm{~d}, J=8.0 \mathrm{~Hz}, 2 \mathrm{H})$, $7.42(\mathrm{t}, J=7.5 \mathrm{~Hz}, 2 \mathrm{H}), 7.32(\mathrm{t}, J=7.4 \mathrm{~Hz}, 1 \mathrm{H}), 7.22(\mathrm{t}, J=8.3 \mathrm{~Hz}, 2 \mathrm{H}), 6.93(\mathrm{td}, J=9.0,2.8 \mathrm{~Hz}, 1 \mathrm{H})$, $6.54(\mathrm{dd}, J=8.0,2.7 \mathrm{~Hz}, 1 \mathrm{H}), 3.81(\mathrm{dd}, J=9.0,4.5 \mathrm{~Hz}, 1 \mathrm{H}), 3.51(\mathrm{dd}, J=13.8,4.5 \mathrm{~Hz}, 1 \mathrm{H}), 2.99(\mathrm{dd}, J$ $=13.8,9.1 \mathrm{~Hz}, 1 \mathrm{H}), 2.25(\mathrm{~m}, 9 \mathrm{H}), 1.69(\mathrm{~m}, 6 \mathrm{H})$.

${ }^{13} \mathrm{C}$ NMR $\left(100 \mathrm{MHz}, \mathrm{CDCl}_{3}\right) \delta 175.0,159.5\left(\mathrm{~d}, \mathrm{C}-\mathrm{F},{ }^{1} J_{\mathrm{C}-\mathrm{F}}=243.1 \mathrm{~Hz}\right), 148.4,140.5,139.8,136.0(\mathrm{~d}, \mathrm{C}-$ $\left.\mathrm{F},{ }^{4} J_{C-F}=2.5 \mathrm{~Hz}\right), 135.9,129.8,129.0\left(\mathrm{~d}, \mathrm{C}-\mathrm{F},{ }^{3} J_{C-F}=8.5 \mathrm{~Hz}\right), 128.8,127.3,127.2,127.0,116.3(\mathrm{~d}, \mathrm{C}-\mathrm{F}$, $\left.{ }^{3} J_{C-F}=8.0 \mathrm{~Hz}\right), 114.7\left(\mathrm{~d}, \mathrm{C}-\mathrm{F},{ }^{2} J_{C-F}=22.7 \mathrm{~Hz}\right), 111.9\left(\mathrm{~d}, \mathrm{C}-\mathrm{F},{ }^{2} J_{C-F}=24.4 \mathrm{~Hz}\right), 84.5,47.6\left(\mathrm{~d}, \mathrm{C}-\mathrm{F},{ }^{4} J_{C-F}=\right.$ $1.8 \mathrm{~Hz}), 41.3,37.1,36.0,31.0$. 
IR (ATR, neat): 2910, 2850, 1782, 1709, 1606, 1482, 1456, 1347, 1313, 1291, 1236, 1155, 1141, 1101.

HRMS (ESI) calcd $(\mathrm{m} / \mathrm{z})$ for $\mathrm{C}_{32} \mathrm{H}_{30} \mathrm{FNO}_{3}:[\mathrm{M}+\mathrm{Na}]^{+}$518.2102, found 518.2101.

Adamantyl 5-fluoro-3-(thiophen-2-ylmethyl)-2-oxoindoline-1-carboxylate (1p)

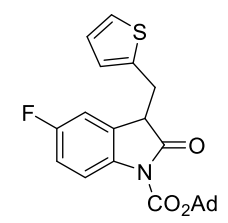

Prepared according to the GP3 to provide the title compound as yellow solid.

m.p. $143-144{ }^{\circ} \mathrm{C}$.

${ }^{1}$ H NMR $\left(400 \mathrm{MHz}, \mathrm{CDCl}_{3}\right) \delta 7.73(\mathrm{dd}, J=9.0,4.6 \mathrm{~Hz}, 1 \mathrm{H}), 7.13(\mathrm{~d}, J=5.0 \mathrm{~Hz}, 1 \mathrm{H}), 6.95(\mathrm{td}, J=9.0$, $2.8 \mathrm{~Hz}, 1 \mathrm{H}), 6.89(\mathrm{dd}, J=5.1,3.5 \mathrm{~Hz}, 1 \mathrm{H}), 6.78(\mathrm{~d}, J=3.4 \mathrm{~Hz}, 1 \mathrm{H}), 6.62(\mathrm{dd}, J=8.1,2.7 \mathrm{~Hz}, 1 \mathrm{H}), 3.80$ (dd, $J=8.4,4.4 \mathrm{~Hz}, 1 \mathrm{H}), 3.60(\mathrm{dd}, J=14.8,4.4 \mathrm{~Hz}, 1 \mathrm{H}), 3.31$ (dd, $J=14.8,8.3 \mathrm{~Hz}, 1 \mathrm{H}), 2.36-2.20$ (m, 9H), $1.71(\mathrm{~m}, 6 \mathrm{H})$.

${ }^{13}$ C NMR $\left(100 \mathrm{MHz}, \mathrm{CDCl}_{3}\right) \delta 174.3,159.6\left(\mathrm{~d}, \mathrm{C}-\mathrm{F},{ }^{1} J_{\mathrm{C}-\mathrm{F}}=243.4 \mathrm{~Hz}\right), 148.4,138.8,136.2\left(\mathrm{~d}, \mathrm{C}-\mathrm{F},{ }^{4} J_{C-}\right.$ $\left.F_{F}=2.3 \mathrm{~Hz}\right), 128.5\left(\mathrm{~d}, \mathrm{C}-\mathrm{F},{ }^{3} J_{\mathrm{C}-F}=8.6 \mathrm{~Hz}\right), 126.9,126.8,124.7,116.2\left(\mathrm{~d}, \mathrm{C}-\mathrm{F},{ }^{3} J_{C-F}=7.9 \mathrm{~Hz}\right), 114.8(\mathrm{~d}$, $\left.\mathrm{C}-\mathrm{F},{ }^{2} J_{C-F}=22.8 \mathrm{~Hz}\right), 111.8\left(\mathrm{~d}, \mathrm{C}-\mathrm{F},{ }^{2} J_{C-F}=24.6 \mathrm{~Hz}\right), 84.5,47.8\left(\mathrm{~d}, \mathrm{C}-\mathrm{F},{ }^{4} J_{C-F}=1.8 \mathrm{~Hz}\right), 41.4,36.1,31.4$, 31.0 .

IR (ATR, neat): 2910, 2850, 1779, 1707, 1604, 1478, 1435, 1313, 1287, 1237, 1205, 1184, 1154, 1101.

HRMS (ESI) calcd $(m / z)$ for $\mathrm{C}_{24} \mathrm{H}_{24} \mathrm{FNO}_{3} \mathrm{~S}:[\mathrm{M}+\mathrm{Na}]^{+} 448.1353$, found 448.1353 .

Adamantyl 5-fluoro-3-(benzo[b]thiophen-2-ylmethyl)-2-oxoindoline-1-carboxylate (1q)

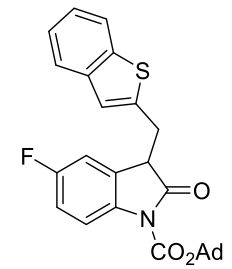

Prepared according to the GP3 to provide the title compound as yellow solid.

m.p. $143-145^{\circ} \mathrm{C}$.

${ }^{1} \mathbf{H}$ NMR $\left(400 \mathrm{MHz}, \mathrm{CDCl}_{3}\right) \delta 7.77(\mathrm{dd}, J=9.0,4.6 \mathrm{~Hz}, 1 \mathrm{H}), 7.58-7.44(\mathrm{~m}, 1 \mathrm{H}), 7.41(\mathrm{~d}, J=7.9 \mathrm{~Hz}, 1 \mathrm{H})$, 7.33-7.14 (m, 2H), $6.94(\mathrm{td}, J=9.0,2.8 \mathrm{~Hz}, 1 \mathrm{H}), 6.64(\mathrm{dd}, J=7.9,2.7 \mathrm{~Hz}, 1 \mathrm{H}), 6.45(\mathrm{~s}, 1 \mathrm{H}), 3.98$ (dd, $J$ $=9.1,4.5 \mathrm{~Hz}, 1 \mathrm{H}), 3.60(\mathrm{dd}, J=15.2,4.5 \mathrm{~Hz}, 1 \mathrm{H}), 3.14(\mathrm{dd}, J=15.2,9.1 \mathrm{~Hz}, 1 \mathrm{H}), 2.27(\mathrm{~m}, 9 \mathrm{H}), 1.71$ $(\mathrm{m}, 6 \mathrm{H})$.

${ }^{13}$ C NMR $\left(100 \mathrm{MHz}, \mathrm{CDCl}_{3}\right) \delta 174.3,159.6\left(\mathrm{~d}, \mathrm{C}-\mathrm{F},{ }^{1} J_{\mathrm{C}-F}=243.3 \mathrm{~Hz}\right), 154.8,154.0,148.5,136.0(\mathrm{~d}, \mathrm{C}-$ $\left.\mathrm{F},{ }^{4} J_{C-F}=2.5 \mathrm{~Hz}\right), 128.6\left(\mathrm{~d}, \mathrm{C}-\mathrm{F},{ }^{3} J_{C-F}=8.5 \mathrm{~Hz}\right), 128.4,124.0,122.8,120.7,116.3\left(\mathrm{~d}, \mathrm{C}-\mathrm{F},{ }^{3} J_{C-F}=8.0 \mathrm{~Hz}\right)$, $114.9\left(\mathrm{~d}, \mathrm{C}-\mathrm{F},{ }^{2} J_{C-F}=22.9 \mathrm{~Hz}\right), 111.8\left(\mathrm{~d}, \mathrm{C}-\mathrm{F},{ }^{2} J_{C-F}=24.6 \mathrm{~Hz}\right), 111.0,104.8,84.7,44.8\left(\mathrm{~d}, \mathrm{C}-\mathrm{F},{ }^{4} J_{C-F}=\right.$ 
$1.9 \mathrm{~Hz}), 41.4,36.1,31.1,30.2$.

IR (ATR, neat): 2904, 2846, 1783, 1709, 1605, 1481, 1456, 1357, 1311, 1288, 1231, 1199, 1156, 1141.

HRMS (ESI) calcd $(m / z)$ for $\mathrm{C}_{28} \mathrm{H}_{26} \mathrm{FNO}_{3} \mathrm{~S}:[\mathrm{M}+\mathrm{Na}]^{+} 498.1510$, found 498.1514 .

Adamantyl 6-chloro-3-(4-(benzyloxy)benzyl)-2-oxoindoline-1-carboxylate (1r)

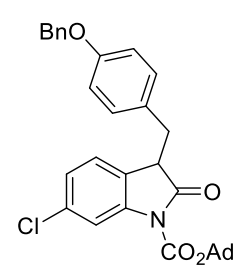

Prepared according to the GP2 to provide the title compound as white solid.

m.p. $131-132{ }^{\circ} \mathrm{C}$.

${ }^{1} \mathbf{H}$ NMR $\left(400 \mathrm{MHz}, \mathrm{CDCl}_{3}\right) \delta 7.80(\mathrm{~d}, J=2.0 \mathrm{~Hz}, 1 \mathrm{H}), 7.52-7.27(\mathrm{~m}, 5 \mathrm{H}), 7.01(\mathrm{~d}, J=8.6 \mathrm{~Hz}, 2 \mathrm{H}), 6.97$ $(\mathrm{dd}, J=8.0,1.9 \mathrm{~Hz}, 1 \mathrm{H}), 6.85(\mathrm{~d}, J=8.6 \mathrm{~Hz}, 2 \mathrm{H}), 6.64(\mathrm{~d}, J=8.0 \mathrm{~Hz}, 1 \mathrm{H}), 5.01(\mathrm{~s}, 2 \mathrm{H}), 3.70(\mathrm{dd}, J=$ 9.2, 4.4 Hz, 1H), 3.39 (dd, $J=13.8,4.6 \mathrm{~Hz}, 1 \mathrm{H}), 2.87(\mathrm{dd}, J=13.8,8.9 \mathrm{~Hz}, 1 \mathrm{H}), 2.31-2.22(\mathrm{~m}, 9 \mathrm{H}), 1.70$ $(\mathrm{m}, 6 \mathrm{H})$.

${ }^{13} \mathbf{C ~ N M R}\left(100 \mathrm{MHz}, \mathrm{CDCl}_{3}\right) \delta 175.0,157.8,148.3,141.0,137.0,133.8,130.4,129.2,128.5,128.0,127.5$, $125.6,125.2,123.9,115.7,114.9,84.8,70.0,47.3,41.3,36.7,36.0,31.0$.

IR (ATR, neat): 2921, 2855, 1758, 1731, 1608, 1585, 1508, 1484, 1454, 1426, 1350, 1313, 1296, 1264, $1234,1157,1104$.

HRMS (ESI) calcd $(m / z)$ for $\mathrm{C}_{33} \mathrm{H}_{32} \mathrm{ClNO}_{4}:[\mathrm{M}+\mathrm{Na}]^{+}$564.1912, found 564.1917.

Adamantyl 6-methoxy-3-(4-chlorobenzyl)-2-oxoindoline-1-carboxylate (1s)

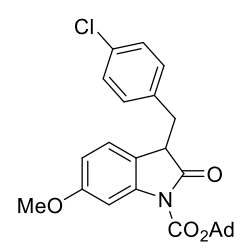

Prepared according to the GP2 to provide the title compound as white solid.

m.p. $143-145^{\circ} \mathrm{C}$.

${ }^{1} \mathbf{H}$ NMR $\left(400 \mathrm{MHz}, \mathrm{CDCl}_{3}\right) \delta 7.36(\mathrm{~d}, J=2.3 \mathrm{~Hz}, 1 \mathrm{H}), 7.20(\mathrm{~d}, J=8.2 \mathrm{~Hz}, 2 \mathrm{H}), 7.04(\mathrm{~d}, J=8.2 \mathrm{~Hz}, 2 \mathrm{H})$, $6.68(\mathrm{~d}, J=8.3 \mathrm{~Hz}, 1 \mathrm{H}), 6.56(\mathrm{dd}, J=8.3,2.4 \mathrm{~Hz}, 1 \mathrm{H}), 3.78(\mathrm{~s}, 3 \mathrm{H}), 3.73(\mathrm{dd}, J=8.6,4.6 \mathrm{~Hz}, 1 \mathrm{H}), 3.37$ $(\mathrm{dd}, J=13.7,4.6 \mathrm{~Hz}, 1 \mathrm{H}), 2.95(\mathrm{dd}, J=13.7,8.6 \mathrm{~Hz}, 1 \mathrm{H}), 2.34-2.21(\mathrm{~m}, 9 \mathrm{H}), 1.71(\mathrm{~m}, 6 \mathrm{H})$.

${ }^{13}$ C NMR $\left(100 \mathrm{MHz}, \mathrm{CDCl}_{3}\right) \delta 175.8,159.8,148.4,141.1,135.8,132.6,130.8,128.5,124.7,118.5,109.5$, $101.9,84.4,55.5,46.9,41.4,37.0,36.0,31.0$.

IR (ATR, neat): 2910, 2851, 1771, 1716, 1622, 1596, 1489, 1459, 1449, 1362, 1352, 1313, 1291, 1249, 
$1200,1162,1111$.

HRMS (ESI) calcd $(m / z)$ for $\mathrm{C}_{27} \mathrm{H}_{28} \mathrm{ClNO}_{4}:[\mathrm{M}+\mathrm{Na}]^{+} 488.1599$, found 488.1597.

Adamantyl 6-methoxy-3-(4-(trifluoromethyl)benzyl)-2-oxoindoline-1-carboxylate (1t)

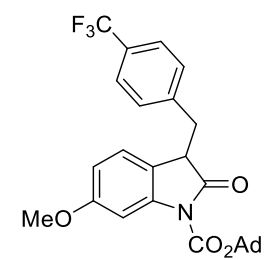

Prepared according to the GP2 to provide the title compound as white solid.

m.p. $141-143{ }^{\circ} \mathrm{C}$.

${ }^{1} \mathrm{H}$ NMR $\left(400 \mathrm{MHz}, \mathrm{CDCl}_{3}\right) \delta 7.39(\mathrm{~d}, J=2.4 \mathrm{~Hz}, 1 \mathrm{H}), 7.21(\mathrm{td}, J=8.0,6.0 \mathrm{~Hz}, 1 \mathrm{H}), 7.00-6.82(\mathrm{~m}, 3 \mathrm{H})$, $6.65(\mathrm{~d}, J=8.3 \mathrm{~Hz}, 1 \mathrm{H}), 6.55(\mathrm{dd}, J=8.3,2.4 \mathrm{~Hz}, 1 \mathrm{H}), 3.78(\mathrm{~s}, 3 \mathrm{H}), 3.74(\mathrm{dd}, J=8.8,4.5 \mathrm{~Hz}, 1 \mathrm{H}), 3.42$ $(\mathrm{dd}, J=13.7,4.6 \mathrm{~Hz}, 1 \mathrm{H}), 2.92(\mathrm{dd}, J=13.7,8.9 \mathrm{~Hz}, 1 \mathrm{H}), 2.27(\mathrm{~m}, 9 \mathrm{H}), 1.72(\mathrm{~m}, 6 \mathrm{H})$.

${ }^{13} \mathrm{C} \mathrm{NMR}\left(100 \mathrm{MHz}, \mathrm{CDCl}_{3}\right) \delta 175.6,159.9,148.3,141.5,141.1,129.8,129.1\left(\mathrm{q}, \mathrm{C}-\mathrm{F},{ }^{2} J_{C-F}=32.3 \mathrm{~Hz}\right)$, $125.3\left(\mathrm{q}, \mathrm{C}-\mathrm{F},{ }^{4} J_{C-F}=3.7 \mathrm{~Hz}\right), 124.6,124.2\left(\mathrm{q}, \mathrm{C}-\mathrm{F},{ }^{1} J_{C-F}=272.0 \mathrm{~Hz}\right), 118.4,109.6,102.0,84.5,55.5$, 46.7, 41.4, 37.4, 36.0, 31.0.

IR (ATR, neat): 2916, 2856, 1764, 1724, 1625, 1600, 1489, 1454, 1364, 1314, 1294, 1251, 1204, 1161, $1125,1107$.

HRMS (ESI) calcd $(m / z)$ for $\mathrm{C}_{28} \mathrm{H}_{28} \mathrm{~F}_{3} \mathrm{NO}_{4}:[\mathrm{M}+\mathrm{Na}]^{+}$522.1863, found 522.1860.

Adamantyl 6-phenyl-3-(4-bromobenzyl)-2-oxoindoline-1-carboxylate (1u)

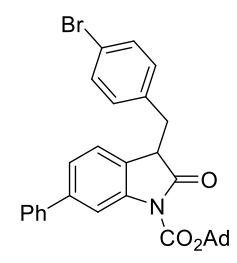

Prepared according to the GP1 and GP2 to provide the title compound as yellow oil.

${ }^{1} \mathbf{H}$ NMR $\left(400 \mathrm{MHz}, \mathrm{CDCl}_{3}\right) \delta 8.03(\mathrm{~d}, J=1.6 \mathrm{~Hz}, 1 \mathrm{H}), 7.56(\mathrm{~d}, J=7.6 \mathrm{~Hz}, 2 \mathrm{H}), 7.47-7.32(\mathrm{~m}, 5 \mathrm{H}), 7.26$ $(\mathrm{dd}, J=7.8,1.6 \mathrm{~Hz}, 1 \mathrm{H}), 7.04(\mathrm{~d}, J=8.2 \mathrm{~Hz}, 2 \mathrm{H}), 6.86(\mathrm{~d}, J=7.8 \mathrm{~Hz}, 1 \mathrm{H}), 3.82(\mathrm{dd}, J=8.7,4.6 \mathrm{~Hz}, 1 \mathrm{H})$, $3.43(\mathrm{dd}, J=13.8,4.7 \mathrm{~Hz}, 1 \mathrm{H}), 2.99$ (dd, $J=13.8,8.7 \mathrm{~Hz}, 1 \mathrm{H}), 2.37-2.22(\mathrm{~m}, 9 \mathrm{H}), 1.71$ (m, 6H).

${ }^{13}$ C NMR $\left(100 \mathrm{MHz}, \mathrm{CDCl}_{3}\right) \delta 175.3,148.5,141.7,140.9,140.7,136.3,131.6,131.3,128.9,127.6,127.2$, $125.8,124.4,122.9,120.9,114.1,84.6,47.2,41.4,37.0,36.1,31.1$.

IR (ATR, neat): 2910, 2851, 1762, 1724, 1616, 1480, 1454, 1422, 1350, 1315, 1256, 1220, 1161, 1103.

HRMS (ESI) calcd $(m / z)$ for $\mathrm{C}_{32} \mathrm{H}_{30} \mathrm{BrNO}_{3}:[\mathrm{M}+\mathrm{Na}]^{+}$578.1301, found 578.1305.

Adamantyl 6-phenyl-3-(4-(tert-butoxycarbonyl)benzyl)-2-oxoindoline-1-carboxylate (1v) 


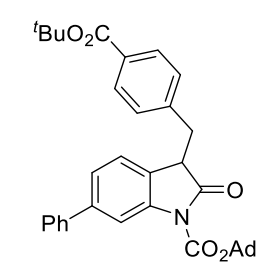

Prepared according to the GP1 and GP2 to provide the title compound as white solid.

m.p. $142-144^{\circ} \mathrm{C}$.

${ }^{1} \mathbf{H}$ NMR $\left(400 \mathrm{MHz}, \mathrm{CDCl}_{3}\right) \delta 8.04(\mathrm{~d}, J=1.6 \mathrm{~Hz}, 1 \mathrm{H}), 7.90(\mathrm{~d}, J=8.2 \mathrm{~Hz}, 2 \mathrm{H}), 7.55(\mathrm{~m}, 2 \mathrm{H}), 7.41(\mathrm{t}, J$ $=7.6 \mathrm{~Hz}, 2 \mathrm{H}), 7.36-7.30(\mathrm{~m}, 1 \mathrm{H}), 7.28-7.20(\mathrm{~m}, 3 \mathrm{H}), 6.80(\mathrm{~d}, J=7.8 \mathrm{~Hz}, 1 \mathrm{H}), 3.86(\mathrm{dd}, J=8.9,4.6 \mathrm{~Hz}$, 1H), $3.53(\mathrm{dd}, J=13.7,4.6 \mathrm{~Hz}, 1 \mathrm{H}), 3.05(\mathrm{dd}, J=13.7,8.9 \mathrm{~Hz}, 1 \mathrm{H}), 2.27(\mathrm{~m}, 9 \mathrm{H}), 1.79-1.66(\mathrm{~m}, 6 \mathrm{H})$, $1.59(\mathrm{~s}, 9 \mathrm{H})$.

${ }^{13} \mathbf{C}$ NMR $\left(100 \mathrm{MHz}, \mathrm{CDCl}_{3}\right) \delta 175.2,165.6,148.5,142.2,141.6,140.8,140.6,130.7,129.6,129.3,128.8$, $127.5,127.1,125.7,124.4,122.9,114.0,84.4,80.9,47.1,41.4,37.4,36.0,31.0,28.2$.

IR (ATR, neat): 2977, 2915, 2853, 1758, 1725, 1702, 1611, 1482, 1455, 1423, 1361, 1314, 1290, 1256, $1222,1166,1114$.

HRMS (ESI) calcd $(\mathrm{m} / z)$ for $\mathrm{C}_{37} \mathrm{H}_{39} \mathrm{NO}_{5}:[\mathrm{M}+\mathrm{Na}]^{+} 600.2720$, found 600.2717.

Adamantyl 3-(3-bromo-4-fluorobenzyl)-6-(naphthalen-2-yl)-2-oxoindoline-1-carboxylate (1w)

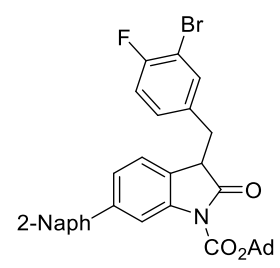

Prepared according to the GP1 and GP2 to provide the title compound as yellow oil.

${ }^{1}$ H NMR $\left(400 \mathrm{MHz}, \mathrm{CDCl}_{3}\right) \delta 8.16(\mathrm{~d}, J=1.6 \mathrm{~Hz}, 1 \mathrm{H}), 8.00(\mathrm{~d}, J=1.8 \mathrm{~Hz}, 1 \mathrm{H}), 7.91-7.82(\mathrm{~m}, 3 \mathrm{H}), 7.70$ $(\mathrm{dd}, J=8.5,1.9 \mathrm{~Hz}, 1 \mathrm{H}), 7.52-7.46(\mathrm{~m}, 2 \mathrm{H}), 7.40(\mathrm{dd}, J=7.8,1.7 \mathrm{~Hz}, 1 \mathrm{H}), 7.35(\mathrm{dd}, J=6.5,2.1 \mathrm{~Hz}, 1 \mathrm{H})$, $7.03(\mathrm{~m} \mathrm{1H}), 6.98$ (t, $J=8.4 \mathrm{~Hz}, 1 \mathrm{H}), 6.91$ (d, $J=7.8 \mathrm{~Hz}, 1 \mathrm{H}), 3.78(\mathrm{dd}, J=8.2,4.8 \mathrm{~Hz}, 1 \mathrm{H}), 3.38$ (dd, $J$ $=13.9,4.8 \mathrm{~Hz}, 1 \mathrm{H}), 3.01(\mathrm{dd}, J=13.9,8.2 \mathrm{~Hz}, 1 \mathrm{H}), 2.28(\mathrm{~m}, 9 \mathrm{H}), 1.75-1.66(\mathrm{~m}, 6 \mathrm{H})$.

${ }^{13} \mathrm{C}$ NMR $\left(100 \mathrm{MHz}, \mathrm{CDCl}_{3}\right) \delta 175.1,158.2\left(\mathrm{~d}, \mathrm{C}-\mathrm{F},{ }^{1} J_{C-F}=246.7 \mathrm{~Hz}\right), 148.5,141.7,140.8,138.1,134.6$ (d, C-F, $\left.{ }^{4} J_{C-F}=3.8 \mathrm{~Hz}\right), 134.3,133.6,132.7,130.0$ (d, C-F, $\left.{ }^{3} J_{C-F}=7.0 \mathrm{~Hz}\right), 128.5,128.2,127.7,126.4$, $126.1,126.0,125.6,125.4,124.4,123.2,116.4\left(\mathrm{~d}, \mathrm{C}-\mathrm{F},{ }^{2} J_{C-F}=22.1 \mathrm{~Hz}\right), 114.3,108.9\left(\mathrm{~d}, \mathrm{C}-\mathrm{F},{ }^{2} J_{C-F}=20.9\right.$ Hz), 84.6, 47.2, 41.4, 36.4, 36.1, 31.1.

IR (ATR, neat): 2915, 2852, 1761, 1725, 1706, 1611, 1480, 1456, 1423, 1314, 1289, 1256, 1227, 1158, 1108 .

HRMS (ESI) calcd $(m / z)$ for $\mathrm{C}_{36} \mathrm{H}_{31} \mathrm{BrFNO}_{3}:[\mathrm{M}+\mathrm{Na}]^{+}$646.1364, found 646.1362 . 
tert-butyl 2-((1-(tert-butoxycarbonyl)-5-fluoro-2-oxoindolin-3-yl)methyl)-1H-indole-1-carboxylate (1x)

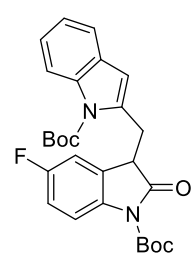

Prepared according to the GP4 to provide the title compound as white solid.

m.p. $140-142{ }^{\circ} \mathrm{C}$.

${ }^{1} \mathbf{H}$ NMR $\left(400 \mathrm{MHz}, \mathrm{CDCl}_{3}\right) \delta 8.09(\mathrm{~d}, J=8.3 \mathrm{~Hz}, 1 \mathrm{H}), 7.81(\mathrm{dd}, J=9.0,4.6 \mathrm{~Hz}, 1 \mathrm{H}), 7.48(\mathrm{~d}, J=7.2$ $\mathrm{Hz}, 1 \mathrm{H}), 7.36-7.17(\mathrm{~m}, 2 \mathrm{H}), 6.96(\mathrm{td}, J=8.9,2.5 \mathrm{~Hz}, 1 \mathrm{H}), 6.56(\mathrm{dd}, J=8.0,2.0 \mathrm{~Hz}, 1 \mathrm{H}), 6.39(\mathrm{~s}, 1 \mathrm{H})$, $4.02(\mathrm{dd}, J=8.9,5.5 \mathrm{~Hz}, 1 \mathrm{H}), 3.94(\mathrm{dd}, J=14.7,5.4 \mathrm{~Hz}, 1 \mathrm{H}), 3.28(\mathrm{dd}, J=14.8,9.1 \mathrm{~Hz}, 1 \mathrm{H}), 1.70$ (s, 9H), $1.64(\mathrm{~s}, 9 \mathrm{H})$.

${ }^{13}$ C NMR $\left(100 \mathrm{MHz}, \mathrm{CDCl}_{3}\right) \delta 174.6,159.5(\mathrm{~d}, J=243.1 \mathrm{~Hz}), 150.6,149.3,136.9,136.7,136.0(\mathrm{~d}, J=$ $2.6 \mathrm{~Hz}), 129.1(\mathrm{~d}, J=8.7 \mathrm{~Hz}), 128.7,124.1,123.0,120.4,116.0(\mathrm{~d}, J=7.8 \mathrm{~Hz}), 115.8,114.6(\mathrm{~d}, J=22.8$ Hz), $112.2(\mathrm{~d}, J=24.7 \mathrm{~Hz}), 110.7,84.7,84.5,45.3$ (d, $J=2.0 \mathrm{~Hz}), 32.1,28.3,28.1$.

IR (ATR, neat): 3120, 2980, 2939, 1934, 1882, 1769, 1712, 1608, 1475, 1453, 1370, 1350, 1327, 1296, 1249, 1115, 1101, 1083, 1068.

HRMS (ESI) calcd $(m / z)$ for $\mathrm{C}_{27} \mathrm{H}_{29} \mathrm{FN}_{2} \mathrm{O}_{5}:[\mathrm{M}+\mathrm{Na}]^{+}$503.1953, found 503.1957.

tert-butyl 2-((1-(tert-butoxycarbonyl)-5-chloro-2-oxoindolin-3-yl)methyl)-6-chloro-1H-indole-1carboxylate (1y)

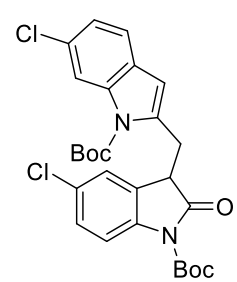

Prepared according to the GP4 to provide the title compound as white solid.

m.p. $156-158^{\circ} \mathrm{C}$.

${ }^{1} \mathbf{H}$ NMR $\left(400 \mathrm{MHz}, \mathrm{CDCl}_{3}\right) \delta 8.12(\mathrm{~d}, J=1.8 \mathrm{~Hz}, 1 \mathrm{H}), 7.78(\mathrm{~d}, J=8.7 \mathrm{~Hz}, 1 \mathrm{H}), 7.39(\mathrm{~d}, J=8.3 \mathrm{~Hz}, 1 \mathrm{H})$, 7.30-7.17 (m, 2H), $6.81(\mathrm{~m}, 1 \mathrm{H}), 6.36(\mathrm{~s}, 1 \mathrm{H}), 3.98(\mathrm{dd}, J=8.8,5.8 \mathrm{~Hz}, 1 \mathrm{H}), 3.85(\mathrm{dd}, J=14.9,5.8 \mathrm{~Hz}$, $1 \mathrm{H}), 3.31(\mathrm{dd}, J=15.0,8.8 \mathrm{~Hz}, 1 \mathrm{H}), 1.71(\mathrm{~s}, 9 \mathrm{H}), 1.64(\mathrm{~s}, 9 \mathrm{H})$.

${ }^{13}$ C NMR $\left(100 \mathrm{MHz}, \mathrm{CDCl}_{3}\right)$ 174.2, 150.2, 149.1, 138.5, 137.6, 137.0, 130.0, 129.5, 128.9, 128.3, 127.2, $124.8,123.5,120.9,116.3,116.1,110.2,85.3,84.7,45.0,32.0,28.2,28.1$.

IR (ATR, neat): 3131, 2979, 2938, 2891, 1874, 1768, 1731, 1605, 1590, 1562, 1473, 1454, 1427, 1371, 
1332, 1295, 1217, 1155, 1120, 1101, 1064.

HRMS (ESI) calcd $(m / z)$ for $\mathrm{C}_{27} \mathrm{H}_{28} \mathrm{Cl}_{2} \mathrm{~N}_{2} \mathrm{O}_{5}$ : [M+Na] $]^{+}$553.1268, found 553.1266. 


\section{Optimization of reaction conditions}

Table S1. Investigation of Lewis acid catalysts. ${ }^{\text {a] }}$

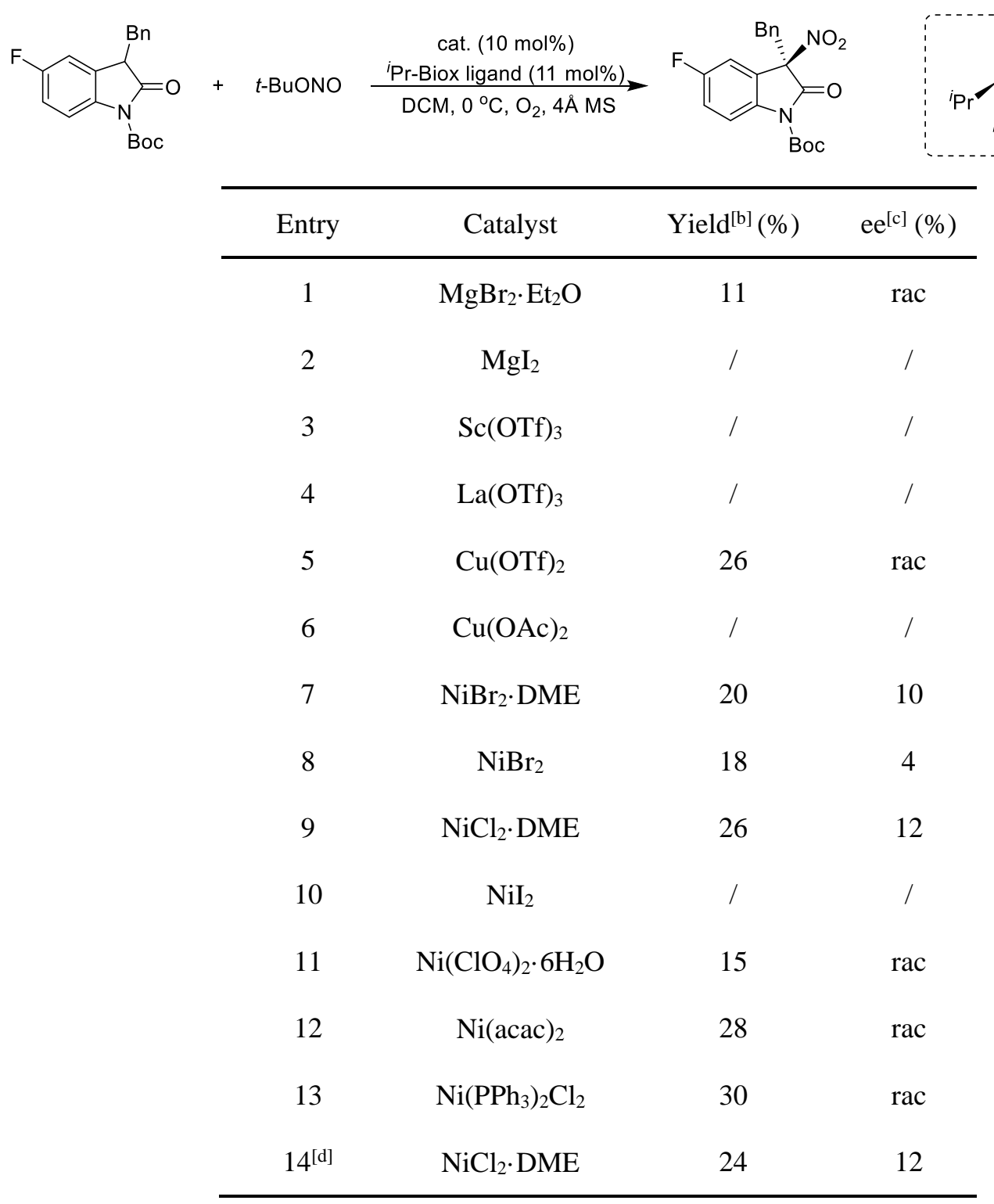

[a] Unless otherwise indicated, all reactions were performed with oxindole $(0.2 \mathrm{mmol})$ and tert-butyl nitrite $(0.3 \mathrm{mmol})$ in the presence of catalyst (10 mol\%), $i \mathrm{Pr}$-Biox ligand $(11 \mathrm{~mol} \%)$ and $4 \AA \mathrm{MS}$ in $1 \mathrm{~mL} \mathrm{DCM}$ at $0{ }^{\circ} \mathrm{C}$ under oxygen atmosphere. [b] Isolated yield. [c] Determined by HPLC analysis. [d] $7.5 \mathrm{~mol} \%$ catalyst and $8 \mathrm{~mol} \%$ ligand used.

We initiated our study with $i$ Pr-Biox ligand as the chiral ligand, a variety of Lewis acids were investigated for this asymmetric nitration. To out delight, the combination of $\mathrm{NiBr}_{2}$. DME with $i$ Pr-Biox ligand afforded the desired product in $20 \%$ yield with $10 \%$ ee. Next, the screening of various nickel catalysts showed that $\mathrm{NiCl}_{2} \cdot \mathrm{DME}$ was the optimal catalyst at this stage. 
Table S2. Investigation of protecting groups. ${ }^{[a]}$

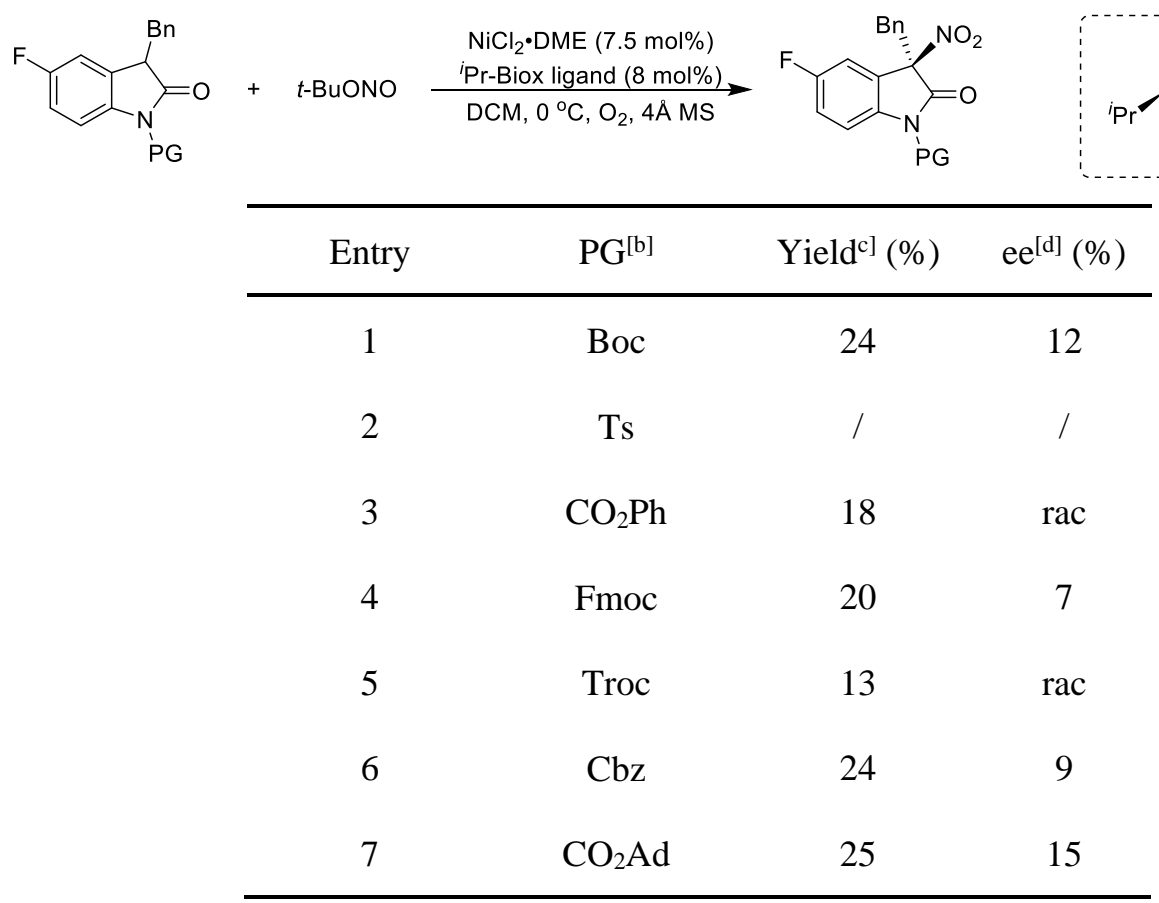

[a] Unless otherwise indicated, all reactions were performed with oxindole $(0.2 \mathrm{mmol})$ and tert-butyl nitrite $(0.3 \mathrm{mmol})$ in the presence of $\mathrm{NiCl}_{2}$. DME (7.5 mol\%), $i \mathrm{Pr}$-Biox ligand $\left(8 \mathrm{~mol} \%\right.$ ) and $4 \AA \mathrm{MS}$ in $1 \mathrm{~mL} \mathrm{DCM}$ at $0{ }^{\circ} \mathrm{C}$ under oxygen atmosphere. [b] PG: Protection group; Boc: tert-butyloxycarbonyl; Ts: p-toluenesulfonamide; Fmoc: fluorenylmethoxycarbonyl; Troc: 2,2,2-Trichloroethoxycarbonyl; Cbz: carboxybenzyl; Ad: Adamantyl. [c] Isolated yield. [d] Determined by HPLC analysis.

The protecting groups of oxindole had dramatic effect on this transformation. The reactions with tertbutyloxycarbonyl group (Boc) or the adamantyl group showed similar reactivities and enantioselective excess, while the later one gave slightly better ee.

Table S3. Investigation of chiral ligands. ${ }^{[a]}$

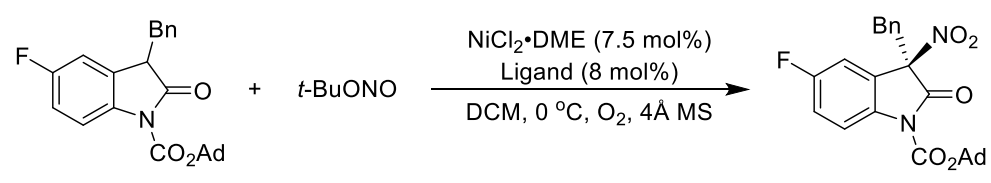<smiles>c1ccc(Nc2ccccc2)cc1</smiles><smiles>[NH3+]C(Nc1ccccc1)c1ccccc1</smiles>

$14 \%$ yield, rac<smiles></smiles>

NR<smiles>CC(C)(C)[C@H]1COC(c2ccccn2)=N1</smiles>

$22 \%$ yield, rac

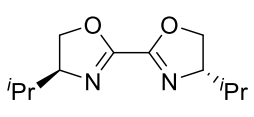<smiles>CC(C)(C1=NC(c2ccccc2)[C@H](c2ccccc2)O1)C1=NC(c2ccccc2)OC1c1ccccc1</smiles>

$24 \%$ yield, $15 \%$ ee 


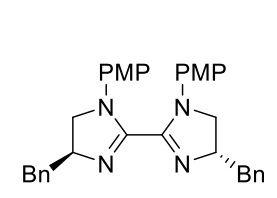

$15 \%$ yield, rac

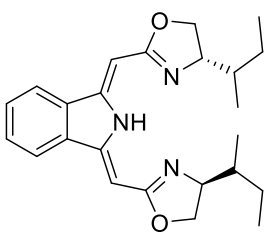

$19 \%$ yield, rac

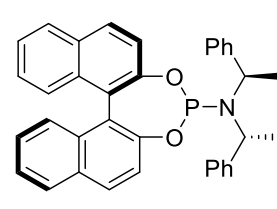

NR

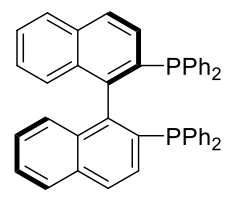

NR

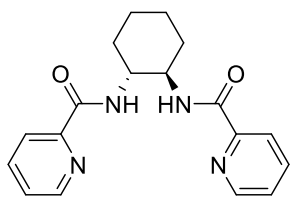

$21 \%$ yield, rac<smiles></smiles>

$10 \%$ yield, rac

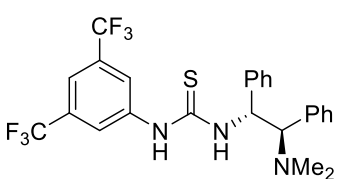

NR

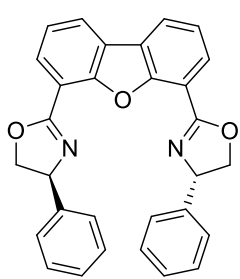

$16 \%$ yield, $5 \%$ ee

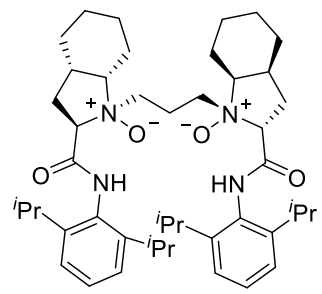

NR

[a] Unless otherwise indicated, all reactions were performed with oxindole $(0.2 \mathrm{mmol})$ and tert-butyl nitrite $(0.3 \mathrm{mmol})$ in the presence of $\mathrm{NiCl}_{2} \cdot \mathrm{DME}(7.5 \mathrm{~mol} \%)$, ligand $(8 \mathrm{~mol} \%)$ and $4 \AA \mathrm{MS}$ in $1 \mathrm{~mL}$ DCM at $0{ }^{\circ} \mathrm{C}$ under oxygen atmosphere. NR: No reaction.

After screening of various chiral ligands, we found that chiral $N, N$-ligands showed certain enantioselective induction effect.

Table S4. Investigation of reaction concentration and amount of tert-butyl nitrite. ${ }^{[a]}$

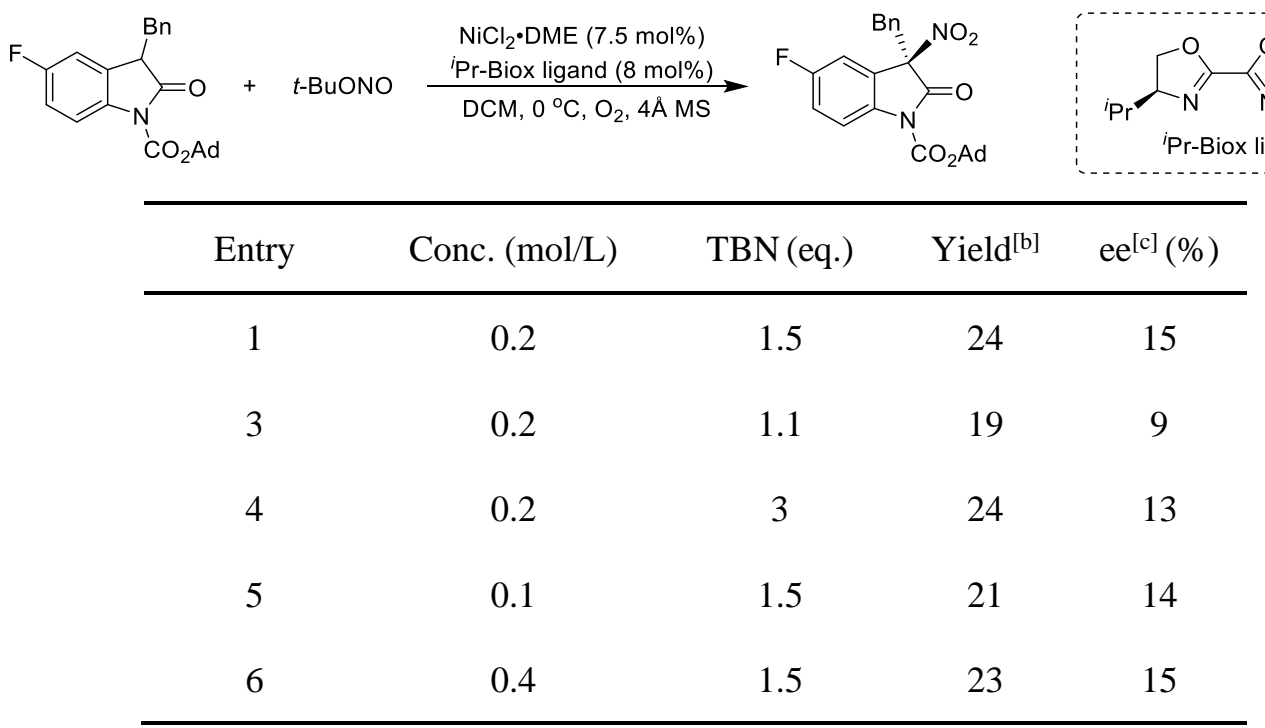

[a] Unless otherwise indicated, all reactions were performed with oxindole $(0.2 \mathrm{mmol})$ and tert-butyl nitrite in the presence of $\mathrm{NiCl}_{2} \cdot \mathrm{DME}(7.5 \mathrm{~mol} \%), i \mathrm{Pr}-\mathrm{Biox}$ ligand $(8 \mathrm{~mol} \%)$ and $4 \AA \mathrm{MS}$ in $1 \mathrm{~mL}$ DCM at $0{ }^{\circ} \mathrm{C}$ under oxygen atmosphere. TBN: tert-butyl nitrite. [b] Isolated yield. [c] Determined by HPLC analysis. 


\section{Synthesis of chiral 3-nitro oxindoles}

\section{General procedure for the enantioselective nitration of oxindoles GP5}
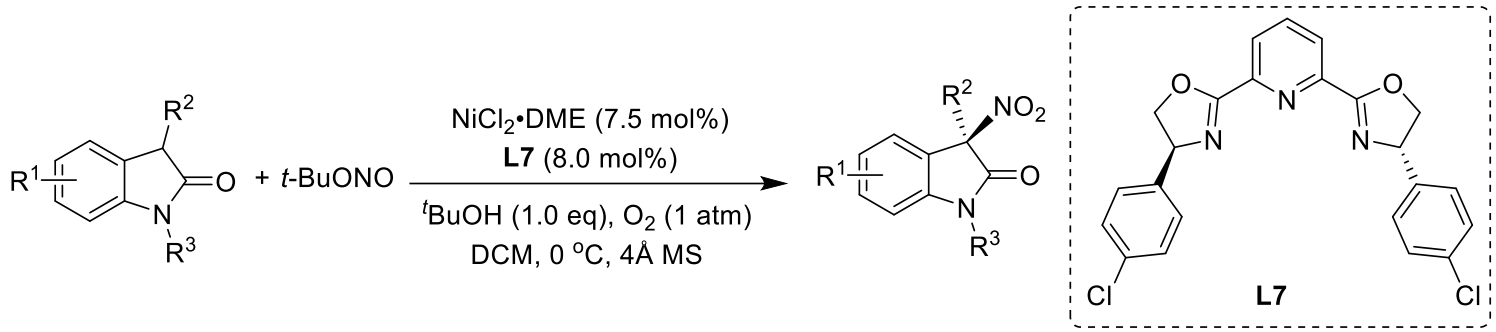

To a mixture of oxindole ( $0.2 \mathrm{mmol}, 1$ equiv.), $\mathrm{NiCl}_{2} \bullet \mathrm{DME}$ (3.3 mg, $0.015 \mathrm{mmol}, 7.5 \mathrm{~mol} \%$ ), $\mathbf{L} 7$ (7.0 mg, $0.016 \mathrm{mmol}, 8 \mathrm{~mol} \%),{ }^{t} \mathrm{BuOH}(14.8 \mathrm{mg}, 0.2 \mathrm{mmol}, 1$ equiv.) and $4 \AA \mathrm{MS}$ in $1 \mathrm{ml} \mathrm{DCM}$ was stirred under $\mathrm{O}_{2}$ balloon at room temperature for $1 \mathrm{~h}$, then the mixture was cooled to $0{ }^{\circ} \mathrm{C}$ and tert-Butyl nitrite $(30.9$ $\mathrm{mg}, 0.3 \mathrm{mmol}, 1.5$ equiv.) was added. The mixture was continued stirred for 10 hours at this temperature. After the solvent was removed under reduced pressure, the residue was purified by column chromatography to afford the nitration product. Enantioselectivity was determined by chiral HPLC analysis.

\section{Adamantyl (R)-5-fluoro-3-benzyl-3-nitro-2-oxoindoline-1-carboxylate (3a)}

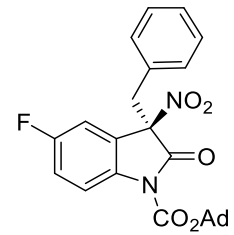

Prepared according to the GP5 to provide the title compound as white solid ( $70 \mathrm{mg}, 77 \%$ yield). Enantiomeric excess was determined by HPLC with a Daicel CHIRALPAK ${ }^{\circledR}$ IC-U column, (Hexane/i$\operatorname{PrOH}=98 / 2, v=0.3 \mathrm{~mL} / \mathrm{min}, \lambda=254 \mathrm{~nm}), \mathrm{t}($ minor $)=6.0 \mathrm{~min}, \mathrm{t}($ major $)=8.8 \mathrm{~min}, 94 \%$ ee. $[\alpha]_{\mathrm{D}}{ }^{20}=$ $42.4\left(c=0.1\right.$ in $\left.\mathrm{CH}_{2} \mathrm{Cl}_{2}\right)$.

m.p. $132-134{ }^{\circ} \mathrm{C}$.

${ }^{1} \mathbf{H}$ NMR $\left(400 \mathrm{MHz}, \mathrm{CDCl}_{3}\right) \delta 7.70(\mathrm{dd}, J=9.0,4.4 \mathrm{~Hz}, 1 \mathrm{H}), 7.24-7.06(\mathrm{~m}, 5 \mathrm{H}), 7.00-6.89$ (m, 2H), 3.88 $(\mathrm{d}, J=13.2 \mathrm{~Hz}, 1 \mathrm{H}), 3.82(\mathrm{~d}, J=13.2 \mathrm{~Hz}, 1 \mathrm{H}), 2.39-2.20(\mathrm{~m}, 9 \mathrm{H}), 1.70(\mathrm{~m}, 6 \mathrm{H})$.

${ }^{13} \mathrm{C}$ NMR $\left(100 \mathrm{MHz}, \mathrm{CDCl}_{3}\right) \delta 166.0,159.7\left(\mathrm{~d}, \mathrm{C}-\mathrm{F},{ }^{1} J_{\mathrm{C}-F}=246.5 \mathrm{~Hz}\right), 147.3,136.9\left(\mathrm{~d}, \mathrm{C}-\mathrm{F},{ }^{4} J_{C-F}=2.7\right.$ $\mathrm{Hz}), 130.6,130.3,128.6,128.1,123.9\left(\mathrm{~d}, \mathrm{C}-\mathrm{F},{ }^{3} J_{C-F}=8.4 \mathrm{~Hz}\right), 118.6\left(\mathrm{~d}, \mathrm{C}-\mathrm{F},{ }^{2} J_{C-F}=22.8 \mathrm{~Hz}\right), 117.4(\mathrm{~d}$, C-F, $\left.{ }^{3} J_{C-F}=7.8 \mathrm{~Hz}\right), 111.8\left(\mathrm{~d}, \mathrm{C}-\mathrm{F},{ }^{2} J_{C-F}=25.2 \mathrm{~Hz}\right), 92.8\left(\mathrm{~d},{ }^{4} J_{C-F}=1.9 \mathrm{~Hz}\right), 85.7,41.3,40.9,36.0,31.1$. IR (ATR, neat): 2921, 2856, 1767, 1744, 1647, 1612, 1556, 1484, 1457, 1442, 1338, 1317, 1295, 1240, $1227,1163,1101$. 
HRMS (ESI) calcd $(\mathrm{m} / \mathrm{z})$ for $\mathrm{C}_{26} \mathrm{H}_{25} \mathrm{FN}_{2} \mathrm{O}_{5}:[\mathrm{M}+\mathrm{Na}]^{+}$487.1640, found 487.1644.

Adamantyl (R)-6-fluoro-3-benzyl-3-nitro-2-oxoindoline-1-carboxylate (3b)

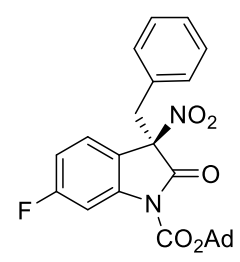

Prepared according to the GP5 to provide the title compound as colorless oil (73 $\mathrm{mg}, 80 \%$ yield). Enantiomeric excess was determined by HPLC with a Daicel CHIRALPAK ${ }^{\circ}$ IC-U column, (Hexane/ $i$ $\operatorname{PrOH}=98 / 2, v=0.3 \mathrm{~mL} / \mathrm{min}, \lambda=254 \mathrm{~nm}), \mathrm{t}($ minor $)=5.8 \mathrm{~min}, \mathrm{t}($ major $)=8.1 \mathrm{~min}, 94 \%$ ee. $[\alpha]_{\mathrm{D}}{ }^{20}=$ $58.2\left(c=0.1\right.$ in $\left.\mathrm{CH}_{2} \mathrm{Cl}_{2}\right)$.

${ }^{1} \mathbf{H}$ NMR $\left(400 \mathrm{MHz}, \mathrm{CDCl}_{3}\right) \delta 7.48(\mathrm{dd}, J=9.9,2.4 \mathrm{~Hz}, 1 \mathrm{H}), 7.37(\mathrm{dd}, J=8.5,5.4 \mathrm{~Hz}, 1 \mathrm{H}), 7.22-7.10$ (m, 3H), 6.99-6.86 (m, 3H), 3.89 (d, $J=13.2 \mathrm{~Hz}, 1 \mathrm{H}), 3.81(\mathrm{~d}, J=13.1 \mathrm{~Hz}, 1 \mathrm{H}), 2.36-2.18(\mathrm{~m}, 9 \mathrm{H}), 1.70$ $(\mathrm{m}, 6 \mathrm{H})$.

${ }^{13}$ C NMR $\left(100 \mathrm{MHz}, \mathrm{CDCl}_{3}\right) \delta 166.2,164.4\left(\mathrm{~d}, \mathrm{C}-\mathrm{F},{ }^{1} J_{\mathrm{C}-F}=250.1 \mathrm{~Hz}\right), 147.1,142.4\left(\mathrm{~d}, \mathrm{C}-\mathrm{F},{ }^{3} J_{C-F}=12.8\right.$ $\mathrm{Hz}), 130.8,130.4,128.5,128.0,125.8\left(\mathrm{~d}, \mathrm{C}-\mathrm{F},{ }^{3} J_{C-F}=10.1 \mathrm{~Hz}\right), 118.2\left(\mathrm{~d}, \mathrm{C}-\mathrm{F},{ }^{4} J_{C-F}=3.1 \mathrm{~Hz}\right), 112.0(\mathrm{~d}$, $\left.\mathrm{C}-\mathrm{F},{ }^{2} J_{\mathrm{C}-\mathrm{F}}=23.4 \mathrm{~Hz}\right), 104.8\left(\mathrm{~d}, \mathrm{C}-\mathrm{F},{ }^{2} J_{C-F}=30.1 \mathrm{~Hz}\right), 92.7,86.0,41.3,40.8,36.0,31.1$.

IR (ATR, neat): 2913, 2853, 1784, 1741, 1609, 1557, 1497, 1456, 1352, 1313, 1292, 1251, 1159, 1109. HRMS (ESI) calcd $(m / z)$ for $\mathrm{C}_{26} \mathrm{H}_{25} \mathrm{FN}_{2} \mathrm{O}_{5}:[\mathrm{M}+\mathrm{Na}]^{+} 487.1640$, found 487.1642 .

Adamantyl (R)-5-chloro-3-benzyl-3-nitro-2-oxoindoline-1-carboxylate (3c)

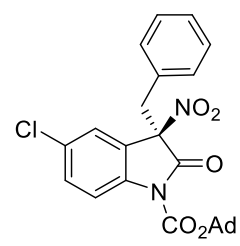

Prepared according to the GP5 to provide the title compound as colorless oil (72 $\mathrm{mg}$, 75\% yield). Enantiomeric excess was determined by HPLC with a Daicel CHIRALPAK® IC-U column, (Hexane/i$\operatorname{PrOH}=98 / 2, v=0.3 \mathrm{~mL} / \mathrm{min}, \lambda=254 \mathrm{~nm}), \mathrm{t}($ minor $)=5.5 \mathrm{~min}, \mathrm{t}($ major $)=8.0 \mathrm{~min}, 95 \%$ ee. $[\alpha]_{\mathrm{D}}{ }^{20}=$ $84.7\left(c=0.1\right.$ in $\left.\mathrm{CH}_{2} \mathrm{Cl}_{2}\right)$.

${ }^{1}$ H NMR $\left(400 \mathrm{MHz}, \mathrm{CDCl}_{3}\right) \delta 7.66(\mathrm{~d}, J=8.7 \mathrm{~Hz}, 1 \mathrm{H}), 7.38(\mathrm{~d}, J=2.2 \mathrm{~Hz}, 1 \mathrm{H}), 7.35(\mathrm{dd}, J=8.7,2.2$ Hz, 1H), 7.22-7.12 (m, 3H), 6.99-6.89 (m, 2H), 3.87 (d, $J=13.1 \mathrm{~Hz}, 1 \mathrm{H}), 3.82(\mathrm{~d}, J=13.2 \mathrm{~Hz}, 1 \mathrm{H}), 2.29$ $2.18(\mathrm{~m}, 9 \mathrm{H}), 1.69(\mathrm{~m}, 6 \mathrm{H})$

${ }^{13}$ C NMR $\left(100 \mathrm{MHz}, \mathrm{CDCl}_{3}\right) \delta 165.8,147.2,139.4,131.9,130.6,130.5,130.3,128.6,128.1,124.4,124.1$, $117.1,92.7,85.9,41.3,40.9,36.0,31.1$. 
IR (ATR, neat): 2912, 2853, 1781, 1739, 1655, 1593, 1557, 1475, 1456, 1330, 1289, 1243, 1214, 1163, $1129,1104$.

HRMS (ESI) calcd $(m / z)$ for $\mathrm{C}_{26} \mathrm{H}_{25} \mathrm{ClN}_{2} \mathrm{O}_{5}:[\mathrm{M}+\mathrm{Na}]^{+}$503.1344, found 503.1345.

Adamantyl (R)-6-chloro-3-benzyl-3-nitro-2-oxoindoline-1-carboxylate (3d)

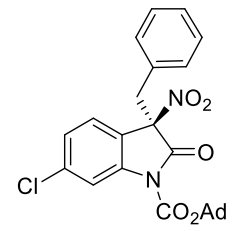

Prepared according to the GP5 to provide the title compound as colorless oil (71 $\mathrm{mg}, 74 \%$ yield). Enantiomeric excess was determined by HPLC with a Daicel CHIRALPAK ${ }^{\circ}$ IC-U column, (Hexane/ $i$ $\mathrm{PrOH}=98 / 2, v=0.3 \mathrm{~mL} / \mathrm{min}, \lambda=254 \mathrm{~nm}), \mathrm{t}($ minor $)=5.8 \mathrm{~min}, \mathrm{t}($ major $)=8.1 \mathrm{~min}, 94 \%$ ee. $[\alpha]_{\mathrm{D}}{ }^{20}=-$ $69.6\left(c=0.1\right.$ in $\left.\mathrm{CH}_{2} \mathrm{Cl}_{2}\right)$.

${ }^{1} \mathbf{H}$ NMR $\left(400 \mathrm{MHz}, \mathrm{CDCl}_{3}\right) \delta 7.78(\mathrm{~d}, J=1.9 \mathrm{~Hz}, 1 \mathrm{H}), 7.32(\mathrm{~d}, J=8.1 \mathrm{~Hz}, 1 \mathrm{H}), 7.22(\mathrm{dd}, J=8.2,2.0$ Hz, 1H), 7.20-7.10 (m, 3H), 6.95-6.89 (m, 2H), 3.89 (d, $J=13.2 \mathrm{~Hz}, 1 \mathrm{H}), 3.80$ (d, $J=13.2 \mathrm{~Hz}, 1 \mathrm{H}), 2.29-$ $2.20(\mathrm{~m}, 9 \mathrm{H}), 1.70(\mathrm{~m}, 6 \mathrm{H})$.

${ }^{13}$ C NMR $\left(100 \mathrm{MHz}, \mathrm{CDCl}_{3}\right) \delta 166.0,147.1,141.8,137.9,130.6,130.4,128.6,128.1,125.2,125.2,120.9$, $116.6,92.7,86.1,41.3,40.7,36.0,31.1$.

IR (ATR, neat): 2912, 2854, 1783, 1740, 1607, 1557, 1483, 1456, 1426, 1342, 1267, 1238, 1163, 1109.

HRMS (ESI) calcd $(m / z)$ for $\mathrm{C}_{26} \mathrm{H}_{25} \mathrm{ClN}_{2} \mathrm{O}_{5}:[\mathrm{M}+\mathrm{Na}]^{+}$503.1344, found 503.1344.

Adamantyl (R)-6-bromo-3-benzyl-3-nitro-2-oxoindoline-1-carboxylate (3e)

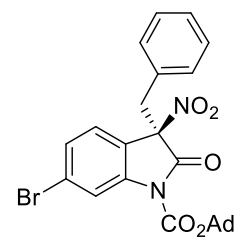

Prepared according to the GP5 to provide the title compound as colorless oil (76 $\mathrm{mg}, 72 \%$ yield). Enantiomeric excess was determined by HPLC with a Daicel CHIRALPAK ${ }^{8}$ IC-U column, (Hexane/ $i$ $\mathrm{PrOH}=98 / 2, v=0.3 \mathrm{~mL} / \mathrm{min}, \lambda=254 \mathrm{~nm}), \mathrm{t}($ minor $)=5.9 \mathrm{~min}, \mathrm{t}$ (major) $=8.3 \mathrm{~min}, 94 \% e e .[\alpha]_{\mathrm{D}}^{20}=-$ $64.4\left(c=0.1\right.$ in $\left.\mathrm{CH}_{2} \mathrm{Cl}_{2}\right)$.

${ }^{1} \mathbf{H}$ NMR $\left(400 \mathrm{MHz}, \mathrm{CDCl}_{3}\right) \delta 7.95(\mathrm{~d}, J=1.8 \mathrm{~Hz}, 1 \mathrm{H}), 7.38(\mathrm{dd}, J=8.1,1.8 \mathrm{~Hz}, 1 \mathrm{H}), 7.24(\mathrm{~d}, J=8.2$ Hz, 1H), 7.20-7.09 (m, 3H), 6.94-6.87 (m, 2H), 3.89 (d, $J=13.2 \mathrm{~Hz}, 1 \mathrm{H}), 3.80(\mathrm{~d}, J=13.2 \mathrm{~Hz}, 1 \mathrm{H}), 2.25-$ $2.19(\mathrm{~m}, 9 \mathrm{H}), 1.70(\mathrm{~m}, 6 \mathrm{H})$.

${ }^{13}$ C NMR $\left(100 \mathrm{MHz}, \mathrm{CDCl}_{3}\right) \delta 165.9,147.1,141.8,130.6,130.4,128.6,128.1,128.1,125.9,125.4,121.5$, 
119.4, 92.7, 86.1, 41.3, 40.7, 36.0, 31.1.

IR (ATR, neat): 2913, 2855, 1809, 1784, 1739, 1603, 1558, 1479, 1422, 1341, 1267, 1237, 1215, 1157.

HRMS (ESI) calcd $(m / z)$ for $\mathrm{C}_{26} \mathrm{H}_{25} \mathrm{BrN}_{2} \mathrm{O}_{5}:\left[\mathrm{M}+\mathrm{NH}_{4}\right]^{+}$542.1285, found 542.1286.

Adamantyl (R)-6-methoxy-3-benzyl-3-nitro-2-oxoindoline-1-carboxylate (3f)

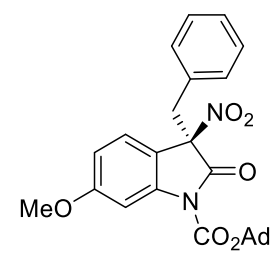

Prepared according to the GP5 to provide the title compound as colorless oil (59 $\mathrm{mg}$, 62\% yield). Enantiomeric excess was determined by HPLC with a Daicel CHIRALPAK® IC-U column, (Hexane/i$\operatorname{PrOH}=95 / 5, v=0.3 \mathrm{~mL} / \mathrm{min}, \lambda=254 \mathrm{~nm}), \mathrm{t}($ minor $)=7.0 \mathrm{~min}, \mathrm{t}($ major $)=11.7 \mathrm{~min}, 91 \% e e .[\alpha]_{\mathrm{D}}{ }^{20}=$ $66.1\left(c=0.1\right.$ in $\left.\mathrm{CH}_{2} \mathrm{Cl}_{2}\right)$.

${ }^{1} \mathbf{H}$ NMR $\left(400 \mathrm{MHz}, \mathrm{CDCl}_{3}\right) \delta 7.31(\mathrm{~d}, J=2.4 \mathrm{~Hz}, 1 \mathrm{H}), 7.29(\mathrm{~d}, J=8.5 \mathrm{~Hz}, 1 \mathrm{H}), 7.19-7.07(\mathrm{~m}, 3 \mathrm{H}), 6.97-$ $6.88(\mathrm{~m}, 2 \mathrm{H}), 6.73(\mathrm{dd}, J=8.5,2.4 \mathrm{~Hz}, 1 \mathrm{H}), 3.89(\mathrm{~d}, J=13.1 \mathrm{~Hz}, 1 \mathrm{H}), 3.80(\mathrm{~s}, 3 \mathrm{H}), 3.79(\mathrm{~d}, J=13.2 \mathrm{~Hz}$, $1 \mathrm{H}), 2.26-2.19(\mathrm{~m}, 9 \mathrm{H}), 1.70(\mathrm{~m}, 6 \mathrm{H})$.

${ }^{13}$ C NMR $\left(100 \mathrm{MHz}, \mathrm{CDCl}_{3}\right) \delta 166.9,162.4,147.4,142.3,131.3,130.5,128.4,127.7,125.2,114.3,110.8$, $102.2,93.1,85.4,55.7,41.3,40.5,36.0,31.1$.

IR (ATR, neat): 2912, 2852, 1801, 1780, 1736, 1616, 1556, 1500, 1455, 1351, 1315, 1289, 1251, 1207, 1163.

HRMS (ESI) calcd $(\mathrm{m} / z)$ for $\mathrm{C}_{27} \mathrm{H}_{28} \mathrm{~N}_{2} \mathrm{O}_{6}:[\mathrm{M}+\mathrm{H}]^{+}$477.2638, found 477.2644.

Adamantyl (R)-6-phenyl-3-benzyl-3-nitro-2-oxoindoline-1-carboxylate (3g)

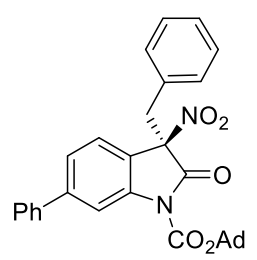

Prepared according to the GP5 to provide the title compound as white solid (76 $\mathrm{mg}, 73 \%$ yield). Enantiomeric excess was determined by HPLC with a Daicel CHIRALPAK $®$ IC-U column, (Hexane/i$\operatorname{PrOH}=98 / 2, v=0.3 \mathrm{~mL} / \mathrm{min}, \lambda=254 \mathrm{~nm}), \mathrm{t}($ minor $)=8.4 \mathrm{~min}, \mathrm{t}($ major $)=13.6 \mathrm{~min} .93 \%$ ee. $[\alpha]_{\mathrm{D}}{ }^{20}=$ $118.2\left(c=0.1\right.$ in $\left.\mathrm{CH}_{2} \mathrm{Cl}_{2}\right)$.

m.p. $144-146^{\circ} \mathrm{C}$.

${ }^{1} \mathbf{H}$ NMR $\left(400 \mathrm{MHz}, \mathrm{CDCl}_{3}\right) \delta 7.99(\mathrm{~s}, 1 \mathrm{H}), 7.56(\mathrm{~d}, J=7.2 \mathrm{~Hz}, 2 \mathrm{H}), 7.49-7.33(\mathrm{~m}, 5 \mathrm{H})$, 7.18-7.08 (m, 3H), 7.00-6.90 (m, 2H), $3.95(\mathrm{~d}, J=13.2 \mathrm{~Hz}, 1 \mathrm{H}), 3.84(\mathrm{~d}, J=13.1 \mathrm{~Hz}, 1 \mathrm{H}), 2.23(\mathrm{~m}, 9 \mathrm{H}), 1.70(\mathrm{~m}, 6 \mathrm{H})$ 
${ }^{13}$ C NMR $\left(100 \mathrm{MHz}, \mathrm{CDCl}_{3}\right) \delta 166.6,147.5,145.2,141.5,140.0,131.0,130.5,129.0,128.5,128.3,127.9$, $127.3,124.5,123.8,121.4,114.7,93.1,85.5,41.3,40.8,36.0,31.1$.

IR (ATR, neat): 2912, 2853, 1780, 1743, 1615, 1599, 1551, 1483, 1455, 1426, 1346, 1253, 1226, 1206, $1161,1127,1105$.

HRMS (ESI) calcd $(m / z)$ for $\mathrm{C}_{32} \mathrm{H}_{30} \mathrm{~N}_{2} \mathrm{O}_{5}:\left[\mathrm{M}+\mathrm{NH}_{4}\right]^{+}$540.2493, found 540.2489.

Adamantyl (R)-3-benzyl-3-nitro-2-oxoindoline-1-carboxylate (3h)

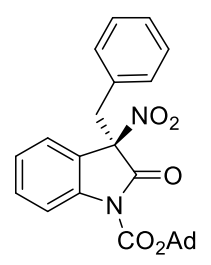

Prepared according to the GP5 to provide the title compound as colorless oil (52 $\mathrm{mg}, 58 \%$ yield). Enantiomeric excess was determined by HPLC with a Daicel CHIRALPAK ${ }^{\circ}$ IC-U column, (Hexane/ $i$ $\mathrm{PrOH}=98 / 2, v=0.3 \mathrm{~mL} / \mathrm{min}, \lambda=254 \mathrm{~nm}), \mathrm{t}($ minor $)=7.4 \mathrm{~min}, \mathrm{t}($ major $)=11.7 \mathrm{~min}, 94 \%$ ee. $[\alpha]_{\mathrm{D}}{ }^{20}=-$ $49.8\left(c=0.1\right.$ in $\left.\mathrm{CH}_{2} \mathrm{Cl}_{2}\right)$.

${ }^{1} \mathbf{H}$ NMR $\left(400 \mathrm{MHz}, \mathrm{CDCl}_{3}\right) \delta 7.68(\mathrm{~d}, J=8.2 \mathrm{~Hz}, 1 \mathrm{H}), 7.45-7.34(\mathrm{~m}, 2 \mathrm{H}), 7.24(\mathrm{~m}, 1 \mathrm{H}), 7.16-7.06(\mathrm{~m}$, 3H), 6.94-6.87 (m, 2H), 3.90 (d, $J=13.0 \mathrm{~Hz}, 1 \mathrm{H}), 3.83$ (d, $J=13.1 \mathrm{~Hz}, 1 \mathrm{H}), 2.25-2.19(\mathrm{~m}, 9 \mathrm{H}), 1.69$ (m, $6 \mathrm{H})$.

${ }^{13}$ C NMR $\left(100 \mathrm{MHz}, \mathrm{CDCl}_{3}\right) \delta 166.5,147.4,140.9,131.9,130.9,130.4,128.4,127.8,125.0,124.1,122.7$, $115.8,93.1,85.4,41.3,40.8,36.0,31.1$.

IR (ATR, neat): 2912, 2853, 1801, 1779, 1740, 1606, 1556, 1496, 1481, 1469, 1346, 1315, 1280, 1243, $1215,1165,1108$.

HRMS (ESI) calcd $(m / z)$ for $\mathrm{C}_{26} \mathrm{H}_{26} \mathrm{~N}_{2} \mathrm{O}_{5}:[\mathrm{M}+\mathrm{Na}]^{+} 469.1734$, found 469.1737 .

Adamantyl (R)-5-fluoro-3-(4-methylbenzyl)-3-nitro-2-oxoindoline-1-carboxylate (3i)

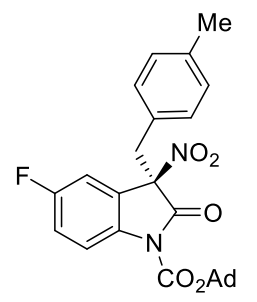

Prepared according to the GP5 to provide the title compound as white solid (68 $\mathrm{mg}, 71 \%$ yield). Enantiomeric excess was determined by HPLC with a Daicel CHIRALPAK ${ }^{\circ}$ IC-U column, (Hexane/ $i$ $\mathrm{PrOH}=98 / 2, v=0.3 \mathrm{~mL} / \mathrm{min}, \lambda=254 \mathrm{~nm}), \mathrm{t}($ minor $)=6.2 \mathrm{~min}, \mathrm{t}$ (major) $=8.8 \mathrm{~min}, 94 \%$ ee. $[\alpha]_{\mathrm{D}}{ }^{20}=-$ $60.5\left(c=0.1\right.$ in $\left.\mathrm{CH}_{2} \mathrm{Cl}_{2}\right)$.

m.p. $133-135^{\circ} \mathrm{C}$. 
${ }^{1} \mathbf{H}$ NMR $\left(400 \mathrm{MHz}, \mathrm{CDCl}_{3}\right) \delta 7.71(\mathrm{dd}, J=8.9,4.4 \mathrm{~Hz}, 1 \mathrm{H}), 7.18-7.06(\mathrm{~m}, 2 \mathrm{H}), 6.93(\mathrm{~d}, J=7.9 \mathrm{~Hz}, 2 \mathrm{H})$, $6.80(\mathrm{~d}, J=8.0 \mathrm{~Hz}, 2 \mathrm{H}), 3.84(\mathrm{~d}, J=13.3 \mathrm{~Hz}, 1 \mathrm{H}), 3.77(\mathrm{~d}, J=13.2 \mathrm{~Hz}, 1 \mathrm{H}), 2.35-2.15(\mathrm{~m}, 12 \mathrm{H}), 1.70$ $(\mathrm{m}, 6 \mathrm{H})$.

${ }^{13} \mathrm{C}$ NMR $\left(100 \mathrm{MHz}, \mathrm{CDCl}_{3}\right) \delta 166.0,159.7\left(\mathrm{~d}, \mathrm{C}-\mathrm{F},{ }^{1} J_{\mathrm{C}-\mathrm{F}}=246.3 \mathrm{~Hz}\right), 147.4,137.8,137.0\left(\mathrm{~d}, \mathrm{C}-\mathrm{F},{ }^{4} J_{C-}\right.$ $F=2.6 \mathrm{~Hz}), 130.2,129.3,127.4,124.0\left(\mathrm{~d}, \mathrm{C}-\mathrm{F},{ }^{3} J_{C-F}=8.5 \mathrm{~Hz}\right), 118.6\left(\mathrm{~d}, \mathrm{C}-\mathrm{F},{ }^{2} J_{C-F}=22.8 \mathrm{~Hz}\right), 117.4(\mathrm{~d}$, C-F, $\left.{ }^{3} J_{C-F}=7.8 \mathrm{~Hz}\right), 111.8\left(\mathrm{~d}, \mathrm{C}-\mathrm{F},{ }^{2} J_{C-F}=25.2 \mathrm{~Hz}\right), 92.8\left(\mathrm{~d}, \mathrm{C}-\mathrm{F},{ }^{4} J_{C-F}=1.8 \mathrm{~Hz}\right), 85.6,41.3,40.5,36.0$, 31.1, 21.1.

IR (ATR, neat): 2916, 2853, 1765, 1741, 1557, 1515, 1485, 1455, 1338, 1316, 1263, 1226, 1164, 1101.

HRMS (ESI) calcd $(\mathrm{m} / \mathrm{z})$ for $\mathrm{C}_{27} \mathrm{H}_{27} \mathrm{FN}_{2} \mathrm{O}_{5}:[\mathrm{M}+\mathrm{Na}]^{+}$501.1796, found 501.1791.

Adamantyl (R)-5-fluoro-3-(3-methylbenzyl)-3-nitro-2-oxoindoline-1-carboxylate (3j)

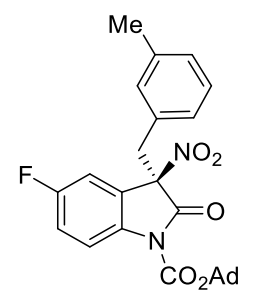

Prepared according to the GP5 to provide the title compound as white solid (67 $\mathrm{mg}, 70 \%$ yield). Enantiomeric excess was determined by HPLC with a Daicel CHIRALPAK® IC-U column, (Hexane/ $i$ $\operatorname{PrOH}=98 / 2, v=0.3 \mathrm{~mL} / \mathrm{min}, \lambda=254 \mathrm{~nm}), \mathrm{t}($ minor $)=5.5 \mathrm{~min}, \mathrm{t}($ major $)=8.1 \mathrm{~min}, 93 \%$ ee. $[\alpha]_{\mathrm{D}}{ }^{20}=$ $54.4\left(c=0.1\right.$ in $\left.\mathrm{CH}_{2} \mathrm{Cl}_{2}\right)$.

m.p. $158-160{ }^{\circ} \mathrm{C}$.

${ }^{1}$ H NMR (400 MHz, $\left.\mathrm{CDCl}_{3}\right) \delta 7.71(\mathrm{dd}, J=8.9,4.4 \mathrm{~Hz}, 1 \mathrm{H}), 7.16-7.04(\mathrm{~m}, 2 \mathrm{H})$, 7.04-6.93 (m, 2H), 6.76 $(\mathrm{d}, J=2.1 \mathrm{~Hz}, 1 \mathrm{H}), 6.69(\mathrm{dd}, J=7.0,2.0 \mathrm{~Hz}, 1 \mathrm{H}), 3.84(\mathrm{~d}, J=13.2 \mathrm{~Hz}, 1 \mathrm{H}), 3.77(\mathrm{~d}, J=13.2 \mathrm{~Hz}, 1 \mathrm{H})$, 2.34-2.18 (m, 12H), $1.70(\mathrm{~m}, 6 \mathrm{H})$.

${ }^{13} \mathrm{C}$ NMR $\left(100 \mathrm{MHz}, \mathrm{CDCl}_{3}\right) \delta 166.1,159.7\left(\mathrm{~d}, \mathrm{C}-\mathrm{F},{ }^{1} J_{\mathrm{C}-F}=246.4 \mathrm{~Hz}\right), 147.4,138.2,136.9\left(\mathrm{~d}, \mathrm{C}-\mathrm{F},{ }^{4} J_{C-}\right.$ $\left.F_{F}=2.6 \mathrm{~Hz}\right), 131.0,130.4,128.7,128.4,127.3,124.0\left(\mathrm{~d}, \mathrm{C}-\mathrm{F},{ }^{3} J_{C-F}=8.5 \mathrm{~Hz}\right), 118.5\left(\mathrm{~d}, \mathrm{C}-\mathrm{F},{ }^{2} J_{C-F}=22.8\right.$ $\mathrm{Hz}), 117.3$ (d, C-F, $\left.{ }^{3} J_{C-F}=7.7 \mathrm{~Hz}\right), 111.8\left(\mathrm{~d}, \mathrm{C}-\mathrm{F},{ }^{2} J_{C-F}=25.2 \mathrm{~Hz}\right), 92.8\left(\mathrm{~d}, \mathrm{C}-\mathrm{F},{ }^{4} J_{C-F}=1.8 \mathrm{~Hz}\right), 85.6$, $41.3,40.9,36.0,31.1,21.3$.

IR (ATR, neat): 2915, 2860, 1769, 1742, 1611, 1553, 1484, 1454, 1337, 1319, 1240, 1226, 1161.

HRMS (ESI) calcd $(\mathrm{m} / \mathrm{z})$ for $\mathrm{C}_{27} \mathrm{H}_{27} \mathrm{FN}_{2} \mathrm{O}_{5}:[\mathrm{M}+\mathrm{Na}]^{+}$501.1796, found 501.1794.

Adamantyl (R)-5-fluoro-3-(2-methylbenzyl)-3-nitro-2-oxoindoline-1-carboxylate (3k) 


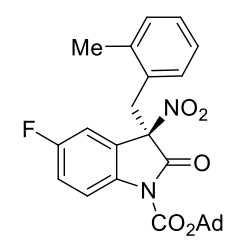

Prepared according to the GP5 to provide the title compound as colorless oil (65 $\mathrm{mg}$, 68\% yield). Enantiomeric excess was determined by HPLC with a Daicel CHIRALPAK ${ }^{\circ}$ IC-U column, (Hexane/ $i$ $\mathrm{PrOH}=98 / 2, v=0.3 \mathrm{~mL} / \mathrm{min}, \lambda=254 \mathrm{~nm}), \mathrm{t}($ minor $)=4.9 \mathrm{~min}, \mathrm{t}($ major $)=9.1 \mathrm{~min}, 89 \%$ ee. $[\alpha]_{\mathrm{D}}^{20}=-$ $36.3\left(c=0.1\right.$ in $\left.\mathrm{CH}_{2} \mathrm{Cl}_{2}\right)$.

${ }^{1}$ H NMR $\left(400 \mathrm{MHz}, \mathrm{CDCl}_{3}\right) \delta 7.77(\mathrm{dd}, J=9.1,4.4 \mathrm{~Hz}, 1 \mathrm{H}), 7.10(\mathrm{~m}, 3 \mathrm{H}), 7.01-6.94(\mathrm{~m}, 1 \mathrm{H}), 6.90(\mathrm{dd}$, $J=7.3,2.8 \mathrm{~Hz}, 1 \mathrm{H}), 6.84(\mathrm{dd}, J=7.8,1.3 \mathrm{~Hz}, 1 \mathrm{H}), 3.99$ (d, $J=14.1 \mathrm{~Hz}, 1 \mathrm{H}), 3.82$ (d, $J=14.1 \mathrm{~Hz}, 1 \mathrm{H})$, 2.28-2.20 (m, 9H), $2.11(\mathrm{~s}, 3 \mathrm{H}), 1.70(\mathrm{~m}, 6 \mathrm{H})$.

${ }^{13}$ C NMR $\left(100 \mathrm{MHz}, \mathrm{CDCl}_{3}\right) \delta 166.2,159.6\left(\mathrm{~d}, \mathrm{C}-\mathrm{F},{ }^{1} J_{\mathrm{C}-\mathrm{F}}=246.4 \mathrm{~Hz}\right), 147.4,137.6,136.8\left(\mathrm{~d}, \mathrm{C}-\mathrm{F},{ }^{4} J_{C-}\right.$ $\left.F_{F}=2.6 \mathrm{~Hz}\right), 131.0,130.0,129.4,128.2,126.1,124.0\left(\mathrm{~d}, \mathrm{C}-\mathrm{F},{ }^{3} J_{C-F}=8.5 \mathrm{~Hz}\right), 118.5\left(\mathrm{~d}, \mathrm{C}-\mathrm{F},{ }^{2} J_{C-F}=22.8\right.$ $\mathrm{Hz}), 117.2\left(\mathrm{~d}, \mathrm{C}-\mathrm{F},{ }^{3} J_{C-F}=7.7 \mathrm{~Hz}\right), 112.3\left(\mathrm{~d}, \mathrm{C}-\mathrm{F},{ }^{2} J_{C-F}=25.4 \mathrm{~Hz}\right), 92.8\left(\mathrm{~d}, \mathrm{C}-\mathrm{F},{ }^{4} J_{C-F}=1.7 \mathrm{~Hz}\right), 85.7$, $41.3,37.3,36.0,31.1,19.9$.

IR (ATR, neat): 2912, 2853, 1781, 1739, 1654, 1610, 1558, 1486, 1457, 1340, 1290, 1264, 1245, 1161, 1098.

HRMS (ESI) calcd $(m / z)$ for $\mathrm{C}_{27} \mathrm{H}_{27} \mathrm{FN}_{2} \mathrm{O}_{5}:[\mathrm{M}+\mathrm{Na}]^{+}$501.1796, found 501.1792.

Adamantyl (R)-5-fluoro-3-(4-methoxybenzyl)-3-nitro-2-oxoindoline-1-carboxylate (3l)

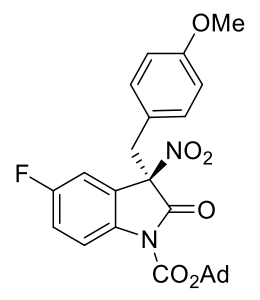

Prepared according to the GP5 to provide the title compound as white solid ( $73 \mathrm{mg}, 74 \%$ yield). Enantiomeric excess was determined by HPLC with a Daicel CHIRALPAK ${ }^{\circ}$ IC-U column, (Hexane/i$\operatorname{PrOH}=98 / 2, v=0.3 \mathrm{~mL} / \mathrm{min}, \lambda=254 \mathrm{~nm}), \mathrm{t}$ (minor) $=9.2 \mathrm{~min}, \mathrm{t}($ major $)=12.6 \mathrm{~min}, 88 \%$ ee. $[\alpha]_{\mathrm{D}}^{20}=$ $52.3\left(c=0.1\right.$ in $\left.\mathrm{CH}_{2} \mathrm{Cl}_{2}\right)$.

m.p. $152-154{ }^{\circ} \mathrm{C}$.

${ }^{1}$ H NMR (400 MHz, $\left.\mathrm{CDCl}_{3}\right) \delta 7.72(\mathrm{dd}, J=8.9,4.4 \mathrm{~Hz}, 1 \mathrm{H}), 7.19-7.03(\mathrm{~m}, 2 \mathrm{H}), 6.84(\mathrm{~d}, J=8.7 \mathrm{~Hz}, 2 \mathrm{H})$, $6.66(\mathrm{~d}, J=8.7 \mathrm{~Hz}, 2 \mathrm{H}), 3.82(\mathrm{~d}, J=13.3 \mathrm{~Hz}, 1 \mathrm{H}), 3.76(\mathrm{~d}, J=13.5 \mathrm{~Hz}, 1 \mathrm{H}), 3.71(\mathrm{~s}, 3 \mathrm{H}), 2.23(\mathrm{~m}, 9 \mathrm{H})$, $1.70(\mathrm{~m}, 6 \mathrm{H})$.

${ }^{13}$ C NMR $\left(100 \mathrm{MHz}, \mathrm{CDCl}_{3}\right) \delta 166.1,159.7\left(\mathrm{~d}, \mathrm{C}-\mathrm{F},{ }^{1} J_{\mathrm{C}-\mathrm{F}}=246.3 \mathrm{~Hz}\right), 159.2,147.4,137.0\left(\mathrm{~d}, \mathrm{C}-\mathrm{F},{ }^{4} J_{C-}\right.$ 
$\left.F_{F}=2.6 \mathrm{~Hz}\right), 131.5,124.0\left(\mathrm{~d}, \mathrm{C}-\mathrm{F},{ }^{3} J_{C-F}=8.5 \mathrm{~Hz}\right), 122.3,118.6\left(\mathrm{~d}, \mathrm{C}-\mathrm{F},{ }^{2} J_{C-F}=22.7 \mathrm{~Hz}\right), 117.4(\mathrm{~d}, \mathrm{C}-\mathrm{F}$, $\left.{ }^{3} J_{C-F}=7.9 \mathrm{~Hz}\right), 114.0,111.9\left(\mathrm{~d}, \mathrm{C}-\mathrm{F},{ }^{2} J_{C-F}=25.2 \mathrm{~Hz}\right), 92.8\left(\mathrm{~d}, \mathrm{C}-\mathrm{F},{ }^{4} J_{C-F}=1.7 \mathrm{~Hz}\right), 85.7,55.2,41.3,40.2$, $36.0,31.1$.

IR (ATR, neat): 2922, 2852, 1780, 1735, 1654, 1610, 1557, 1486, 1341, 1265, 1250, 1130.

HRMS (ESI) calcd $(m / z)$ for $\mathrm{C}_{27} \mathrm{H}_{27} \mathrm{FN}_{2} \mathrm{O}_{6}:[\mathrm{M}+\mathrm{Na}]^{+}$517.1745, found 517.1749.

Adamantyl (R)-5-fluoro-3-(3,5-dimethylbenzyl)-3-nitro-2-oxoindoline-1-carboxylate (3m)

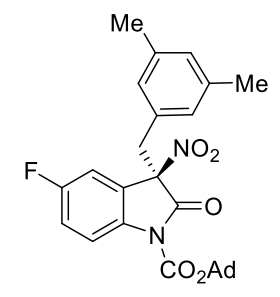

Prepared according to the GP5 to provide the title compound as white solid (74 $\mathrm{mg}, 75 \%$ yield). Enantiomeric excess was determined by HPLC with a Daicel CHIRALPAK ${ }^{\circledR}$ IC-U column, (Hexane $/ i$ $\mathrm{PrOH}=98 / 2, v=0.3 \mathrm{~mL} / \mathrm{min}, \lambda=254 \mathrm{~nm}), \mathrm{t}($ minor $)=8.1 \mathrm{~min}, \mathrm{t}($ major $)=10.6 \mathrm{~min}, 88 \%$ ee. $[\alpha]_{\mathrm{D}}{ }^{20}=-$ $58.3\left(c=0.1\right.$ in $\left.\mathrm{CH}_{2} \mathrm{Cl}_{2}\right)$.

m.p. $143-145^{\circ} \mathrm{C}$.

${ }^{1}$ H NMR $\left(400 \mathrm{MHz}, \mathrm{CDCl}_{3}\right) \delta 7.71(\mathrm{ddd}, J=8.3,4.4,1.6 \mathrm{~Hz}, 1 \mathrm{H}), 7.08(\mathrm{t}, J=8.2 \mathrm{~Hz}, 2 \mathrm{H}), 6.79(\mathrm{~s}, 1 \mathrm{H})$, $6.53(\mathrm{~s}, 2 \mathrm{H}), 3.80(\mathrm{~d}, J=13.2 \mathrm{~Hz}, 1 \mathrm{H}), 3.73(\mathrm{~d}, J=13.1 \mathrm{~Hz}, 1 \mathrm{H}), 2.32-2.20(\mathrm{~m}, 9 \mathrm{H}), 2.14(\mathrm{~s}, 6 \mathrm{H}), 1.70$ $(\mathrm{m}, 6 \mathrm{H})$.

${ }^{13} \mathrm{C} \mathrm{NMR}\left(100 \mathrm{MHz}, \mathrm{CDCl}_{3}\right) \delta 166.1,159.7\left(\mathrm{~d}, \mathrm{C}-\mathrm{F},{ }^{1} J_{C-F}=246.2 \mathrm{~Hz}\right), 147.4,138.1,136.9\left(\mathrm{~d}, \mathrm{C}-\mathrm{F},{ }^{4} J_{C-}\right.$ $\left.F_{F}=2.6 \mathrm{~Hz}\right), 130.3,129.6,128.1,124.1\left(\mathrm{~d}, \mathrm{C}-\mathrm{F},{ }^{3} J_{C-F}=8.4 \mathrm{~Hz}\right), 118.4\left(\mathrm{~d}, \mathrm{C}-\mathrm{F},{ }^{2} J_{C-F}=22.8 \mathrm{~Hz}\right), 117.2(\mathrm{~d}$, C-F, $\left.{ }^{3} J_{C-F}=7.8 \mathrm{~Hz}\right), 111.9\left(\mathrm{~d}, \mathrm{C}-\mathrm{F},{ }^{2} J_{C-F}=25.2 \mathrm{~Hz}\right), 92.9\left(\mathrm{~d}, \mathrm{C}-\mathrm{F},{ }^{4} J_{C-F}=1.9 \mathrm{~Hz}\right), 85.6,41.3,40.9,36.0$, 31.1, 21.2.

IR (ATR, neat): 2914, 2853, 1779, 1741, 1610, 1559, 1487, 1340, 1319, 1264, 1244, 1159.

HRMS (ESI) calcd $(m / z)$ for $\mathrm{C}_{28} \mathrm{H}_{29} \mathrm{FN}_{2} \mathrm{O}_{5}:[\mathrm{M}+\mathrm{Na}]^{+}$515.1953, found 515.1949.

Adamantyl (R)-5-fluoro-3-(3,5-dimethoxybenzyl)-3-nitro-2-oxoindoline-1-carboxylate (3n)

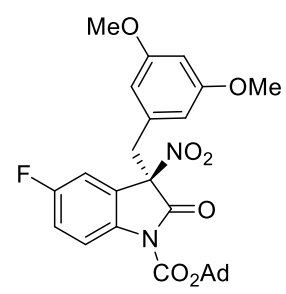

Prepared according to the GP5 to provide the title compound as colorless oil ( $82 \mathrm{mg}$, $78 \%$ yield). Enantiomeric excess was determined by HPLC with a Daicel CHIRALPAK ${ }^{\circ}$ IC-U column, (Hexane/i- 
$\mathrm{PrOH}=98 / 2, v=0.3 \mathrm{~mL} / \mathrm{min}, \lambda=254 \mathrm{~nm}), \mathrm{t}($ minor $)=8.3 \mathrm{~min}, \mathrm{t}($ major $)=10.8 \mathrm{~min}, 94 \%$ ee. $[\alpha]_{\mathrm{D}}{ }^{20}=-$ $55.0\left(c=0.1\right.$ in $\left.\mathrm{CH}_{2} \mathrm{Cl}_{2}\right)$.

${ }^{1}$ H NMR (400 MHz, $\left.\mathrm{CDCl}_{3}\right) \delta 7.75(\mathrm{dd}, J=9.0,4.4 \mathrm{~Hz}, 1 \mathrm{H}), 7.18-7.06(\mathrm{~m}, 2 \mathrm{H}), 6.25(\mathrm{t}, J=2.3 \mathrm{~Hz}, 1 \mathrm{H})$, $6.06(\mathrm{~d}, J=2.3 \mathrm{~Hz}, 2 \mathrm{H}), 3.79(\mathrm{~s}, 2 \mathrm{H}), 3.62(\mathrm{~s}, 6 \mathrm{H}), 2.31-2.17(\mathrm{~m}, 9 \mathrm{H}), 1.70(\mathrm{~m}, 6 \mathrm{H})$.

${ }^{13} \mathrm{C}$ NMR $\left(100 \mathrm{MHz}, \mathrm{CDCl}_{3}\right) \delta 166.1,160.7,159.7\left(\mathrm{~d}, \mathrm{C}-\mathrm{F},{ }^{1} J_{C-F}=246.7 \mathrm{~Hz}\right), 147.4,137.1\left(\mathrm{~d}, \mathrm{C}-\mathrm{F},{ }^{4} J_{C-}\right.$ $F=2.6 \mathrm{~Hz}), 132.6,124.1\left(\mathrm{~d}, \mathrm{C}-\mathrm{F},{ }^{3} J_{C-F}=8.5 \mathrm{~Hz}\right), 118.6\left(\mathrm{~d}, \mathrm{C}-\mathrm{F},{ }^{2} J_{C-F}=22.8 \mathrm{~Hz}\right), 117.5\left(\mathrm{~d}, \mathrm{C}-\mathrm{F},{ }^{3} J_{C-F}=\right.$ $7.7 \mathrm{~Hz}), 111.7\left(\mathrm{~d}, \mathrm{C}-\mathrm{F},{ }^{2} J_{\mathrm{C}-F}=25.3 \mathrm{~Hz}\right), 108.1,100.5,92.7$ (d, C-F, $\left.{ }^{4} J_{C-F}=1.7 \mathrm{~Hz}\right), 85.7,55.3,41.3,41.1$, $36.0,31.1$.

IR (ATR, neat): 2913, 2853, 1779, 1739, 1608, 1598, 1557, 1486, 1459, 1431, 1340, 1294, 1262, 1244, 1207, 1157, 1106.

HRMS (ESI) calcd $(m / z)$ for $\mathrm{C}_{28} \mathrm{H}_{29} \mathrm{FN}_{2} \mathrm{O}_{7}:[\mathrm{M}+\mathrm{Na}]^{+}$547.1934, found 547.1938.

Adamantyl (R)-5-fluoro-3-([1,1'-biphenyl]-4-ylmethyl)-3-nitro-2-oxoindoline-1-carboxylate (3o)

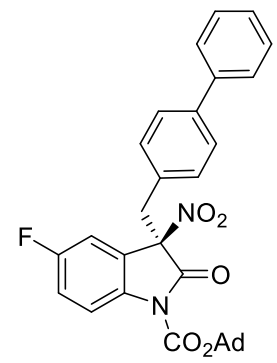

Prepared according to the GP5 to provide the title compound as colorless oil (78 $\mathrm{mg}, 72 \%$ yield). Enantiomeric excess was determined by HPLC with a Daicel CHIRALPAK ${ }^{\text {IC }}$-U column, (Hexane/ $i$ $\mathrm{PrOH}=98 / 2, v=0.3 \mathrm{~mL} / \mathrm{min}, \lambda=254 \mathrm{~nm}), \mathrm{t}($ minor $)=9.2 \mathrm{~min}, \mathrm{t}($ major $)=12.7 \mathrm{~min}, 94 \%$ ee. $[\alpha]_{\mathrm{D}}{ }^{20}=-$ $32.5\left(c=0.1\right.$ in $\left.\mathrm{CH}_{2} \mathrm{Cl}_{2}\right)$.

${ }^{1}$ H NMR (400 MHz, $\left.\mathrm{CDCl}_{3}\right) \delta 7.71(\mathrm{dd}, J=9.0,4.4 \mathrm{~Hz}, 1 \mathrm{H}), 7.51-7.46(\mathrm{~m}, 2 \mathrm{H}), 7.42-7.35$ (m, 4H), 7.34$7.29(\mathrm{~m}, 1 \mathrm{H}), 7.17(\mathrm{dd}, J=7.1,2.7 \mathrm{~Hz}, 1 \mathrm{H}), 7.10(\mathrm{td}, J=8.9,2.8 \mathrm{~Hz}, 1 \mathrm{H}), 6.99(\mathrm{~d}, J=8.2 \mathrm{~Hz}, 2 \mathrm{H}), 3.91$ $(\mathrm{d}, J=13.2 \mathrm{~Hz}, 1 \mathrm{H}), 3.86(\mathrm{~d}, J=13.2 \mathrm{~Hz}, 1 \mathrm{H}), 2.25-2.16(\mathrm{~m}, 9 \mathrm{H}), 1.66(\mathrm{~m}, 6 \mathrm{H})$.

${ }^{13} \mathrm{C}$ NMR $\left(100 \mathrm{MHz}, \mathrm{CDCl}_{3}\right) \delta 166.0,159.7\left(\mathrm{~d}, \mathrm{C}-\mathrm{F},{ }^{1} J_{\mathrm{C}-F}=246.6 \mathrm{~Hz}\right), 147.3,140.8,140.1,137.0(\mathrm{~d}, \mathrm{C}-$ $\left.\mathrm{F},{ }^{4} J_{C-F}=2.3 \mathrm{~Hz}\right), 130.8,129.5,128.9,127.6,127.2,127.0,123.9\left(\mathrm{~d}, \mathrm{C}-\mathrm{F},{ }^{3} J_{C-F}=8.3 \mathrm{~Hz}\right), 118.7(\mathrm{~d}, \mathrm{C}-\mathrm{F}$, $\left.{ }^{2} J_{C-F}=22.7 \mathrm{~Hz}\right), 117.5\left(\mathrm{~d}, \mathrm{C}-\mathrm{F},{ }^{3} J_{C-F}=7.8 \mathrm{~Hz}\right), 111.8\left(\mathrm{~d}, \mathrm{C}-\mathrm{F},{ }^{2} J_{C-F}=25.4 \mathrm{~Hz}\right), 92.7\left(\mathrm{~d}, \mathrm{C}-\mathrm{F},{ }^{4} J_{C-F}=1.5\right.$ Hz), 85.8, 41.3, 40.5, 36.0, 31.1.

IR (ATR, neat): 2912, 2853, 1804, 1780, 1736, 1701, 1655, 1557, 1486, 1456, 1340, 1313, 1263, 1243, 1159, 1103.

HRMS (ESI) calcd $(\mathrm{m} / \mathrm{z})$ for $\mathrm{C}_{32} \mathrm{H}_{29} \mathrm{FN}_{2} \mathrm{O}_{5}:[\mathrm{M}+\mathrm{Na}]^{+}$563.1949, found 563.1942. 


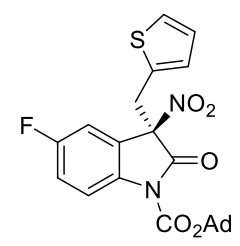

Prepared according to the GP5 to provide the title compound as colorless oil (78 $\mathrm{mg}$, 83\% yield). Enantiomeric excess was determined by HPLC with a Daicel CHIRALPAK ${ }^{\circ}$ IC-U column, (Hexane/ $i$ $\operatorname{PrOH}=98 / 2, v=0.3 \mathrm{~mL} / \mathrm{min}, \lambda=254 \mathrm{~nm}), \mathrm{t}($ minor $)=5.5 \mathrm{~min}, \mathrm{t}($ major $)=8.6 \mathrm{~min}, 93 \%$ ee. $[\alpha]_{\mathrm{D}}{ }^{20}=$ $52.6\left(c=0.1\right.$ in $\left.\mathrm{CH}_{2} \mathrm{Cl}_{2}\right)$.

${ }^{1}$ H NMR $\left(400 \mathrm{MHz}, \mathrm{CDCl}_{3}\right) \delta 7.80(\mathrm{dd}, J=9.1,4.4 \mathrm{~Hz}, 1 \mathrm{H}), 7.19-7.03(\mathrm{~m}, 3 \mathrm{H}), 6.82(\mathrm{dd}, J=5.1,3.5$ $\mathrm{Hz}, 1 \mathrm{H}), 6.73(\mathrm{~d}, J=3.5 \mathrm{~Hz}, 1 \mathrm{H}), 4.11(\mathrm{~d}, J=14.5 \mathrm{~Hz}, 1 \mathrm{H}), 4.04(\mathrm{~d}, J=14.4 \mathrm{~Hz}, 1 \mathrm{H}), 2.24(\mathrm{~m}, 9 \mathrm{H}), 1.70$ $(\mathrm{m}, 6 \mathrm{H})$.

${ }^{13} \mathrm{C}$ NMR $\left(100 \mathrm{MHz}, \mathrm{CDCl}_{3}\right) \delta 165.7,159.8\left(\mathrm{~d}, \mathrm{C}-\mathrm{F},{ }^{1} J_{C-F}=246.8 \mathrm{~Hz}\right), 147.4,137.3\left(\mathrm{~d}, \mathrm{C}-\mathrm{F},{ }^{4} J_{C-F}=2.7\right.$ $\mathrm{Hz}), 131.7,129.1,127.1,126.4,123.8\left(\mathrm{~d}, \mathrm{C}-\mathrm{F},{ }^{3} J_{C-F}=8.5 \mathrm{~Hz}\right), 118.9$ (d, C-F, $\left.{ }^{2} J_{C-F}=22.8 \mathrm{~Hz}\right), 117.5(\mathrm{~d}$, C-F, $\left.{ }^{3} J_{C-F}=7.9 \mathrm{~Hz}\right), 111.8\left(\mathrm{~d}, \mathrm{C}-\mathrm{F},{ }^{2} J_{C-F}=25.3 \mathrm{~Hz}\right), 92.0\left(\mathrm{~d}, \mathrm{C}-\mathrm{F},{ }^{4} J_{C-F}=1.8 \mathrm{~Hz}\right), 85.9,41.3,36.0,35.0$, 31.1 .

IR (ATR, neat): 2914, 2854, 1805, 1782, 1739, 1620, 1558, 1486, 1457, 1431, 1340, 1313, 1292, 1261, 1243, 1166, 1132, 1104.

HRMS (ESI) calcd $(m / z)$ for $\mathrm{C}_{24} \mathrm{H}_{23} \mathrm{FN}_{2} \mathrm{O}_{5} \mathrm{~S}:[\mathrm{M}+\mathrm{Na}]^{+}$493.1204, found 493.1201.

Adamantyl (R)-5-fluoro-3-(benzo[b]thiophen-2-ylmethyl)-3-nitro-2-oxoindoline-1-carboxylate (3q)

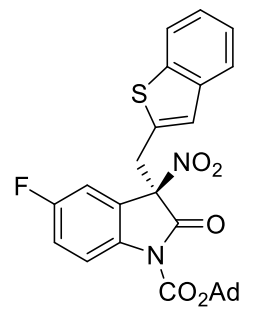

Prepared according to the GP5 to provide the title compound as colorless oil ( $81 \mathrm{mg}$, 78\% yield). Enantiomeric excess was determined by HPLC with a Daicel CHIRALPAK® IC-U column, (Hexane/ $i$ $\operatorname{PrOH}=98 / 2, v=0.3 \mathrm{~mL} / \mathrm{min}, \lambda=254 \mathrm{~nm}), \mathrm{t}($ minor $)=7.2 \mathrm{~min}, \mathrm{t}($ major $)=9.1 \mathrm{~min}, 90 \%$ ee. $[\alpha]_{\mathrm{D}}{ }^{20}=$ $45.4\left(c=0.1\right.$ in $\left.\mathrm{CH}_{2} \mathrm{Cl}_{2}\right)$.

${ }^{1} \mathbf{H}$ NMR $\left(400 \mathrm{MHz}, \mathrm{CDCl}_{3}\right) \delta 7.78(\mathrm{dd}, J=8.8,4.4 \mathrm{~Hz}, 1 \mathrm{H}), 7.69-7.59(\mathrm{~m}, 2 \mathrm{H}), 7.28(\mathrm{ddd}, J=14.3,7.1$, 1.6 Hz, 2H), 7.20-7.09 (m, 2H), 7.03 (s, 1H), 4.16 (s, 2H), 2.27-2.16 (m, 9H), 1.67 (m, 6H).

${ }^{13} \mathrm{C}$ NMR $\left(100 \mathrm{MHz}, \mathrm{CDCl}_{3}\right) \delta 165.7,159.9\left(\mathrm{~d}, \mathrm{C}-\mathrm{F},{ }^{1} J_{\mathrm{C}-\mathrm{F}}=247.0 \mathrm{~Hz}\right), 147.3,140.2,139.2,137.5(\mathrm{~d}, \mathrm{C}-$ $\left.\mathrm{F},{ }^{4} J_{C-F}=2.7 \mathrm{~Hz}\right), 132.9,126.1,124.7,124.5,123.6\left(\mathrm{~d}, \mathrm{C}-\mathrm{F},{ }^{3} J_{C-F}=8.3 \mathrm{~Hz}\right), 123.6,122.1,119.2(\mathrm{~d}, \mathrm{C}-\mathrm{F}$, 
$\left.{ }^{2} J_{C-F}=22.7 \mathrm{~Hz}\right), 117.8\left(\mathrm{~d}, \mathrm{C}-\mathrm{F},{ }^{3} J_{C-F}=7.8 \mathrm{~Hz}\right), 111.8\left(\mathrm{~d}, \mathrm{C}-\mathrm{F},{ }^{2} J_{C-F}=25.3 \mathrm{~Hz}\right), 91.8\left(\mathrm{~d}, \mathrm{C}-\mathrm{F},{ }^{4} J_{C-F}=1.8\right.$ $\mathrm{Hz}), 86.0,41.2,36.0,35.6,31.1$.

IR (ATR, neat): 2914, 2853, 1807, 1778, 1735, 1657, 1615, 1555, 1487, 1457, 1339, 1313, 1265, 1239, $1164,1126,1109$.

HRMS (ESI) calcd $(m / z)$ for $\mathrm{C}_{28} \mathrm{H}_{25} \mathrm{FN}_{2} \mathrm{O}_{5} \mathrm{~S}:\left[\mathrm{M}+\mathrm{NH}_{4}\right]^{+}$538.1806, found 538.1810.

Adamantyl (R)-6-chloro-3-(4-(benzyloxy)benzyl)-3-nitro-2-oxoindoline-1-carboxylate (3r)

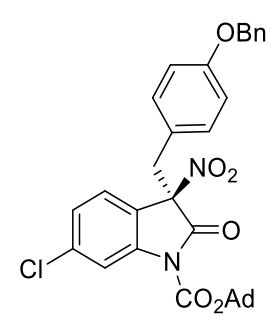

Prepared according to the GP5 to provide the title compound as colorless oil ( $89 \mathrm{mg}$, $76 \%$ yield). Enantiomeric excess was determined by HPLC with a Daicel CHIRALPAK® IC-U column, (Hexane/i$\operatorname{PrOH}=98 / 2, v=0.3 \mathrm{~mL} / \mathrm{min}, \lambda=254 \mathrm{~nm}), \mathrm{t}$ (minor) $=9.5 \mathrm{~min}, \mathrm{t}($ major $)=12.1 \mathrm{~min}, 93 \%$ ee. $[\alpha]_{\mathrm{D}}^{20}=$ $50.1\left(c=0.1\right.$ in $\left.\mathrm{CH}_{2} \mathrm{Cl}_{2}\right)$.

${ }^{1}$ H NMR $\left(400 \mathrm{MHz}, \mathrm{CDCl}_{3}\right) \delta 7.80(\mathrm{~d}, J=1.9 \mathrm{~Hz}, 1 \mathrm{H}), 7.36(\mathrm{~d}, J=4.3 \mathrm{~Hz}, 5 \mathrm{H}), 7.30(\mathrm{~d}, J=8.2 \mathrm{~Hz}, 1 \mathrm{H})$, $7.21(\mathrm{dd}, J=8.1,1.9 \mathrm{~Hz}, 1 \mathrm{H}), 6.86-6.79(\mathrm{~m}, 2 \mathrm{H}), 6.78-6.70(\mathrm{~m}, 2 \mathrm{H}), 4.96$ (s, 2H), 3.83 (d, $J=13.3 \mathrm{~Hz}$, $1 \mathrm{H}), 3.74(\mathrm{~d}, J=13.4 \mathrm{~Hz}, 1 \mathrm{H}), 2.26-2.17(\mathrm{~m}, 9 \mathrm{H}), 1.70(\mathrm{~m}, 6 \mathrm{H})$.

${ }^{13}$ C NMR $\left(100 \mathrm{MHz}, \mathrm{CDCl}_{3}\right) \delta 166.0,158.5,147.2,141.8,137.8,136.7,131.5,128.6,128.1,127.5,125.2$, $125.2,122.7,121.0,116.7,115.0,92.6,86.0,70.0,41.3,40.0,36.0,31.1$.

IR (ATR, neat): 2912, 2854, 1809, 1782, 1737, 1608, 1556, 1512, 1483, 1455, 1426, 1342, 1267, 1238, $1157,1108$.

HRMS (ESI) calcd $(m / z)$ for $\mathrm{C}_{33} \mathrm{H}_{31} \mathrm{ClN}_{2} \mathrm{O}_{6}:\left[\mathrm{M}+\mathrm{NH}_{4}\right]^{+}$604.2119, found 604.2113.

Adamantyl (R)-6-methoxy-3-(4-chlorobenzyl)-3-nitro-2-oxoindoline-1-carboxylate (3s)

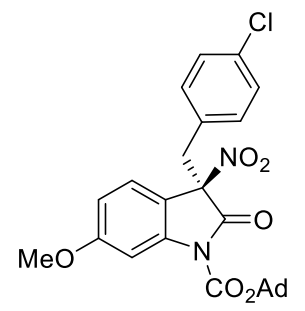

Prepared according to the GP5 to provide the title compound as colorless oil (60 $\mathrm{mg}, 59 \%$ yield). Enantiomeric excess was determined by HPLC with a Daicel CHIRALPAK® IC-U column, (Hexane/i$\mathrm{PrOH}=95 / 5, v=0.3 \mathrm{~mL} / \mathrm{min}, \lambda=254 \mathrm{~nm}), \mathrm{t}$ (minor) $=6.6 \mathrm{~min}, \mathrm{t}$ (major) $=8.4 \mathrm{~min}, 88 \% e e .[\alpha]_{\mathrm{D}}^{20}=-$ $66.9\left(c=0.1\right.$ in $\left.\mathrm{CH}_{2} \mathrm{Cl}_{2}\right)$. 
${ }^{1} \mathbf{H}$ NMR $\left(400 \mathrm{MHz}, \mathrm{CDCl}_{3}\right) \delta 7.33(\mathrm{~d}, J=2.4 \mathrm{~Hz}, 1 \mathrm{H}), 7.30(\mathrm{~d}, J=8.5 \mathrm{~Hz}, 1 \mathrm{H}), 7.10(\mathrm{~d}, J=8.4 \mathrm{~Hz}, 2 \mathrm{H})$, $6.86(\mathrm{~d}, J=8.4 \mathrm{~Hz}, 2 \mathrm{H}), 6.74(\mathrm{dd}, J=8.5,2.4 \mathrm{~Hz}, 1 \mathrm{H}), 3.84(\mathrm{~d}, J=13.2 \mathrm{~Hz}, 1 \mathrm{H}), 3.82(\mathrm{~s}, 3 \mathrm{H}), 3.76(\mathrm{~d}, J$ $=13.2 \mathrm{~Hz}, 1 \mathrm{H}), 2.27-2.18(\mathrm{~m}, 9 \mathrm{H}), 1.70(\mathrm{~m}, 6 \mathrm{H})$.

${ }^{13} \mathrm{C} \mathrm{NMR}\left(100 \mathrm{MHz}, \mathrm{CDCl}_{3}\right) \delta 166.7,162.6,147.3,142.4,133.9,131.8,129.8,128.7,125.0,114.0,110.9$, $102.3,92.8,85.7,55.7,41.3,39.7,36.0,31.1$.

IR (ATR, neat): 2914, 2854, 1802, 1779, 1736, 1617, 1554, 1493, 1449, 1345, 1316, 1345, 1316, 1247 , 1207, 1125, 1113.

HRMS (ESI) calcd $(m / z)$ for $\mathrm{C}_{27} \mathrm{H}_{27} \mathrm{ClN}_{2} \mathrm{O}_{6}:[\mathrm{M}+\mathrm{H}]^{+}$511.1630, found 511.1635.

Adamantyl (R)-6-methoxy-3-(4-(trifluoromethyl)benzyl)-3-nitro-2-oxoindoline-1-carboxylate (3t)

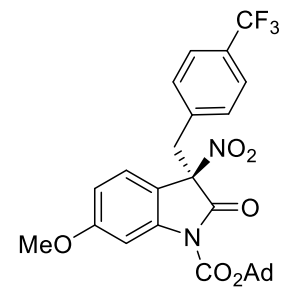

Prepared according to the GP5 to provide the title compound as colorless oil (67 $\mathrm{mg}, 61 \%$ yield). Enantiomeric excess was determined by HPLC with a Daicel CHIRALPAK ${ }^{\circ}$ IC-U column, (Hexane/i$\mathrm{PrOH}=95 / 5, v=0.3 \mathrm{~mL} / \mathrm{min}, \lambda=254 \mathrm{~nm}), \mathrm{t}$ (minor) $=5.2 \mathrm{~min}, \mathrm{t}$ (major) $=5.8 \mathrm{~min}, 89 \% e e .[\alpha]_{\mathrm{D}}^{20}=-$ $64.4\left(c=0.1\right.$ in $\left.\mathrm{CH}_{2} \mathrm{Cl}_{2}\right)$.

${ }^{1} \mathbf{H}$ NMR $\left(400 \mathrm{MHz}, \mathrm{CDCl}_{3}\right) \delta 7.39(\mathrm{~d}, J=8.1 \mathrm{~Hz}, 2 \mathrm{H}), 7.33(\mathrm{dd}, J=5.5,3.0 \mathrm{~Hz}, 2 \mathrm{H}), 7.06(\mathrm{~d}, J=8.0$ $\mathrm{Hz}, 2 \mathrm{H}), 6.75(\mathrm{dd}, J=8.6,2.4 \mathrm{~Hz}, 1 \mathrm{H}), 3.93(\mathrm{~d}, J=13.1 \mathrm{~Hz}, 1 \mathrm{H}), 3.85$ (d, $J=13.2 \mathrm{~Hz}, 1 \mathrm{H}), 3.82(\mathrm{~s}, 3 \mathrm{H})$, $2.21(\mathrm{~m}, 9 \mathrm{H}), 1.69(\mathrm{~m}, 6 \mathrm{H})$.

${ }^{13} \mathrm{C}$ NMR $\left(100 \mathrm{MHz}, \mathrm{CDCl}_{3}\right) \delta 166.6,162.7,147.2,142.4,135.6(\mathrm{~d}, J=1.0 \mathrm{~Hz}), 130.9,130.0(\mathrm{q}, \mathrm{C}-\mathrm{F}$, $\left.{ }^{2} J_{C-F}=32.6 \mathrm{~Hz}\right), 125.3\left(\mathrm{q}, \mathrm{C}-\mathrm{F},{ }^{4} J_{C-F}=3.3 \mathrm{~Hz}\right), 124.9,123.9\left(\mathrm{q}, \mathrm{C}-\mathrm{F},{ }^{1} J_{C-F}=273.1 \mathrm{~Hz}\right), 113.8,111.0,102.4$, $92.7,85.8,55.7,41.3,40.1,36.0,31.1$.

IR (ATR, neat): 2913, 2852, 1802, 1777, 1735, 1610, 1554, 1492, 1448, 1421, 1325, 1289, 1245, 1209 , $1164,1123$.

HRMS (ESI) calcd $(m / z)$ for $\mathrm{C}_{28} \mathrm{H}_{27} \mathrm{~F}_{3} \mathrm{~N}_{2} \mathrm{O}_{6}:[\mathrm{M}+\mathrm{Na}]^{+}$567.1713, found 567.1709.

Adamantyl (R)-6-phenyl-3-(4-bromobenzyl)-3-nitro-2-oxoindoline-1-carboxylate (3u)

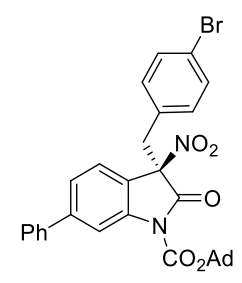

Prepared according to the GP5 to provide the title compound as yellow solid ( $83 \mathrm{mg}$, 69\% yield). 
Enantiomeric excess was determined by HPLC with a Daicel CHIRALPAK ${ }^{\circledR}$ IC-U column, (Hexane $/ i$ $\operatorname{PrOH}=98 / 2, v=0.3 \mathrm{~mL} / \mathrm{min}, \lambda=254 \mathrm{~nm}), \mathrm{t}($ minor $)=10.2 \mathrm{~min}, \mathrm{t}($ major $)=14.1 \mathrm{~min}, 91 \% e e .[\alpha]_{\mathrm{D}}{ }^{20}=$ $-71.0\left(c=0.1\right.$ in $\left.\mathrm{CH}_{2} \mathrm{Cl}_{2}\right)$.

m.p. $169-171^{\circ} \mathrm{C}$.

${ }^{1} \mathbf{H}$ NMR $\left(400 \mathrm{MHz}, \mathrm{CDCl}_{3}\right) \delta 8.01(\mathrm{~s}, 1 \mathrm{H}), 7.61-7.53(\mathrm{~m}, 2 \mathrm{H}), 7.49-7.35(\mathrm{~m}, 5 \mathrm{H})$, 7.30-7.22 (m, 2H), 6.89-6.80 (m, 2H), 3.89 (d, $J=13.3 \mathrm{~Hz}, 1 \mathrm{H}), 3.80$ (d, $J=13.3 \mathrm{~Hz}, 1 \mathrm{H}), 2.24$ (m, 9H), 1.70 (m, 6H).

${ }^{13}$ C NMR (100 MHz, $\left.\mathrm{CDCl}_{3}\right) \delta 166.4,147.3,145.5,141.5,139.9,132.1,131.7,130.1,129.0,128.4,127.3$, $124.3,123.9,122.3,121.0,114.8,92.7,85.8,41.3,39.9,36.0,31.1$.

IR (ATR, neat): 2917, 2854, 1777, 1741, 1615, 1552, 1483, 1457, 1425, 1345, 1318, 1250, 1207, 1163, $1129,1103$.

HRMS (ESI) calcd $(m / z)$ for $\mathrm{C}_{32} \mathrm{H}_{29} \mathrm{BrN}_{2} \mathrm{O}_{5}:[\mathrm{M}+\mathrm{H}]^{+}$601.1333, found 601.1334.

Adamantyl (R)-6-phenyl-3-(4-(tert-butoxycarbonyl)benzyl)-3-nitro-2-oxoindoline-1-carboxylate (3v)

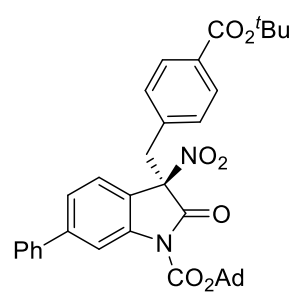

Prepared according to the GP5 to provide the title compound as white solid ( $95 \mathrm{mg}, 77 \%$ yield). Enantiomeric excess was determined by HPLC with a Daicel CHIRALPAK ${ }^{\circ}$ IC-U column, (Hexane/i$\operatorname{PrOH}=98 / 2, v=0.3 \mathrm{~mL} / \mathrm{min}, \lambda=254 \mathrm{~nm}), \mathrm{t}$ (minor) $=7.4 \mathrm{~min}, \mathrm{t}($ major $)=10.3 \mathrm{~min}, 93 \%$ ee. $[\alpha]_{\mathrm{D}}^{20}=-$ $99.7\left(c=0.1\right.$ in $\left.\mathrm{CH}_{2} \mathrm{Cl}_{2}\right)$.

m.p. $100-102{ }^{\circ} \mathrm{C}$.

${ }^{1} \mathbf{H}$ NMR $\left(400 \mathrm{MHz}, \mathrm{CDCl}_{3}\right) \delta$ 8.08-7.97 (m, 1H), $7.75(\mathrm{~d}, J=8.1 \mathrm{~Hz}, 2 \mathrm{H}), 7.57(\mathrm{dd}, J=8.2,1.4 \mathrm{~Hz}, 2 \mathrm{H})$, 7.50-7.33 (m, 5H), $7.02(\mathrm{~d}, J=8.0 \mathrm{~Hz}, 2 \mathrm{H}), 3.99(\mathrm{~d}, J=13.1 \mathrm{~Hz}, 1 \mathrm{H}), 3.90(\mathrm{~d}, J=13.1 \mathrm{~Hz}, 1 \mathrm{H}), 2.23(\mathrm{~m}$, 9H), $1.70(\mathrm{~m}, 6 \mathrm{H}), 1.54(\mathrm{~s}, 9 \mathrm{H})$.

${ }^{13}$ C NMR $\left(100 \mathrm{MHz}, \mathrm{CDCl}_{3}\right) \delta 166.4,165.3,147.4,145.4,141.5,139.9,135.7,131.6,130.4,129.5,129.0$, $128.4,127.3,124.3,123.9,121.0,114.8,92.8,85.7,81.2,41.3,40.3,36.0,31.1,28.1$.

IR (ATR, neat): 2915, 2852, 1777, 1735, 1616, 1553, 1492, 1445, 1423, 1338, 1313, 1242, 1211, 1167 , $1125,1105$.

HRMS (ESI) calcd $(m / z)$ for $\mathrm{C}_{37} \mathrm{H}_{38} \mathrm{~N}_{2} \mathrm{O}_{7}:\left[\mathrm{M}+\mathrm{NH}_{4}\right]^{+}$640.3017, found 640.3017 .

Adamantyl (R)-3-(3-bromo-4-fluorobenzyl)-6-(naphthalen-2-yl)-2-oxoindoline-1-carboxylate (3w) 


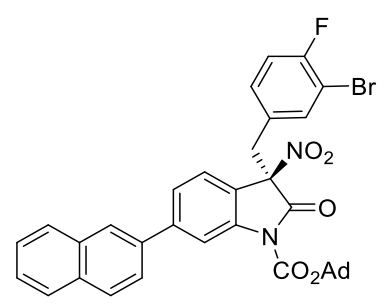

Prepared according to the GP5 to provide the title compound as yellow solid (96 $\mathrm{mg}, 72 \%$ yield). Enantiomeric excess was determined by HPLC with a Daicel CHIRALPAK® IC-U column, (Hexane/i$\mathrm{PrOH}=98 / 2, v=0.3 \mathrm{~mL} / \mathrm{min}, \lambda=254 \mathrm{~nm}), \mathrm{t}($ minor $)=10.5 \mathrm{~min}, \mathrm{t}$ (major) $=12.6 \mathrm{~min}, 88 \%$ ee. $[\alpha]_{\mathrm{D}}{ }^{20}=$ $-103.7\left(c=0.1\right.$ in $\left.\mathrm{CH}_{2} \mathrm{Cl}_{2}\right)$.

m.p. $146-148^{\circ} \mathrm{C}$.

${ }^{1}$ H NMR $\left(400 \mathrm{MHz}, \mathrm{CDCl}_{3}\right) \delta 8.16(\mathrm{~d}, J=1.6 \mathrm{~Hz}, 1 \mathrm{H}), 8.03(\mathrm{~d}, J=1.8 \mathrm{~Hz}, 1 \mathrm{H}), 7.95-7.85(\mathrm{~m}, 3 \mathrm{H}), 7.69$ $(\mathrm{dd}, J=8.5,1.9 \mathrm{~Hz}, 1 \mathrm{H}), 7.60(\mathrm{dd}, J=7.9,1.6 \mathrm{~Hz}, 1 \mathrm{H}), 7.57-7.48(\mathrm{~m}, 3 \mathrm{H}), 7.24-7.17(\mathrm{~m}, 1 \mathrm{H}), 6.87(\mathrm{dd}$, $J=5.9,1.9 \mathrm{~Hz}, 2 \mathrm{H}), 3.88(\mathrm{~d}, J=13.3 \mathrm{~Hz}, 1 \mathrm{H}), 3.82(\mathrm{~d}, J=13.4 \mathrm{~Hz}, 1 \mathrm{H}), 2.26(\mathrm{~m}, 9 \mathrm{H}), 1.71(\mathrm{~m}, 6 \mathrm{H})$.

${ }^{13} \mathrm{C}$ NMR $\left(100 \mathrm{MHz}, \mathrm{CDCl}_{3}\right) \delta 166.3,158.7\left(\mathrm{~d}, \mathrm{C}-\mathrm{F},{ }^{1} J_{C-F}=248.9 \mathrm{~Hz}\right), 147.4,145.7,141.6,137.2,135.5$, 133.5, 133.1, 131.1 (d, C-F, $\left.{ }^{3} J_{C-F}=7.3 \mathrm{~Hz}\right), 128.8,128.6\left(\mathrm{~d}, \mathrm{C}-\mathrm{F},{ }^{3} J_{C-F}=4.0 \mathrm{~Hz}\right), 128.4,127.7,126.7$, $126.6,126.5,125.2,124.3,124.3120 .9,116.5\left(\mathrm{~d}, \mathrm{C}-\mathrm{F},{ }^{2} J_{C-F}=22.4 \mathrm{~Hz}\right), 115.1,109.1\left(\mathrm{~d}, \mathrm{C}-\mathrm{F},{ }^{2} J_{C-F}=21.1\right.$ Hz), 92.7 (d, C-F, $\left.{ }^{4} J_{C-F}=1.6 \mathrm{~Hz}\right), 85.9,41.3,39.5,36.0,31.1$.

IR (ATR, neat): 2913, 2581, 1802, 1776, 1735, 1614, 1553, 1494, 1443, 1422, 1351, 1335, 1313, 1260, $1240,1210,1166,1126,1104$.

HRMS (ESI) calcd $(m / z)$ for $\mathrm{C}_{36} \mathrm{H}_{30} \mathrm{BrFN}_{2} \mathrm{O}_{5}:\left[\mathrm{M}+\mathrm{NH}_{4}\right]^{+}$686.1660, found 686.1656.

tert-butyl (R)-2-((1-(tert-butoxycarbonyl)-5-chloro-3-nitro-2-oxoindolin-3-yl)methyl)-1H-indole-1carboxylate (3x)

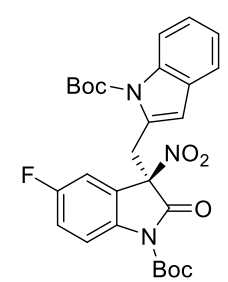

Prepared according to the GP5 to provide the title compound as white solid (71 $\mathrm{mg}, 68 \%$ yield). Enantiomeric excess was determined by HPLC with a Daicel CHIRALPAK ${ }^{\circ}$ IC-U column, (Hexane/ $i$ $\mathrm{PrOH}=98 / 2, v=0.3 \mathrm{~mL} / \mathrm{min}, \lambda=254 \mathrm{~nm}), \mathrm{t}($ minor $)=4.4 \mathrm{~min}, \mathrm{t}($ major $)=5.4 \mathrm{~min}, 90 \% e e .[\alpha]_{\mathrm{D}}^{20}=-$ $12.8\left(c=0.1\right.$ in $\left.\mathrm{CH}_{2} \mathrm{Cl}_{2}\right)$.

m.p. $134-136^{\circ} \mathrm{C}$. 
${ }^{1}$ H NMR $\left(400 \mathrm{MHz}, \mathrm{CDCl}_{3}\right) \delta$ 7.92-7.80 (m, 2H), $7.45(\mathrm{~d}, J=7.5 \mathrm{~Hz}, 1 \mathrm{H}), 7.30-7.15(\mathrm{~m}, 2 \mathrm{H}), 7.08(\mathrm{td}$, $J=8.8,2.8 \mathrm{~Hz}, 1 \mathrm{H}), 6.95(\mathrm{dd}, J=7.4,2.8 \mathrm{~Hz}, 1 \mathrm{H}), 6.55(\mathrm{~s}, 1 \mathrm{H}), 4.80(\mathrm{~d}, J=15.3 \mathrm{~Hz}, 1 \mathrm{H}), 4.40(\mathrm{~d}, J=$ $15.3 \mathrm{~Hz}, 1 \mathrm{H}), 1.60(\mathrm{~s}, 9 \mathrm{H}), 1.59$ (s, 9H).

${ }^{13} \mathrm{C}$ NMR $\left(100 \mathrm{MHz}, \mathrm{CDCl}_{3}\right) \delta 166.1,159.6\left(\mathrm{~d}, \mathrm{C}-\mathrm{F},{ }^{1} J_{C-F}=246.0 \mathrm{~Hz}\right), 150.7,148.3,137.1\left(\mathrm{~d}, \mathrm{C}-\mathrm{F},{ }^{4} J_{C-F}\right.$ $=2.7 \mathrm{~Hz}), 136.2,131.7,128.6,124.6,124.4\left(\mathrm{~d}, \mathrm{C}-\mathrm{F},{ }^{3} J_{C-F}=8.7 \mathrm{~Hz}\right), 123.1,120.7,118.5\left(\mathrm{~d}, \mathrm{C}-\mathrm{F},{ }^{2} J_{C-F}=\right.$ $22.8 \mathrm{~Hz}), 117.3\left(\mathrm{~d}, \mathrm{C}-\mathrm{F},{ }^{3} J_{C-F}=7.7 \mathrm{~Hz}\right), 116.0,112.1\left(\mathrm{~d}, \mathrm{C}-\mathrm{F},{ }^{2} J_{C-F}=25.6 \mathrm{~Hz}\right), 112.0,92.2\left(\mathrm{~d}, \mathrm{C}-\mathrm{F},{ }^{4} J_{C-F}\right.$ $=1.8 \mathrm{~Hz}), 85.5,84.7,33.6,28.2,28.1$.

IR (ATR, neat): 3122, 3044, 2982, 2935, 2852, 2295, 1952, 1766, 1725, 1653, 1611, 1558, 1474, 1424, $1368,1292,1123,1081$.

HRMS (ESI) calcd $(m / z)$ for $\mathrm{C}_{27} \mathrm{H}_{28} \mathrm{FN}_{3} \mathrm{O}_{7}:\left[\mathrm{M}+\mathrm{NH}_{4}\right]^{+}$543.2250, found 543.2253.

tert-butyl (R)-2-((1-(tert-butoxycarbonyl)-5-chloro-3-nitro-2-oxoindolin-3-yl)methyl)-1H-indole-1carboxylate (3y)

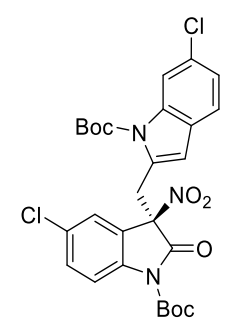

Prepared according to the GP5 to provide the title compound as white solid (76 $\mathrm{mg}$, 66\% yield). Enantiomeric excess was determined by HPLC with a Daicel CHIRALPAK® IC-U column, (Hexane/ $i$ $\mathrm{PrOH}=98 / 2, v=0.3 \mathrm{~mL} / \mathrm{min}, \lambda=254 \mathrm{~nm}), \mathrm{t}($ minor $)=4.0 \mathrm{~min}, \mathrm{t}($ major $)=4.4 \mathrm{~min}, 87 \% e e .[\alpha]_{\mathrm{D}}^{20}=-$ $42.1\left(c=0.1\right.$ in $\left.\mathrm{CH}_{2} \mathrm{Cl}_{2}\right)$.

m.p. $132-134{ }^{\circ} \mathrm{C}$.

${ }^{1}$ H NMR $\left(400 \mathrm{MHz}, \mathrm{CDCl}_{3}\right) \delta 7.94(\mathrm{~d}, J=1.8 \mathrm{~Hz}, 1 \mathrm{H}), 7.84(\mathrm{~d}, J=8.7 \mathrm{~Hz}, 1 \mathrm{H}), 7.41-7.34(\mathrm{~m}, 2 \mathrm{H}), 7.18$ $(\mathrm{dt}, J=5.0,2.2 \mathrm{~Hz}, 2 \mathrm{H}), 6.52(\mathrm{~s}, 1 \mathrm{H}), 4.69$ (d, $J=15.4 \mathrm{~Hz}, 1 \mathrm{H}), 4.40(\mathrm{~d}, J=15.4 \mathrm{~Hz}, 1 \mathrm{H}), 1.61(\mathrm{~s}, 9 \mathrm{H})$, $1.60(\mathrm{~s}, 9 \mathrm{H})$.

${ }^{13} \mathrm{C} \mathrm{NMR}\left(100 \mathrm{MHz}, \mathrm{CDCl}_{3}\right) \delta 165.7,150.2,148.2,139.4,136.5,132.4,132.0,130.5,130.5,127.0,124.6$, $124.4,123.7,121.3,117.1,116.5,111.7,91.8,85.9,85.5,33.6,28.2,28.0$.

IR (ATR, neat): 3134, 3080, 2984, 2934, 2294, 1917, 1898, 1786, 1722, 1646, 1605, 1561, 1475, 1454, 1374, 1330, 1289, 1164, 1127, 1083.

HRMS (ESI) calcd $(m / z)$ for $\mathrm{C}_{27} \mathrm{H}_{28} \mathrm{Cl}_{2} \mathrm{~N}_{2} \mathrm{O}_{5}:\left[\mathrm{M}+\mathrm{NH}_{4}\right]^{+}$553.1564, found 553.1567.

tert-butyl (R)-3-nitro-2-oxo-3-phenylindoline-1-carboxylate (3za) 


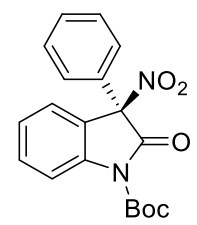

Prepared according to the GP5 to provide the title compound as white solid ( $41 \mathrm{mg}$, 58\% yield). Enantiomeric excess was determined by HPLC with a Daicel CHIRALPAK ${ }^{\circledR}$ ID-U column, (Hexane/i$\operatorname{PrOH}=99.5 / 0.5, v=0.3 \mathrm{~mL} / \mathrm{min}, \lambda=254 \mathrm{~nm}), \mathrm{t}($ minor $)=13.9 \mathrm{~min}, \mathrm{t}$ (major) $=16.1 \mathrm{~min}, 80 \% e e .[\alpha]_{\mathrm{D}}^{20}$ $=-28.2\left(c=0.1\right.$ in $\left.\mathrm{CH}_{2} \mathrm{Cl}_{2}\right)$.

m.p. $85-87^{\circ} \mathrm{C}$.

${ }^{1} \mathbf{H}$ NMR $\left(400 \mathrm{MHz}, \mathrm{CDCl}_{3}\right) \delta 8.06(\mathrm{~d}, J=8.2 \mathrm{~Hz}, 1 \mathrm{H}), 7.63-7.53(\mathrm{~m}, 2 \mathrm{H}), 7.52-7.39(\mathrm{~m}, 5 \mathrm{H}), 7.34(\mathrm{td}$, $J=7.7,1.1 \mathrm{~Hz}, 1 \mathrm{H}), 1.63(\mathrm{~s}, 9 \mathrm{H})$.

${ }^{13}$ C NMR $\left(100 \mathrm{MHz}, \mathrm{CDCl}_{3}\right) \delta 165.7,148.6,141.2,132.5,131.8,130.5,128.9,128.7,126.1,125.4,122.4$, $116.2,94.9,85.7,28.1$.

IR (ATR, neat): 3139, 2985, 2935, 2879, 1974, 1957, 1786, 1737, 1604, 1553, 1475, 1393, 1374, 1340, $1287,1254,1161$.

HRMS (ESI) calcd $(m / z)$ for $\mathrm{C}_{19} \mathrm{H}_{18} \mathrm{~N}_{2} \mathrm{O}_{5}:[\mathrm{M}+\mathrm{Na}]^{+} 337.1108$, found 337.1105.

tert-butyl (R)-5-methyl-3-nitro-2-oxo-3-phenylindoline-1-carboxylate (3zb)

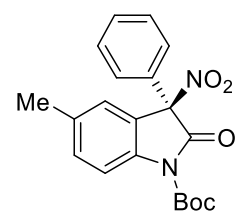

Prepared according to the GP5 to provide the title compound as colorless oil (40 mg, 54\% yield). Enantiomeric excess was determined by HPLC with a Daicel CHIRALPAK ${ }^{\circledR}$ ID-U column, (Hexane/i$\mathrm{PrOH}=99.5 / 0.5, v=0.3 \mathrm{~mL} / \mathrm{min}, \lambda=254 \mathrm{~nm}), \mathrm{t}($ minor $)=15.5 \mathrm{~min}, \mathrm{t}($ major $)=17.0 \mathrm{~min}, 80 \%$ ee. $[\alpha]_{\mathrm{D}}{ }^{20}$ $=-24.3\left(c=0.1\right.$ in $\left.\mathrm{CH}_{2} \mathrm{Cl}_{2}\right)$.

${ }^{1} \mathbf{H}$ NMR $\left(400 \mathrm{MHz}, \mathrm{CDCl}_{3}\right) \delta 7.84(\mathrm{~d}, J=8.3 \mathrm{~Hz}, 1 \mathrm{H}), 7.42-7.26(\mathrm{~m}, 7 \mathrm{H}), 2.33(\mathrm{~s}, 3 \mathrm{H}), 1.54(\mathrm{~s}, 9 \mathrm{H})$.

${ }^{13}$ C NMR (100 MHz, $\left.\mathrm{CDCl}_{3}\right) \delta 165.9,148.6,138.7,135.4,133.0,131.9,130.4,128.9,128.7,126.3,122.4$, $116.0,95.1,85.5,28.1,21.1$.

IR (ATR, neat): 3122, 2978, 2961, 2917, 1907, 1779, 1753, 1601, 1543, 1466, 1390, 1278, 1244, 1159, 1087.

HRMS (ESI) calcd $(m / z)$ for $\mathrm{C}_{20} \mathrm{H}_{20} \mathrm{~N}_{2} \mathrm{O}_{5}:[\mathrm{M}+\mathrm{Na}]^{+}$391.1264, found 391.1265.

tert-butyl (R)-3-nitro-2-oxo-3-(p-tolyl)indoline-1-carboxylate (3zc) 


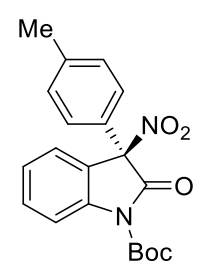

Prepared according to the GP5 to provide the title compound as white solid (45 $\mathrm{mg}, 62 \%$ yield). Enantiomeric excess was determined by HPLC with a Daicel CHIRALPAK ${ }^{\circ}$ IC-U column, (Hexane/ $i$ $\mathrm{PrOH}=99.5 / 0.5, v=0.3 \mathrm{~mL} / \mathrm{min}, \lambda=254 \mathrm{~nm}), \mathrm{t}$ (major) $=13.8 \mathrm{~min}, \mathrm{t}($ minor $)=16.0 \mathrm{~min}, 81 \%$ ee. $[\alpha]_{\mathrm{D}}{ }^{20}$ $=-17.4\left(c=0.1\right.$ in $\left.\mathrm{CH}_{2} \mathrm{Cl}_{2}\right)$.

m.p. $82-84{ }^{\circ} \mathrm{C}$.

${ }^{1} \mathbf{H}$ NMR $\left(400 \mathrm{MHz}, \mathrm{CDCl}_{3}\right) \delta 8.04(\mathrm{~d}, J=8.2 \mathrm{~Hz}, 1 \mathrm{H}), 7.64-7.49(\mathrm{~m}, 2 \mathrm{H}), 7.31(\mathrm{~m}, 3 \mathrm{H}), 7.23(\mathrm{~d}, J=8.1$ $\mathrm{Hz}, 2 \mathrm{H}), 2.37$ (s, 3H), $1.62(\mathrm{~s}, 9 \mathrm{H})$.

${ }^{13} \mathbf{C ~ N M R}\left(100 \mathrm{MHz}, \mathrm{CDCl}_{3}\right) \delta 165.9,148.6,141.1,140.9,132.4,129.6,128.8,128.6,126.0,125.3,122.6$, $116.2,94.8,85.6,28.1,21.3$.

IR (ATR, neat): 3125, 2984, 2959, 2923, 1918, 1776, 1743, 1605, 1558, 1478, 1392, 1368, 1341, 1289, $1253,1154,1096$.

HRMS (ESI) calcd $(m / z)$ for $\mathrm{C}_{20} \mathrm{H}_{20} \mathrm{~N}_{2} \mathrm{O}_{5}:[\mathrm{M}+\mathrm{Na}]^{+}$391.1264, found 391.1262. 


\section{Derivatization of chiral 3-nitro oxindole products}

\section{Large-scale experiment}

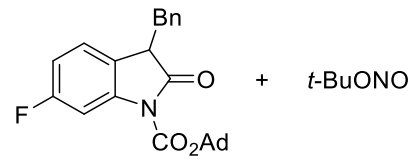

2b (2 mmol)

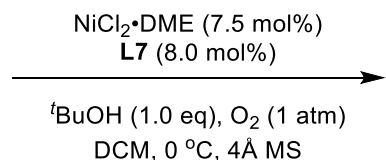

$\mathrm{DCM}, 0^{\circ} \mathrm{C}, 4 \AA \mathrm{MS}$

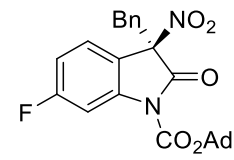

3b, $66 \%$ yield, $94 \%$ ee

To a mixture of $\mathbf{2 b}$ ( $839 \mathrm{mg}, 2 \mathrm{mmol}, 1$ equiv.), $\mathrm{NiCl}_{2} \cdot \mathrm{DME}$ (33 mg, $0.15 \mathrm{mmol}, 7.5 \mathrm{~mol} \%$ ), $\mathbf{L} 7$ (70 mg, $0.16 \mathrm{mmol}, 8 \mathrm{~mol} \%),{ }^{t} \mathrm{BuOH}$ (148 mg, $2 \mathrm{mmol}, 1$ equiv.) and $4 \AA \mathrm{MS}$ in $10 \mathrm{ml} \mathrm{DCM}$ was stirred under $\mathrm{O}_{2}$ balloon at room temperature for $1 \mathrm{~h}$, then the mixture was cooled to $0{ }^{\circ} \mathrm{C}$ and tert-Butyl nitrite $(309 \mathrm{mg}, 3$ mmol, 1.5 equiv.) was added. The mixture was continued stirred for 20 hours at this temperature. After the solvent was removed under reduced pressure, the residue was purified by column chromatography to afford 3b as colorless oil (608 mg, 66\% yield). Enantiomeric excess was determined by HPLC with a Daicel CHIRALPAK ${ }^{\circledR}$ IC-U column, $(H e x a n e / i-\mathrm{PrOH}=98 / 2, v=0.3 \mathrm{~mL} / \mathrm{min}, \lambda=254 \mathrm{~nm}), \mathrm{t}(\operatorname{minor})=$ $6.2 \mathrm{~min}, \mathrm{t}$ (major) $=8.1 \mathrm{~min}, 94 \%$ ee

\section{(R)-3-amino-3-benzyl-5-fluoroindolin-2-one (4a)}

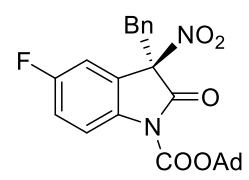

3a

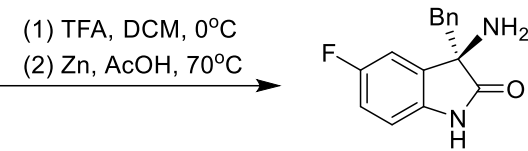

$4 a$

To a solution of 3a ( $46 \mathrm{mg}, 0.1 \mathrm{mmol}, 1$ equiv.) in $2 \mathrm{ml}$ DCM was added TFA ( $46 \mathrm{mg}, 0.4 \mathrm{mmol}, 4$ equiv.) dropwise at $0{ }^{\circ} \mathrm{C}$. After stirring for $1 \mathrm{~h}$, the reaction mixture was concentrated under reduced pressure to afford afford the crude product. The crude product was dissolved in acetic acid and Zn dust (196 mg, 3.0 mmol, 30 equiv) was added, the mixture was stirred at $70{ }^{\circ} \mathrm{C}$ for $2 \mathrm{~h}$, the reaction mixture was concentrated under reduced pressure and a solution of aq. $\mathrm{NaHCO}_{3}$ was added to neutralize the solution to $\mathrm{pH} 7$ and extracted with DCM $(2 \times 5 \mathrm{ml})$. The organic phase was dried over $\mathrm{Na}_{2} \mathrm{SO}_{4}$, filtered and solvent was evaporated under reduced pressure. The crude product was purified by column chromatography on silicagel to give the desired product as a white solid (21 mg, 83\% yield). Enantiomeric excess was determined by HPLC with a Daicel CHIRALPAK ${ }^{\circledR}$ IA-U column, $(H e x a n e / i-P r O H=90 / 10, v=0.3 \mathrm{~mL} / \mathrm{min}, \lambda=254$ $\mathrm{nm}), \mathrm{t}($ major $)=7.6 \mathrm{~min}, \mathrm{t}($ minor $)=11.6 \min , 94 \%$ ee. $[\alpha]_{\mathrm{D}}{ }^{20}=-34.3(c=0.1$ in $\mathrm{MeOH})$.

m.p. $207-209^{\circ} \mathrm{C}$.

${ }^{1}$ H NMR (400 MHz, DMSO-d6) $\delta 10.00(\mathrm{~s}, 1 \mathrm{H})$, 7.24-7.02 (m, 4H), 6.98-6.80 (m, 3H), $6.55(\mathrm{dd}, J=8.4$, $4.4 \mathrm{~Hz}, 1 \mathrm{H}), 3.05(\mathrm{~d}, J=12.7 \mathrm{~Hz}, 1 \mathrm{H}), 2.95(\mathrm{~d}, J=12.7 \mathrm{~Hz}, 1 \mathrm{H}), 2.28(\mathrm{~s}, 2 \mathrm{H})$. 
${ }^{13}$ C NMR (100 MHz, DMSO-d6) $\delta 180.8,157.8\left(\mathrm{~d}, \mathrm{C}-\mathrm{F},{ }^{1} J_{C-F}=236.2 \mathrm{~Hz}\right), 137.8,135.6,134.3$ (d, C-F, $\left.{ }^{3} J_{C-F}=7.9 \mathrm{~Hz}\right), 129.9,127.5,126.4,114.4\left(\mathrm{~d}, \mathrm{C}-\mathrm{F},{ }^{2} J_{C-F}=23.2 \mathrm{~Hz}\right), 112.1\left(\mathrm{~d}, \mathrm{C}-\mathrm{F},{ }^{2} J_{C-F}=24.2 \mathrm{~Hz}\right), 109.8$ $\left(\mathrm{d}, \mathrm{C}-\mathrm{F},{ }^{3} J_{\mathrm{C}-\mathrm{F}}=8.1 \mathrm{~Hz}\right), 63.1,44.8$.

IR (ATR, neat): 3030, 2920, 2877, 1705, 1670, 1631, 1585, 1486, 1454, 1312, 1257, 1187, 1153, 1124.

HRMS (ESI) calcd $(m / z)$ for $\mathrm{C}_{36} \mathrm{H}_{30} \mathrm{BrFN}_{2} \mathrm{O}_{5}:[\mathrm{M}+\mathrm{Na}]^{+} 279.1541$, found 279.1536.

(R)-N-((3-benzyl-5-fluoro-2-oxoindolin-3-yl)carbamoyl)-4 methylbenzenesulfonamide (4b)

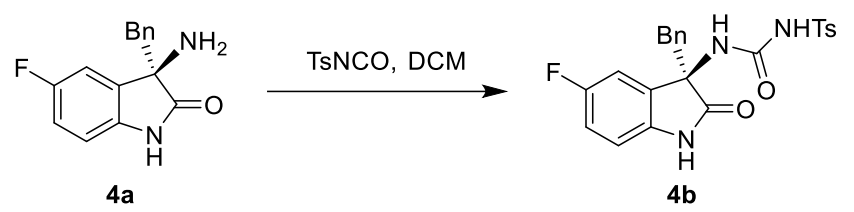

To a solution of $4 \mathbf{a}(26 \mathrm{mg}, 0.1 \mathrm{mmol}, 1$ equiv.) in $1 \mathrm{ml} \mathrm{DCM}$ was added TsNCO (20 mg, $0.1 \mathrm{mmol}, 1$ equiv.) dropwise at room temperature. The mixture was stirred for $30 \mathrm{~min}$, then the solvent was removed in vacuo, and the residue was purified by silica gel column chromatography to give $\mathbf{4 b}$ as a white solid (41 mg, 91\% yield). Enantiomeric excess was determined by HPLC with a Daicel CHIRALPAK® ID-U column, $($ Hexane $/ i-\mathrm{PrOH}=60 / 40, v=0.3 \mathrm{~mL} / \mathrm{min}, \lambda=254 \mathrm{~nm}), \mathrm{t}($ minor $)=10.2 \mathrm{~min}, \mathrm{t}($ major $)=18.6$ $\min , 94 \%$ ee. $[\alpha]^{\mathrm{D}_{20}}=-3.3(c=0.1$ in $\mathrm{MeOH})$.

m.p. $162-162{ }^{\circ} \mathrm{C}$.

${ }^{1}$ H NMR (400 MHz, DMSO-d6) $\delta 10.75$ (s, 1H), 10.13 (s, 1H), $7.68(\mathrm{dd}, J=8.2,2.3 \mathrm{~Hz}, 2 \mathrm{H}), 7.36$ (d, $J$ $=8.0 \mathrm{~Hz}, 2 \mathrm{H}), 7.26(\mathrm{~d}, J=5.3 \mathrm{~Hz}, 1 \mathrm{H}), 7.12(\mathrm{~m}, 3 \mathrm{H}), 7.01-6.76(\mathrm{~m}, 4 \mathrm{H}), 6.59-6.38(\mathrm{~m}, 1 \mathrm{H}), 3.18(\mathrm{~d}$, $J=12.5 \mathrm{~Hz}, 1 \mathrm{H}), 2.95(\mathrm{~d}, J=12.5 \mathrm{~Hz}, 1 \mathrm{H}), 2.38(\mathrm{~s}, 3 \mathrm{H})$.

${ }^{13}$ C NMR (100 MHz, DMSO-d6) $\delta 176.8,158.1$ (d, C-F, $\left.{ }^{1} J_{C-F}=236.5 \mathrm{~Hz}\right), 150.3,144.3,138.5,137.5$, 133.5, 131.9 (d, C-F, $\left.{ }^{3} J_{C-F}=7.8 \mathrm{~Hz}\right), 130.7,129.9,128.1,127.6,127.5,115.0\left(\mathrm{~d}, \mathrm{C}-\mathrm{F},{ }^{2} J_{C-F}=23.3 \mathrm{~Hz}\right)$, $111.5\left(\mathrm{~d}, \mathrm{C}-\mathrm{F},{ }^{2} J_{\mathrm{C}-F}=24.7 \mathrm{~Hz}\right), 110.3\left(\mathrm{~d}, \mathrm{C}-\mathrm{F},{ }^{3} J_{C-F}=7.8 \mathrm{~Hz}\right), 63.1,42.4,21.5$.

HRMS (ESI) calcd $(m / z)$ for $\mathrm{C}_{23} \mathrm{H}_{20} \mathrm{FN}_{3} \mathrm{O}_{4} \mathrm{~S}$ : $[\mathrm{M}+\mathrm{H}]^{+}$454.1231, found 454.1236.

(R)-3-amino-3-benzyl-6-fluoroindolin-2-one (4c)

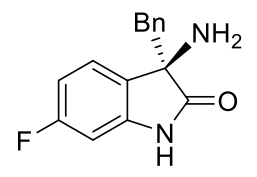

Prepared according to the similar procedure for the synthesis of $\mathbf{4 a}$ to provide the title compound as white solid (20 mg, 79\% yield). Enantiomeric excess was determined by HPLC with a Daicel CHIRALPAK® IA-U column, $($ Hexane $/ i-\mathrm{PrOH}=90 / 10, v=0.3 \mathrm{~mL} / \mathrm{min}, \lambda=254 \mathrm{~nm}), \mathrm{t}($ major $)=8.7 \mathrm{~min}, \mathrm{t}($ minor $)=$ $12.3 \min , 94 \%$ ee. $[\alpha]^{\mathrm{D}_{20}}=-12.1(c=0.1$ in $\mathrm{MeOH})$.

m.p. $214-216^{\circ} \mathrm{C}$. 
${ }^{1}$ H NMR (400 MHz, DMSO-d6) $\delta 10.30$ (s, 1H), 7.33 (dd, $J=8.2,5.7$ Hz, 1H), $7.13-7.04$ (m, 3H), 6.84 $(\mathrm{m}, 2 \mathrm{H}), 6.77(\mathrm{~m}, 1 \mathrm{H}), 6.42(\mathrm{dd}, J=9.3,2.3 \mathrm{~Hz}, 1 \mathrm{H}), 4.16(\mathrm{~s}, 2 \mathrm{H}), 3.11(\mathrm{~d}, J=12.7 \mathrm{~Hz}, 1 \mathrm{H}), 3.01(\mathrm{~d}, J=$ $12.7 \mathrm{~Hz}, 1 \mathrm{H})$.

${ }^{13}$ C NMR (100 MHz, DMSO-d6) $\delta$ 177.9, 163.4 (d, C-F, ${ }^{1} J_{C-F}=243.6$ Hz), 144.3 (d, C-F, ${ }^{3} J_{C-F}=12.7$ $\mathrm{Hz}), 134.3,130.4,128.3,127.4,127.0\left(\mathrm{~d}, \mathrm{C}-\mathrm{F},{ }^{3} J_{C-F}=10.2 \mathrm{~Hz}\right), 124.7,108.4\left(\mathrm{~d}, \mathrm{C}-\mathrm{F},{ }^{2} J_{C-F}=22.4 \mathrm{~Hz}\right)$, $98.5\left(\mathrm{~d}, \mathrm{C}-\mathrm{F},{ }^{2} J_{\mathrm{C}-\mathrm{F}}=27.1 \mathrm{~Hz}\right), 62.0,42.9$.

HRMS (ESI) calcd $(\mathrm{m} / \mathrm{z})$ for $\mathrm{C}_{15} \mathrm{H}_{13} \mathrm{FN}_{2} \mathrm{O}:[\mathrm{M}+\mathrm{H}]^{+} 257.1085$, found 257.1089 .

(R)- $N$-(3-benzyl-6-fluoro-2-oxoindolin-3-yl)benzamide (4d)
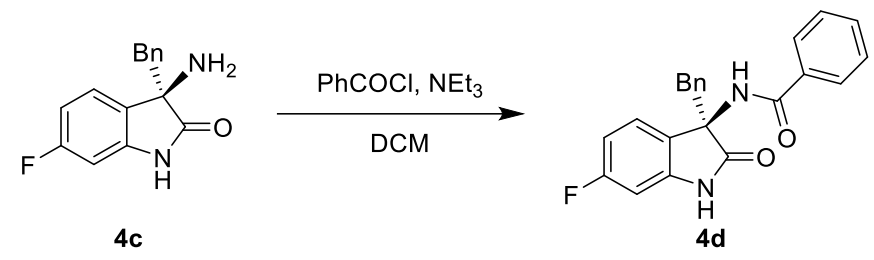

To a solution of $4 \mathbf{c}\left(26 \mathrm{mg}, 0.1 \mathrm{mmol}, 1\right.$ equiv.) and $\mathrm{Et}_{3} \mathrm{~N}$ (15 mg, $0.15 \mathrm{mmol}, 1.5$ equiv.) in $1 \mathrm{ml} \mathrm{DCM}$ was added benzoyl chloride ( $14 \mathrm{mg}, 0.1 \mathrm{mmol}, 1$ equiv.) dropwise at room temperature. The mixture was stirred for $1 \mathrm{~h}$, then the solvent was removed in vacuo, and the residue was purified by silica gel column chromatography to give $\mathbf{4 d}$ as a white solid (31 mg, 85\% yield). Enantiomeric excess was determined by HPLC with a Daicel CHIRALPAK ${ }^{\circ}$ IB-U column, (Hexane $/ i-\mathrm{PrOH}=90 / 10, v=0.3 \mathrm{~mL} / \mathrm{min}, \lambda=254$ $\mathrm{nm}), \mathrm{t}($ minor $)=8.1 \mathrm{~min}, \mathrm{t}($ major $)=13.5 \mathrm{~min}, 94 \% e e .[\alpha]^{\mathrm{D}_{20}}=-76.3(c=0.1$ in $\mathrm{MeOH})$.

m.p. $244-246{ }^{\circ} \mathrm{C}$.

${ }^{1}$ H NMR (400 MHz, DMSO-d6) $\delta 10.24(\mathrm{~s}, 1 \mathrm{H}), 9.12(\mathrm{~s}, 1 \mathrm{H}), 7.88(\mathrm{~d}, J=6.9 \mathrm{~Hz}, 2 \mathrm{H}), 7.57$ (t, $J=7.2$ $\mathrm{Hz}, 1 \mathrm{H}), 7.49(\mathrm{t}, J=7.5 \mathrm{~Hz}, 2 \mathrm{H}), 7.26(\mathrm{t}, J=6.6 \mathrm{~Hz}, 1 \mathrm{H}), 7.10(\mathrm{q}, J=6.4 \mathrm{~Hz}, 3 \mathrm{H}), 6.81(\mathrm{~d}, J=6.6 \mathrm{~Hz}$, 2H), $6.71(\mathrm{t}, J=9.0 \mathrm{~Hz}, 1 \mathrm{H}), 6.31(\mathrm{~d}, J=9.3 \mathrm{~Hz}, 1 \mathrm{H}), 3.38(\mathrm{~d}, J=12.4 \mathrm{~Hz}, 1 \mathrm{H}), 3.22(\mathrm{~d}, J=12.4 \mathrm{~Hz}$, $1 \mathrm{H})$.

${ }^{13}$ C NMR (100 MHz, DMSO-d6) $\delta$ 177.4, 165.9, $162.6\left(\mathrm{~d}, \mathrm{C}-\mathrm{F},{ }^{1} J_{C-F}=241.2 \mathrm{~Hz}\right), 144.5\left(\mathrm{~d}, \mathrm{C}-\mathrm{F},{ }^{3} J_{C-F}=\right.$ $12.5 \mathrm{~Hz}), 134.0,133.8,132.1,130.6,128.7,128.2,127.9,127.2,126.6\left(\mathrm{~d}, \mathrm{C}-\mathrm{F},{ }^{4} J_{C-F}=2.6 \mathrm{~Hz}\right), 124.4(\mathrm{~d}$, $\left.\mathrm{C}-\mathrm{F},{ }^{3} J_{C-F}=10.1 \mathrm{~Hz}\right), 107.5\left(\mathrm{~d}, \mathrm{C}-\mathrm{F},{ }^{2} J_{C-F}=22.2 \mathrm{~Hz}\right), 97.4\left(\mathrm{~d}, \mathrm{C}-\mathrm{F},{ }^{2} J_{C-F}=27.1 \mathrm{~Hz}\right), 63.3,42.6$.

HRMS (ESI) calcd $(m / z)$ for $\mathrm{C}_{22} \mathrm{H}_{17} \mathrm{FN}_{2} \mathrm{O}_{2}:[\mathrm{M}+\mathrm{H}]^{+}$361.1347, found 361.1351.

$(1 ' R, 3 R)-5$-chloro-1'-methyl-1',2',4',5'-tetrahydrospiro[indoline-3,3'-pyrido[4,3-b]indol]-2-one (4x) 

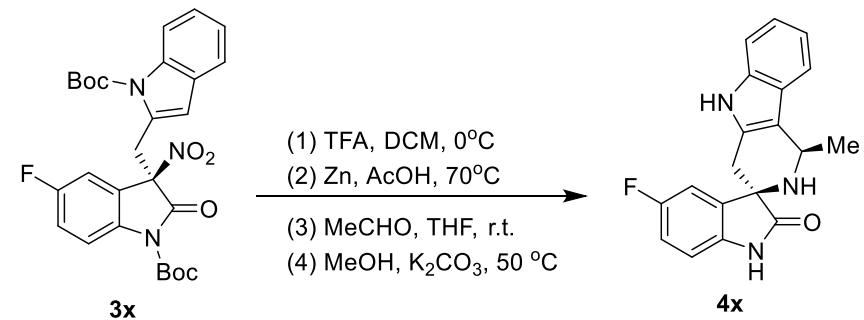

To a solution of $\mathbf{3 x}$ (53 mg, $0.1 \mathrm{mmol}, 1$ equiv.) in $2 \mathrm{ml} \mathrm{DCM}$ was added TFA (46 mg, $0.4 \mathrm{mmol}$, 4 equiv.) dropwise at $0{ }^{\circ} \mathrm{C}$. After stirring for $1 \mathrm{~h}$, the reaction mixture was concentrated under reduced pressure to afford the crude product. The crude product was dissolved in acetic acid and Zn dust (196 mg, $3.0 \mathrm{mmol}$, 30 equiv) was added, the mixture was stirred at $70{ }^{\circ} \mathrm{C}$ for $2 \mathrm{~h}$, the reaction mixture was concentrated under reduced pressure and a solution of aq. $\mathrm{NaHCO}_{3}$ was added to neutralize the solution to $\mathrm{pH} 7$ and extracted with DCM $(2 \times 5 \mathrm{ml})$. The organic phase was dried over $\mathrm{Na}_{2} \mathrm{SO}_{4}$, filtered and solvent was evaporated under reduced pressure. The crude product was dissolved in $1 \mathrm{ml}$ THF and acetaldehyde $(4.4 \mathrm{mg}, 0.1 \mathrm{mmol}, 1$ equiv.) was added, the mixture was stirred at room temperature. for $1 \mathrm{~h}$. And the solvent was removed in vacuo, the residue was dissolved in $1 \mathrm{ml} \mathrm{MeOH}$ followed by add $\mathrm{K}_{2} \mathrm{CO}_{3}$, the result mixture was heated to $50{ }^{\circ} \mathrm{C}$. After 4 hours, the reaction mixture was concentrated in vacuo and purified by silica gel column chromatography to give $4 \mathbf{x}$ as a white solid (15 mg, 46\% yield). Enantiomeric excess was determined by HPLC with a Daicel CHIRALPAK $®$ IA-U column, (Hexane $/ i$-PrOH $=80 / 20, v=0.3 \mathrm{~mL} / \mathrm{min}, \lambda=254$ $\mathrm{nm}), \mathrm{t}($ minor $)=8.3 \min , \mathrm{t}($ major $)=13.1 \mathrm{~min}, 90 \% e e .[\alpha]_{\mathrm{D}}{ }^{20}=-21.0(c=0.1$ in $\mathrm{MeOH})$.

m.p. $176-178^{\circ} \mathrm{C}$.

${ }^{1}$ H NMR (400 MHz, DMSO-d $\left.d_{6}\right) \delta 10.74(\mathrm{~s}, 1 \mathrm{H}), 10.21(\mathrm{~s}, 1 \mathrm{H}), 7.49(\mathrm{~d}, J=7.8 \mathrm{~Hz}, 1 \mathrm{H}), 7.29(\mathrm{td}, J=6.0$, $2.8 \mathrm{~Hz}, 2 \mathrm{H}), 7.14-6.88(\mathrm{~m}, 3 \mathrm{H}), 6.81(\mathrm{dd}, J=8.4,4.4 \mathrm{~Hz}, 1 \mathrm{H}), 4.64(\mathrm{q}, J=6.4 \mathrm{~Hz}, 1 \mathrm{H}), 3.17(\mathrm{~d}, J=16.4$ $\mathrm{Hz}, 1 \mathrm{H}), 3.05$ (s, 1H), 2.73 (d, $J=16.4 \mathrm{~Hz}, 1 \mathrm{H}), 1.47$ (d, $J=6.4 \mathrm{~Hz}, 3 \mathrm{H})$.

${ }^{13}$ C NMR $\left(100 \mathrm{MHz}, \mathrm{DMSO}-d_{6}\right) \delta 178.8,158.5\left(\mathrm{~d}, \mathrm{C}-\mathrm{F},{ }^{1} J_{C-F}=236.4 \mathrm{~Hz}\right), 138.3,136.3,135.6(\mathrm{~d}, \mathrm{C}-\mathrm{F}$, $\left.{ }^{3} J_{C-F}=7.8 \mathrm{~Hz}\right), 130.5,125.8,120.2,118.9,118.6,115.3\left(\mathrm{~d}, \mathrm{C}-\mathrm{F},{ }^{2} J_{C-F}=23.3 \mathrm{~Hz}\right), 112.5,112.4(\mathrm{~d}, \mathrm{C}-\mathrm{F}$, $\left.{ }^{2} J_{C-F}=24.5 \mathrm{~Hz}\right), 111.3,110.3\left(\mathrm{~d}, \mathrm{C}-\mathrm{F},{ }^{3} J_{C-F}=8.1 \mathrm{~Hz}\right), 60.1,44.7,30.8,22.0$.

IR (ATR, neat): 3121, 2983, 2935, 1952, 1881, 1770, 1730, 1654, 1611, 1560, 1484, 1452, 1370, 1334, $1298,1277,1147,1125,1079$.

HRMS (ESI) calcd $(m / z)$ for $\mathrm{C}_{19} \mathrm{H}_{16} \mathrm{FN}_{3} \mathrm{O}:[\mathrm{M}+\mathrm{H}]^{+} 322.1350$, found 322.1331 .

(1'R,3R)-5,7'-dichloro-1'-methyl-1',2',4',5'-tetrahydrospiro[indoline-3,3'-pyrido[4,3-b]indol]-2-one (4y) 


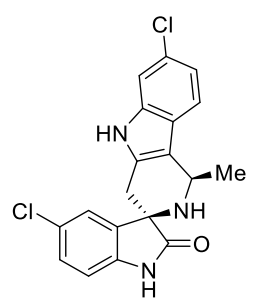

Prepared according to the procedure for synthesis of $\mathbf{4 x}$ to provide the title compound as white solid (20 mg, 53\% yield). Enantiomeric excess was determined by HPLC with a Daicel CHIRALPAK® IA-U column, $($ Hexane $/ i-\mathrm{PrOH}=80 / 20, v=0.3 \mathrm{~mL} / \mathrm{min}, \lambda=254 \mathrm{~nm}), \mathrm{t}($ minor $)=9.8 \mathrm{~min}, \mathrm{t}$ (major) $=18.7$ $\min , 87 \%$ ee. $[\alpha]_{\mathrm{D}}{ }^{20}=-47.3(c=0.1$ in $\mathrm{MeOH})$.

m.p. $189-191{ }^{\circ} \mathrm{C}$.

${ }^{1}$ H NMR (400 MHz, DMSO-d $\left.d_{6}\right) \delta 10.95(\mathrm{~s}, 1 \mathrm{H}), 10.33(\mathrm{~s}, 1 \mathrm{H}), 7.48(\mathrm{~d}, J=8.4 \mathrm{~Hz}, 1 \mathrm{H}), 7.44$ (d, $J=2.0$ $\mathrm{Hz}, 1 \mathrm{H}), 7.32(\mathrm{~d}, J=2.0 \mathrm{~Hz}, 1 \mathrm{H}), 7.28(\mathrm{dd}, J=8.4,2.0 \mathrm{~Hz}, 1 \mathrm{H}), 6.95(\mathrm{dd}, J=8.4,2.0 \mathrm{~Hz}, 1 \mathrm{H}), 6.82(\mathrm{~d}$, $J=8.4 \mathrm{~Hz}, 1 \mathrm{H}), 4.68-4.49(\mathrm{~m}, 1 \mathrm{H}), 3.16(\mathrm{~d}, J=16.5 \mathrm{~Hz}, 1 \mathrm{H}), 3.14(\mathrm{~s}, 1 \mathrm{H}), 2.72(\mathrm{~d}, J=16.5 \mathrm{~Hz}, 1 \mathrm{H})$, $1.42(\mathrm{~d}, J=6.5 \mathrm{~Hz}, 3 \mathrm{H})$.

${ }^{13}$ C NMR (100 MHz, DMSO-d $\left.d_{6}\right) \delta 178.4,141.1,136.7,135.7,131.8,129.0,126.0,124.9,124.9,124.5$, $120.1,118.8,112.7,111.1,110.9,59.8,44.5,30.6,21.9$.

IR (ATR, neat): 3140, 2962, 2926, 2854, 1863, 1708, 1622, 1560, 1467, 1375, 1350, 1315, 1260, 1198, $1165,1092$.

HRMS (ESI) calcd $(m / z)$ for $\mathrm{C}_{19} \mathrm{H}_{15} \mathrm{Cl}_{2} \mathrm{~N}_{3} \mathrm{O}$ : $[\mathrm{M}+\mathrm{H}]^{+} 372.0665$, found 372.0664 . 


\section{Control experiments}

Adamantyl-5-fluoro-3-benzyl-3-(2,2,6,6-tetramethylpiperidin-1-yl)-2-oxoindoline-1-carboxylate (5)

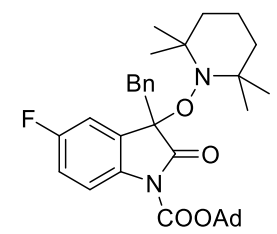

To a mixture of $\mathbf{1 a}\left(88 \mathrm{mg}, 0.2 \mathrm{mmol}, 1\right.$ equiv.), $\mathrm{NiCl}_{2} \cdot \mathrm{DME}$ (3.3 mg, $\left.0.015 \mathrm{mmol}, 7.5 \mathrm{~mol} \%\right)$, $\mathbf{L} 7$ (7.0 $\mathrm{mg}, 0.016 \mathrm{mmol}, 8 \mathrm{~mol} \%),{ }^{t} \mathrm{BuOH}(14.8 \mathrm{mg}, 0.2 \mathrm{mmol}, 1$ equiv.) and $4 \AA \mathrm{MS}$ in $1 \mathrm{ml} \mathrm{DCM}$ was stirred under $\mathrm{O}_{2}$ balloon at room temperature for $1 \mathrm{~h}$, then the mixture was cooled to $0{ }^{\circ} \mathrm{C}$ and tert-Butyl nitrite (30.9 mg, $0.3 \mathrm{mmol}, 1.5$ equiv.) and TEMPO (62 mg, $0.4 \mathrm{mmol}, 2$ equiv.) was added. The mixture was continued stirred for 10 hours at this temperature. The reaction mixture was filtered, and the filtrate was concentrated in vacuo, then detected by HRMS.

HRMS (ESI) calcd $(m / z)$ for $\mathrm{C}_{35} \mathrm{H}_{43} \mathrm{FN}_{2} \mathrm{O}_{4}:[\mathrm{M}+\mathrm{H}]^{+}$575.3280, found 575.3280.

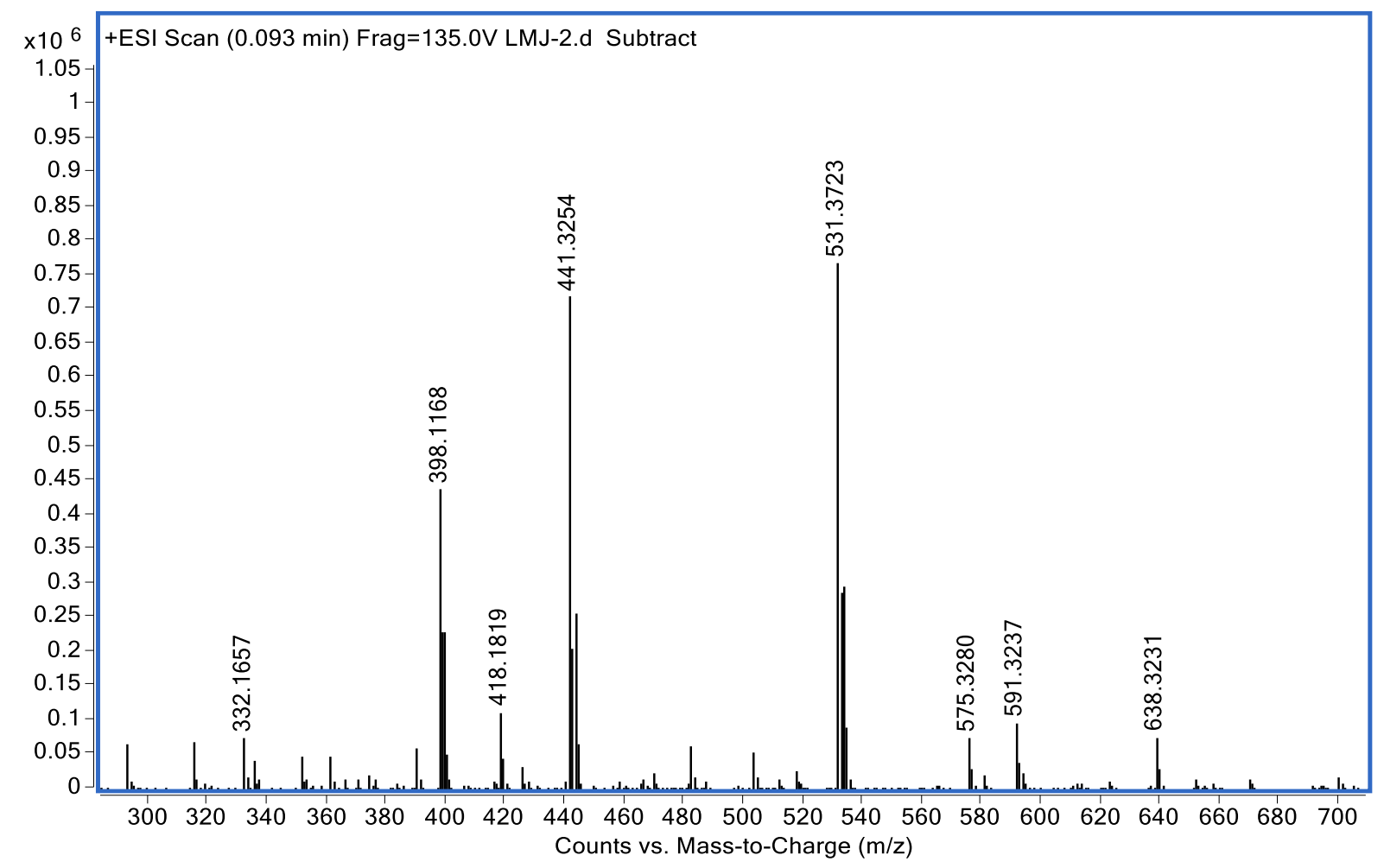




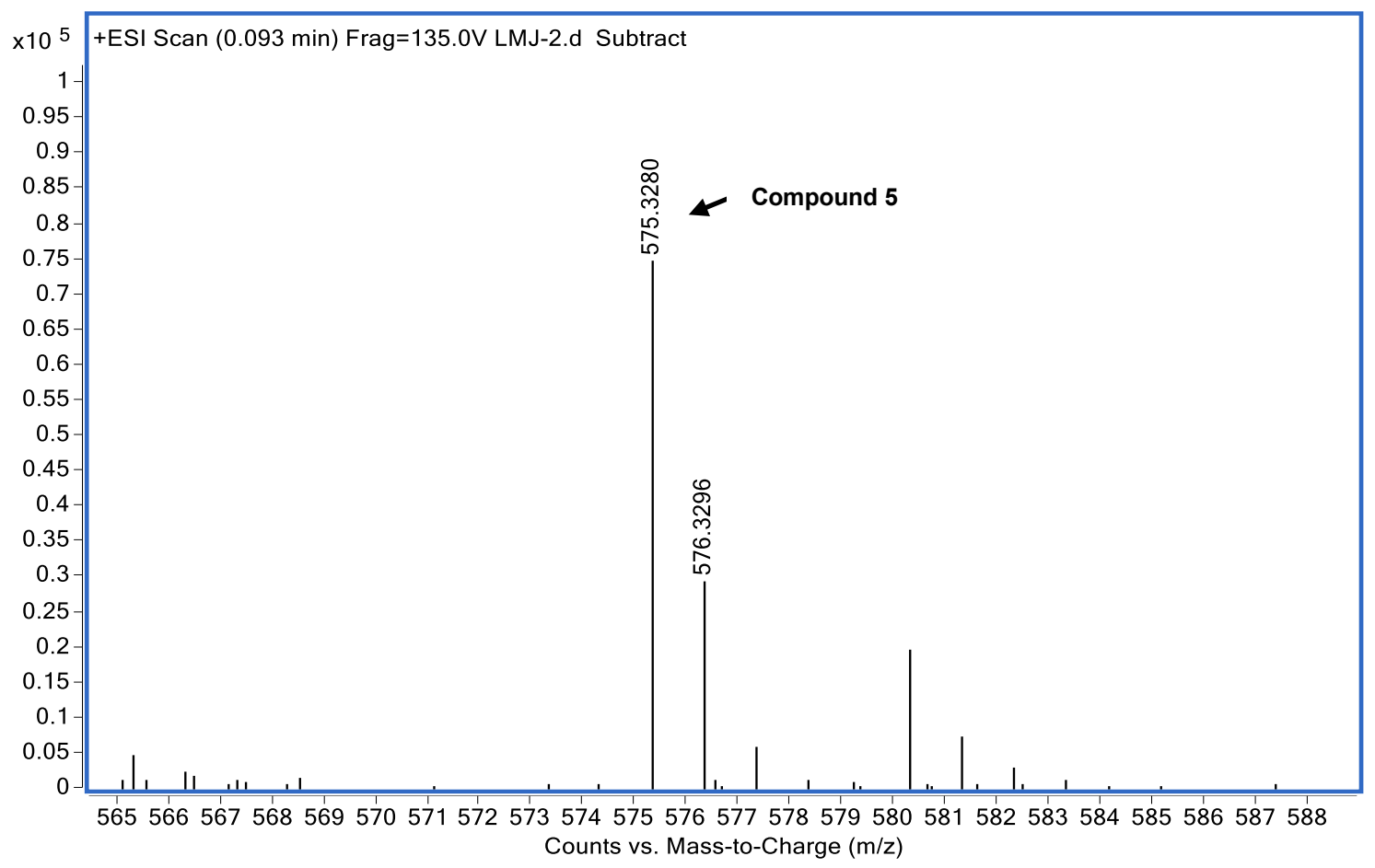

Di-tert-butyl 5,5'-dimethyl-2,2'-dioxo-3,3'-diphenyl-[3,3'-biindoline]-1,1'-dicarboxylate (6)

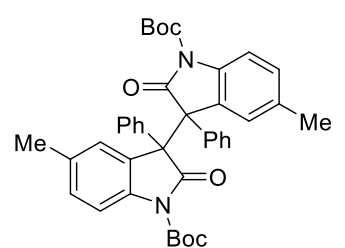

To a mixture of $\mathbf{1 z b}$ (64 mg, 0.2 mmol, 1 equiv.), $\mathrm{NiCl}_{2} \bullet \mathrm{DME}$ (3.3 mg, $0.015 \mathrm{mmol}, 7.5 \mathrm{~mol} \%$ ), $\mathbf{L} 7$ (7.0 $\mathrm{mg}, 0.016 \mathrm{mmol}, 8 \mathrm{~mol} \%),{ }^{t} \mathrm{BuOH}(14.8 \mathrm{mg}, 0.2 \mathrm{mmol}, 1$ equiv.) and $4 \AA \mathrm{MS}$ in $1 \mathrm{ml} \mathrm{DCM}$ was stirred under $\mathrm{O}_{2}$ balloon at room temperature for $1 \mathrm{~h}$, then the mixture was cooled to $0{ }^{\circ} \mathrm{C}$ and tert-Butyl nitrite (30.9 mg, $0.3 \mathrm{mmol}, 1.5$ equiv.) was added. The mixture was continued stirred for 10 hours at this temperature. After the solvent was removed under reduced pressure, the residue was purified by column chromatography (Petroleum ether/EtOAc $=20: 1)$ to afford the compound $\mathbf{6}$ as white solid $(5 \mathrm{mg}, 4 \%$ yield).

m.p. $186-188^{\circ} \mathrm{C}$.

${ }^{1} \mathbf{H}$ NMR $\left(400 \mathrm{MHz}, \mathrm{CDCl}_{3}\right) \delta 7.67(\mathrm{~d}, J=8.3 \mathrm{~Hz}, 2 \mathrm{H}), 7.42(\mathrm{dd}, J=8.3,2.3 \mathrm{~Hz}, 4 \mathrm{H}), 7.34(\mathrm{t}, J=7.3$ $\mathrm{Hz}, 2 \mathrm{H}), 7.26(\mathrm{~m}, 6 \mathrm{H}), 7.12(\mathrm{~d}, J=8.4 \mathrm{~Hz}, 2 \mathrm{H}), 2.15(\mathrm{~s}, 6 \mathrm{H}), 1.54(\mathrm{~s}, 18 \mathrm{H})$.

${ }^{13} \mathrm{C} \mathrm{NMR}\left(100 \mathrm{MHz}, \mathrm{CDCl}_{3}\right) \delta 172.8,149.0,138.4,132.4,131.5,131.3,129.6,128.2,128.0,127.3,127.1$, $114.9,84.0,28.1,21.3$.

HRMS (ESI) calcd $(\mathrm{m} / \mathrm{z})$ for $\mathrm{C}_{40} \mathrm{H}_{40} \mathrm{~N}_{2} \mathrm{O}_{6}:[\mathrm{M}+\mathrm{H}]^{+}$667.2779, found 667.2774. 


\section{NMR Spectra}

1a ${ }^{1} \mathrm{H}$ NMR (400M Hz, $\left.\mathrm{CDCl}_{3}\right)$

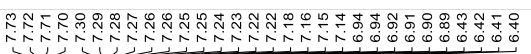

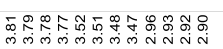

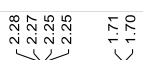

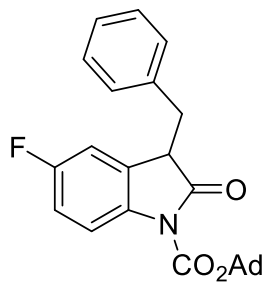

1a

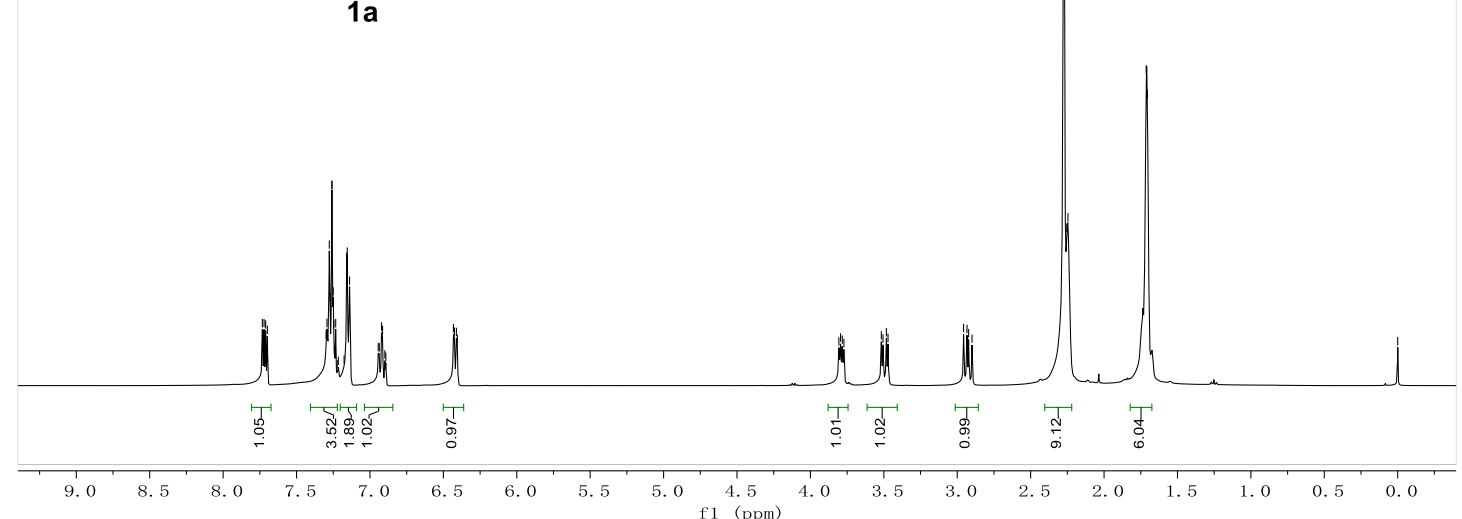

1a ${ }^{13} \mathrm{C}$ NMR $\left(100 \mathrm{M} \mathrm{Hz}, \mathrm{CDCl}_{3}\right)$

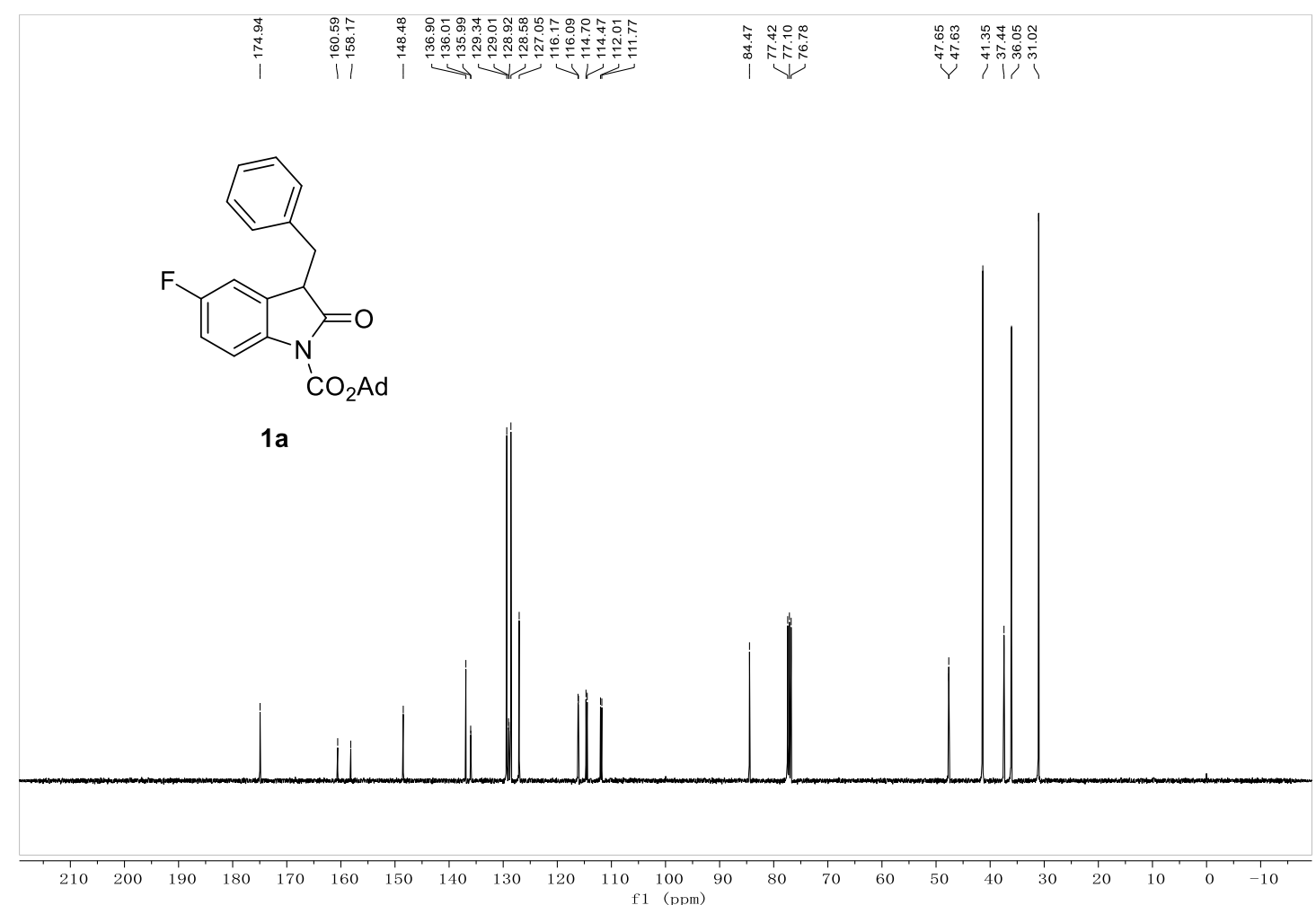


1b ${ }^{1} \mathrm{H}$ NMR (400M Hz, $\mathrm{CDCl}_{3}$ )

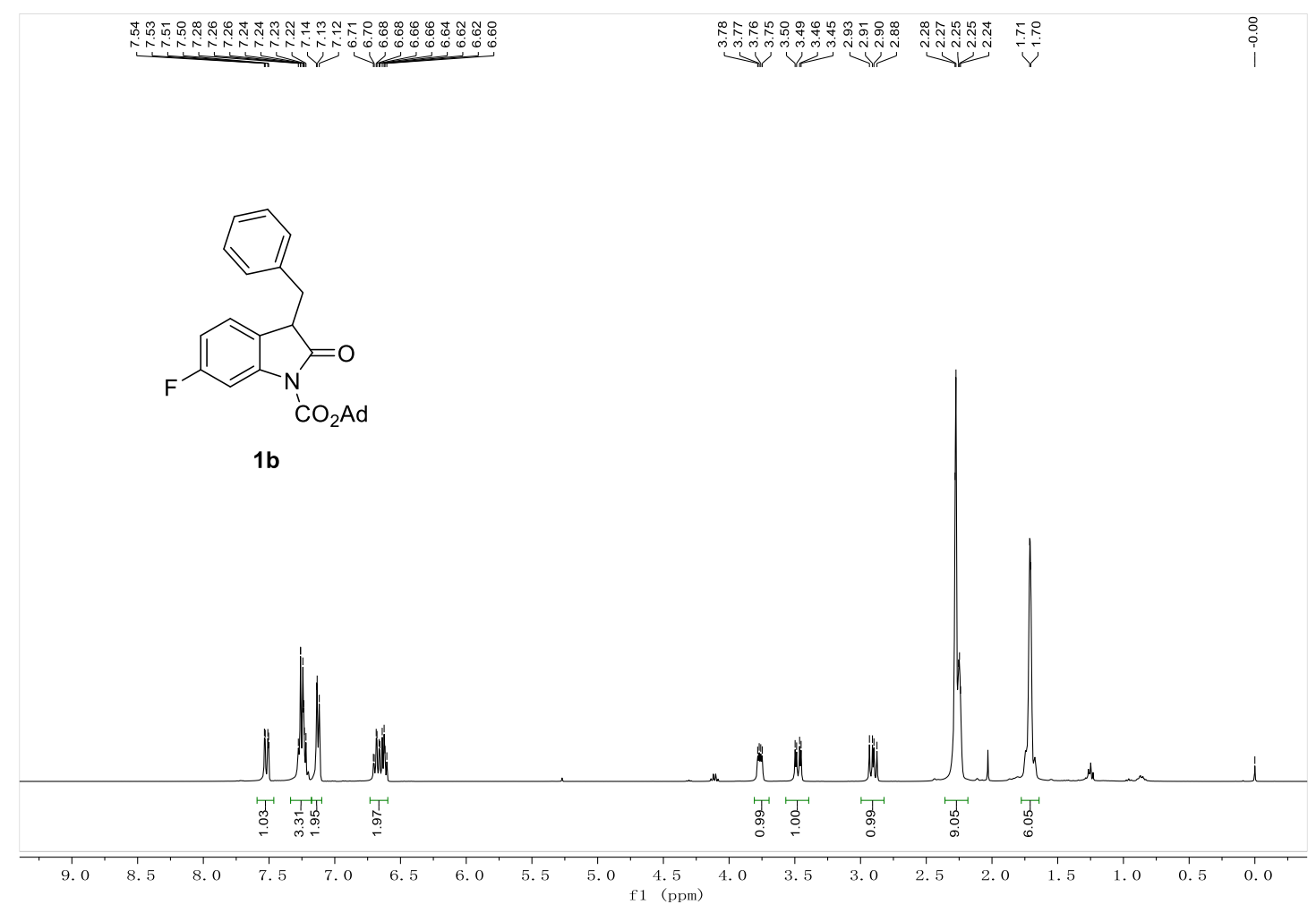

1b ${ }^{13} \mathrm{C}$ NMR $\left(100 \mathrm{M} \mathrm{Hz}, \mathrm{CDCl}_{3}\right)$

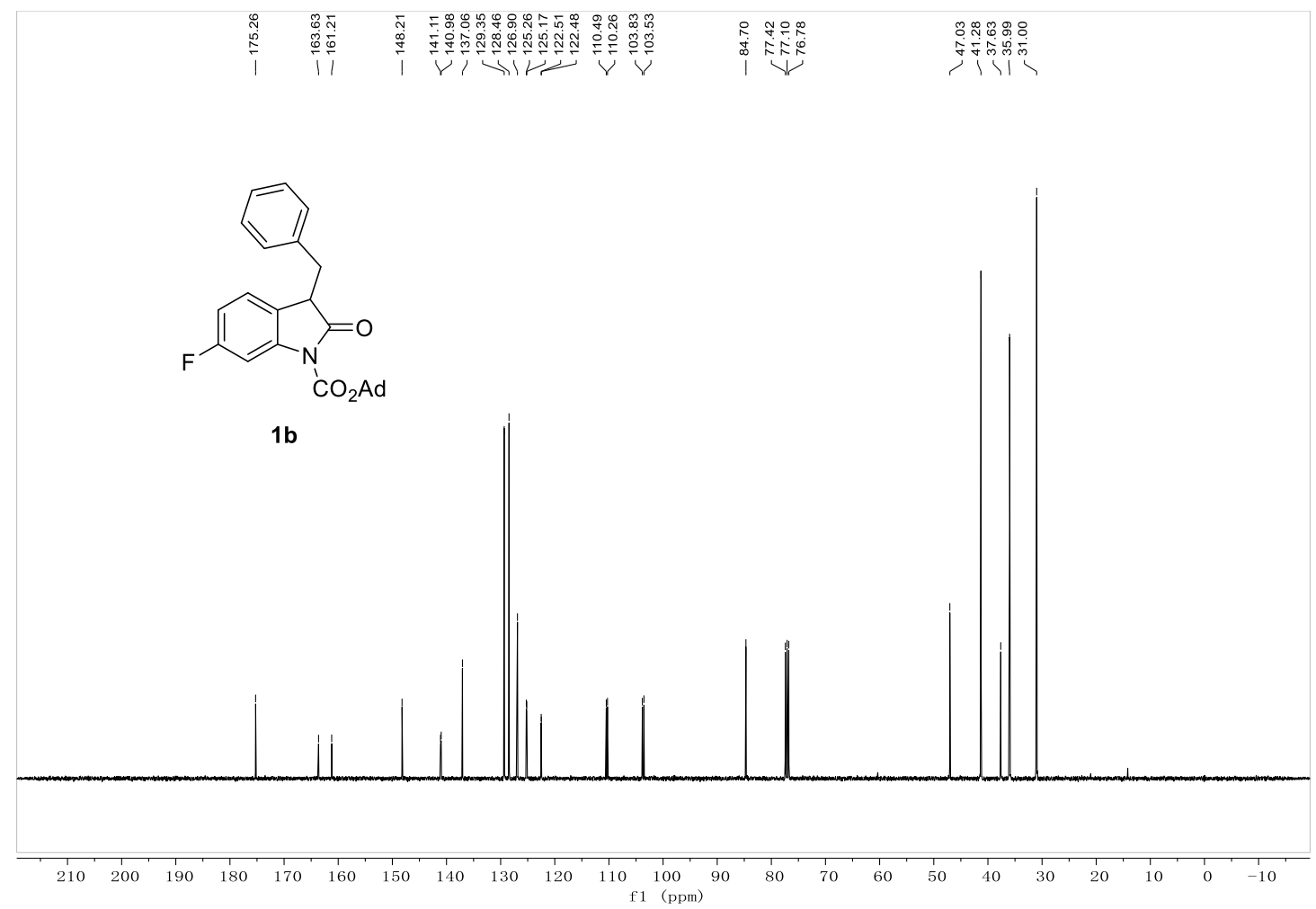


1c ${ }^{1} \mathrm{H}$ NMR $\left(400 \mathrm{M} \mathrm{Hz}, \mathrm{CDCl}_{3}\right)$

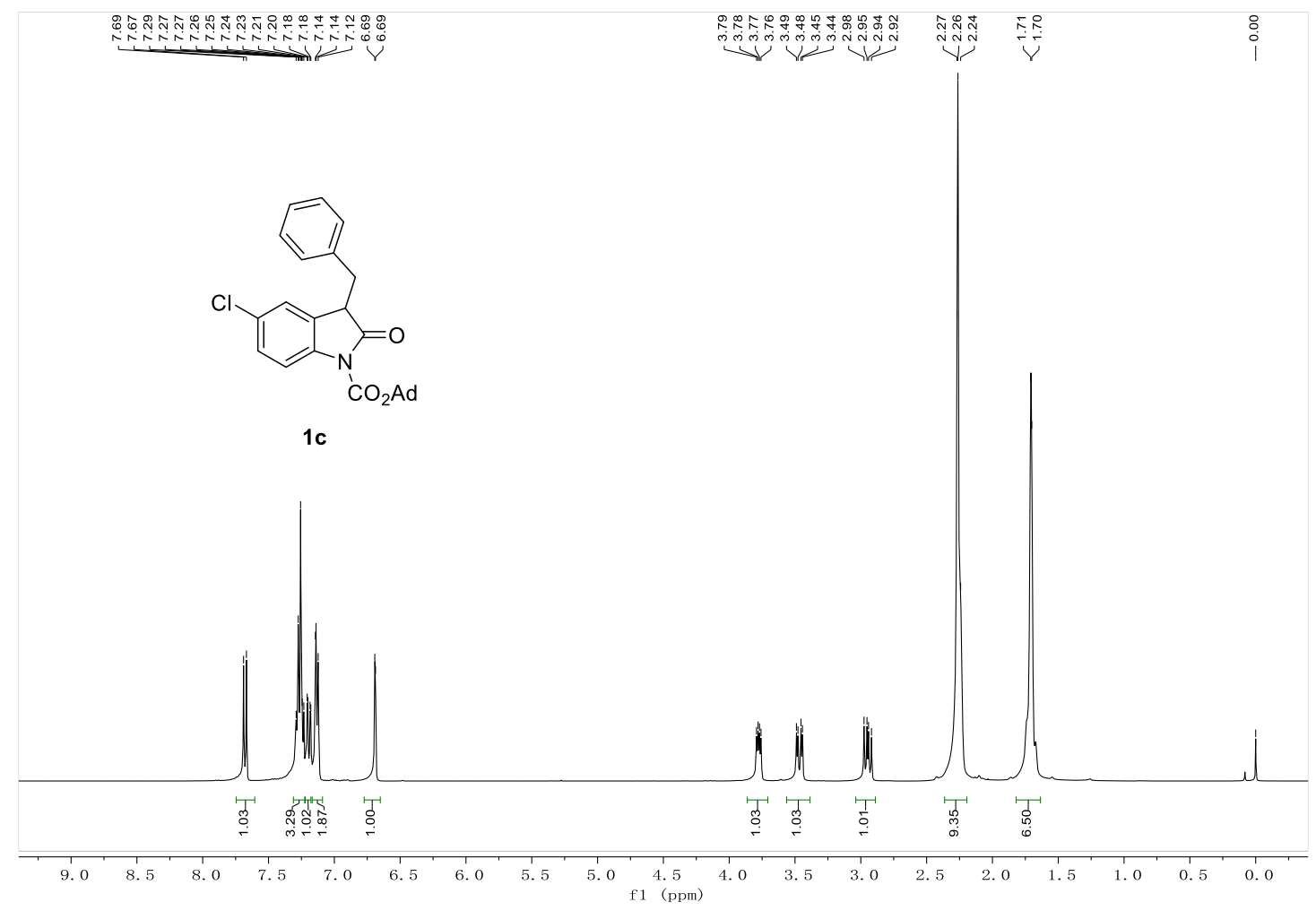

1c ${ }^{13} \mathrm{C} \mathrm{NMR}\left(100 \mathrm{M} \mathrm{Hz}, \mathrm{CDCl}_{3}\right)$

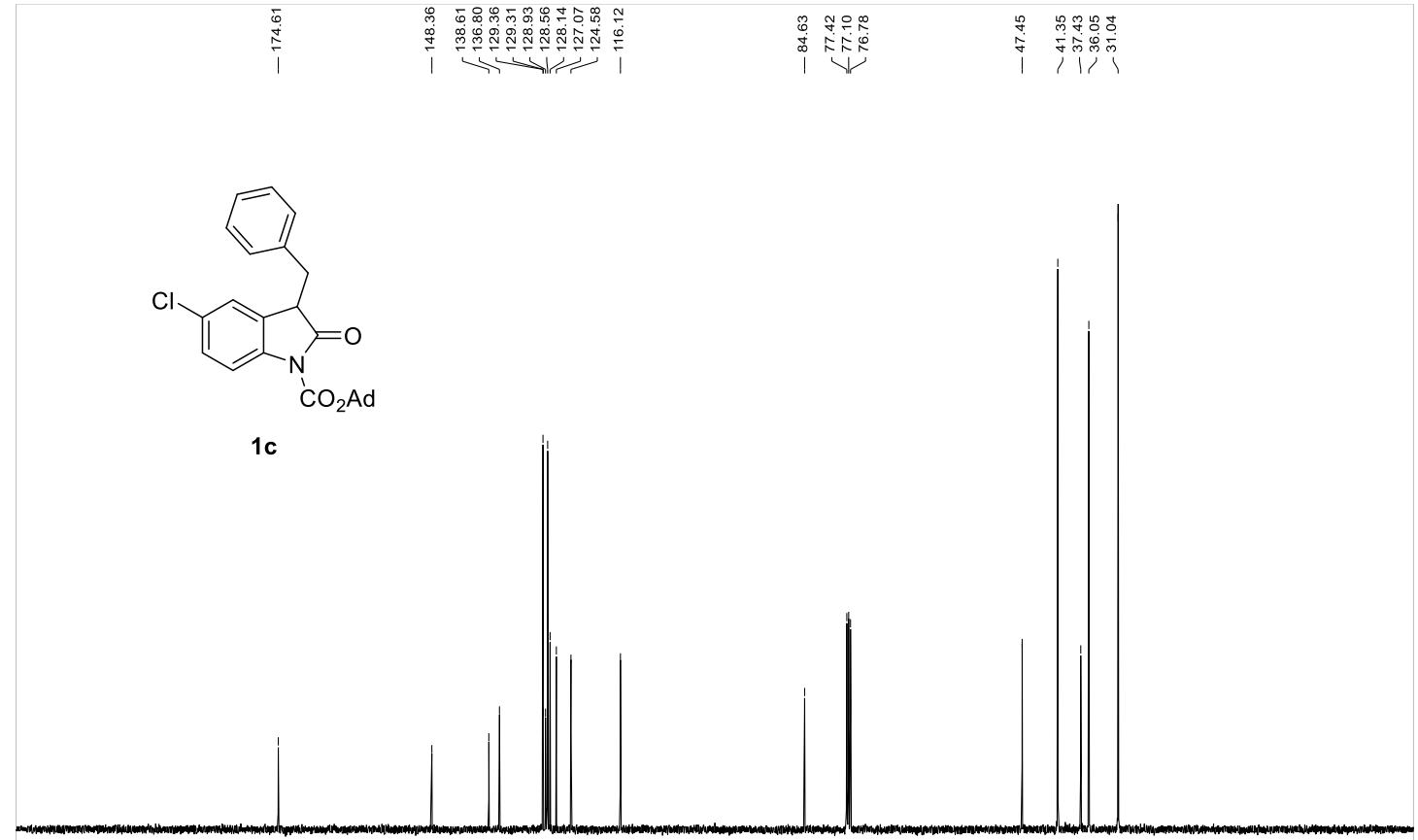

$\begin{array}{rlllllllllllllllllllllllll}210 & 200 & 190 & 180 & 170 & 160 & 150 & 140 & 130 & 120 & 110 & 100 & 90 & 80 & 70 & 60 & 50 & 40 & 30 & 20 & 10 & 0 & -10\end{array}$ 
1d ${ }^{1} \mathrm{H}$ NMR (400M Hz, $\left.\mathrm{CDCl}_{3}\right)$

$\underbrace{5}$

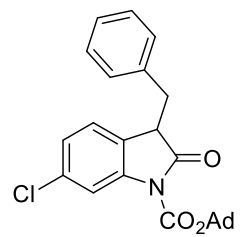

$1 d$

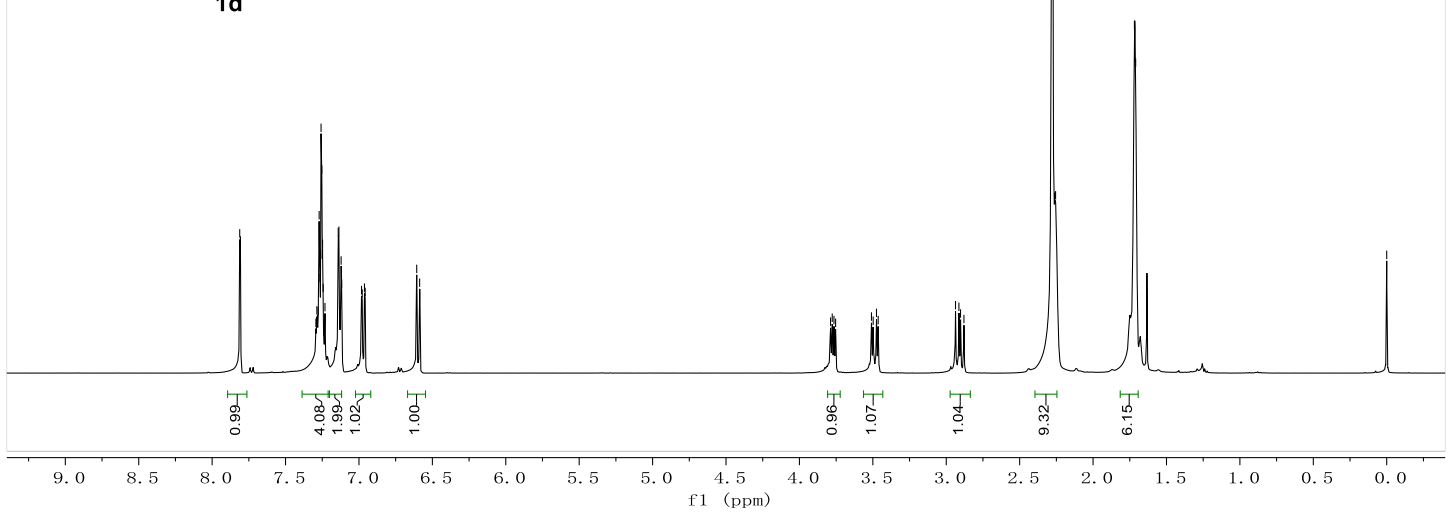

1d ${ }^{13} \mathrm{C}$ NMR $\left(100 \mathrm{M} \mathrm{Hz}, \mathrm{CDCl}_{3}\right)$

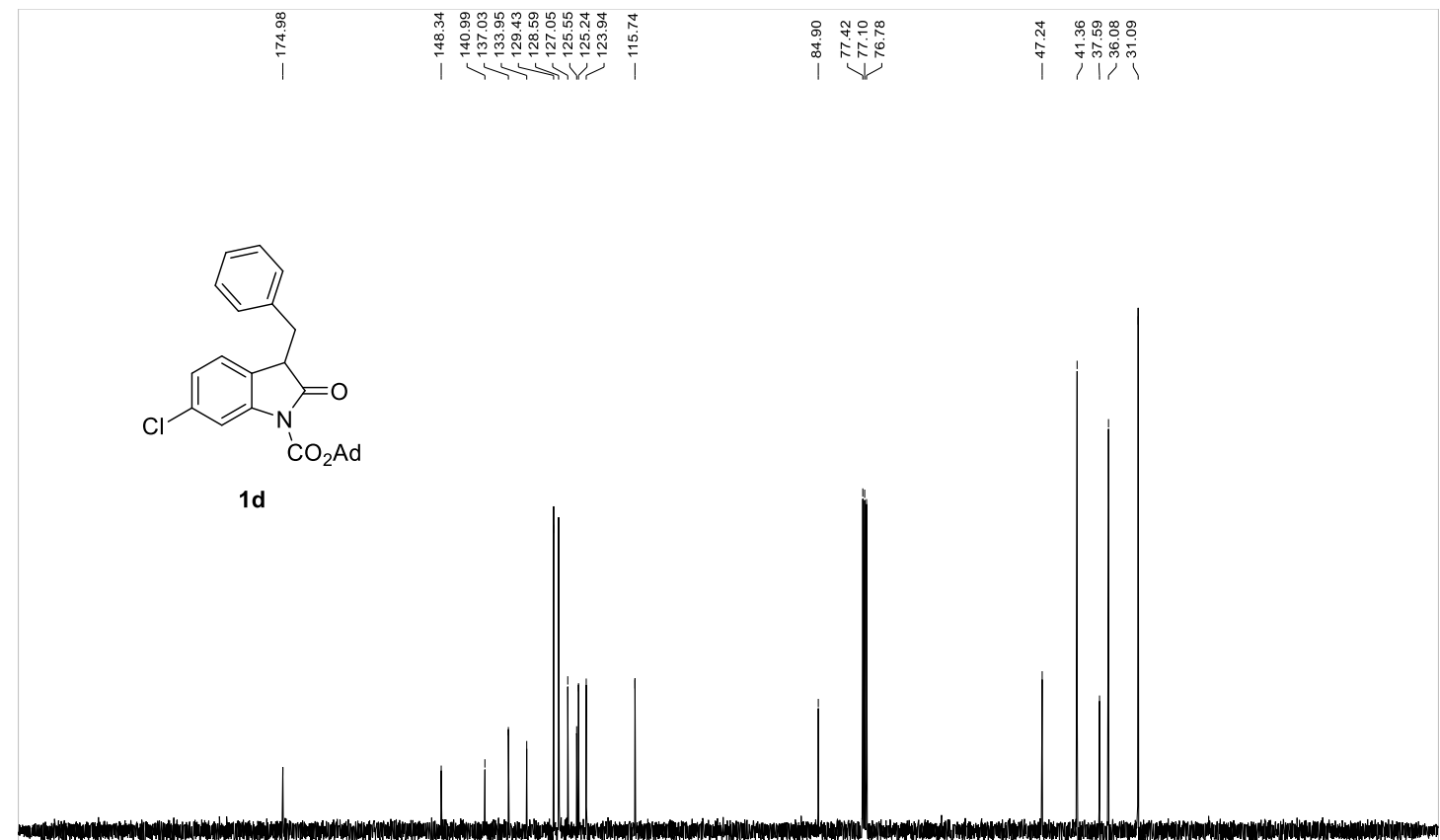

$\begin{array}{lllllllllllllllllllllllll}1 & 1 \\ 210 & 200 & 190 & 180 & 170 & 160 & 150 & 140 & 130 & 120 & 110 & 100 & 90 & 80 & 70 & 60 & 50 & 40 & 30 & 20 & 10 & 0 & -10\end{array}$ 
1e ${ }^{1} \mathrm{H}$ NMR $\left(400 \mathrm{M} \mathrm{Hz}, \mathrm{CDCl}_{3}\right)$

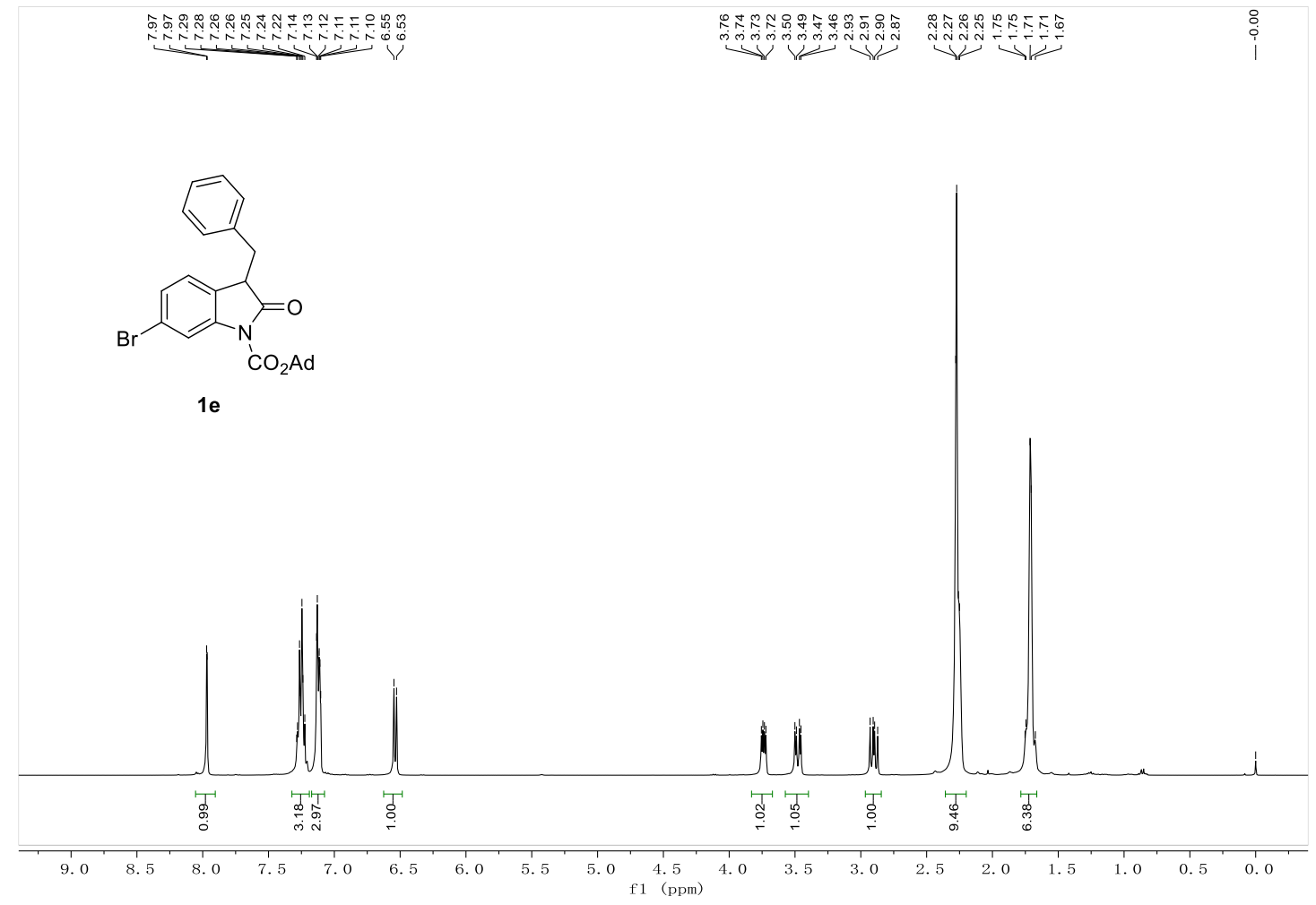

1e ${ }^{13} \mathrm{C} \mathrm{NMR}\left(100 \mathrm{M} \mathrm{Hz}, \mathrm{CDCl}_{3}\right)$

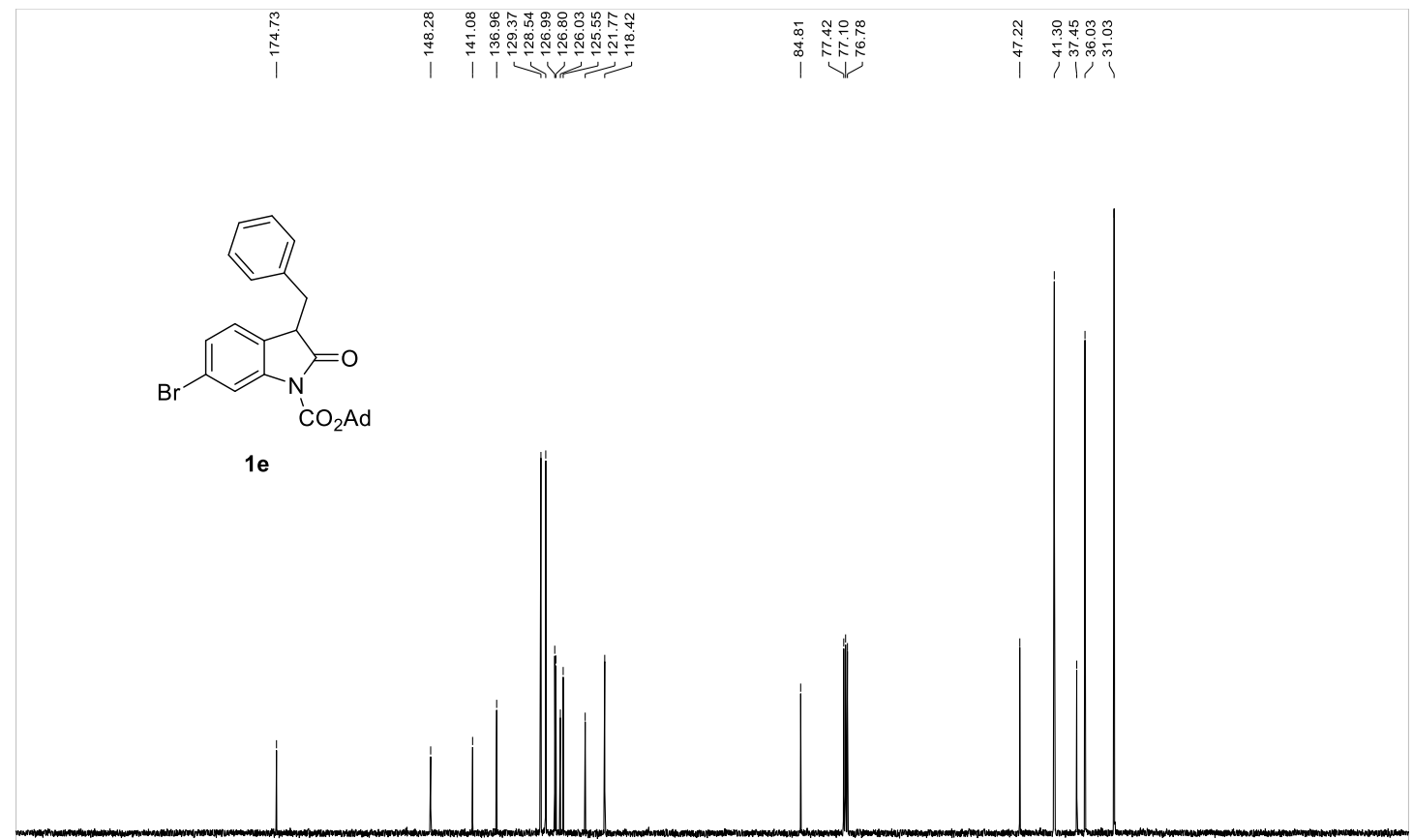

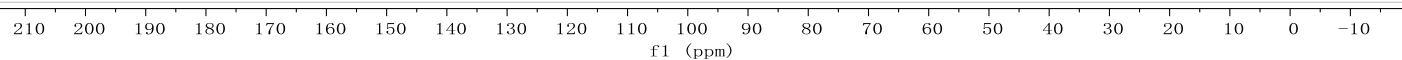


1f ${ }^{1} \mathrm{H} \mathrm{NMR}\left(400 \mathrm{M} \mathrm{Hz}, \mathrm{CDCl}_{3}\right)$

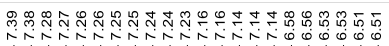

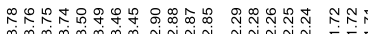

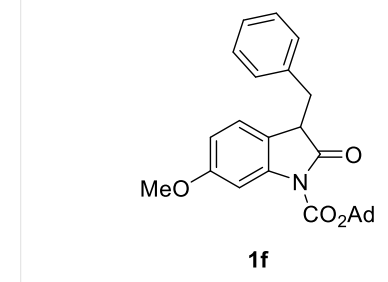

1f
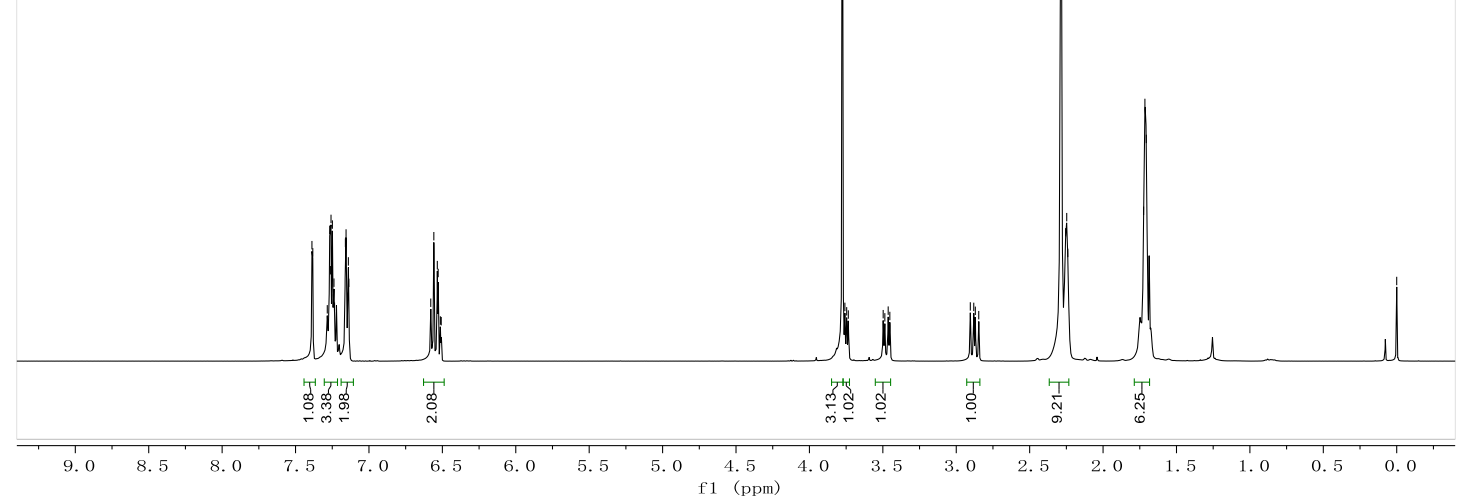

1f ${ }^{13} \mathrm{C} \mathrm{NMR}\left(100 \mathrm{M} \mathrm{Hz}, \mathrm{CDCl}_{3}\right)$

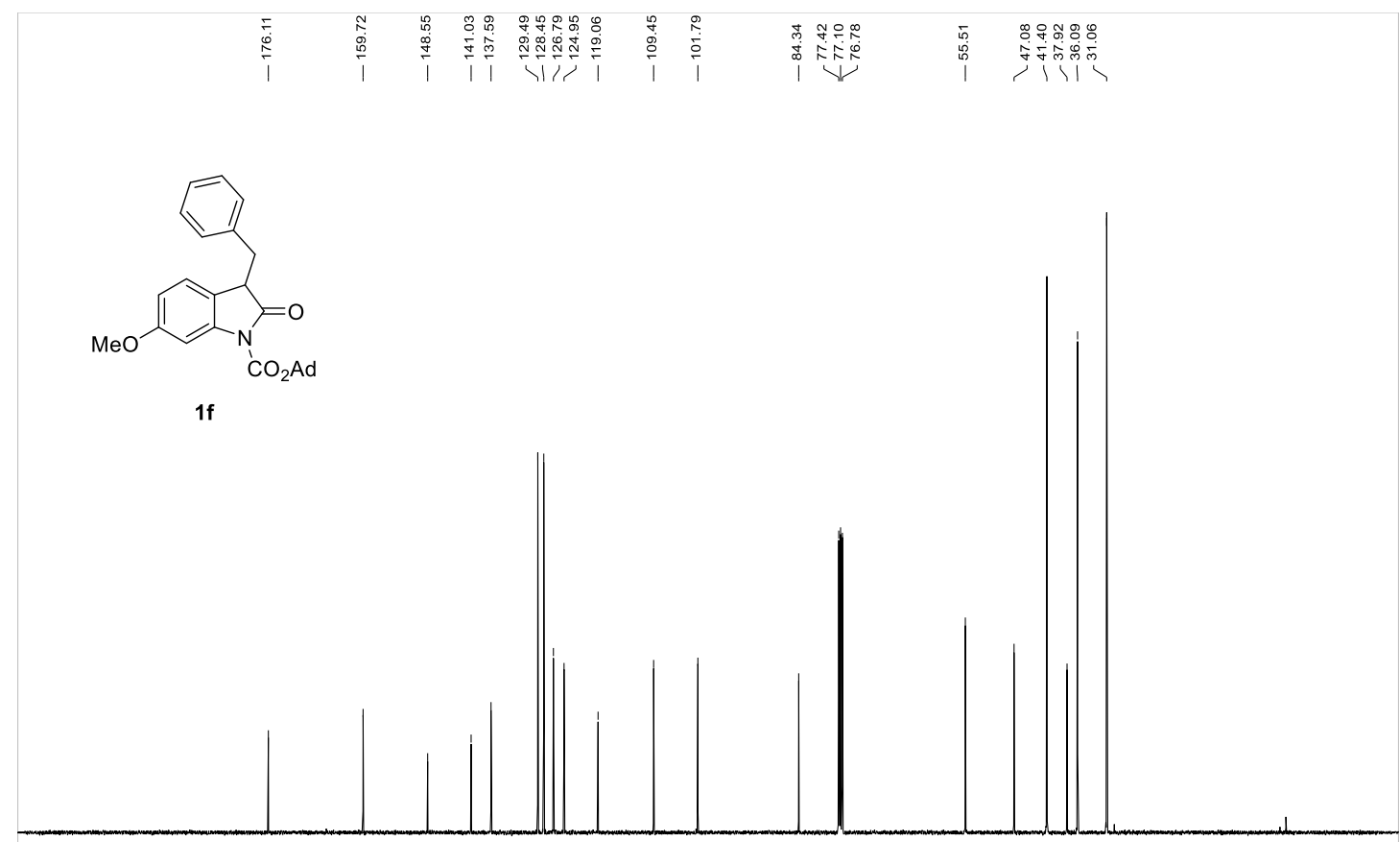

$\begin{array}{rllllllllllllllllllllllll}1 & 10 & 200 & 190 & 180 & 170 & 160 & 150 & 140 & 130 & 120 & 110 & 100 & 90 & 80 & 70 & 60 & 50 & 40 & 30 & 20 & 10 & 0 & -10\end{array}$ 
1g ${ }^{1} \mathrm{H}$ NMR (400M Hz, $\left.\mathrm{CDCl}_{3}\right)$

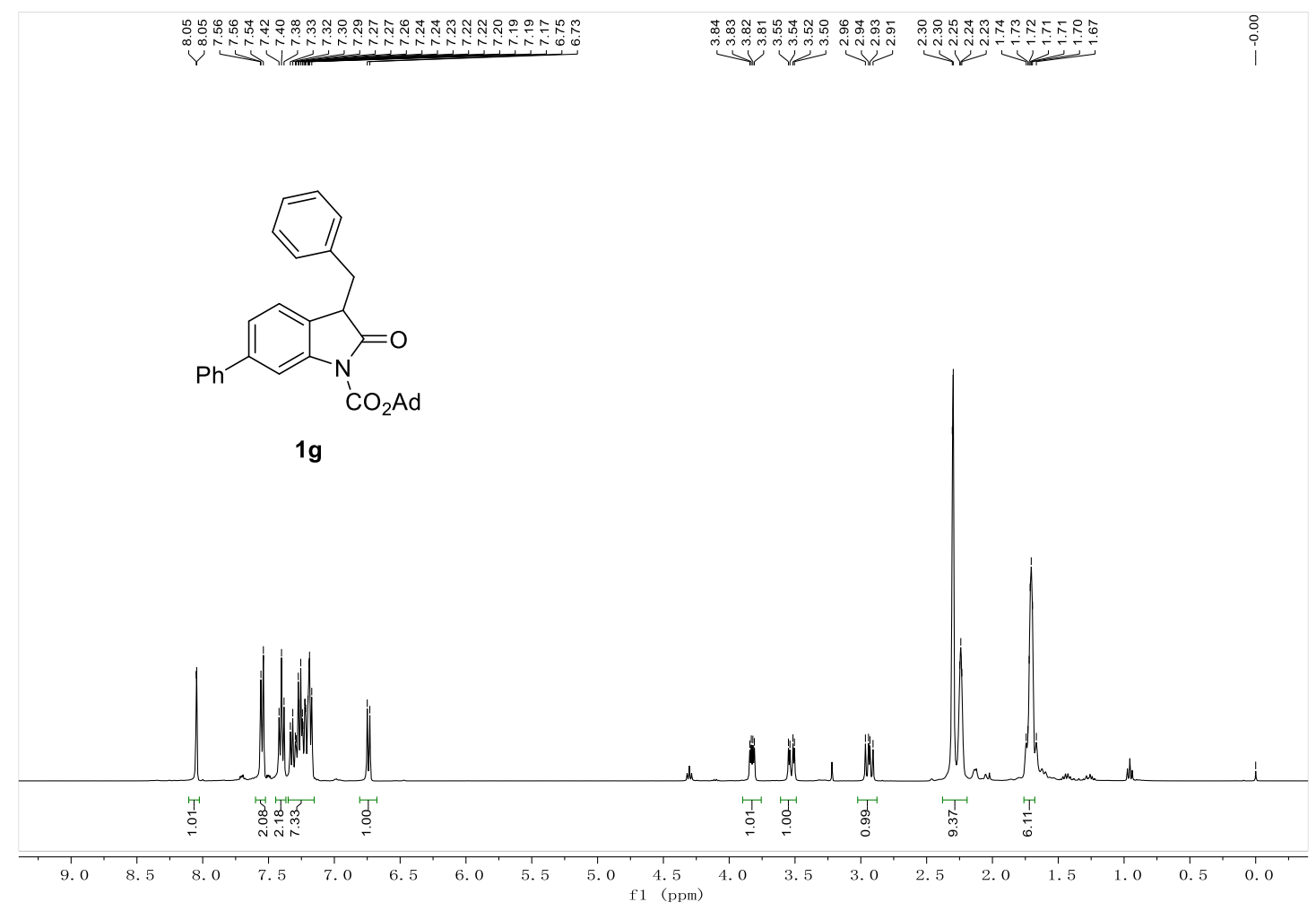

1 g ${ }^{13} \mathrm{C} \mathrm{NMR}\left(100 \mathrm{M} \mathrm{Hz}, \mathrm{CDCl}_{3}\right)$

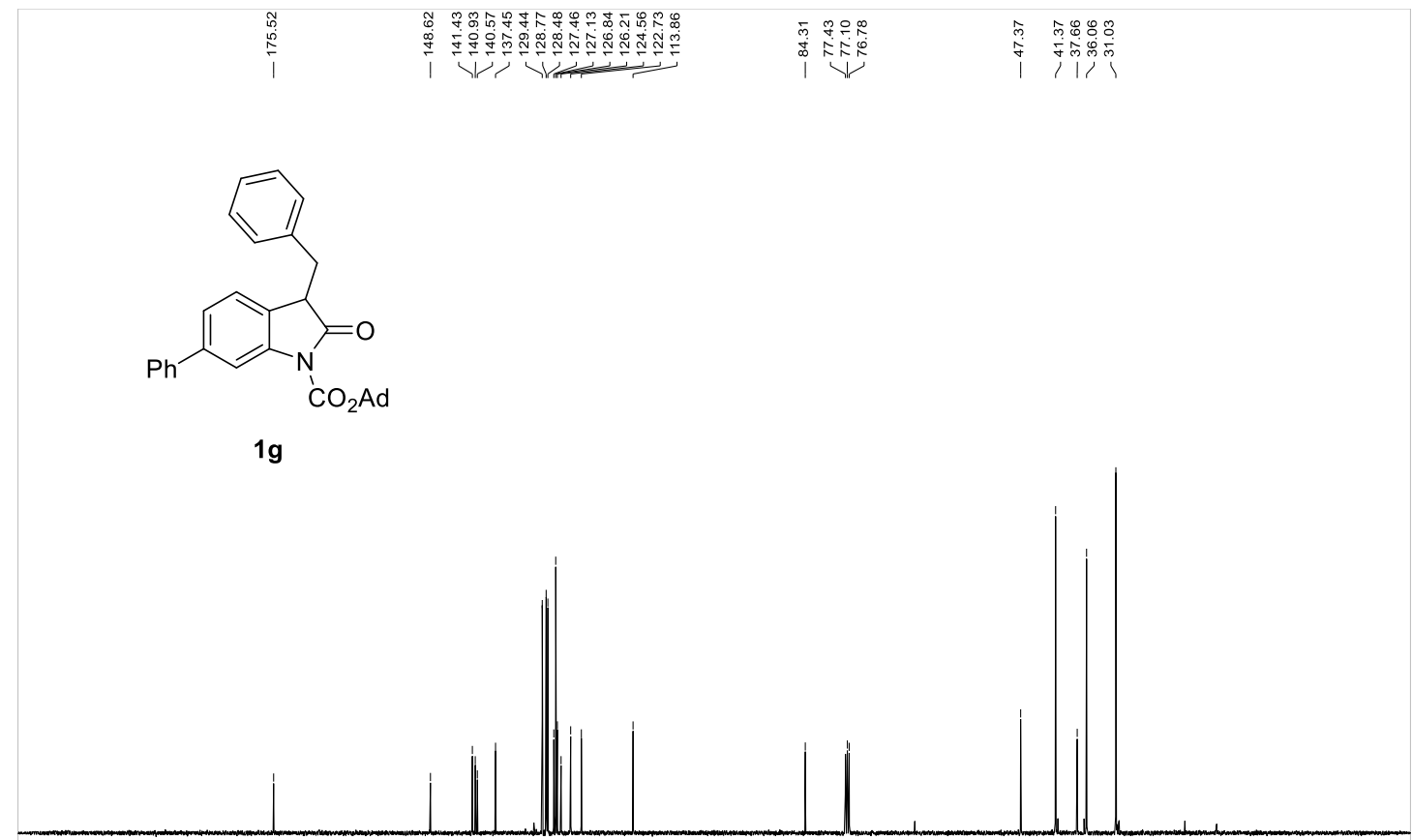

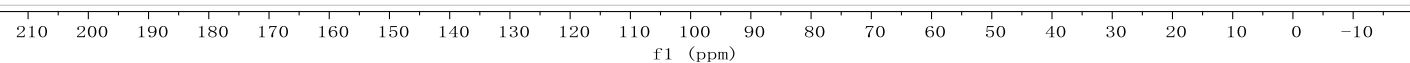


1h ${ }^{1} \mathrm{H}$ NMR (400M Hz, $\mathrm{CDCl}_{3}$ )

0.5
4

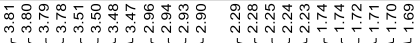

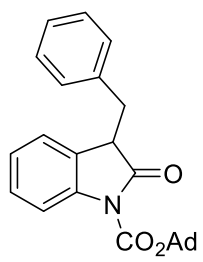

$1 \mathrm{~h}$

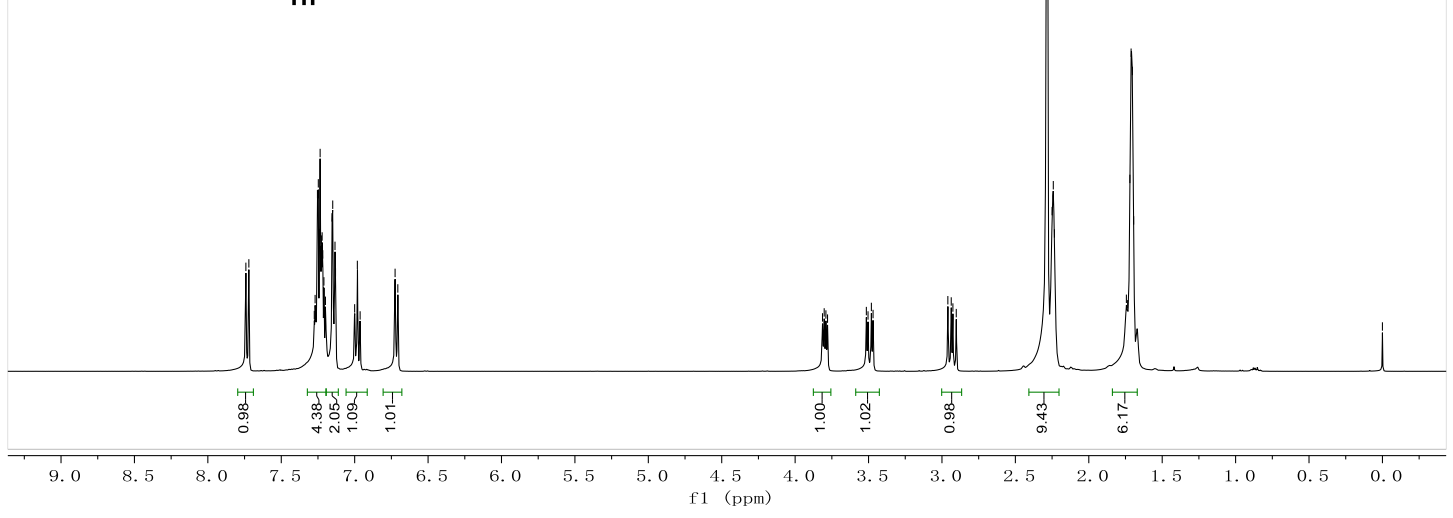

1h ${ }^{13} \mathrm{C}$ NMR (100M Hz, $\left.\mathrm{CDCl}_{3}\right)$

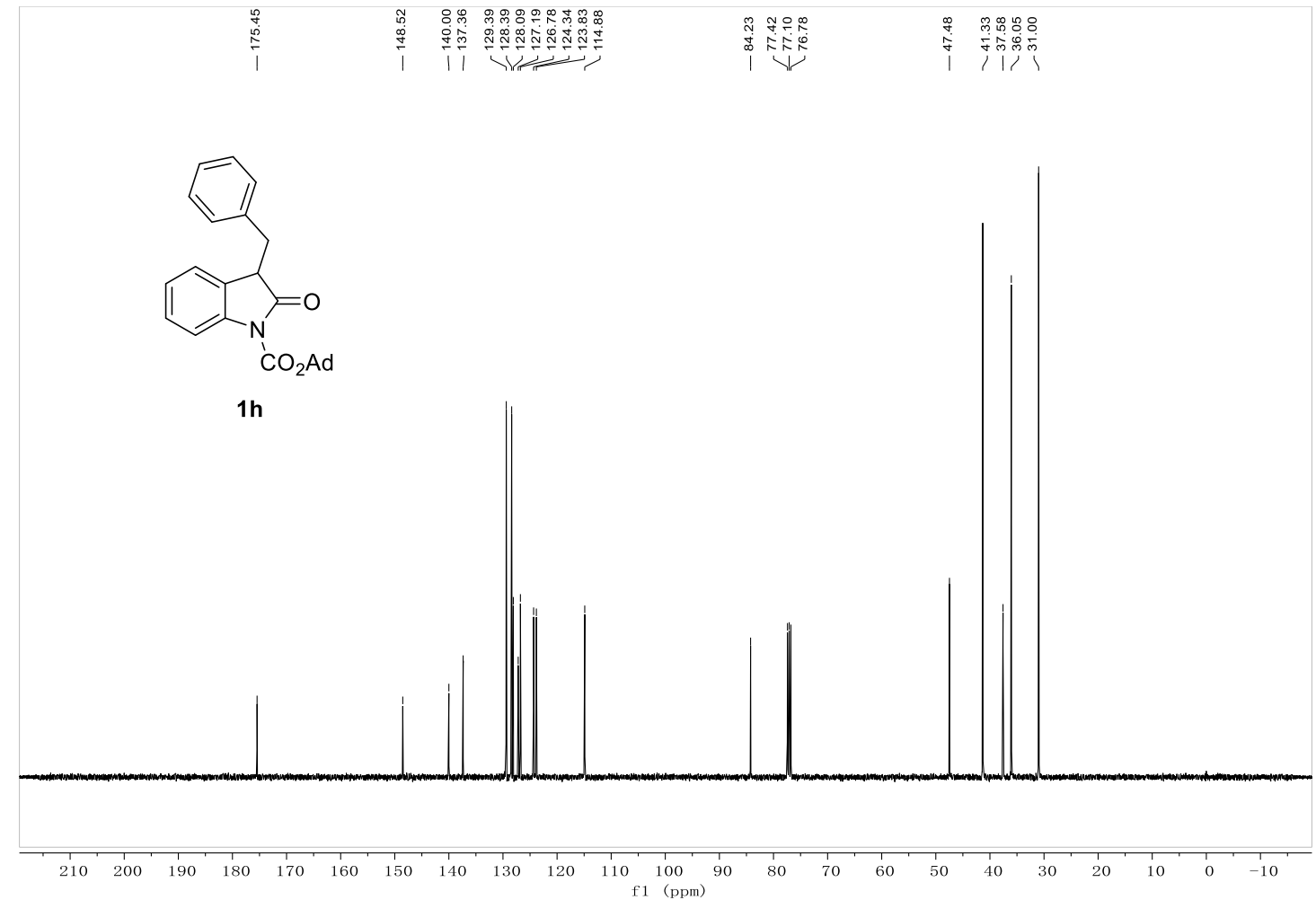


1i ${ }^{1} \mathrm{H}$ NMR (400M Hz, $\left.\mathrm{CDCl}_{3}\right)$

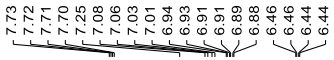

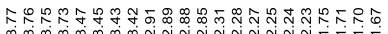

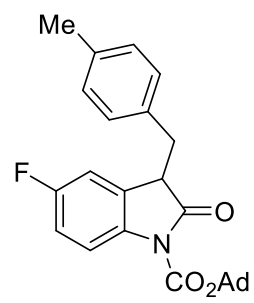

$1 \mathbf{i}$

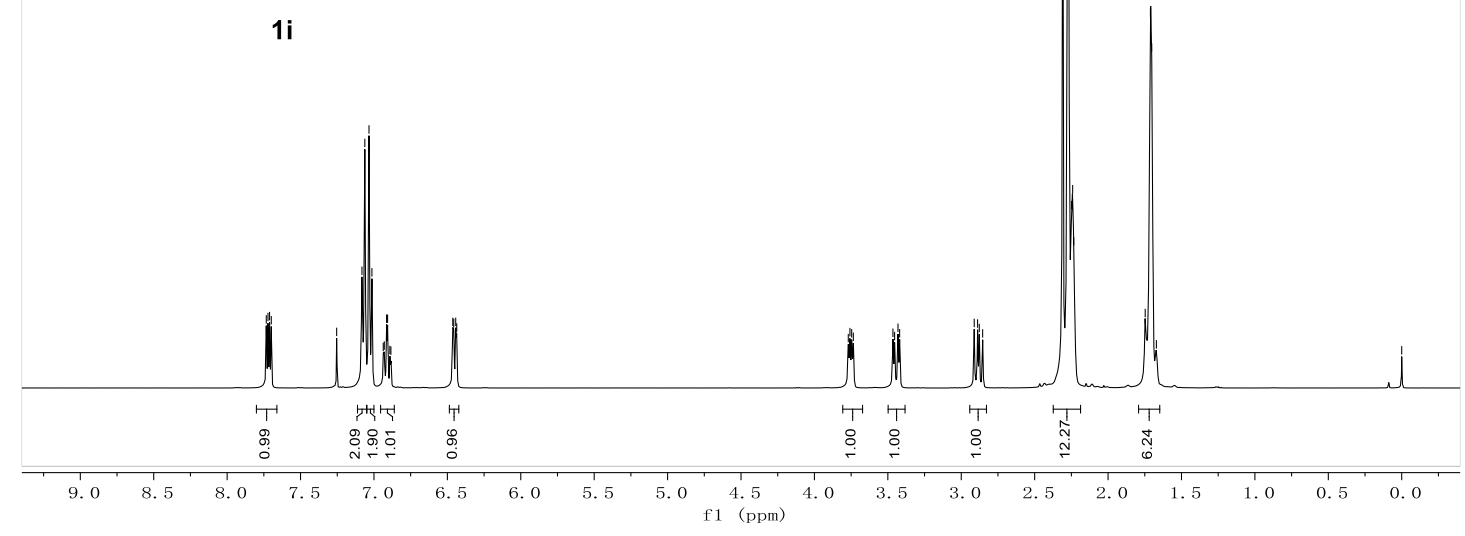

1i ${ }^{13} \mathrm{C} \mathrm{NMR}\left(100 \mathrm{M} \mathrm{Hz}, \mathrm{CDCl}_{3}\right)$

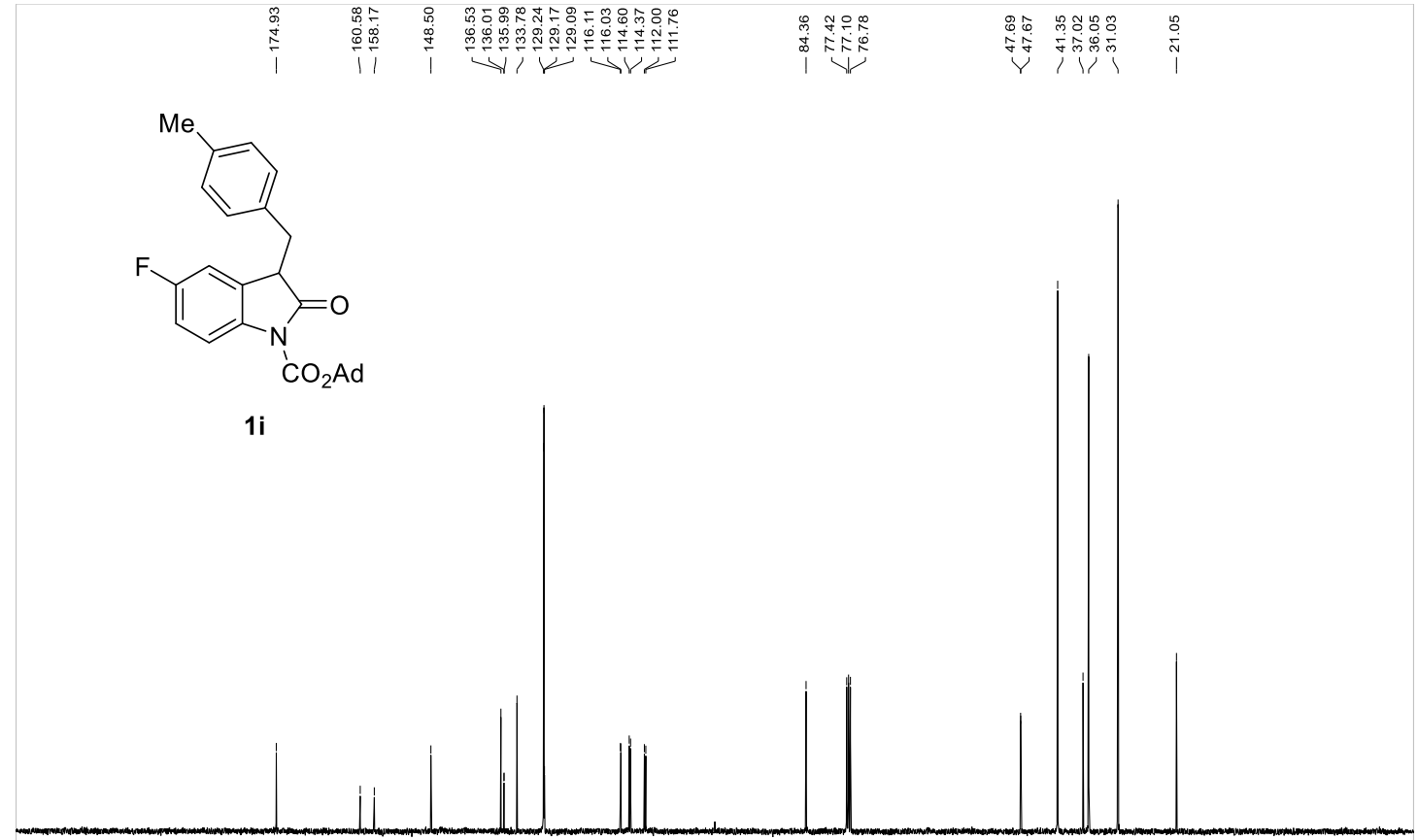

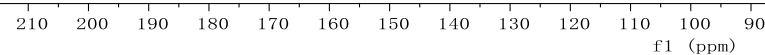


1j ${ }^{1} \mathrm{H}$ NMR (400M Hz, $\left.\mathrm{CDCl}_{3}\right)$

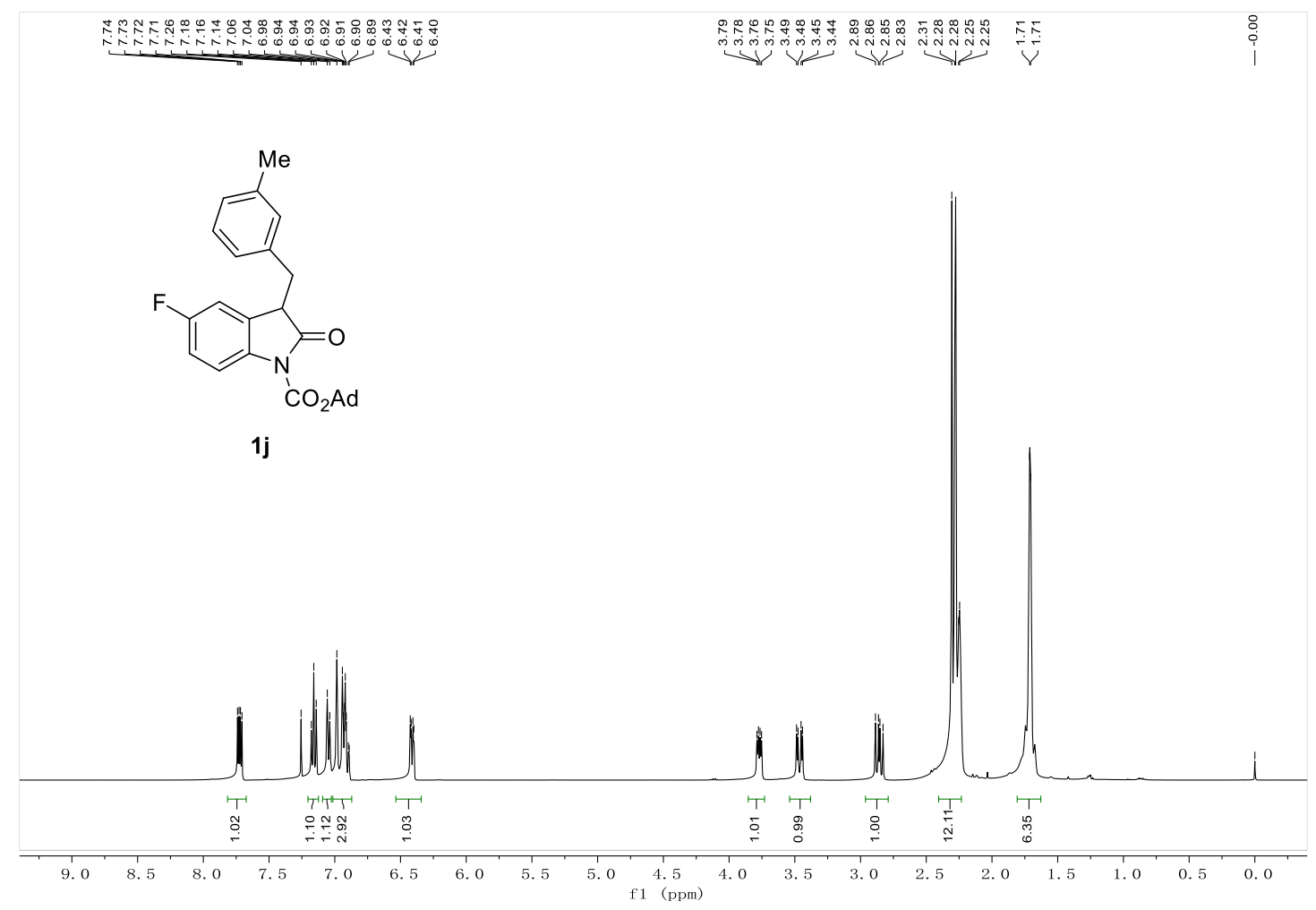

1j ${ }^{13} \mathrm{C}$ NMR $\left(100 \mathrm{M} \mathrm{Hz}, \mathrm{CDCl}_{3}\right)$

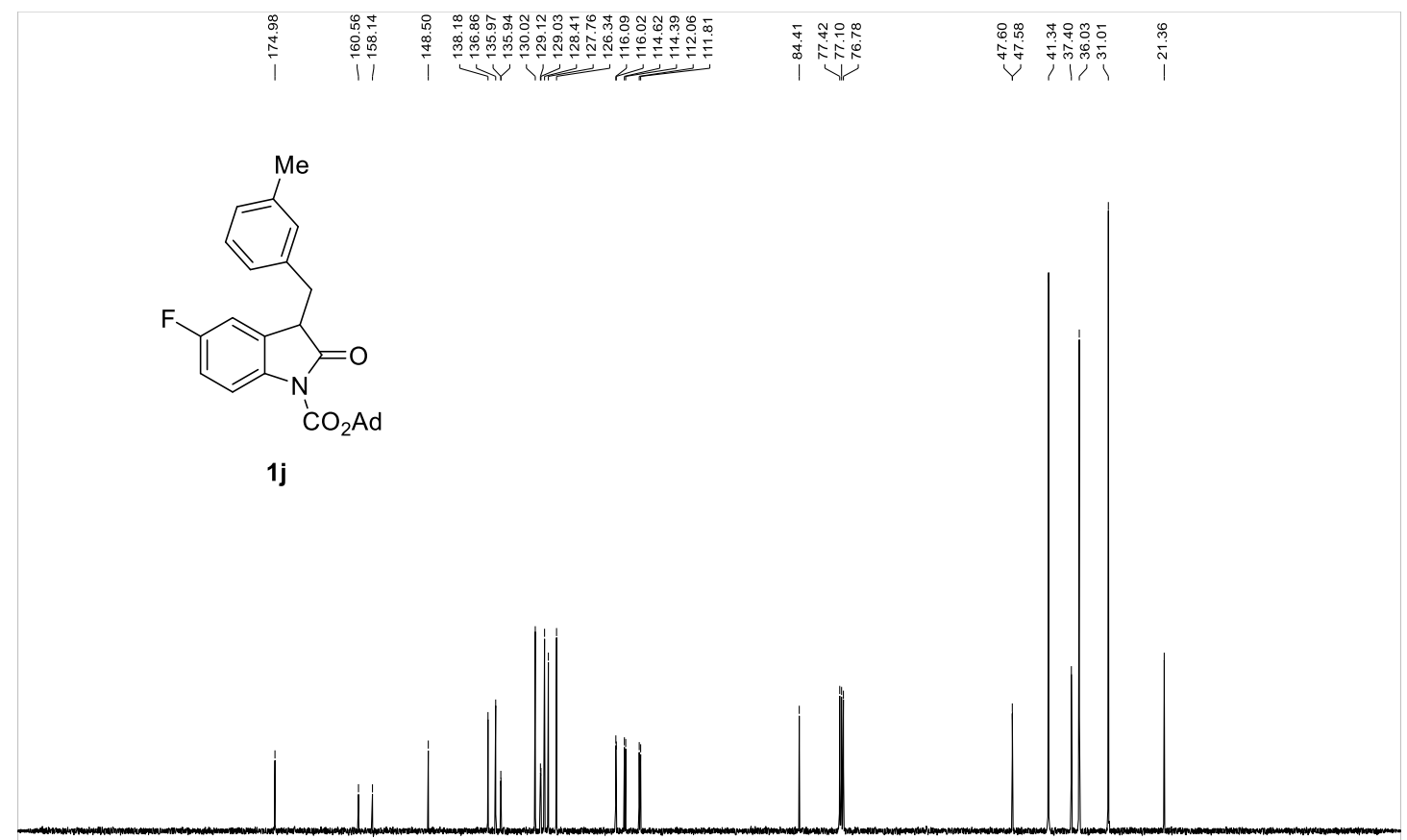

$\begin{array}{lllllllllllllllllllllllllllll}1 & 1 & 200 & 190 & 180 & 170 & 160 & 150 & 140 & 130 & 120 & 110 & 100 & 90 & 80 & 70 & 60 & 50 & 40 & 30 & 20 & 10 & 0 & -10\end{array}$ 
1k ${ }^{1} \mathrm{H}$ NMR (400M Hz, $\left.\mathrm{CDCl}_{3}\right)$

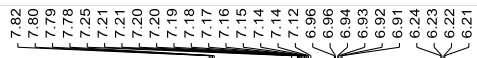

$\underbrace{\text { non }}$

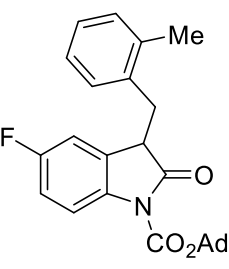

$1 \mathrm{k}$

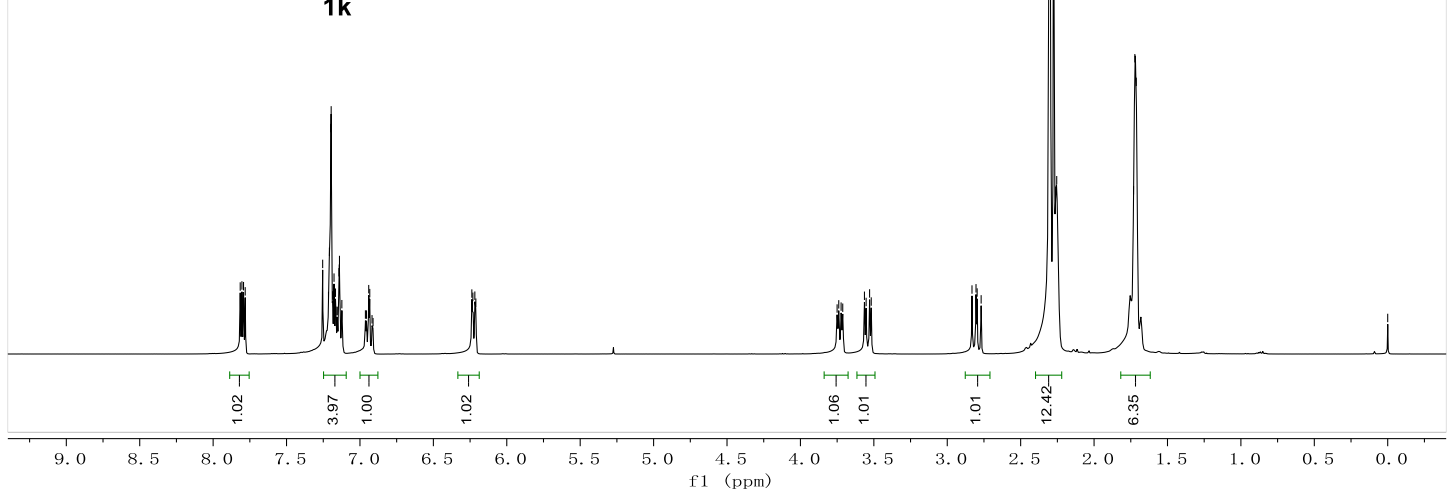

1k ${ }^{13} \mathrm{C}$ NMR $\left(100 \mathrm{M} \mathrm{Hz}, \mathrm{CDCl}_{3}\right)$

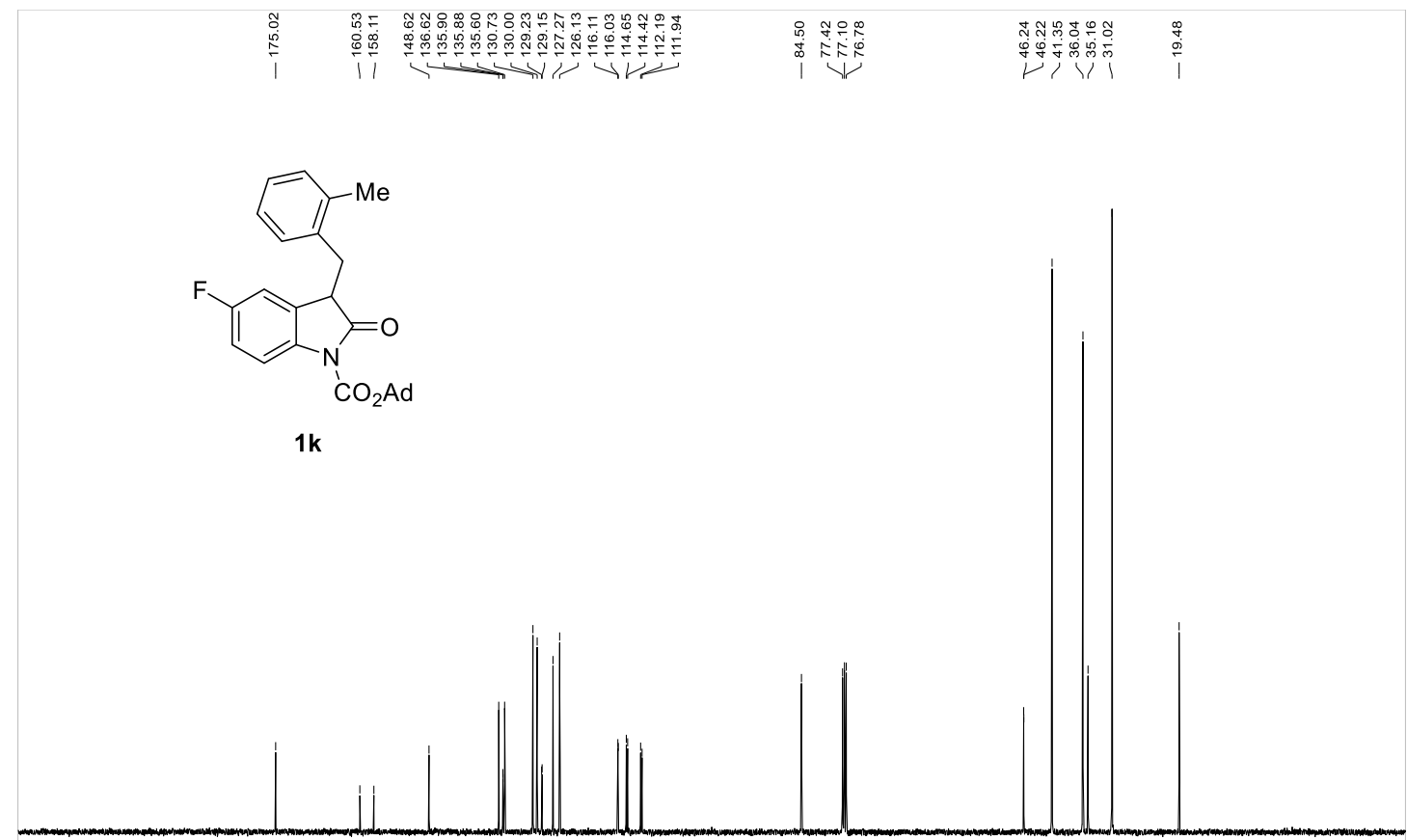

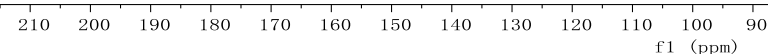


$1{ }^{1} \mathrm{H}$ NMR $\left(400 \mathrm{M} \mathrm{Hz}, \mathrm{CDCl}_{3}\right)$

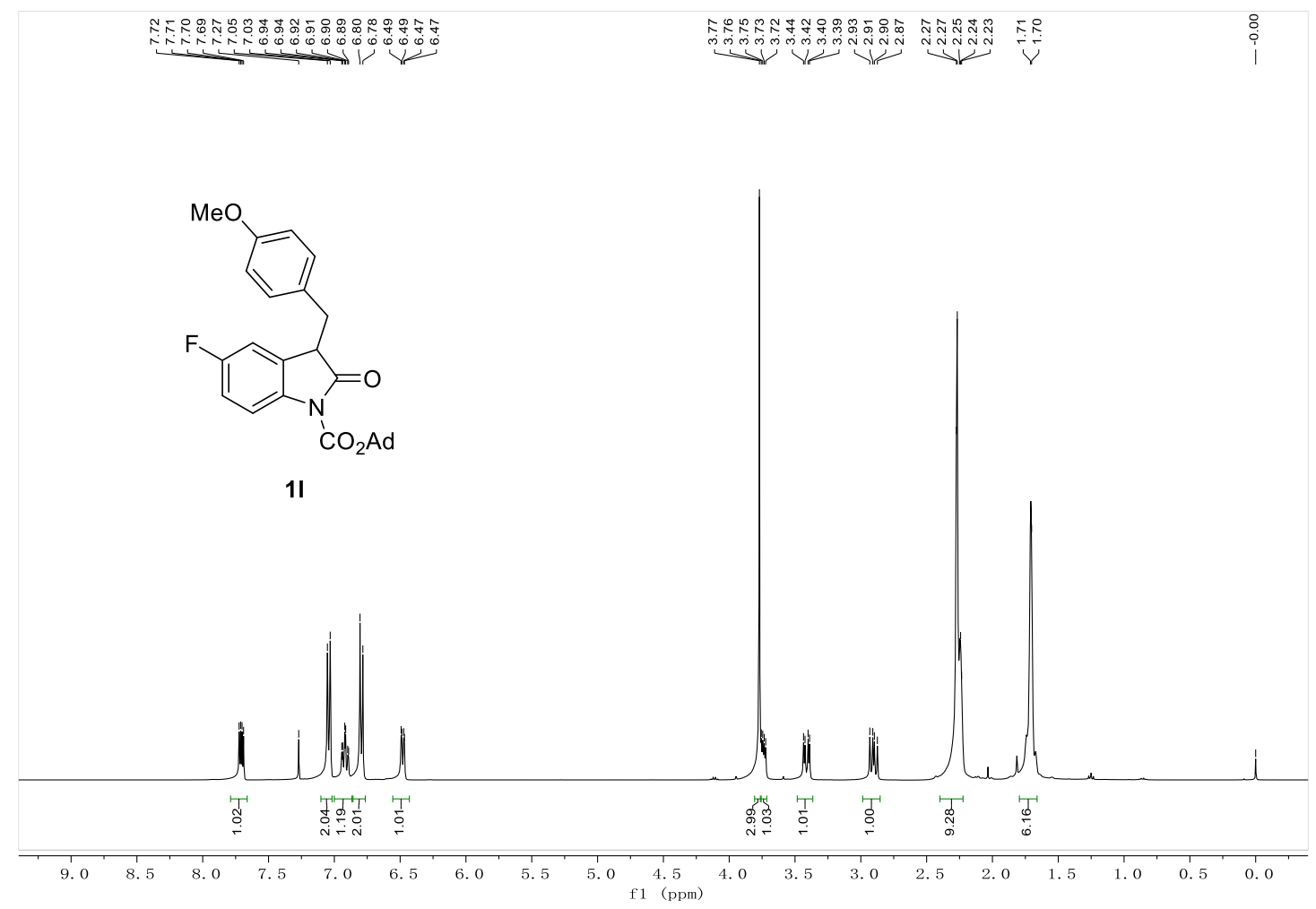

$1{ }^{13} \mathrm{C}$ NMR $\left(100 \mathrm{M} \mathrm{Hz}, \mathrm{CDCl}_{3}\right)$

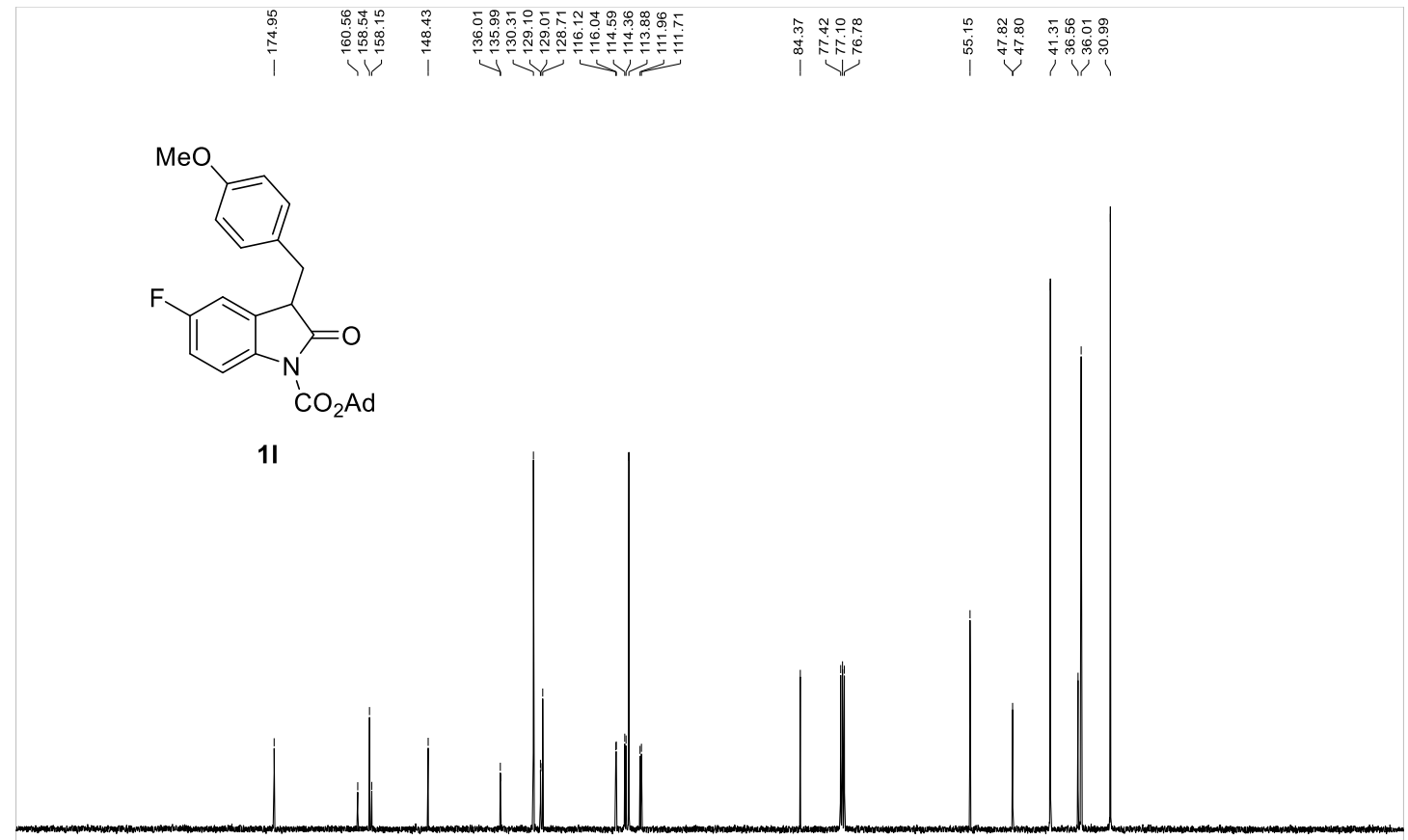

$\begin{array}{lllllllllllllllllllllllll}1 & 1 & 200 & 190 & 180 & 170 & 160 & 150 & 140 & 130 & 120 & 110 & 100 & 90 & 80 & 70 & 60 & 50 & 40 & 30 & 20 & 10 & 0 & -10\end{array}$ 
$1 \mathbf{m}{ }^{1} \mathrm{H}$ NMR $\left(400 \mathrm{M} \mathrm{Hz}, \mathrm{CDCl}_{3}\right)$

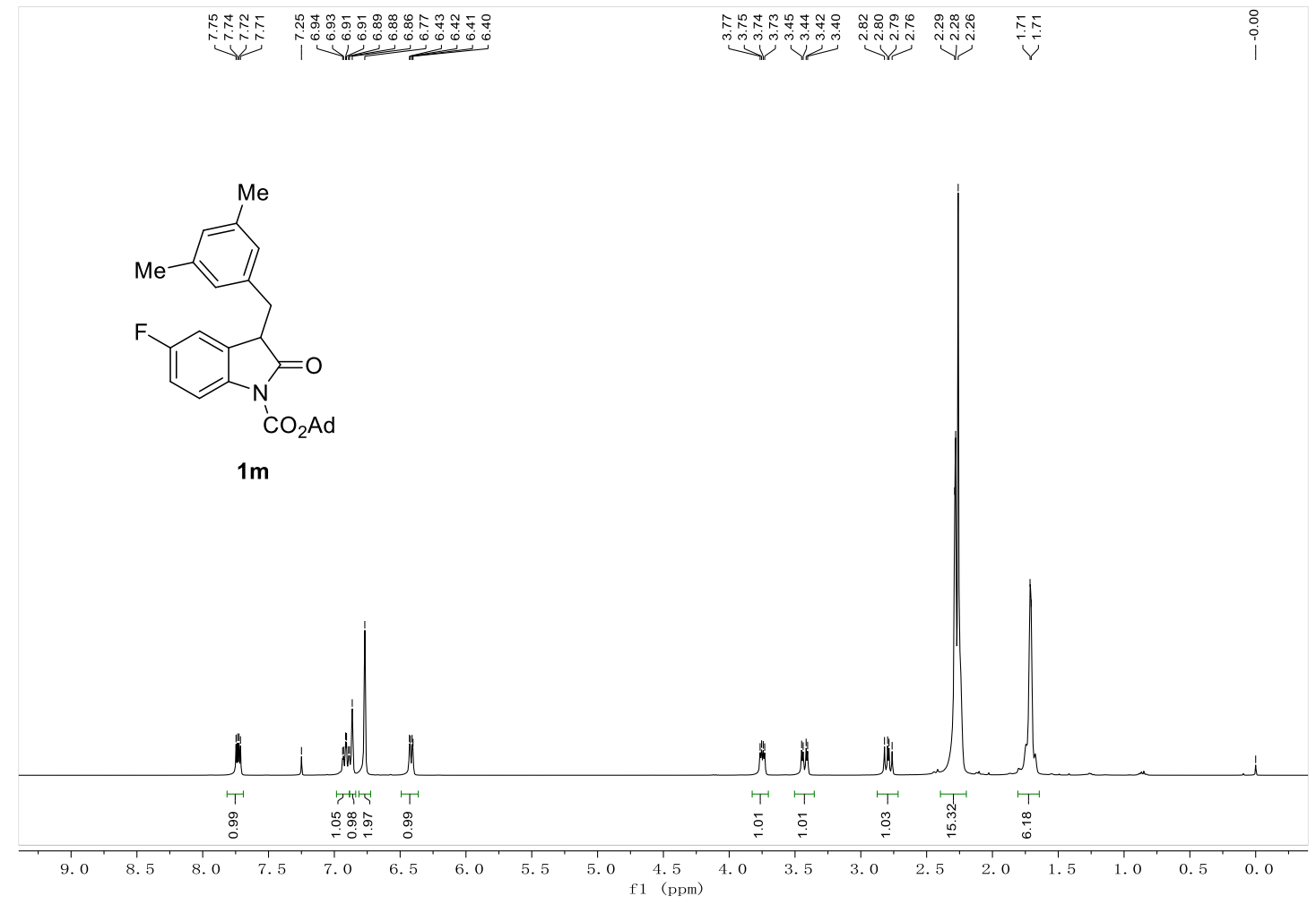

$1 \mathbf{m}{ }^{13} \mathrm{C}$ NMR $\left(100 \mathrm{M} \mathrm{Hz}, \mathrm{CDCl}_{3}\right)$

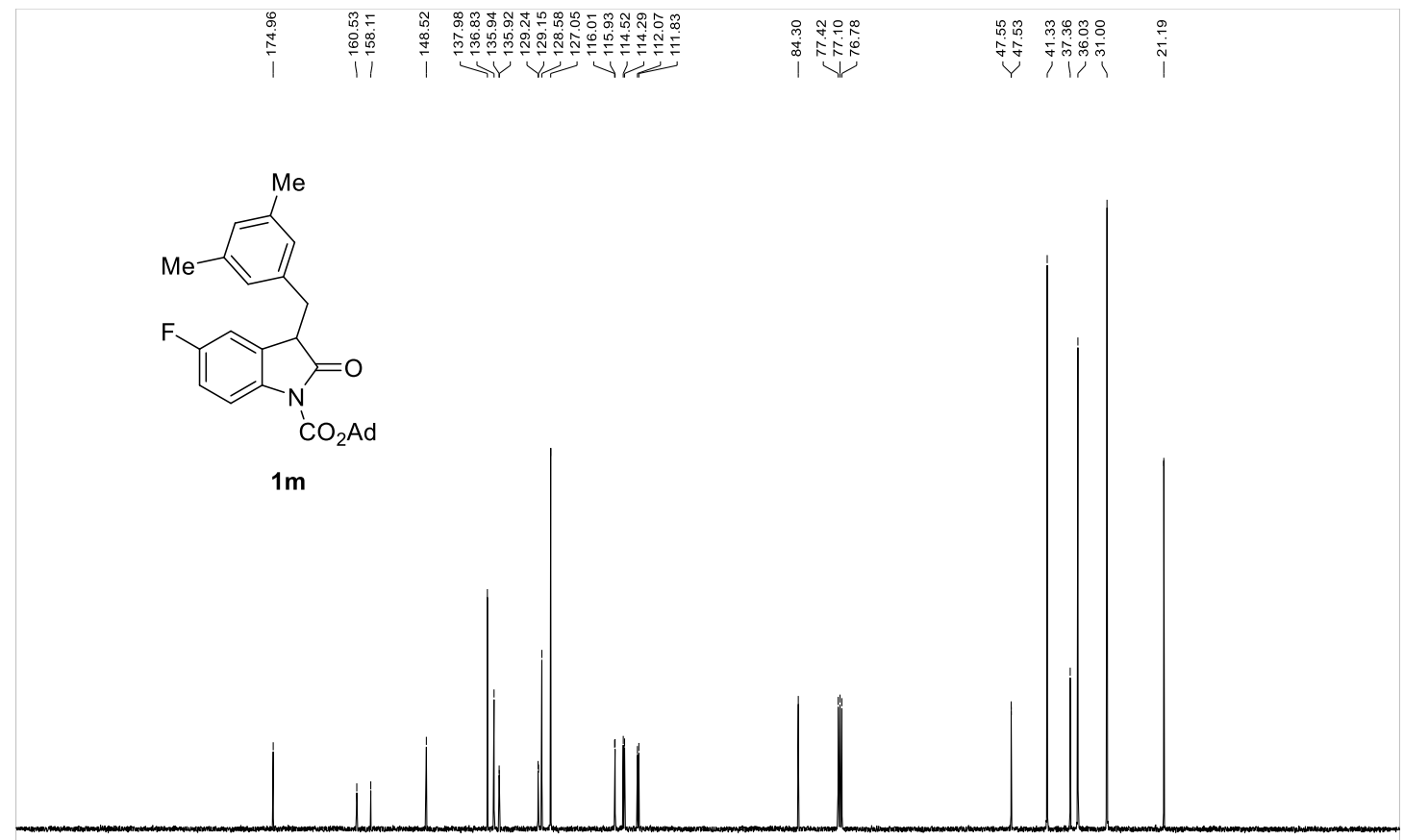

$\begin{array}{rlllllllllllllllllllllllll}1 & 10 & 200 & 190 & 180 & 170 & 160 & 150 & 140 & 130 & 120 & 110 & 100 & 90 & 80 & 70 & 60 & 50 & 40 & 30 & 20 & 10 & 0 & -10\end{array}$ 
1n ${ }^{1} \mathrm{H}$ NMR (400M Hz, $\left.\mathrm{CDCl}_{3}\right)$

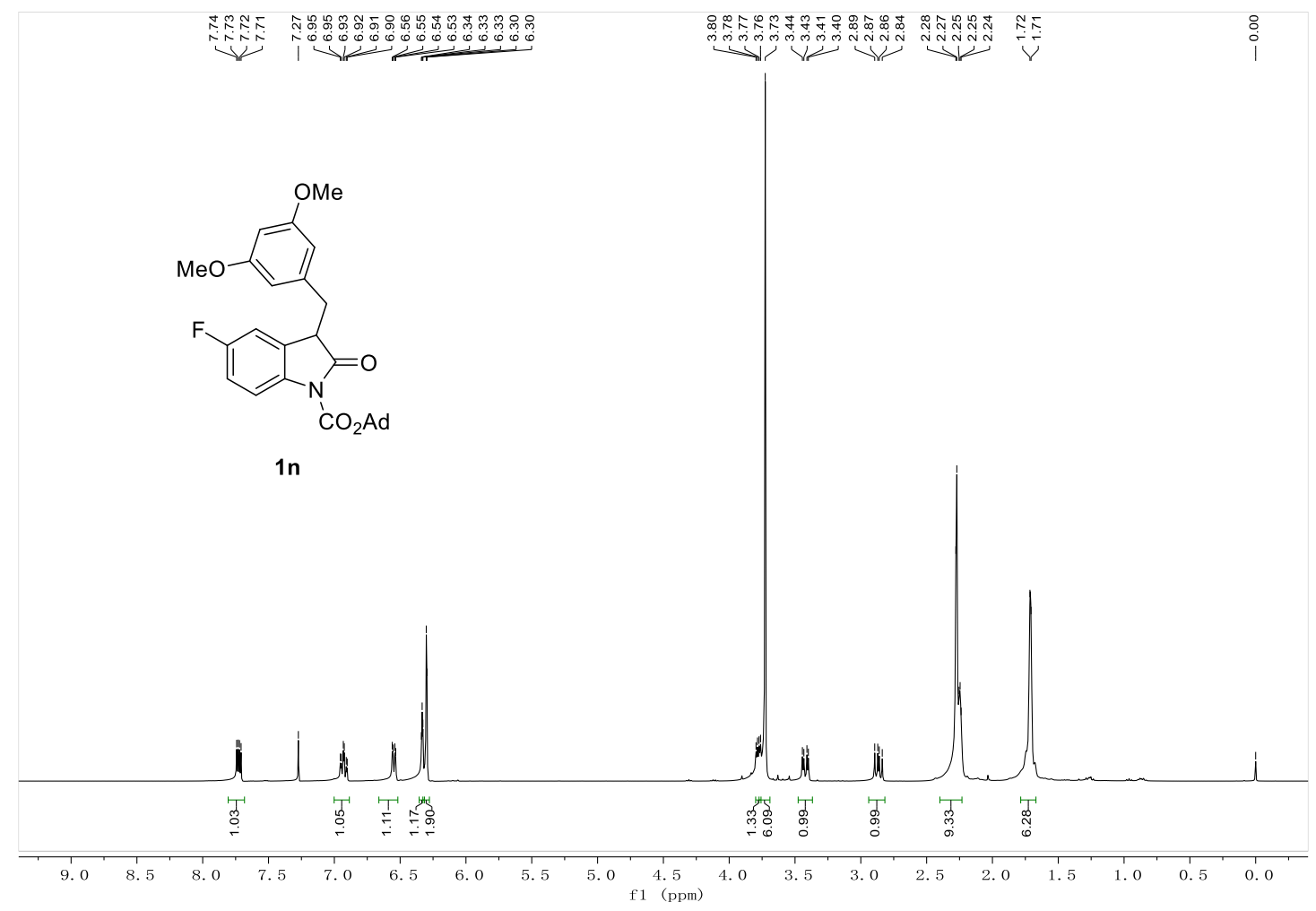

1n ${ }^{13} \mathrm{C} \mathrm{NMR}\left(100 \mathrm{M} \mathrm{Hz}, \mathrm{CDCl}_{3}\right)$

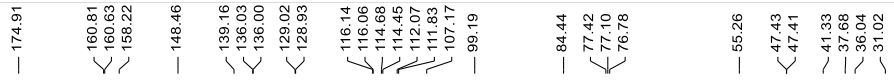

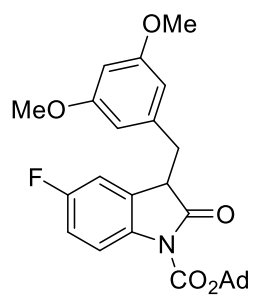

$1 n$

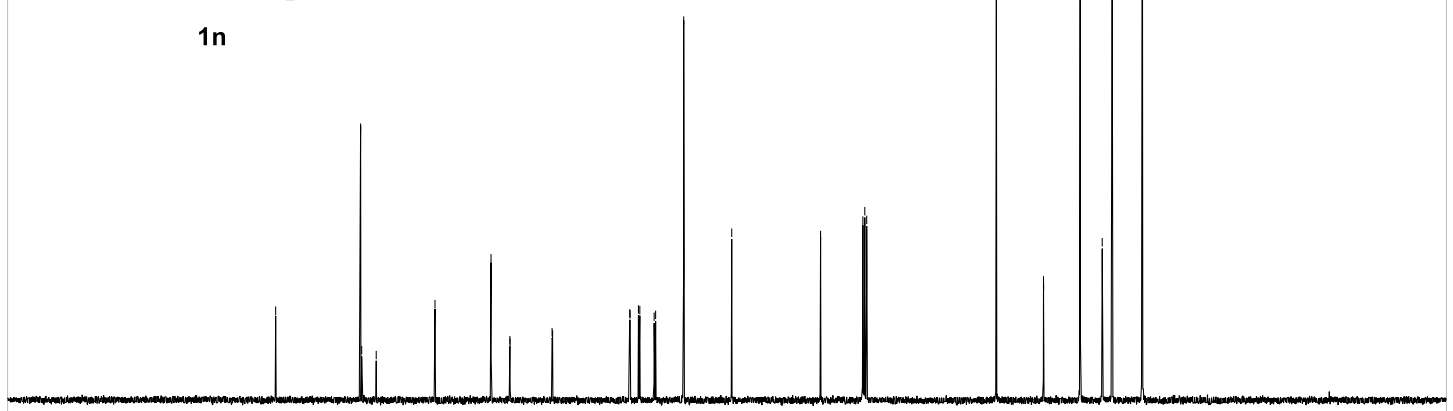

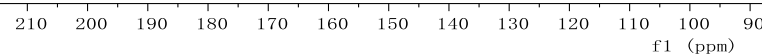


10 ${ }^{1} \mathrm{H}$ NMR (400M Hz, $\left.\mathrm{CDCl}_{3}\right)$

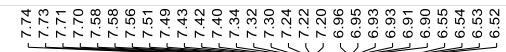

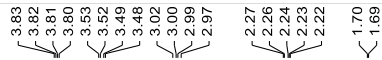

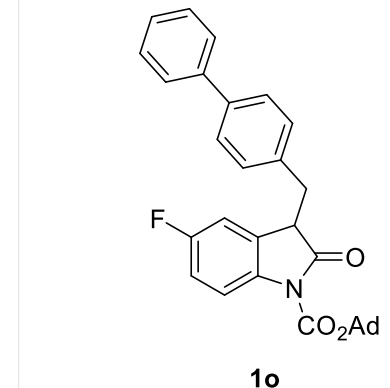

10
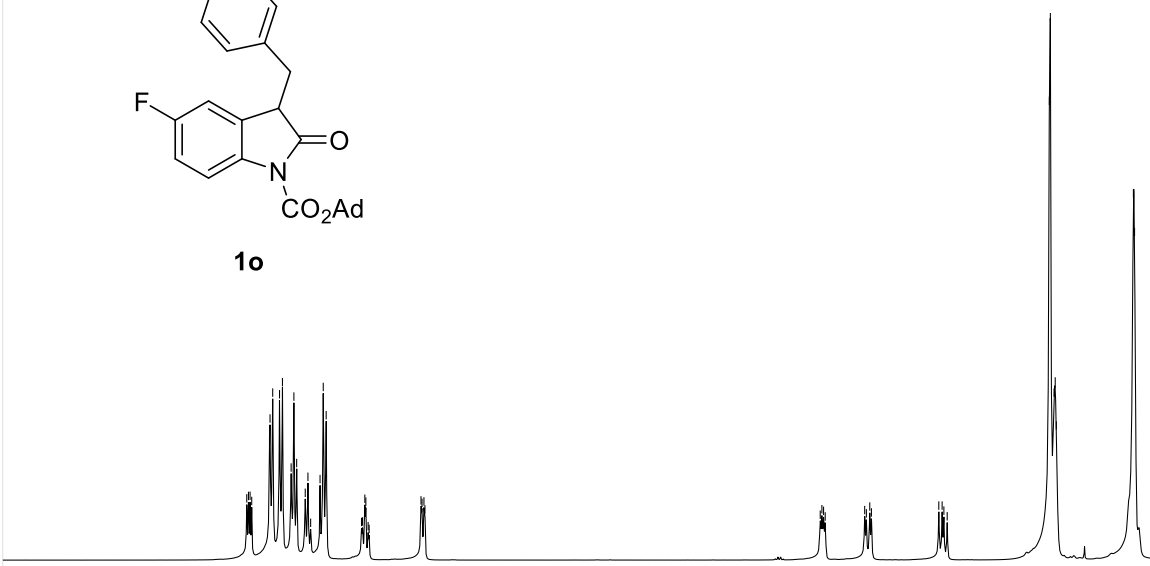

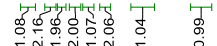

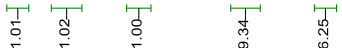

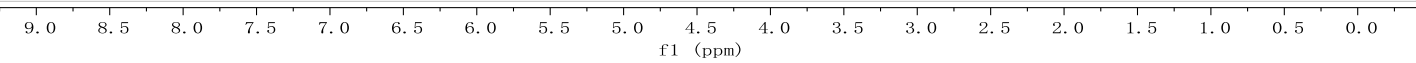

1o ${ }^{13} \mathrm{C} \mathrm{NMR}\left(100 \mathrm{M} \mathrm{Hz}, \mathrm{CDCl}_{3}\right)$

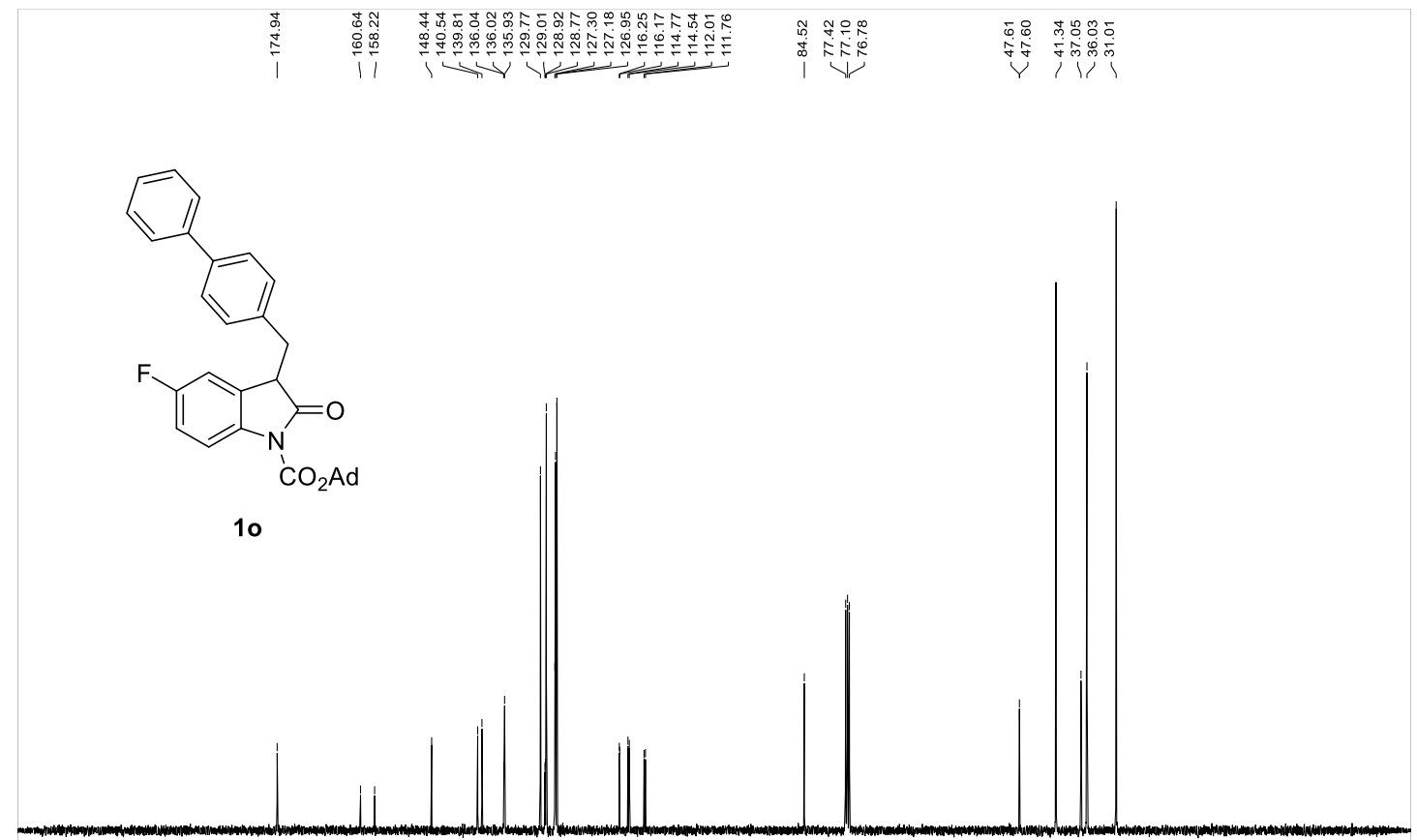

$\begin{array}{lllllllllllll}210 & 200 & 190 & 180 & 170 & 160 & 150 & 140 & 130 & 120 & 110 & 100 & 90 \\ \mathrm{f} 1 & (\mathrm{ppm})\end{array}$ 
1p ${ }^{1} \mathrm{H}$ NMR $\left(400 \mathrm{M} \mathrm{Hz}, \mathrm{CDCl}_{3}\right)$

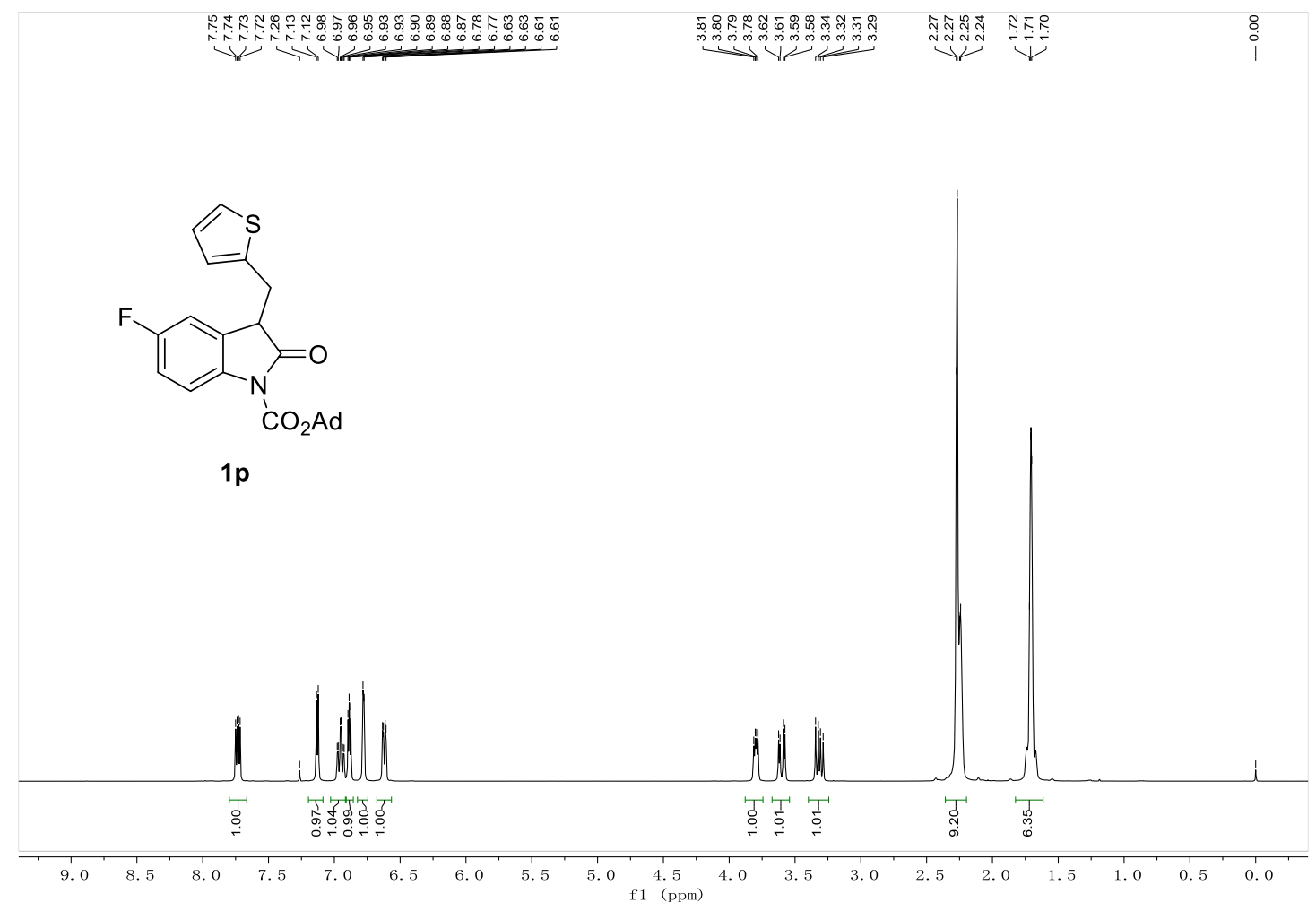

1 p ${ }^{13} \mathrm{C}$ NMR $\left(100 \mathrm{M} \mathrm{Hz}, \mathrm{CDCl}_{3}\right)$

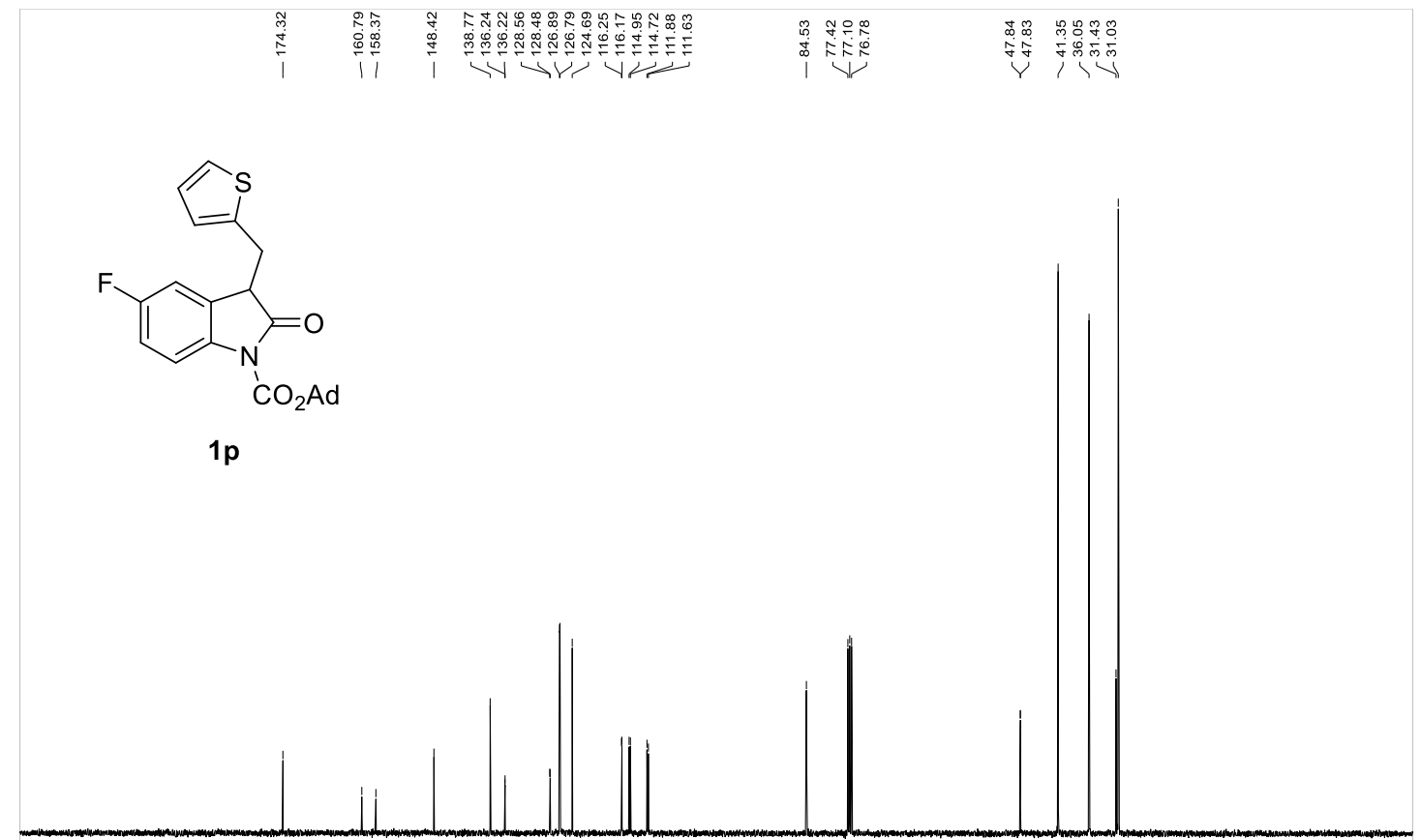

$\begin{array}{rllllllllllllllllllllllll}210 & 200 & 190 & 180 & 170 & 160 & 150 & 140 & 130 & 120 & 110 & 100 & 90 & 80 & 70 & 60 & 50 & 40 & 30 & 20 & 10 & 0 & -10\end{array}$ 
$1 \mathbf{q}^{1} \mathrm{H}$ NMR $\left(400 \mathrm{M} \mathrm{Hz}, \mathrm{CDCl}_{3}\right)$

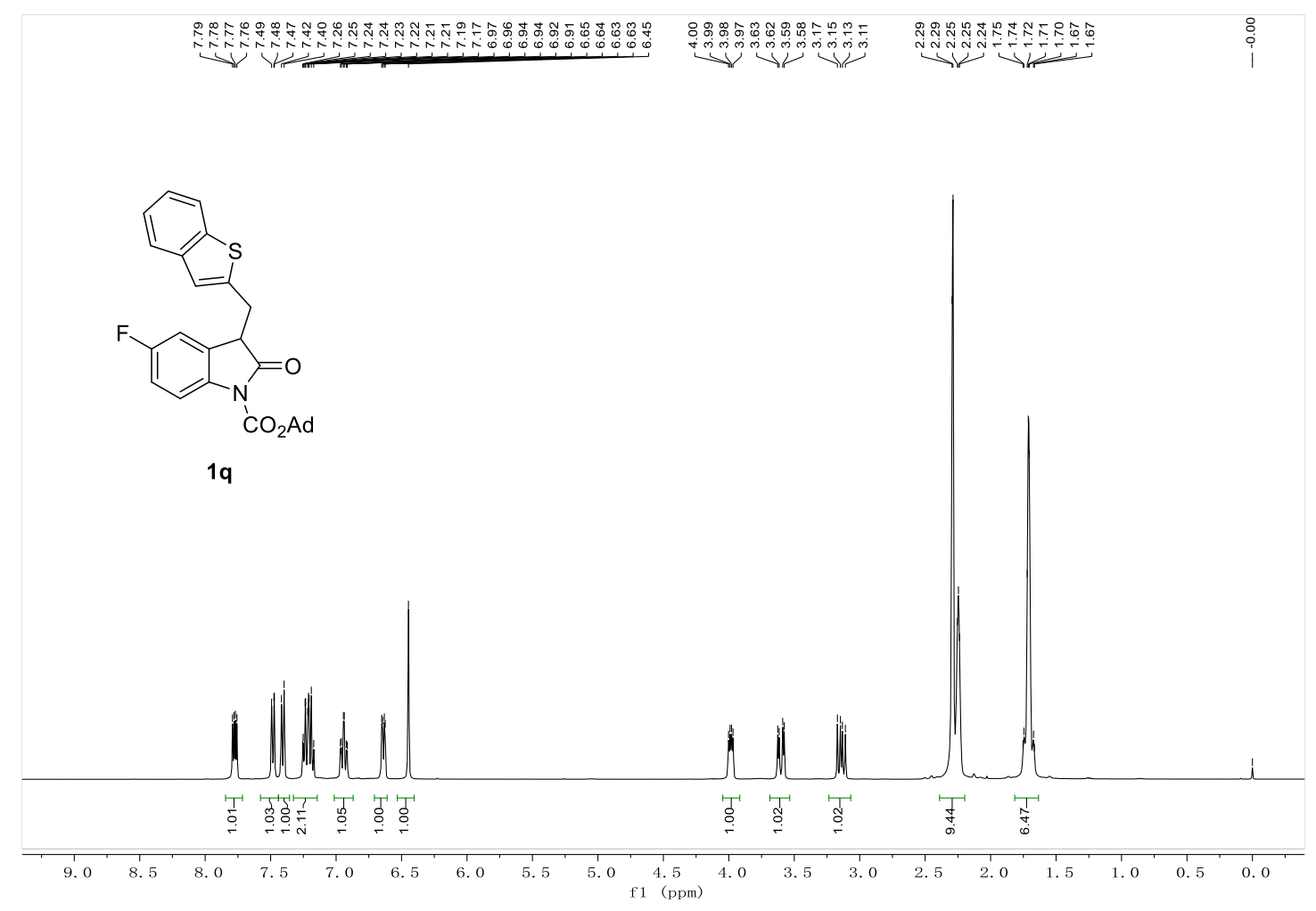

$1 \mathbf{q}^{13} \mathrm{C} \mathrm{NMR}\left(100 \mathrm{M} \mathrm{Hz}, \mathrm{CDCl}_{3}\right)$

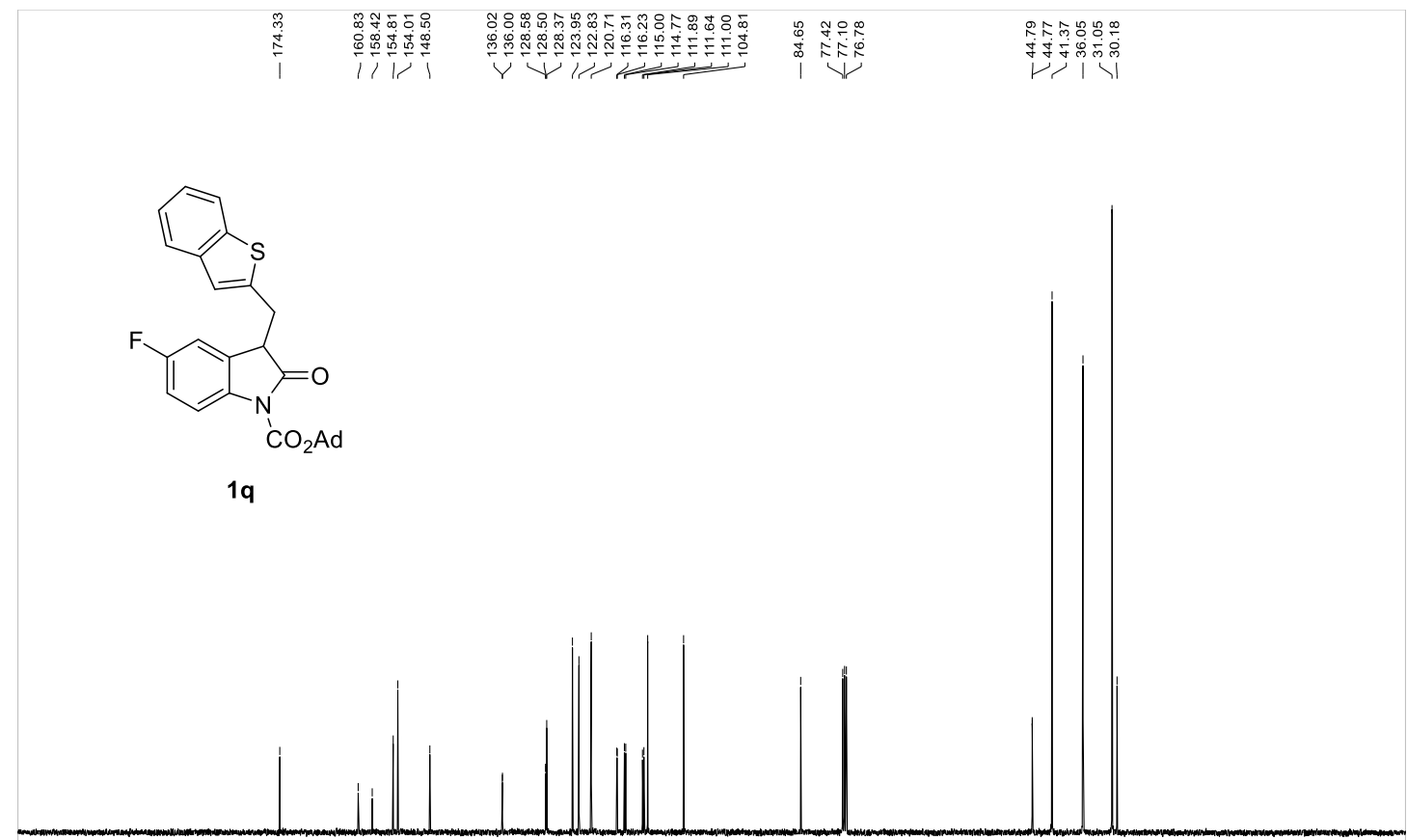

$\begin{array}{rlllllllllllllllllllllll}1 & 10 & 200 & 190 & 180 & 170 & 160 & 150 & 140 & 130 & 120 & 110 & 100 & 90 & 80 & 70 & 60 & 50 & 40 & 30 & 20 & 10 & 0 & -10\end{array}$ 
1r ${ }^{1} \mathrm{H}$ NMR (400M Hz, $\left.\mathrm{CDCl}_{3}\right)$

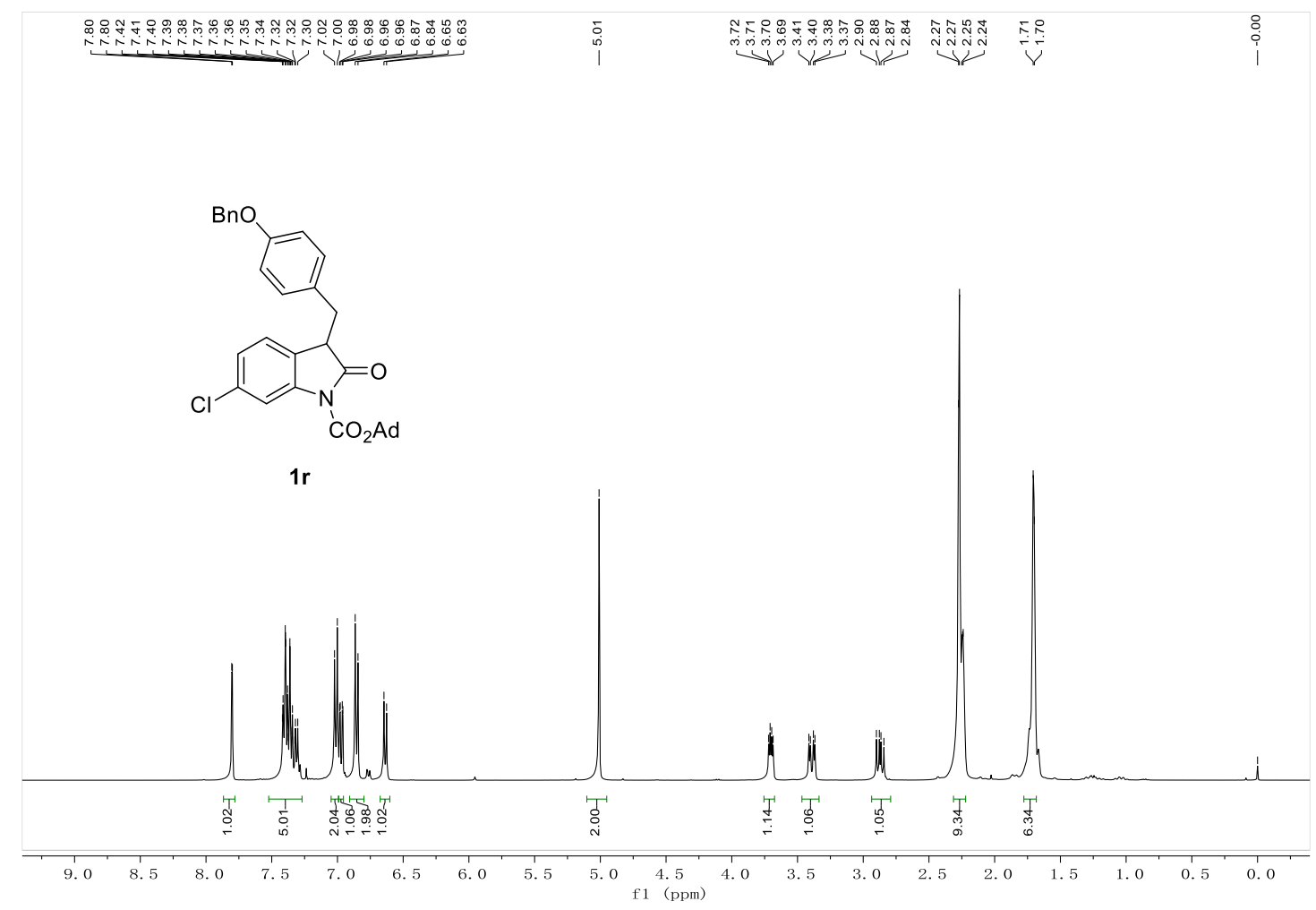

1r ${ }^{13} \mathrm{C} \mathrm{NMR}\left(100 \mathrm{M} \mathrm{Hz}, \mathrm{CDCl}_{3}\right)$

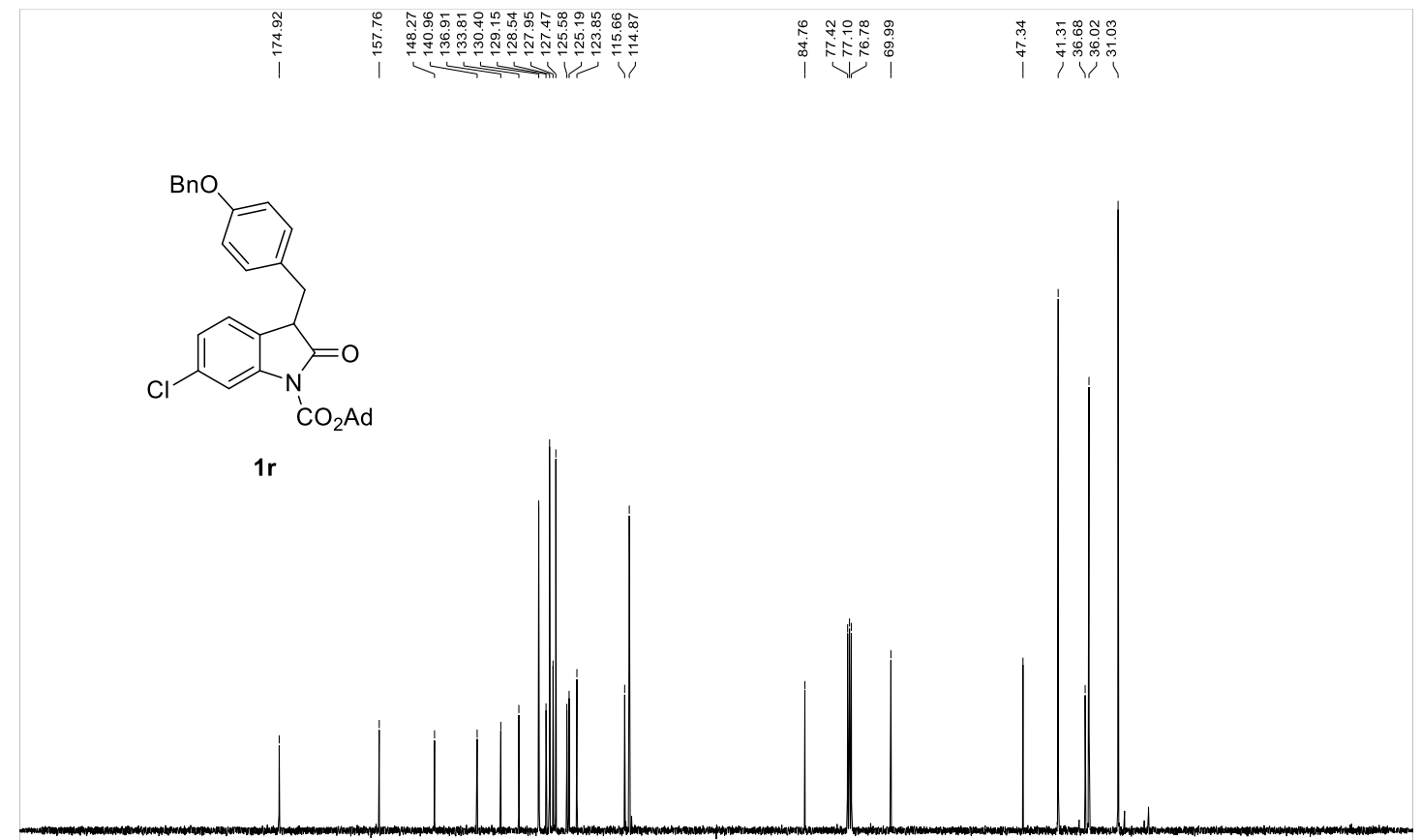

\begin{tabular}{lllllllllllllllllllllllll}
\hline & 1 & 200 & 190 & 180 & 170 & 160 & 150 & 140 & 130 & 120 & 110 & 100 & 90 & 80 & 70 & 60 & 50 & 40 & 30 & 20 & 10 & 0 & -10
\end{tabular} 
1s ${ }^{1} \mathrm{H}$ NMR (400M Hz, $\left.\mathrm{CDCl}_{3}\right)$

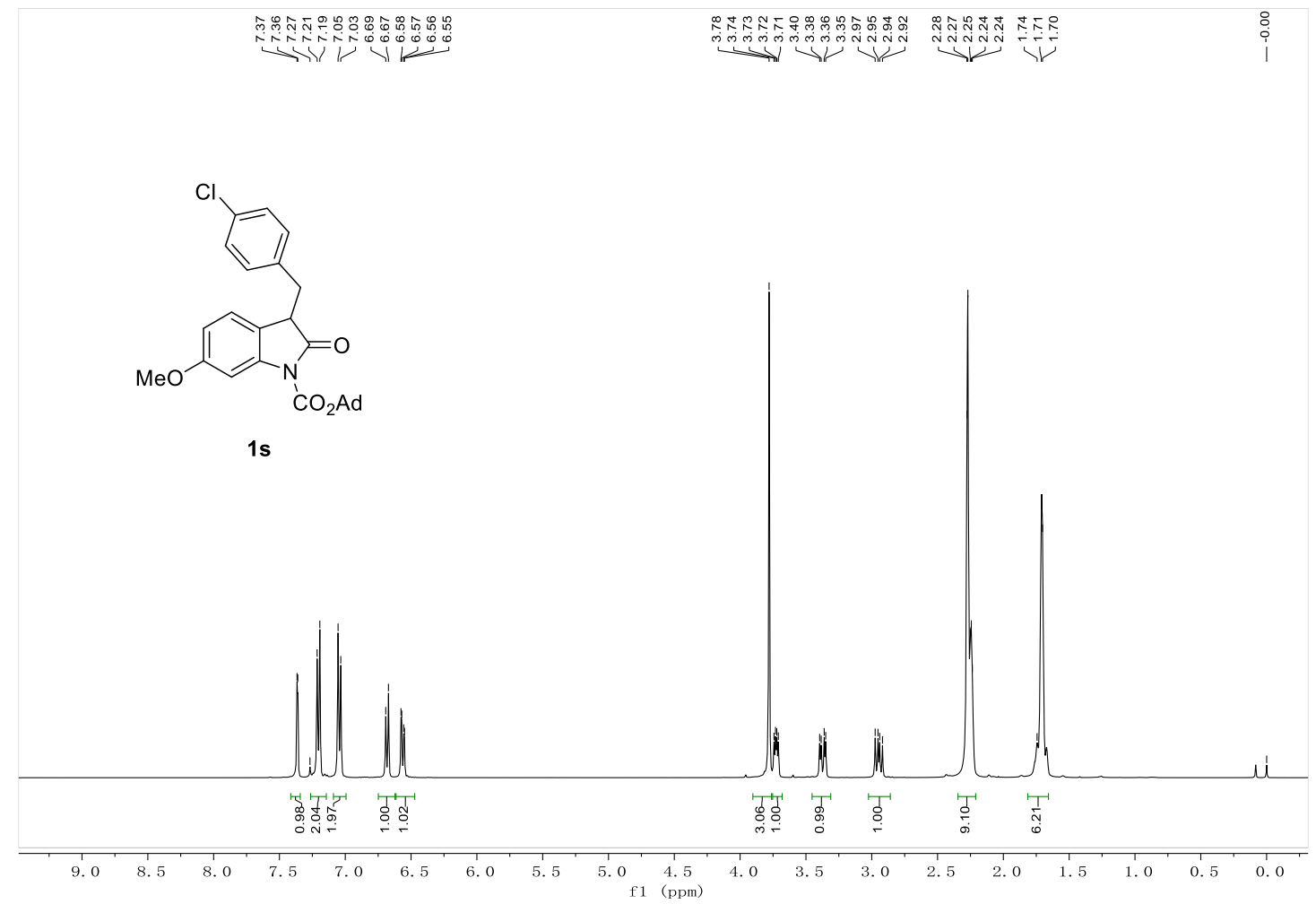

1s ${ }^{13} \mathrm{C} \mathrm{NMR}\left(100 \mathrm{M} \mathrm{Hz}, \mathrm{CDCl}_{3}\right)$

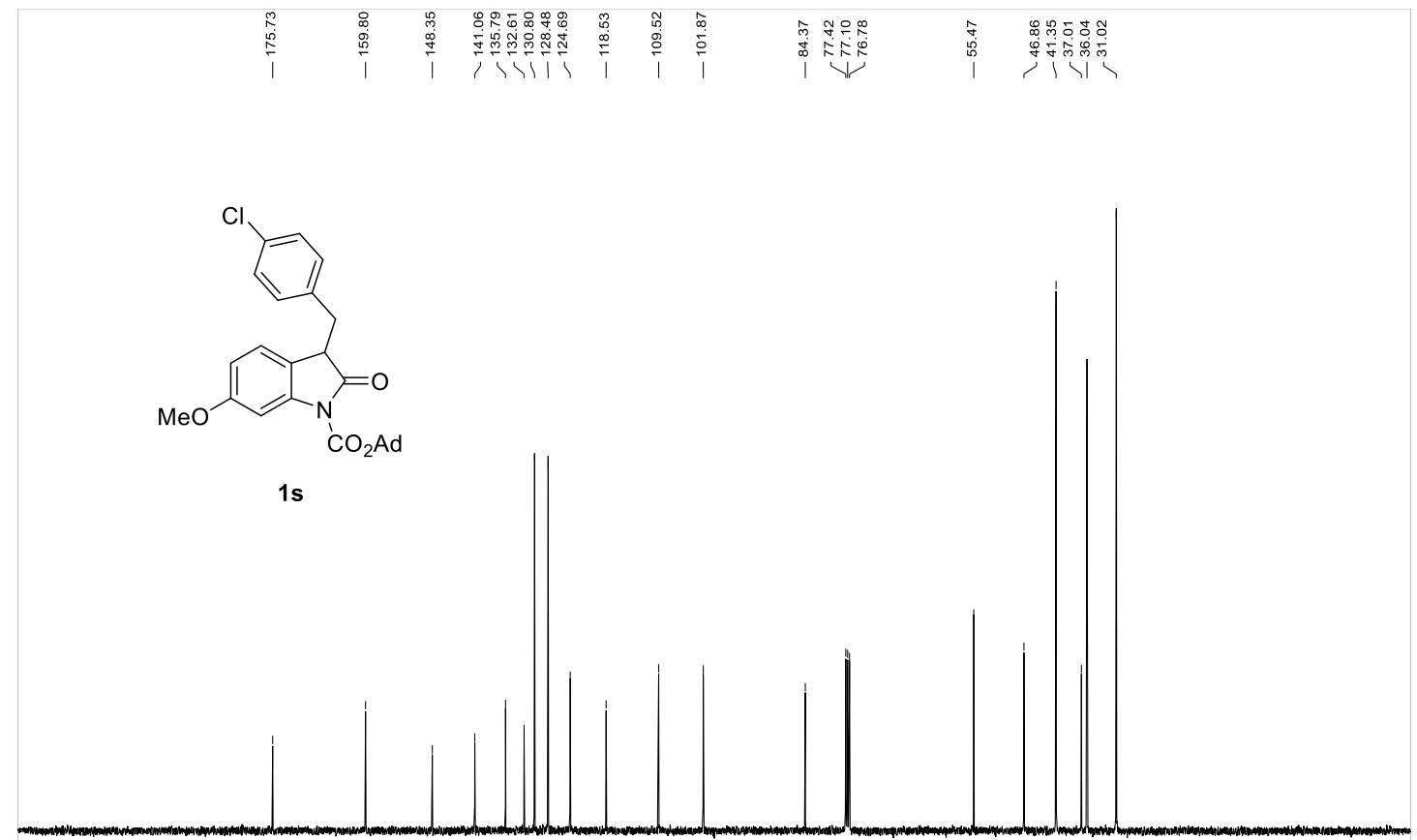

$\begin{array}{lllllllllllll}210 & 200 & 190 & 180 & 170 & 160 & 150 & 140 & 130 & 120 & 110 & 100 & 90 \\ \mathrm{f} 1 & & 1 \mathrm{ppm})\end{array}$ 
1t ${ }^{1} \mathrm{H}$ NMR (400M Hz, $\left.\mathrm{CDCl}_{3}\right)$

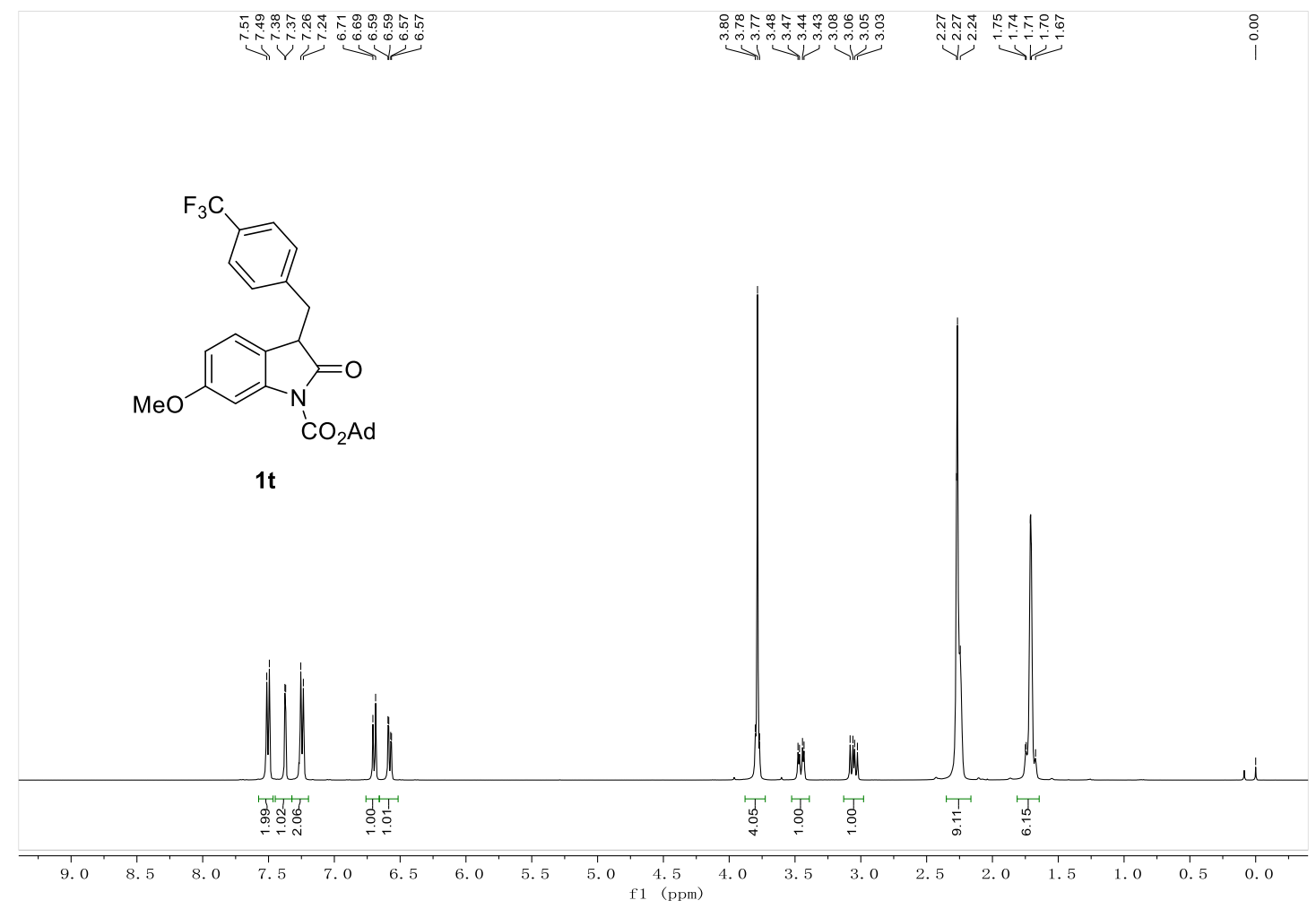

1t ${ }^{13} \mathrm{C}$ NMR $\left(100 \mathrm{M} \mathrm{Hz}, \mathrm{CDCl}_{3}\right)$

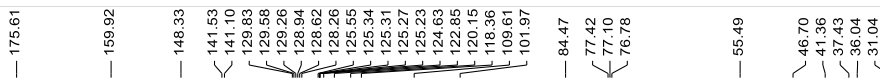

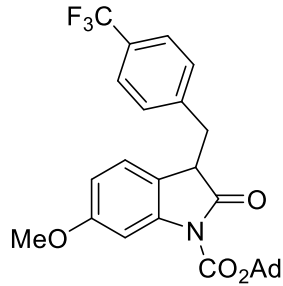

$1 \mathrm{t}$

$\mathrm{CO}_{2} \mathrm{Ad}$

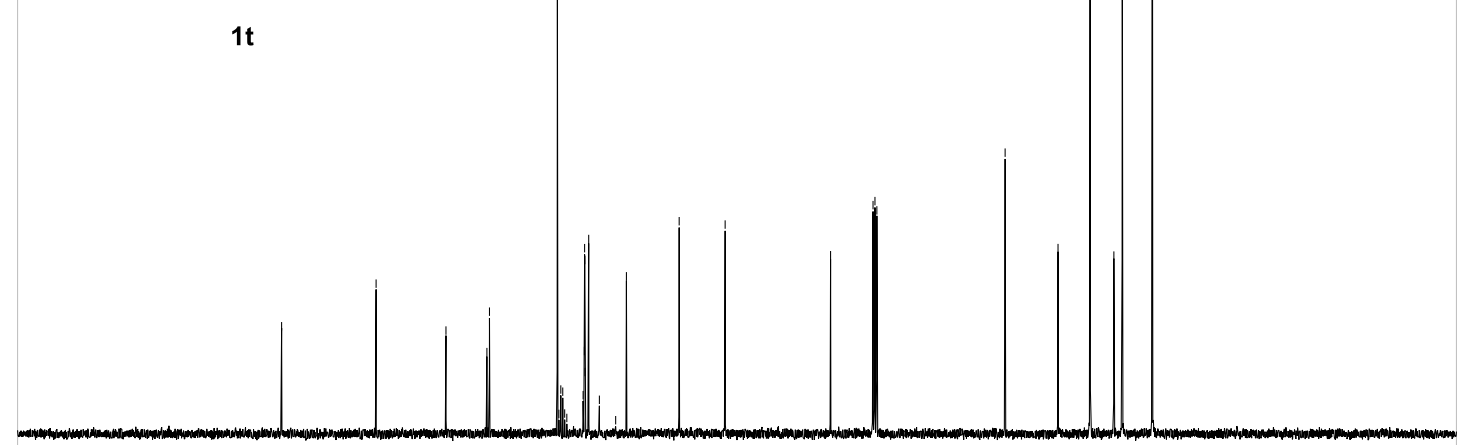

$\begin{array}{lllllllllllll}210 & 200 & 190 & 180 & 170 & 160 & 150 & 140 & 130 & 120 & 110 & 100 & 90\end{array}$ f1 (ppm) 
1u ${ }^{1} \mathrm{H}$ NMR (400M Hz, $\left.\mathrm{CDCl}_{3}\right)$

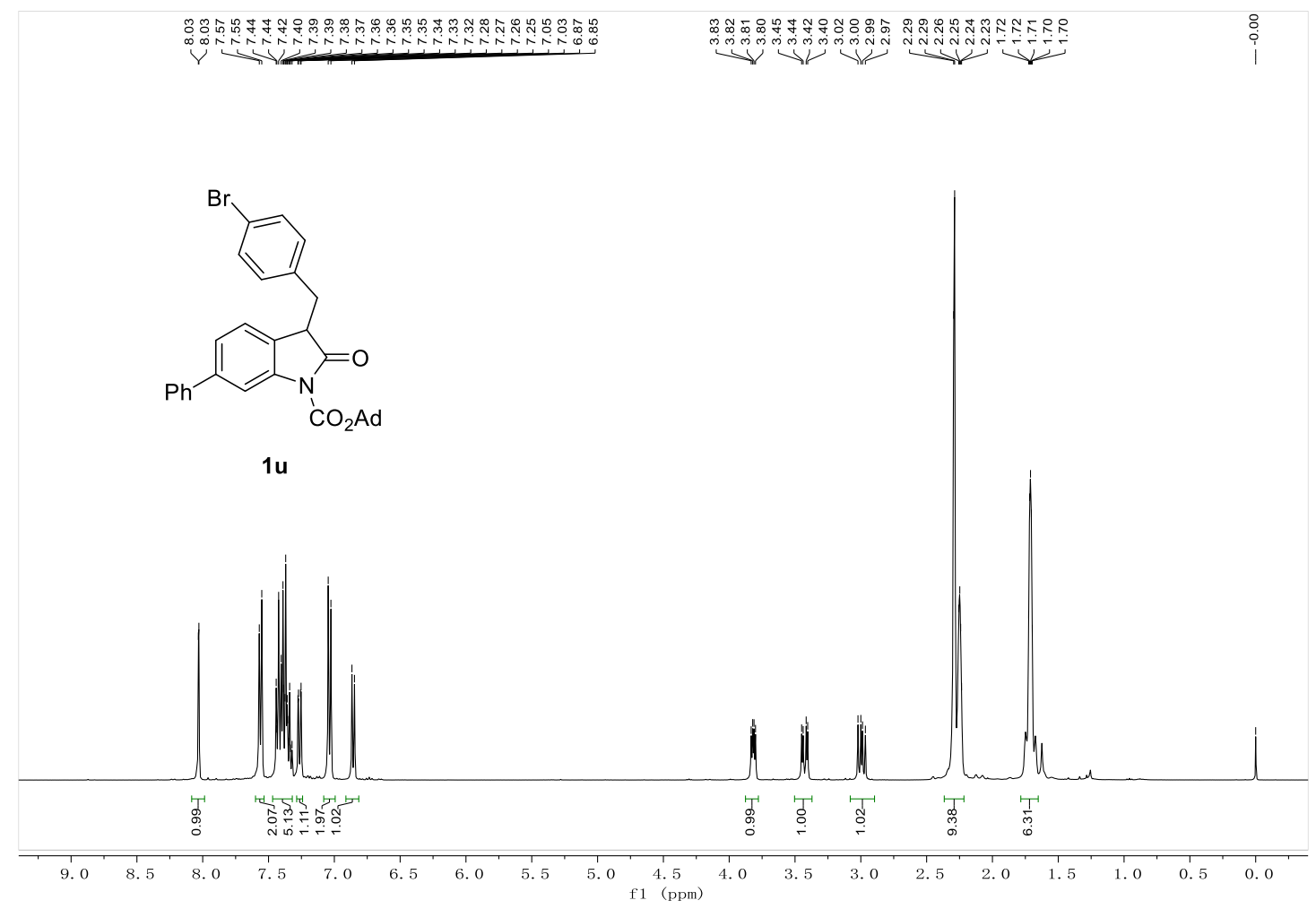

1u ${ }^{13} \mathrm{C}$ NMR $\left(100 \mathrm{M} \mathrm{Hz}, \mathrm{CDCl}_{3}\right)$

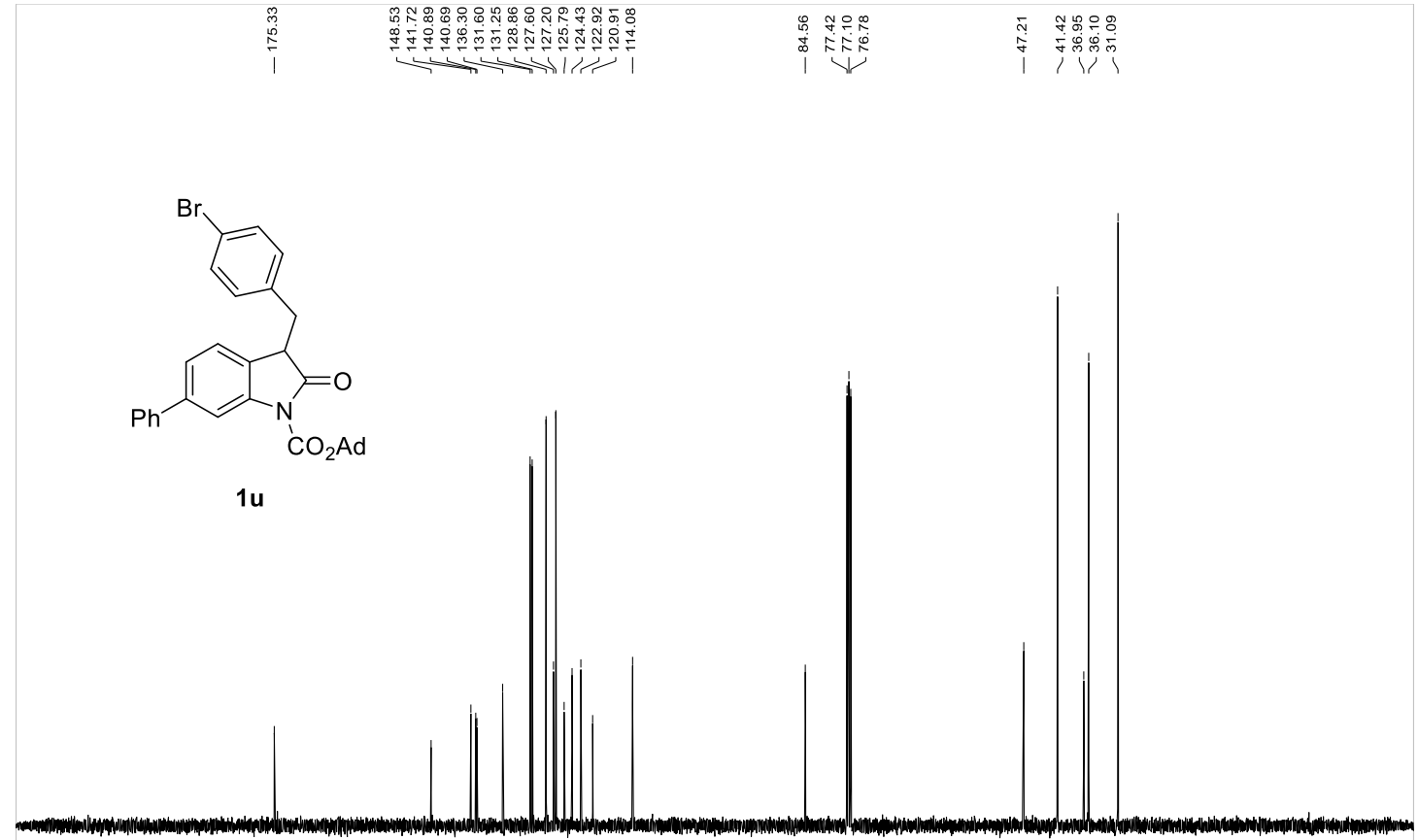

$\begin{array}{lllllllllllll}210 & 200 & 190 & 180 & 170 & 160 & 150 & 140 & 130 & 120 & 110 & 100 & 90\end{array}$ 
1v ${ }^{1} \mathrm{H}$ NMR (400M Hz, $\left.\mathrm{CDCl}_{3}\right)$

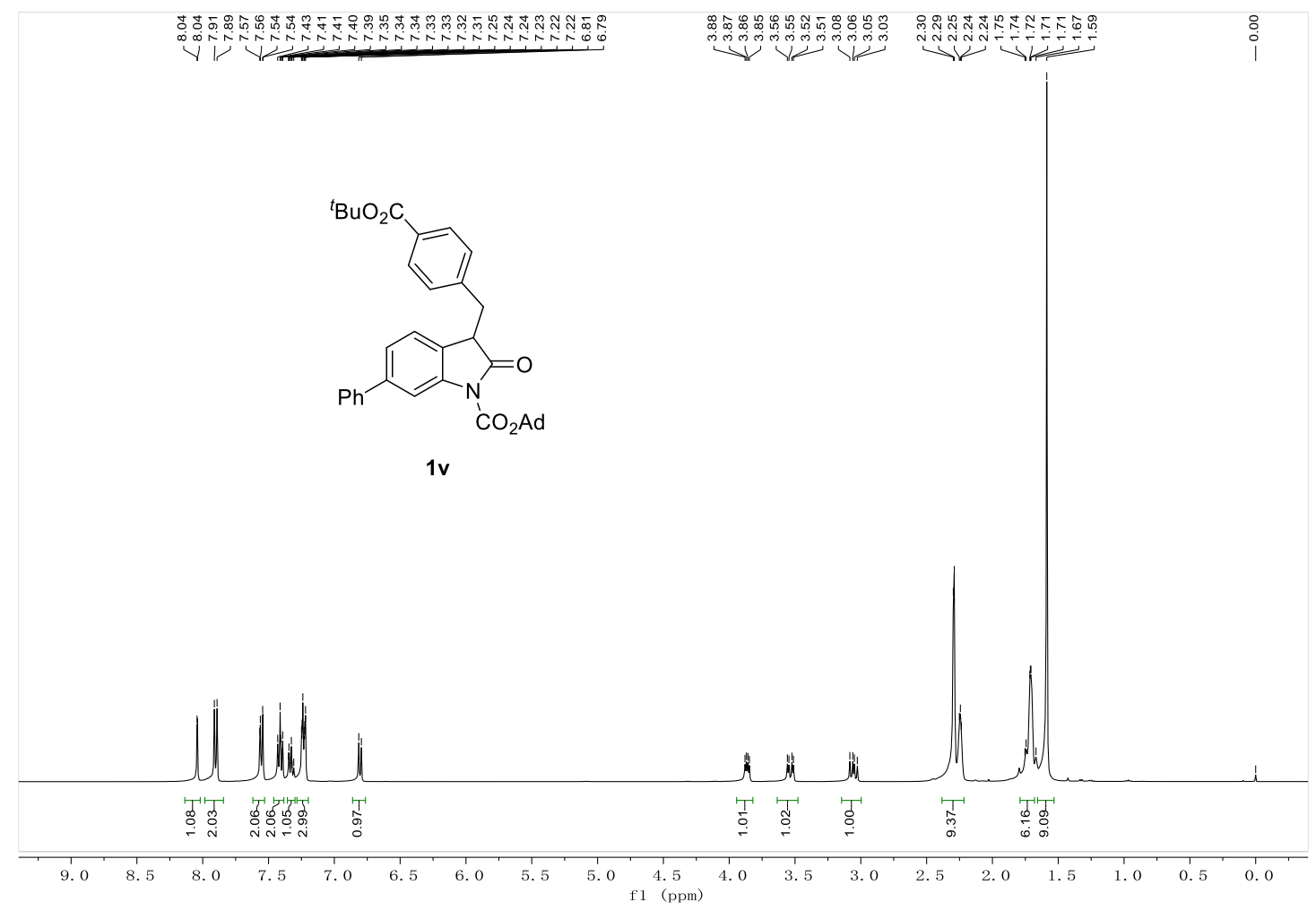

1v ${ }^{13} \mathrm{C} \mathrm{NMR}\left(100 \mathrm{M} \mathrm{Hz}, \mathrm{CDCl}_{3}\right)$

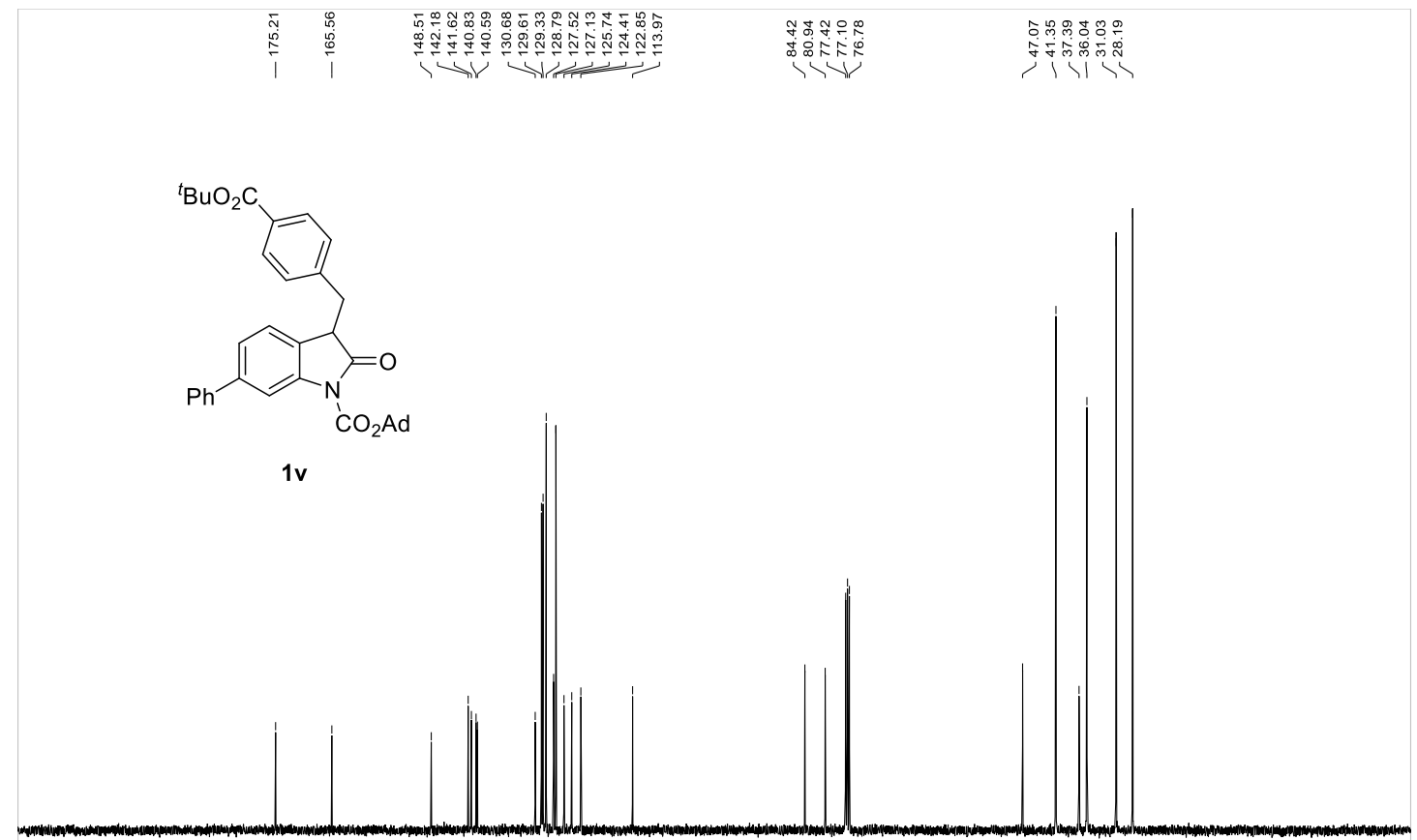

$\begin{array}{lllllllllllll}210 & 200 & 190 & 180 & 170 & 160 & 150 & 140 & 130 & 120 & 110 & 100 & 100 \\ \mathrm{f} 1 & (\mathrm{ppm}) & 1\end{array}$ 
1w ${ }^{1} \mathrm{H}$ NMR $\left(400 \mathrm{M} \mathrm{Hz}, \mathrm{CDCl}_{3}\right)$

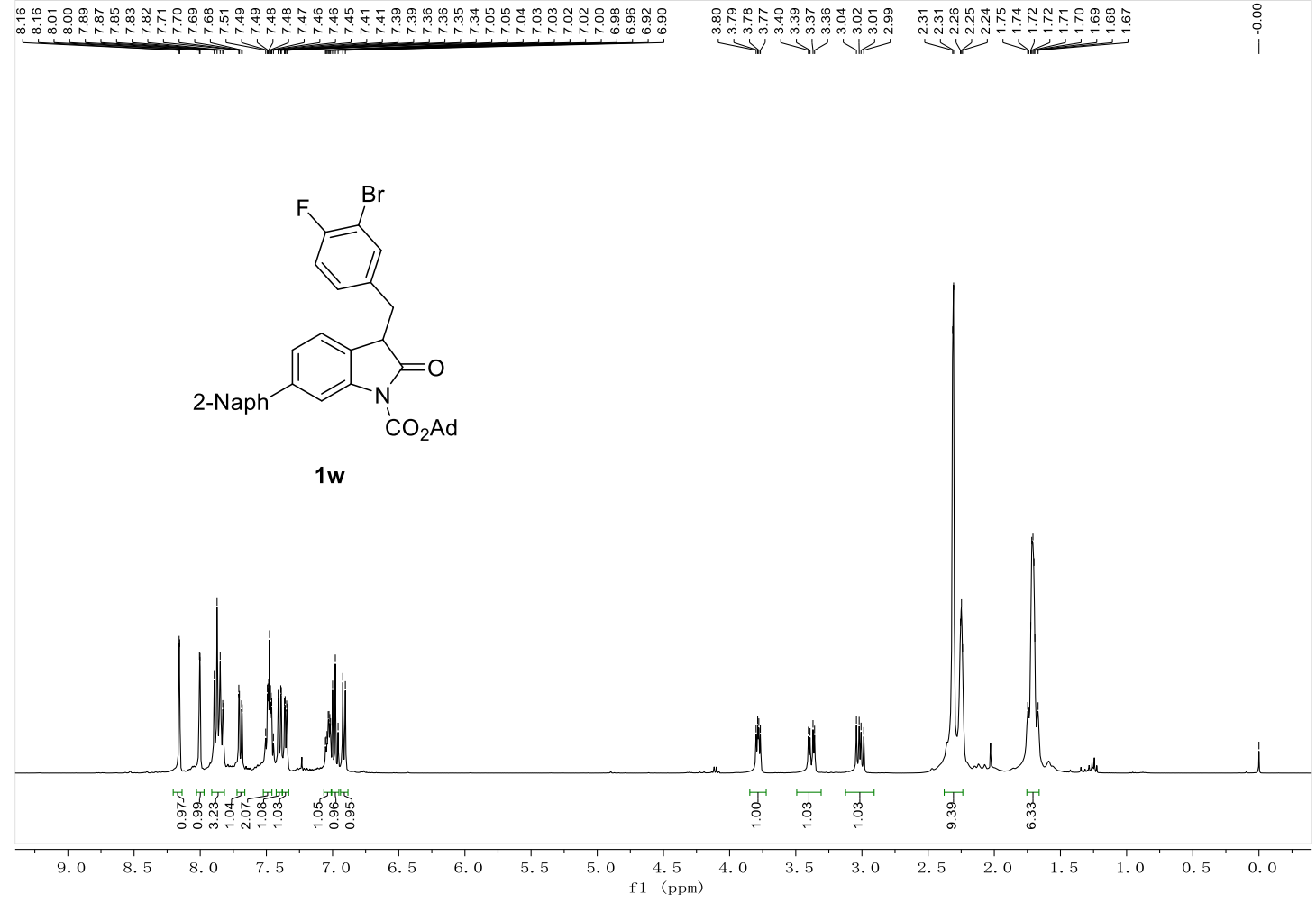

1w ${ }^{13} \mathrm{C}$ NMR $\left(100 \mathrm{M} \mathrm{Hz}, \mathrm{CDCl}_{3}\right)$

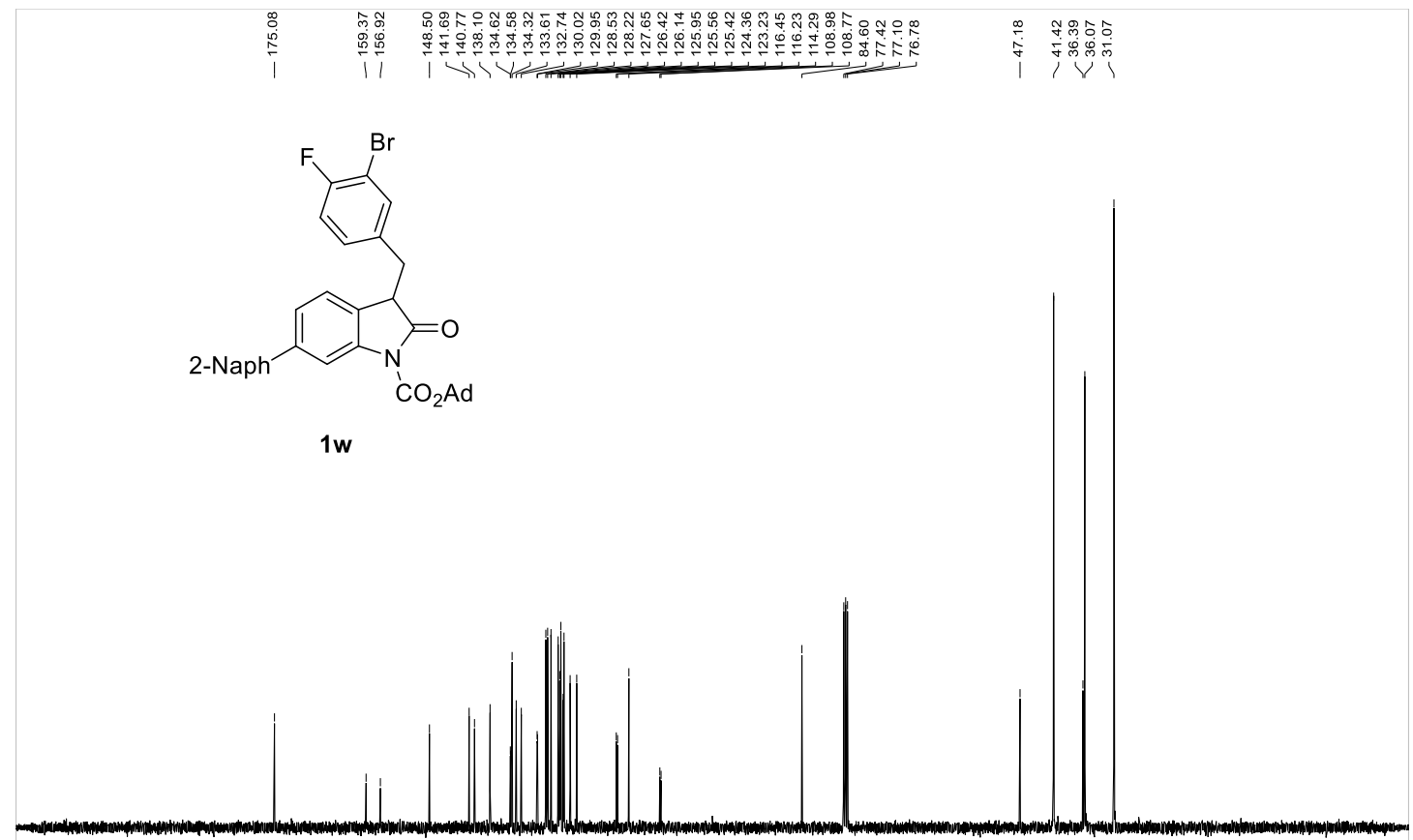

$\begin{array}{lllllllllllll}210 & 200 & 190 & 180 & 170 & 160 & 150 & 140 & 130 & 120 & 110 & 100 & 100 \\ \mathrm{f} 1 & & 1 \mathrm{ppm})\end{array}$ 
1x ${ }^{1} \mathrm{H}$ NMR (400M Hz, $\left.\mathrm{CDCl}_{3}\right)$

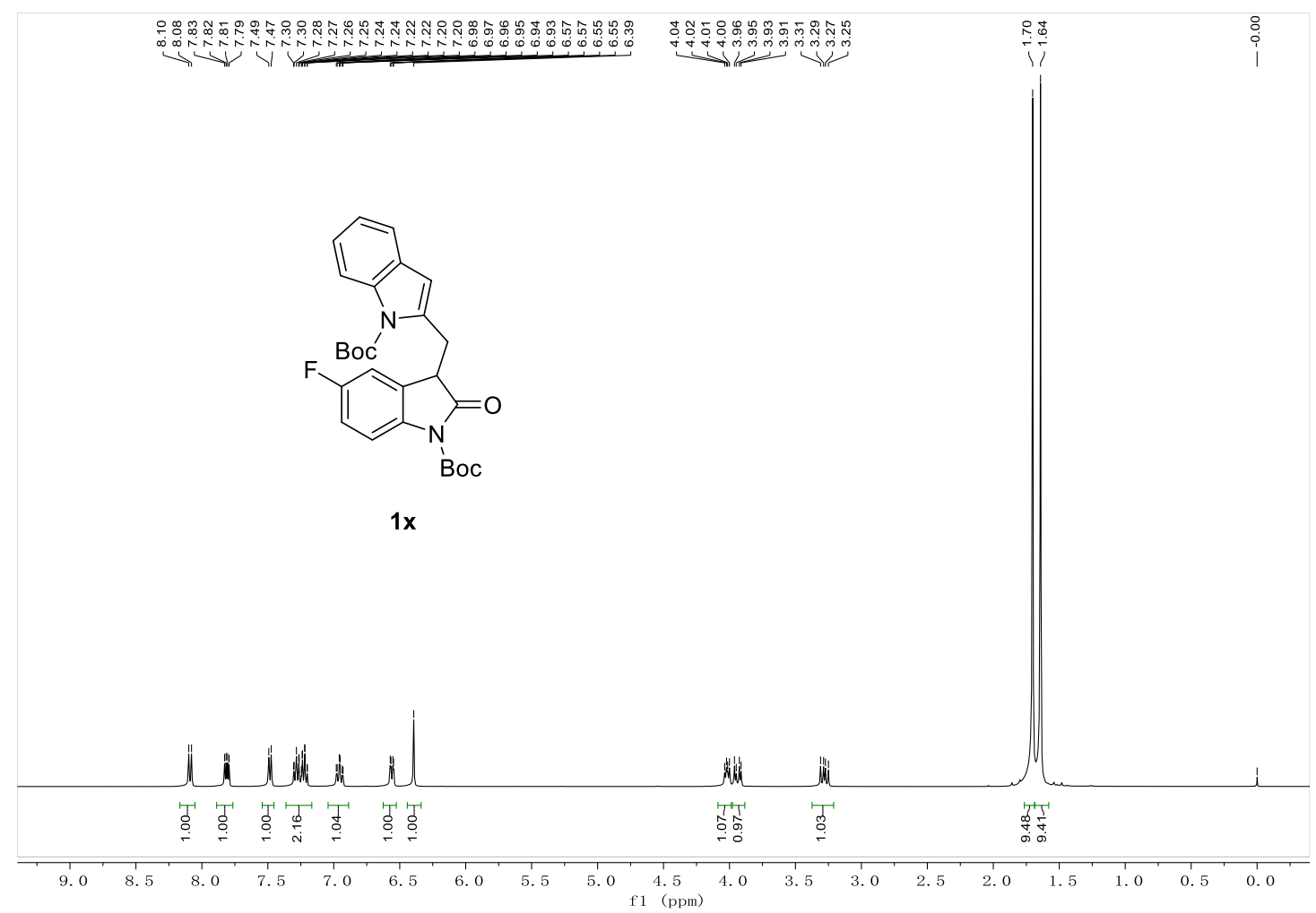

$\mathbf{1 x}{ }^{13} \mathrm{C} \mathrm{NMR}\left(100 \mathrm{M} \mathrm{Hz}, \mathrm{CDCl}_{3}\right)$

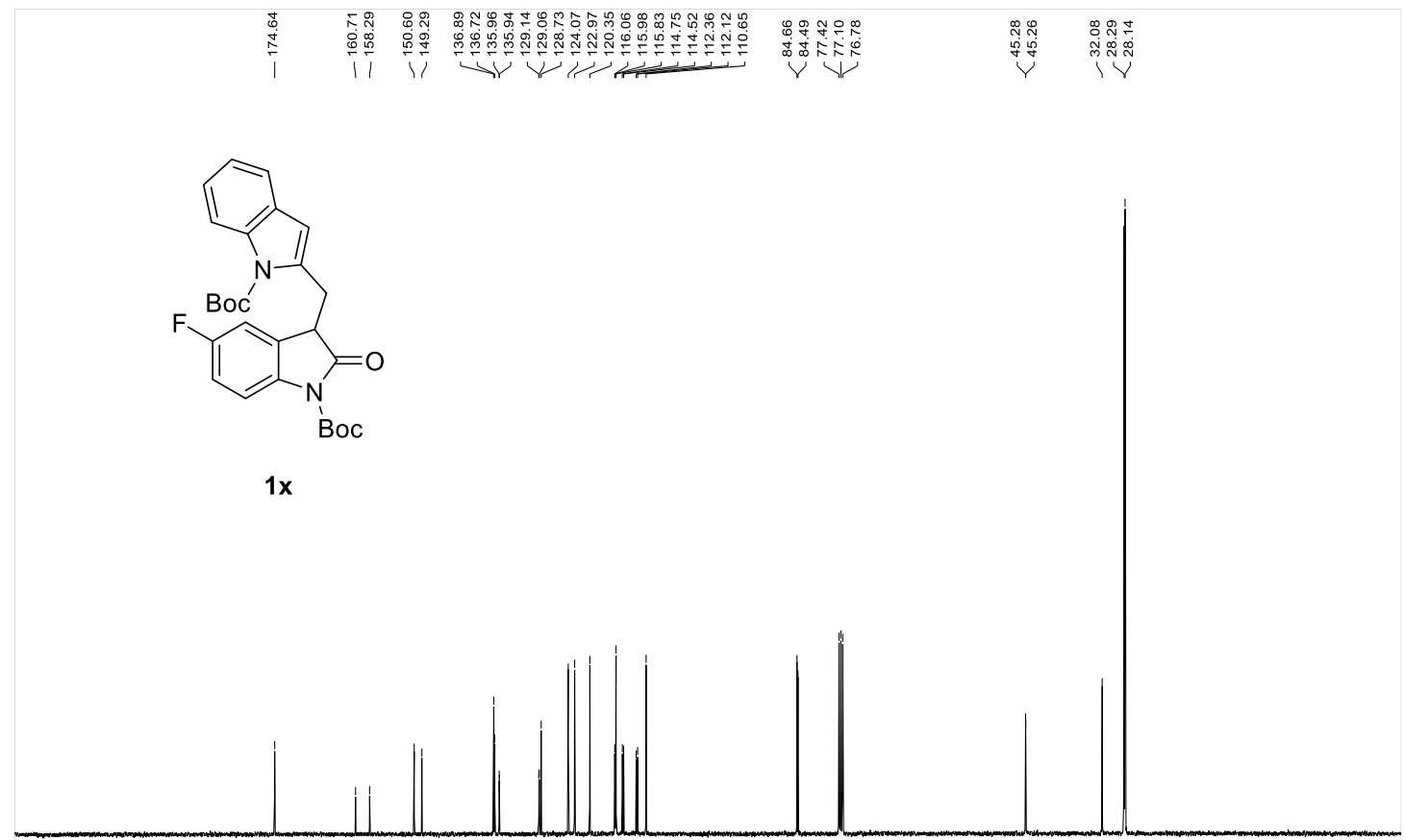

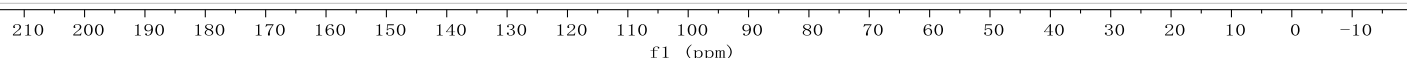


$1 \mathbf{y}{ }^{1} \mathrm{H}$ NMR (400M Hz, $\left.\mathrm{CDCl}_{3}\right)$

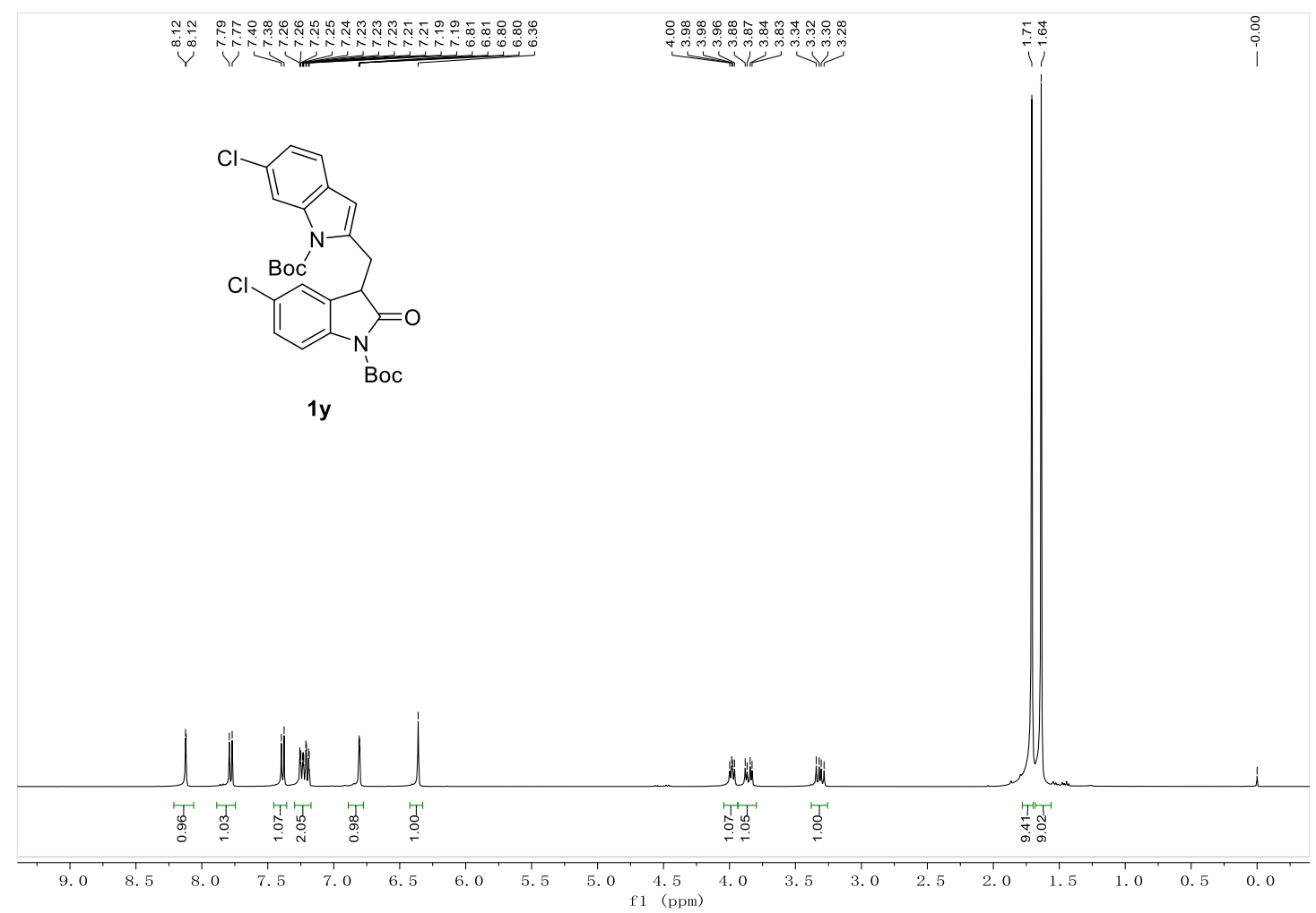

$1 \mathbf{y}{ }^{13} \mathrm{C}$ NMR $\left(100 \mathrm{M} \mathrm{Hz}, \mathrm{CDCl}_{3}\right)$

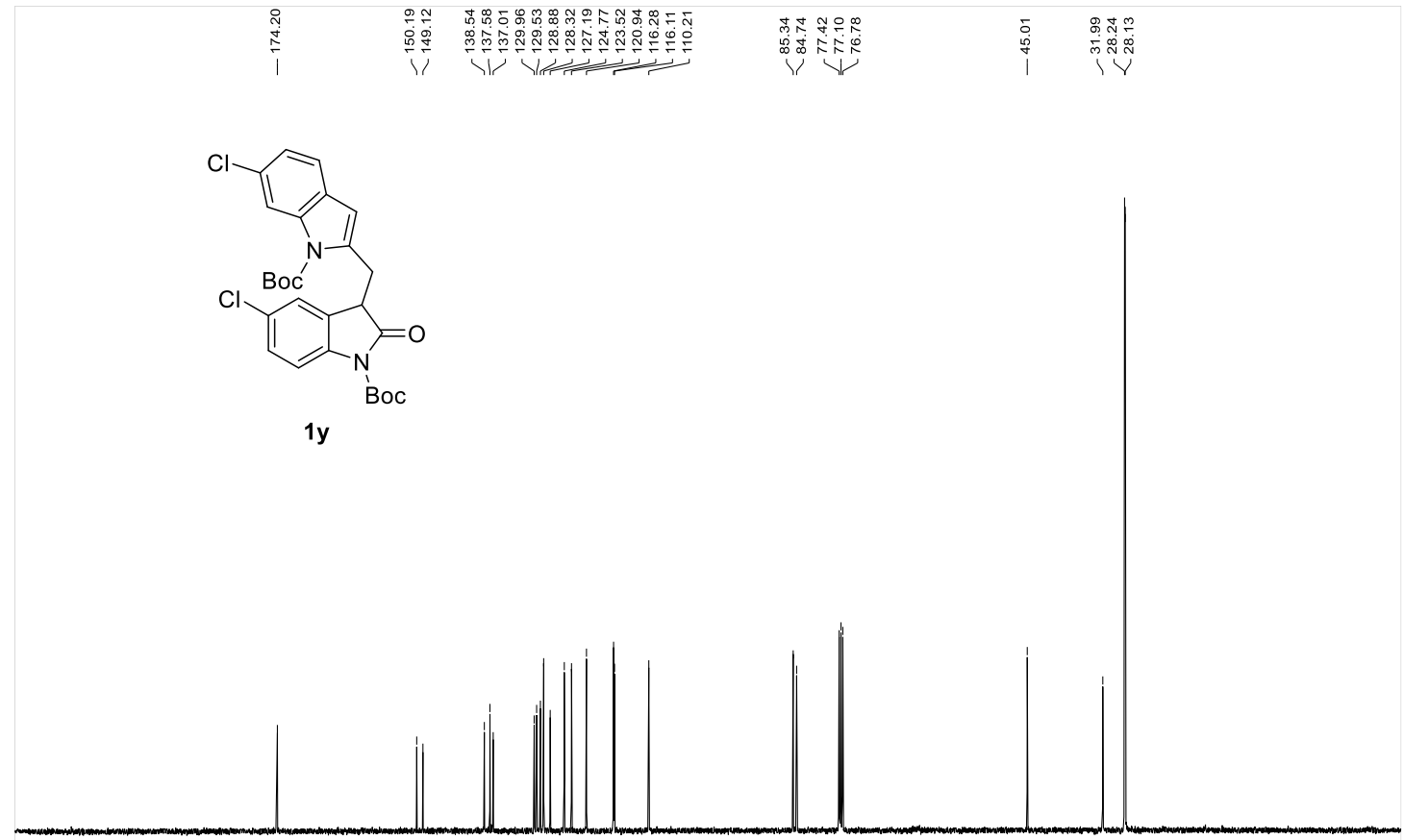

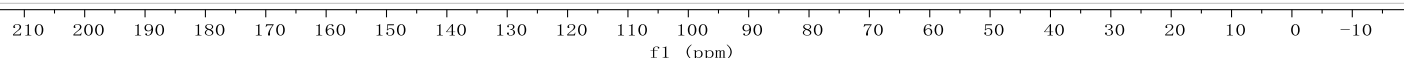


3a ${ }^{1} \mathrm{H}$ NMR $\left(400 \mathrm{M} \mathrm{Hz}, \mathrm{CDCl}_{3}\right)$

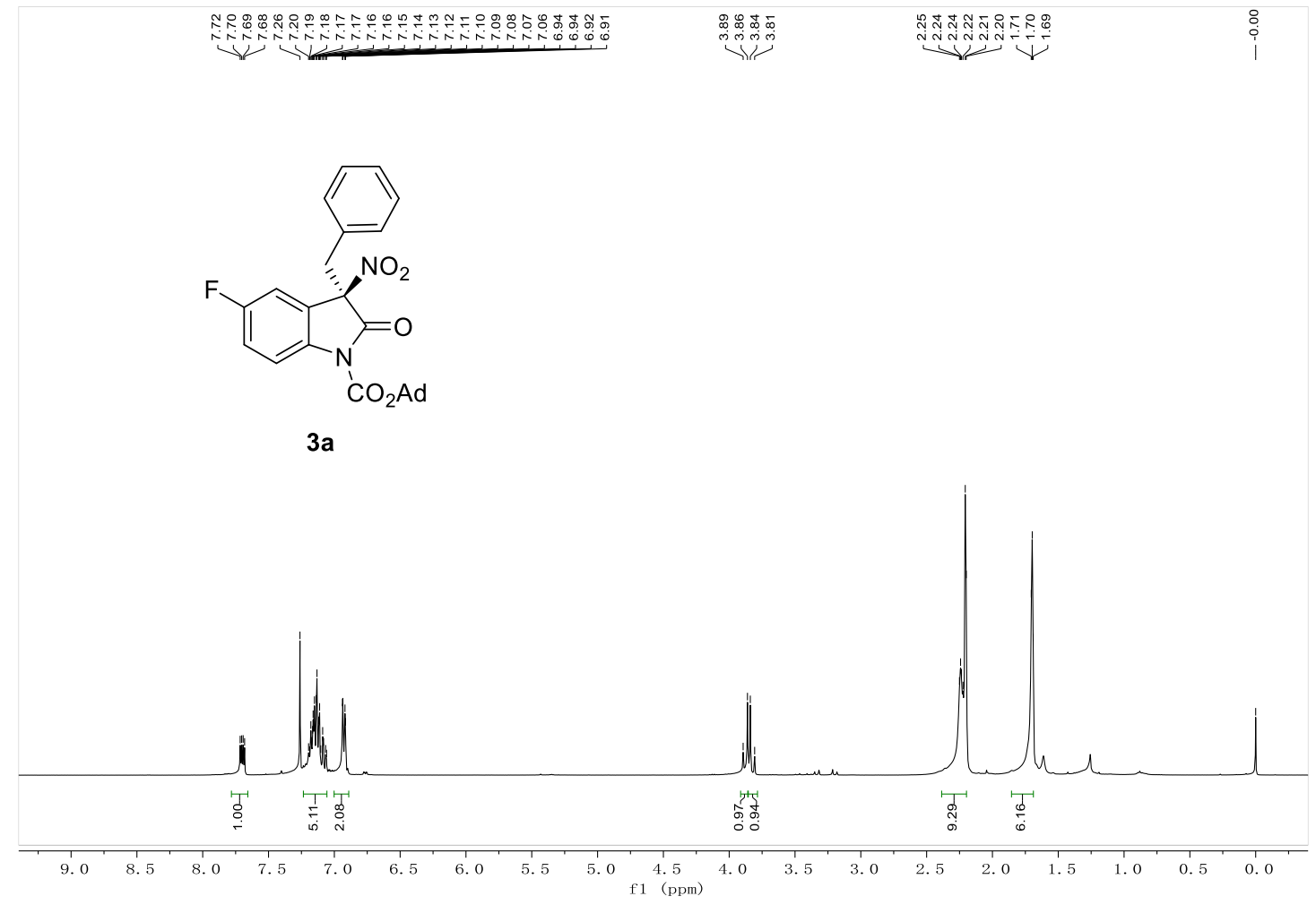

3a ${ }^{13} \mathrm{C}$ NMR $\left(100 \mathrm{M} \mathrm{Hz}, \mathrm{CDCl}_{3}\right)$

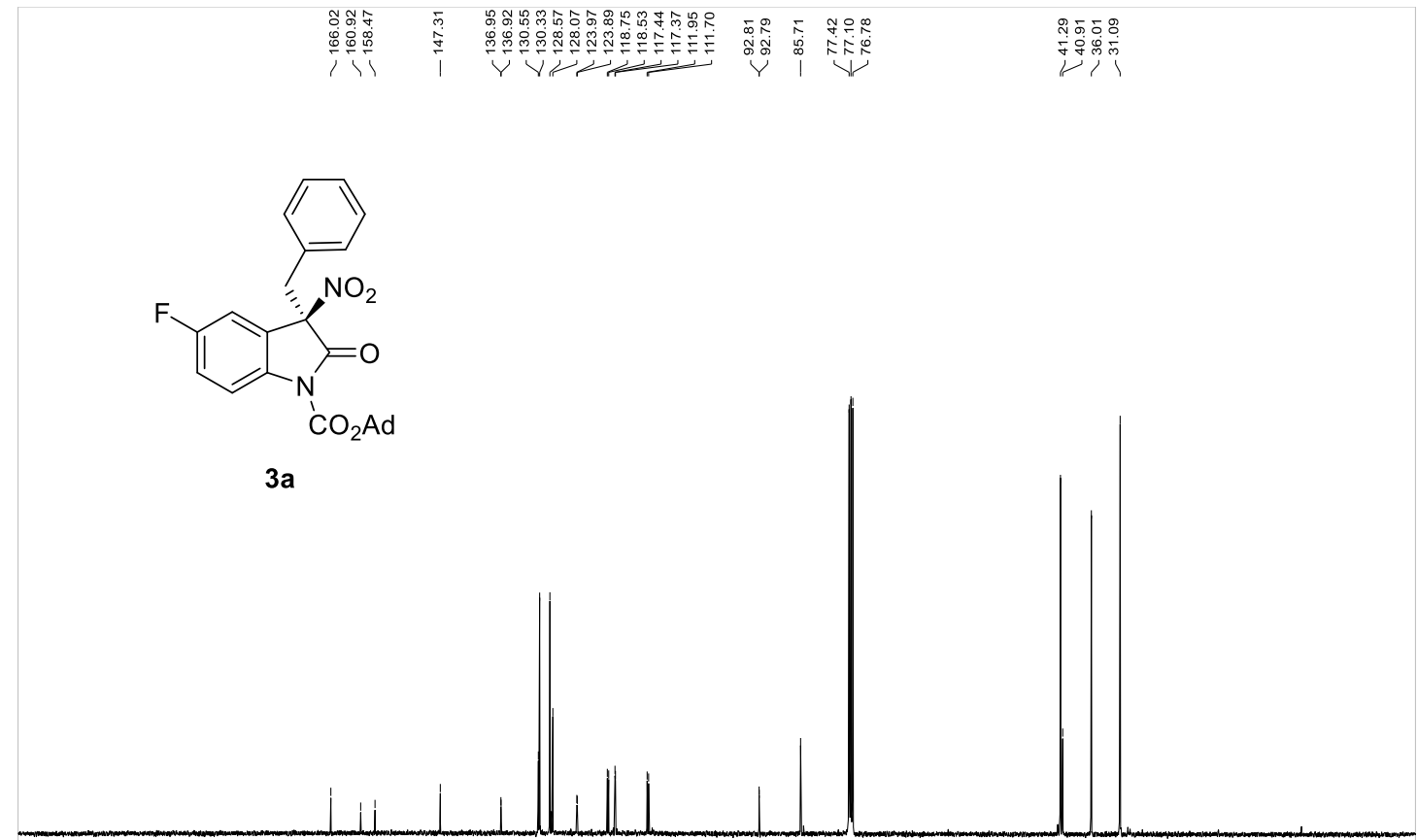

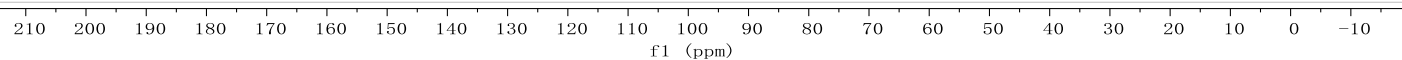


3b ${ }^{1} \mathrm{H}$ NMR (400M Hz, $\left.\mathrm{CDCl}_{3}\right)$

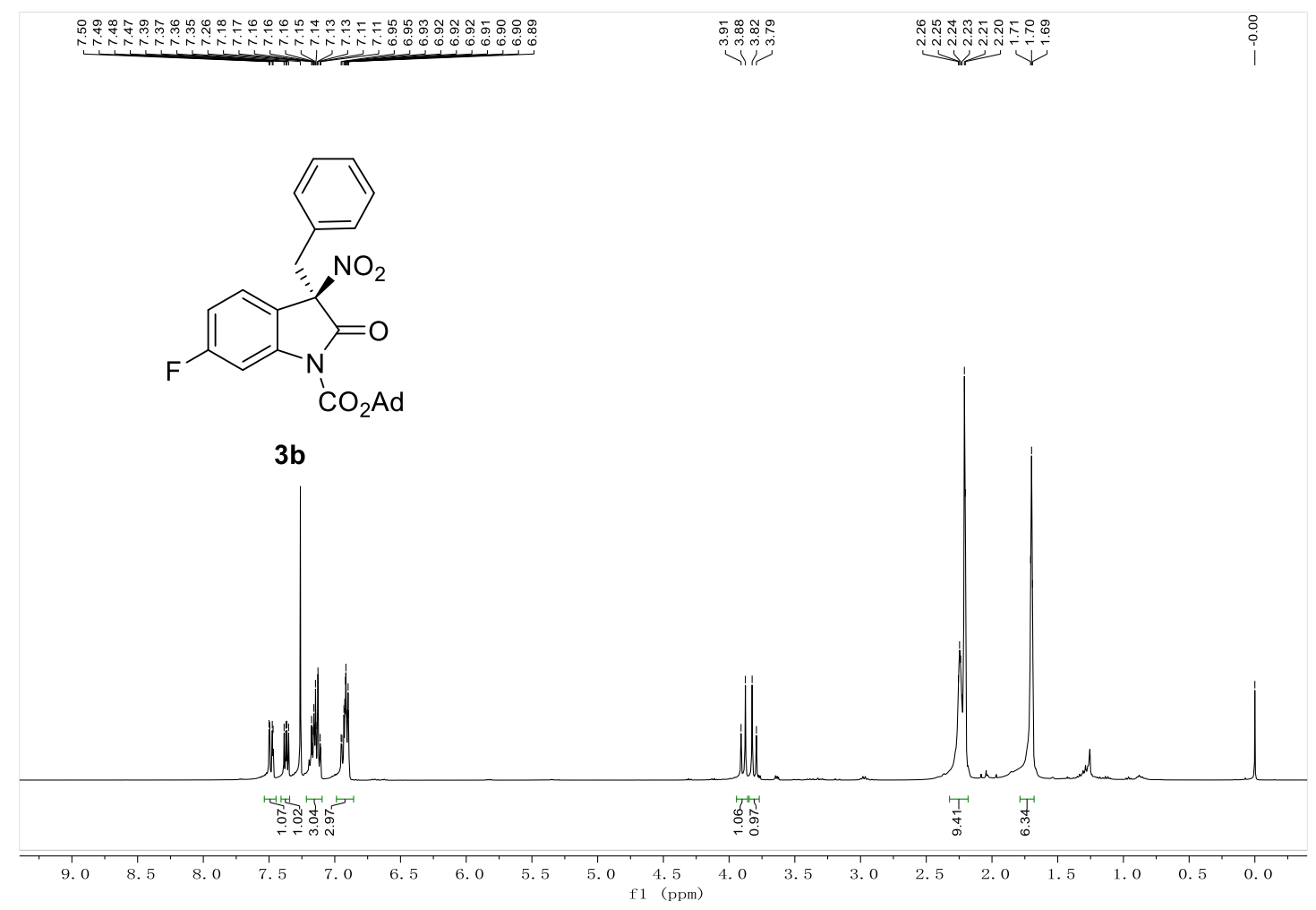

3b ${ }^{13} \mathrm{C}$ NMR $\left(100 \mathrm{M} \mathrm{Hz}, \mathrm{CDCl}_{3}\right)$

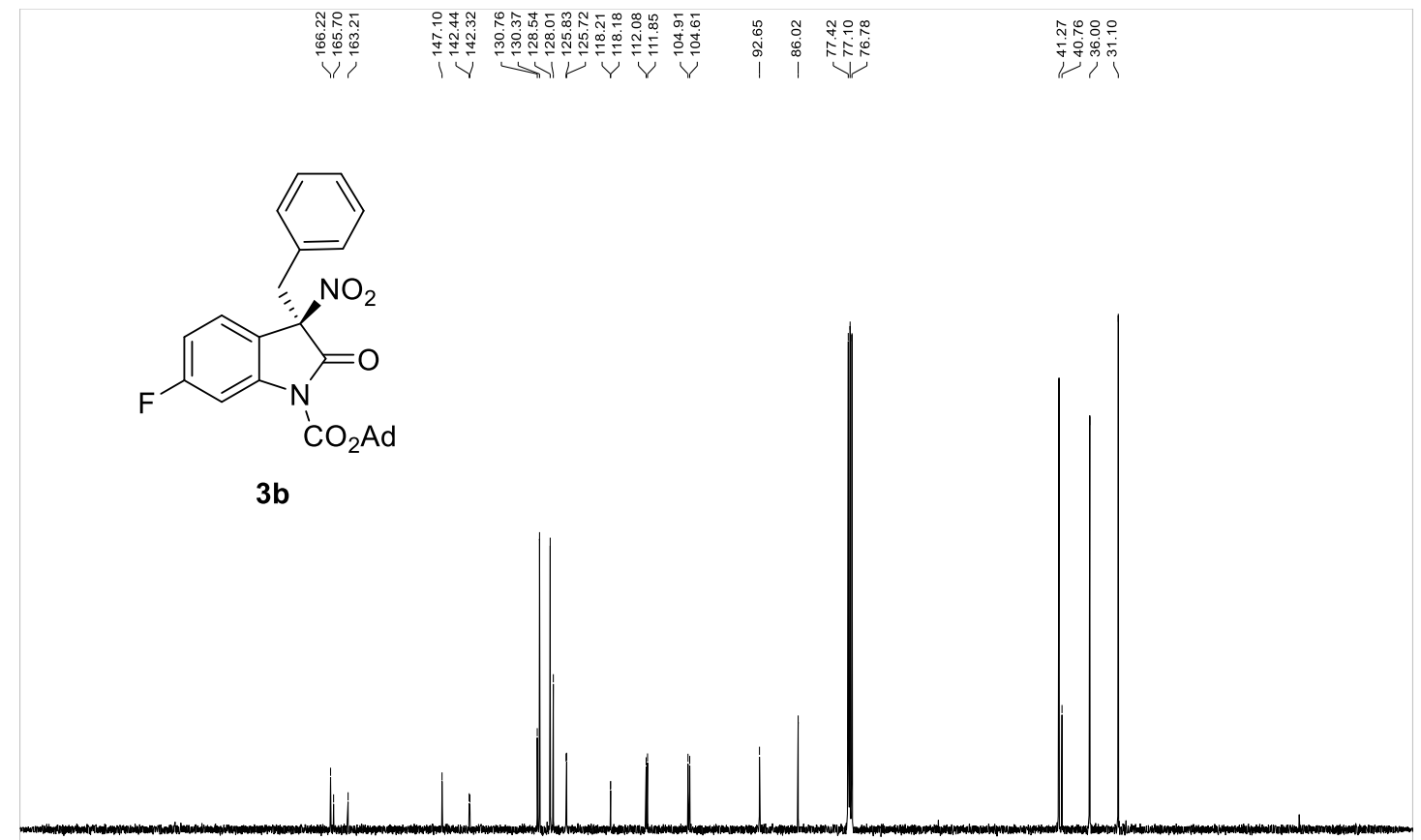

$\begin{array}{rllllllllllllllllllllllll}1 & 10 & 200 & 190 & 180 & 170 & 160 & 150 & 140 & 130 & 120 & 110 & 100 & 90 & 80 & 70 & 60 & 50 & 40 & 30 & 20 & 10 & 0 & -10 & 10\end{array}$ 
3c ${ }^{1} \mathrm{H}$ NMR $\left(400 \mathrm{M} \mathrm{Hz}, \mathrm{CDCl}_{3}\right)$

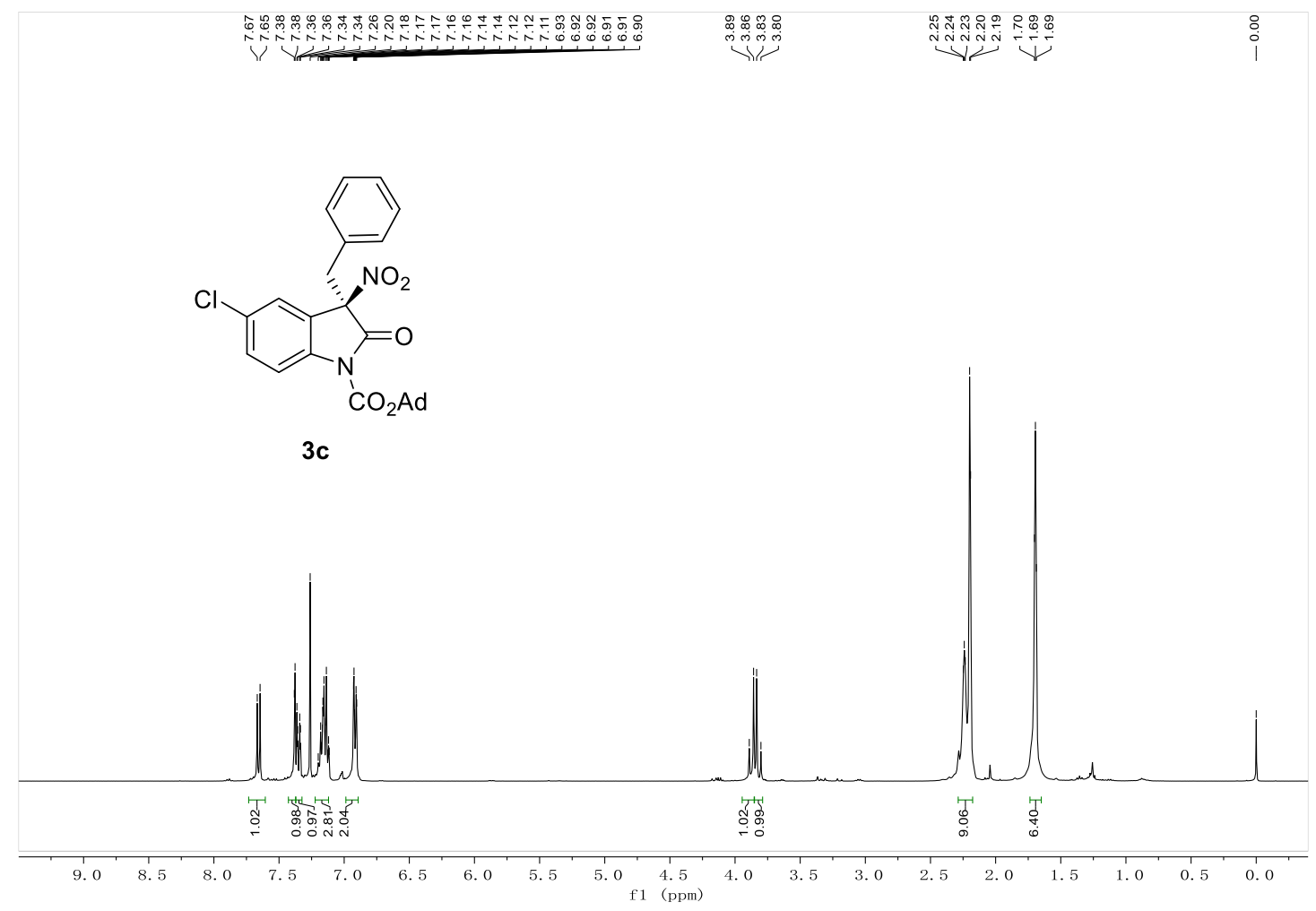

3c ${ }^{13} \mathrm{C}$ NMR $\left(100 \mathrm{M} \mathrm{Hz}, \mathrm{CDCl}_{3}\right)$

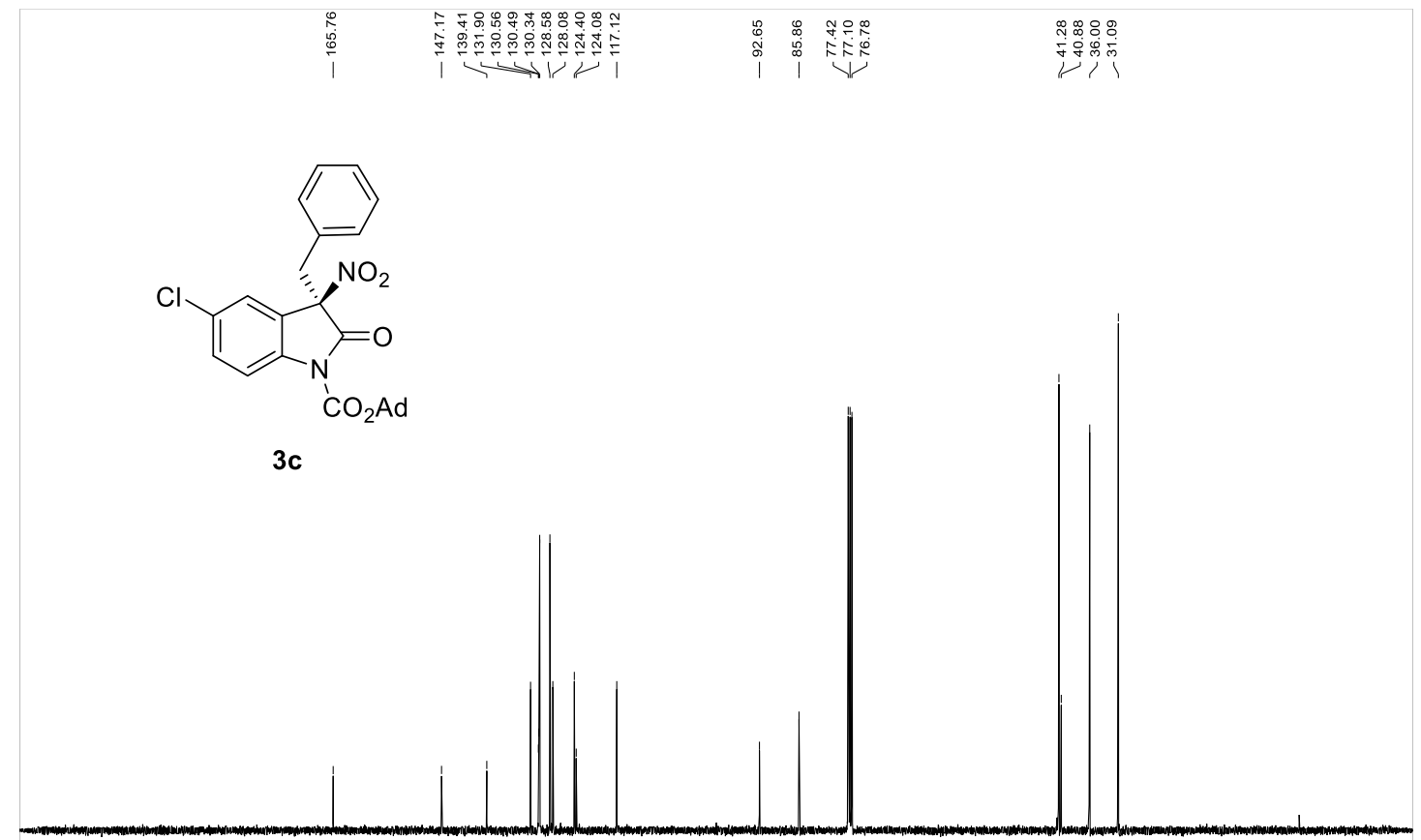

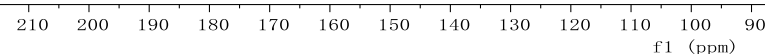


3d ${ }^{1} \mathrm{H}$ NMR $\left(400 \mathrm{M} \mathrm{Hz}, \mathrm{CDCl}_{3}\right)$

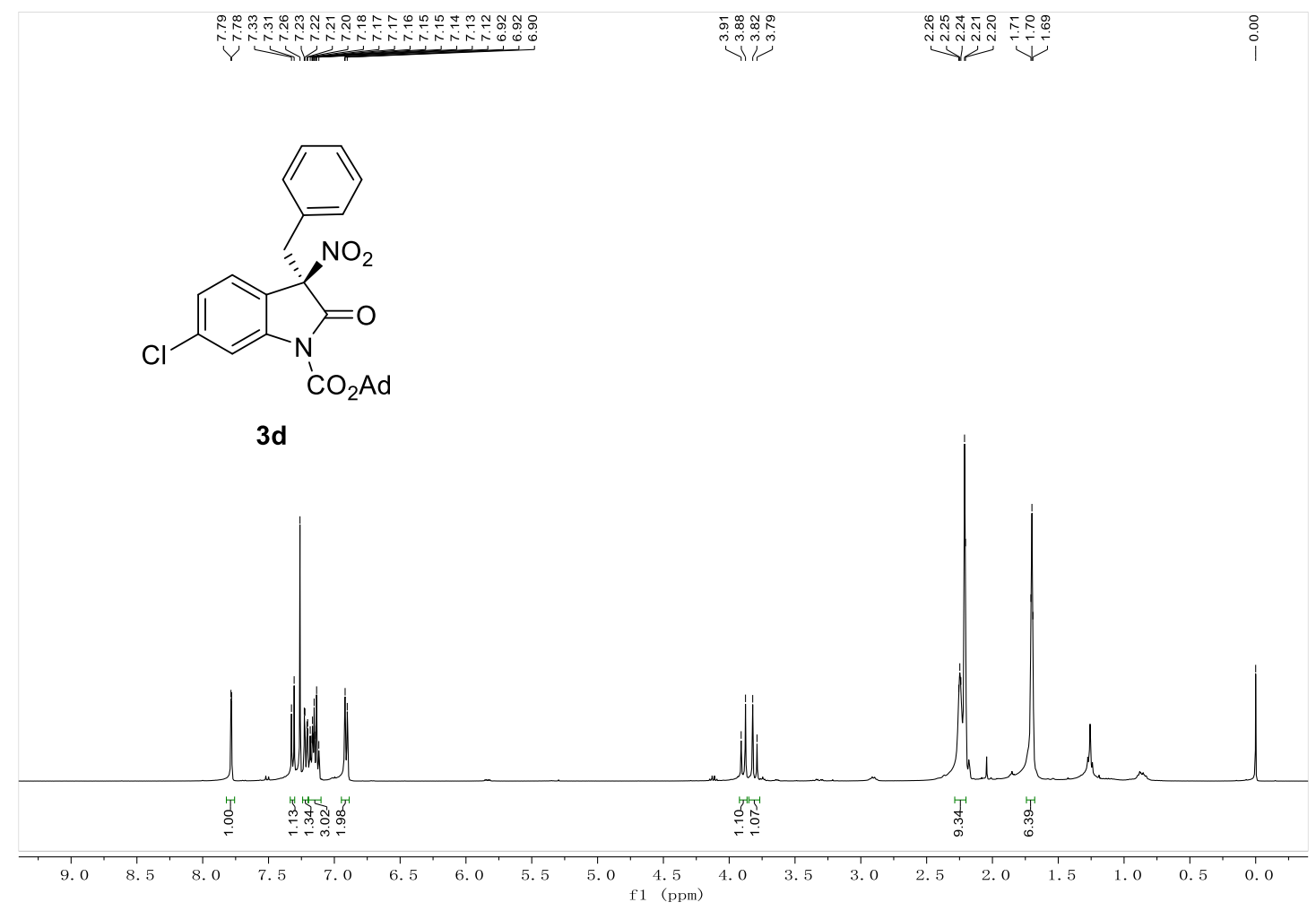

3d ${ }^{13} \mathrm{C} \mathrm{NMR}\left(100 \mathrm{M} \mathrm{Hz}, \mathrm{CDCl}_{3}\right)$

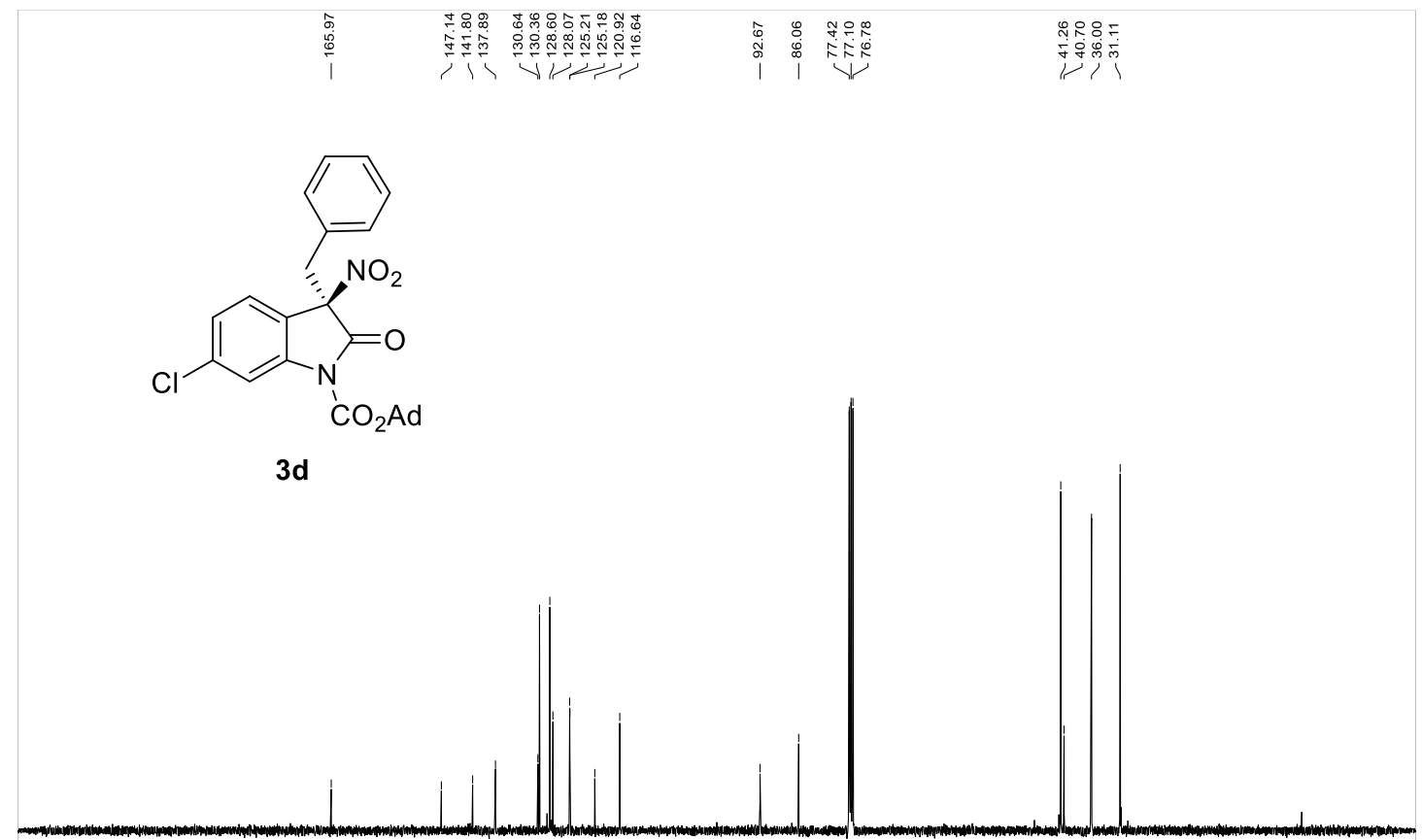

$\begin{array}{rllllllllllllllllllllllll}1 & 10 & 200 & 190 & 180 & 170 & 160 & 150 & 140 & 130 & 120 & 110 & 100 & 90 & 80 & 70 & 60 & 50 & 40 & 30 & 20 & 10 & 0 & -10\end{array}$ 
3e ${ }^{1} \mathrm{H}$ NMR (400M Hz, $\left.\mathrm{CDCl}_{3}\right)$

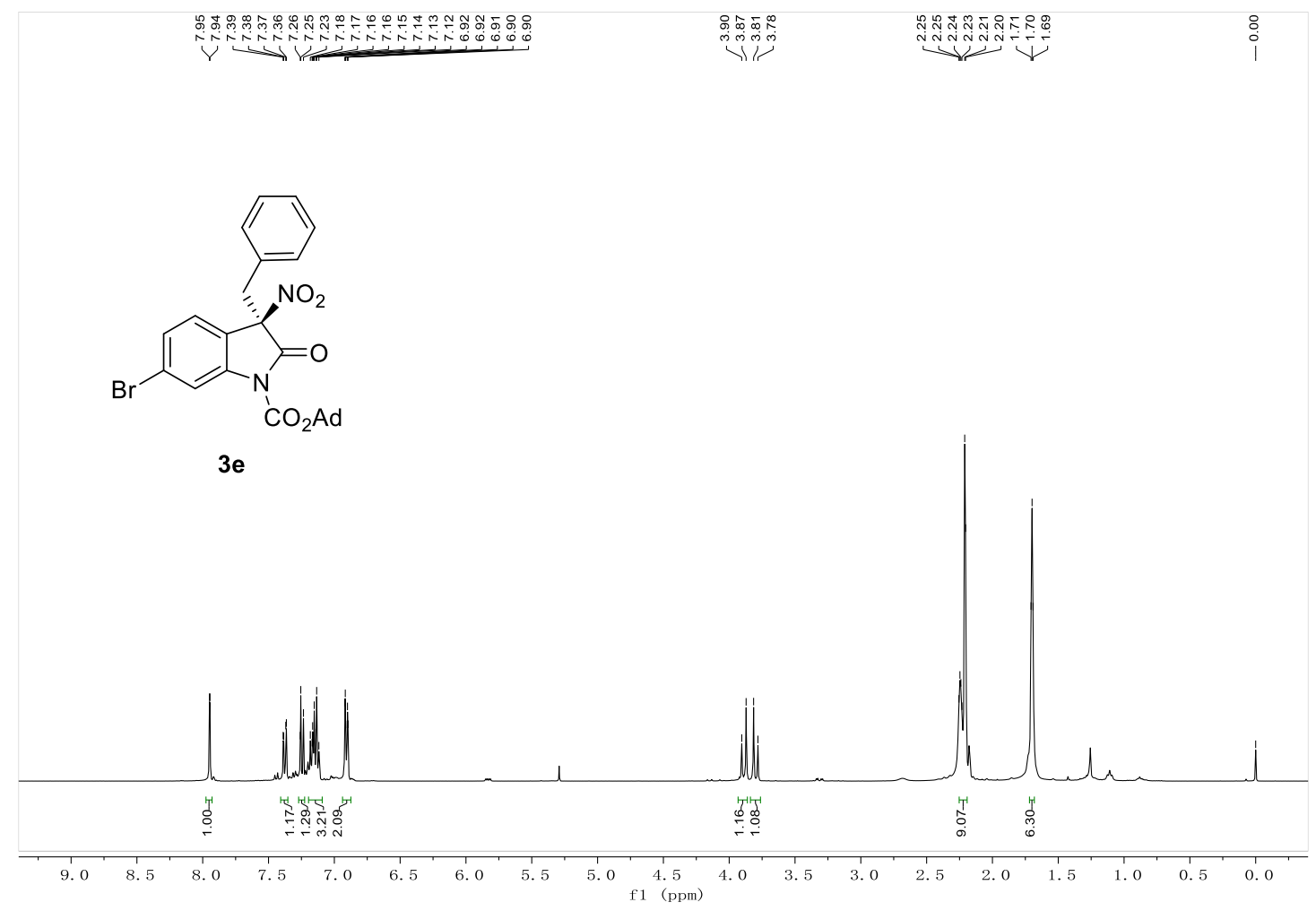

$3 e^{13} \mathrm{C} \mathrm{NMR}\left(100 \mathrm{M} \mathrm{Hz}, \mathrm{CDCl}_{3}\right)$

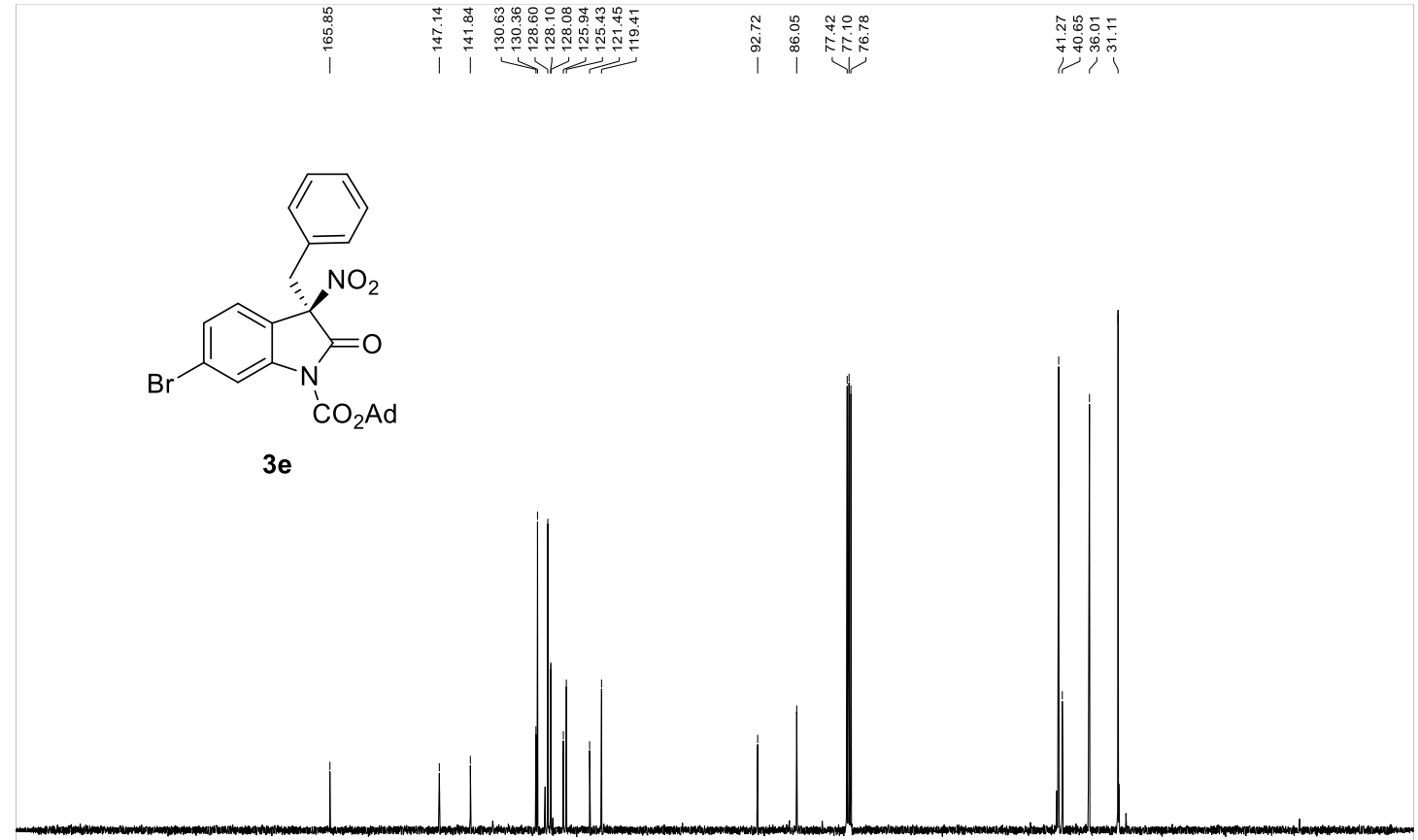

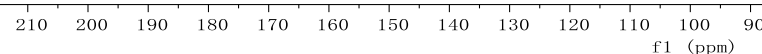


3f ${ }^{1} \mathrm{H}$ NMR (400M Hz, $\left.\mathrm{CDCl}_{3}\right)$

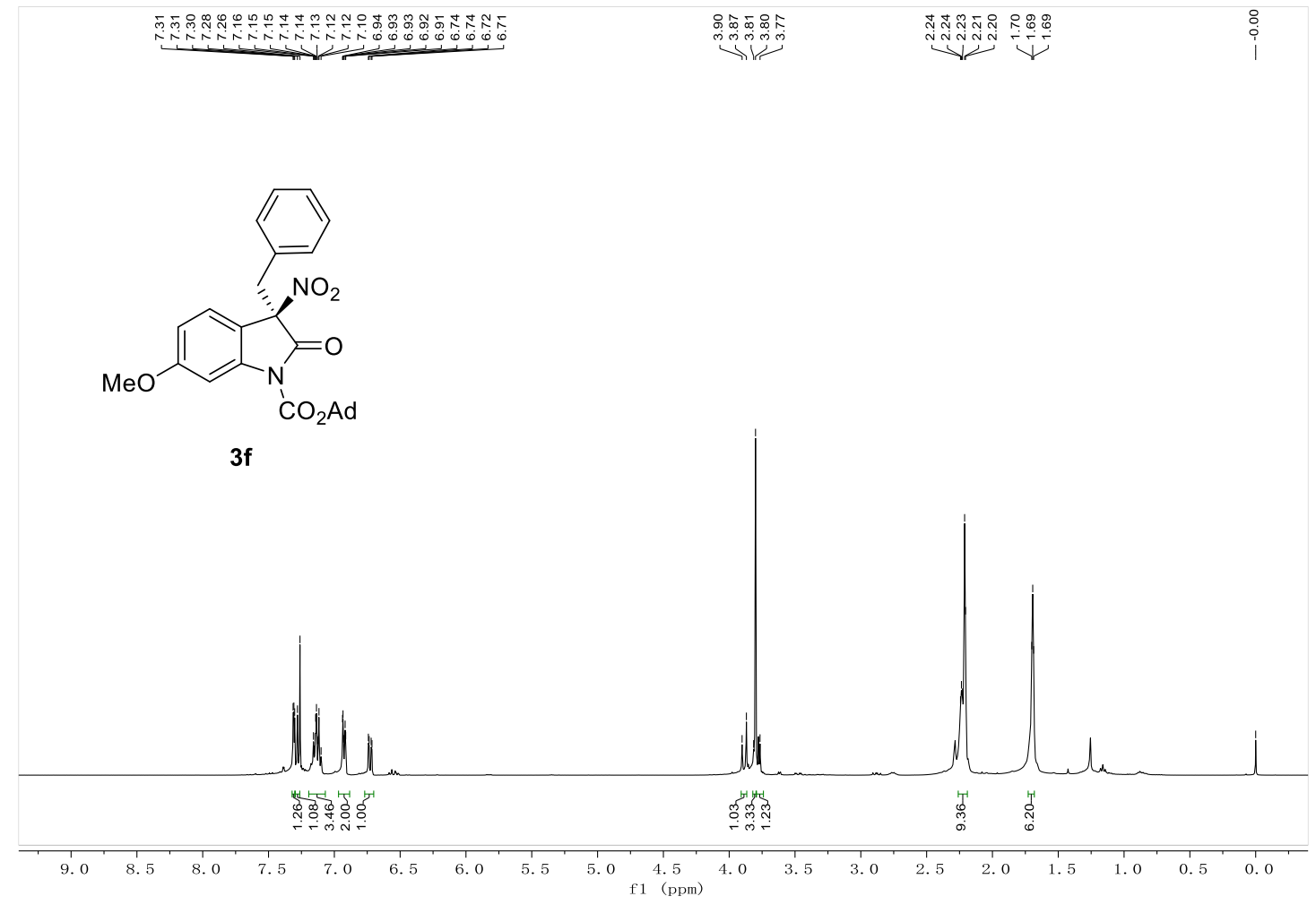

3f ${ }^{13} \mathrm{C}$ NMR $\left(100 \mathrm{M} \mathrm{Hz}, \mathrm{CDCl}_{3}\right)$

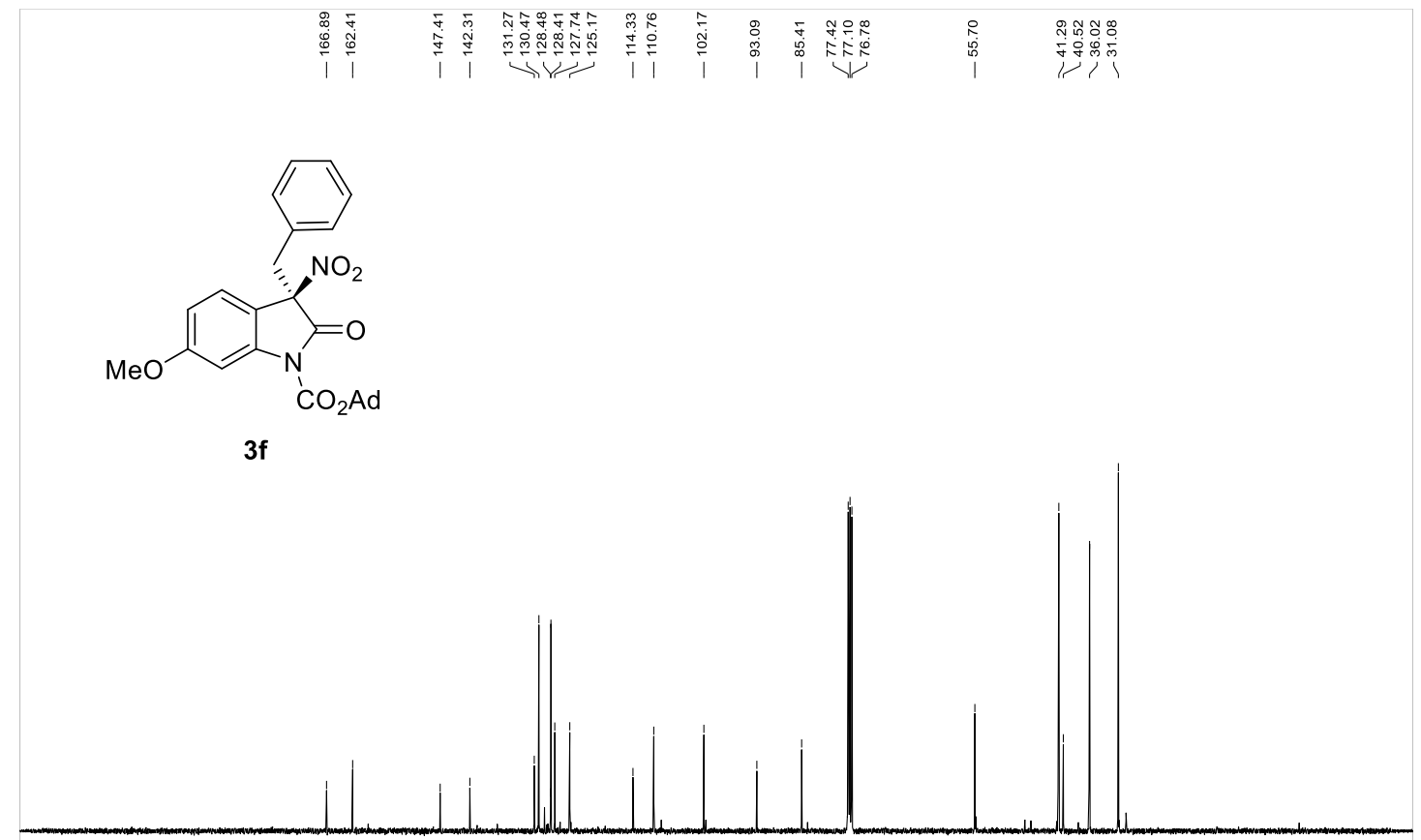

$\begin{array}{lllllllllllll}210 & 200 & 190 & 180 & 170 & 160 & 150 & 140 & 130 & 120 & 110 & 100 & 90 \\ \mathrm{fl} 1 & (\mathrm{pmm}) & 1\end{array}$ 
$3{ }^{1} \mathrm{H}$ NMR $\left(400 \mathrm{M} \mathrm{Hz}, \mathrm{CDCl}_{3}\right)$

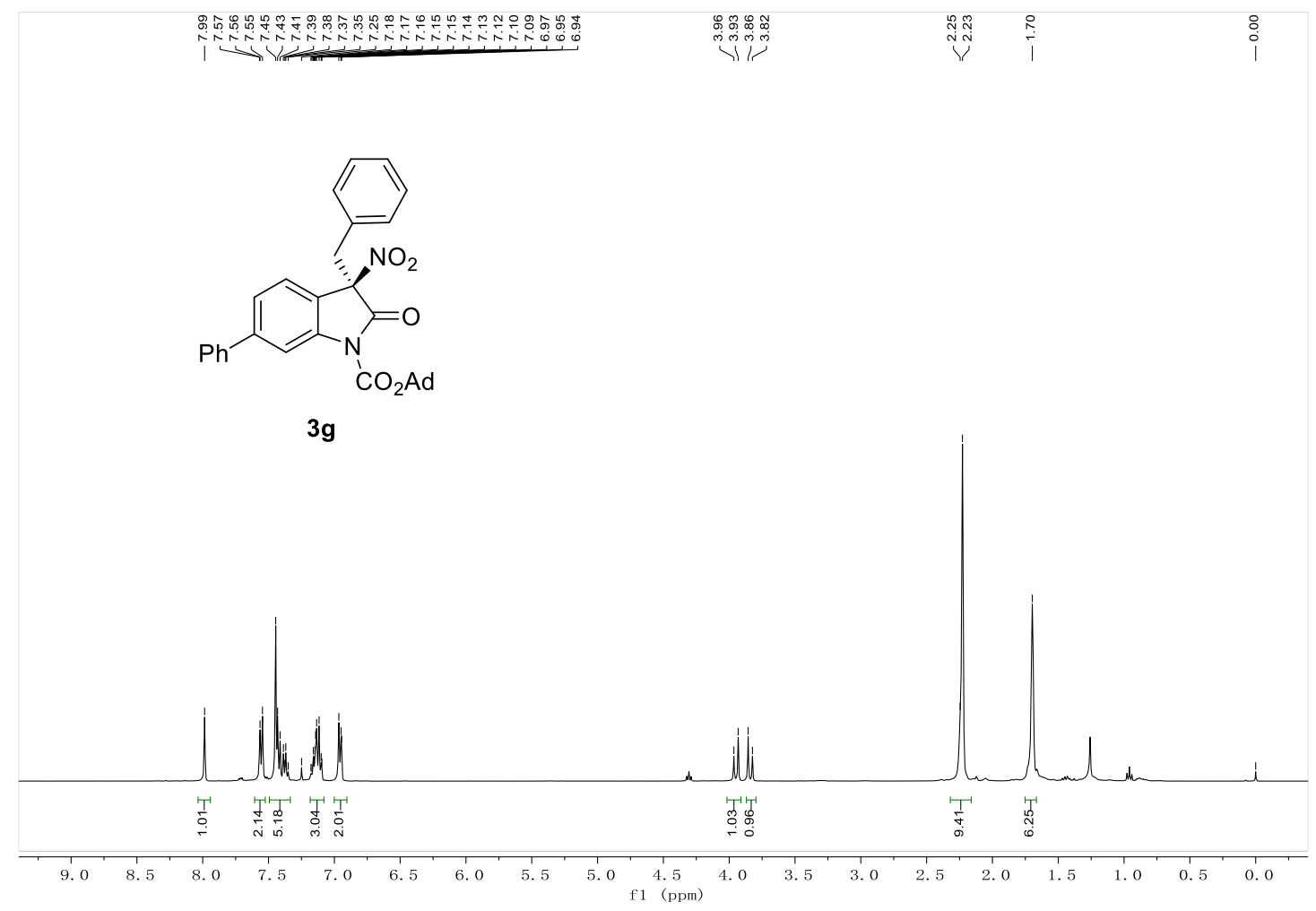

$3 g{ }^{13} \mathrm{C} \mathrm{NMR}\left(100 \mathrm{M} \mathrm{Hz}, \mathrm{CDCl}_{3}\right)$

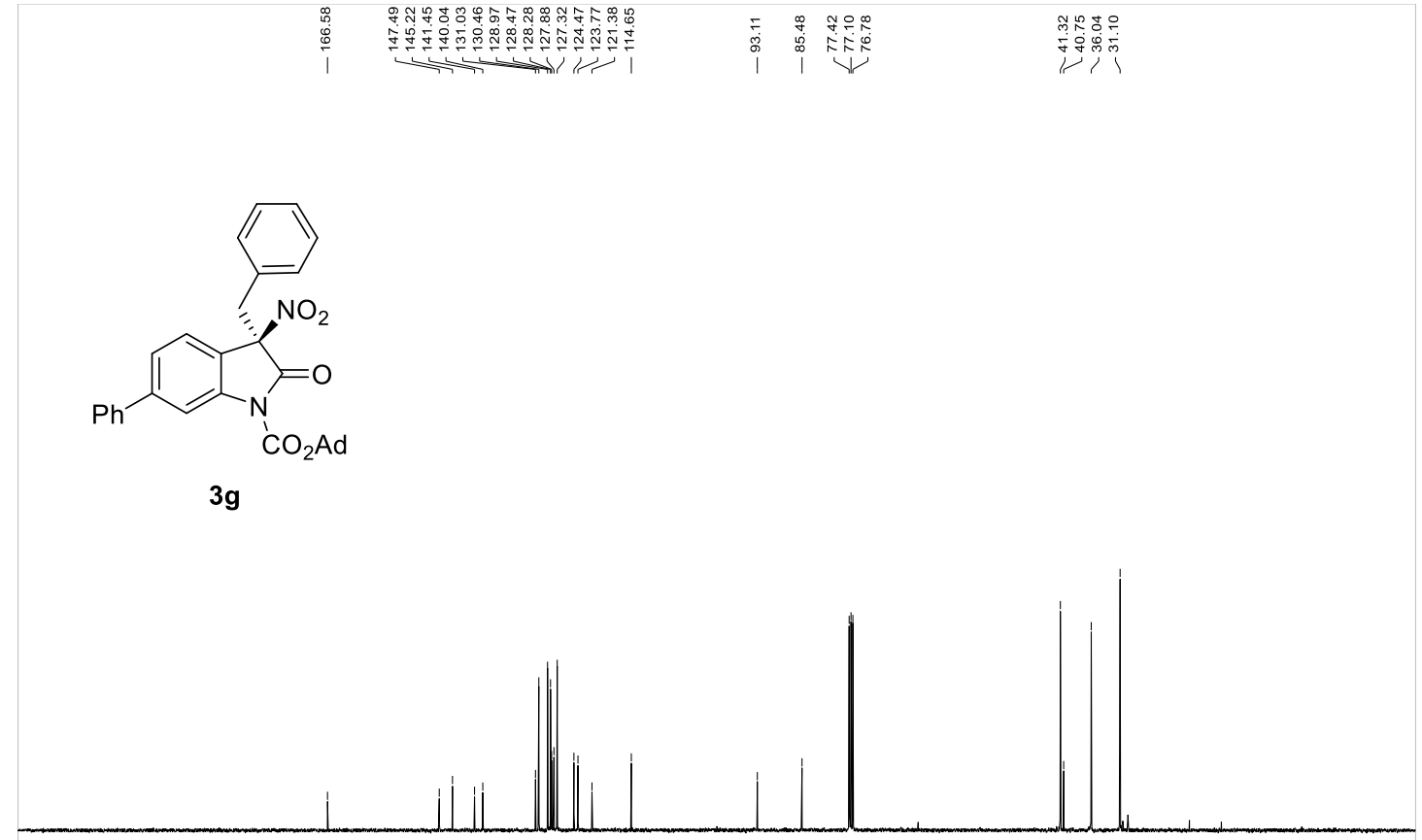

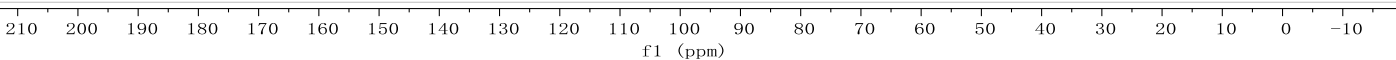


3h ${ }^{1} \mathrm{H}$ NMR (400M Hz, $\left.\mathrm{CDCl}_{3}\right)$

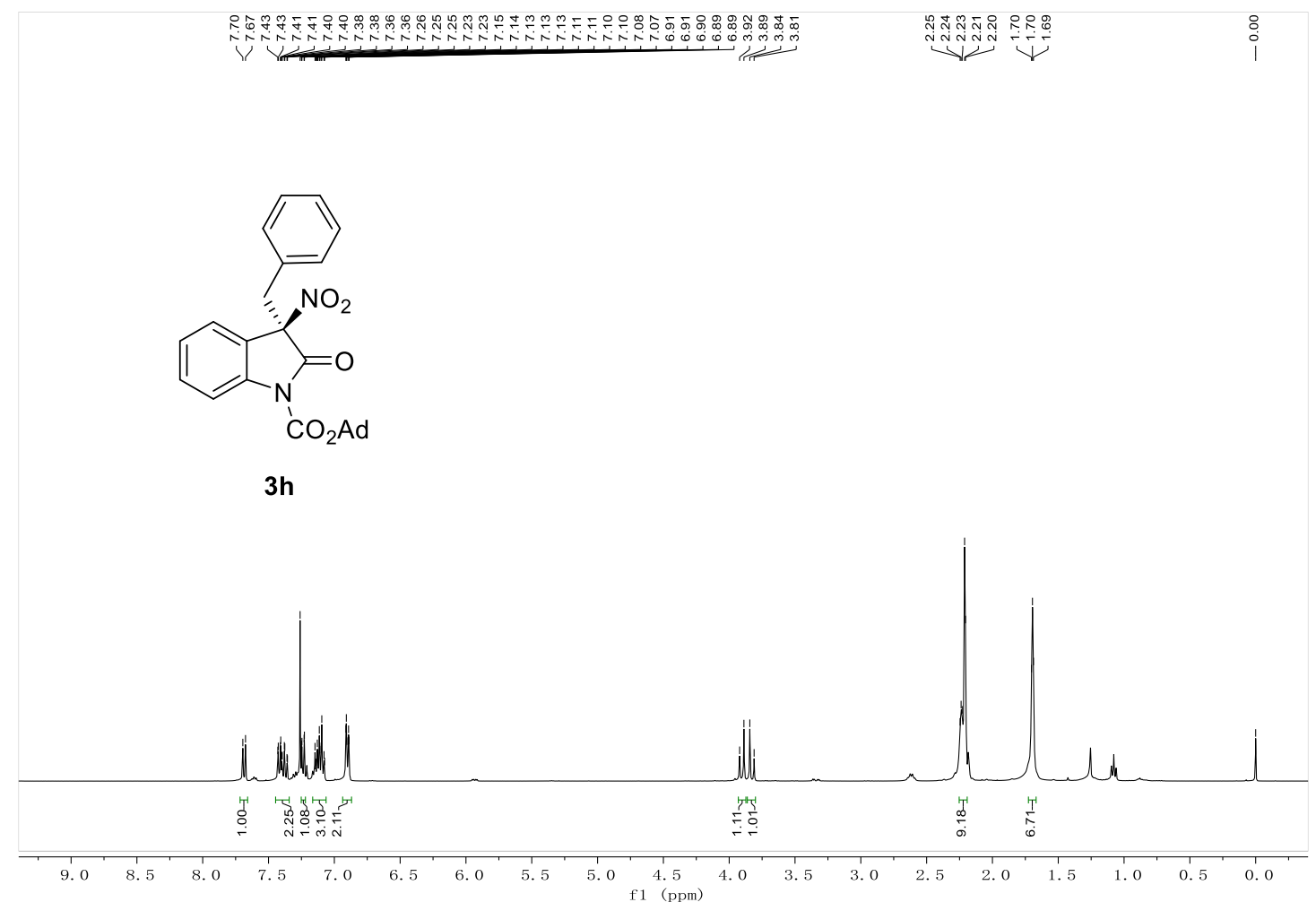

3h ${ }^{13} \mathrm{C}$ NMR $\left(100 \mathrm{M} \mathrm{Hz}, \mathrm{CDCl}_{3}\right)$

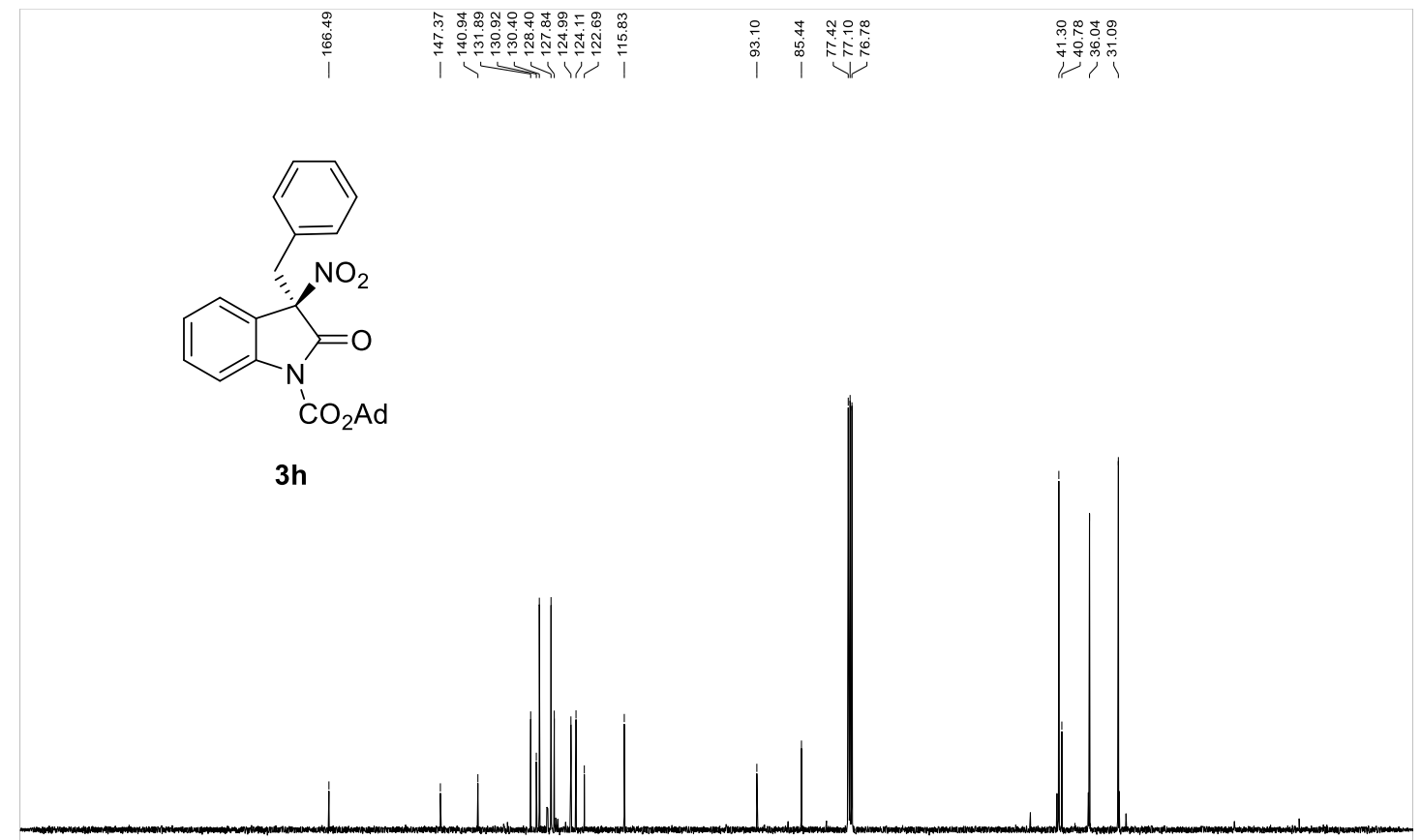

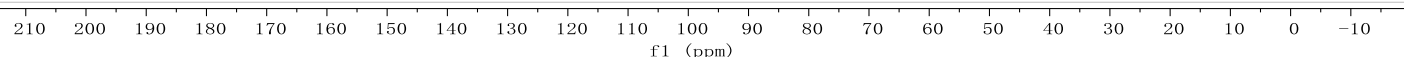


3i ${ }^{1} \mathrm{H}$ NMR (400M Hz, $\left.\mathrm{CDCl}_{3}\right)$

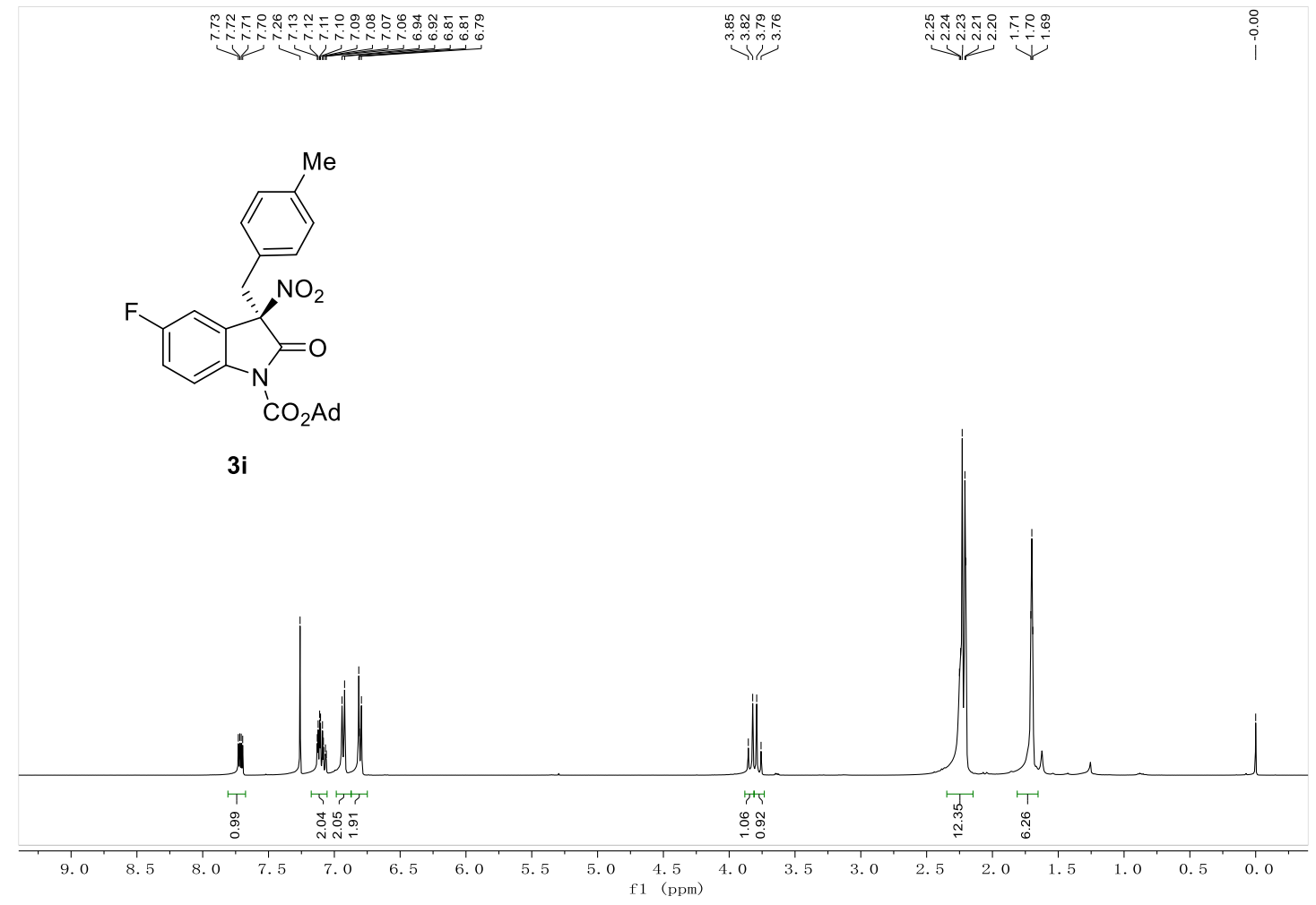

$3 \mathbf{i}^{13} \mathrm{C}$ NMR $\left(100 \mathrm{M} \mathrm{Hz}, \mathrm{CDCl}_{3}\right)$

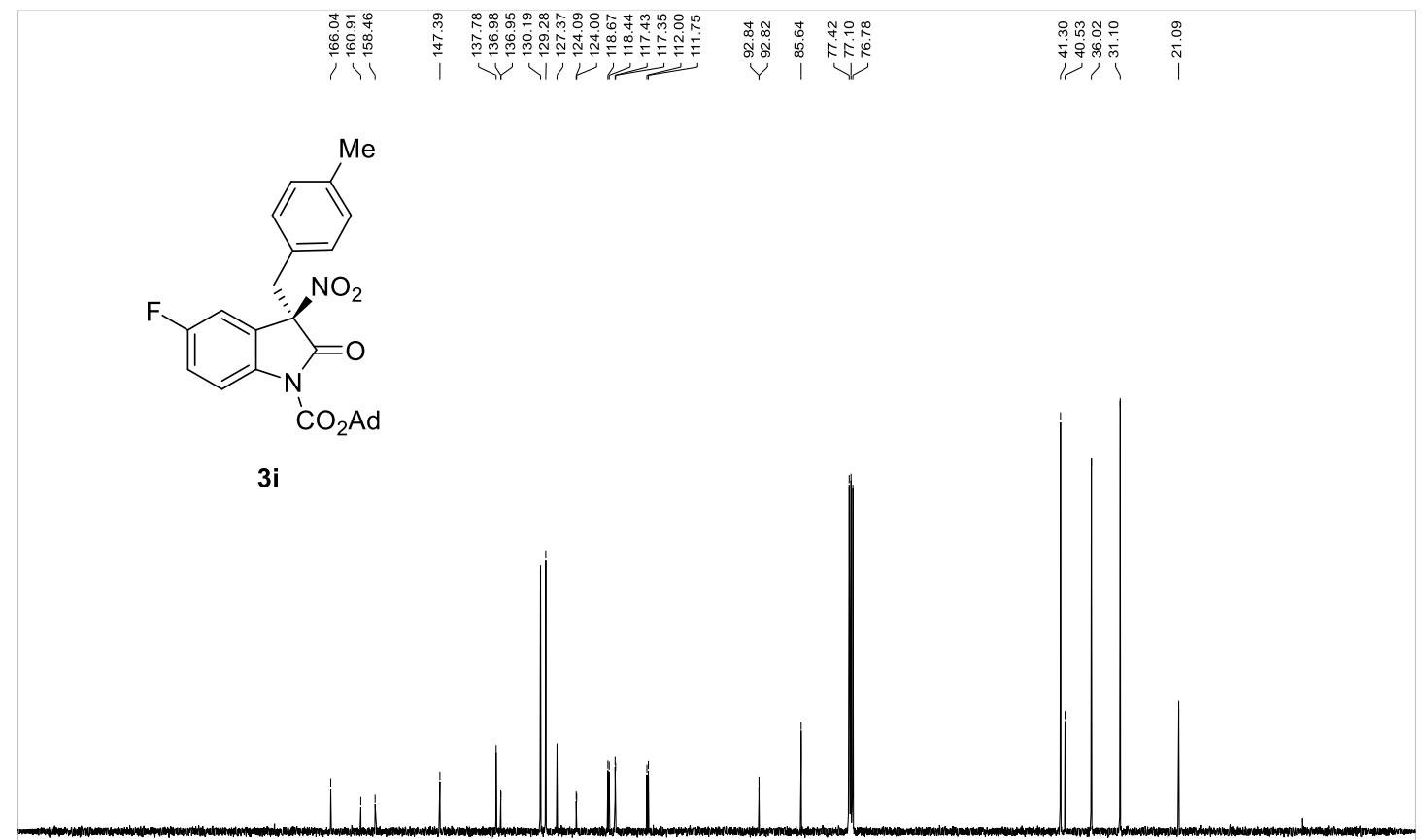

$\begin{array}{lllllllllllll}210 & 200 & 190 & 180 & 170 & 160 & 150 & 140 & 130 & 120 & 110 & 100 & 100 \\ \mathrm{f} 1 & (\mathrm{ppm})\end{array}$ 
3j ${ }^{1} \mathrm{H}$ NMR $\left(400 \mathrm{M} \mathrm{Hz}, \mathrm{CDCl}_{3}\right)$

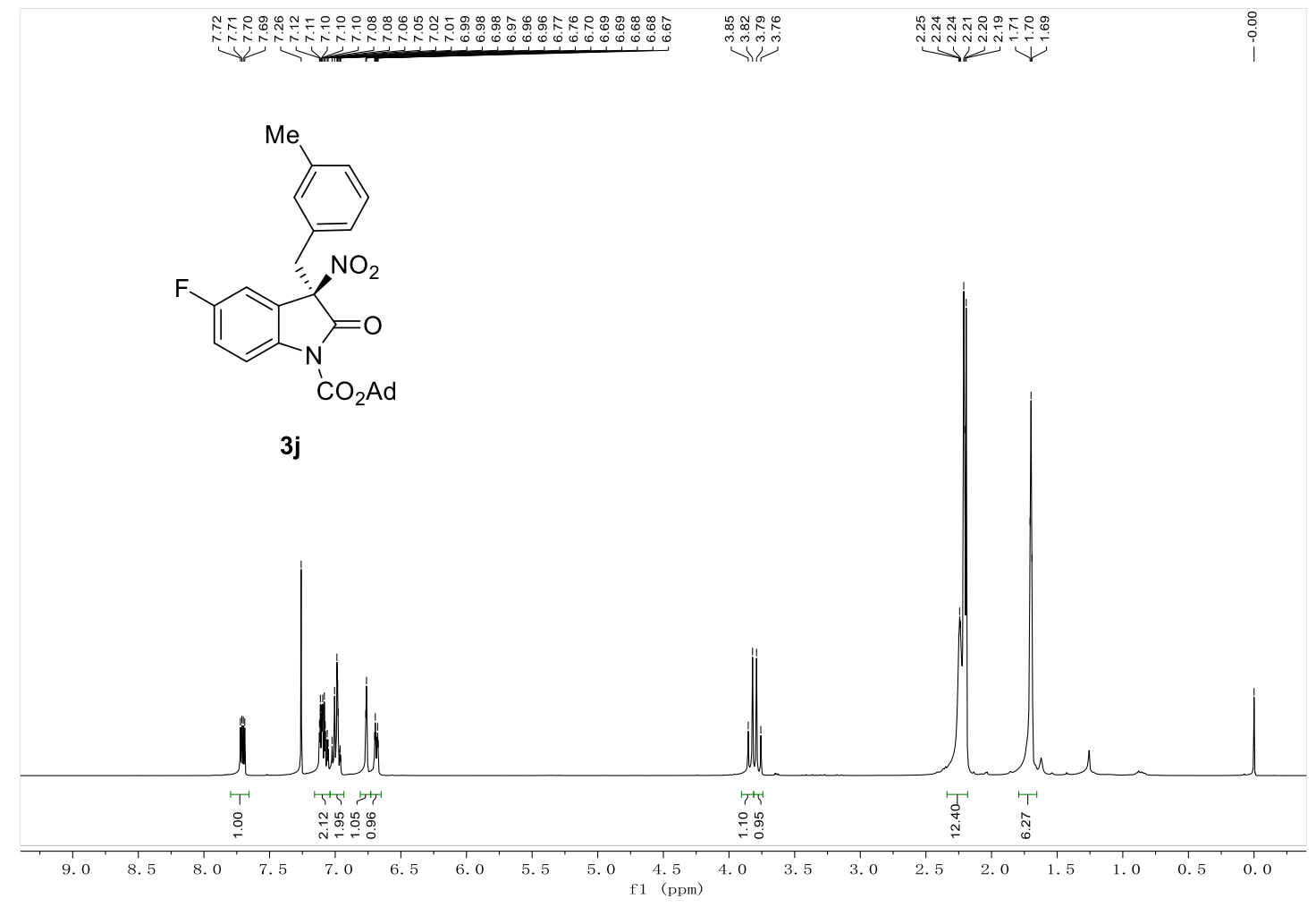

$3 \mathbf{j}{ }^{13} \mathrm{C}$ NMR $\left(100 \mathrm{M} \mathrm{Hz}, \mathrm{CDCl}_{3}\right)$

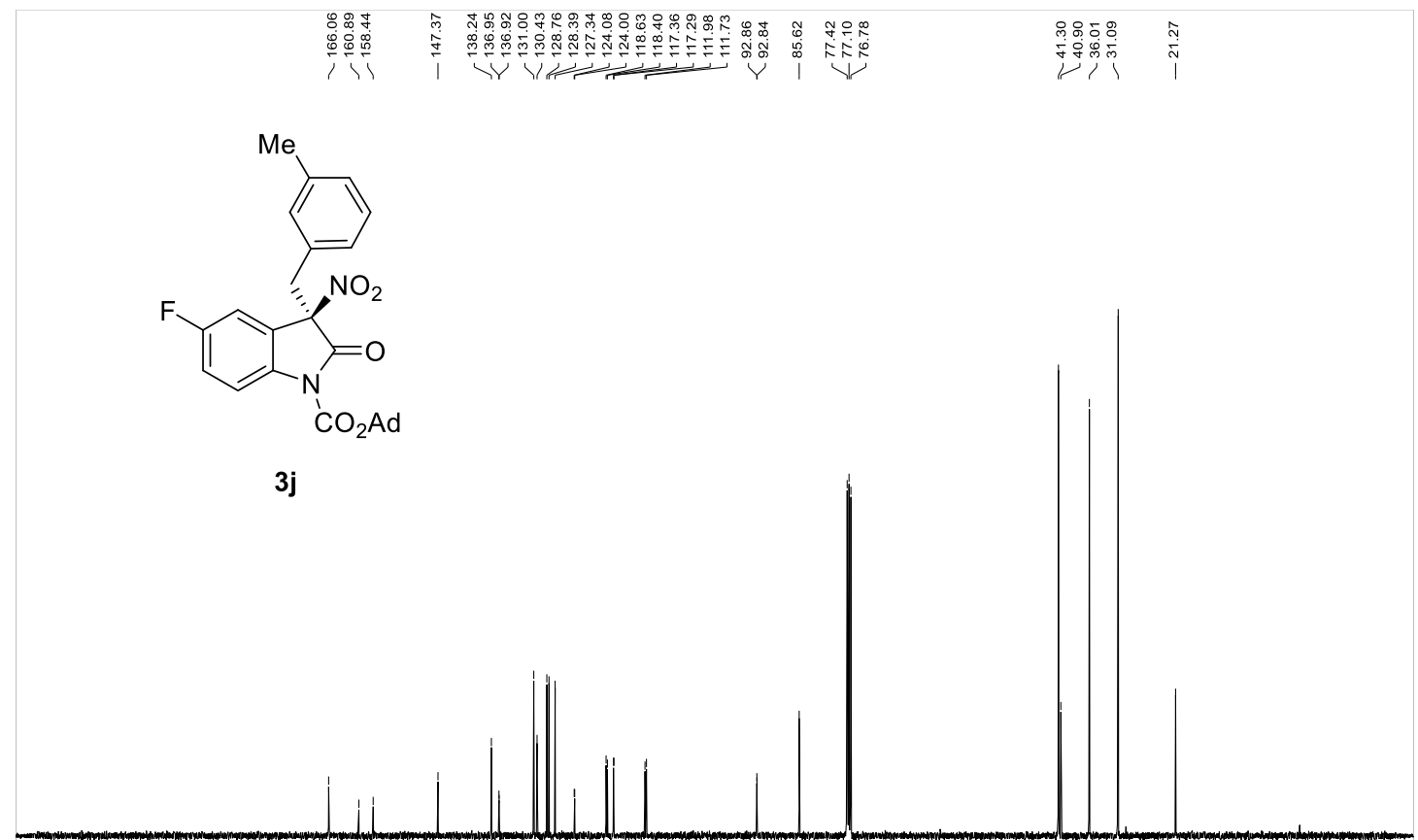

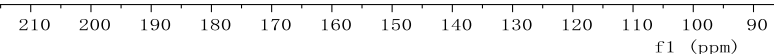


3k ${ }^{1} \mathrm{H}$ NMR (400M Hz, $\left.\mathrm{CDCl}_{3}\right)$

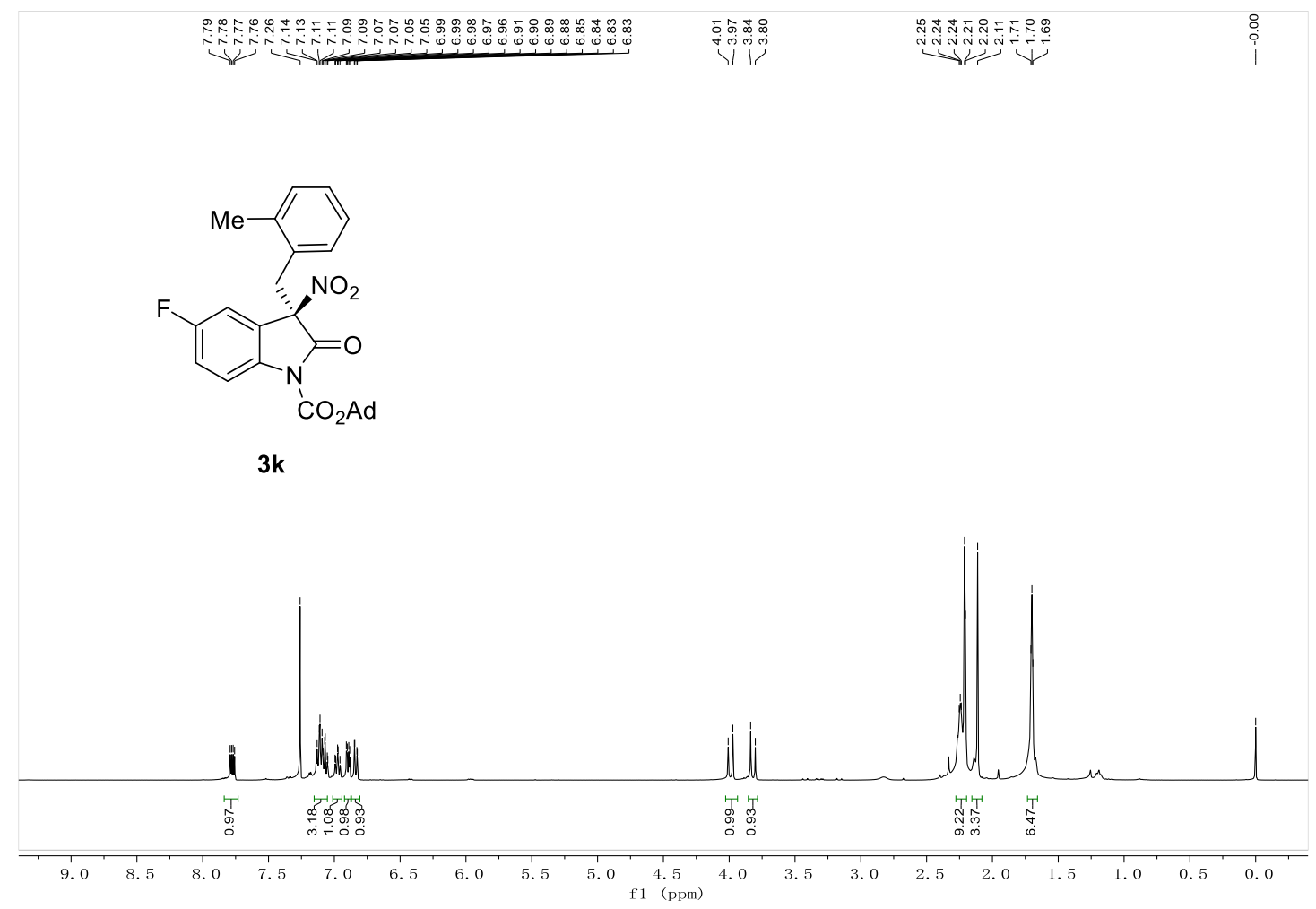

3k ${ }^{13} \mathrm{C} \mathrm{NMR}\left(100 \mathrm{M} \mathrm{Hz}, \mathrm{CDCl}_{3}\right)$

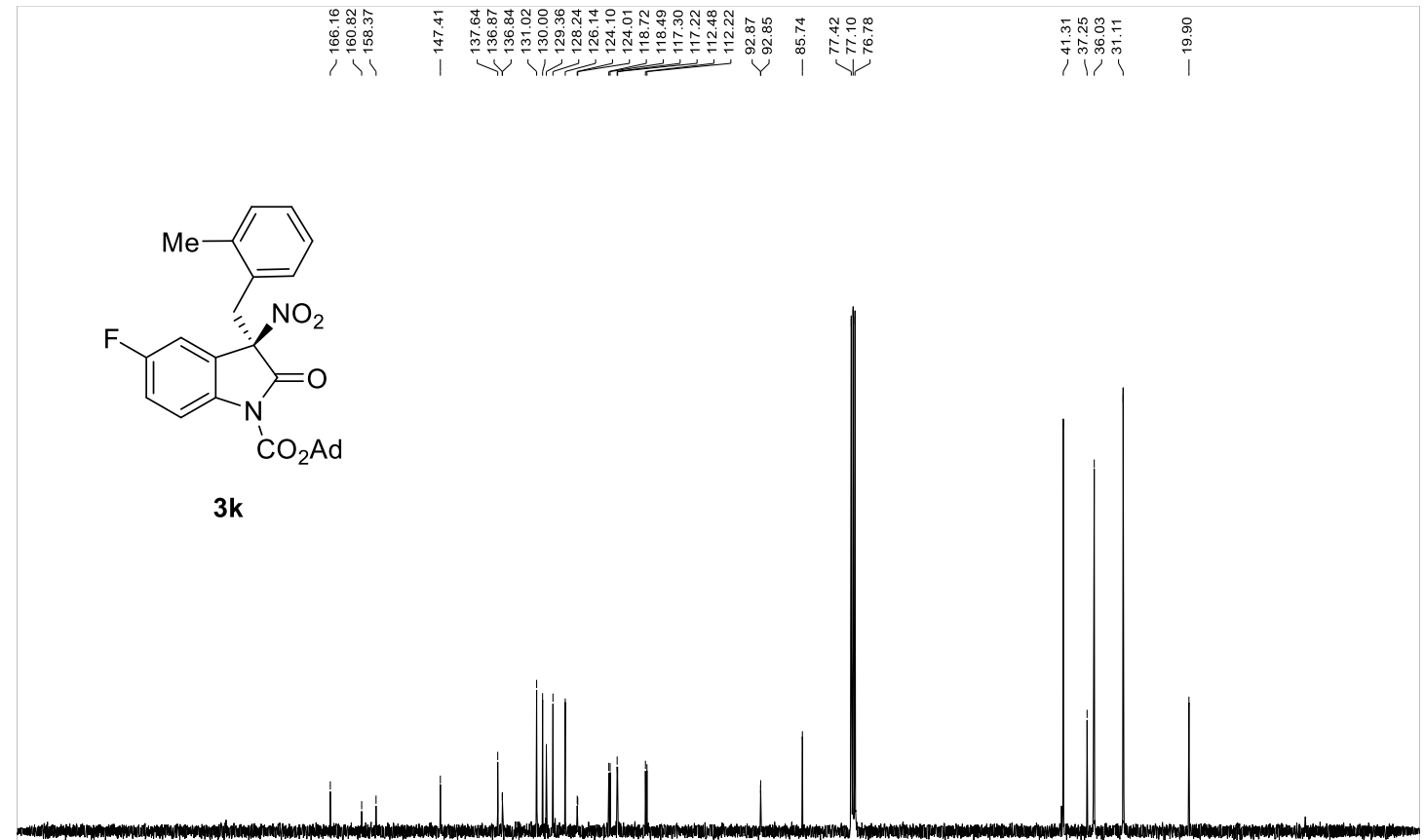

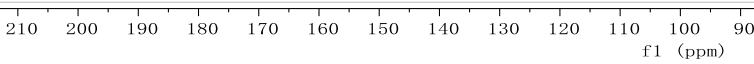


3I ${ }^{1} \mathrm{H}$ NMR (400M Hz, $\left.\mathrm{CDCl}_{3}\right)$

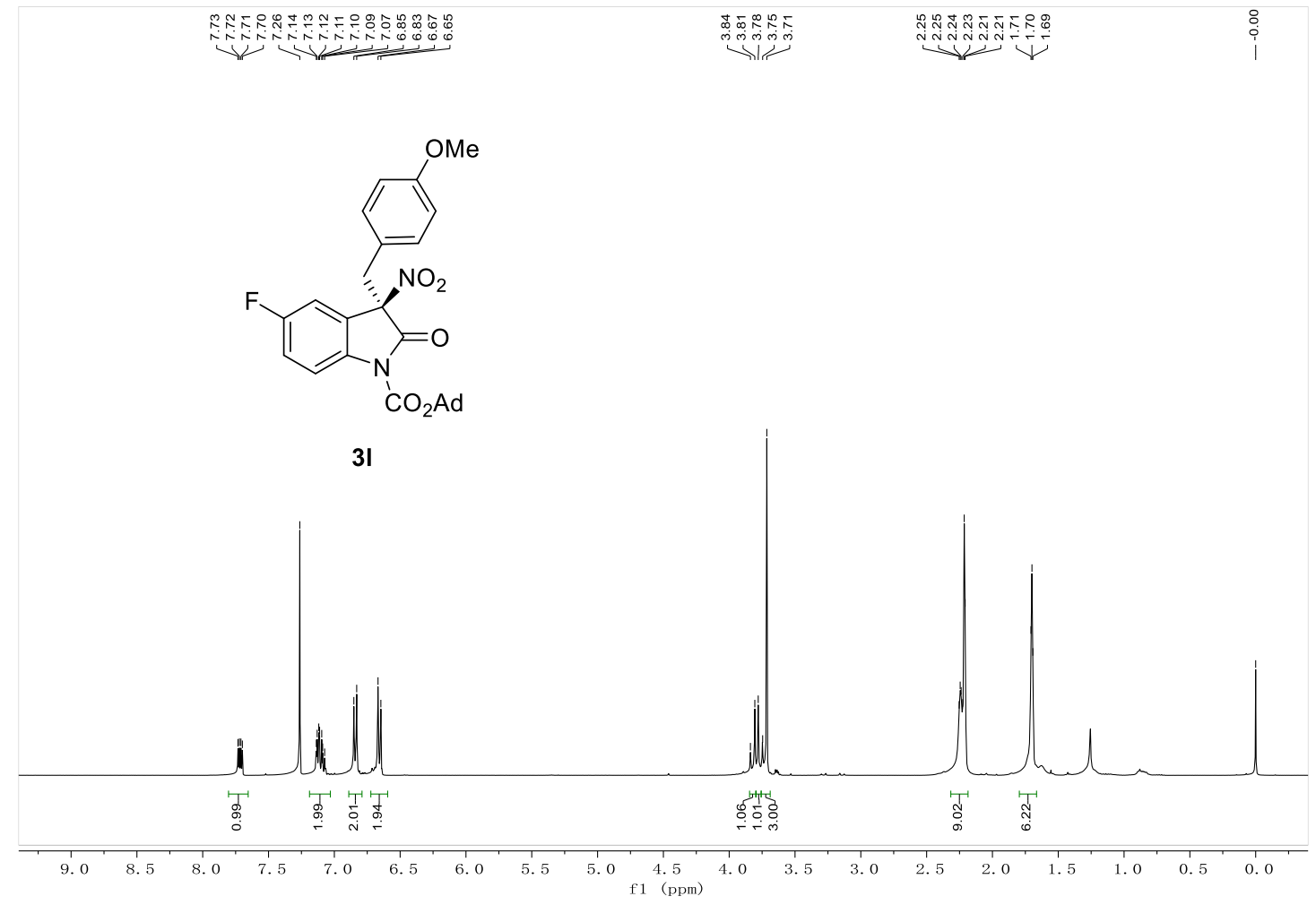

$3 \mathbf{I}^{13} \mathrm{C}$ NMR $\left(100 \mathrm{M} \mathrm{Hz}, \mathrm{CDCl}_{3}\right)$

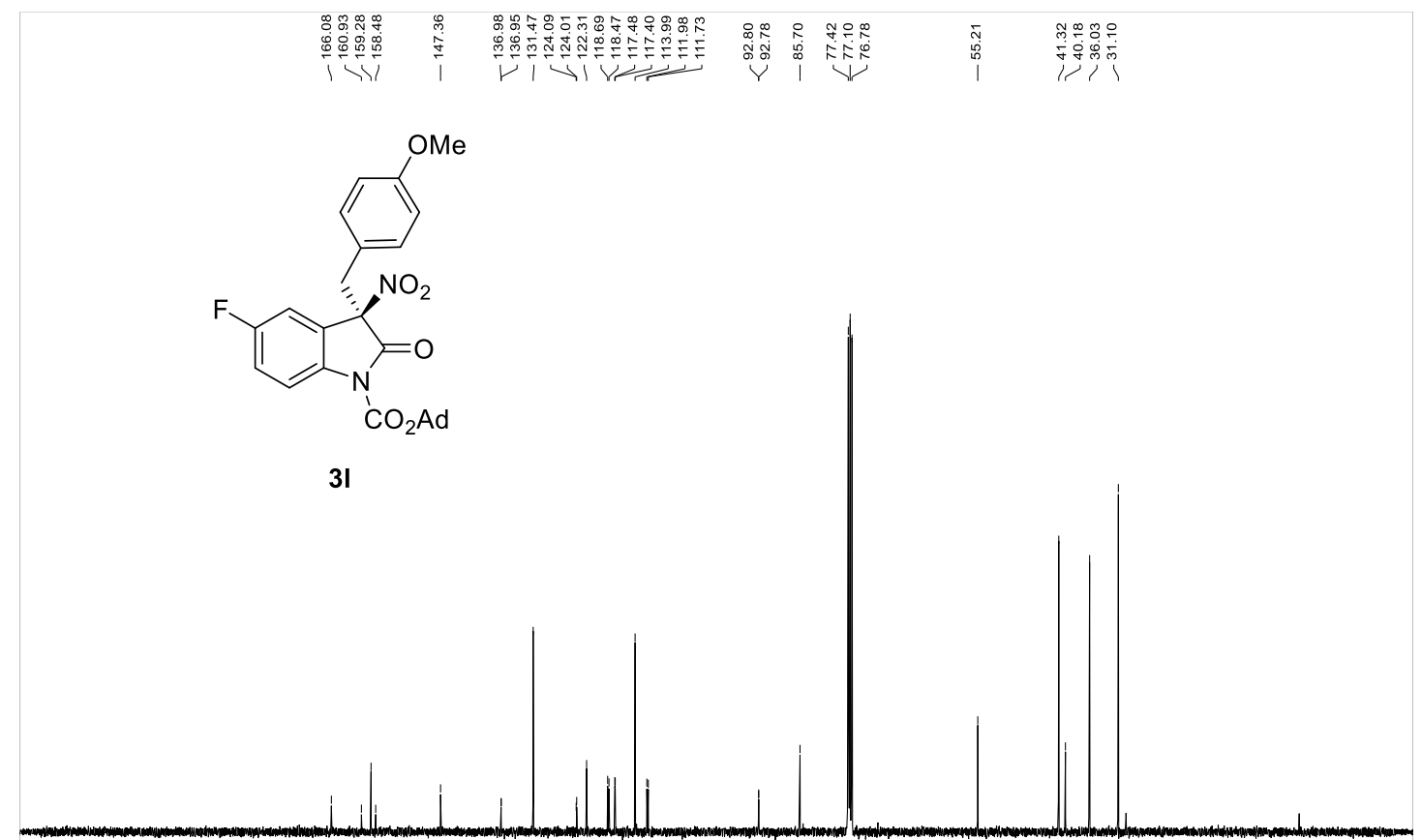

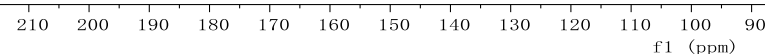


3m ${ }^{1} \mathrm{H}$ NMR (400M Hz, $\left.\mathrm{CDCl}_{3}\right)$

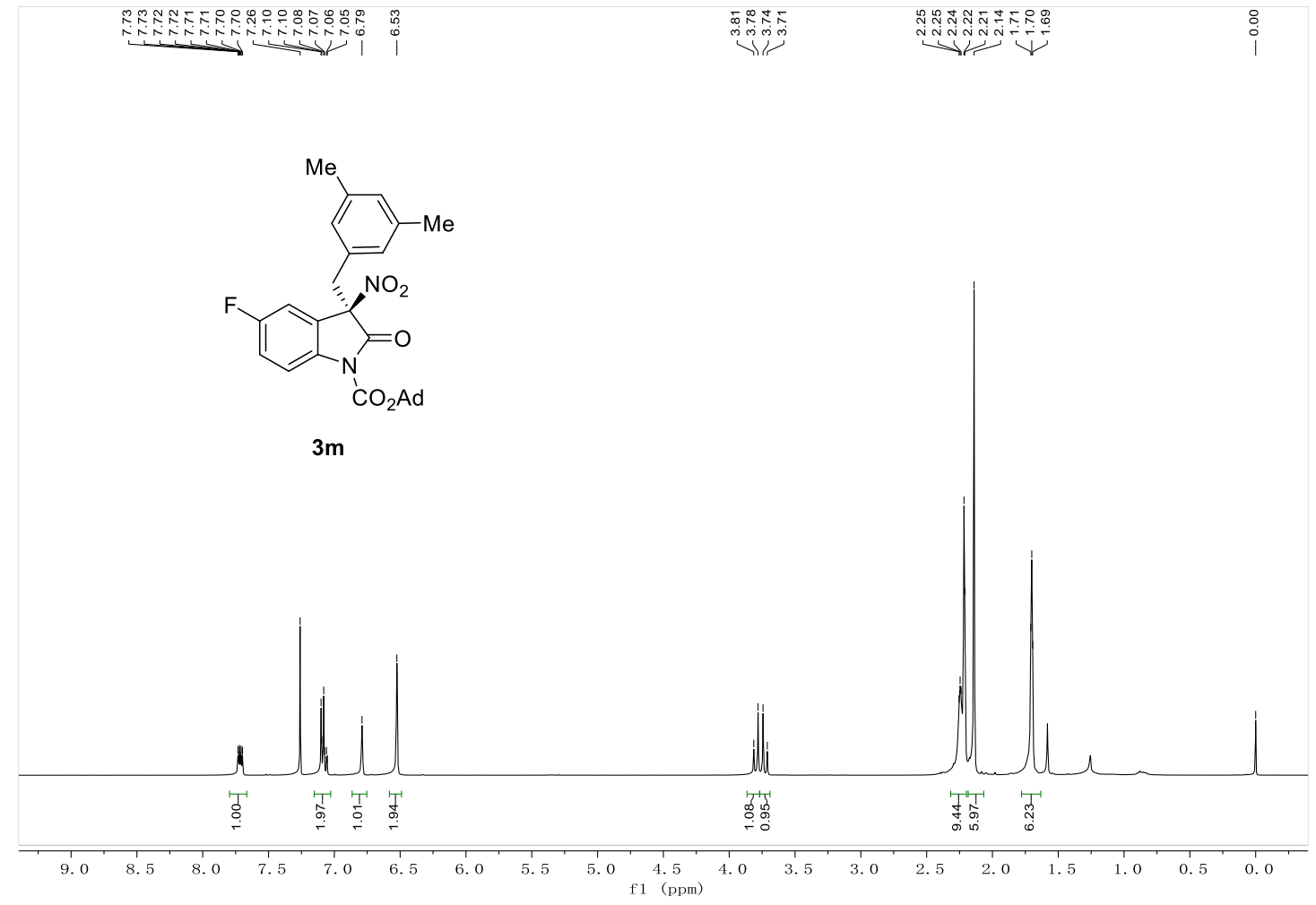

3m ${ }^{13} \mathrm{C}$ NMR $\left(100 \mathrm{M} \mathrm{Hz}, \mathrm{CDCl}_{3}\right)$

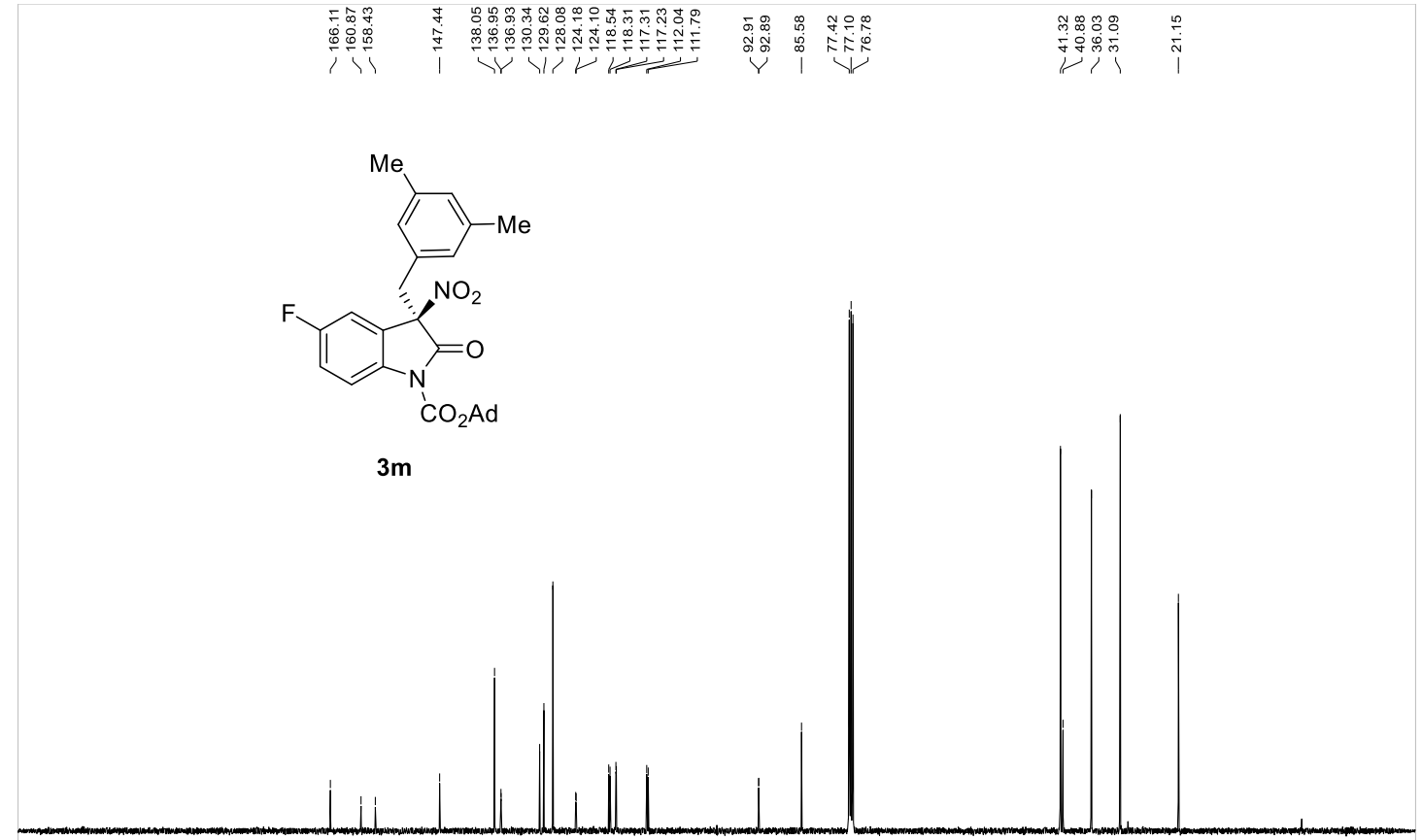

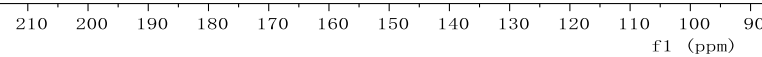


3n ${ }^{1} \mathrm{H}$ NMR (400M Hz, $\left.\mathrm{CDCl}_{3}\right)$

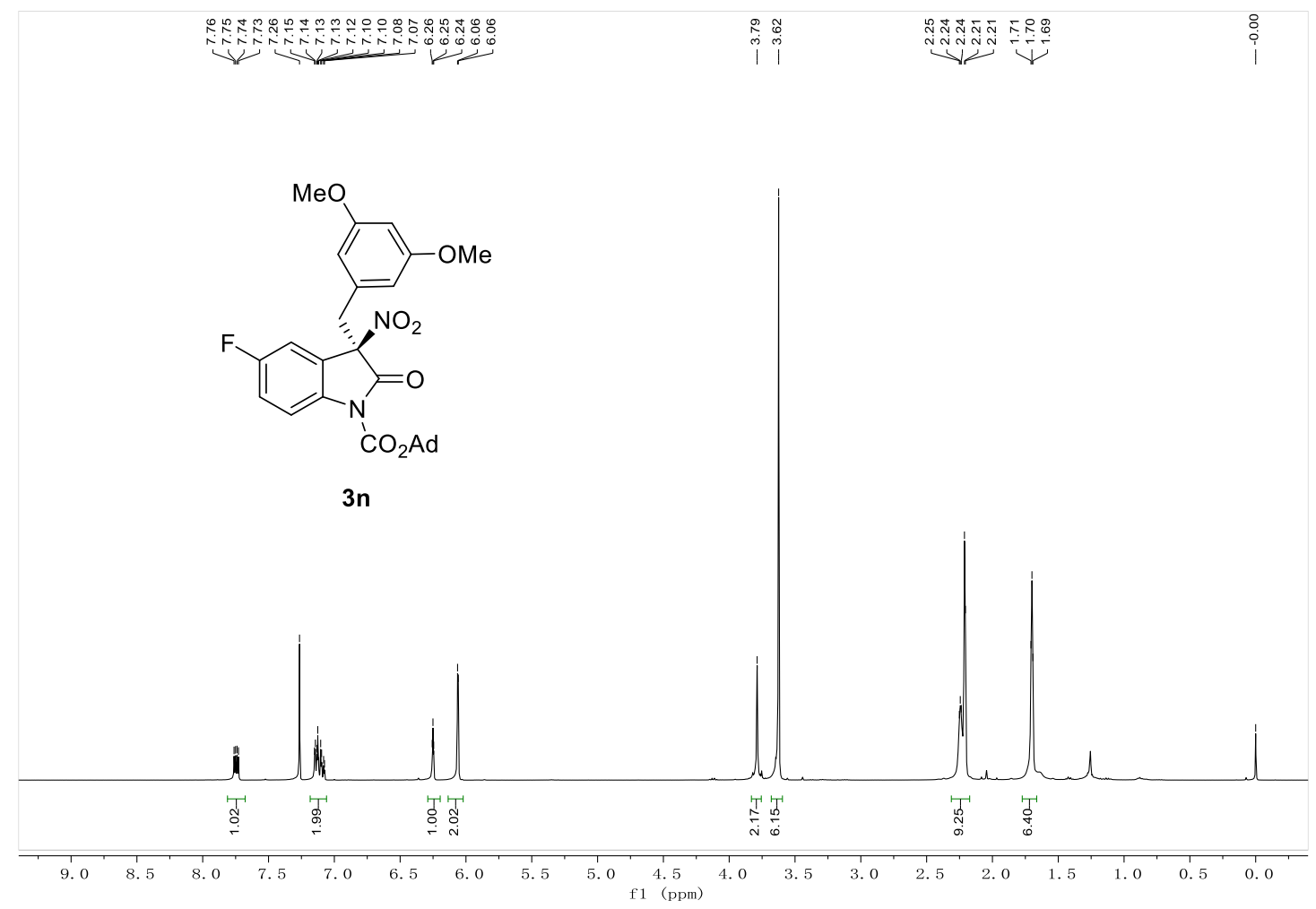

3n ${ }^{13} \mathrm{C} \mathrm{NMR}\left(100 \mathrm{M} \mathrm{Hz}, \mathrm{CDCl}_{3}\right)$

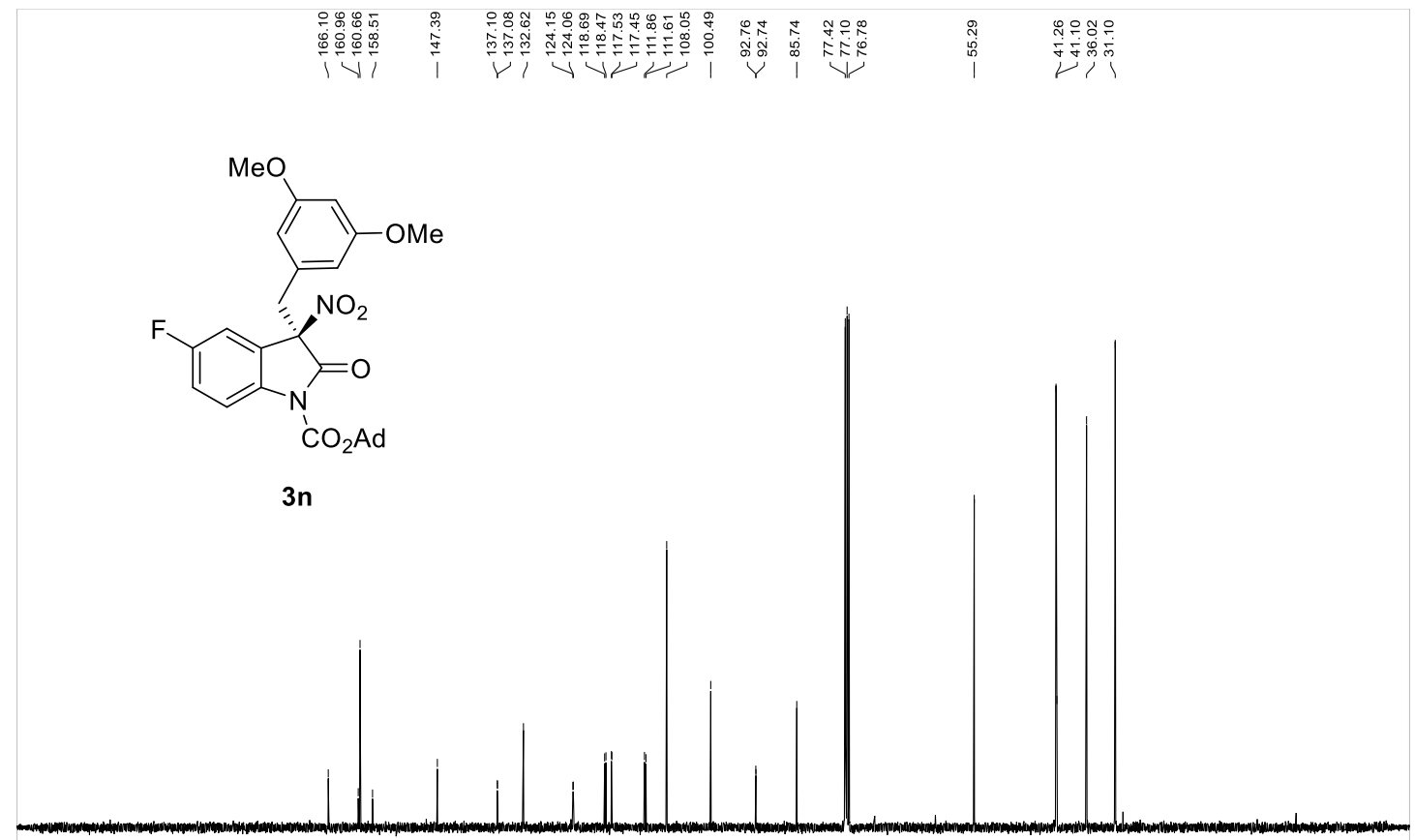

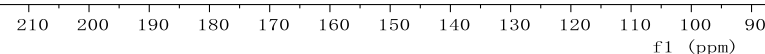


3o ${ }^{1} \mathrm{H}$ NMR (400M Hz, $\left.\mathrm{CDCl}_{3}\right)$

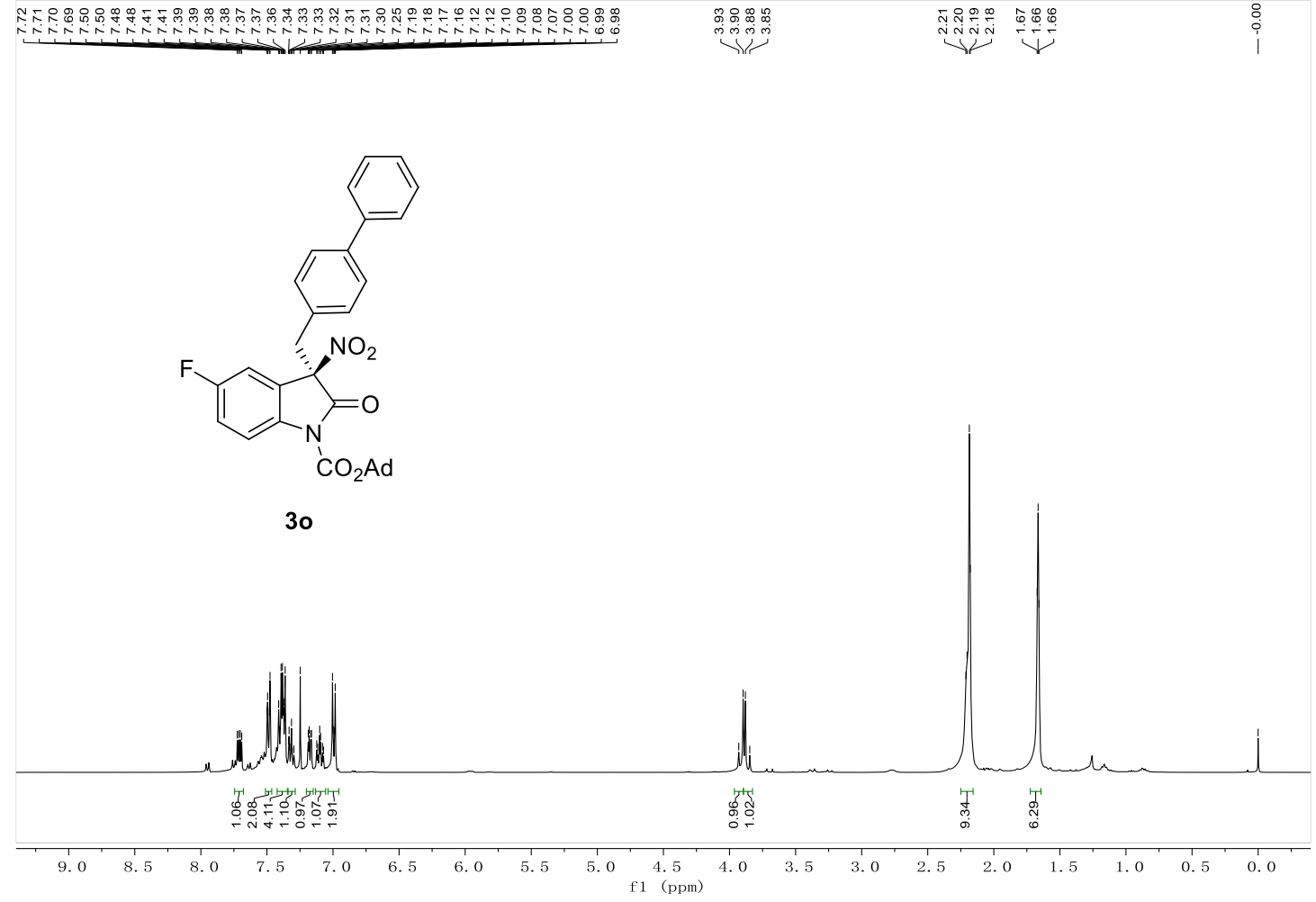

3o ${ }^{13} \mathrm{C}$ NMR $\left(100 \mathrm{M} \mathrm{Hz}, \mathrm{CDCl}_{3}\right)$

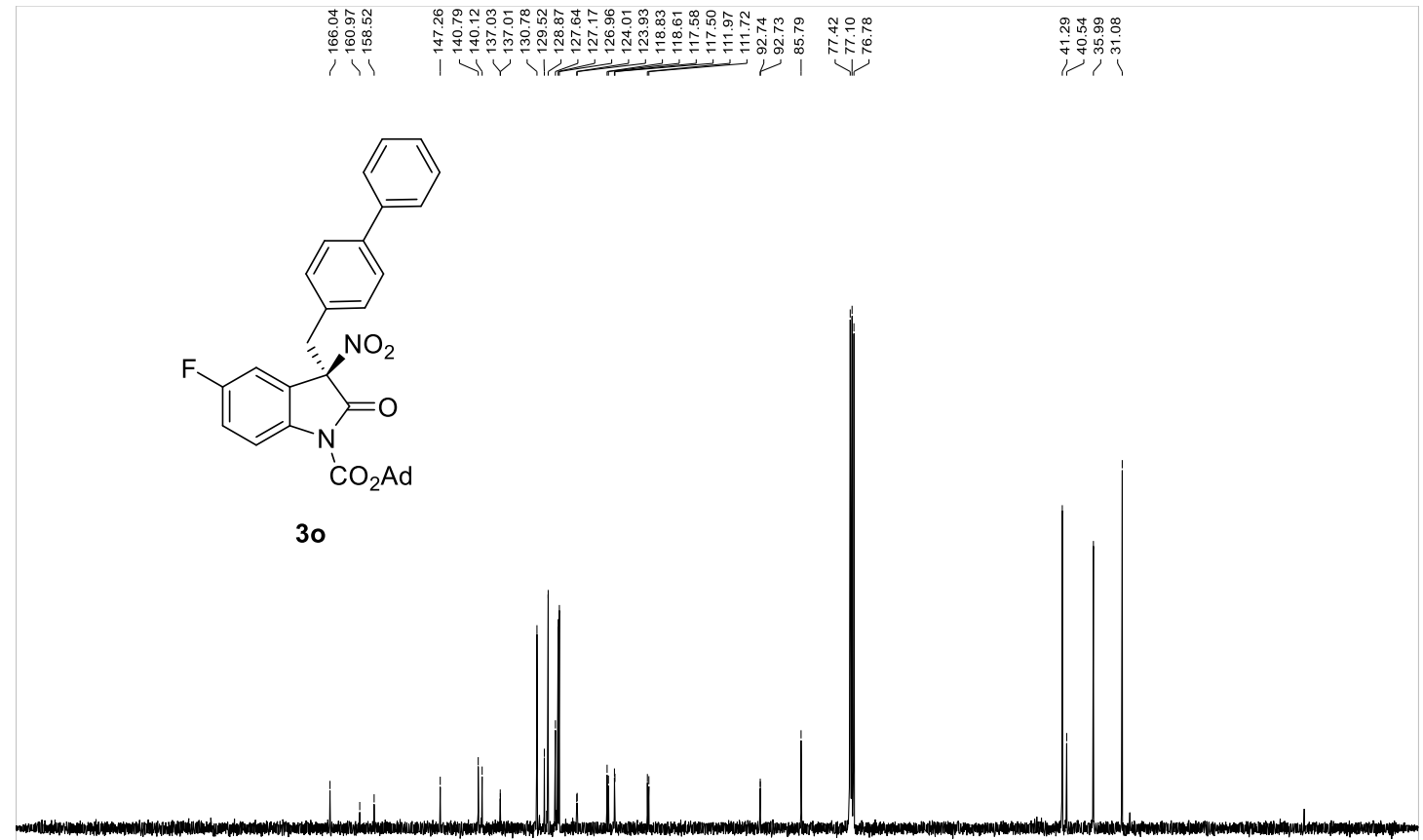

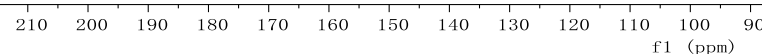


3p ${ }^{1} \mathrm{H}$ NMR $\left(400 \mathrm{M} \mathrm{Hz}, \mathrm{CDCl}_{3}\right)$

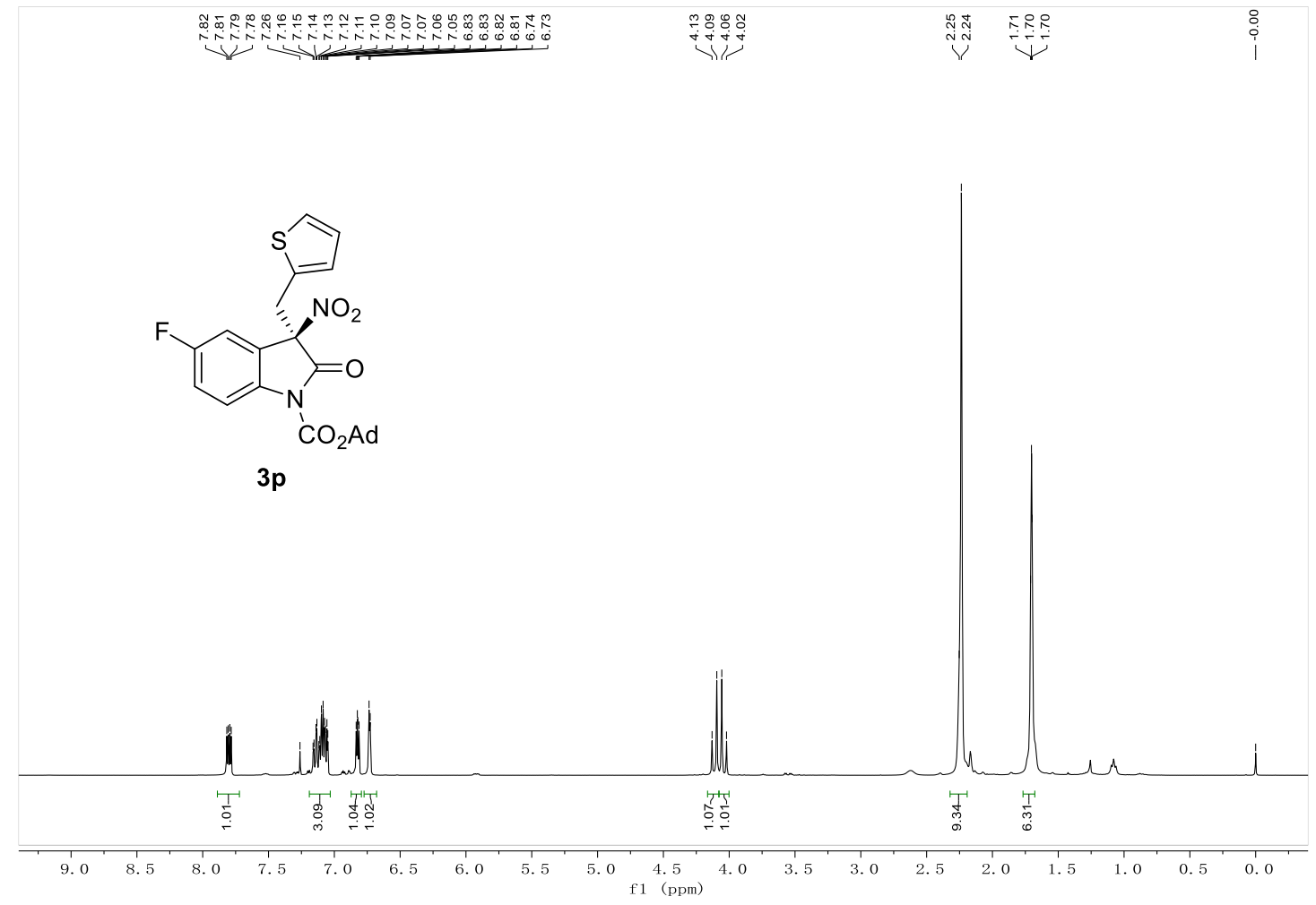

3p ${ }^{13} \mathrm{C} \mathrm{NMR}\left(100 \mathrm{M} \mathrm{Hz}, \mathrm{CDCl}_{3}\right)$

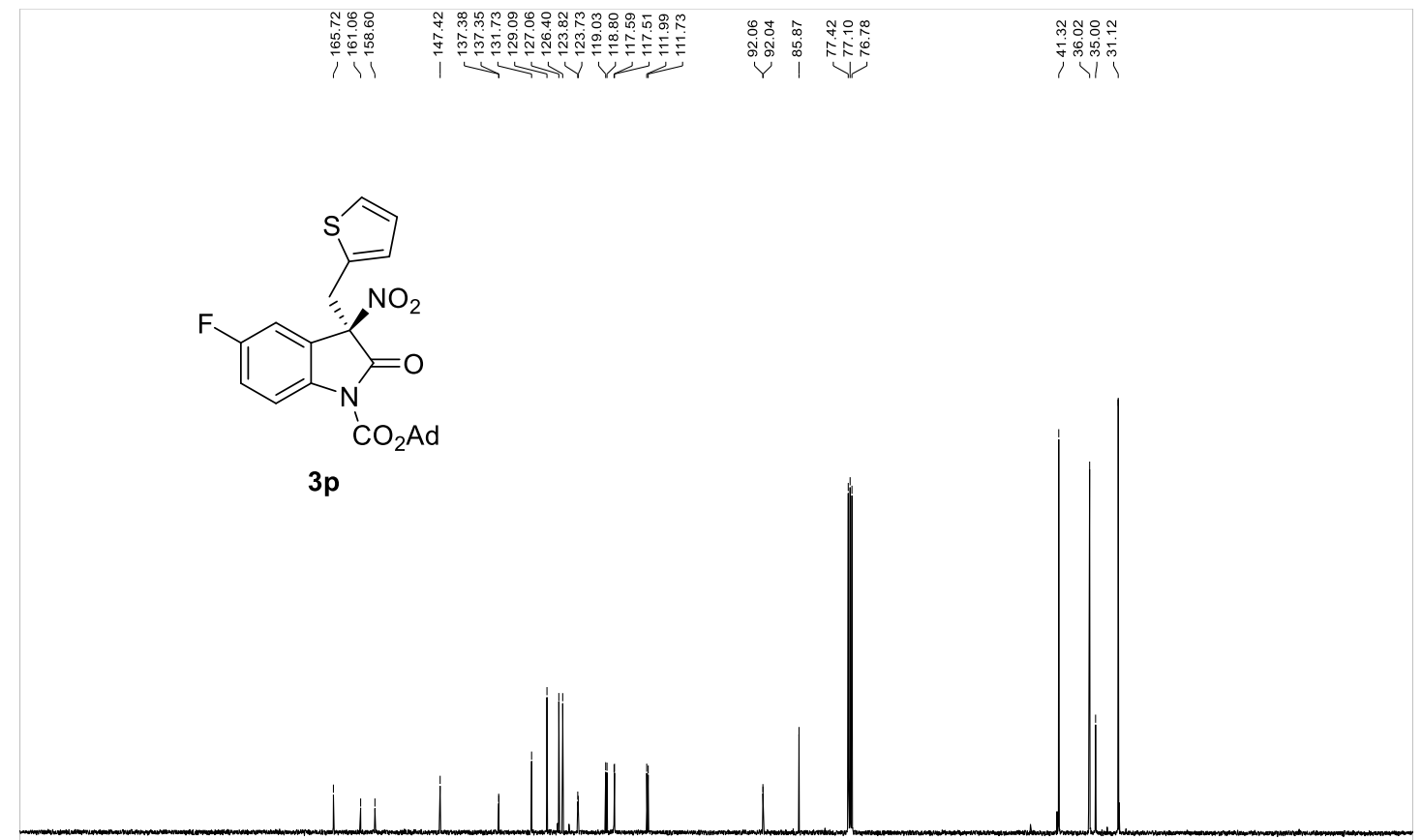

$\begin{array}{rllllllllllllllllllllllll}1 & 1 \\ 210 & 200 & 190 & 180 & 170 & 160 & 150 & 140 & 130 & 120 & 110 & 100 & 90 & 80 & 70 & 60 & 50 & 40 & 30 & 20 & 10 & 0 & -10\end{array}$ 
3q ${ }^{1} \mathrm{H}$ NMR $\left(400 \mathrm{M} \mathrm{Hz}, \mathrm{CDCl}_{3}\right)$

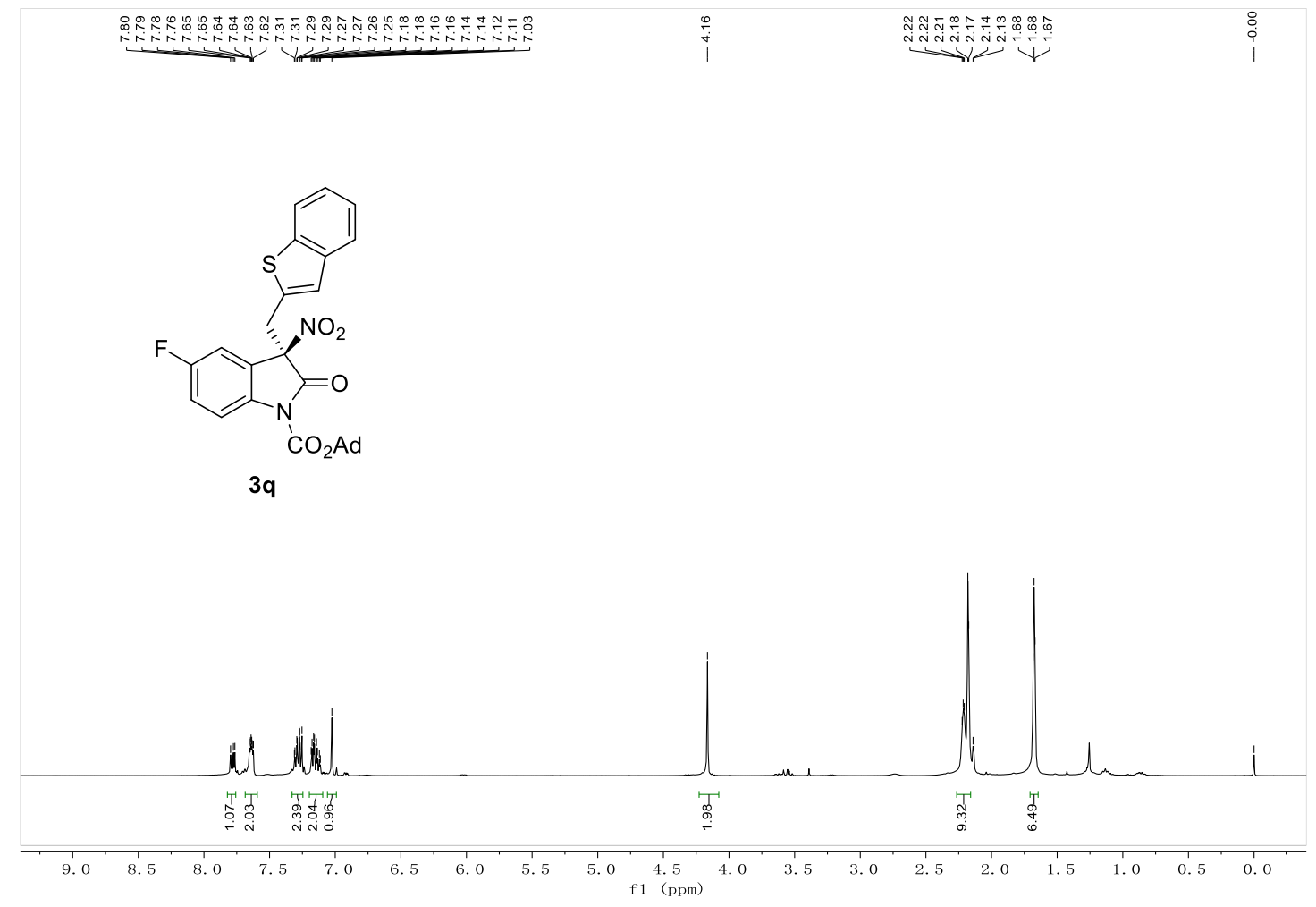

$3 \mathbf{q}^{13} \mathrm{C}$ NMR $\left(100 \mathrm{M} \mathrm{Hz}, \mathrm{CDCl}_{3}\right)$

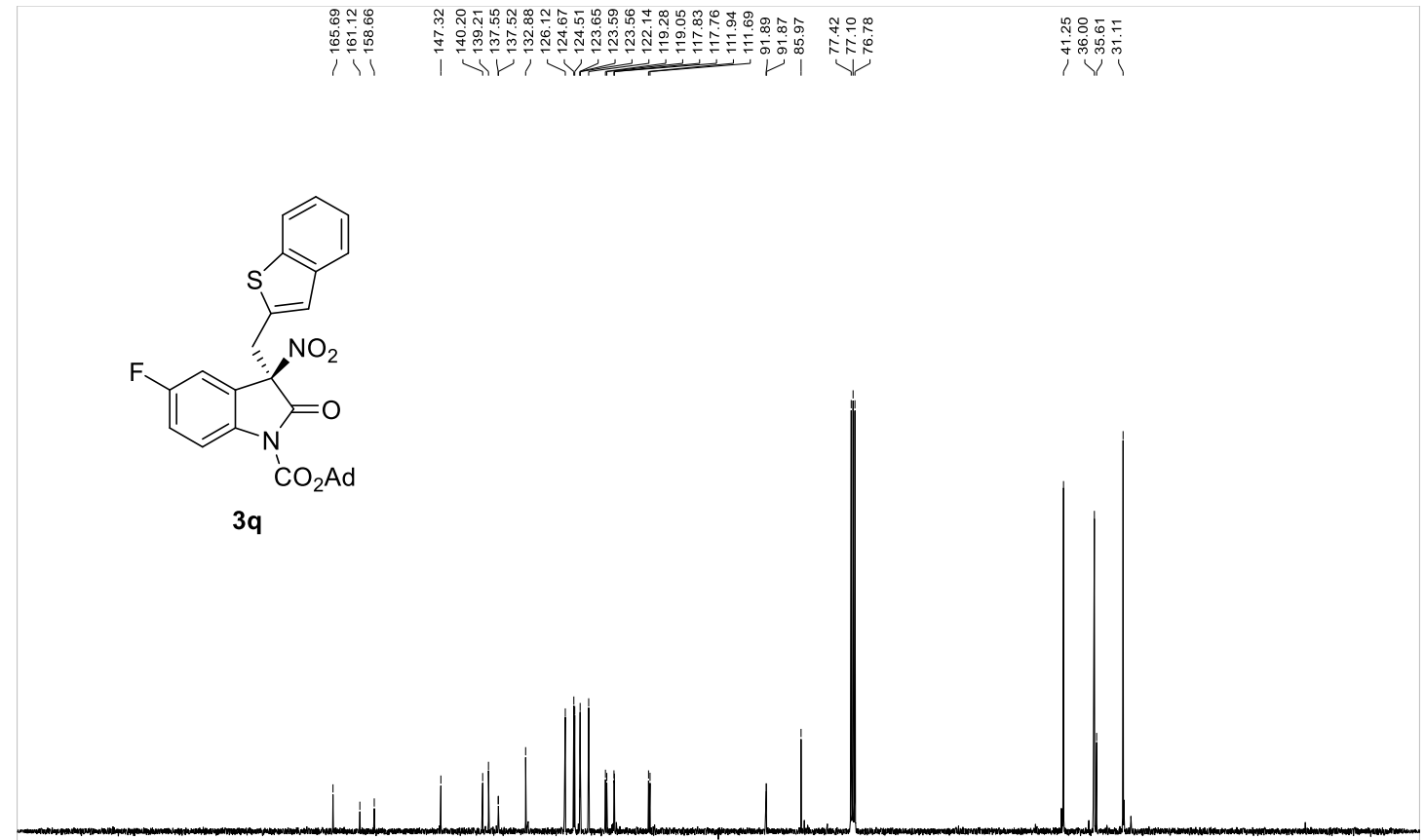

$\begin{array}{rllllllllllllllllllllllll}1 & 10 & 200 & 190 & 180 & 170 & 160 & 150 & 140 & 130 & 120 & 110 & 100 & 90 & 80 & 70 & 60 & 50 & 40 & 30 & 20 & 10 & 0 & -10 & 10\end{array}$ 
3r ${ }^{1} \mathrm{H}$ NMR $\left(400 \mathrm{M} \mathrm{Hz}, \mathrm{CDCl}_{3}\right)$

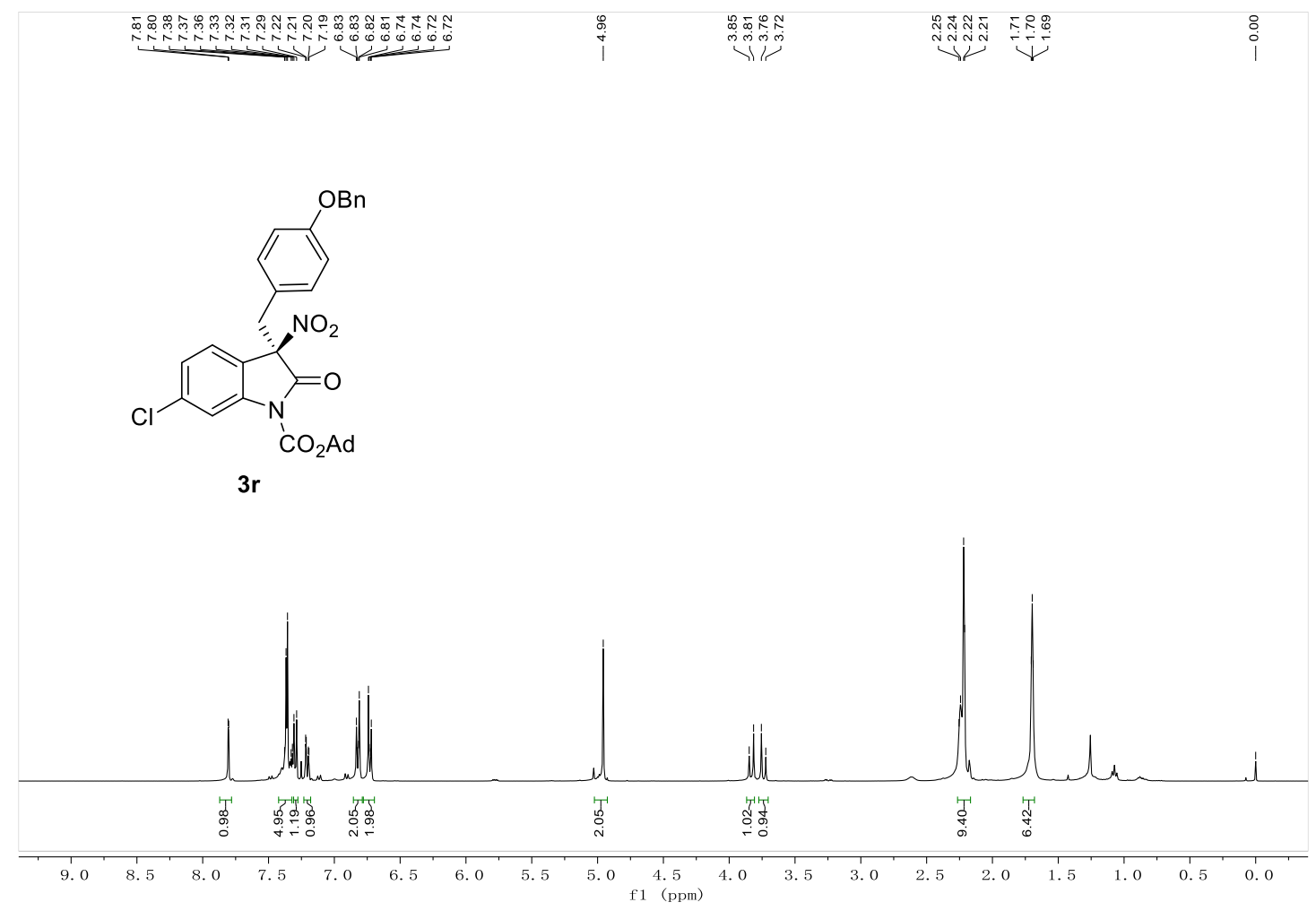

$3{ }^{13} \mathrm{C}$ NMR $\left(100 \mathrm{M} \mathrm{Hz}, \mathrm{CDCl}_{3}\right)$

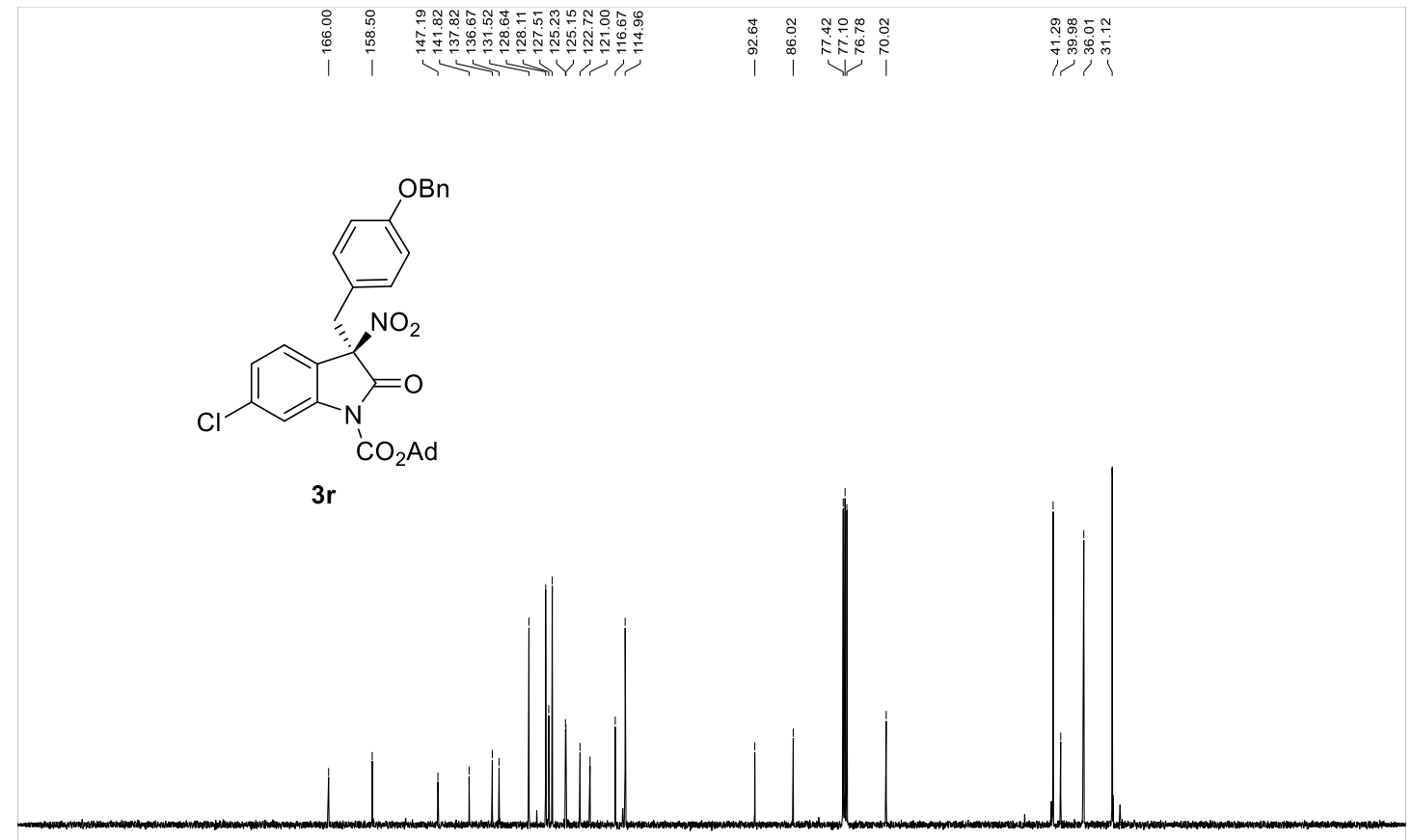

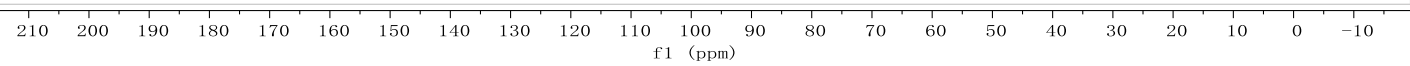


3s ${ }^{1} \mathrm{H}$ NMR $\left(400 \mathrm{M} \mathrm{Hz}, \mathrm{CDCl}_{3}\right)$

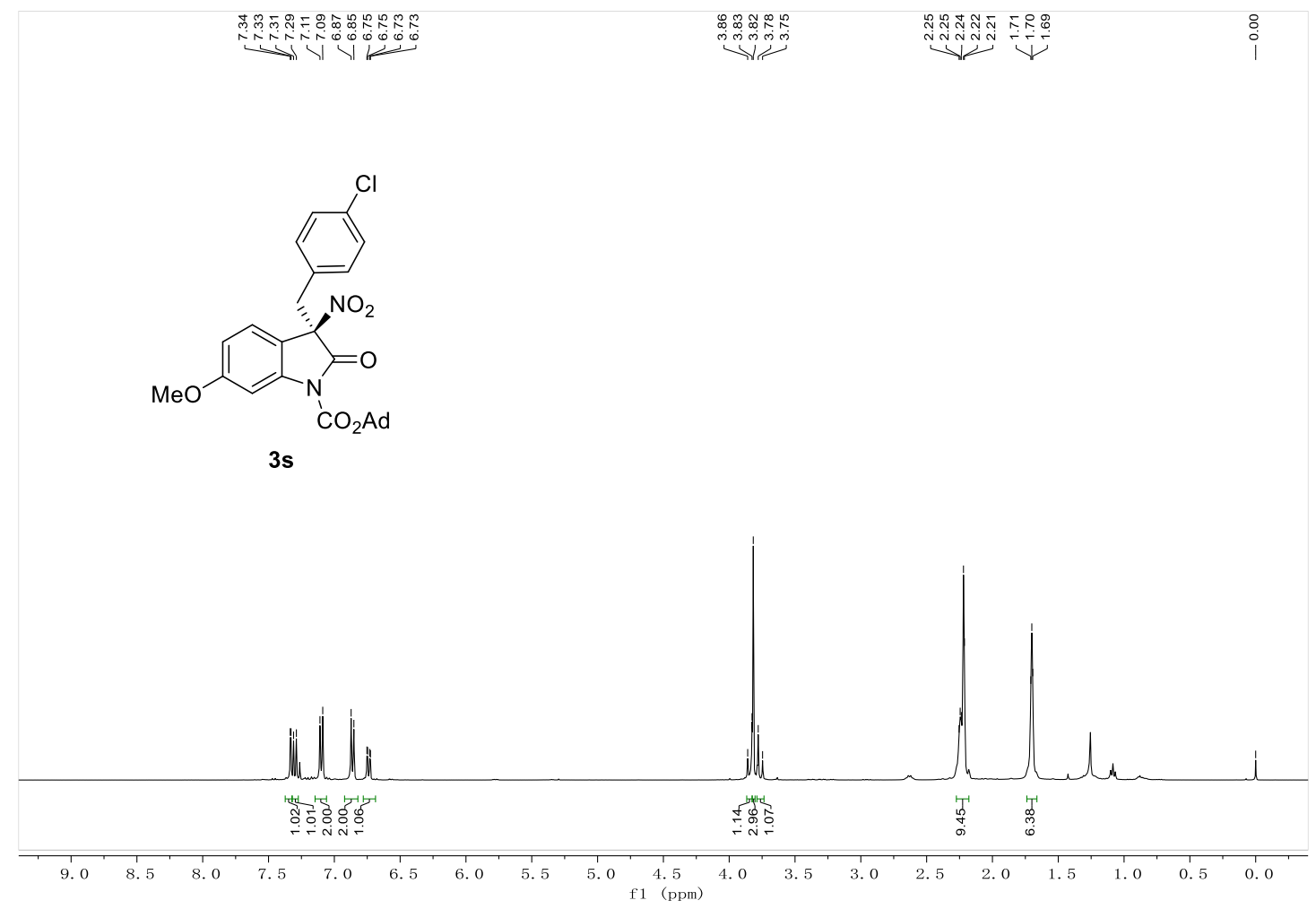

3s ${ }^{13} \mathrm{C} \mathrm{NMR}\left(100 \mathrm{M} \mathrm{Hz}, \mathrm{CDCl}_{3}\right)$

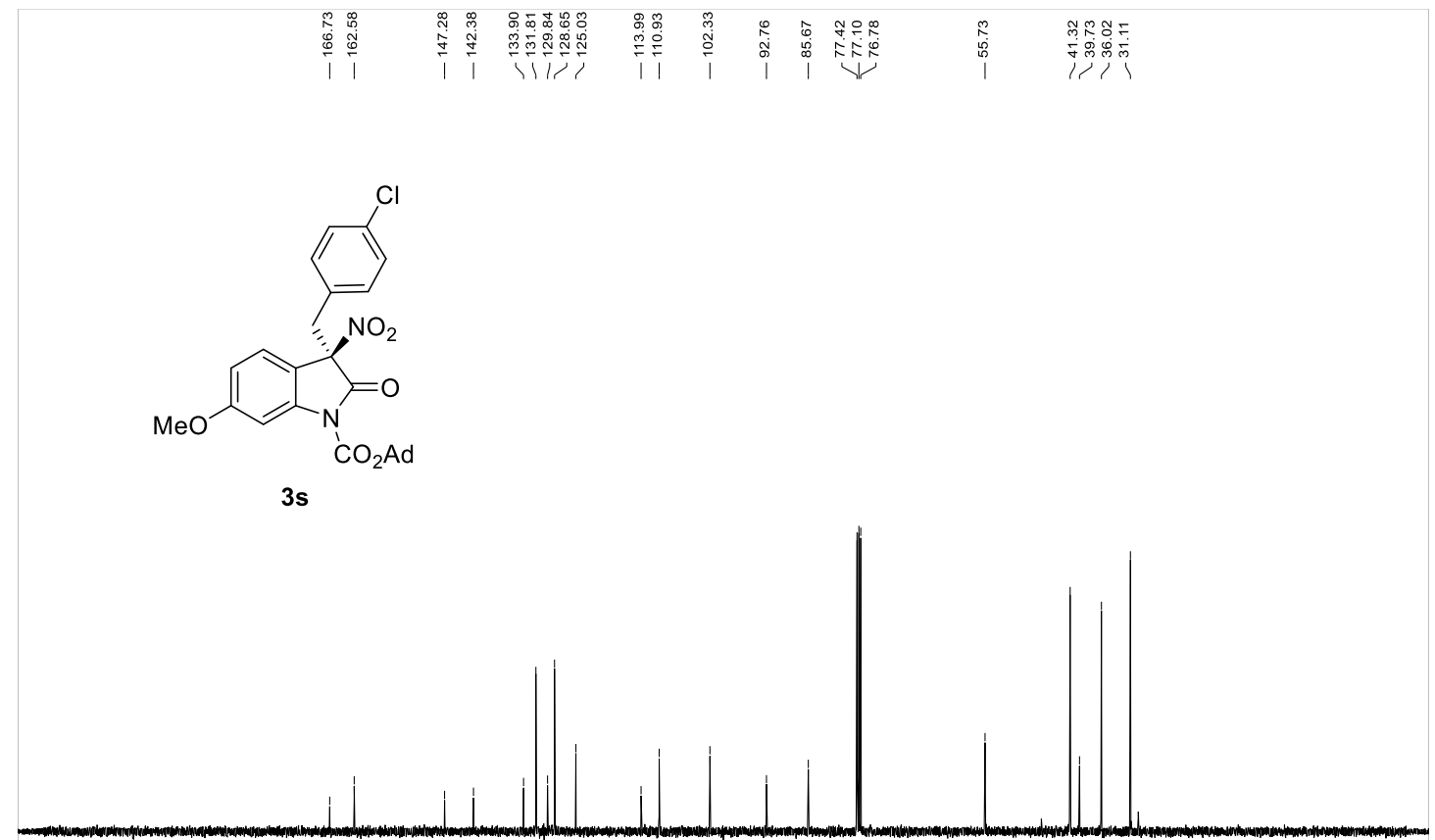

\begin{tabular}{|lllllllllllllllllllllllll}
1 & 10 & 200 & 190 & 180 & 170 & 160 & 150 & 140 & 130 & 120 & 110 & 100 & 90 & 80 & 70 & 60 & 50 & 40 & 30 & 20 & 10 & 0 & -10
\end{tabular} 
3t ${ }^{1} \mathrm{H}$ NMR (400M Hz, $\left.\mathrm{CDCl}_{3}\right)$

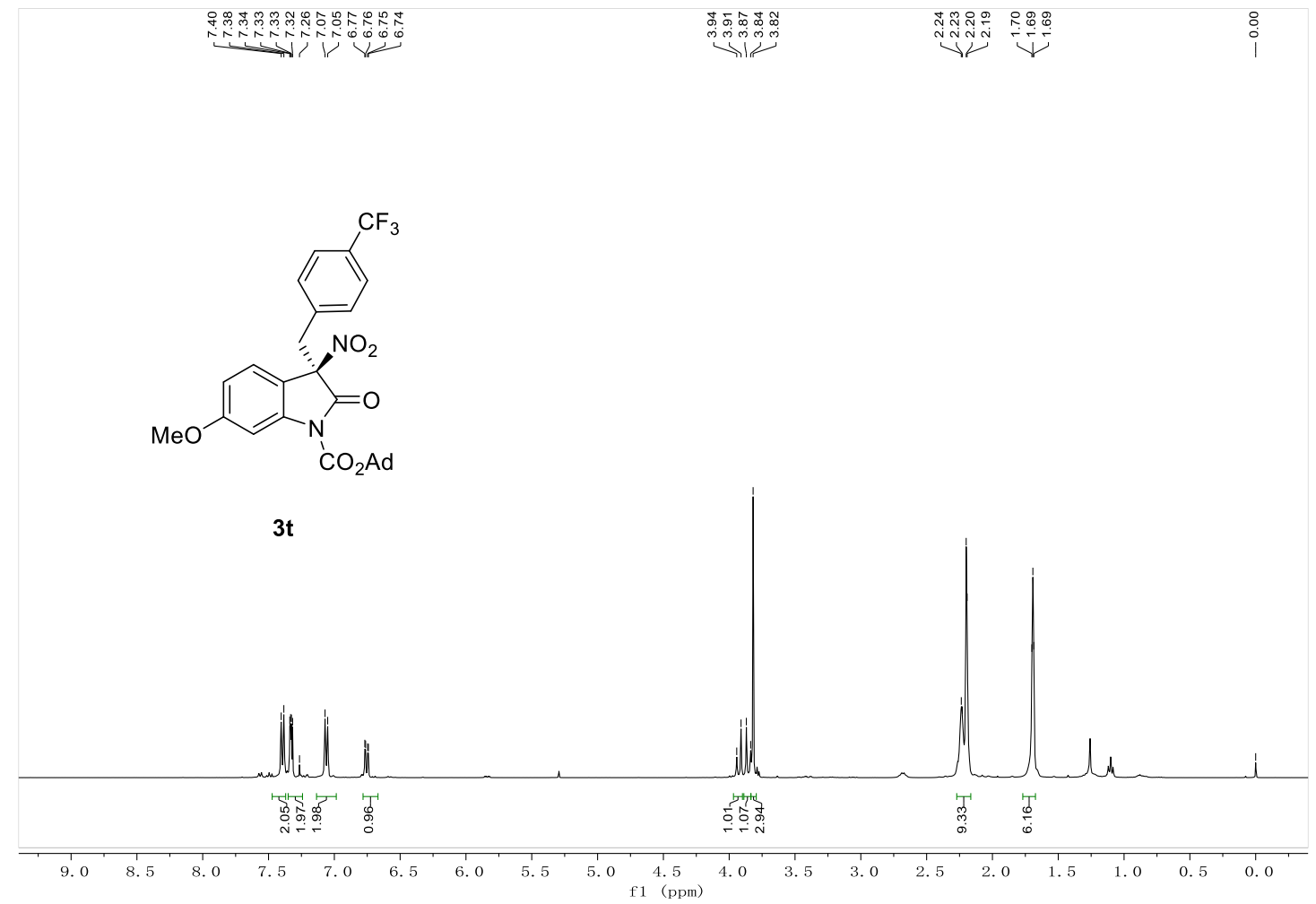

$3 \mathrm{t}^{13} \mathrm{C}$ NMR $\left(100 \mathrm{M} \mathrm{Hz}, \mathrm{CDCl}_{3}\right)$

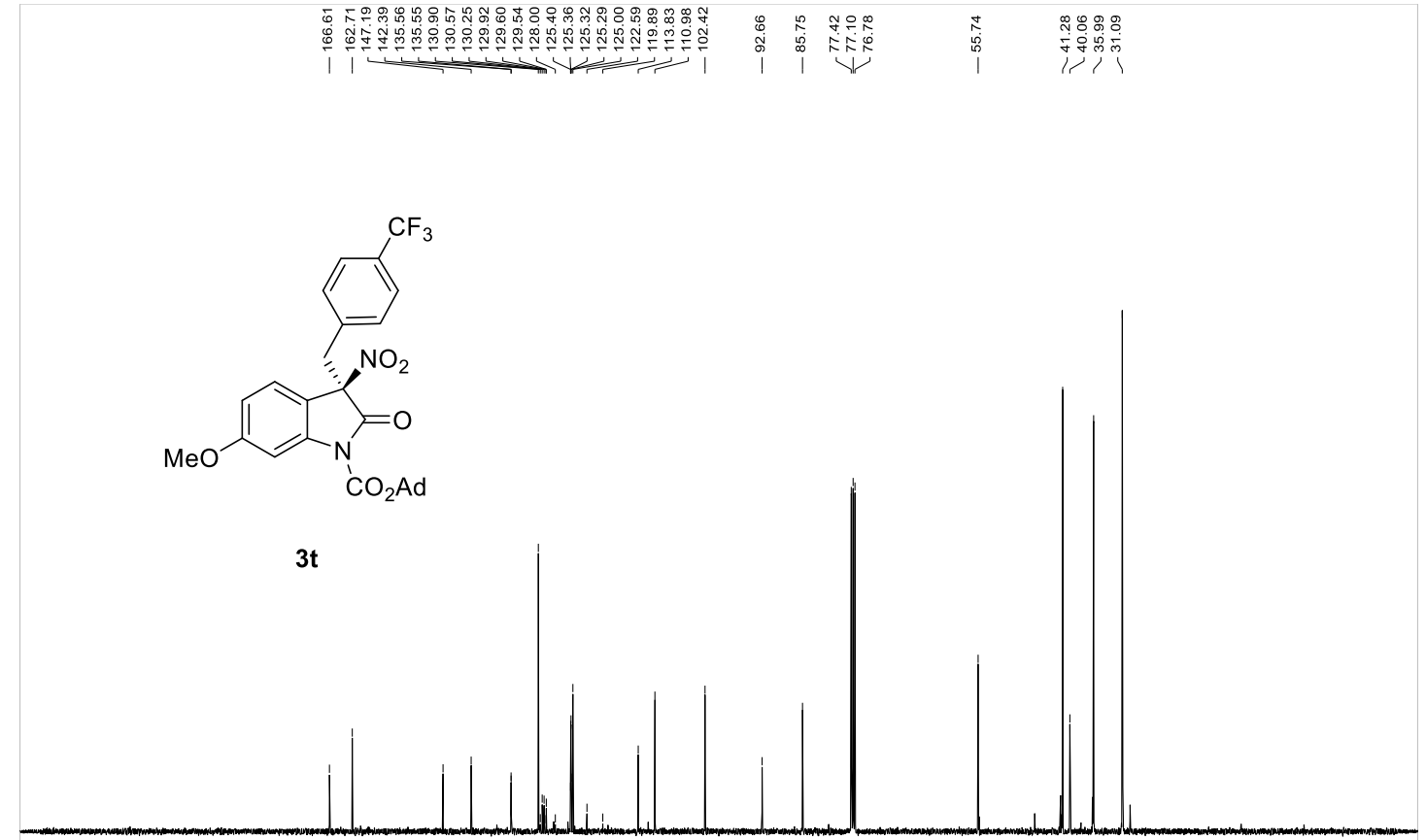

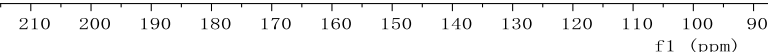


3u ${ }^{1} \mathrm{H}$ NMR (400M Hz, $\left.\mathrm{CDCl}_{3}\right)$

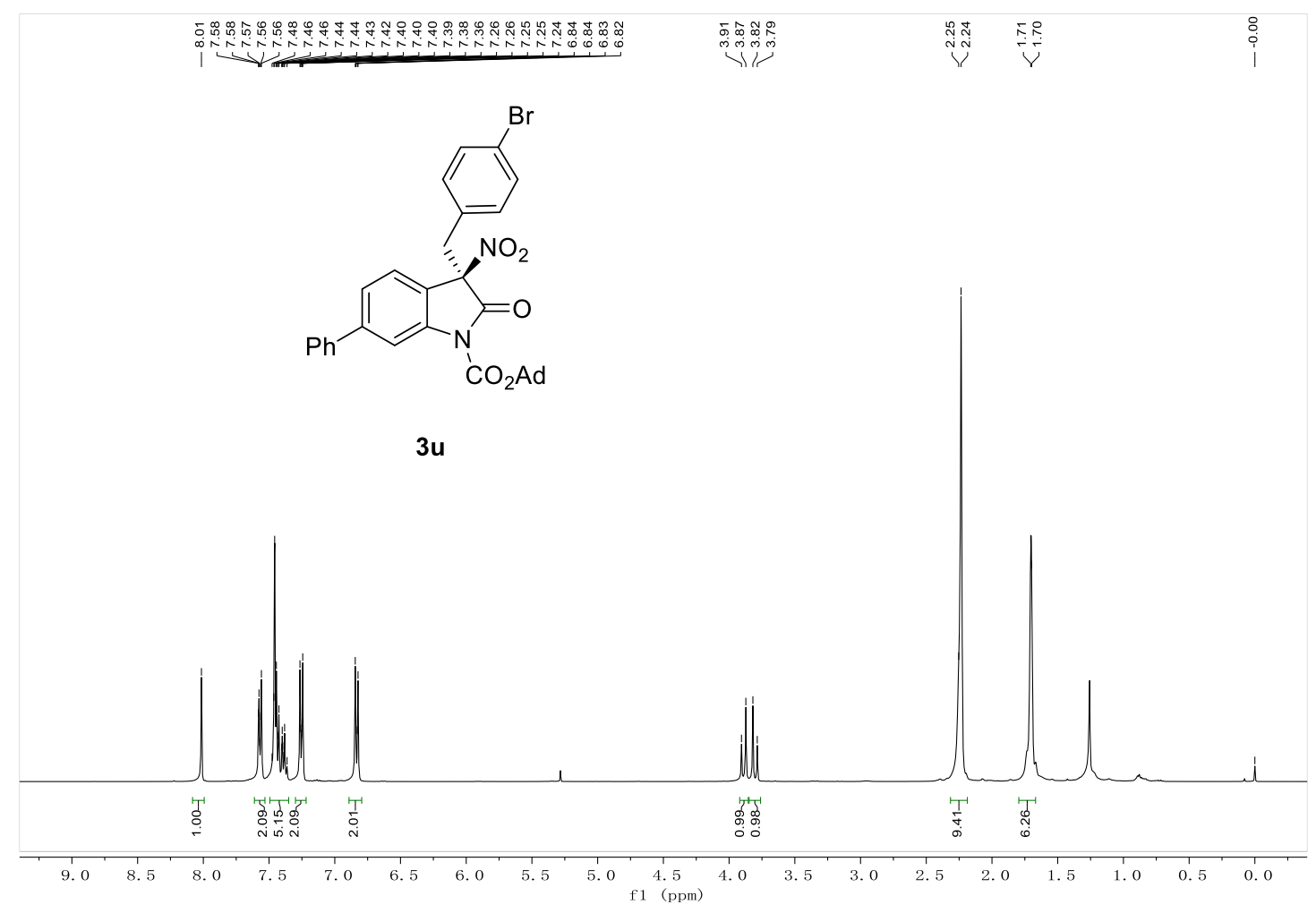

3u ${ }^{13} \mathrm{C} \mathrm{NMR}\left(100 \mathrm{M} \mathrm{Hz}, \mathrm{CDCl}_{3}\right)$

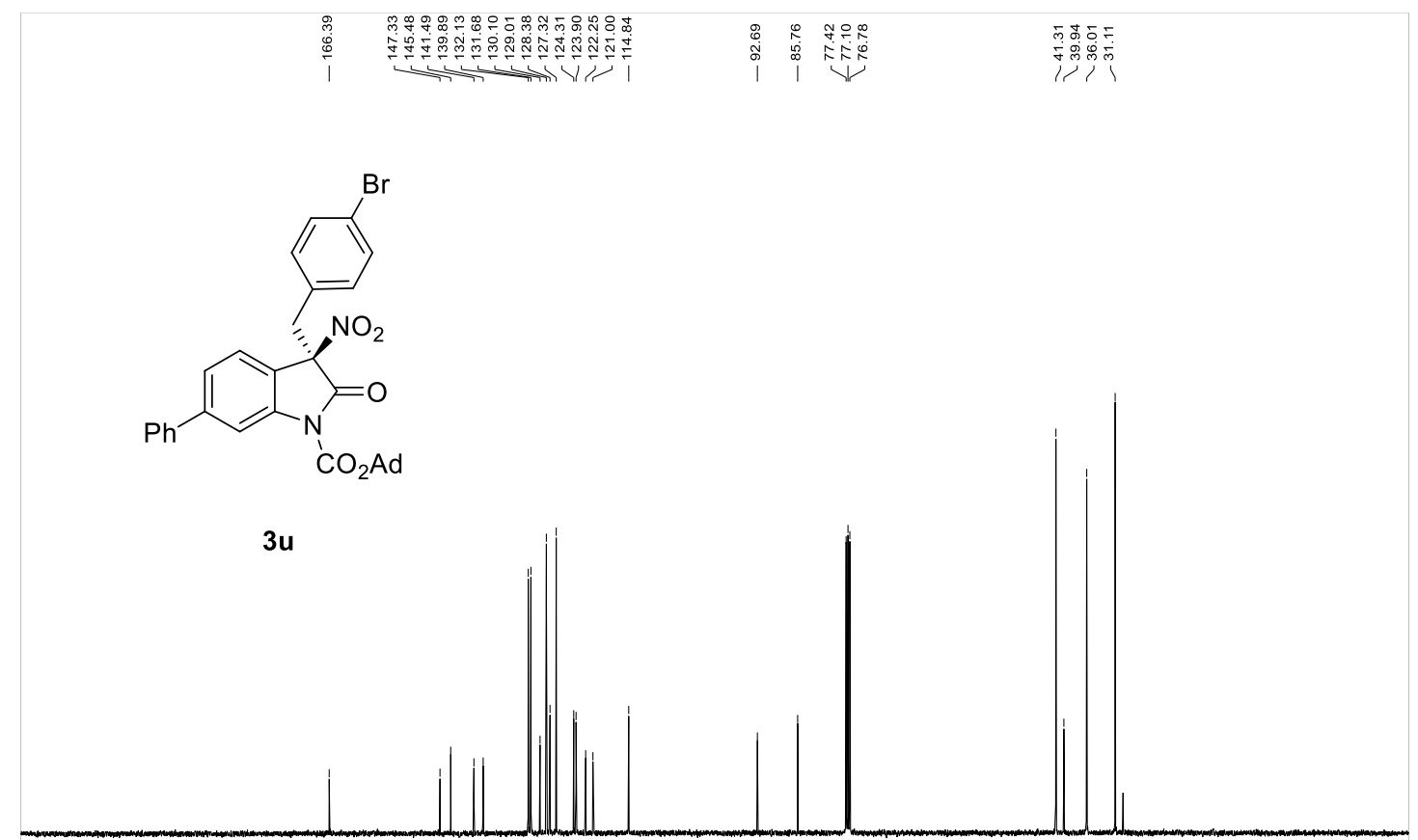

$\begin{array}{rllllllllllllllllllllllll}1 & 10 & 200 & 190 & 180 & 170 & 160 & 150 & 140 & 130 & 120 & 110 & 100 & 90 & 80 & 70 & 60 & 50 & 40 & 30 & 20 & 10 & 0 & -10\end{array}$ 
3v ${ }^{1} \mathrm{H}$ NMR $\left(400 \mathrm{M} \mathrm{Hz}, \mathrm{CDCl}_{3}\right)$

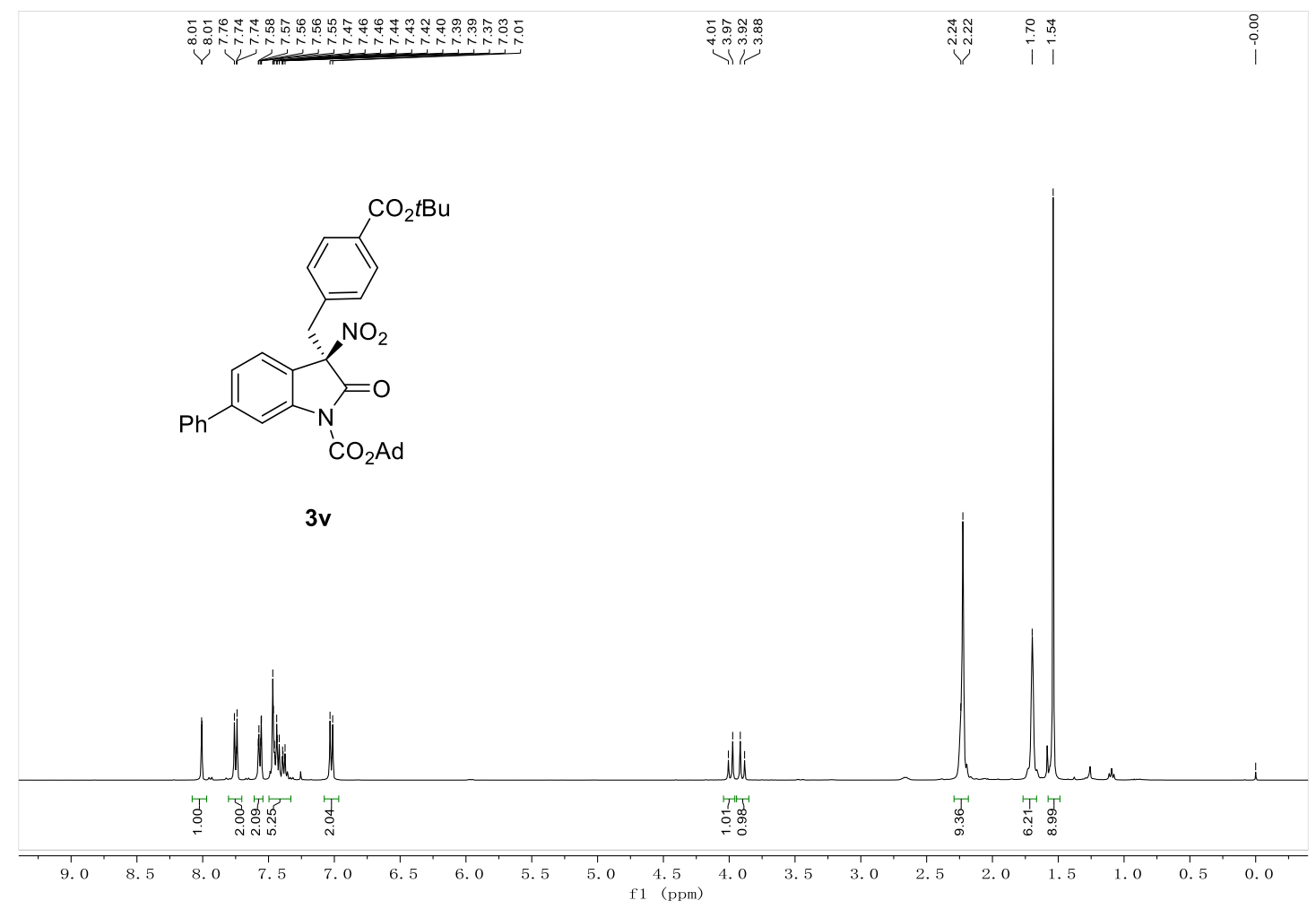

3v ${ }^{13} \mathrm{C}$ NMR $\left(100 \mathrm{M} \mathrm{Hz}, \mathrm{CDCl}_{3}\right)$

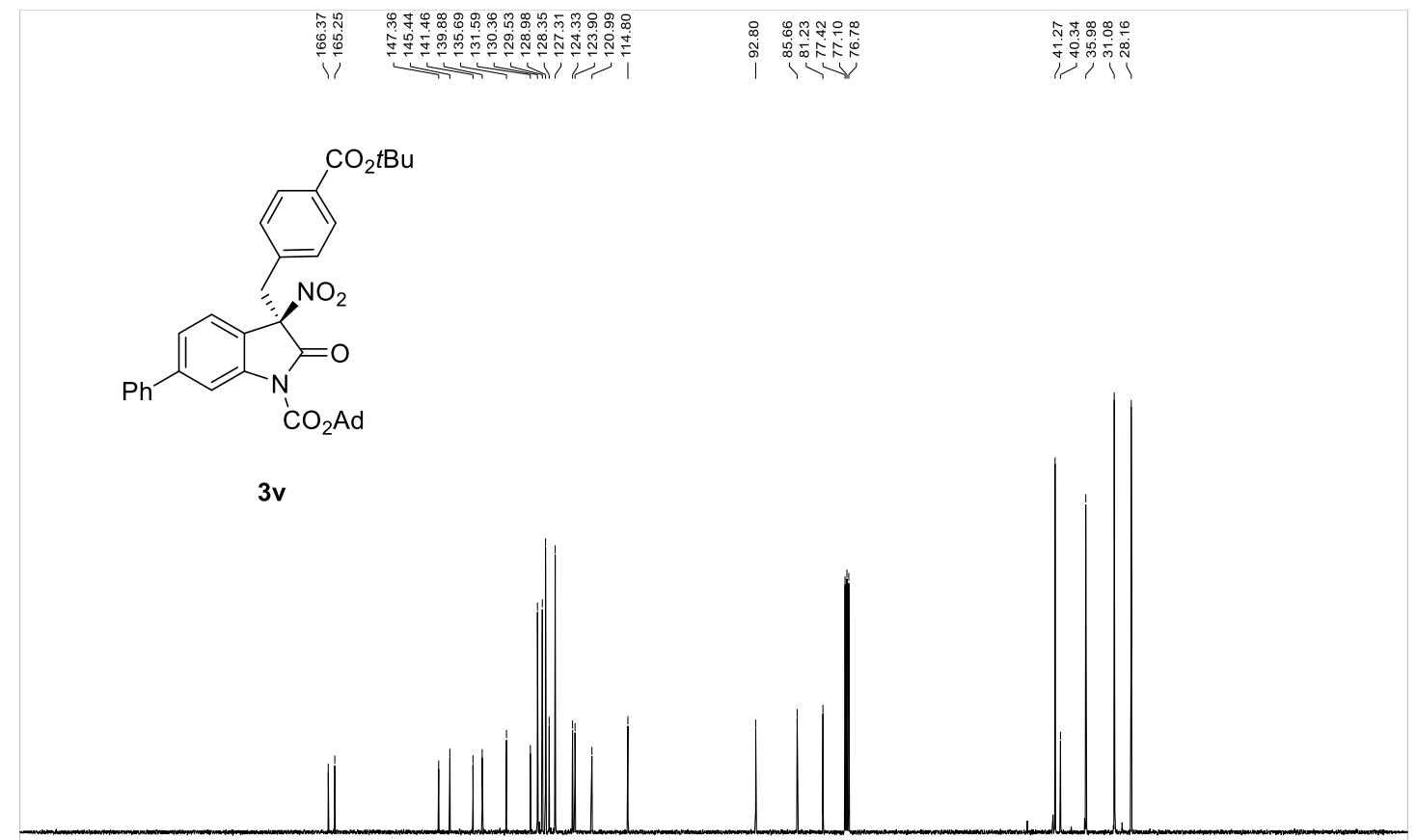

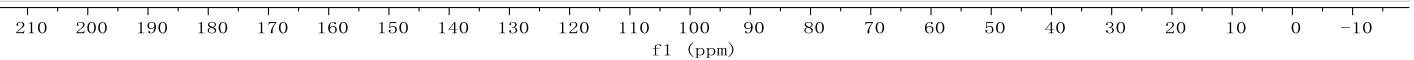


3w ${ }^{1} \mathrm{H}$ NMR $\left(400 \mathrm{M} \mathrm{Hz}, \mathrm{CDCl}_{3}\right)$

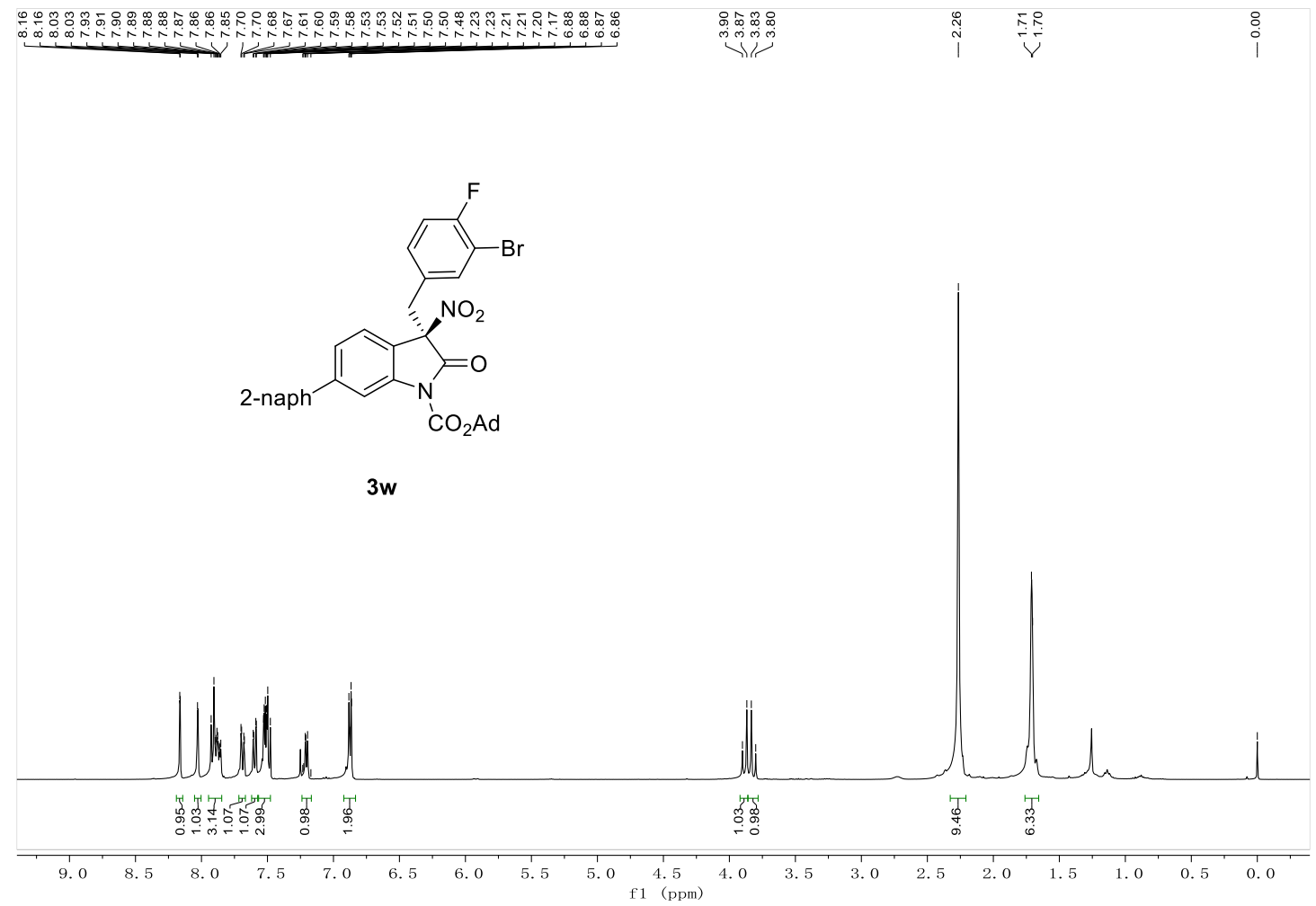

3w ${ }^{13} \mathrm{C}$ NMR $\left(100 \mathrm{M} \mathrm{Hz}, \mathrm{CDCl}_{3}\right)$

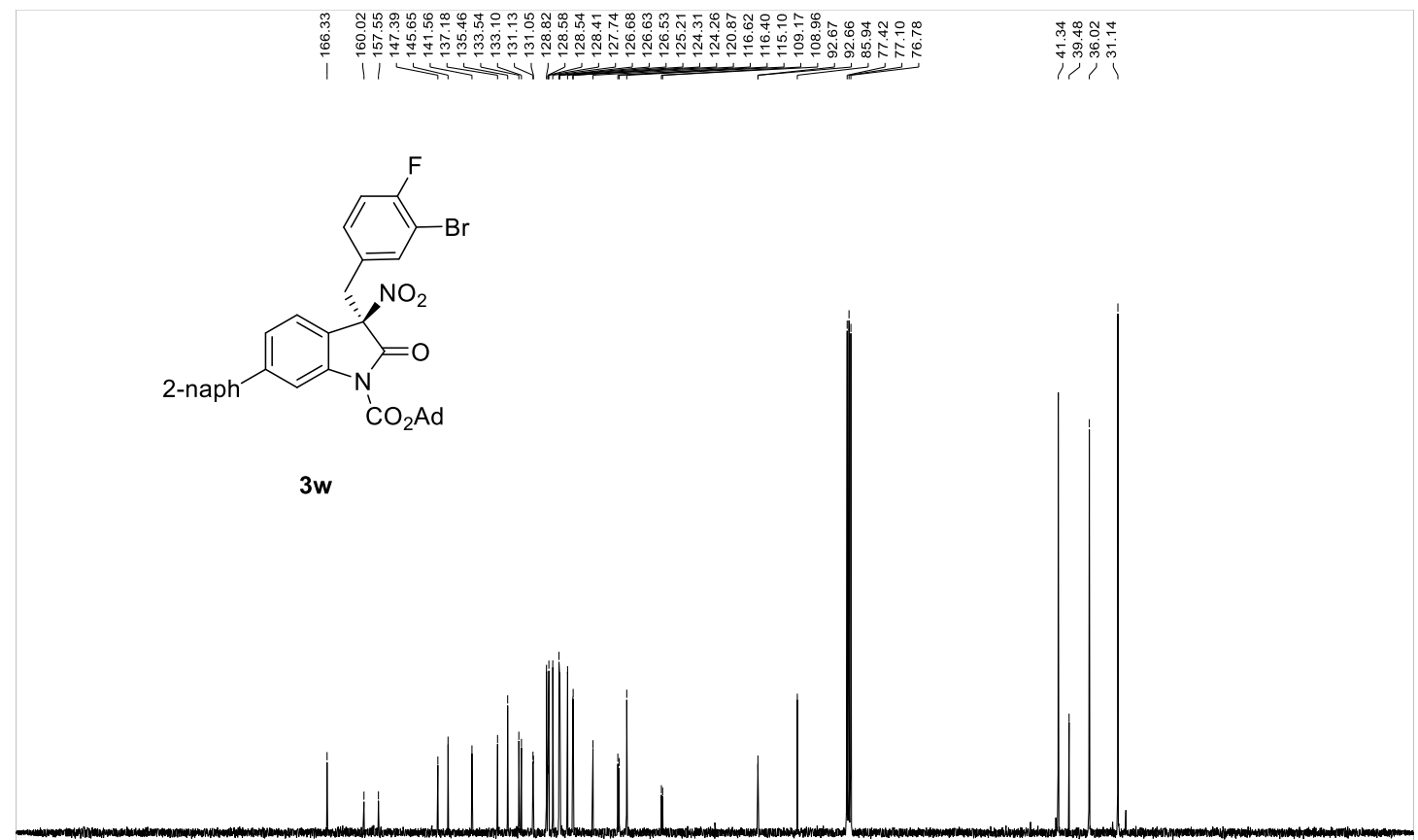

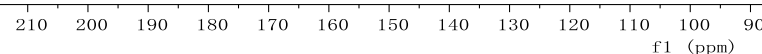


3x ${ }^{1} \mathrm{H}$ NMR (400M Hz, $\left.\mathrm{CDCl}_{3}\right)$

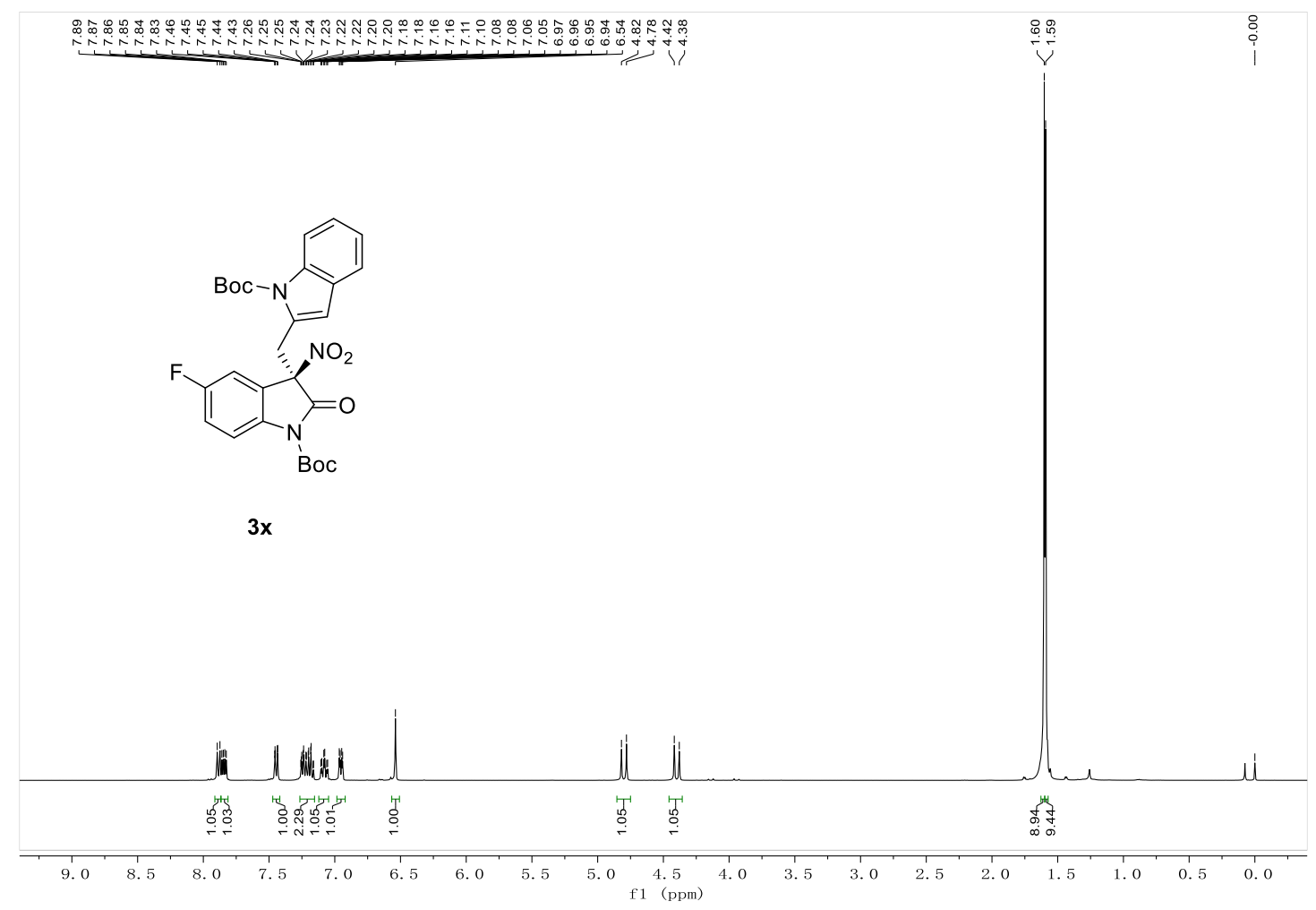

3x ${ }^{13} \mathrm{C}$ NMR $\left(100 \mathrm{M} \mathrm{Hz}, \mathrm{CDCl}_{3}\right)$

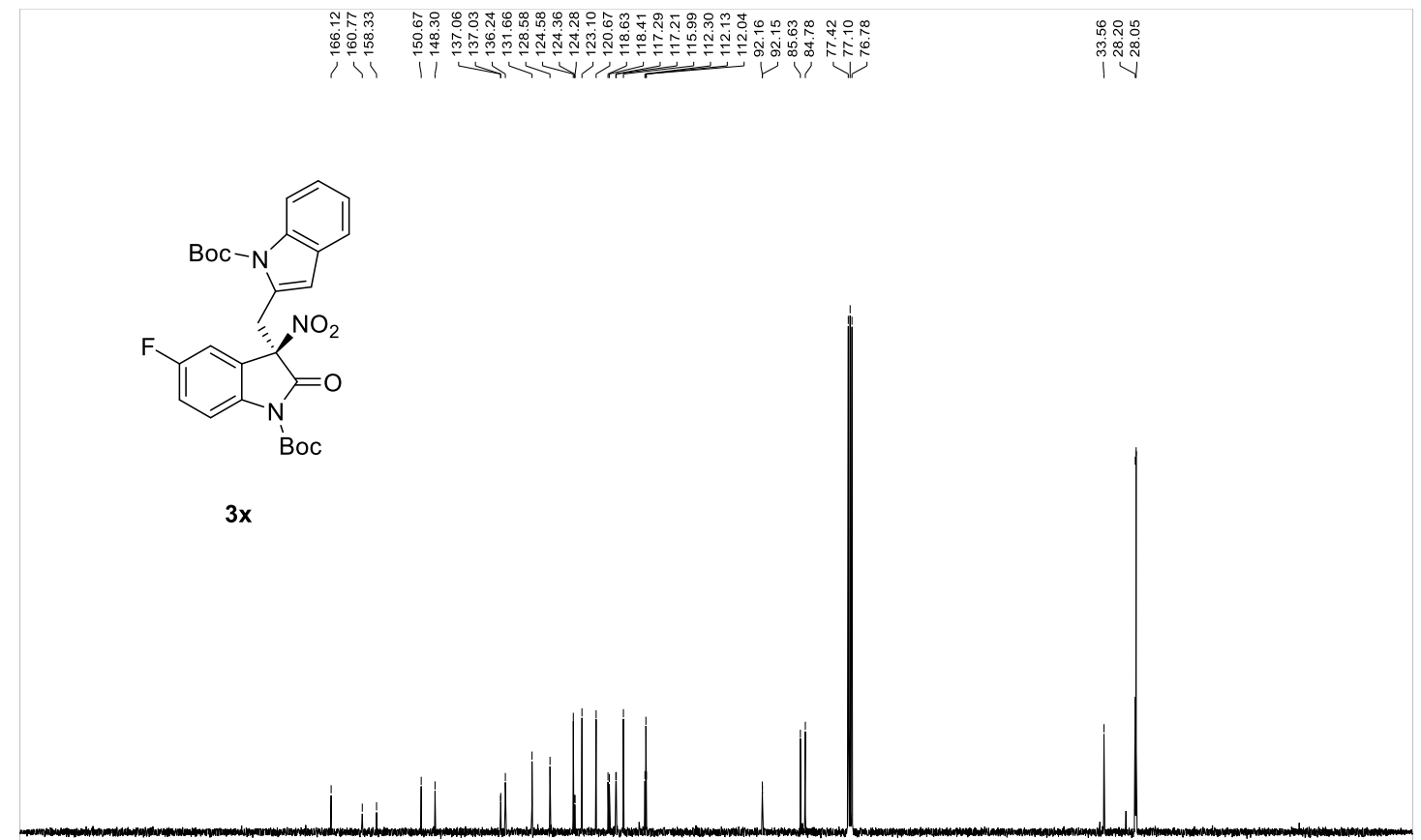

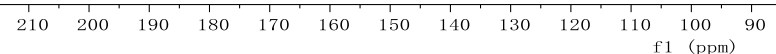


3y ${ }^{1} \mathrm{H}$ NMR (400M Hz, $\left.\mathrm{CDCl}_{3}\right)$

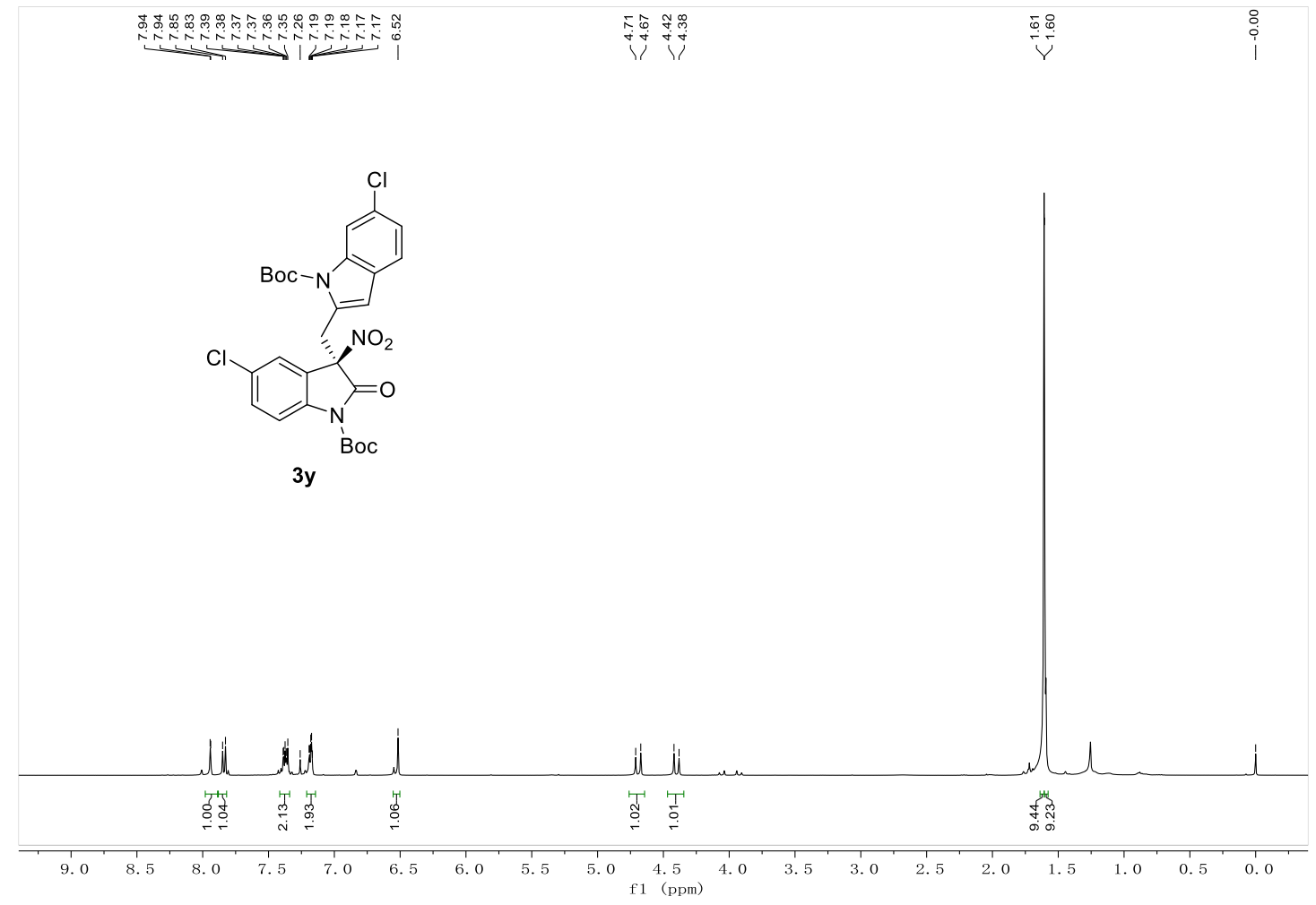

3y ${ }^{13} \mathrm{C}$ NMR $\left(100 \mathrm{M} \mathrm{Hz}, \mathrm{CDCl}_{3}\right)$

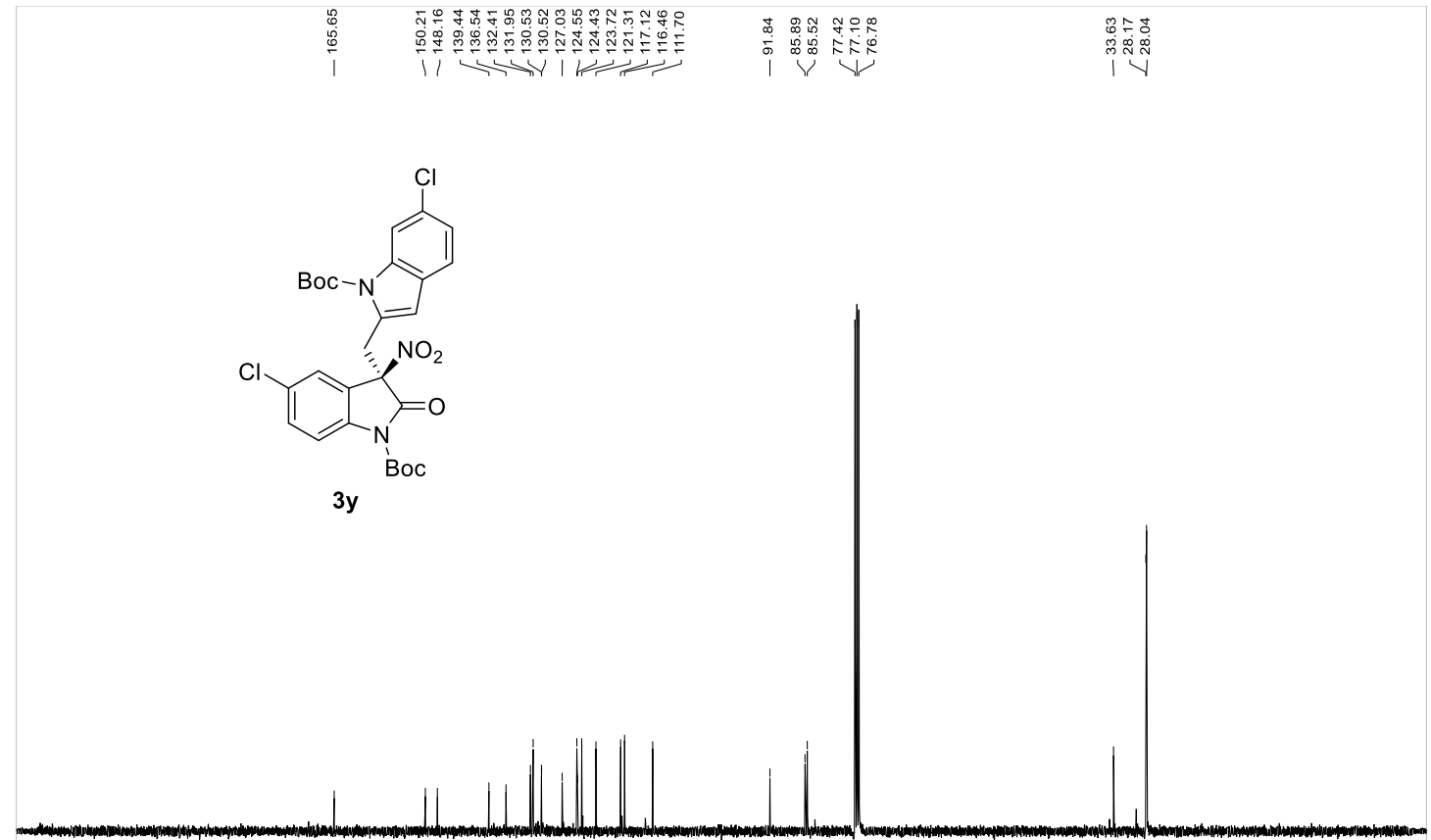

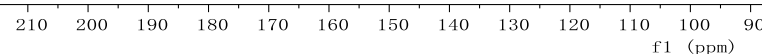


3za ${ }^{1} \mathrm{H}$ NMR $\left(400 \mathrm{M} \mathrm{Hz}, \mathrm{CDCl}_{3}\right)$

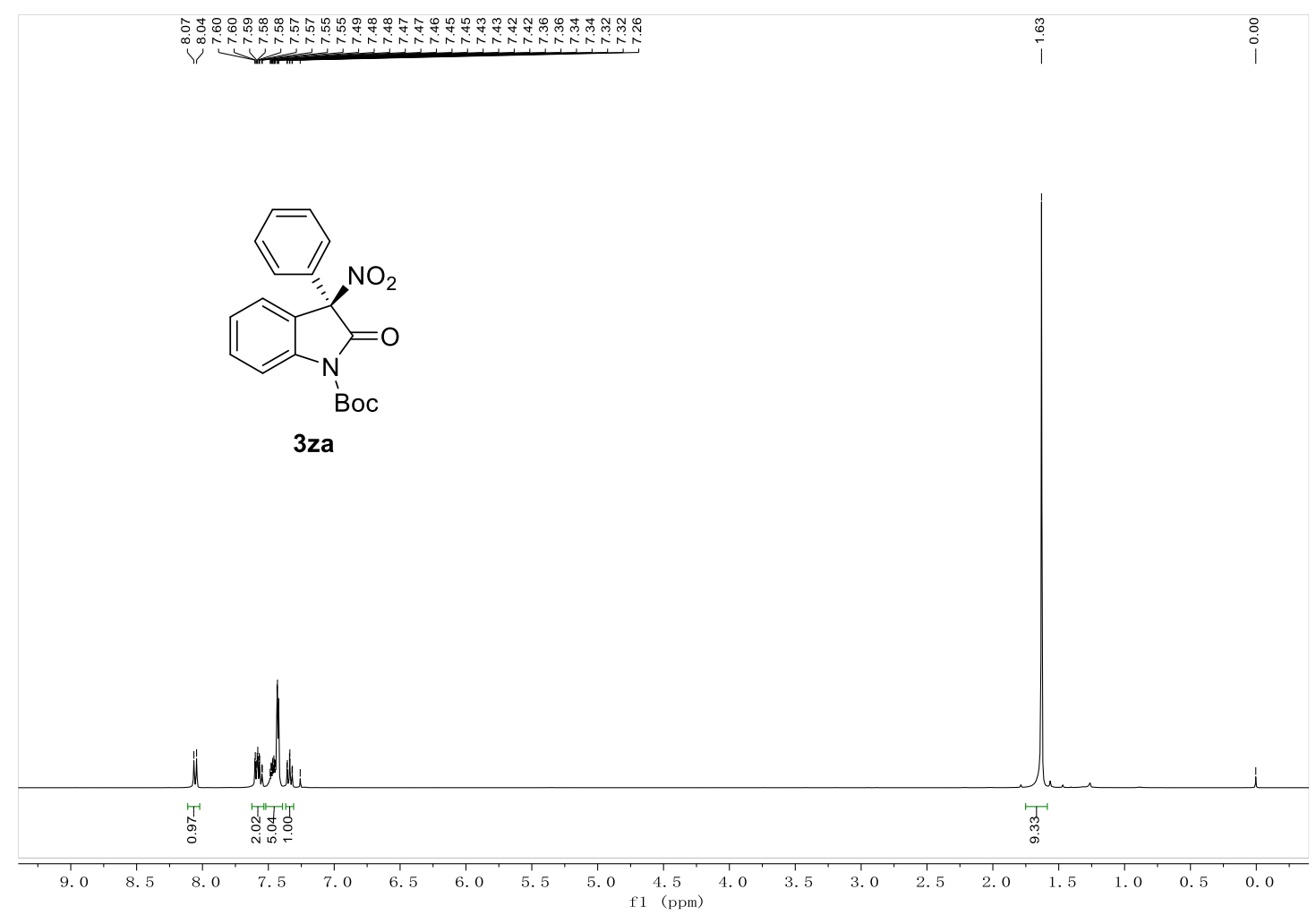

3za ${ }^{13} \mathrm{C} \mathrm{NMR}\left(100 \mathrm{M} \mathrm{Hz}, \mathrm{CDCl}_{3}\right)$

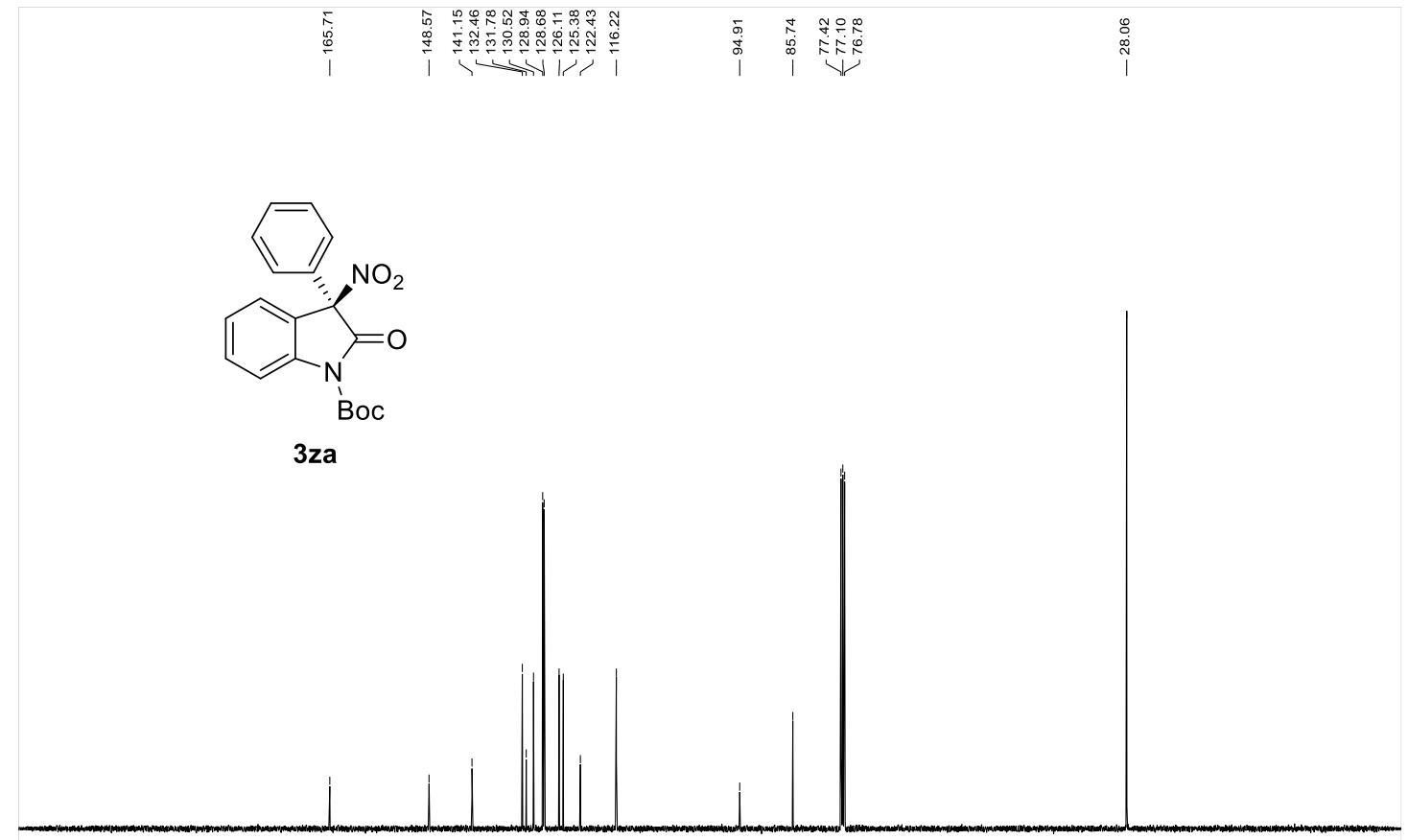

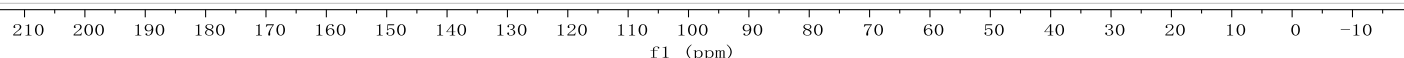


3zb ${ }^{1} \mathrm{H}$ NMR (400M Hz, $\mathrm{CDCl}_{3}$ )

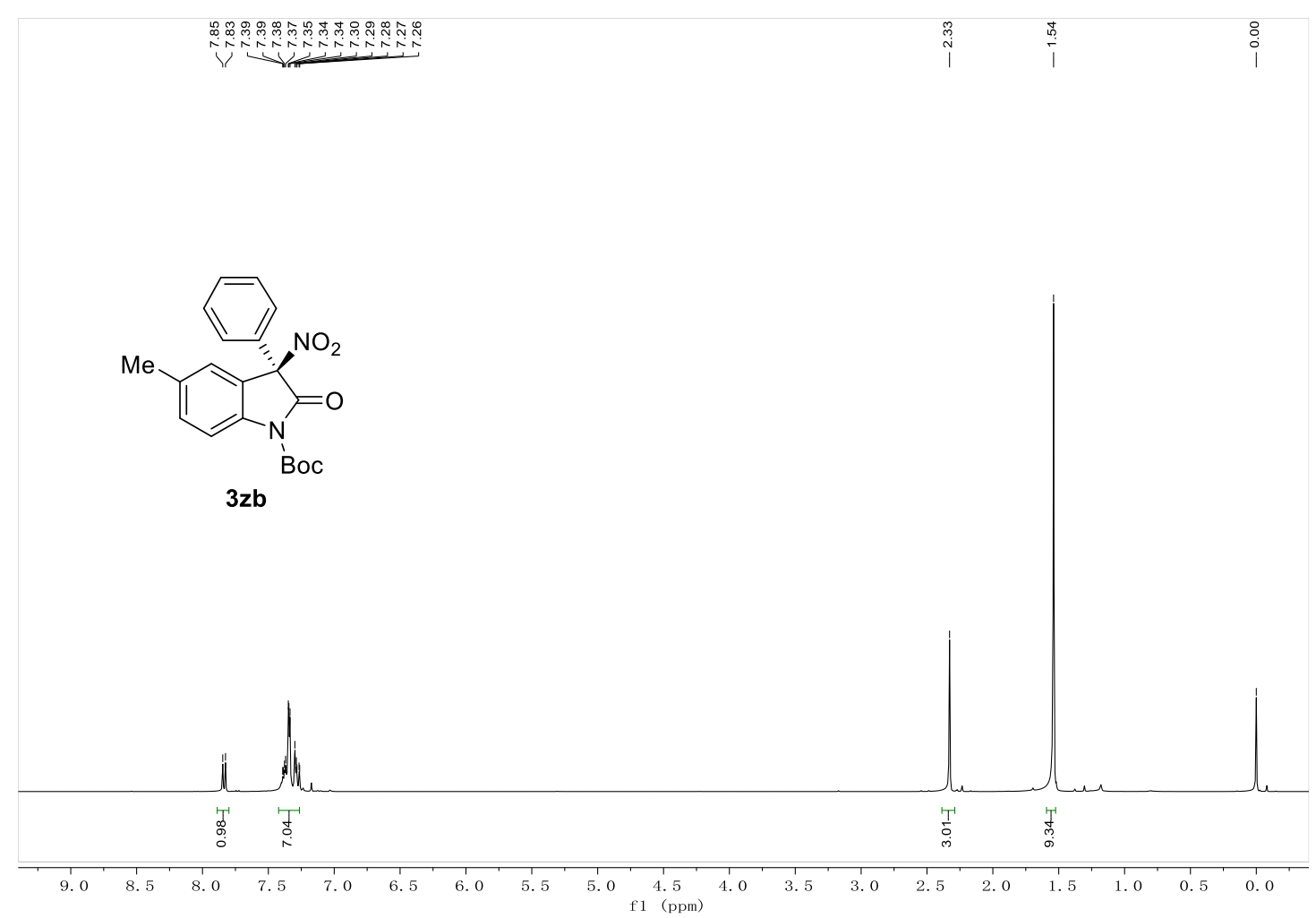

3zb ${ }^{13} \mathrm{C}$ NMR $\left(100 \mathrm{M} \mathrm{Hz}, \mathrm{CDCl}_{3}\right)$

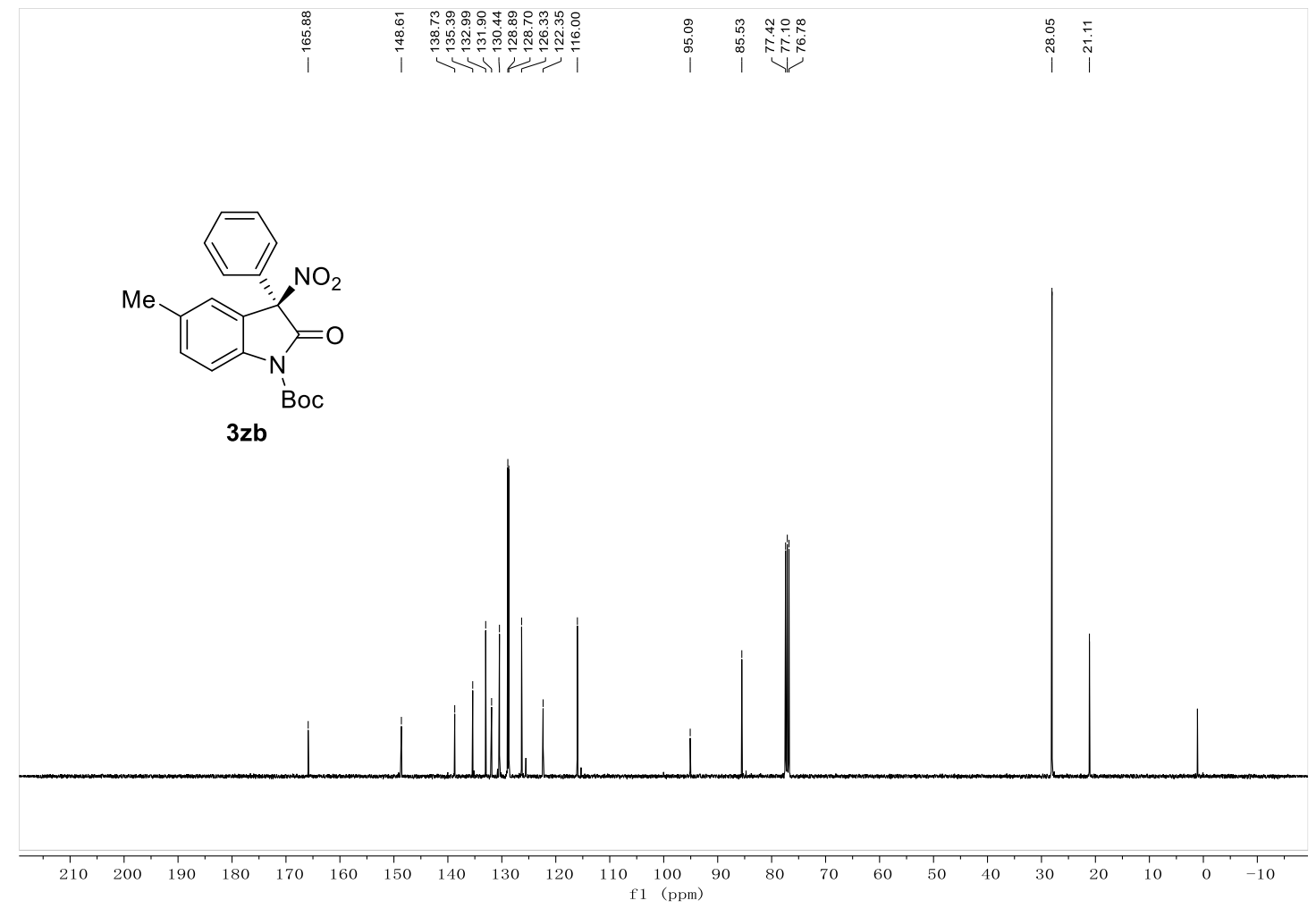


3zc ${ }^{1} \mathrm{H}$ NMR $\left(400 \mathrm{M} \mathrm{Hz}, \mathrm{CDCl}_{3}\right)$

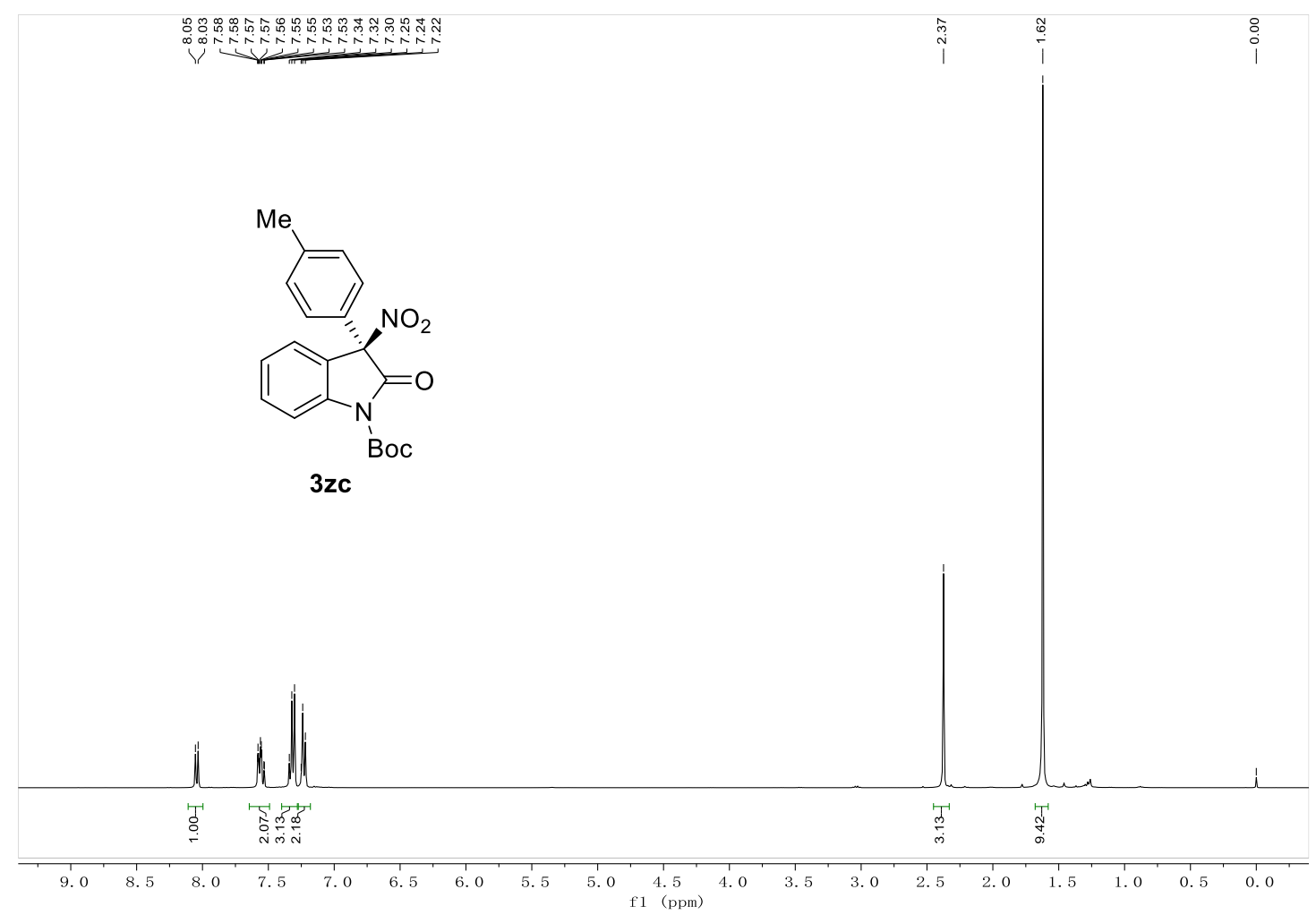

3zc ${ }^{13} \mathrm{C}$ NMR $\left(100 \mathrm{M} \mathrm{Hz}, \mathrm{CDCl}_{3}\right)$

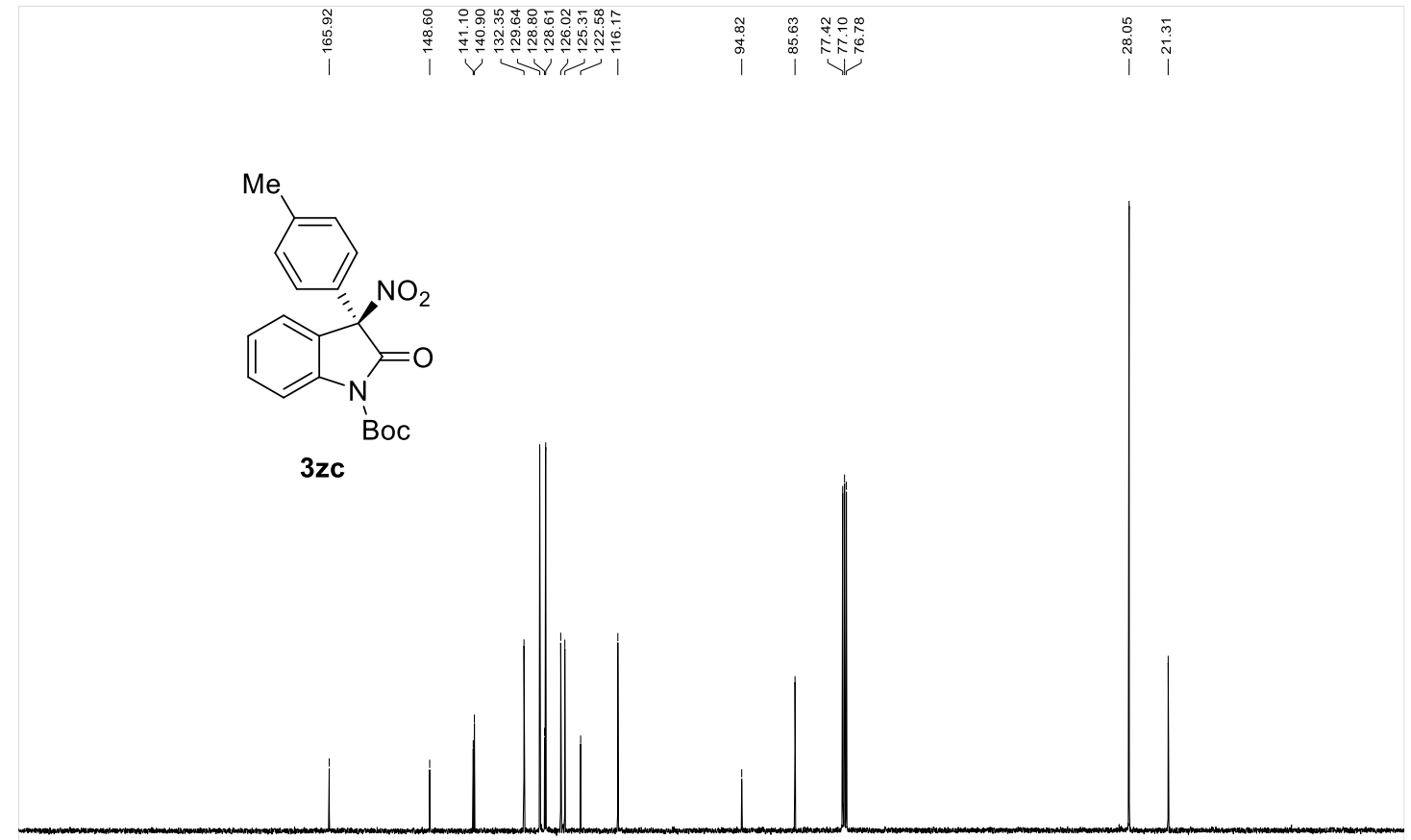

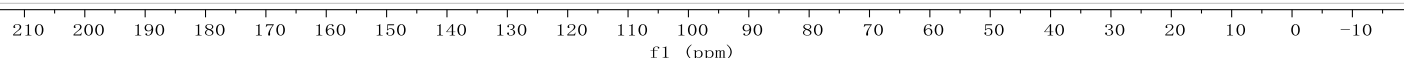


4a ${ }^{1} \mathrm{H}$ NMR (400M Hz, DMSO-d6)

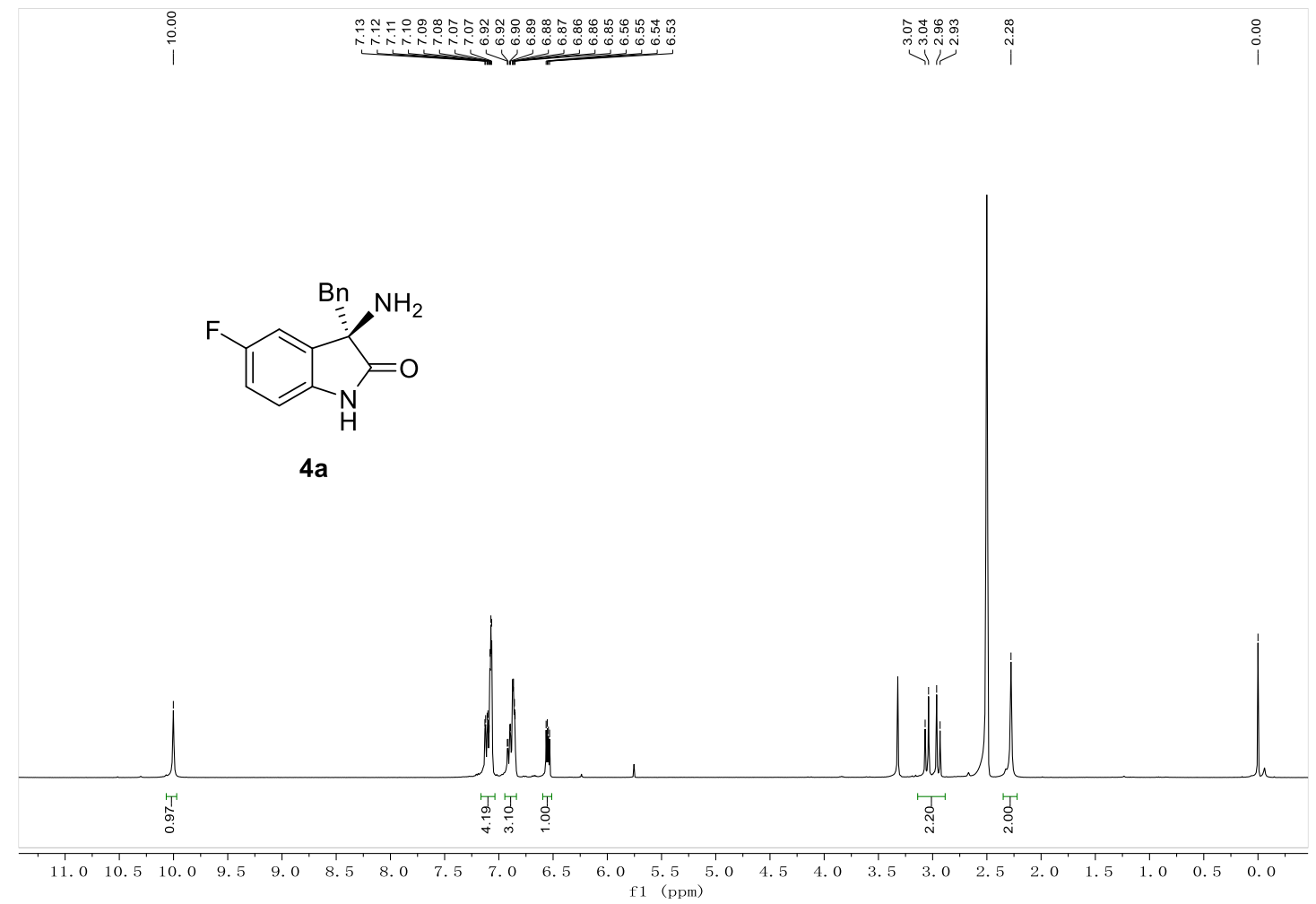

4a ${ }^{13} \mathrm{C}$ NMR (100M Hz, DMSO- $d 6$ )

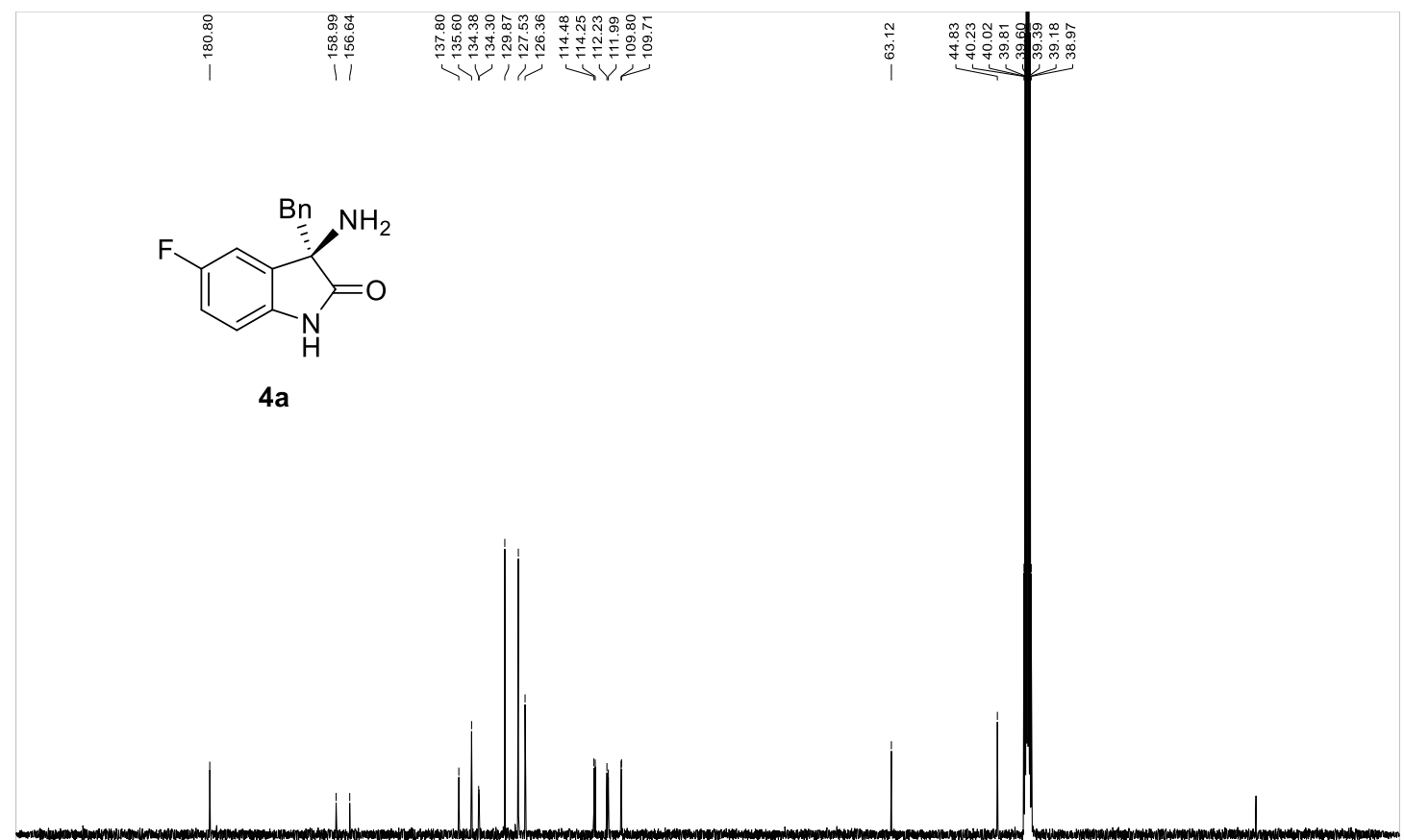

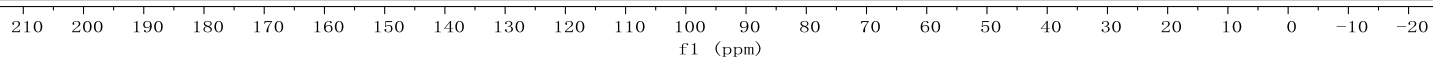


4b ${ }^{1} \mathrm{H}$ NMR (400M Hz, DMSO- $d 6$ )

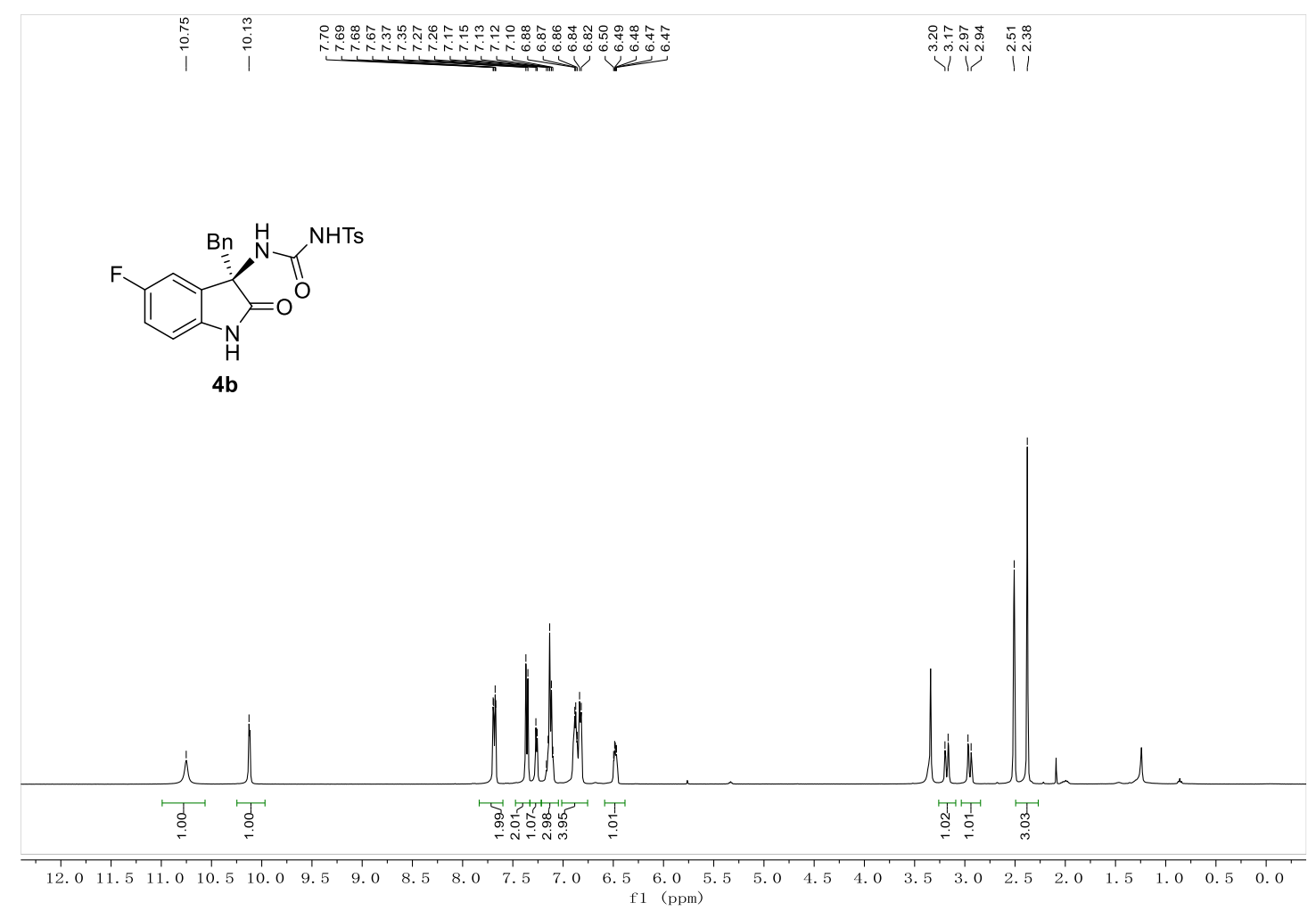

4b ${ }^{13} \mathrm{C}$ NMR (100M Hz, DMSO- $d 6$ )

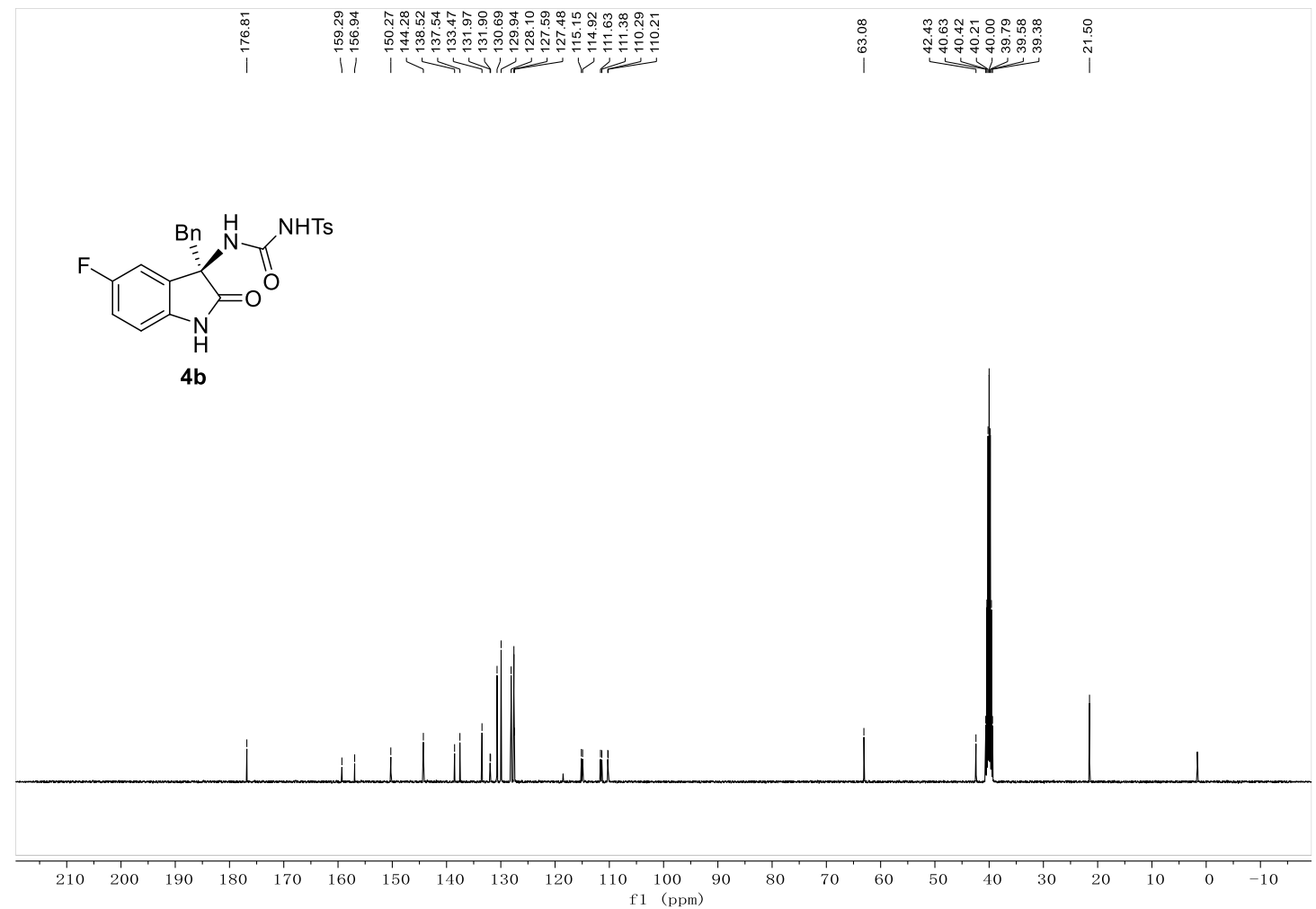


4c ${ }^{1} \mathrm{H}$ NMR (400M Hz, DMSO-d6)

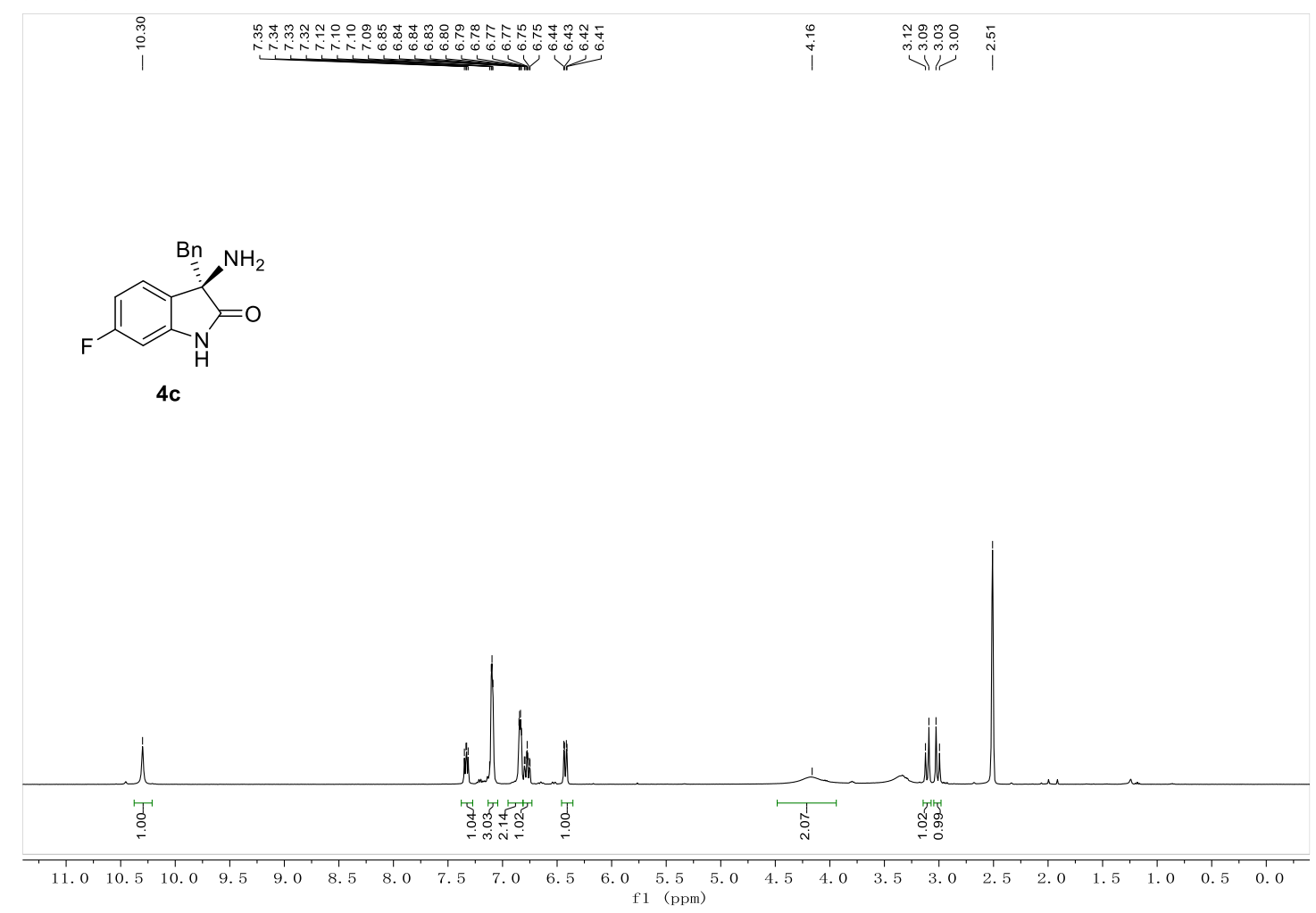

$4 c^{13} \mathrm{C}$ NMR (100M Hz, DMSO-d6)

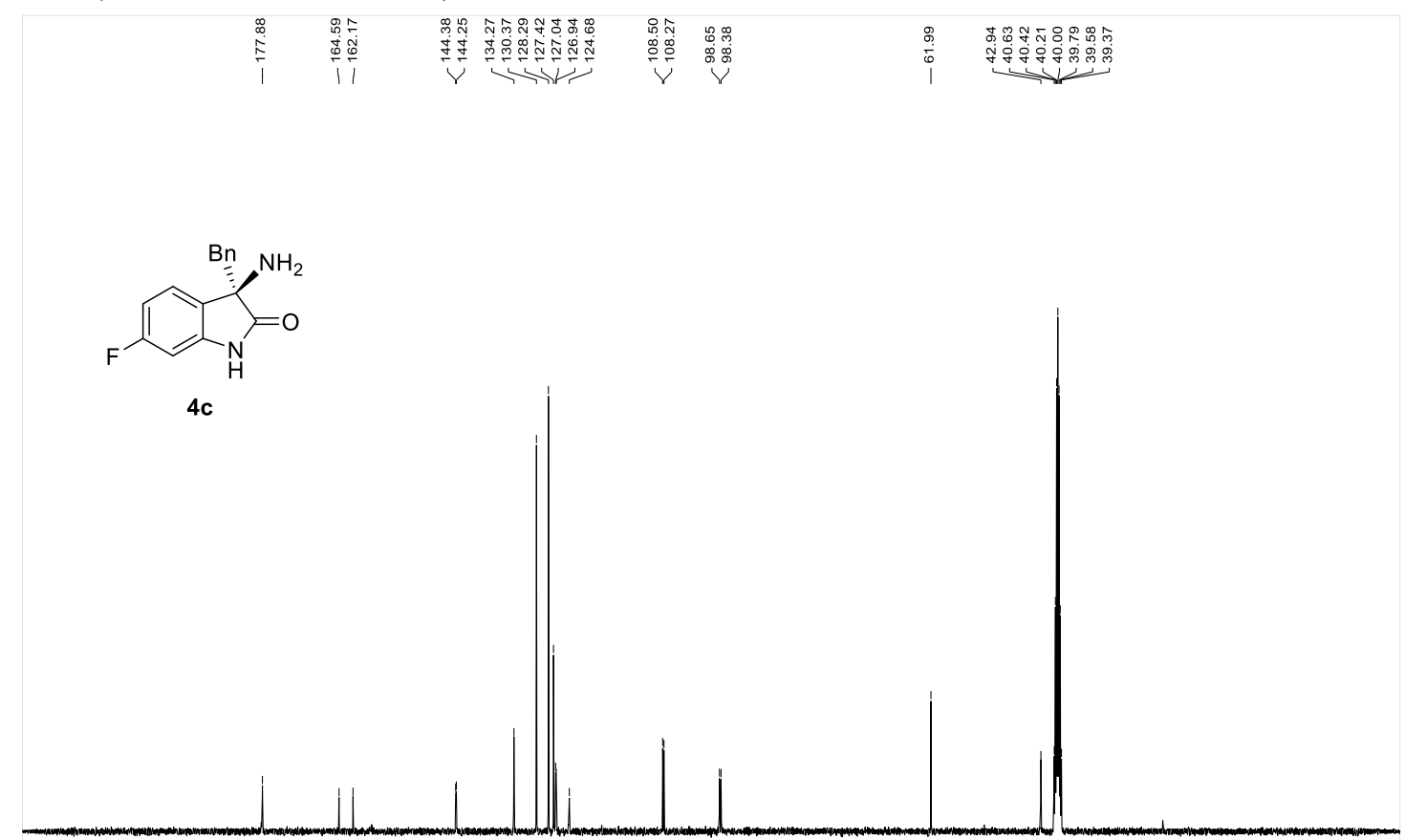

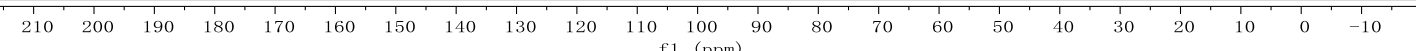


4d ${ }^{1} \mathrm{H}$ NMR (400M Hz, DMSO-d6)

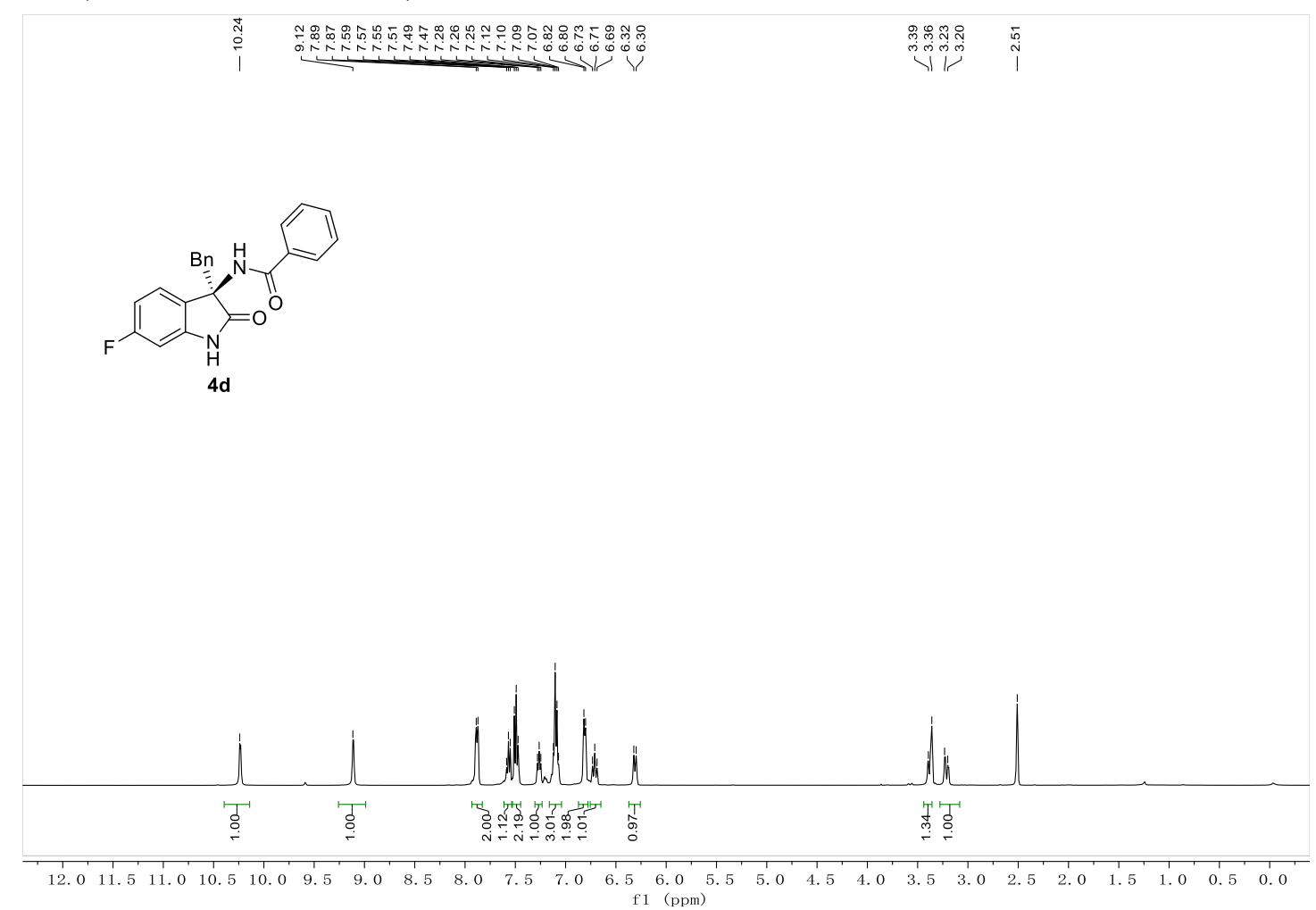

4d ${ }^{13} \mathrm{C}$ NMR (100M Hz, DMSO-d6)

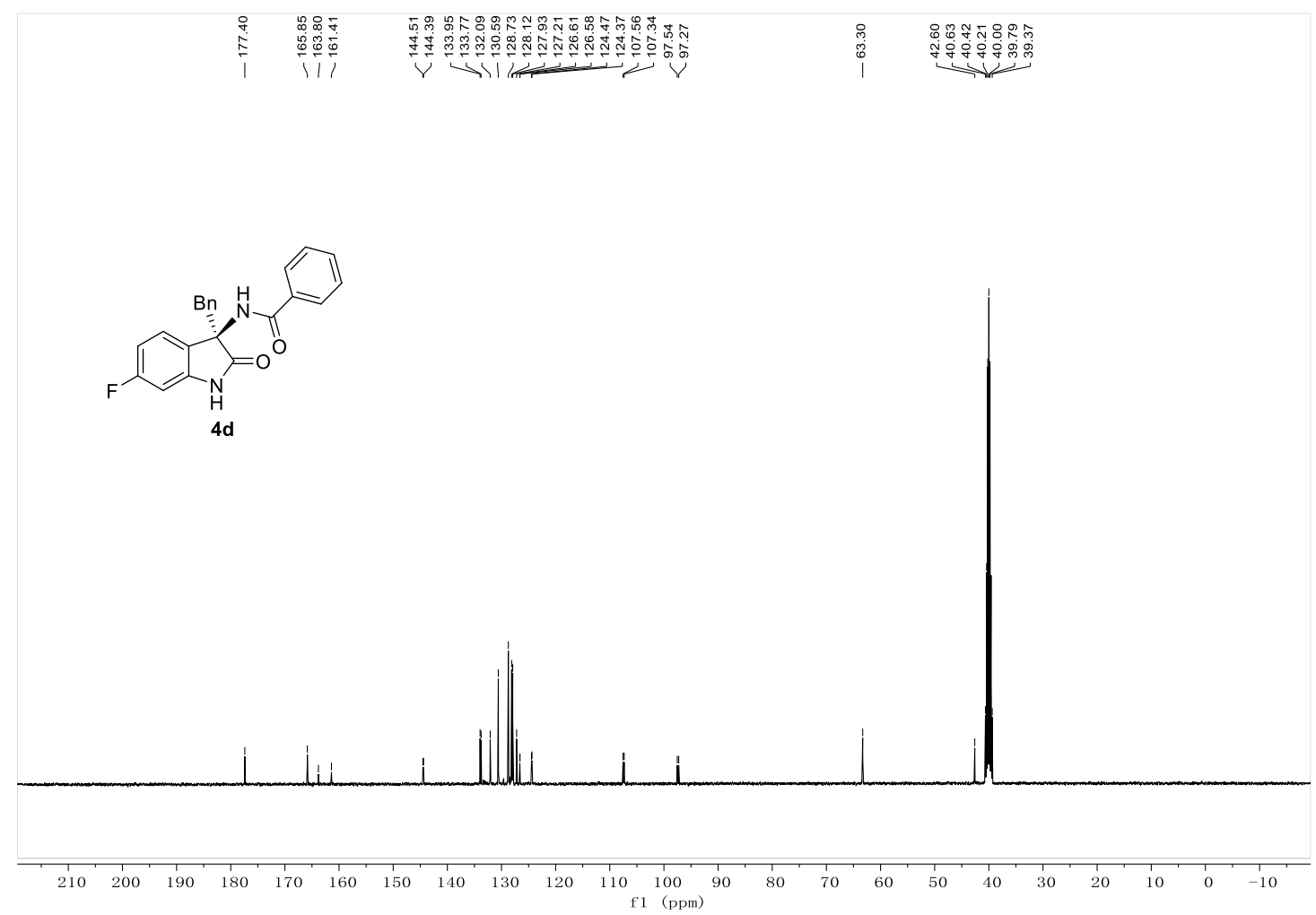


4x ${ }^{1} \mathrm{H}$ NMR (400M Hz, DMSO-d6)

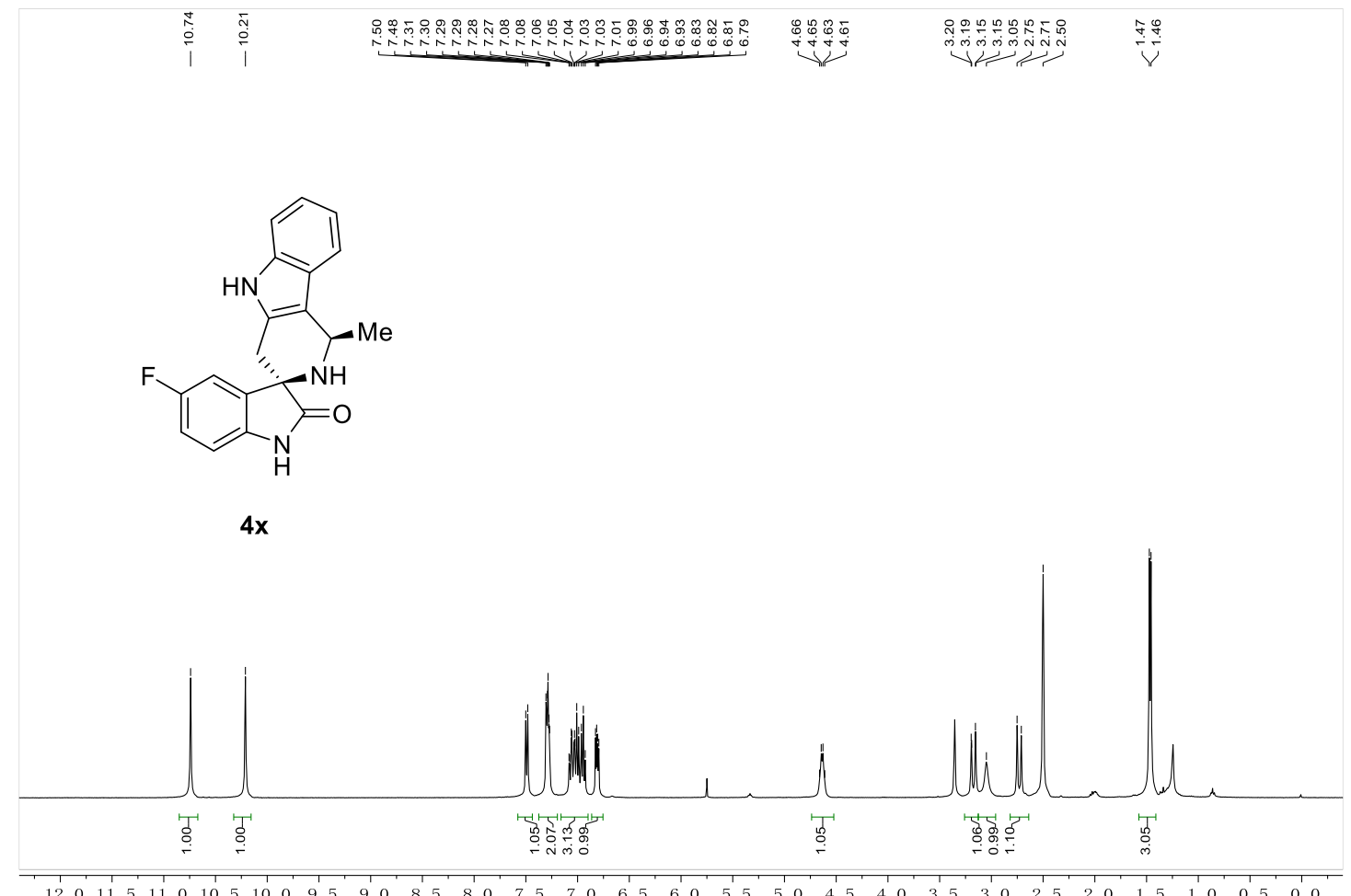

$\begin{array}{lllllllllllllllllllllllllllllll}12.0 & 11.5 & 11.0 & 10.5 & 10.0 & 9.5 & 9.0 & 8.5 & 8.0 & 7.5 & 7.0 & 6.5 & 6.0 & 5.5 & 5.0 & 4.5 & 4.0 & 3.5 & 3.0 & 2.5 & 2.0 & 1.5 & 1.0 & 0.5 & 0.0\end{array}$

4x ${ }^{13} \mathrm{C}$ NMR (100M Hz, DMSO-d6)

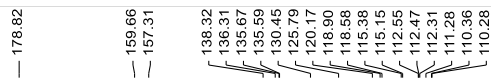

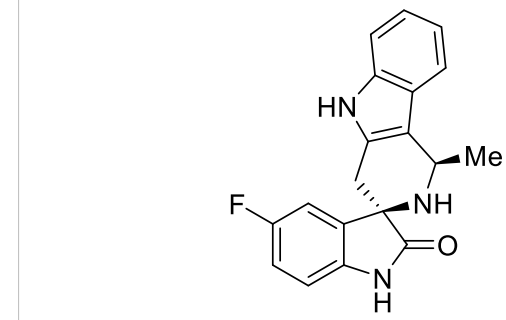

$4 x$

然等

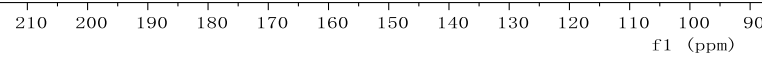


4y ${ }^{1} \mathrm{H}$ NMR (400M Hz, DMSO-d6)

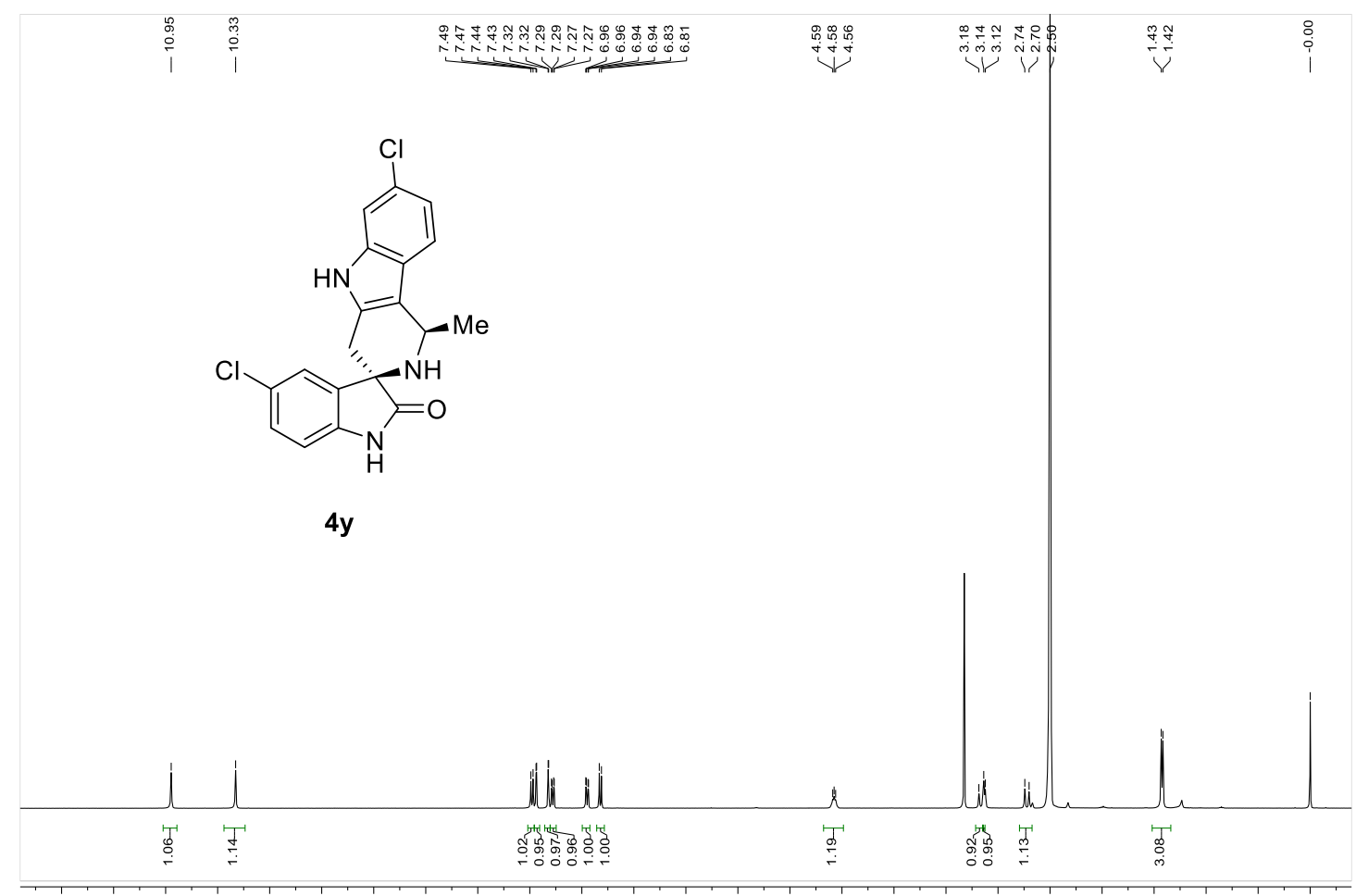

$\begin{array}{rlllllllllllllllllllllllllllllll}12.0 & 11.5 & 11.0 & 10.5 & 10.0 & 9.5 & 9.0 & 8.5 & 8.0 & 7.5 & 7.0 & 6.5 & 6.0 & 5.5 & 5.0 & 4.5 & 4.0 & 3.5 & 3.0 & 2.5 & 2.0 & 1.5 & 1.0 & 0.5 & 0.0\end{array}$

$4 \mathbf{y}{ }^{13} \mathrm{C}$ NMR (100M Hz, DMSO- $\left.d 6\right)$

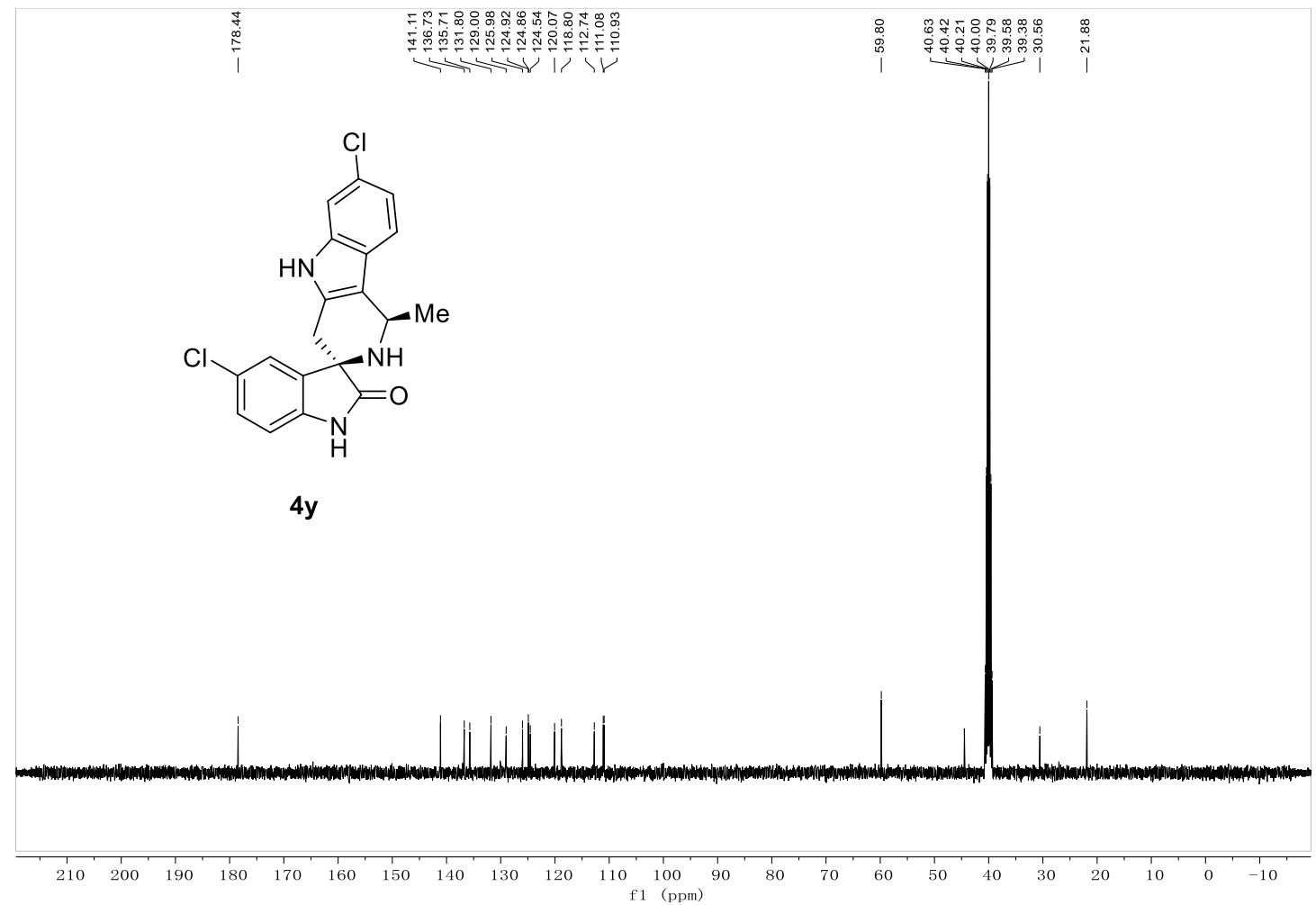


$6{ }^{1} \mathbf{H}$ NMR (400M Hz, $\left.\mathrm{CDCl}_{3}\right)$

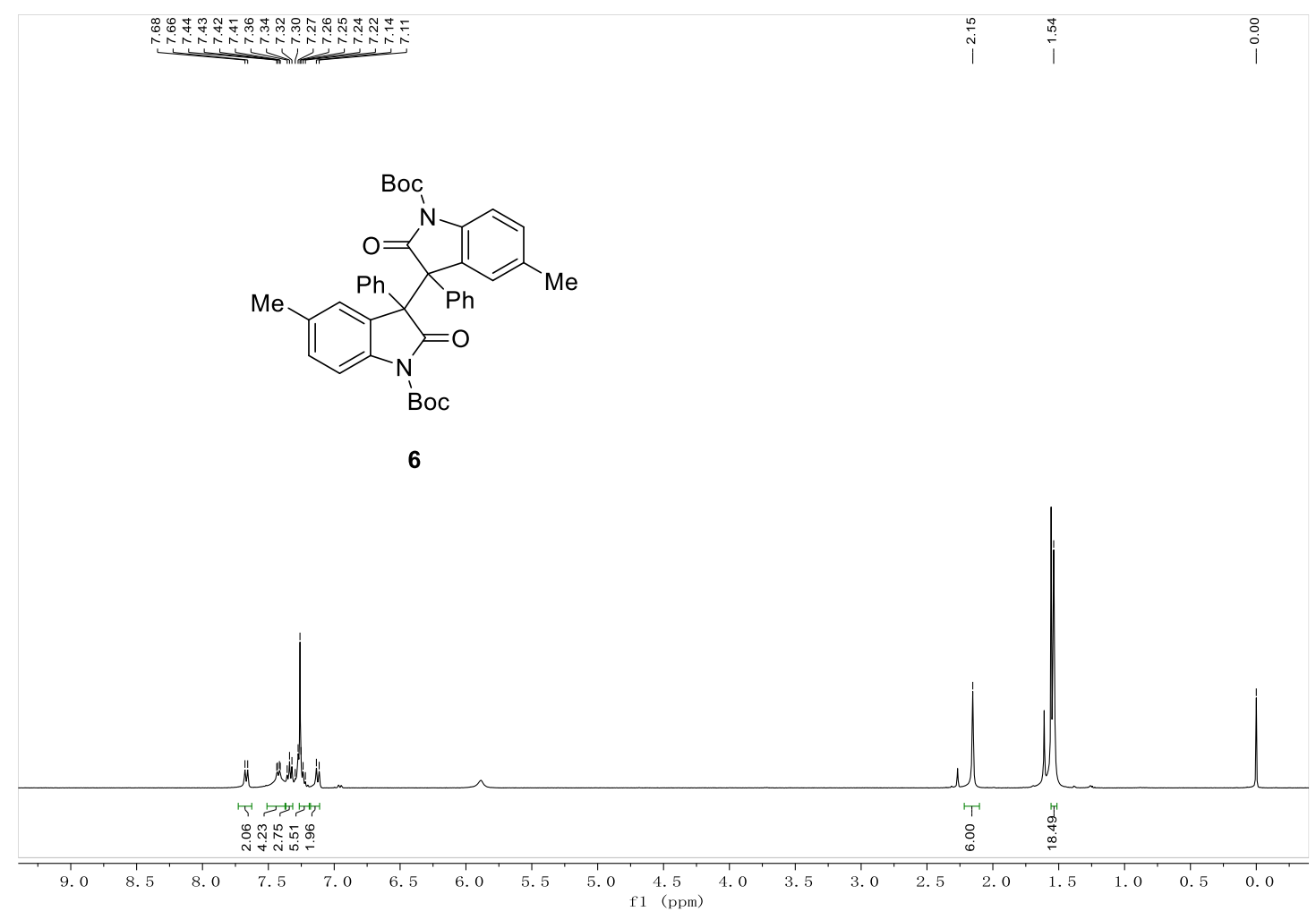

6 ${ }^{13} \mathbf{C}$ NMR $\left(100 \mathrm{M} \mathrm{Hz}, \mathrm{CDCl}_{3}\right)$

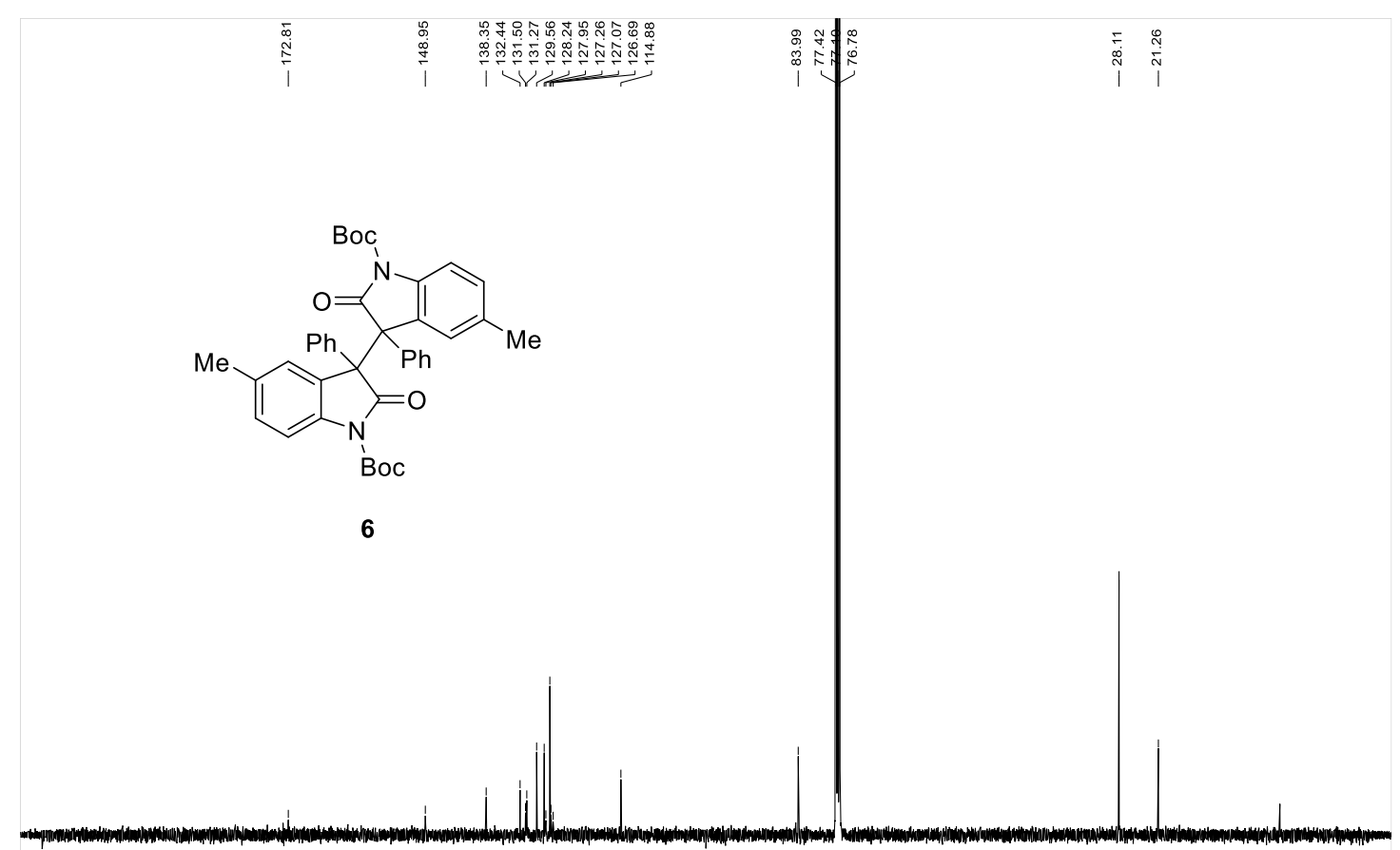

\begin{tabular}{|l|lllllllllllllllllllllllllll}
1 & 100 & 200 & 190 & 180 & 170 & 160 & 150 & 140 & 130 & 120 & 110 & 100 & 90 & 80 & 70 & 60 & 50 & 40 & 30 & 20 & 10 & 0 & -10
\end{tabular} 


\section{HPLC data}

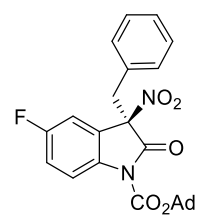

3a

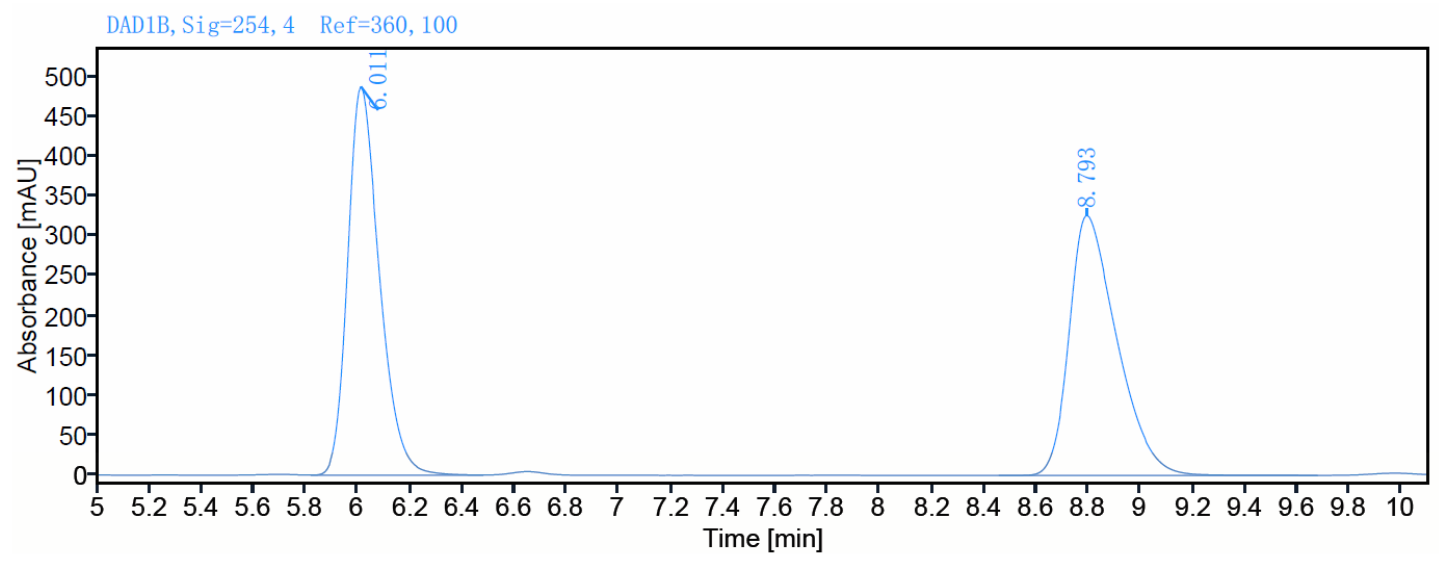

Signal: $\quad$ DAD1B, Sig=254, $4 \quad \operatorname{Ref}=360,100$

$\begin{array}{rrrr}\text { Rentention Time[min] } & \text { Area[mAU*min] } & \text { Height [mAU] } & \text { Relative Area[\%] } \\ 6.011 & 4119.69 & 487.40 & 49.97 \\ 8.793 & 4124.67 & 326.03 & 50.03\end{array}$

DAD1B, Sig=254, $4 \quad$ Ref $=360,100$

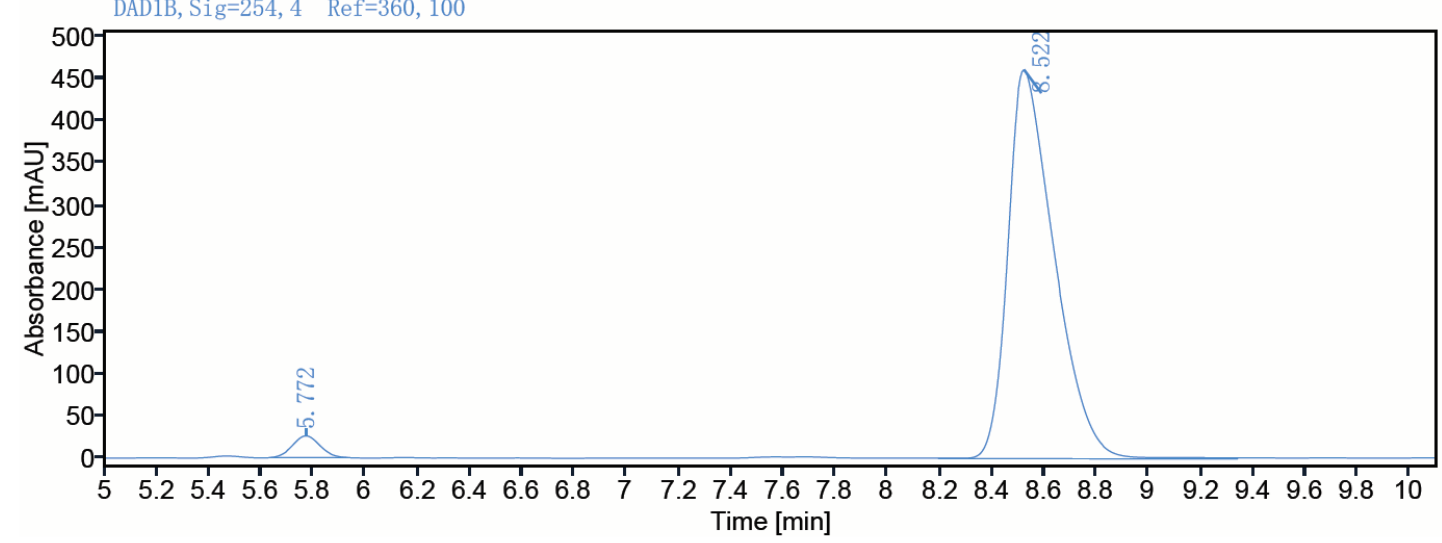

Signal: $\quad$ DAD1B, Sig=254, $4 \quad$ Ref $=360,100$

Rentention Time[min]

5. 772

8.522
Area [mAU*min]

188. 05

5716. 86
Height [mAU]

25.90

461. 72
Relative Area[\%]

3. 18

96.82 


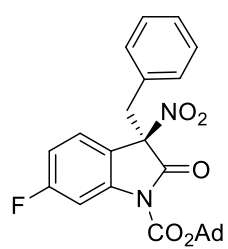

3b

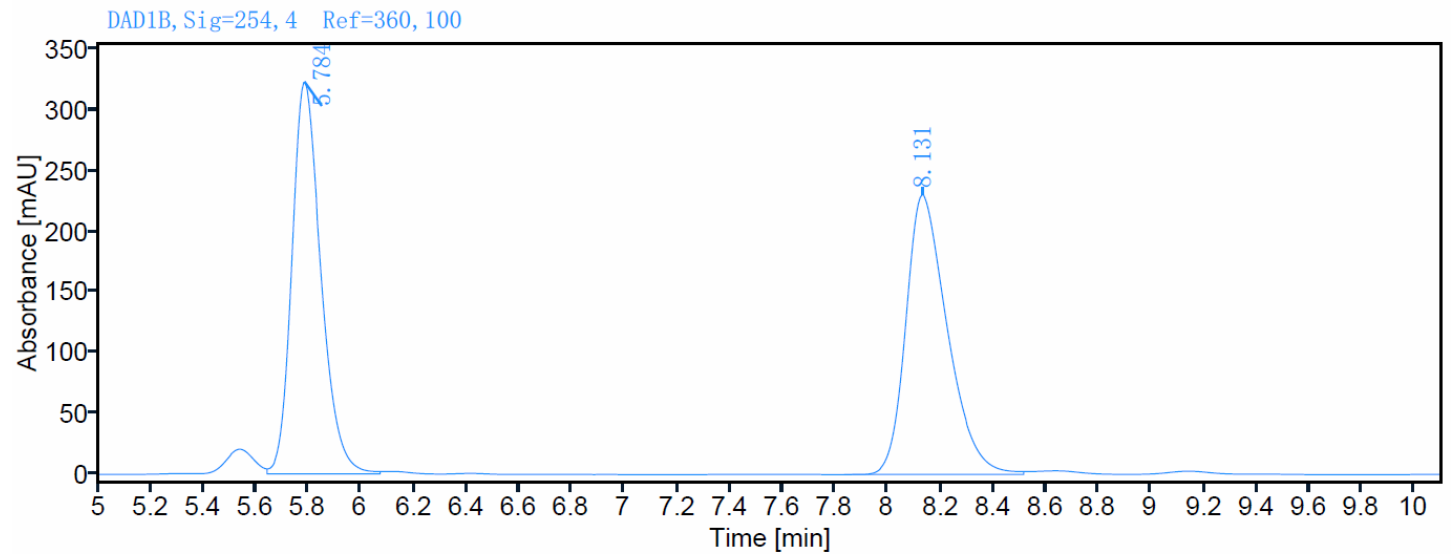

Signal: DAD1B, Sig=254, $4 \quad \operatorname{Ref}=360,100$

$\begin{array}{rrrr}\text { Rentention Time[min] } & \text { Area[mAU*min] } & \text { Height[mAU] } & \text { Relative Area[\%] } \\ 5.784 & 2462.78 & 322.58 & 49.43 \\ 8.131 & 2519.37 & 230.17 & 50.57\end{array}$

DAD1B, Sig=254, $4 \quad$ Ref=360, 100

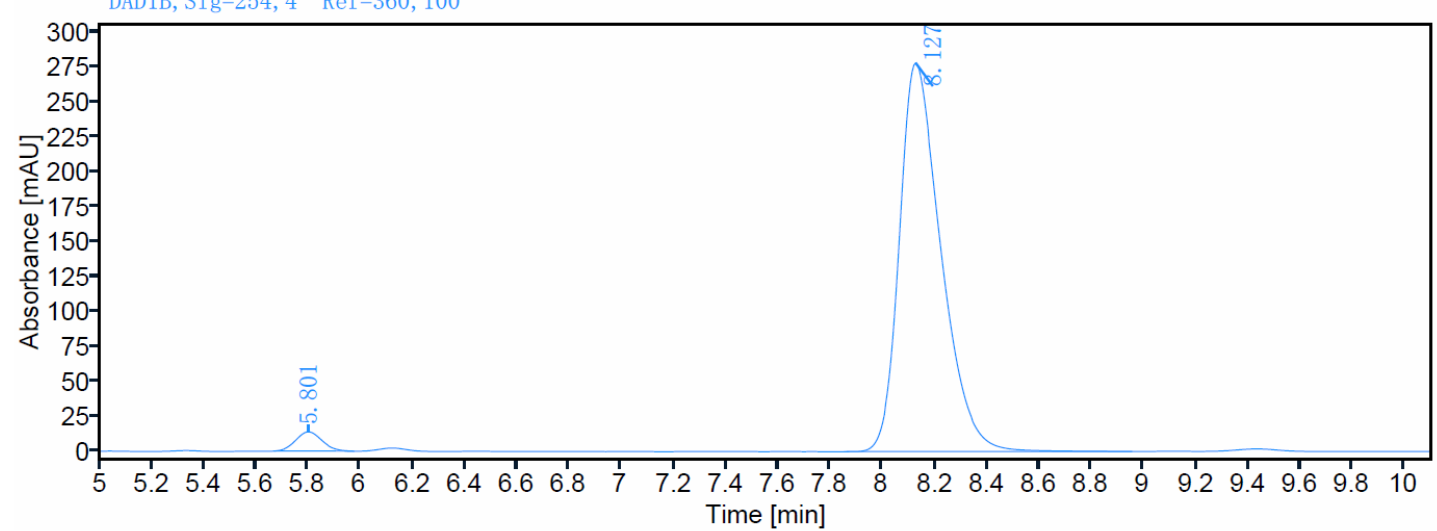

Signal: $\quad$ DAD1B, Sig=254, $4 \quad \operatorname{Ref}=360,100$

$\begin{array}{rrrr}\text { Rentention Time[min] } & \text { Area[mAU*min] } & \text { Height [mAU] } & \text { Relative Area[\%] } \\ 5.801 & 95.84 & 13.78 & 3.03 \\ 8.127 & 3068.95 & 278.10 & 96.97\end{array}$




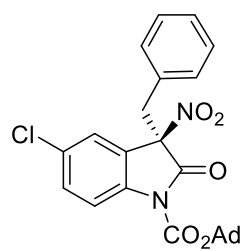

$3 c$

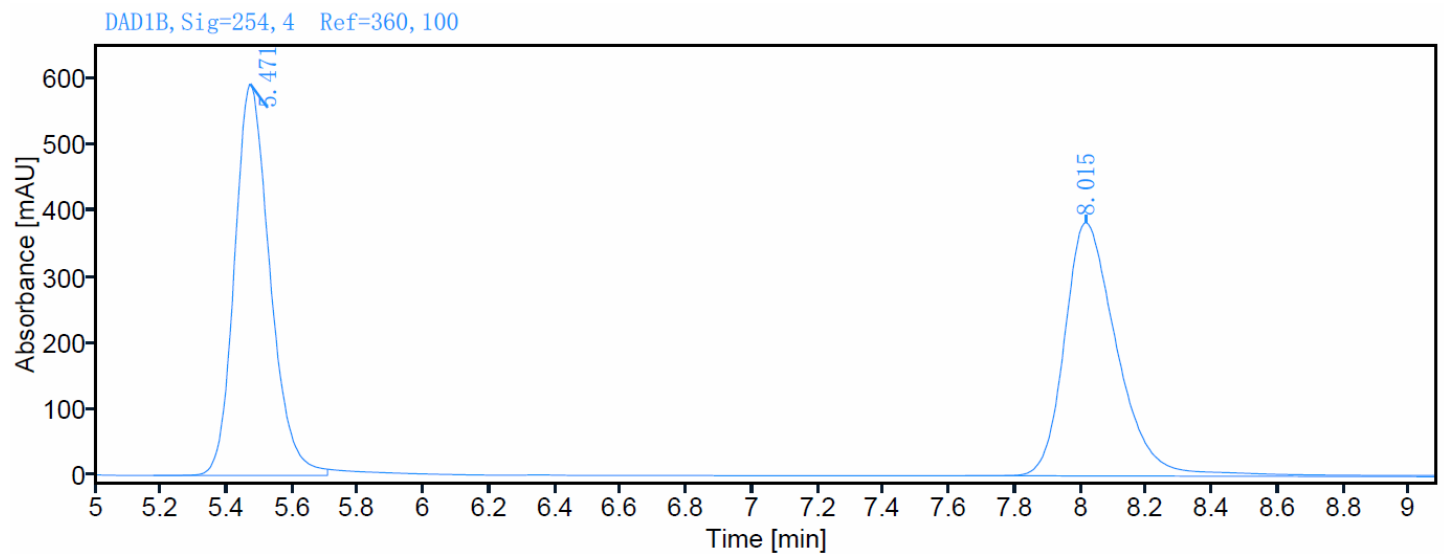

Signal: DAD1B, Sig=254, $4 \quad$ Ref $=360,100$

Rentention Time[min]

5. 471

8.015

4438. 44

4321.80
Height [mAU]

593. 02

383. 92
Relative Area[\%]

50.67

49. 33

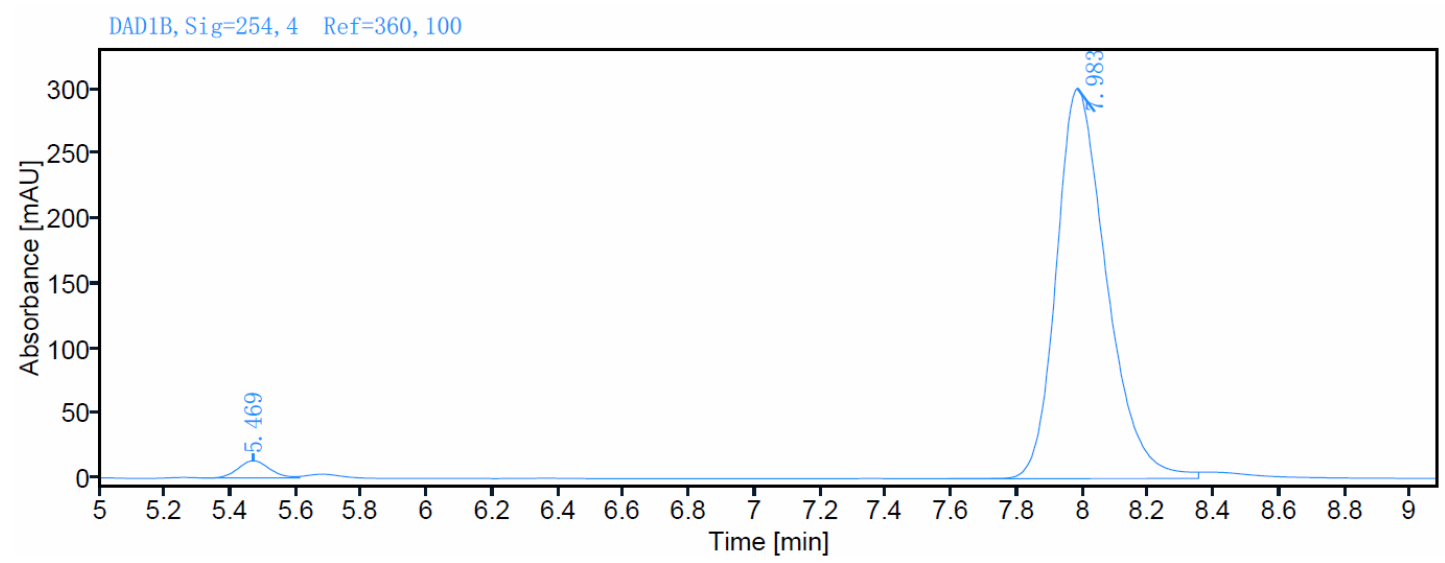

Signal: $\quad$ DAD1B, $\operatorname{Sig}=254,4 \quad$ Ref $=360,100$

Rentention Time[min]

5. 469

7. 983
Area $[\mathrm{mAU} * \min ]$

88.68

3194. 58
Height [mAU]

13. 28

300.88
Relative Area[\%]

2. 70

97.30 


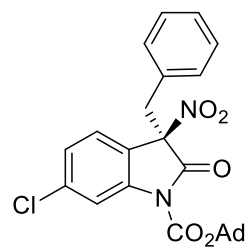

3d

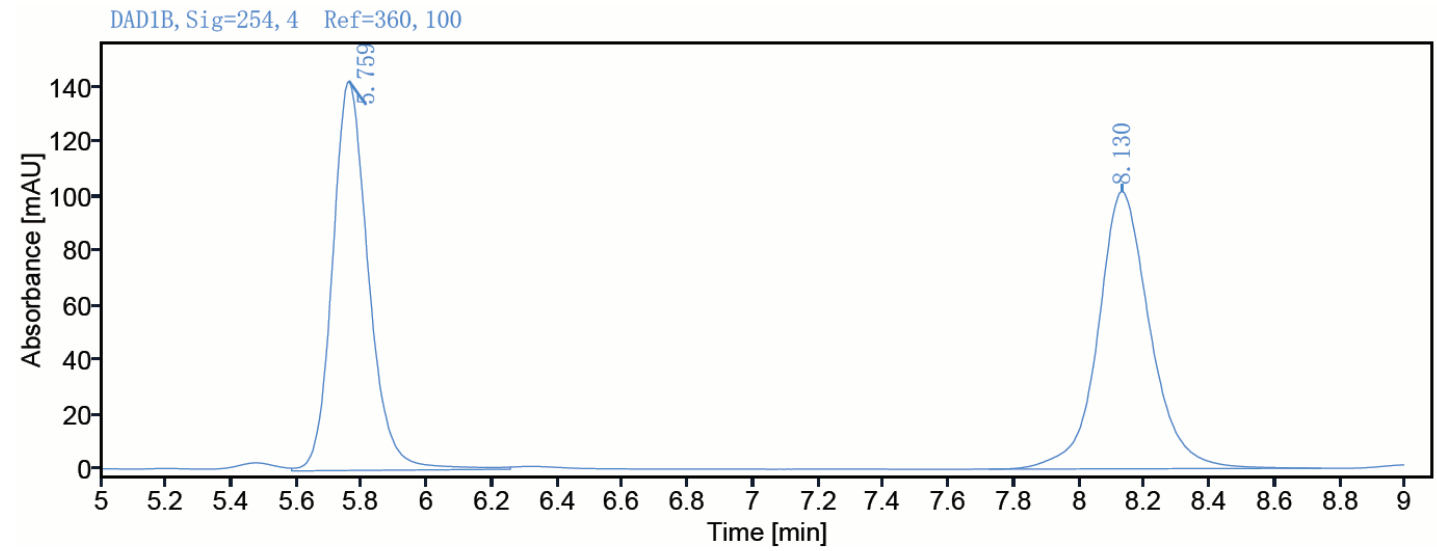

Signa1: DAD1B, Sig=254, 4 Ref=360, 100

$\begin{array}{rrrr}\text { Rentention Time[min] } & \text { Area[mAU*min] } & \text { Height [mAU] } & \text { Relative Area[\%] } \\ 5.759 & 1096.36 & 143.16 & 49.33 \\ 8.130 & 1126.13 & 101.95 & 50.67\end{array}$

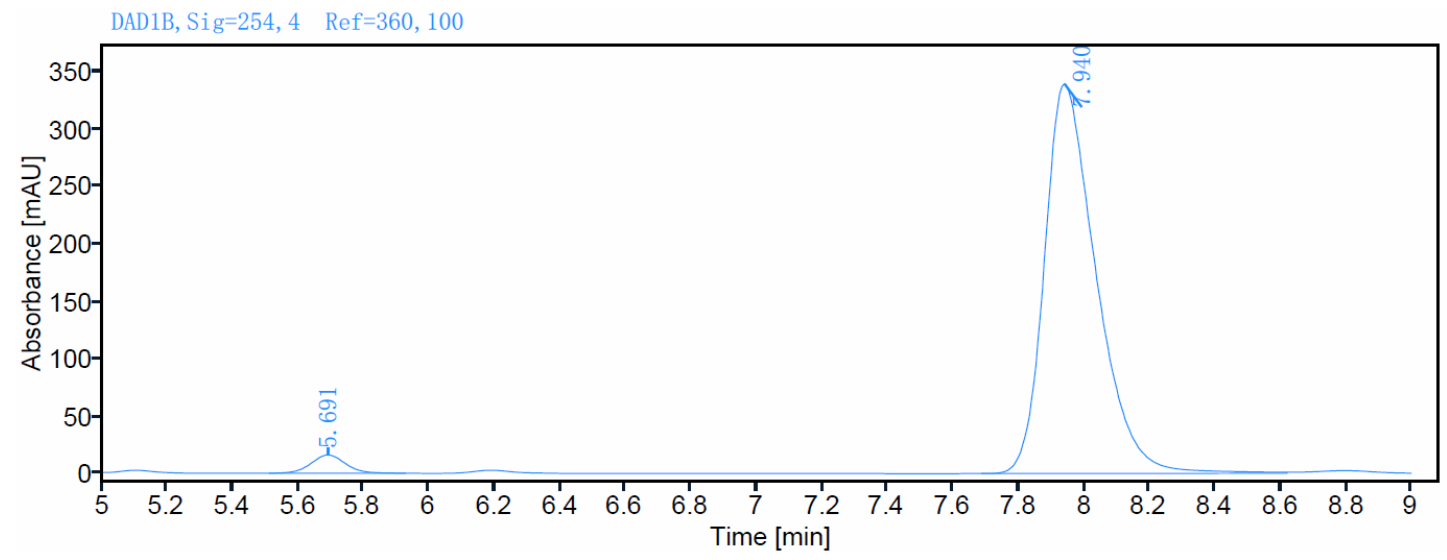

Signal: $\quad$ DAD1B, Sig=254, 4 Ref $=360,100$

$\begin{array}{rrrr}\text { Rentention Time[min] } & \text { Area[mAU*min] } & \text { Height [mAU] } & \text { Relative Area[\%] } \\ 5.691 & 115.96 & 15.95 & 3.02 \\ 7.940 & 3724.88 & 340.02 & 96.98\end{array}$


<smiles>CC(=O)N1C(=O)C(Cc2ccccc2)([N+](=O)[O-])c2ccc(Br)cc2N1C(C)=O</smiles>

$3 e$

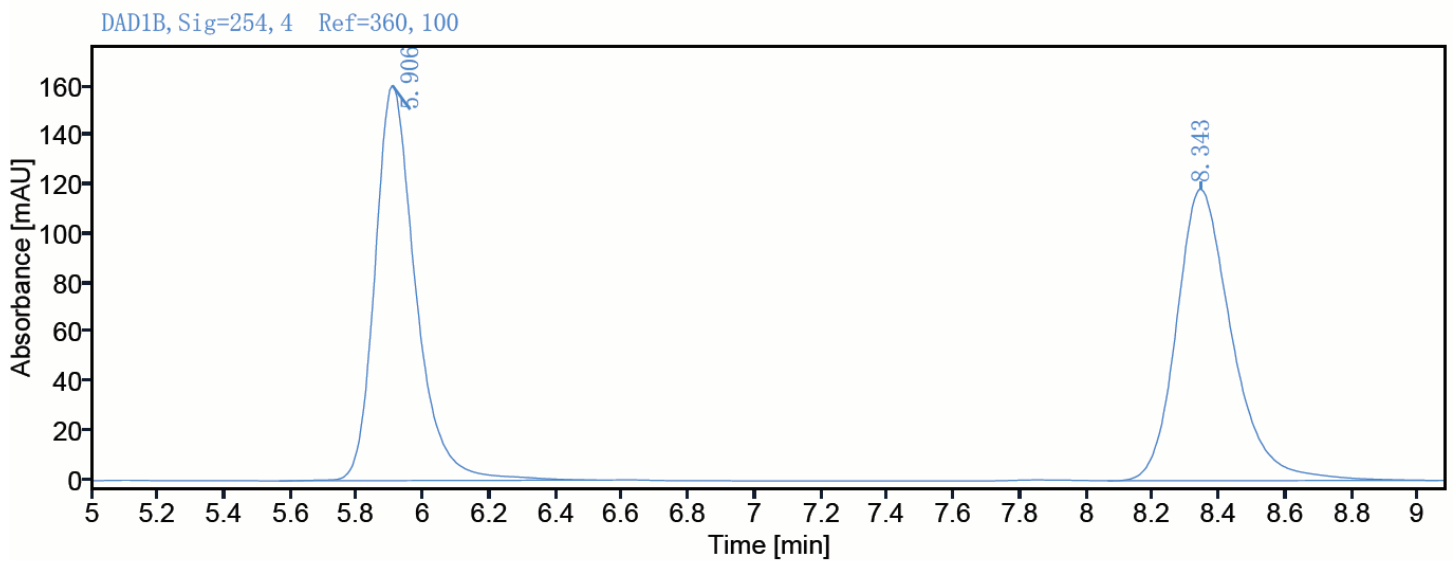

Signal: $\quad$ DAD1B, Sig=254, $4 \quad$ Ref $=360,100$

Rentention Time[min]

5. 906

8. 343

DAD1B, Sig=254, $4 \quad$ Ref=360, 100

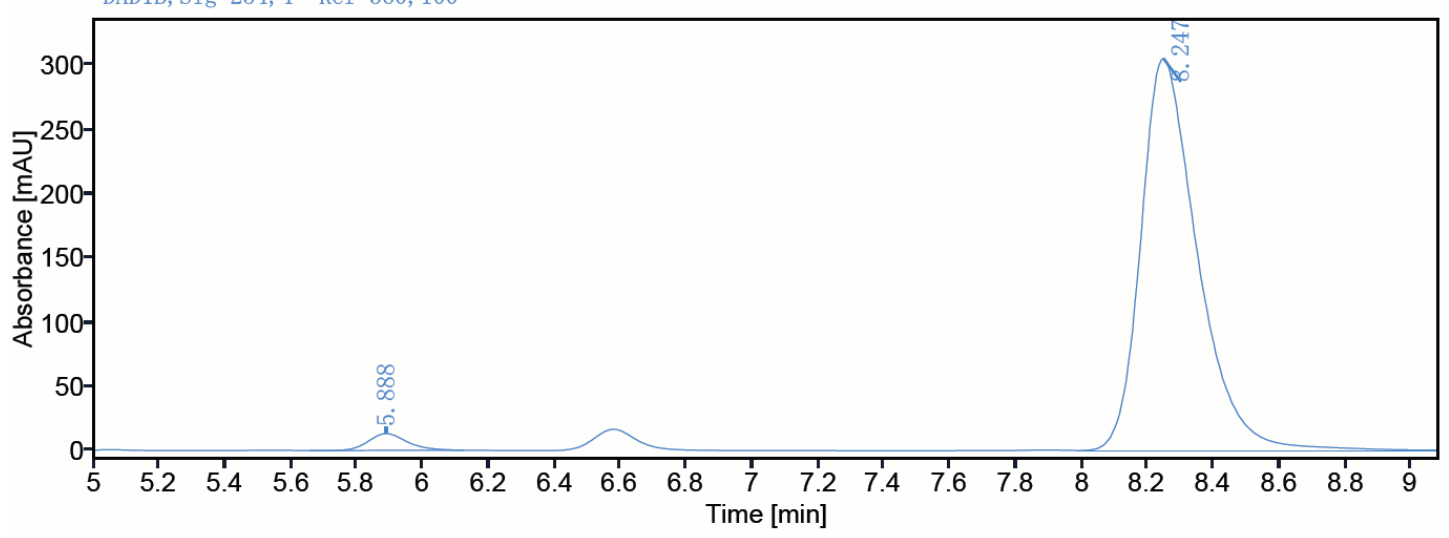

Signa1: $\quad$ DAD1B, $\operatorname{Sig}=254,4 \quad \operatorname{Ref}=360,100$

Rentention Time[min]

5. 888

8. 247
Area [mAU*min]

1363. 90

1368. 79
Height [mAU]

160. 46

118. 44
Relative Area[\%]

49.91

50.09 ime [min]

Area [mAU*min]

106. 69

3662. 32
Height [mAU]

13. 10

305.82
Relative Area[\%]

2. 83

97. 17 


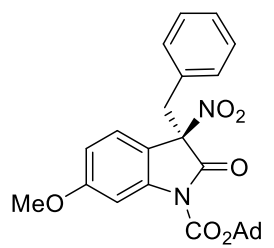

$3 f$

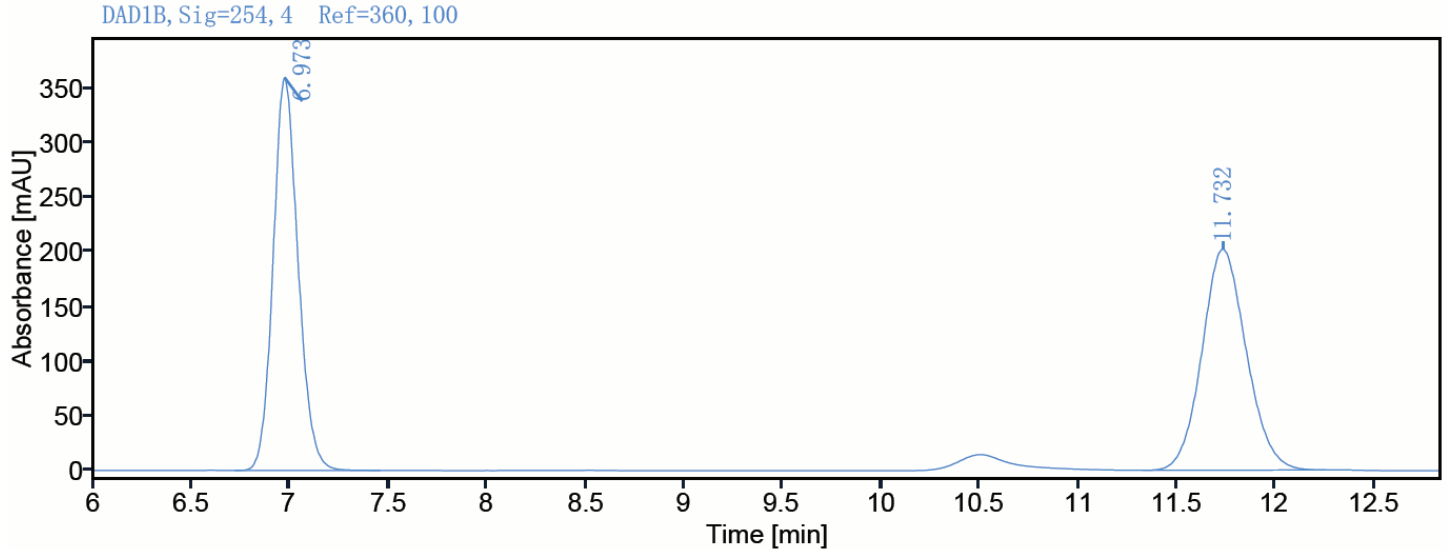

Signal: $\quad$ DAD1B, Sig=254, $4 \quad \operatorname{Ref}=360,100$ Rentention Time[min]

Area [mAU*min]

3164.68

3166.88

11. 732
Height [mAU]

360. 22

202. 78
Relative Area[\%]

49.98

50.02

DAD1B, Sig=254, $4 \quad$ Ref=360, 100

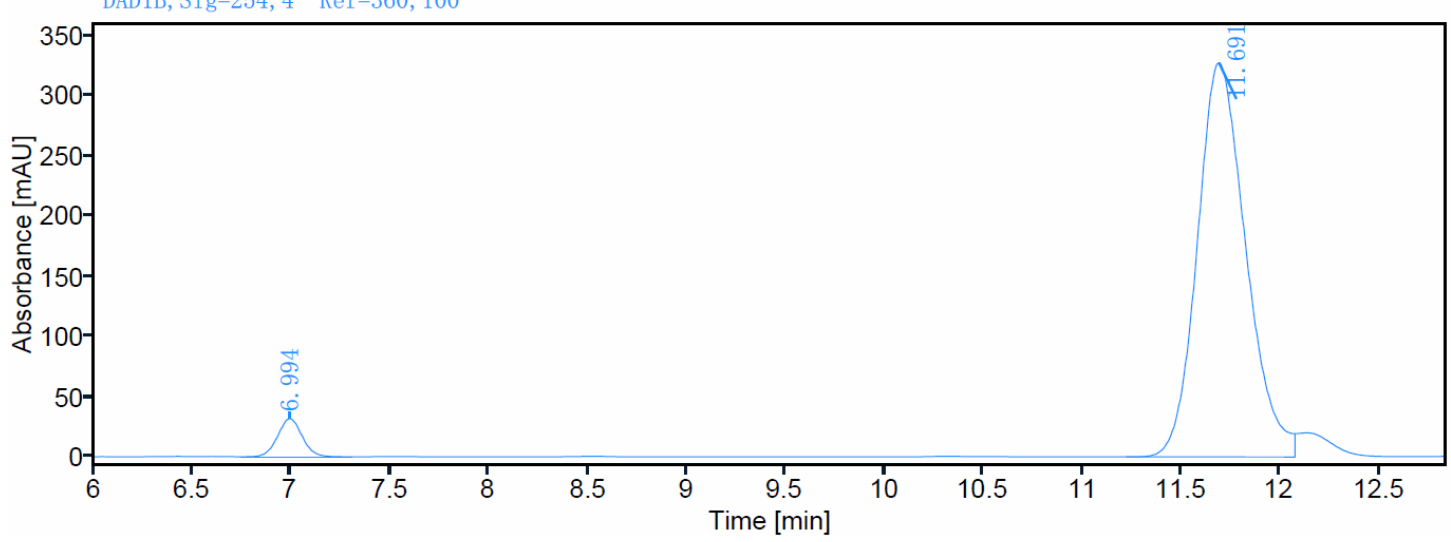

Signal: $\quad$ DAD1B, Sig=254, $4 \quad$ Ref=360, 100

Rentention Time[min]

6. 994

11. 691
Area [mAU*min]

272.88

5573. 02
Height [mAU]

31. 69

327. 72
Relative Area[\%]

4. 67

95. 33 
<smiles>O=C(O)N1C(=O)[C@@](Cc2ccccc2)([N+](=O)[O-])c2ccc(-c3ccccc3)cc21</smiles>

$3 \mathrm{~g}$

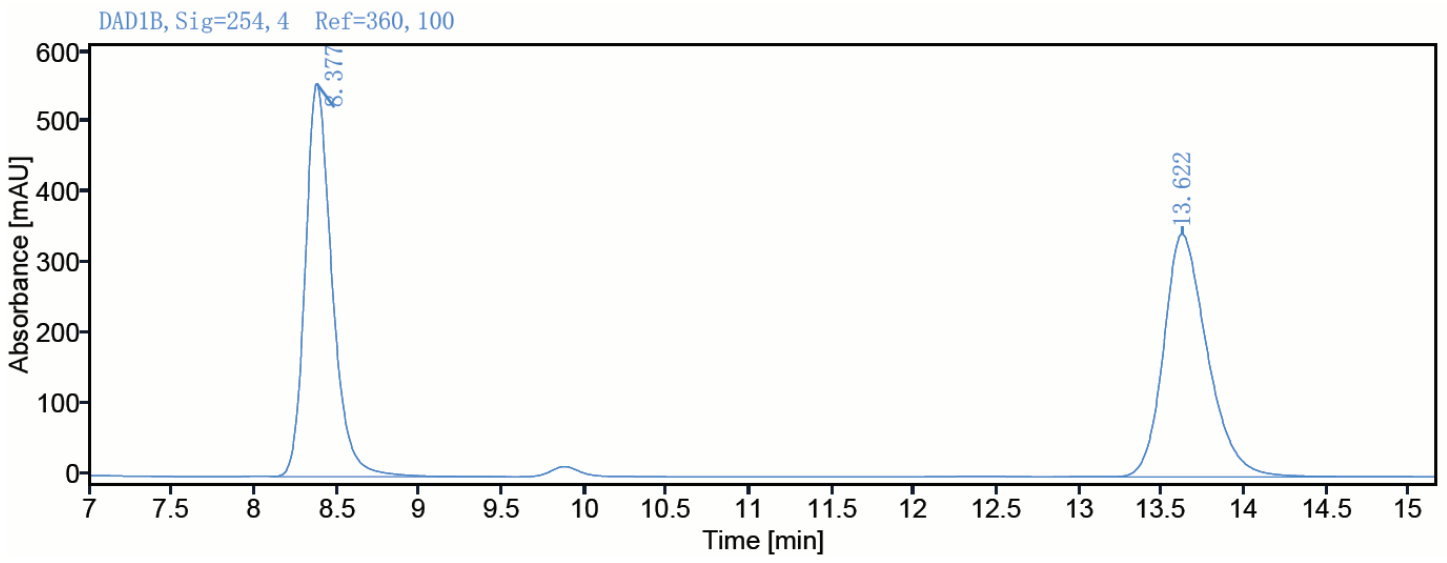

Signa1: $\quad$ DAD1B, Sig=254, $4 \quad \operatorname{Ref}=360,100$

Rentention Time[min]

8. 377

13. 622
Area $[\mathrm{mAU} * \min ]$

6286. 36

6352.74
Height [mAU]

557. 29

344. 30
Relative Area[\%]

49. 74

50.26

DAD1B, Sig=254, $4 \quad$ Ref $=360,100$

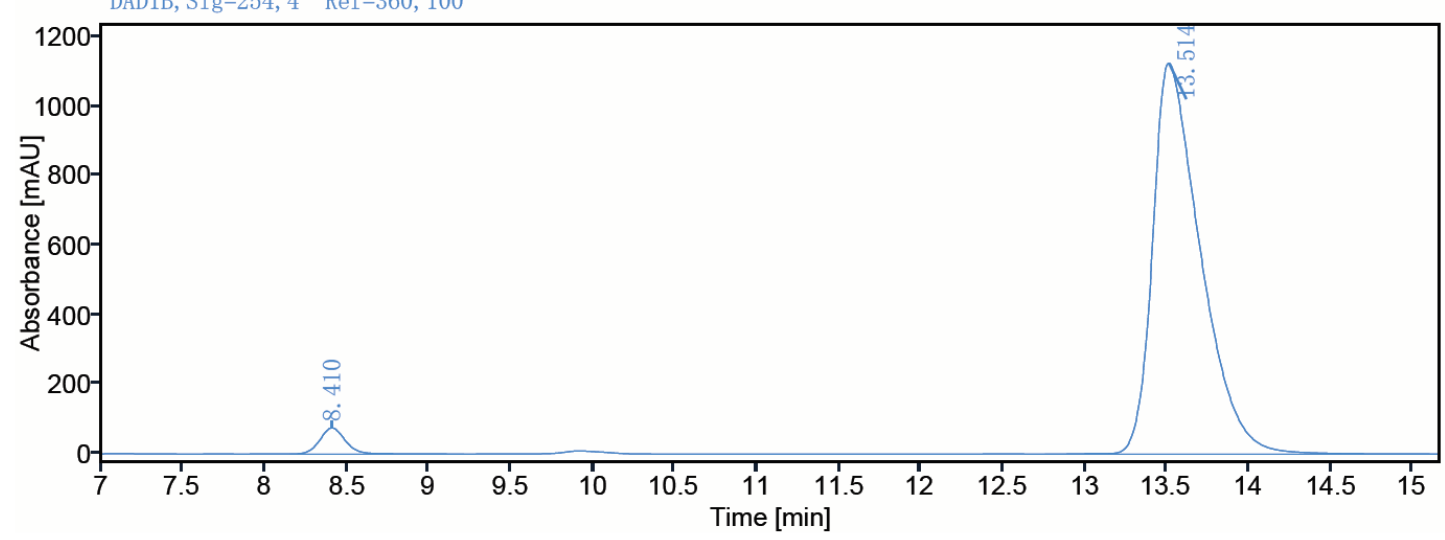

Signal: $\quad$ DAD1B, Sig=254, $4 \quad$ Ref=360, 100

Rentention Time[min]

8. 410

13. 514
Area [mAU*min]

823.30

22776. 45
Height [mAU]

75. 14

1123. 42
Relative Area[\%]

3. 49

96.51 


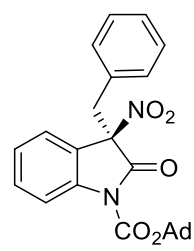

3h

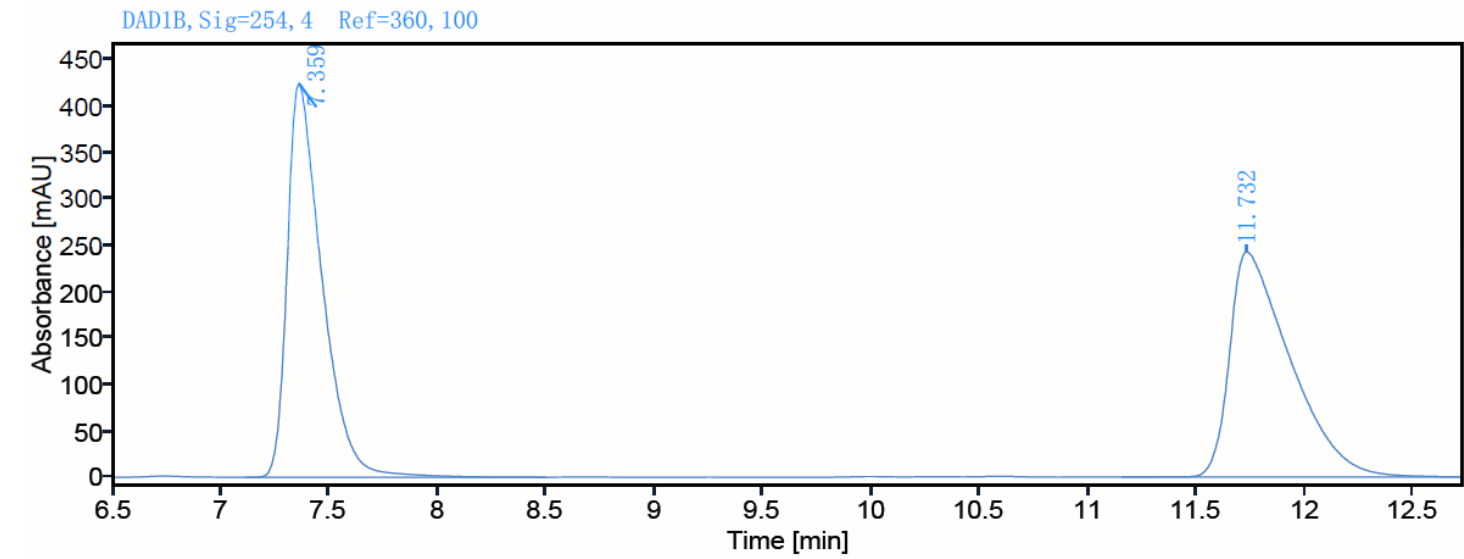

Signa1: DAD1B, Sig=254, 4 Ref $=360,100$

Rentention Time[min]

7. 359

11. 732
Area [mAU*min]

4733. 02

4734. 04
Height [mAU]

424. 94

242. 90
Relative Area[\%]

49. 99

50.01

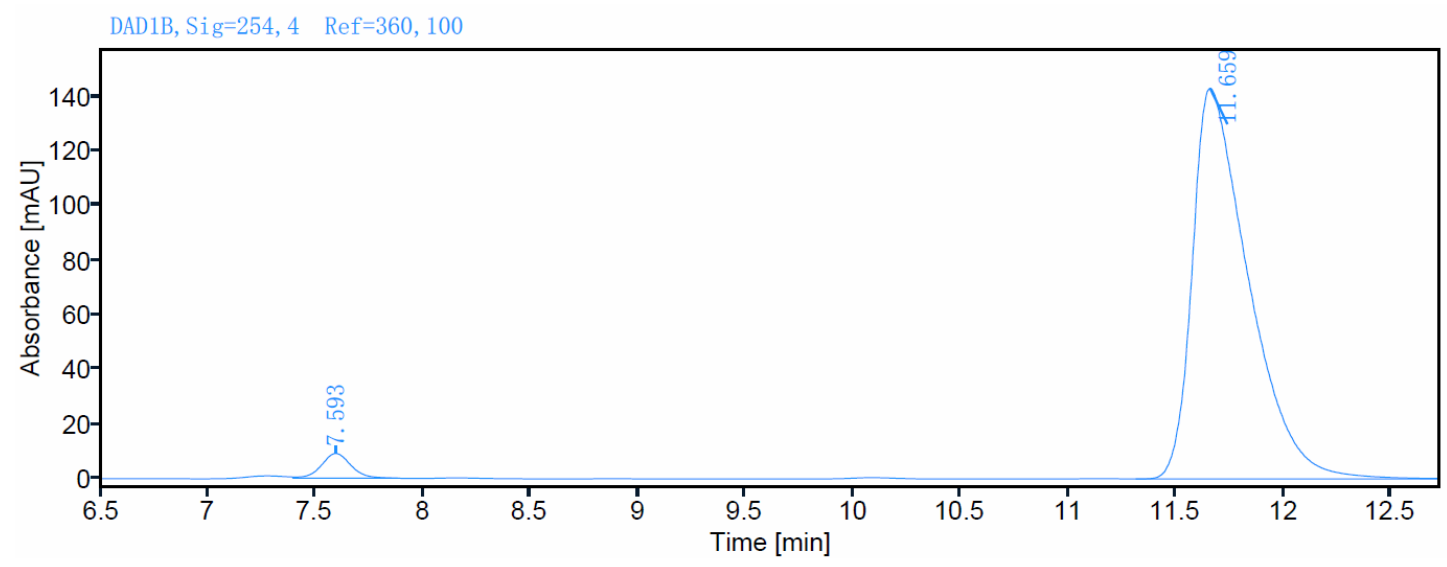

Signal: $\quad$ DAD1B, Sig=254, 4 Ref $=360,100$

Rentention Time[min]

7. 593

11. 659
Area $[\mathrm{mAU} * \min ]$

85.57

2599. 96
Height [mAU]

9. 05

143. 11
Relative Area[\%]

3. 19

96.81 


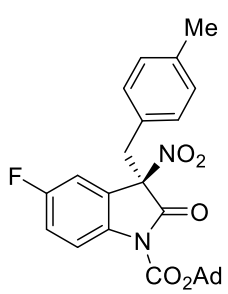

3i

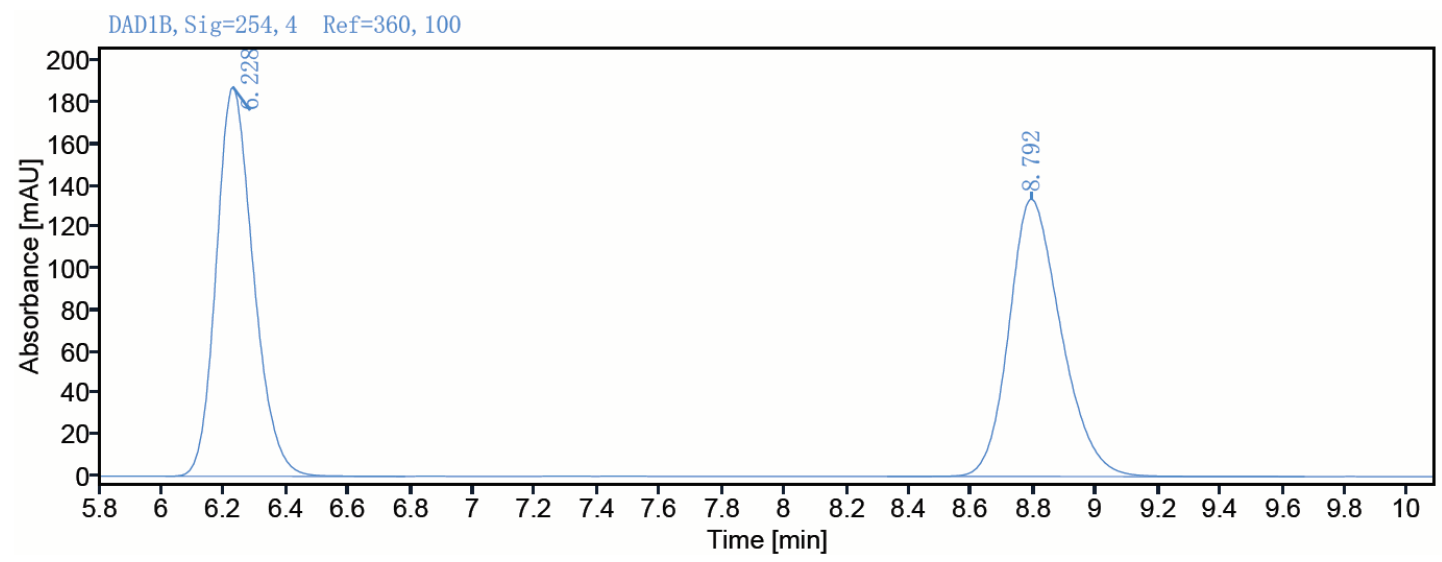

Signal: $\quad$ DAD1B, Sig=254, 4 Ref=360, 100

$\begin{array}{rrrr}\text { Rentention Time[min] } & \text { Area[mAU*min] } & \text { Height [mAU] } & \text { Relative Area[\%] } \\ 6.228 & 1552.72 & 187.84 & 49.97 \\ 8.792 & 1554.50 & 133.80 & 50.03\end{array}$

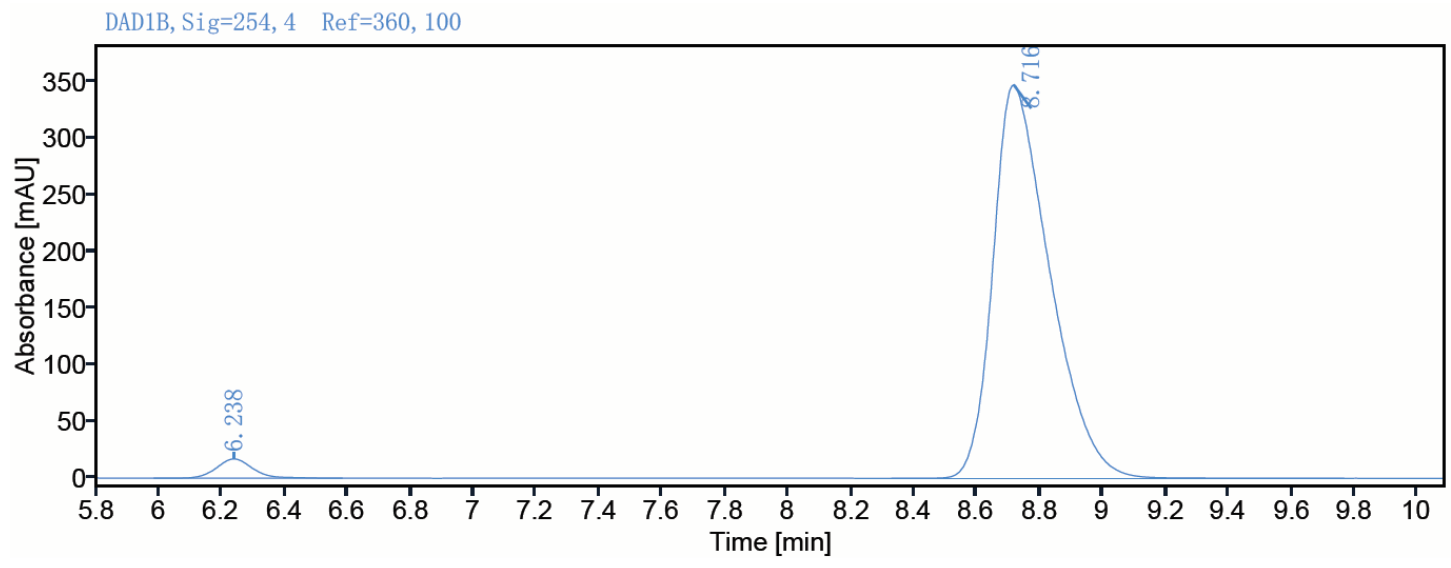

Signal: $\quad$ DAD1B, Sig=254, $4 \quad \operatorname{Ref}=360,100$

$\begin{array}{rrrr}\text { Rentention Time[min] } & \text { Area[mAU*min] } & \text { Height [mAU] } & \text { Relative Area[\%] } \\ 6.238 & 136.89 & 17.05 & 3.04 \\ 8.716 & 4360.29 & 347.56 & 96.96\end{array}$




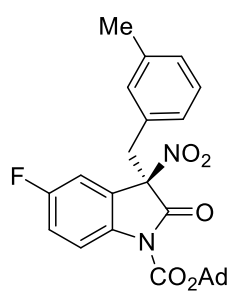

3j

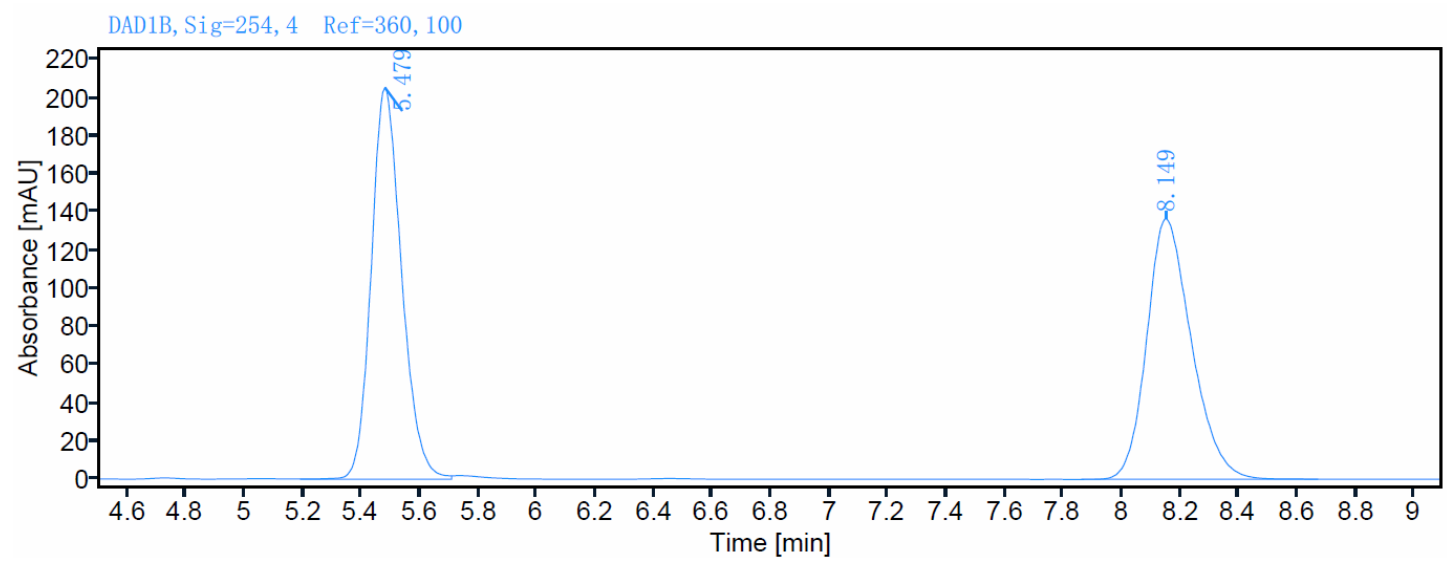

Signa1: DAD1B, $\operatorname{Sig}=254,4 \quad \operatorname{Ref}=360,100$

$\begin{array}{rrrr}\text { Rentention Time[min] } & \text { Area[mAU*min] } & \text { Height [mAU] } & \text { Relative Area[\%] } \\ 5.479 & 1483.22 & 205.53 & 50.58 \\ 8.149 & 1449.44 & 136.85 & 49.42\end{array}$

DAD1B, Sig=254, $4 \quad$ Ref $=360,100$

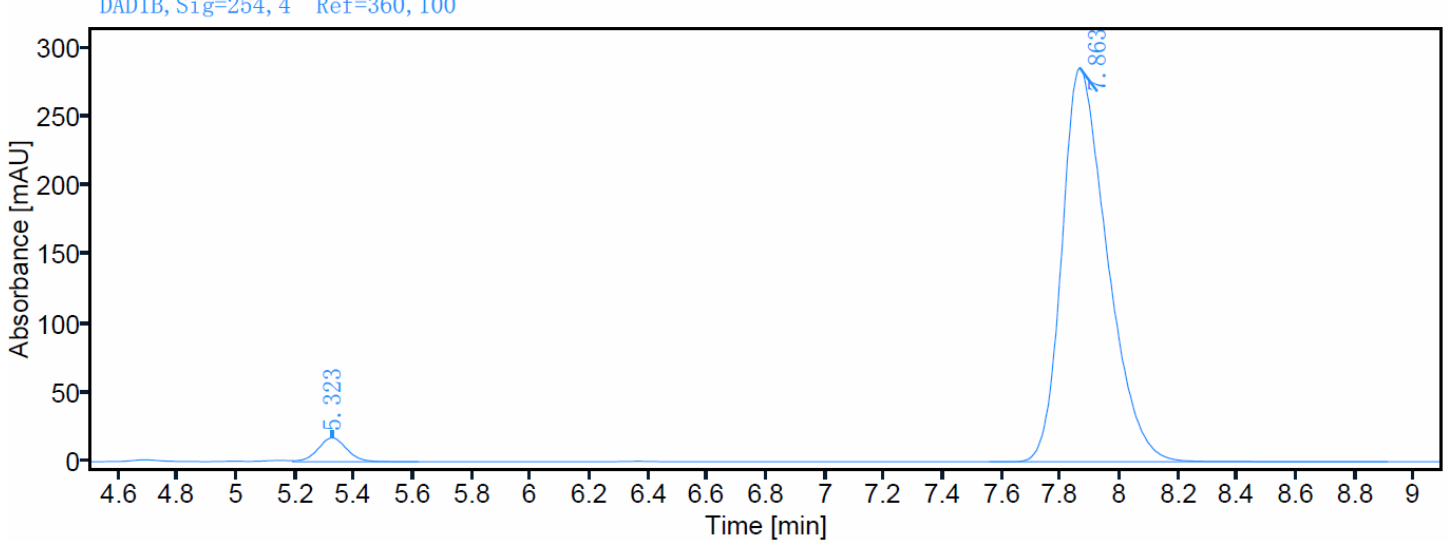

Signal: $\quad$ DAD1B, $\operatorname{Sig}=254,4 \quad \operatorname{Ref}=360,100$

Rentention Time[min]

5. 323

7. 863
Area $[\mathrm{mAU} * \min ]$

117.08

3050. 12
Height $[\mathrm{mAU}]$

17.32

285. 52
Relative Area[\%]

3. 70

96. 30 


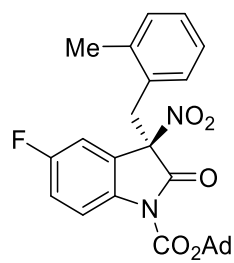

$3 \mathbf{k}$

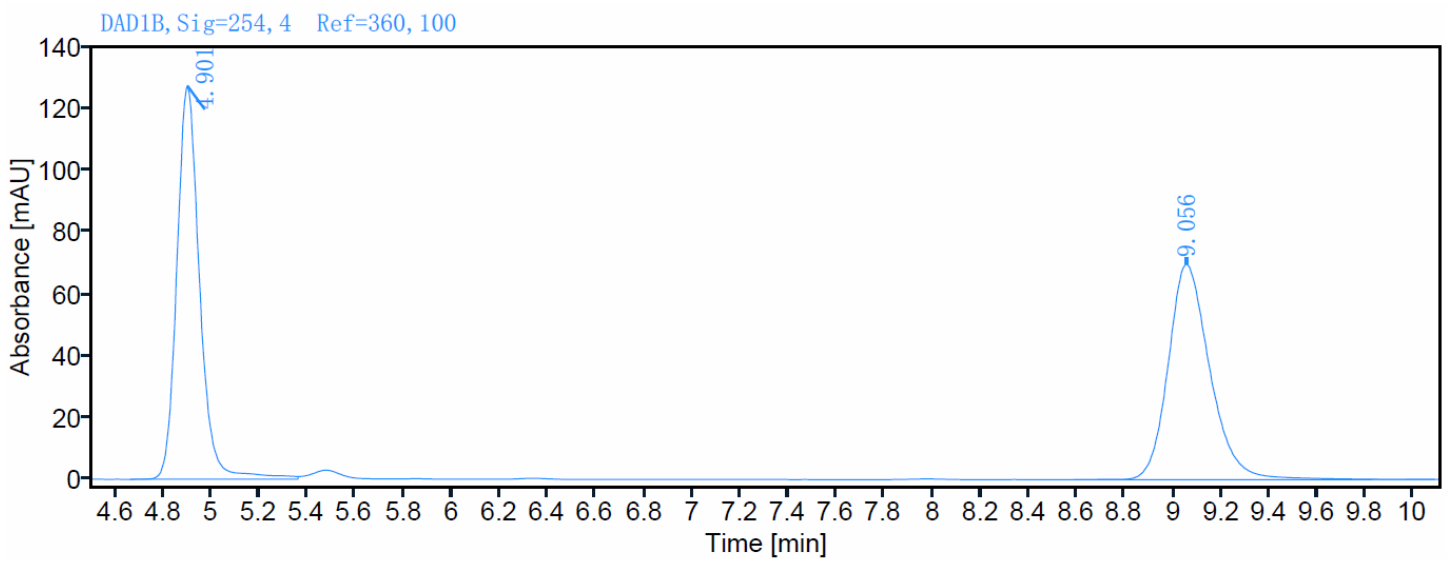

Signal: $\quad$ DAD1B, Sig=254, $4 \quad$ Ref $=360,100$

Rentention Time[min]

Area $[\mathrm{mAU} * \min ]$

Height [mAU]

Relative Area[\%]
4. 901
840.21
127. 46
49. 99
9. 056
840.63
69. 74
50. 01

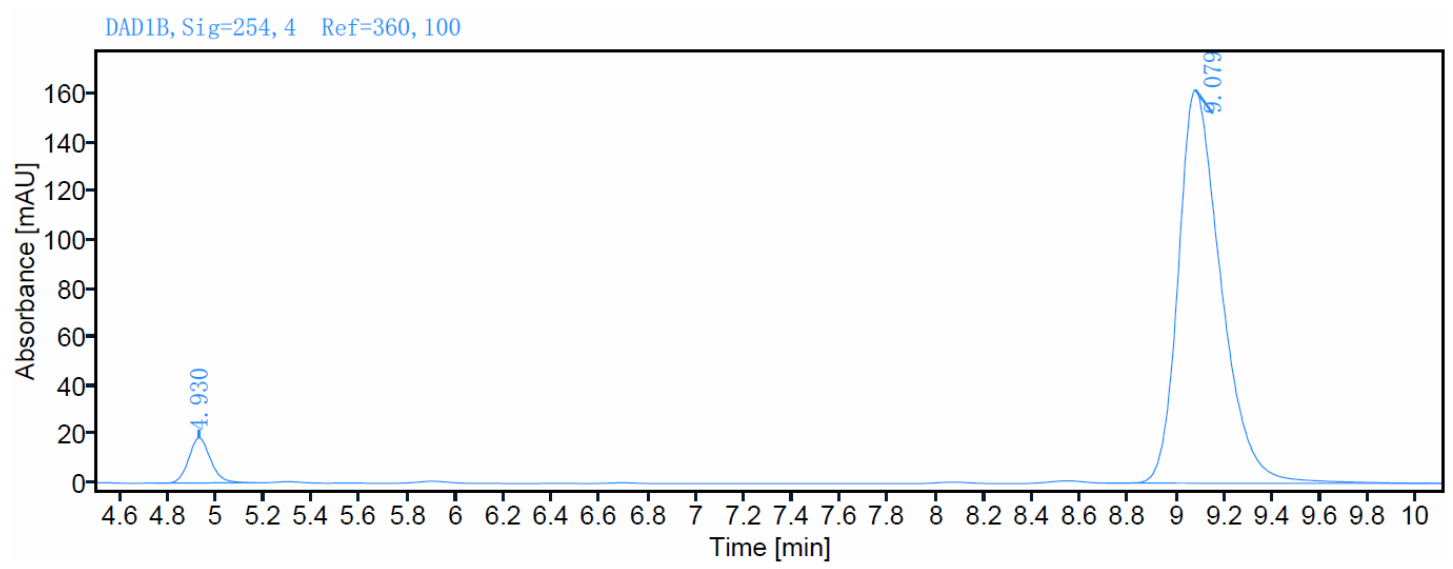

Signal: $\quad$ DAD1B, $\operatorname{Sig}=254,4 \quad$ Ref $=360,100$

$\begin{array}{rr}\text { Rentention Time[min] } & \text { Area[mAU*min] } \\ 4.930 & 116.84 \\ 9.079 & 1999.78\end{array}$

$\begin{array}{rr}\text { Height }[\mathrm{mAU}] & \text { Relative Area[\%] } \\ 18.60 & 5.52 \\ 162.03 & 94.48\end{array}$




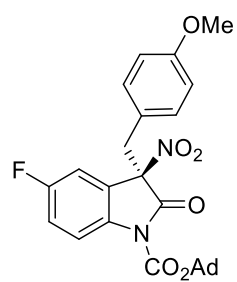

31

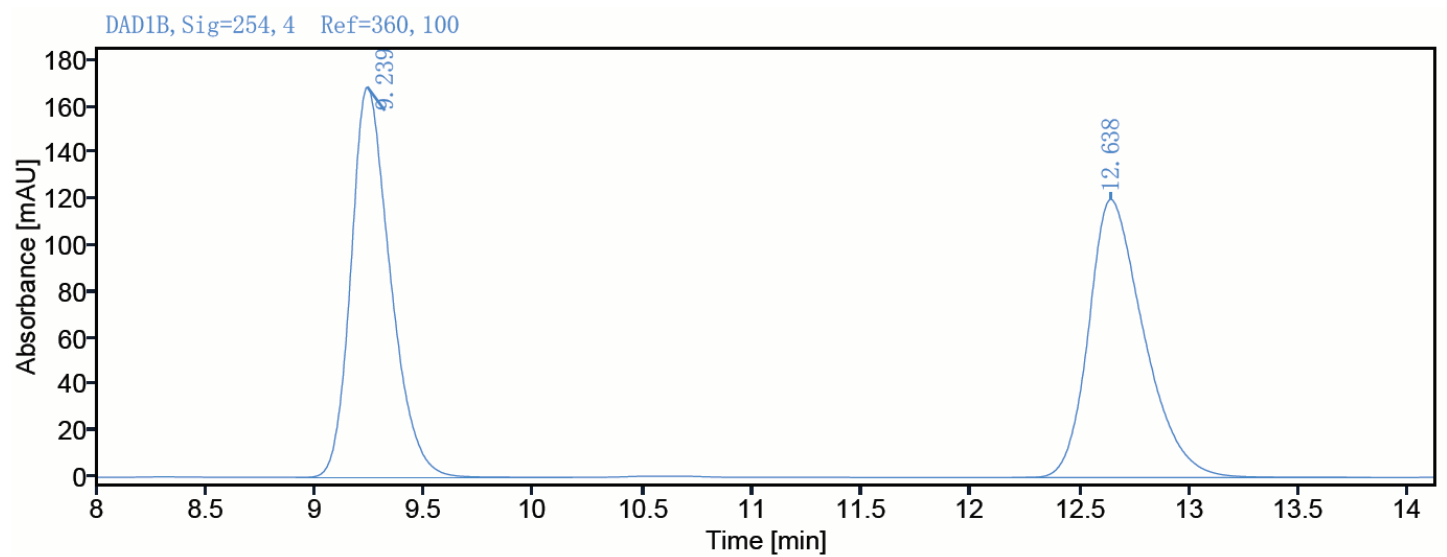

Signa1: $\quad$ DAD1B, Sig=254, 4 Ref=360, 100

$\begin{array}{rrrr}\text { Rentention Time[min] } & \text { Area[mAU*min] } & \text { Height [mAU] } & \text { Relative Area[\%] } \\ 9.239 & 2101.97 & 168.48 & 49.96 \\ 12.638 & 2105.06 & 120.11 & 50.04\end{array}$

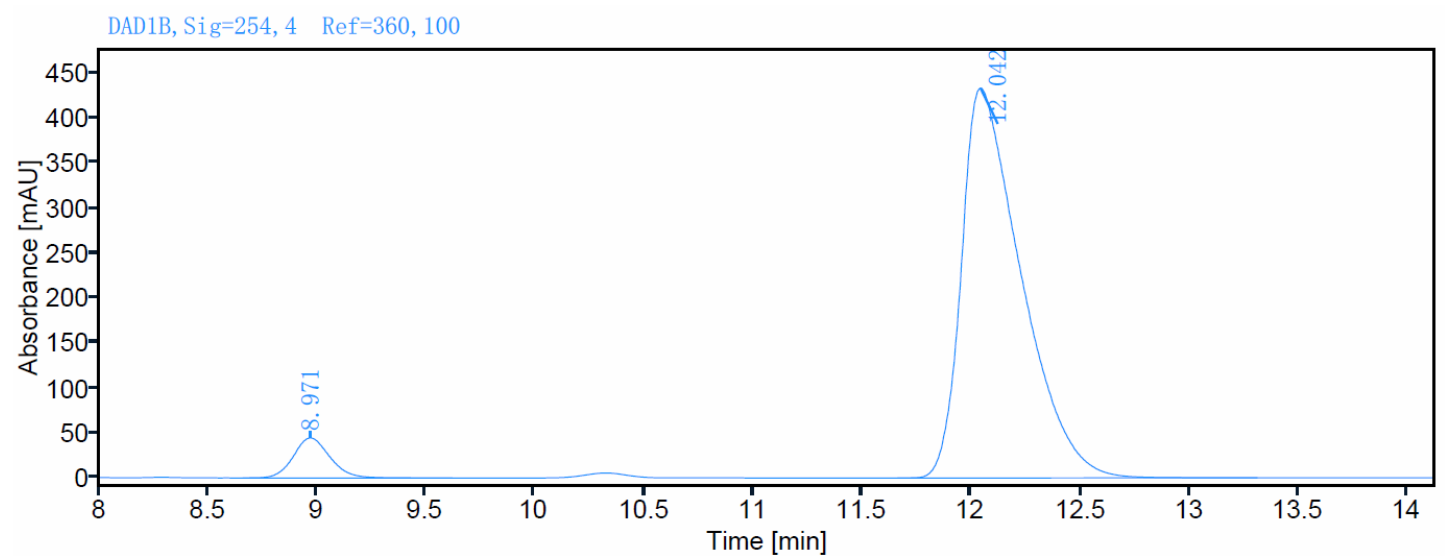

Signal: $\quad$ DAD1B, Sig=254, 4 Ref $=360,100$

$\begin{array}{rrrr}\text { Rentention Time[min] } & \text { Area[mAU*min] } & \text { Height [mAU] } & \text { Relative Area[\%] } \\ 8.971 & 517.61 & 44.49 & 5.92 \\ 12.042 & 8228.66 & 433.14 & 94.08\end{array}$


<smiles>CC(=O)N1C(=O)[C@](Cc2cc(C)cc(C)c2)([N+](=O)[O-])c2cc(F)ccc21</smiles>

$3 \mathrm{~m}$

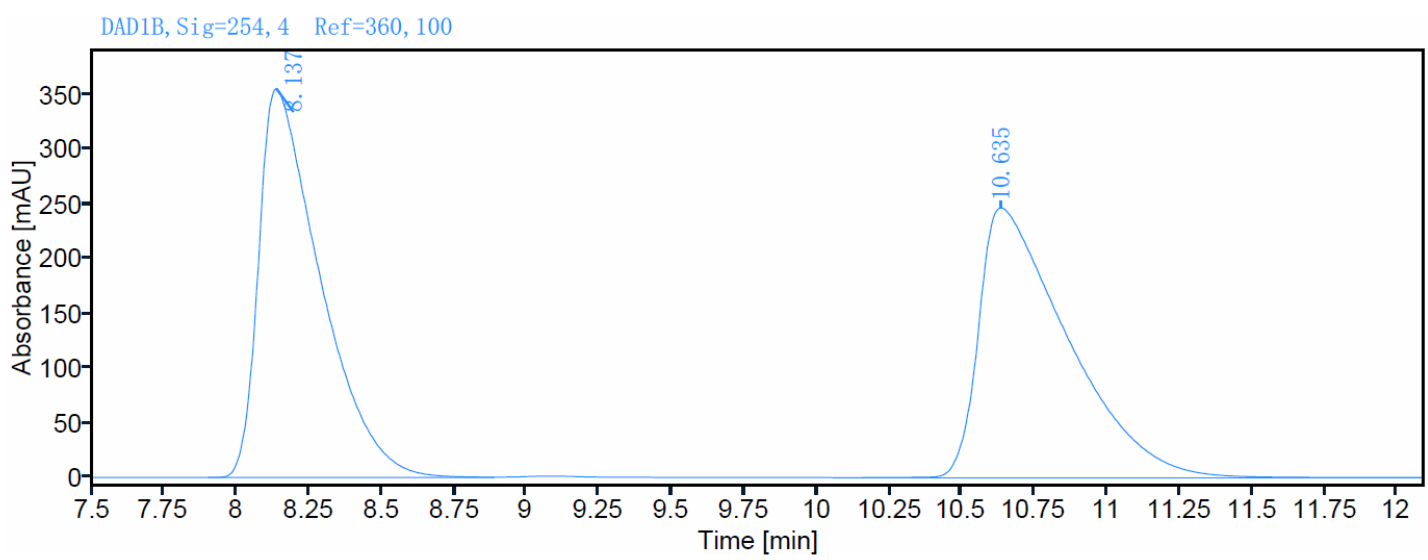

Signal: DAD1B, $\operatorname{Sig}=254,4 \quad \operatorname{Ref}=360,100$

Rentention Time[min]

8. 137

10. 635

DADIB, Sig $=254,4$ Ref $=360,100$

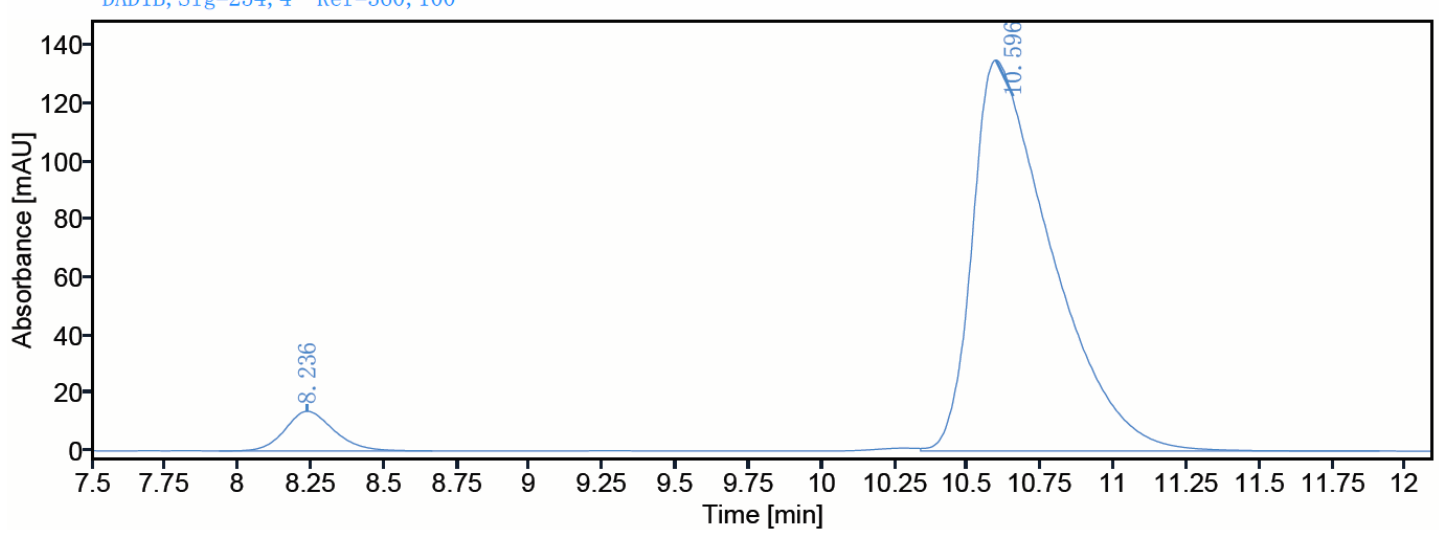

Signal: $\quad$ DAD1B, $\operatorname{Sig}=254,4 \quad \operatorname{Ref}=360,100$

Rentention Time[min]

8. 236

10. 596
Area [mAU*min]

159. 24

2549. 19
Height $[\mathrm{mAU}]$

13. 72

135. 53
Relative Area[\%]

5. 88

94.12 
<smiles>COc1cc(CS[C@@]2([N+](=O)[O-])C(=O)N(C(=O)OCc3ccccc3)c3ccc(F)cc32)cc(OC)c1</smiles>

3n

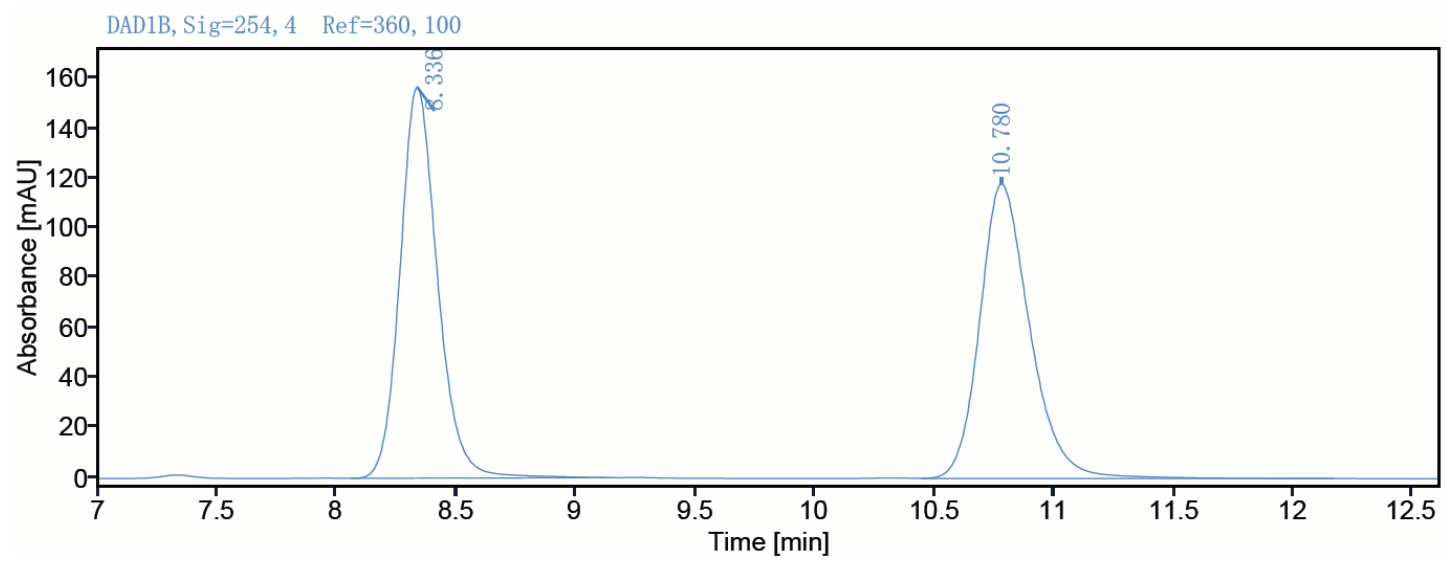

Signal: $\quad$ DAD1B, $S i g=254,4 \quad \operatorname{Ref}=360,100$

Rentention Time[min]

8. 336

10. 780

DAD1B, Sig=254, $4 \quad$ Ref $=360,100$

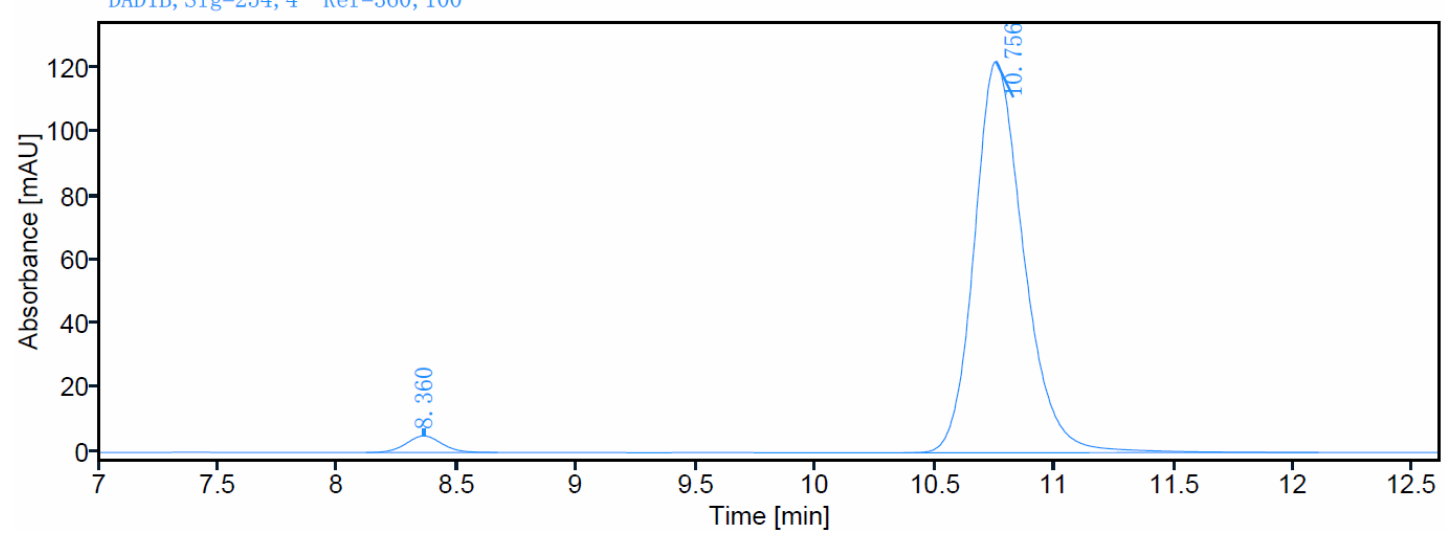

Signal: DAD1B, Sig=254, $4 \quad \operatorname{Ref}=360,100$

Rentention Time[min]
8. 360
10. 756

Area [mAU*min]

1706. 65

1721. 52
Height [mAU]

156. 75

118. 01
Relative Area[\%]

49. 78

50. 22 


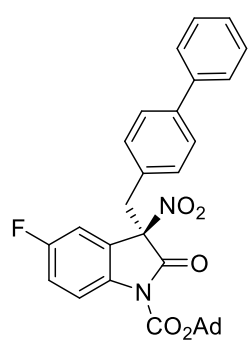

30

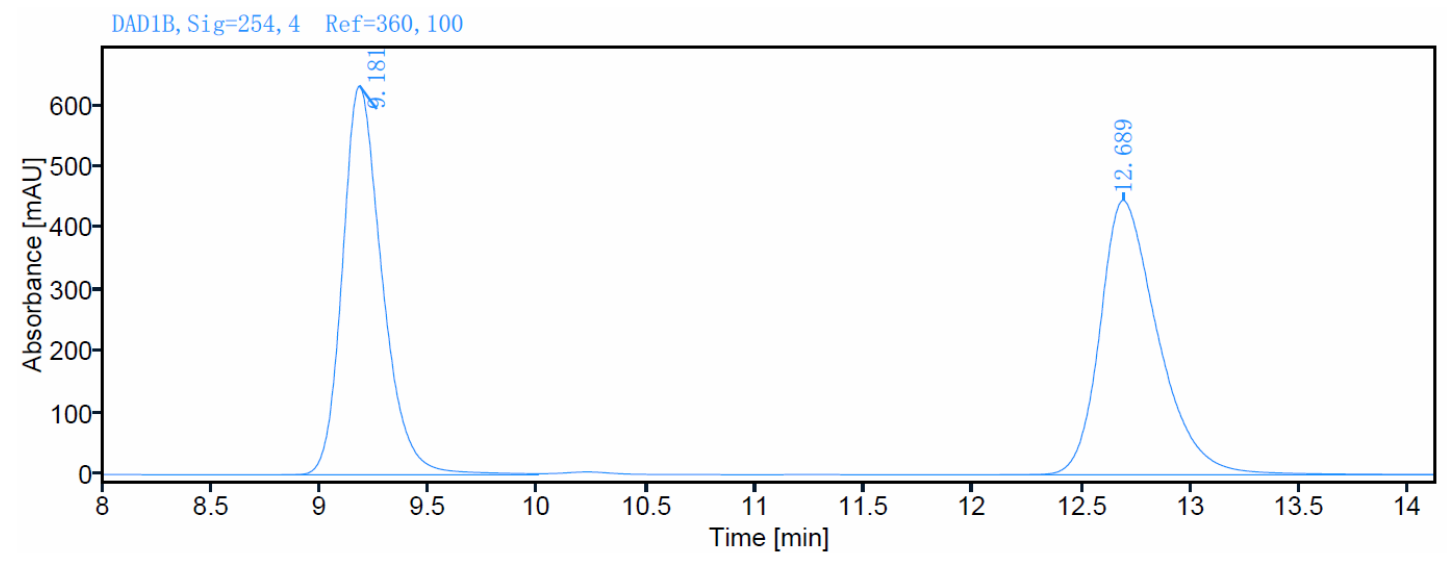

Signal: DAD1B, Sig=254, 4 Ref=360, 100

$\begin{array}{rrrr}\text { Rentention Time[min] } & \text { Area[mAU*min] } & \text { Height[mAU] } & \text { Relative Area[\%] } \\ 9.181 & 8010.33 & 631.78 & 49.28 \\ 12.689 & 8244.52 & 445.80 & 50.72\end{array}$

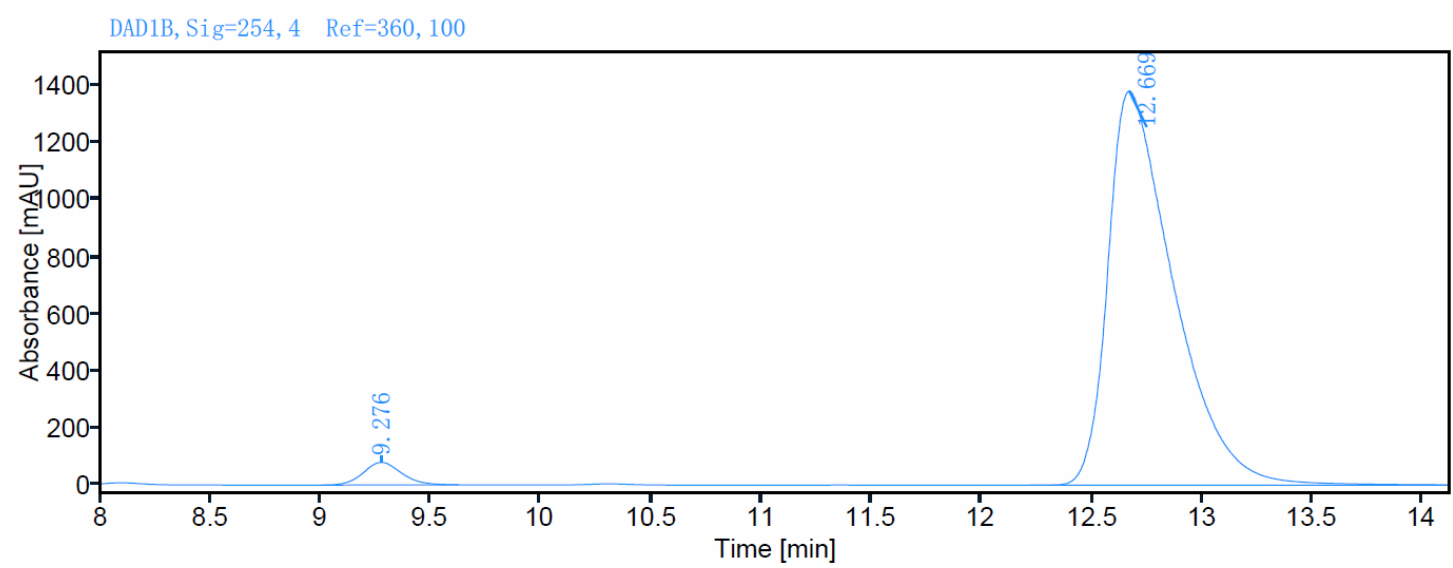

Signal: DAD1B, Sig=254, 4 Ref $=360,100$

Rentention Time[min]

9. 276

12. 669

Area [mAU*min]

950.94

28620.84
Height [mAU]

79.28

1382. 75
Relative Area[\%]

3. 22

96. 78 
<smiles></smiles>

$3 p$

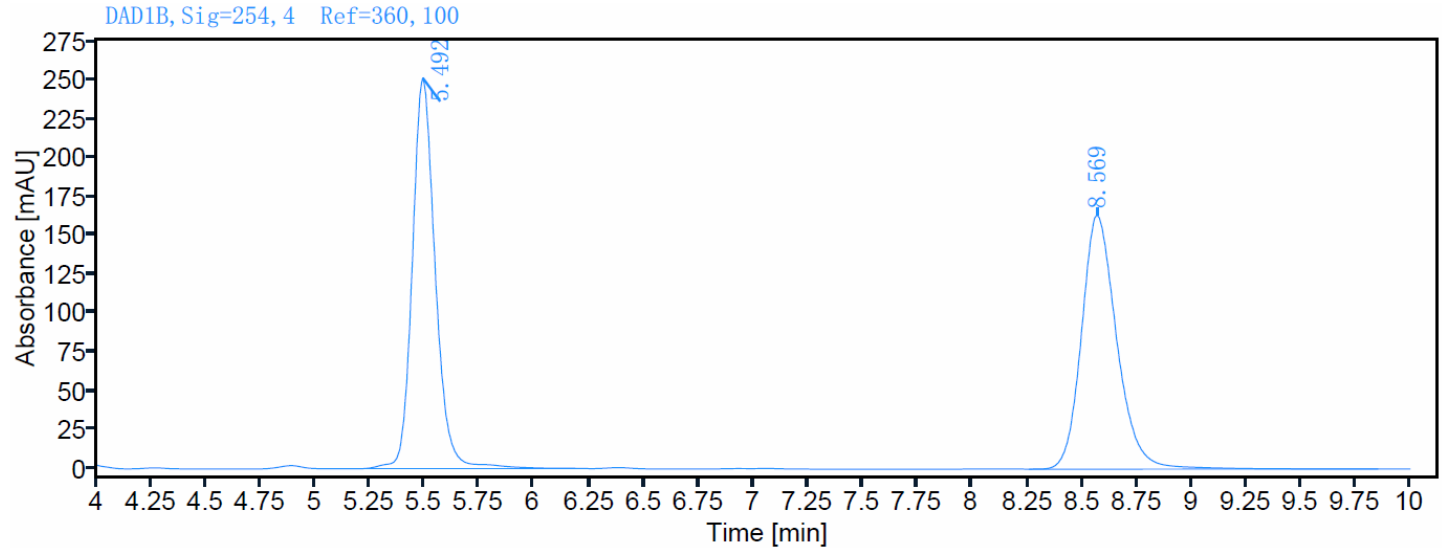

Signal: DAD1B, Sig $=254,4 \quad \operatorname{Ref}=360,100$

$\begin{array}{rrrr}\text { Rentention Time[min] } & \text { Area[mAU*min] } & \text { Height[mAU] } & \text { Relative Area[\%] } \\ 5.492 & 1861.42 & 252.36 & 50.36 \\ 8.569 & 1834.87 & 163.79 & 49.64\end{array}$

DAD1B, Sig=254, $4 \quad \operatorname{Ref}=360,100$

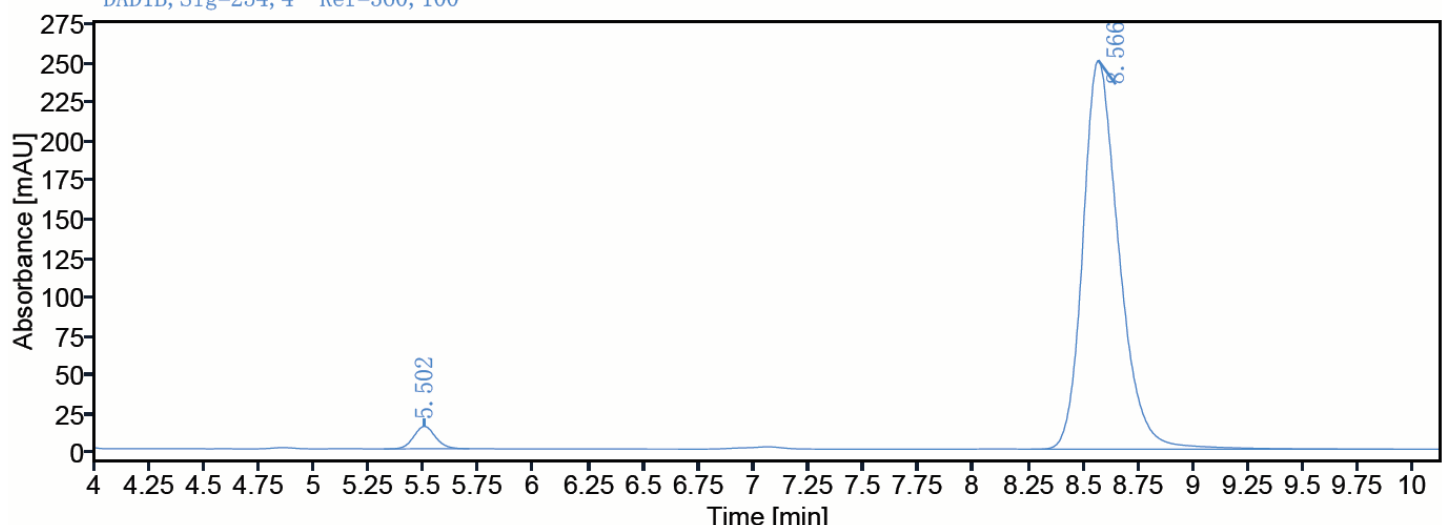

Signa1: DAD1B, Sig=254, $4 \quad \operatorname{Ref}=360,100$

Rentention Time[min]

5. 502

8. 566
Area [mAU*min]

98. 46

2840. 40
Height [mAU]

14. 39

249. 38

\section{Relative Area[\%]}

3. 35

96.65 
<smiles>CC(=O)OC1Nc2ccc(F)cc2[C@@]1(Cc1cc2ccccc2s1)[N+](=O)[O-]</smiles>

$3 q$

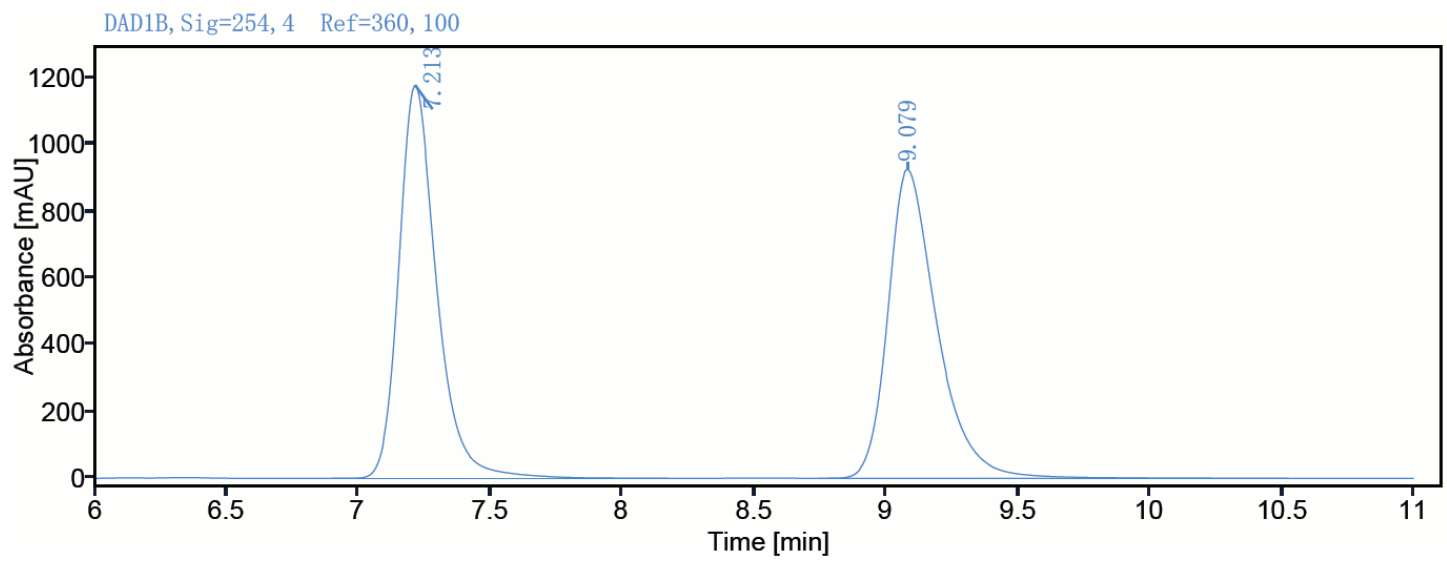

Signal: DAD1B, Sig=254, $4 \quad$ Ref $=360,100$

$\begin{array}{rrrr}\text { Rentention Time[min] } & \text { Area[mAU*min] } & \text { Height [mAU] } & \text { Relative Area[\%] } \\ 7.213 & 11722.74 & 1177.47 & 49.45 \\ 9.079 & 11982.82 & 925.73 & 50.55\end{array}$

DAD1B, Sig=254, $4 \quad$ Ref $=360,100$

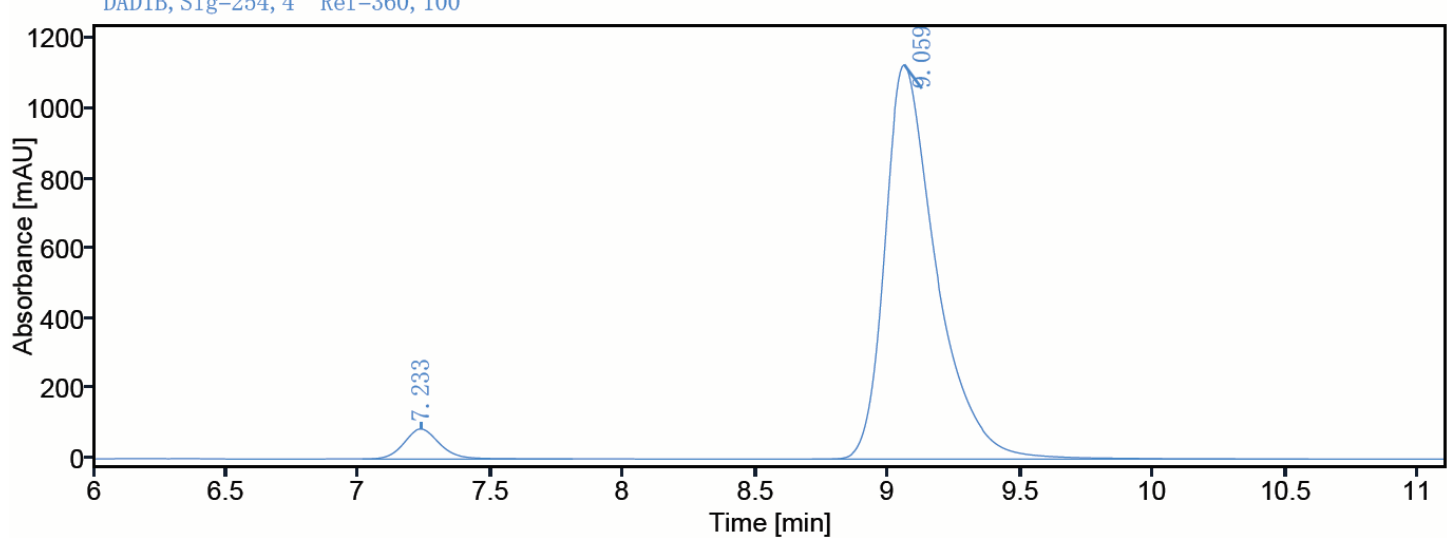

Signal: $\quad$ DAD1B, Sig=254, $4 \quad$ Ref $=360,100$

Rentention Time[min]

7. 233

9. 059
Area [mAU*min]

816. 89

15179.63
Height [mAU]

85. 98

1126. 16
Relative Area[\%]

5. 11

94.89 


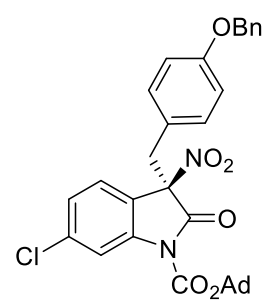

$3 r$

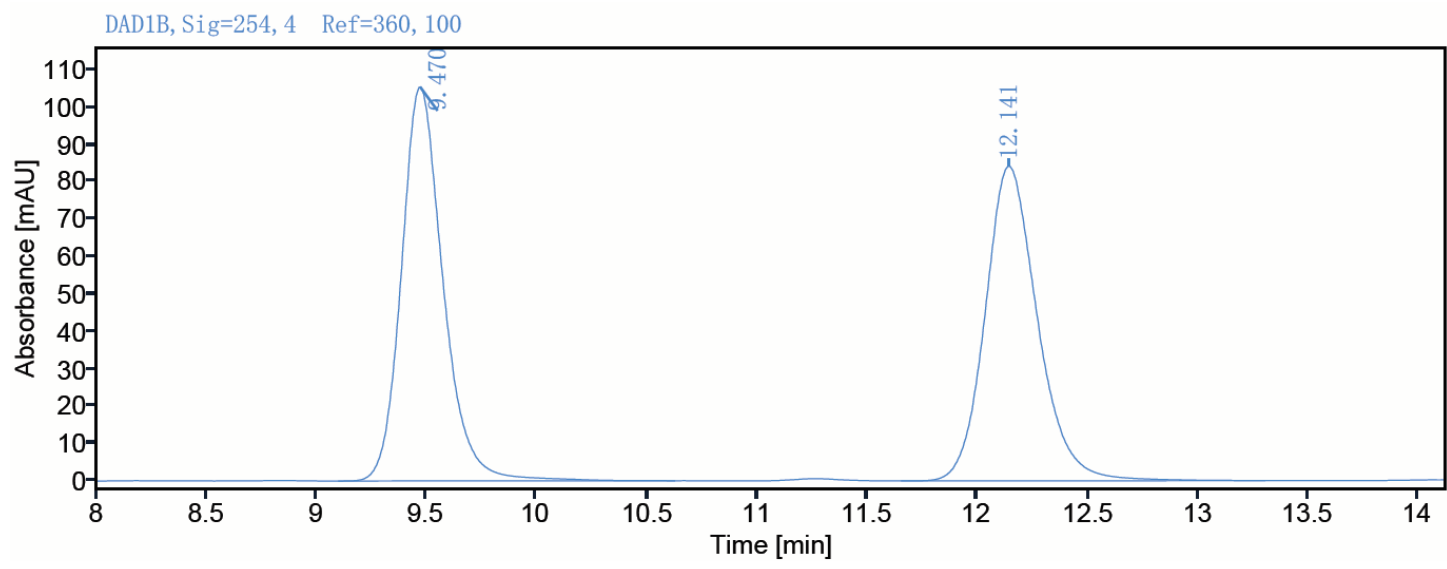

Signal: $\quad$ DAD1B, Sig=254, $4 \quad \operatorname{Ref}=360,100$

Rentention Time[min]

9. 470

12. 141

$=360,100$

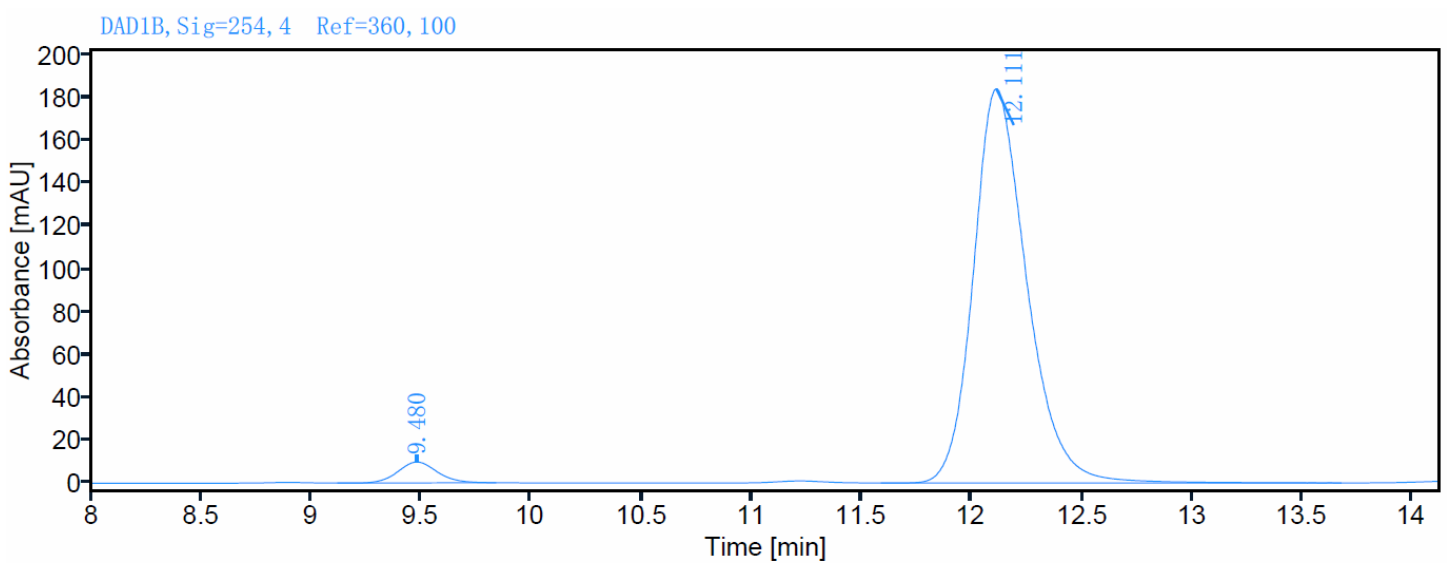

Height [mAU]

105. 40

84.17
Relative Area[\%]

49. 94

50.06
1385. 62

1389. 15

Signal: $\quad$ DAD1B, Sig=254, 4 Ref $=360,100$

Rentention Time[min]

9. 480

12. 111
Area [mAU*min]

120.90

3106.62 


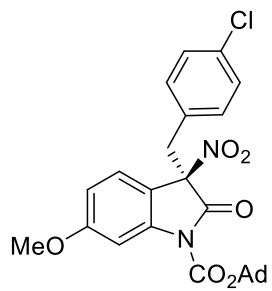

$3 s$

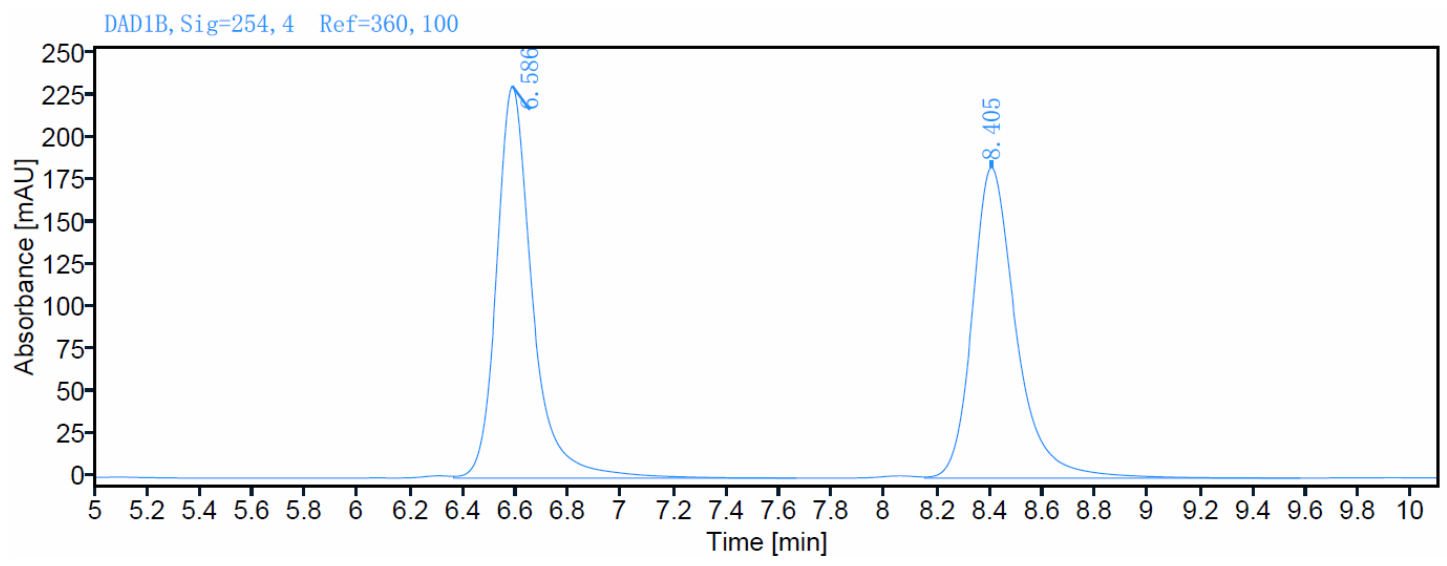

Signal: DAD1B, Sig=254, $4 \quad$ Ref $=360,100$

$\begin{array}{rrrr}\text { Rentention Time[min] } & \text { Area[mAU*min] } & \text { Height[mAU] } & \text { Relative Area[\%] } \\ 6.586 & 2185.34 & 231.70 & 50.75 \\ 8.405 & 2120.54 & 183.73 & 49.25\end{array}$

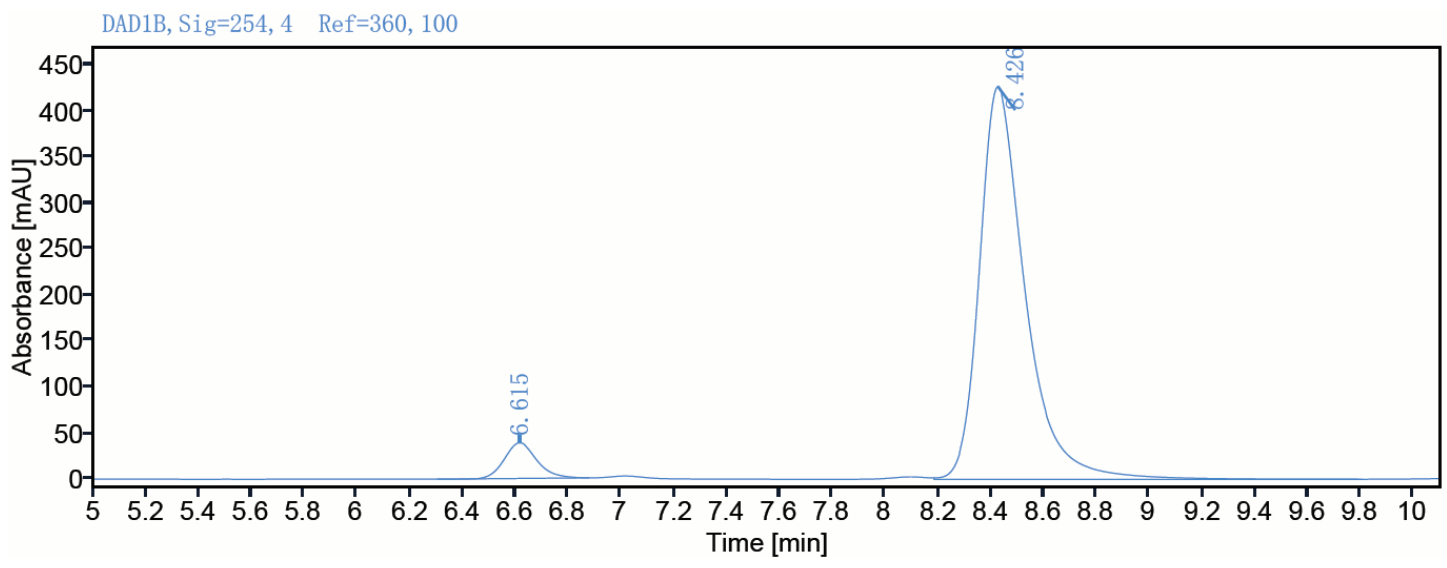

Signal: $\quad$ DAD1B, Sig=254, $4 \quad$ Ref $=360,100$

\section{Rentention Time[min]}

6.615

8. 426
Area $[\mathrm{mAU} * \min ]$

329. 47

5123. 04
Height [mAU]

38.92

427. 39
Relative Area[\%]

6. 04

93. 96 


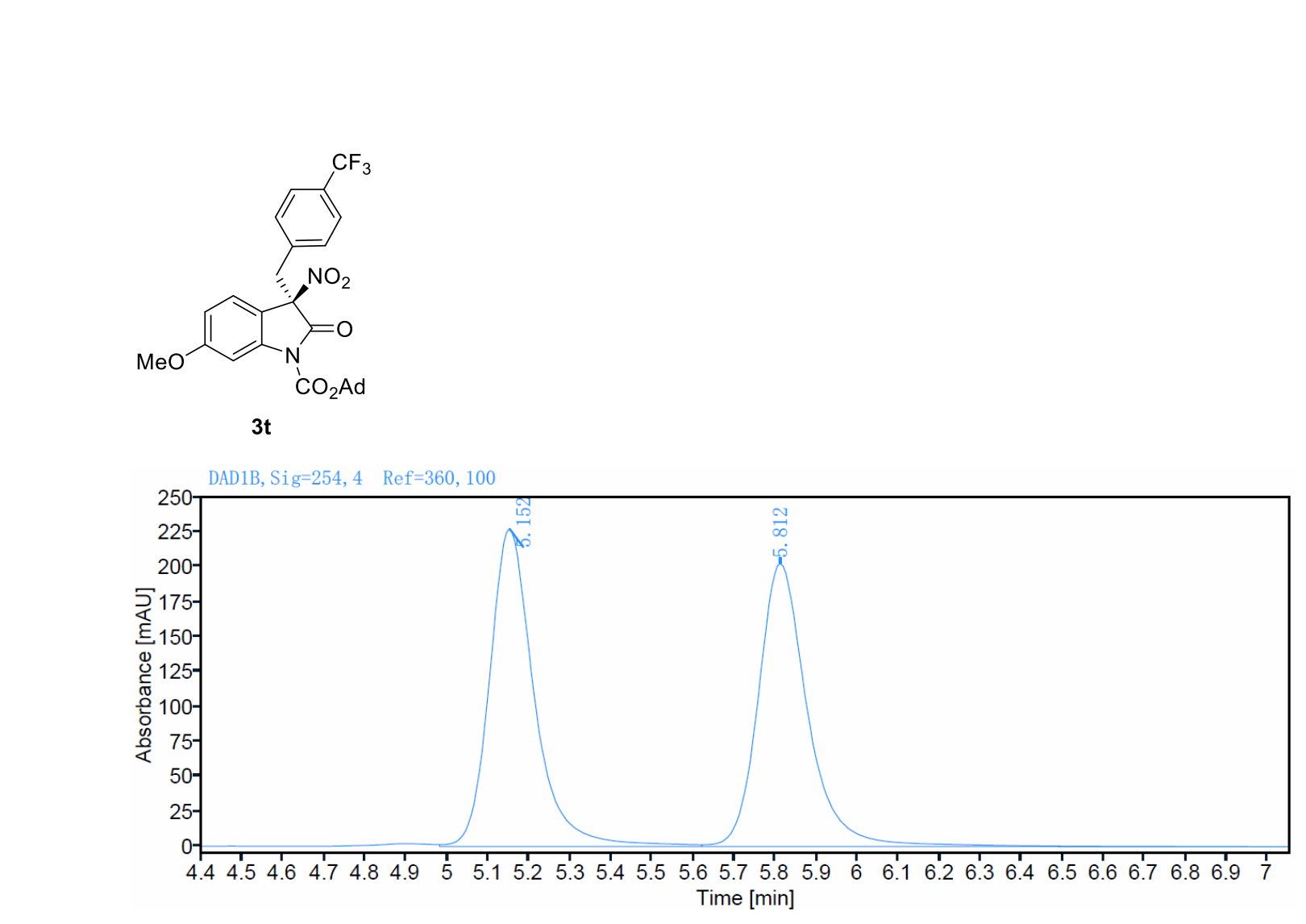

$3 t$

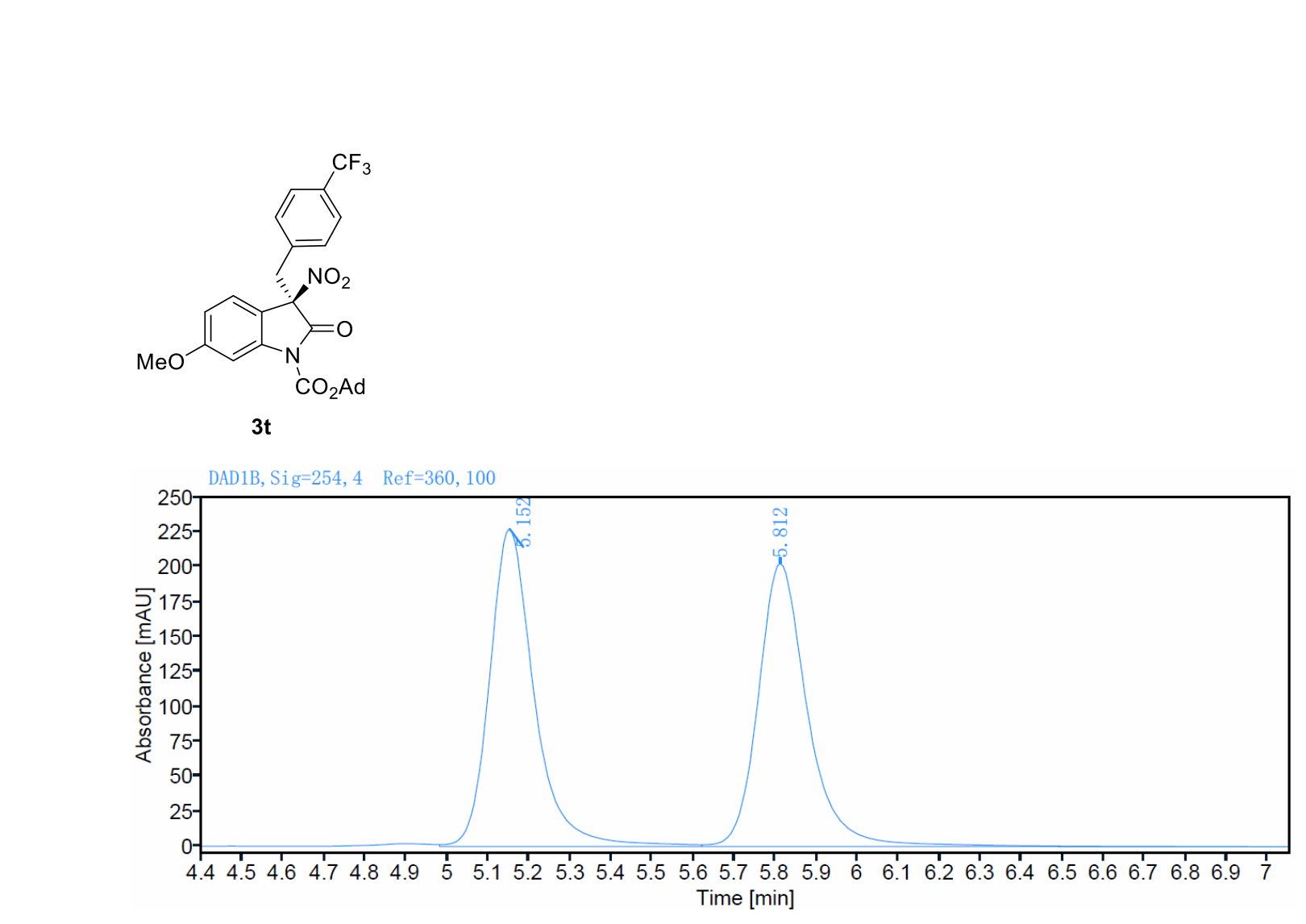

Signal: $\quad$ DAD1B, $\operatorname{Sig}=254,4 \quad \operatorname{Ref}=360,100$

Rentention Time[min]

5. 152

5.812

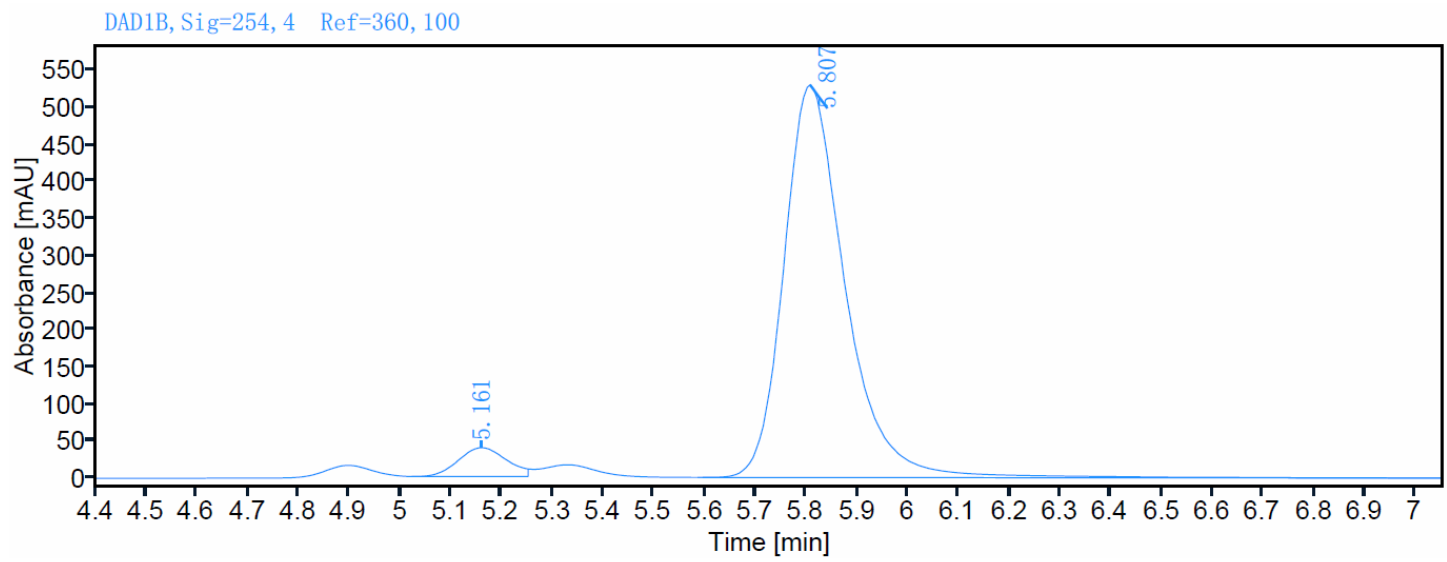

Relative Area[\%]

50.38

49. 62
227. 30

202. 36

Signal: $\quad$ DAD1B, Sig=254, $4 \quad \operatorname{Ref}=360,100$

Rentention Time[min]

5. 161

5. 807
Area [mAU*min]

254.78

4456. 71
Height [mAU]

38.88

529.96

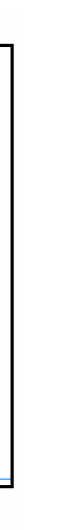

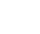


<smiles>O=C([O-])OC(=O)N1C(=O)[C@](Cc2ccc(Br)cc2)(c2ccccc2)c2ccc(-c3ccccc3)cc21</smiles>

$3 u$

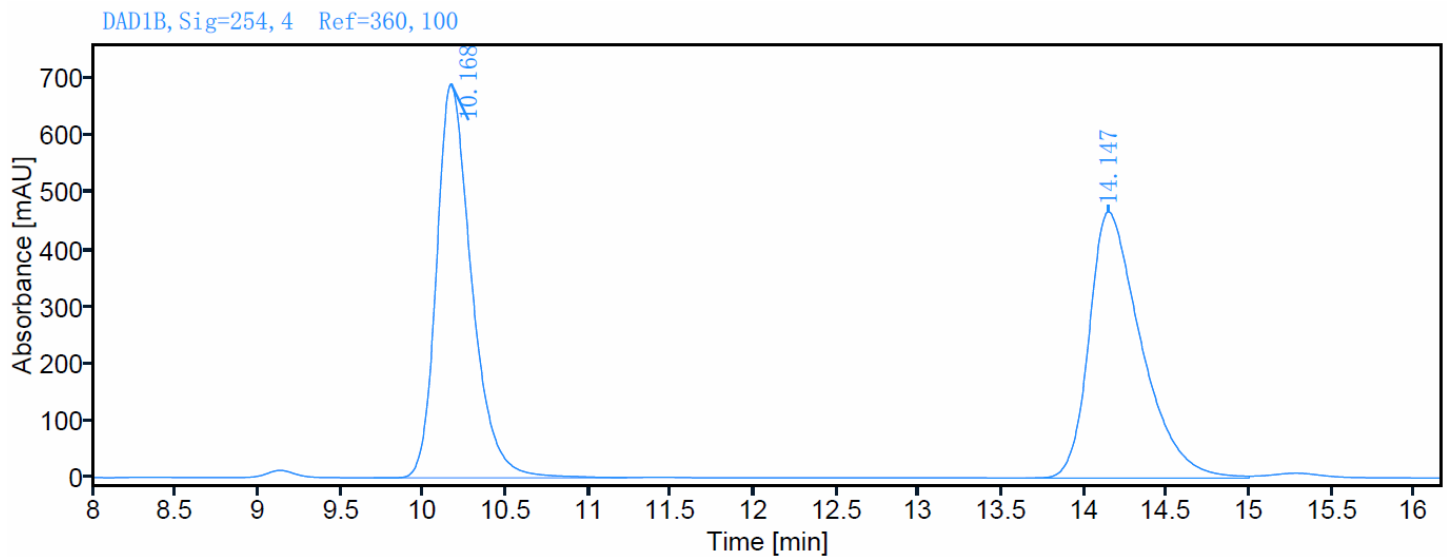

Signal: $\quad$ DAD1B, $\operatorname{Sig}=254,4 \quad$ Ref $=360,100$

Rentention Time[min]

10. 168

14. 147

(4)

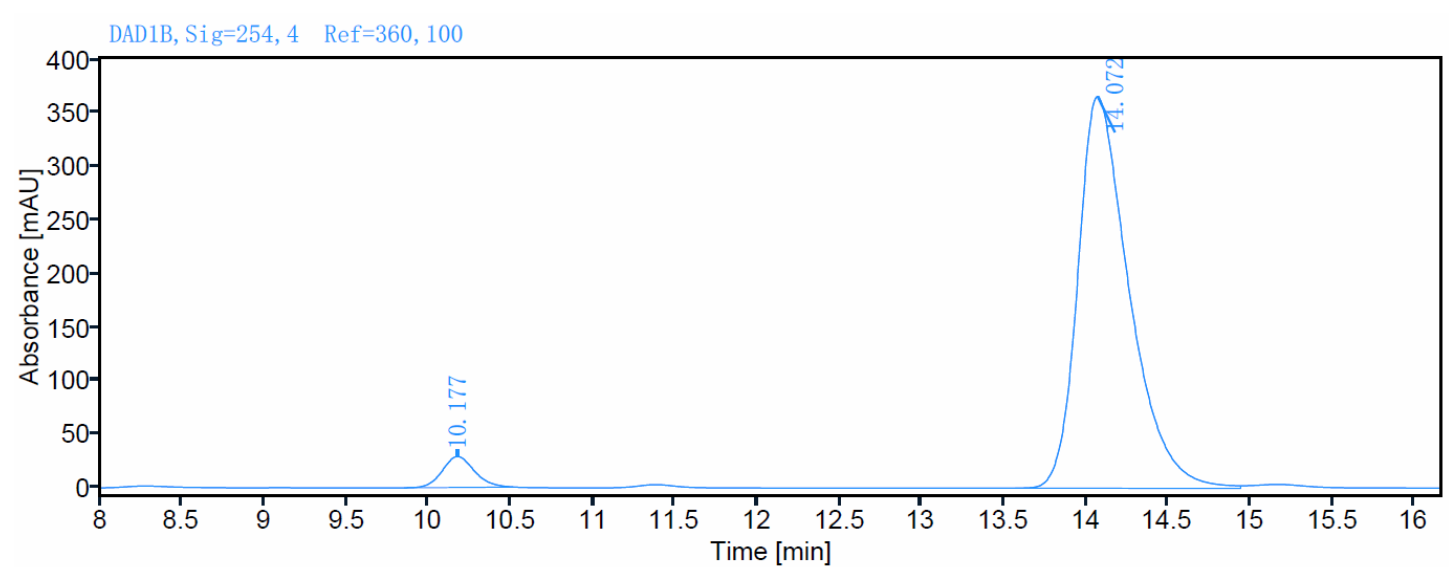

Signal: DAD1B, $\operatorname{Sig}=254,4 \quad \operatorname{Ref}=360,100$

$\begin{array}{rrrr}\text { Rentention Time[min] } & \text { Area[mAU*min] } & \text { Height }[\mathrm{mAU}] & \text { Relative Area[\%] } \\ 10.177 & 392.69 & 28.99 & 4.70 \\ 14.072 & 7962.27 & 366.50 & 95.30\end{array}$




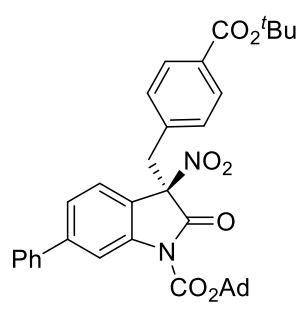

$3 v$

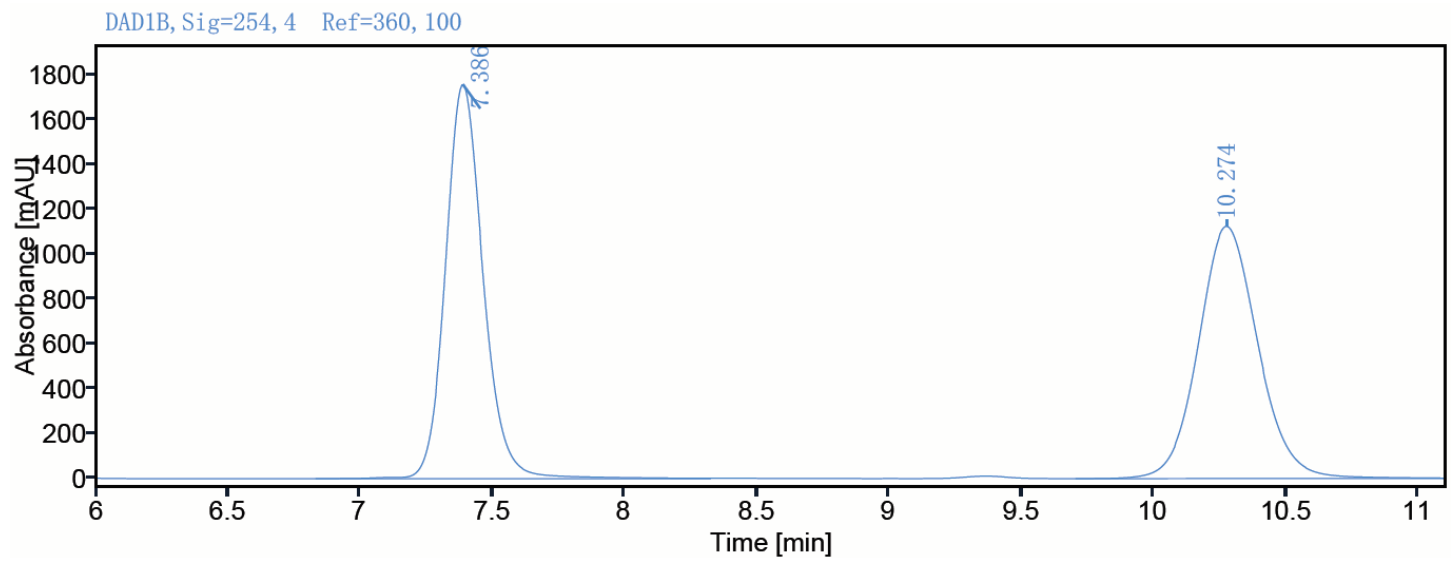

Signal: $\quad$ DAD1B, Sig=254, $4 \quad \operatorname{Ref}=360,100$

Rentention Time[min]

7. 386

10. 274
Area [mAU*min]

17412.15

17587.68
Height [mAU]

1755. 37

1123. 16
Relative Area[\%]

49. 75

50.25

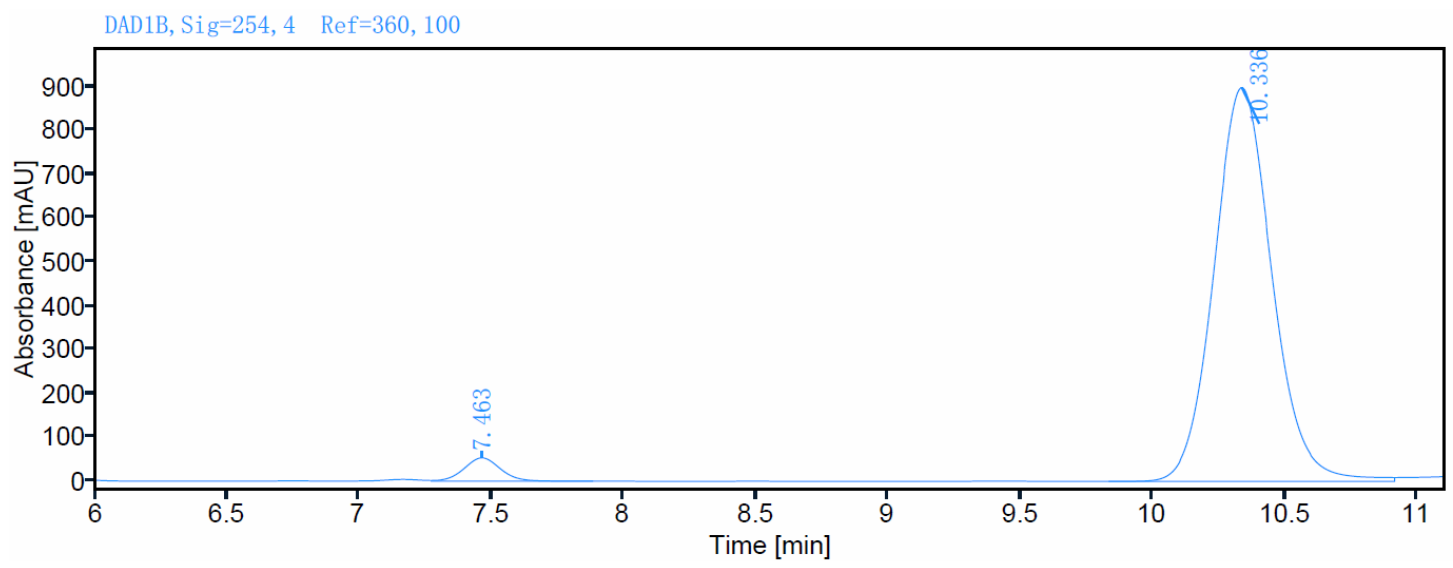

Signal: $\quad$ DAD1B, Sig=254, 4 Ref $=360,100$

Rentention Time[min]

7.463

10. 336
Area [mAU*min]

514.04

13680.84
Height [mAU]

53. 46

897.63
Relative Area[\%]

3. 62

96.38 


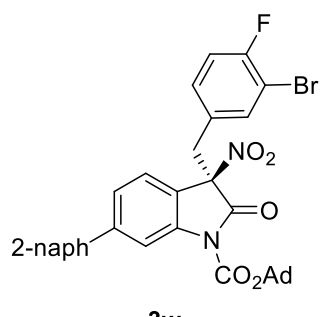

$3 w$

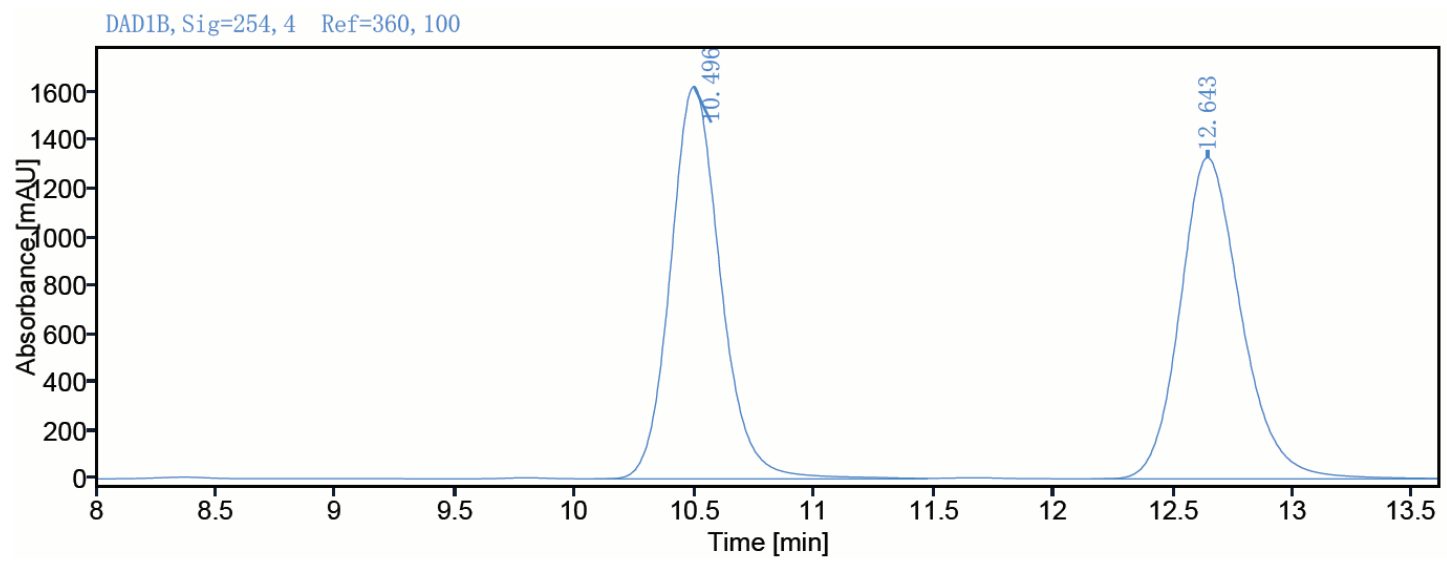

Signal: $\quad$ DAD1B, Sig=254, $4 \quad$ Ref=360, 100

Rentention Time[min]

10. 496

12. 643
Area [mAU*min]

23675. 17

23646. 14
Height [mAU]

1627. 29

1331.83
Relative Area[\%]

50.03

49. 97

DAD1B, Sig=254, $4 \quad$ Ref $=360,100$

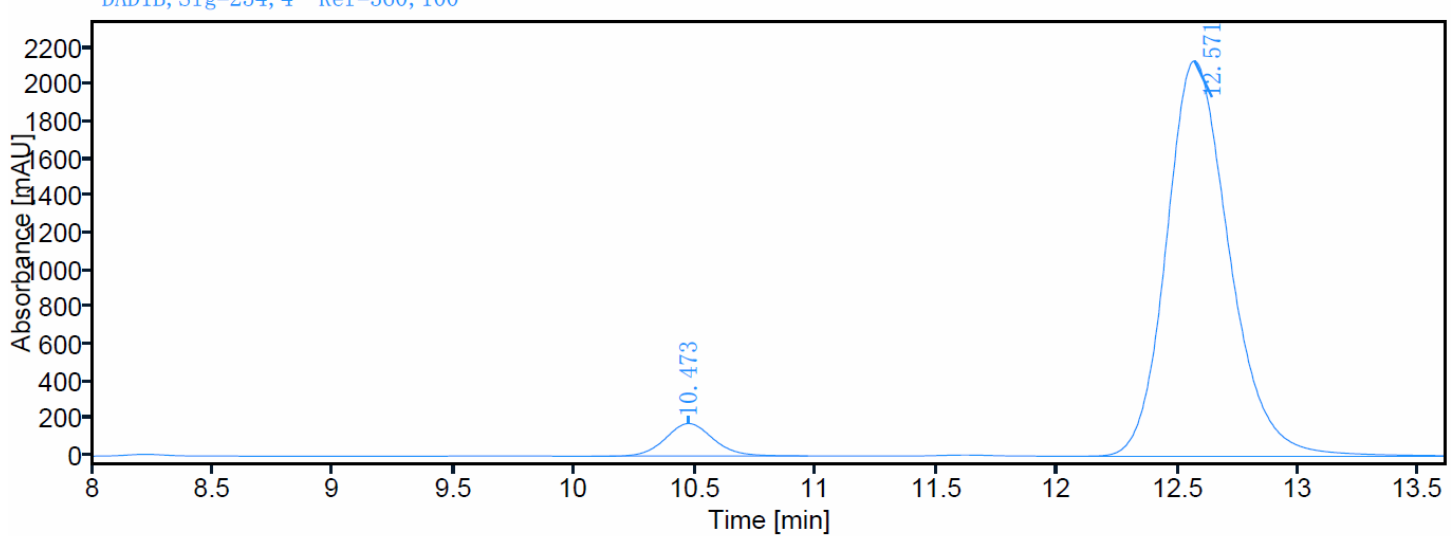

Signa1: DAD1B, Sig=254, 4 Ref=360, 100

Rentention Time[min]

10. 473

12.571
Area $[\mathrm{mAU} * \min ]$

2420. 42

38655. 13
Height [mAU]

175. 37

2130. 26
Relative Area[\%]

5.89

94. 11 

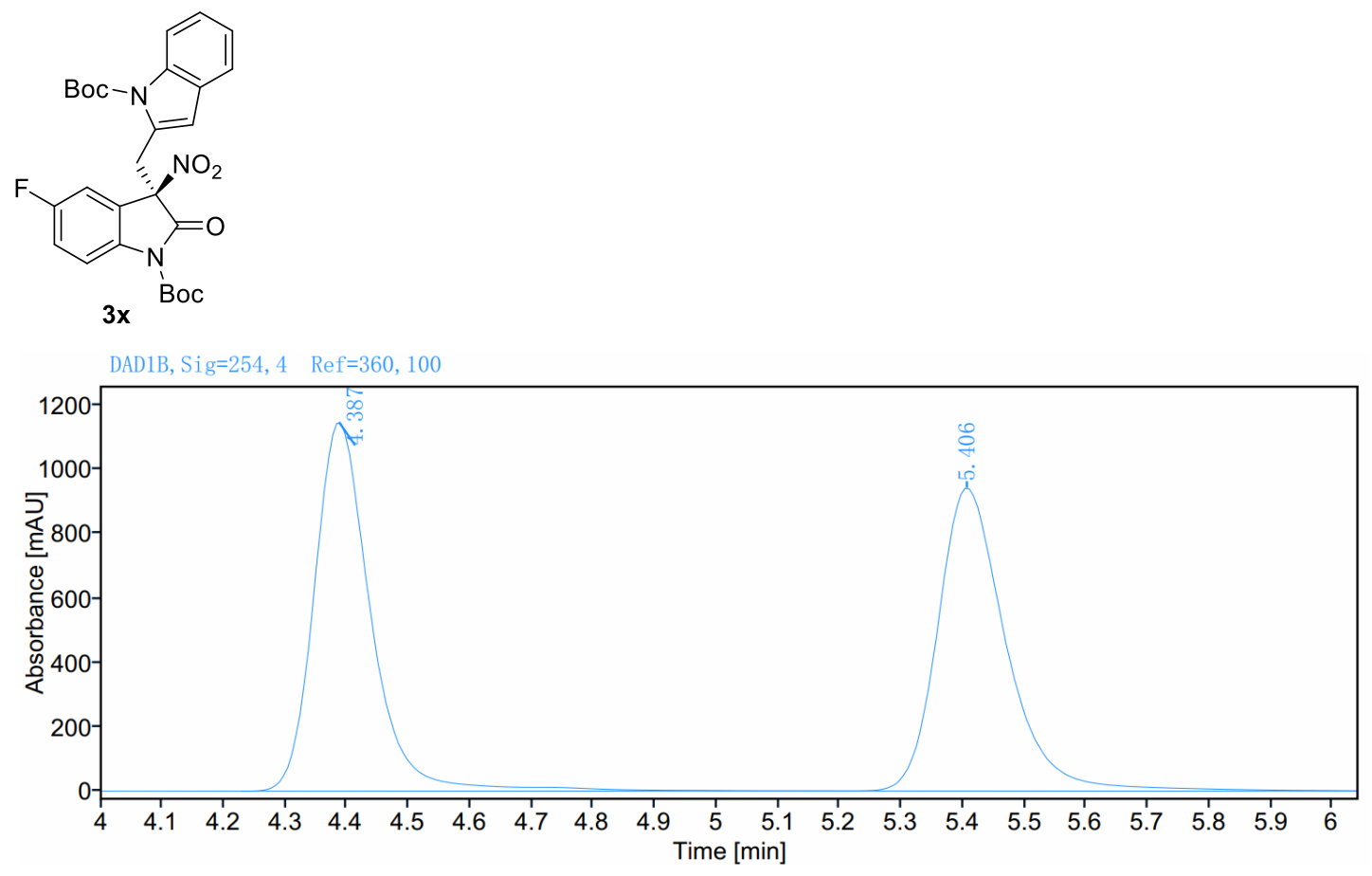

Signa1: DAD1B, Sig=254, 4 Ref=360, 100

$\begin{array}{rrrr}\text { Rentention Time[min] } & \text { Area[mAU*min] } & \text { Height [mAU] } & \text { Relative Area[\%] } \\ 4.387 & 7297.52 & 1147.20 & 50.12 \\ 5.406 & 7261.40 & 942.43 & 49.88\end{array}$

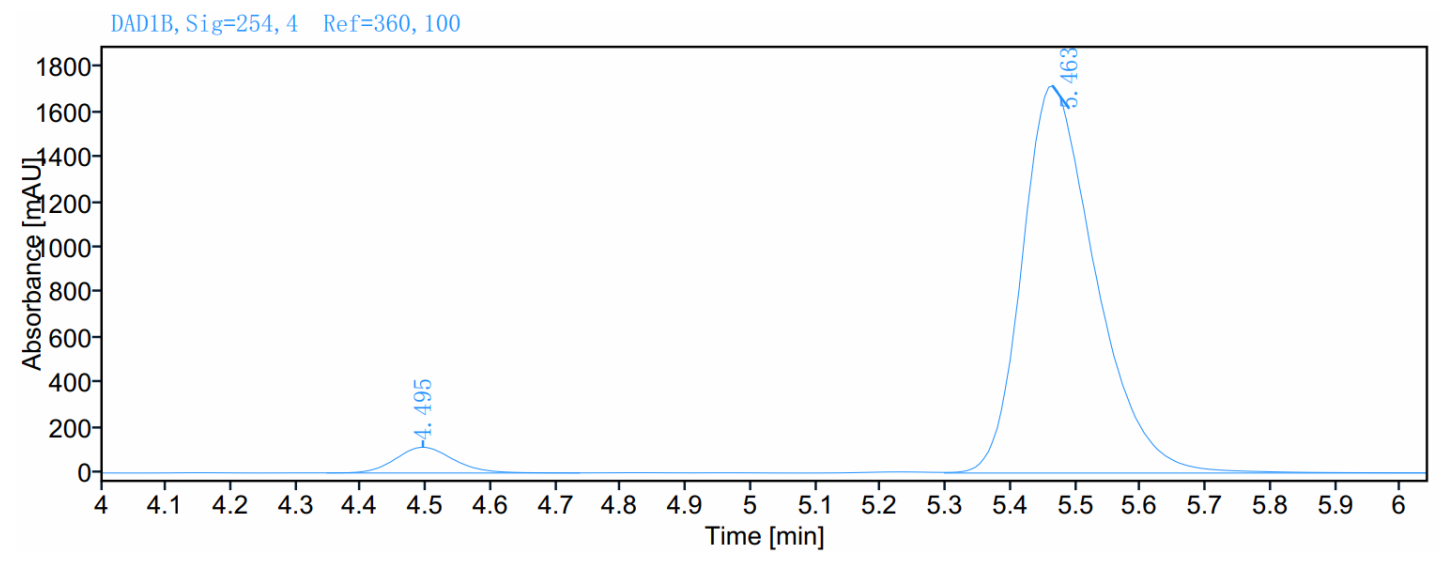

Signal: $\quad$ DAD1B, Sig=254, $4 \quad$ Ref $=360,100$

$\begin{array}{rrrr}\text { Rentention Time[min] } & \text { Area[mAU*min] } & \text { Height[mAU] } & \text { Relative Area[\%] } \\ 4.495 & 710.40 & 114.38 & 5.00 \\ 5.463 & 13491.70 & 1720.30 & 95.00\end{array}$




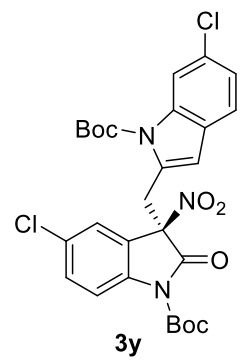

DAD1B, Sig=254, 4 Ref=360, 100

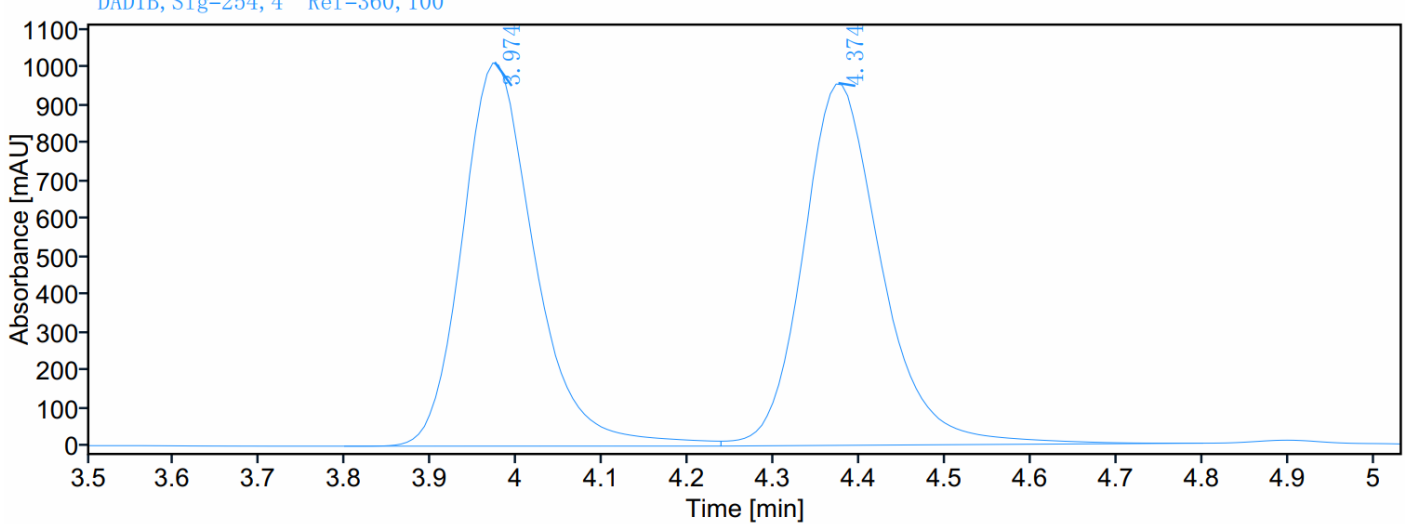

Signal: $\quad$ DAD1B, Sig=254, $4 \quad$ Ref=360, 100

$\begin{array}{rrrr}\text { Rentention Time[min] } & \text { Area[mAU*min] } & \text { Height[mAU] } & \text { Relative Area[\%] } \\ 3.974 & 5956.12 & 1013.08 & 49.51 \\ 4.374 & 6072.86 & 956.02 & 50.49\end{array}$

DAD1B, Sig=254, $4 \quad \operatorname{Ref}=360,100$

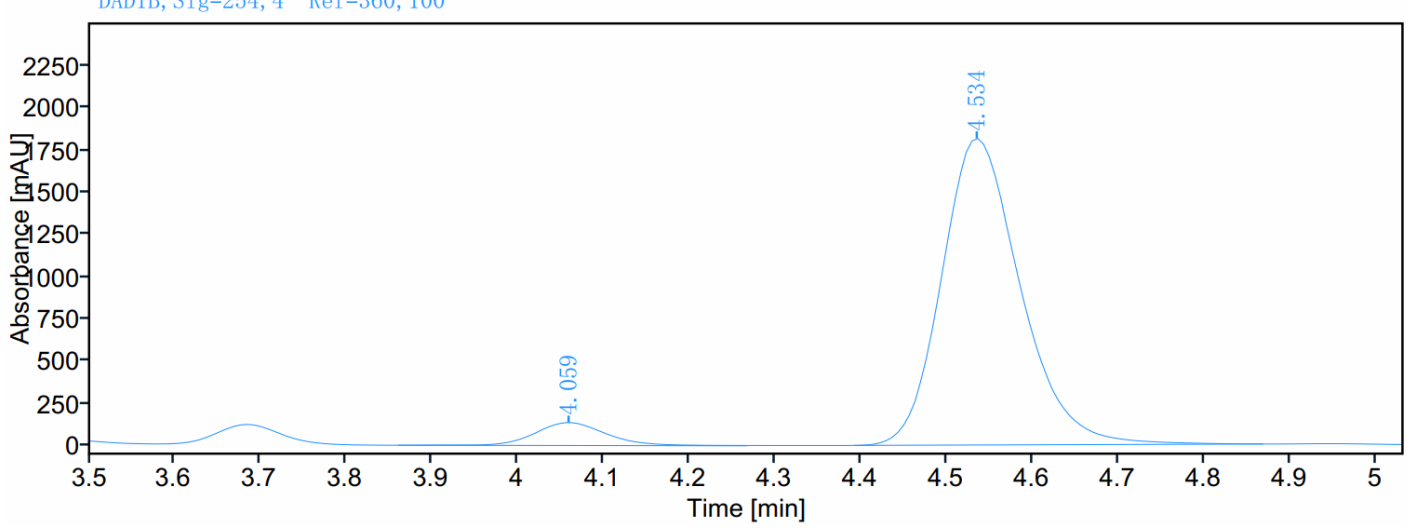

Signal: $\quad$ DAD1B, Sig=254, $4 \quad$ Ref $=360,100$

Rentention Time[min]

4. 059

4. 534
Area[mAU*min]

787.09

11531.54
Height [mAU]

135. 66

1813. 99
Relative Area[\%]

6. 39

93.61 


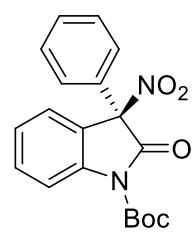

3za

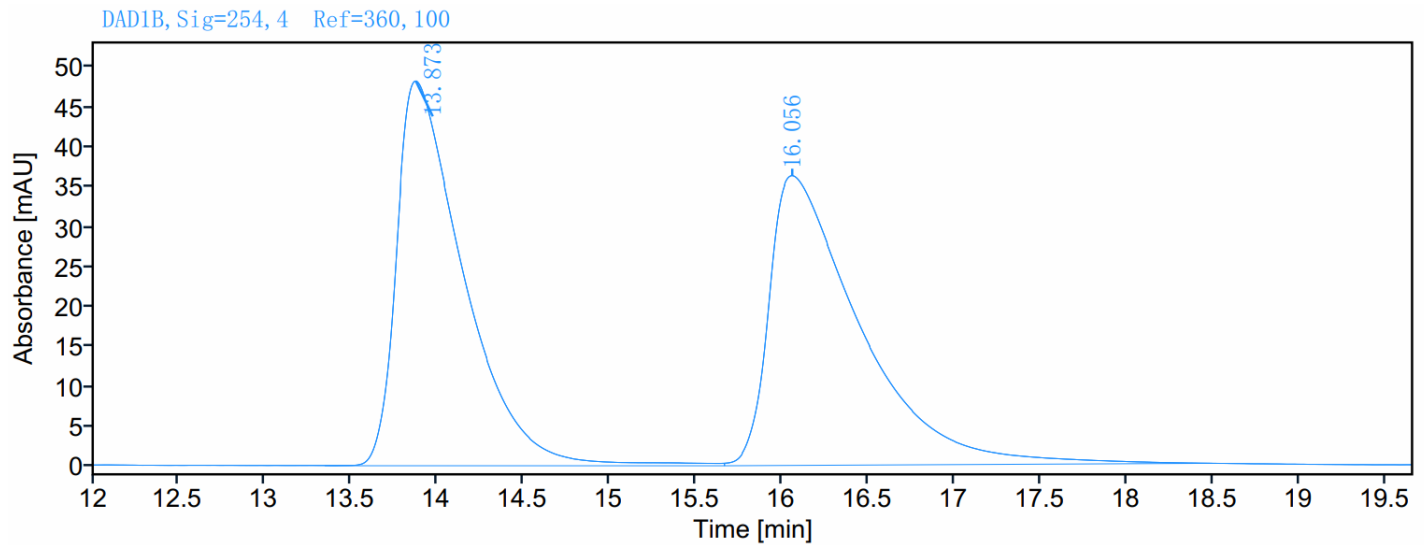

Signal: $\quad$ DAD1B, Sig=254, 4 Ref=360, 100

$\begin{array}{rrrr}\text { Rentention Time[min] } & \text { Area[mAU*min] } & \text { Height[mAU] } & \text { Relative Area[\%] } \\ 13.873 & 1304.14 & 48.24 & 49.48 \\ 16.056 & 1331.61 & 36.33 & 50.52\end{array}$

DAD1B, Sig=254, $4 \quad$ Ref $=360,100$

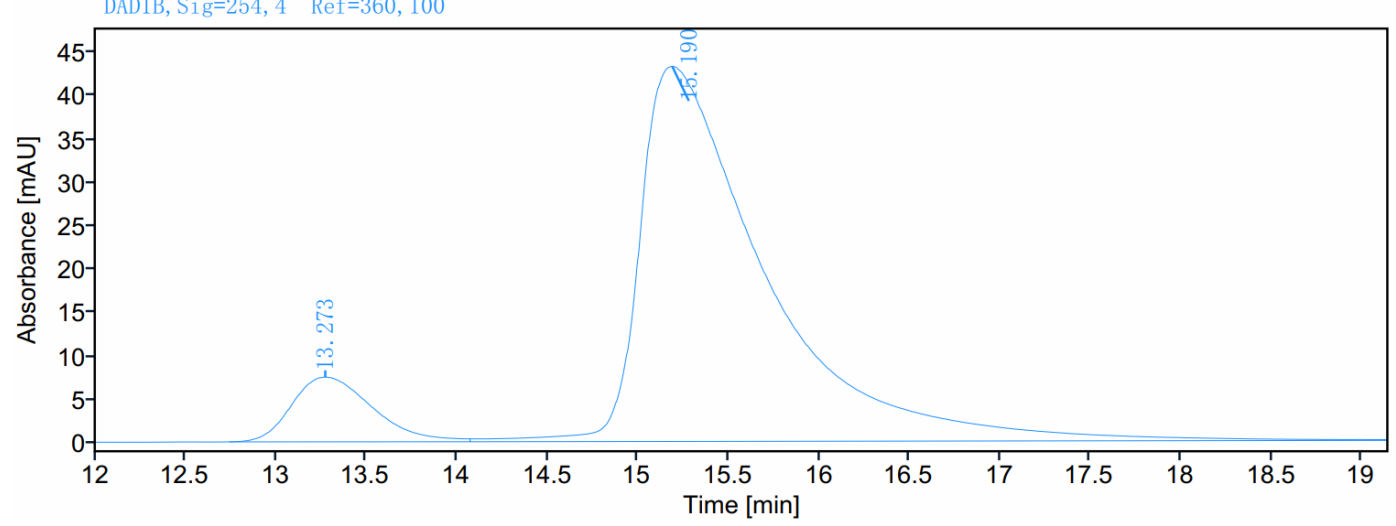

Signal: $\quad$ DAD1B, Sig=254, $4 \quad$ Ref $=360,100$

Rentention Time[min]

13. 273

15. 190

Area $[\mathrm{mAU} * \min ]$

230.67

2084. 59
Height [mAU]

7. 45

43. 18

Relative Area[\%]

9. 96

90. 04 
<smiles>Cc1ccc2c(c1)C(c1ccccc1)([N+](=O)[O-])C(=O)N2C(=O)O</smiles>

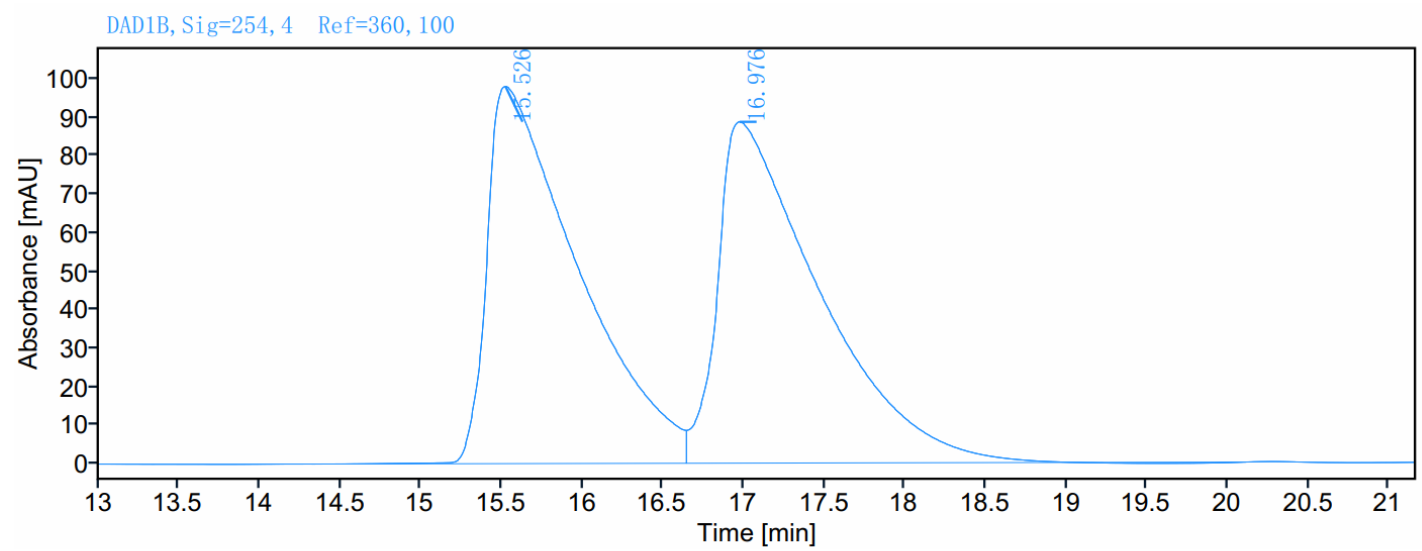

Signal: $\quad$ DAD1B, Sig=254, $4 \quad$ Ref $=360,100$

$\begin{array}{rrrr}\text { Rentention Time[min] } & \text { Area[mAU*min] } & \text { Height [mAU] } & \text { Relative Area[\%] } \\ 15.526 & 3841.45 & 98.06 & 49.80 \\ 16.976 & 3872.32 & 88.73 & 50.20\end{array}$

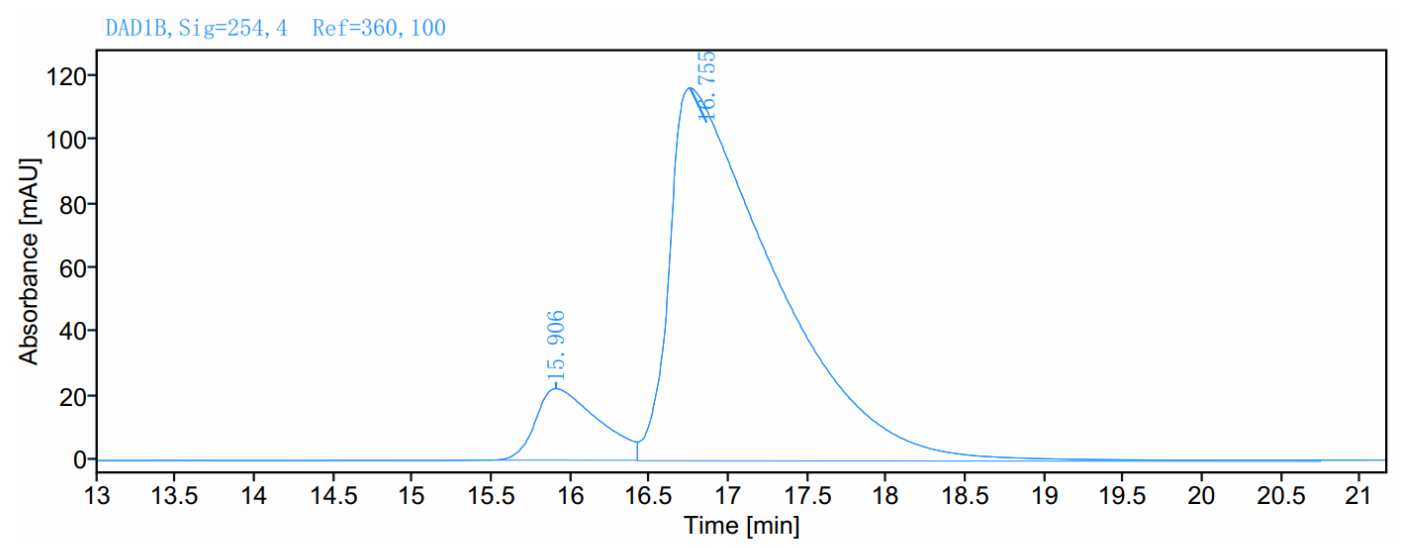

Signa1: $\quad$ DAD1B, Sig $=254,4 \quad \operatorname{Ref}=360,100$

Rentention Time[min]

15. 906

16. 755
Area[mAU*min]

615.83

5404. 07
Height [mAU]

22. 30

116. 33
Relative Area[\%]

10. 23

89. 77 


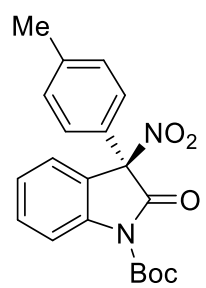

3zc

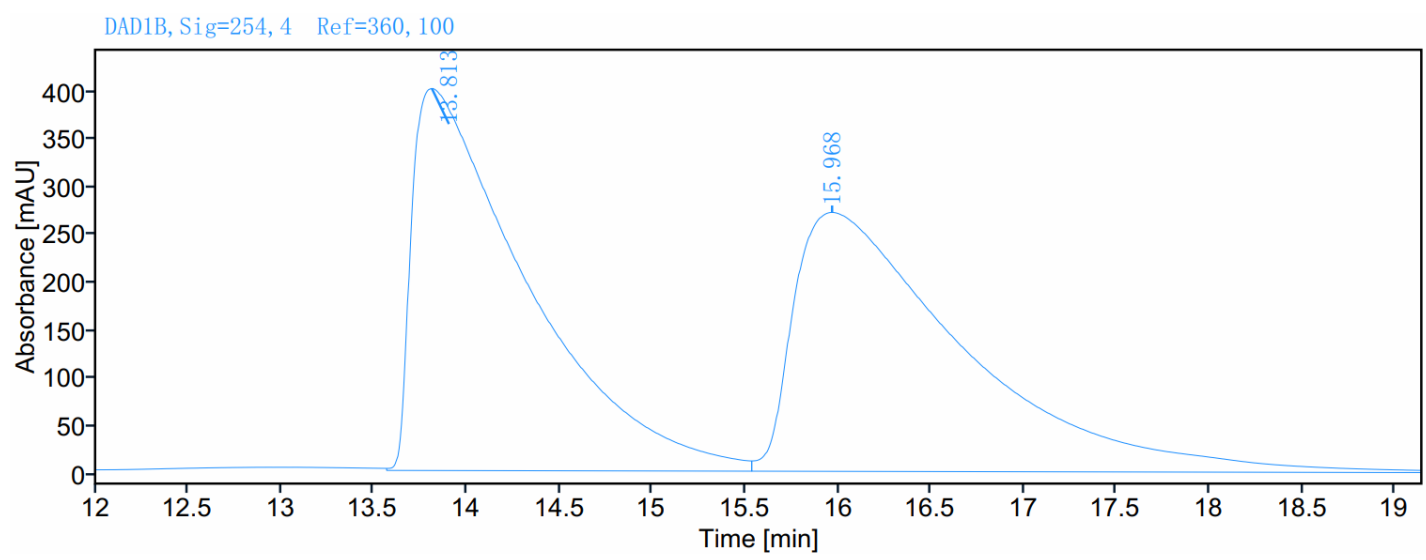

Signal: $\quad$ DAD1B, $\operatorname{Sig}=254,4 \quad \operatorname{Ref}=360,100$

Rentention Time[min]

13. 813

15. 968

DAD1B, Sig $=254,4 \quad$ Ref $=360,100$

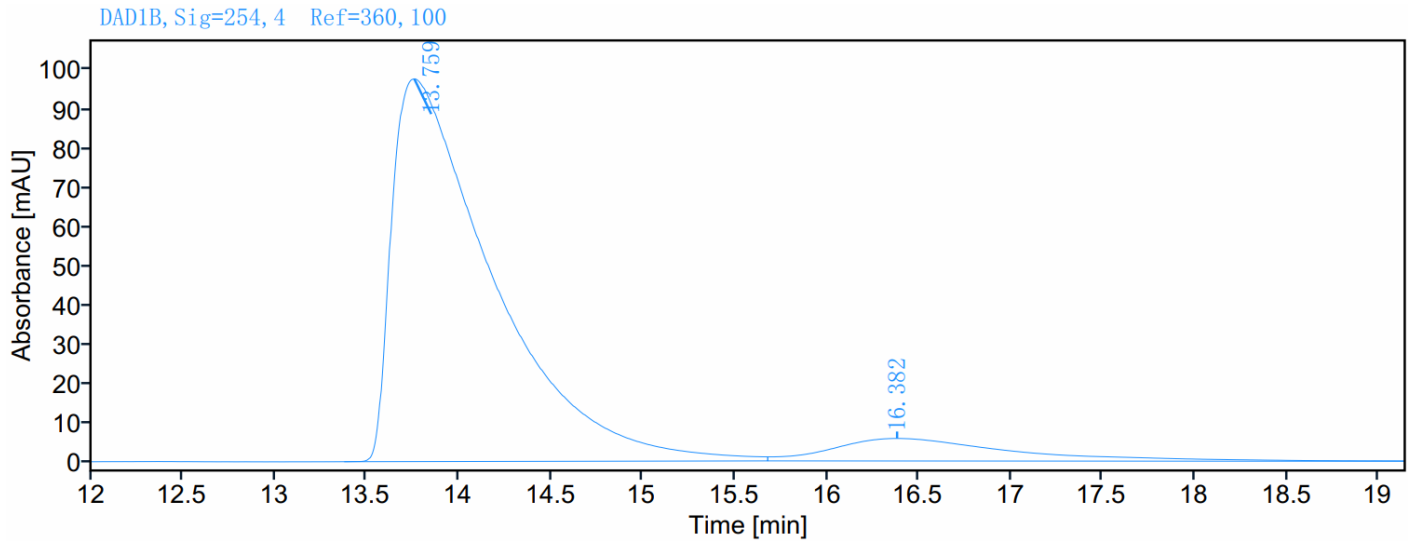

Height [mAU] Relative Area[\%]

$\begin{array}{ll}399.34 & 49.93 \\ 270.59 & 50.07\end{array}$

399.34

49.93

17230. 39

17276. 69

Time $[\mathrm{min}]$

Signal: DAD1B, Sig=254, $4 \quad \operatorname{Ref}=360,100$

$\begin{array}{rr}\text { Rentention Time[min] } & \text { Area[mAU*min] } \\ 13.759 & 3720.35 \\ 16.382 & 393.72\end{array}$

393.72

Height [mAU]

Relative Area[\%]

97. 42

90.43

5.74

9. 57 


\section{Large-scale experiment}<smiles>CC(=O)N1C(=O)[C@](Cc2ccccc2)(c2ccc(F)cc2[N+](=O)[O-])c2ccccc21</smiles>

3b

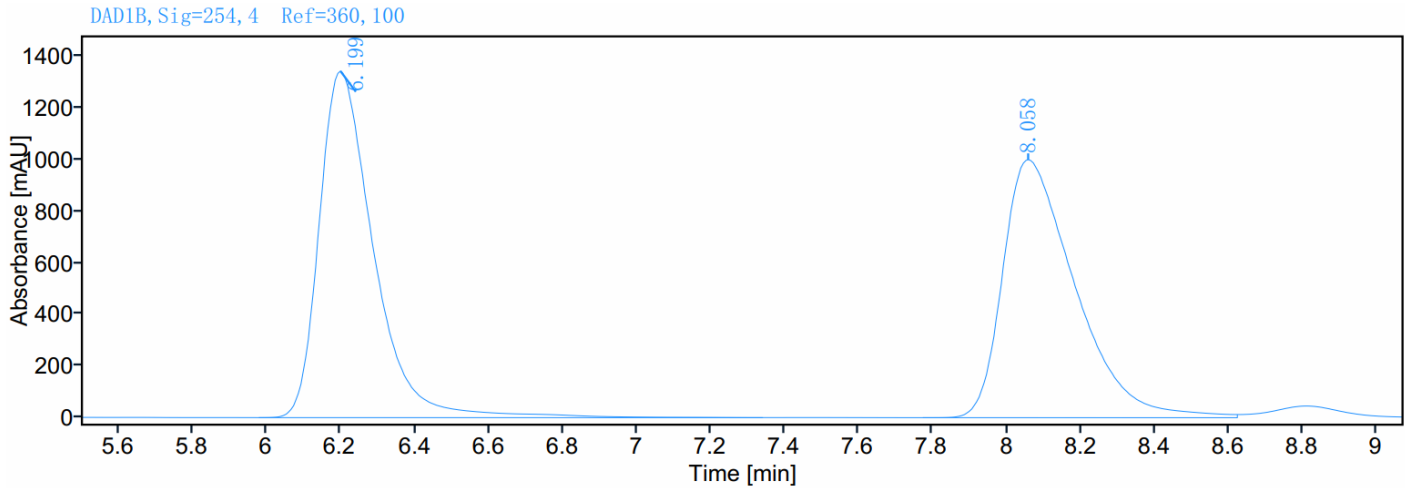

Signa1: DAD1B, Sig=254, 4 Ref $=360,100$

Rentention Time[min]

6. 199

8. 058
Area[mAU*min]

13708. 85

13583. 78
Height [mAU]

1345.50

1001. 90
Relative Area[\%]

50.23

49. 77

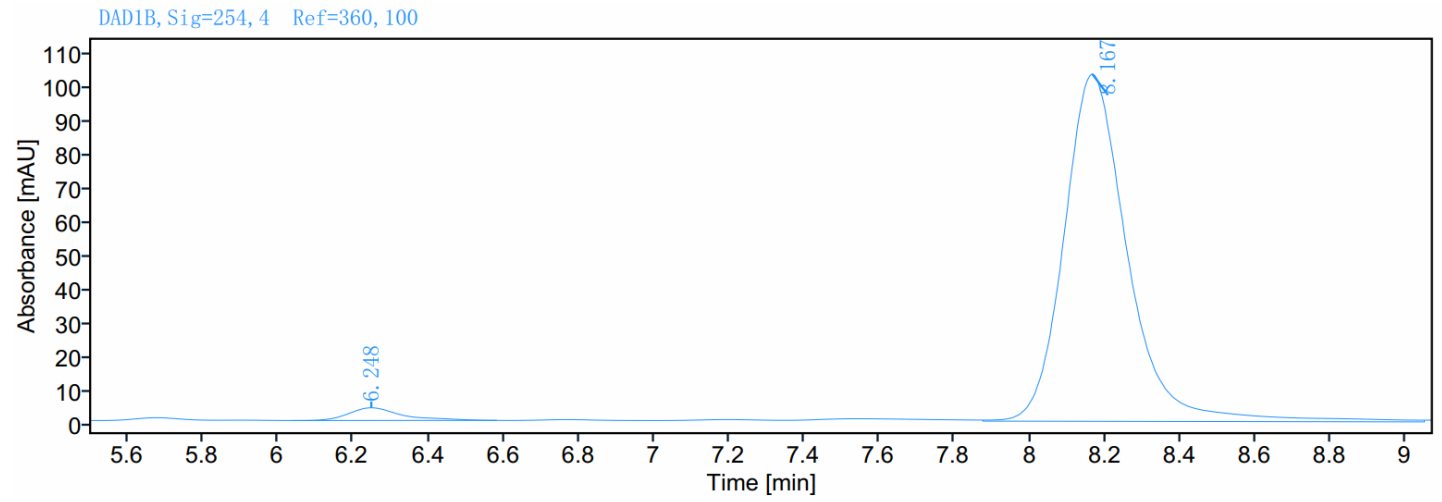

Signal: $\quad$ DAD1B, $\mathrm{Sig}=254,4 \quad$ Ref $=360,100$

Rentention Time[min]

6. 248

8. 167
Area [mAU*min]

36. 66

1209. 79
Height [mAU]

3. 75

102.68
Relative Area[\%]

2.94

97.06 
<smiles>N[C@@]1(Br)C(=O)Nc2ccc(F)cc21</smiles>

$4 a$

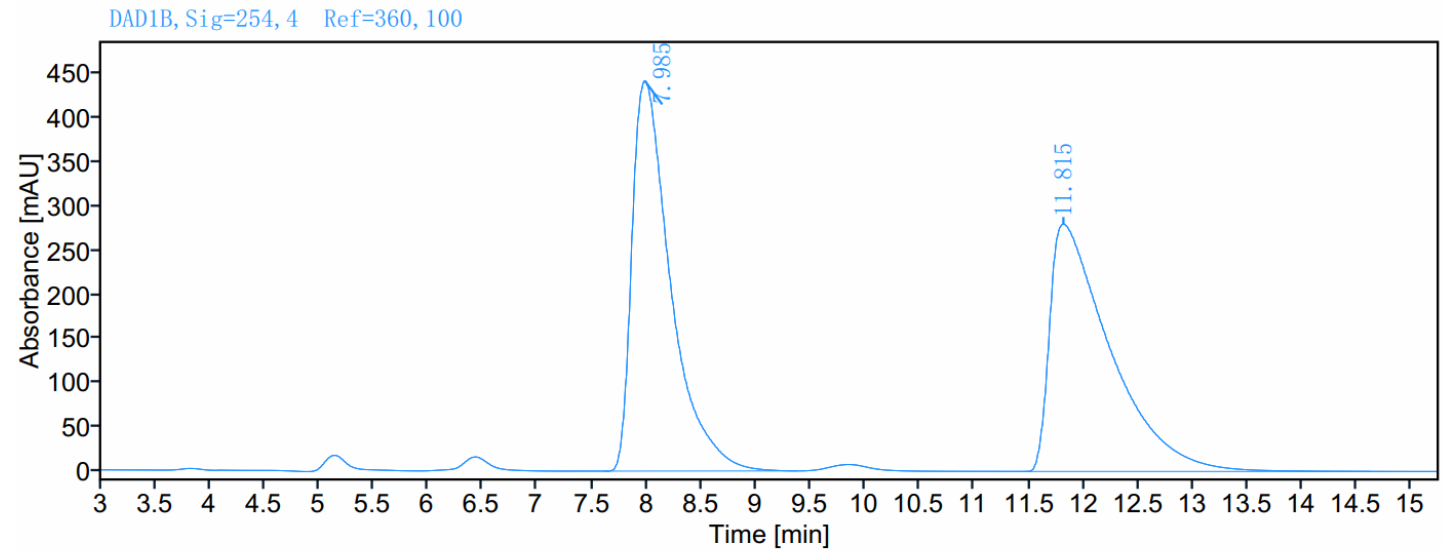

Signal: $\quad$ DAD1B, $\operatorname{Sig}=254,4 \quad$ Ref $=360,100$

$\begin{array}{rrrr}\text { Rentention Time[min] } & \text { Area[mAU*min] } & \text { Height [mAU] } & \text { Relative Area[\%] } \\ 7.985 & 10864.18 & 442.80 & 50.18 \\ 11.815 & 10787.77 & 280.75 & 49.82\end{array}$

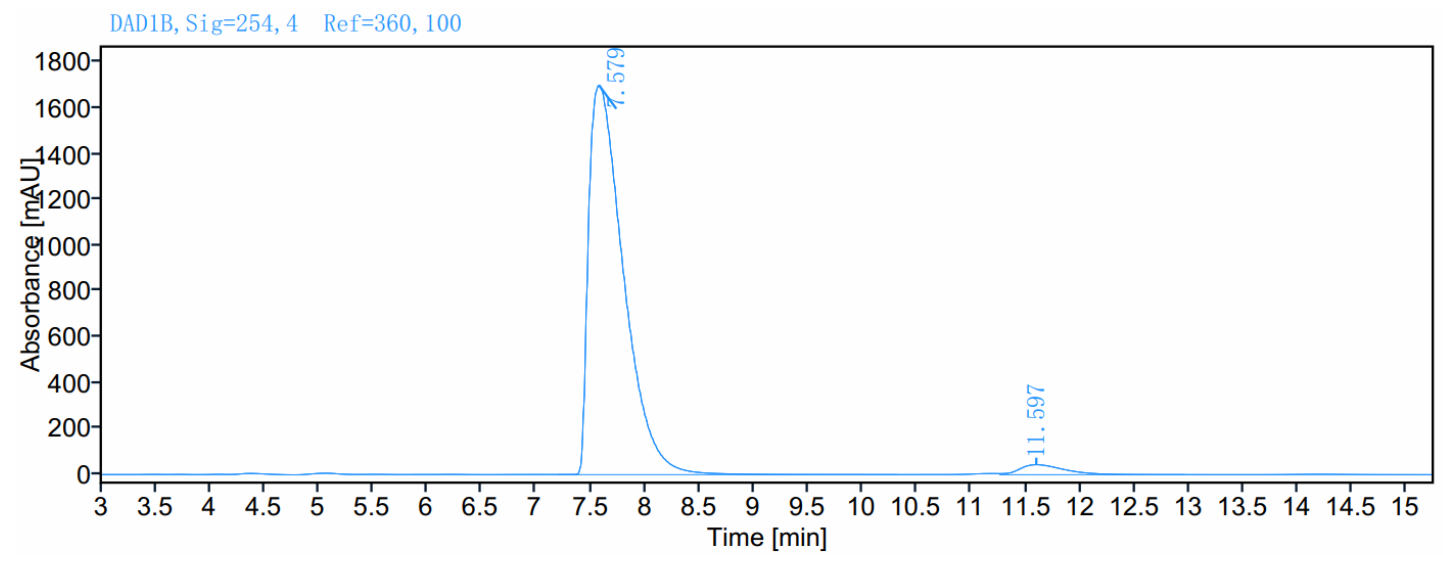

Signal: DAD1B, Sig=254, $4 \quad$ Ref $=360,100$

$\begin{array}{rrrr}\text { Rentention Time[min] } & \text { Area[mAU*min] } & \text { Height [mAU] } & \text { Relative Area[\%] } \\ 7.579 & 37783.19 & 1698.62 & 96.82 \\ 11.597 & 1240.57 & 43.62 & 3.18\end{array}$


<smiles>NC(=O)N[C@]1(Cc2ccccc2)C(=O)Nc2ccc(F)cc21</smiles>

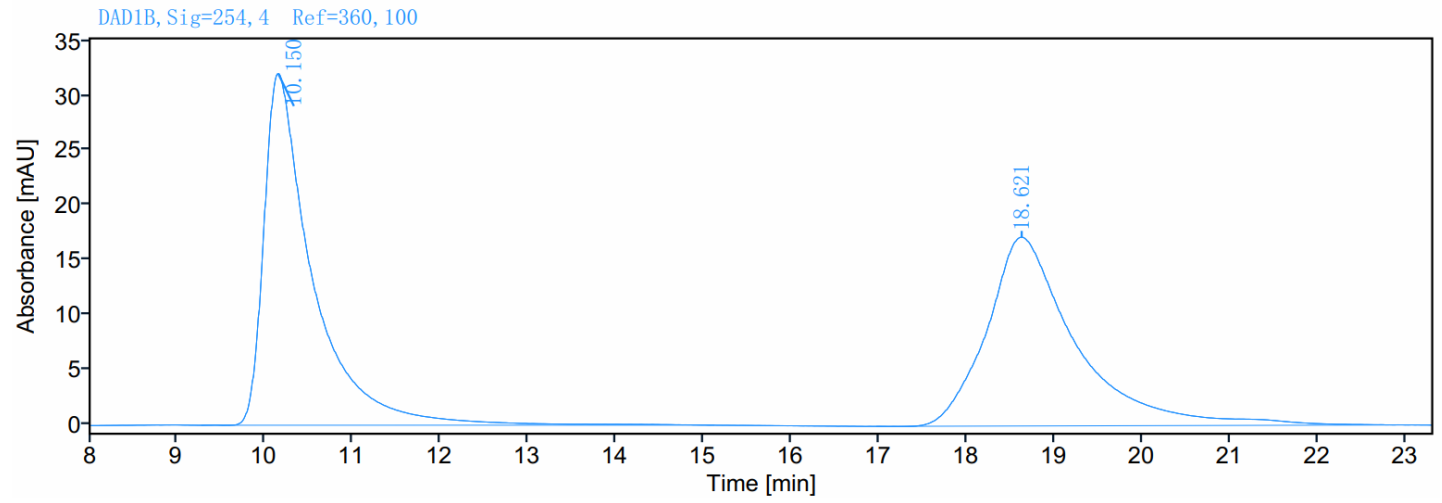

Signal: DAD1B, Sig=254, $4 \quad$ Ref $=360,100$

$\begin{array}{rrrr}\text { Rentention Time[min] } & \text { Area[mAU*min] } & \text { Height [mAU] } & \text { Relative Area[\%] } \\ 10.150 & 1241.14 & 32.21 & 50.39 \\ 18.621 & 1221.84 & 17.29 & 49.61\end{array}$

DAD1B, Sig=254, $4 \quad$ Ref $=360,100$

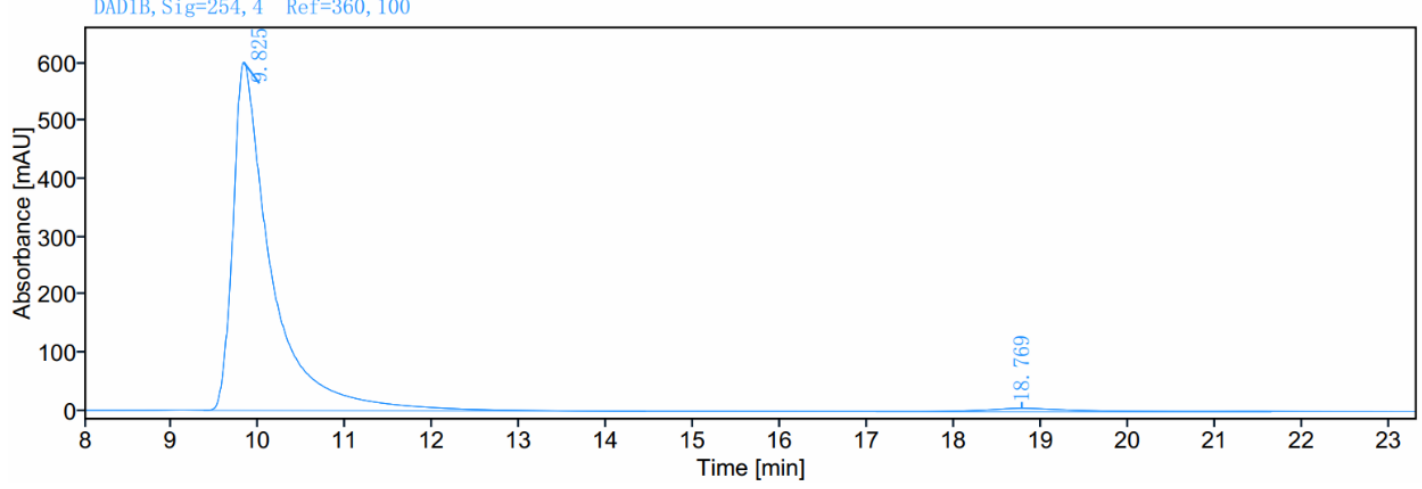

Signal: $\quad$ DAD1B, Sig=254, $4 \quad$ Ref $=360,100$

$\begin{array}{rrrr}\text { Rentention Time[min] } & \text { Area[mAU*min] } & \text { Height[mAU] } & \text { Relative Area[\%] } \\ 9.825 & 18366.20 & 602.46 & 96.97 \\ 18.769 & 574.80 & 6.24 & 3.03\end{array}$




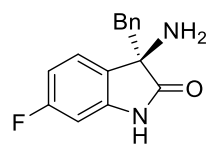

4c

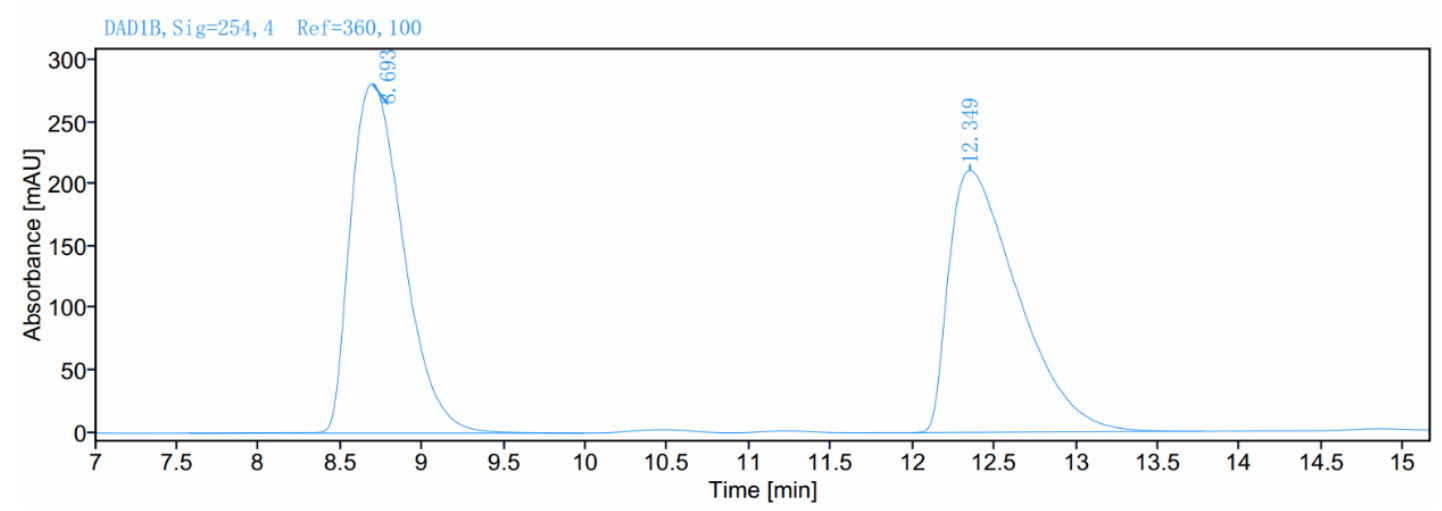

Signal: $\quad$ DAD1B, Sig=254, $4 \quad$ Ref $=360,100$

$\begin{array}{rrrr}\text { Rentention Time[min] } & \text { Area[mAU*min] } & \text { Height[mAU] } & \text { Relative Area[\%] } \\ 8.693 & 6322.35 & 281.57 & 50.47 \\ 12.349 & 6203.76 & 210.96 & 49.53\end{array}$

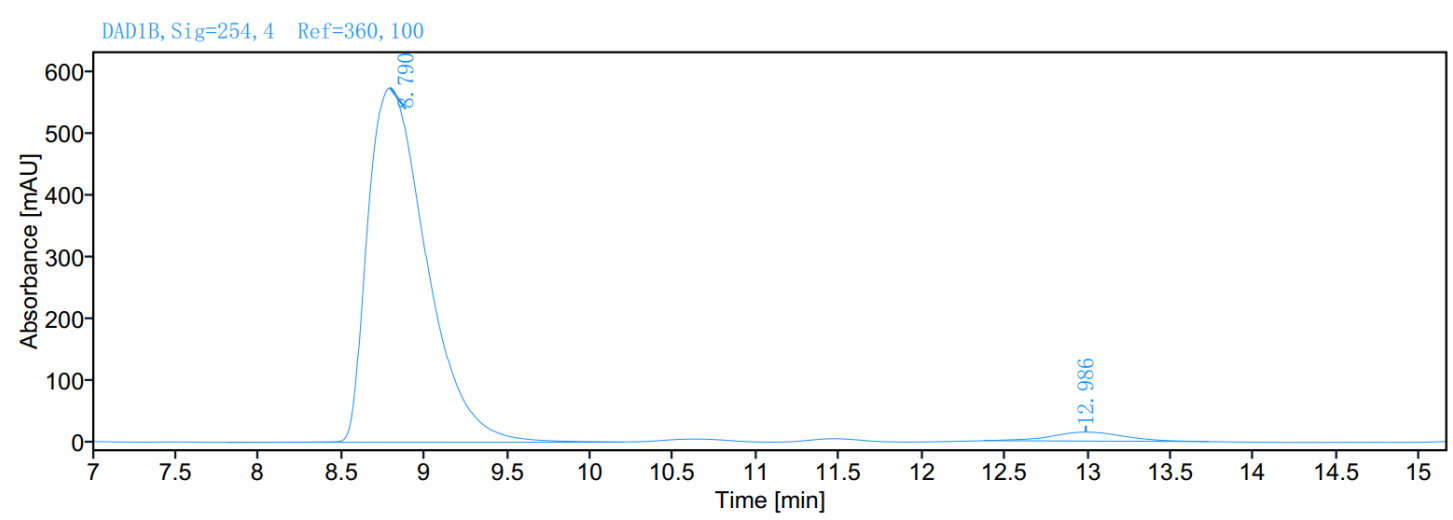

Signal: $\quad$ DAD1B, Sig $=254,4 \quad$ Ref $=360,100$

$\begin{array}{rrrr}\text { Rentention Time[min] } & \text { Area[mAU*min] } & \text { Height[mAU] } & \text { Relative Area[\%] } \\ 8.790 & 14082.87 & 573.47 & 96.92 \\ 12.986 & 448.10 & 14.54 & 3.08\end{array}$



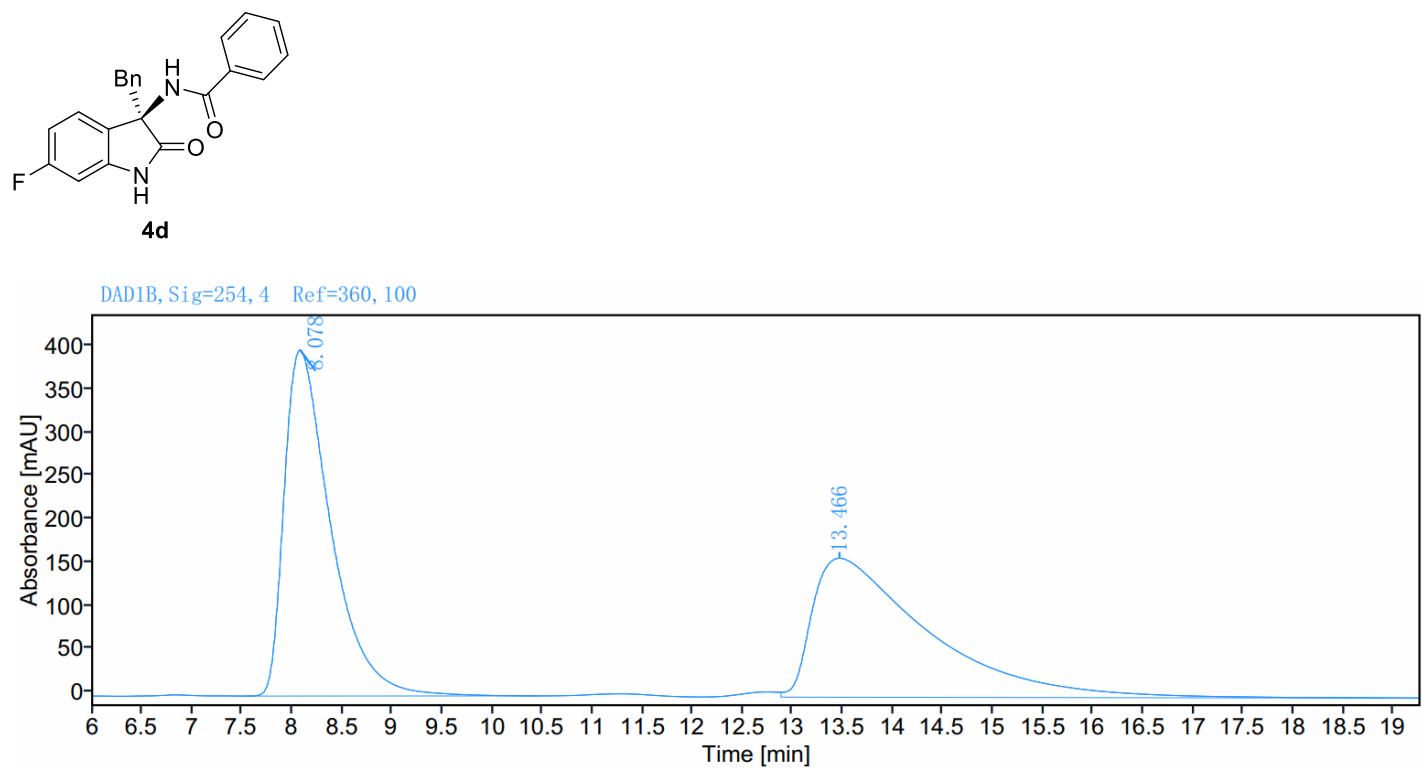

Signa1: DAD1B, Sig=254, $4 \quad \operatorname{Ref}=360,100$ Rentention Time[min]

Area[mAU*min]

Height [mAU]

Relative Area[\%]

8. 078

12923. 19

400. 12

50. 01

13. 466

12919. 12

160. 68

49. 99

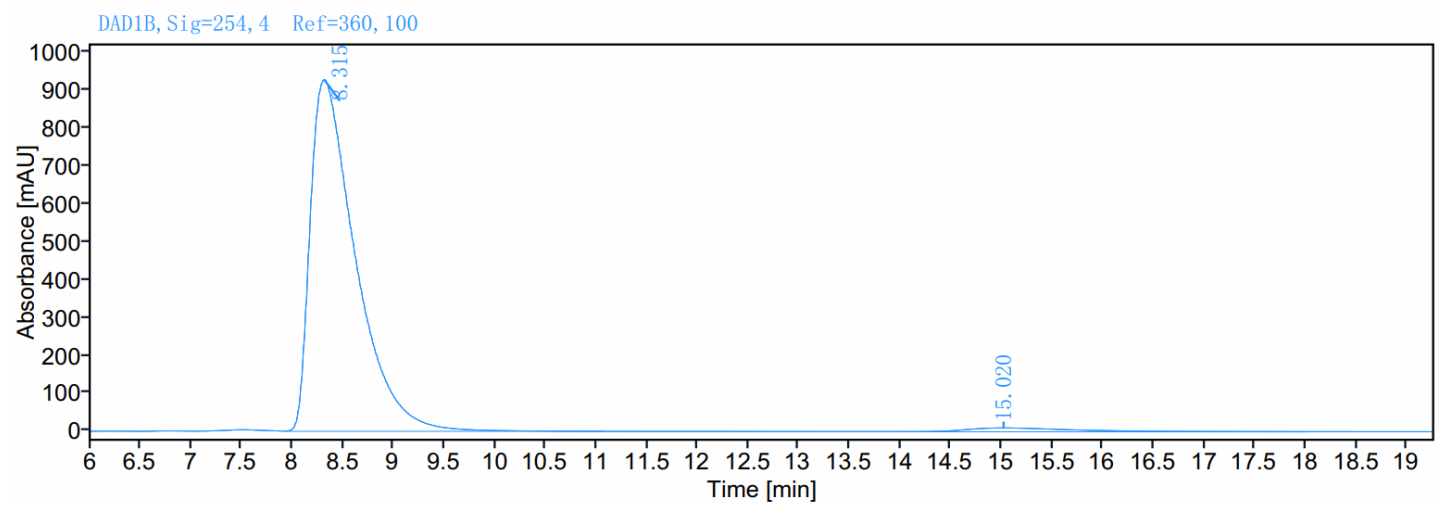

Signal: $\quad$ DAD1B, Sig=254, $4 \quad$ Ref $=360,100$

$\begin{array}{rrrr}\text { Rentention Time[min] } & \text { Area[mAU*min] } & \text { Height[mAU] } & \text { Relative Area[\%] } \\ 8.315 & 28733.70 & 927.98 & 97.21 \\ 15.020 & 824.89 & 10.16 & 2.79\end{array}$




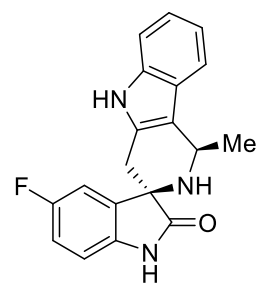

$4 x$

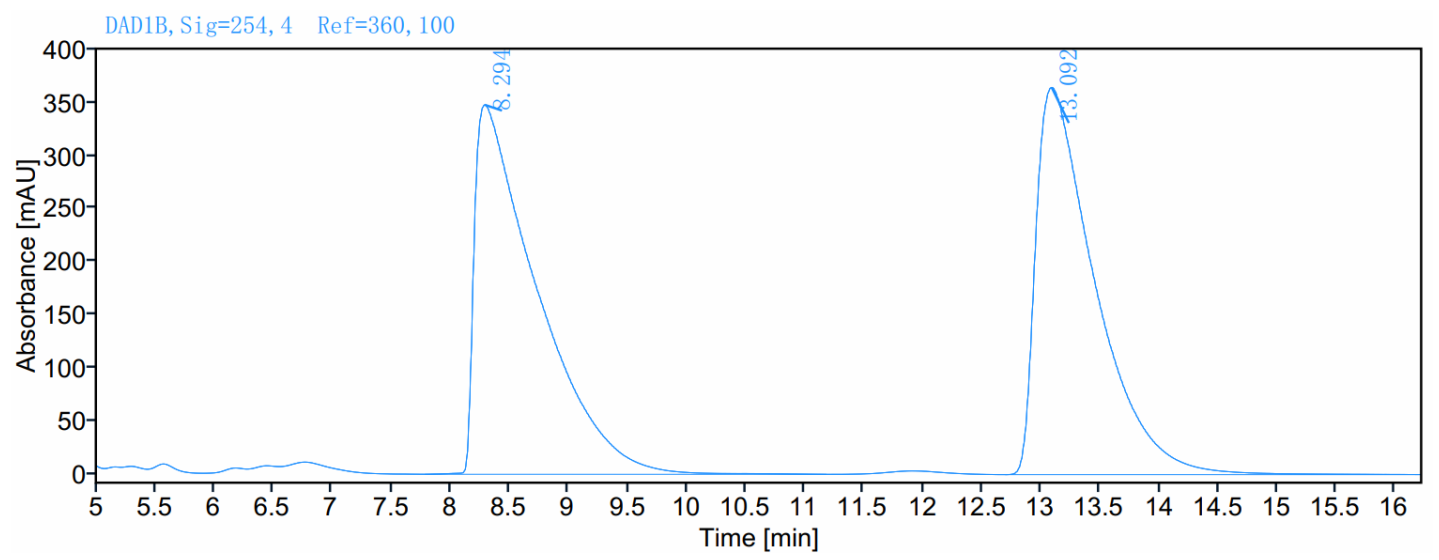

Signal: $\quad$ DAD1B, Sig=254, 4 Ref $=360,100$

Rentention Time[min]

Area $[\mathrm{mAU} * \mathrm{~min}]$

Height [mAU]

Relative Area[\%]

8. 294

12795.55

347. 19

50. 22

13. 092

12681. 34

363.92

49. 78

DAD1B, Sig=254, $4 \quad$ Ref=360, 100

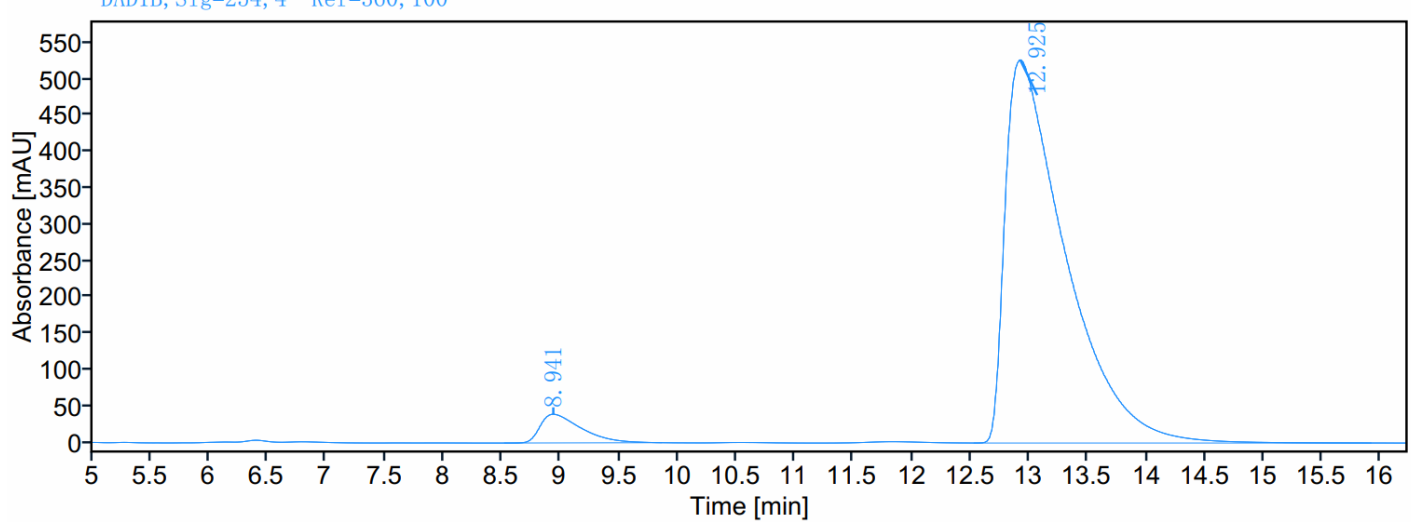

Signal: $\quad$ DAD1B, Sig=254, 4 Ref=360, 100

$\begin{array}{rrrr}\text { Rentention Time[min] } & \text { Area[mAU*min] } & \text { Height [mAU] } & \text { Relative Area[\%] } \\ 8.941 & 956.56 & 39.41 & 4.84 \\ 12.925 & 18821.88 & 526.52 & 95.16\end{array}$




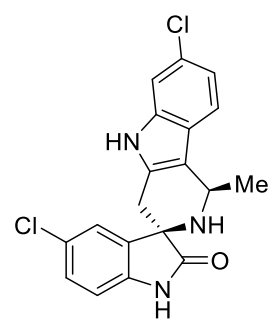

$4 y$

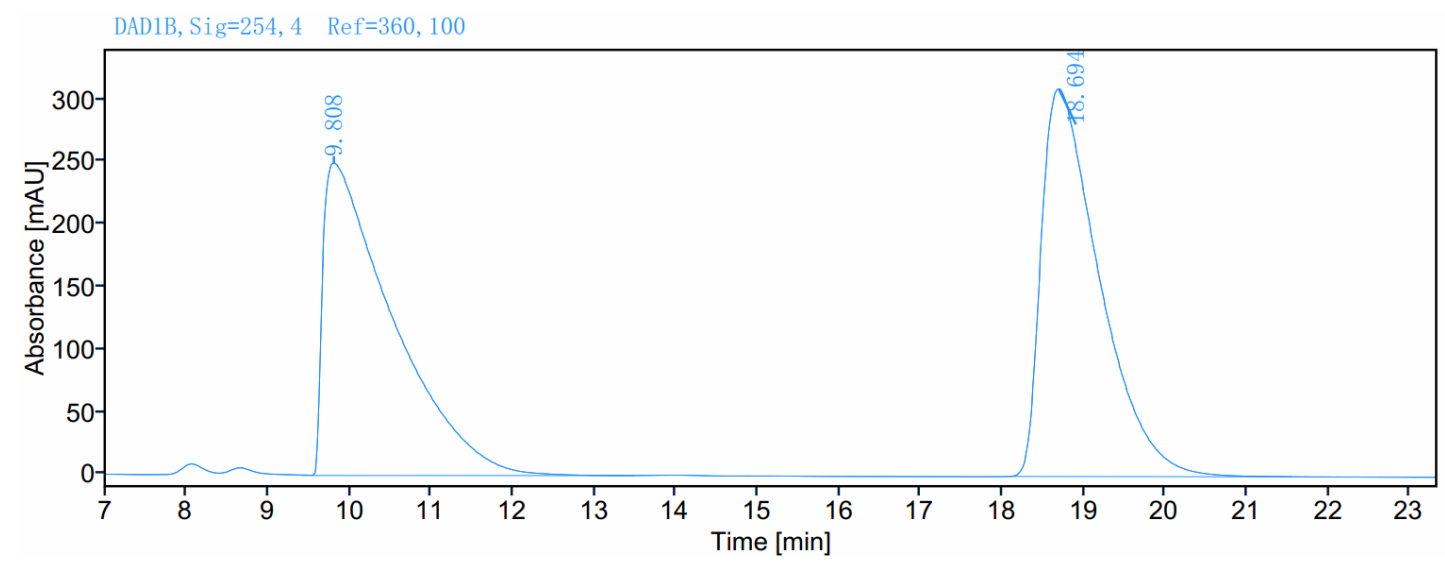

Signal: $\quad$ DAD1B, Sig=254, $4 \quad$ Ref=360, 100

Rentention Time[min]

9.808

18. 694
Area $[\mathrm{mAU} * \min ]$

14824.03

15360.51
Height [mAU]

249. 92

310. 23
Relative Area[\%]

49. 11

50.89

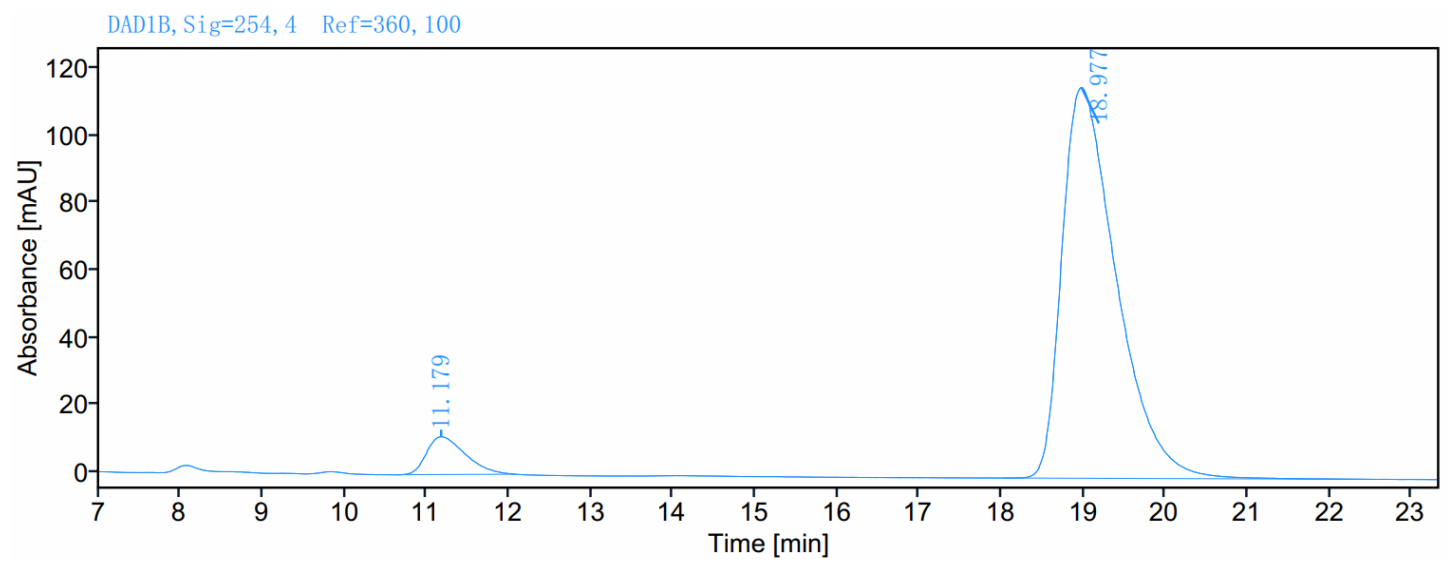

Signal: $\quad$ DAD1B, Sig=254, $4 \quad$ Ref=360, 100

$\begin{array}{rrrr}\text { Rentention Time[min] } & \text { Area[mAU*min] } & \text { Height [mAU] } & \text { Relative Area[\%] } \\ 11.179 & 367.55 & 11.23 & 6.44 \\ 18.977 & 5343.81 & 116.20 & 93.56\end{array}$




\section{X-ray Crystallography data}

\section{X-ray structure of product 3a}

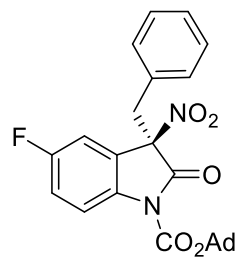

$3 a$

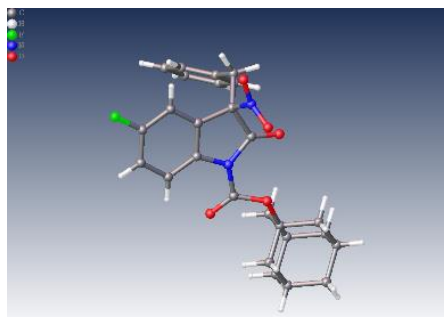

CCDC: 2097341

Table S5 Crystal data and structure refinement for 3a.

Identification code

Empirical formula

Formula weight

Temperature/K

Crystal system

Space group

$\mathrm{a} / \AA$

$\mathrm{b} / \AA$

$\mathrm{c} / \AA$

$\alpha /^{\circ}$

$\beta /{ }^{\circ}$

$\gamma /{ }^{\circ}$

Volume $/ \AA^{3}$

Z

$\rho_{\text {calc }} \mathrm{g} / \mathrm{cm}^{3}$

$\mu / \mathrm{mm}^{-1}$

$\mathrm{F}(000)$

Crystal size $/ \mathrm{mm}^{3}$

Radiation
$3 a$

$\mathrm{C}_{26} \mathrm{H}_{25} \mathrm{FN}_{2} \mathrm{O}_{5}$

464.48

293(2)

monoclinic

$\mathrm{P} 21$

$8.7046(3)$

11.9983(4)

11.5160(4)

90

$100.577(3)$

90

1182.30(6)

2

1.305

0.798

488.0

$0.04 \times 0.03 \times 0.02$

$\mathrm{CuK} \alpha(\lambda=1.54184)$

$2 \Theta$ range for data collection/ ${ }^{\circ} 7.81$ to 134.056 
Index ranges

Reflections collected

Independent reflections

Data/restraints/parameters

Goodness-of-fit on $\mathrm{F}^{2}$

Final $R$ indexes $[\mathrm{I}>=2 \sigma(\mathrm{I})] \quad \mathrm{R}_{1}=0.0663, \mathrm{wR}_{2}=0.1535$

Final $\mathrm{R}$ indexes [all data] $\quad \mathrm{R}_{1}=0.0744, \mathrm{wR}_{2}=0.1627$

Largest diff. peak/hole / e $\AA^{-3} 0.46 /-0.24$

Flack parameter $0.0(4)$

Table S6 Fractional Atomic Coordinates $\left(\times 10^{4}\right)$ and Equivalent Isotropic Displacement Parameters $\left(\AA^{2} \times 10^{3}\right)$ for $3 \mathrm{a}$. U $\mathrm{U}_{\text {eq }}$ is defined as $1 / 3$ of of the trace of the orthogonalised UIJ tensor.

$\begin{array}{ccccc}\text { Atom } & \boldsymbol{x} & \boldsymbol{y} & z & \mathbf{U}(\mathbf{e q}) \\ \mathrm{F} 1 & 4151(4) & 9609(2) & 5101(3) & 85.7(9) \\ \mathrm{O} 1 & 3949(4) & 3510(3) & 6939(2) & 60.4(8) \\ \mathrm{O} 2 & 5444(4) & 4905(3) & 7859(3) & 76.5(10) \\ \text { O3 } & 1115(4) & 4193(3) & 5846(4) & 82.6(11) \\ \text { O4 } & 347(7) & 6530(7) & 3146(4) & 139(3) \\ \text { O5 } & 1765(8) & 5103(5) & 3524(5) & 125.0(19) \\ \mathrm{N} 1 & 3379(4) & 5269(3) & 6379(3) & 52.1(8) \\ \mathrm{N} 2 & 1029(5) & 5844(5) & 3831(4) & 76.4(12) \\ \mathrm{C} 1 & 3774(4) & 6389(3) & 6159(4) & 50.0(9) \\ \mathrm{C} 2 & 4377(5) & 4554(4) & 7150(4) & 54.0(10) \\ \mathrm{C} 3 & 2521(5) & 6911(4) & 5458(4) & 52.7(9) \\ \mathrm{C} 4 & 1199(5) & 6108(4) & 5167(4) & 55.7(10) \\ \mathrm{C} 5 & 1841(5) & 5030(4) & 5811(4) & 59.5(11) \\ \mathrm{C} 6 & 4460(5) & 2622(4) & 7831(4) & 52.3(9)\end{array}$




\begin{tabular}{|c|c|c|c|c|}
\hline $\mathrm{C} 7$ & $5179(5)$ & $6938(4)$ & $6513(4)$ & $61.0(11)$ \\
\hline $\mathrm{C} 8$ & $2616(5)$ & 7998(4) & $5091(4)$ & $59.2(10)$ \\
\hline $\mathrm{C} 9$ & $4018(6)$ & $8535(4)$ & $5450(4)$ & $63.1(11)$ \\
\hline $\mathrm{C} 10$ & $3850(6)$ & $2869(4)$ & $8957(4)$ & $64.3(12)$ \\
\hline $\mathrm{C} 11$ & $3997(7)$ & $587(4)$ & $8066(5)$ & $73.8(14)$ \\
\hline $\mathrm{C} 12$ & $-321(5)$ & $6684(5)$ & $6732(5)$ & $66.7(12)$ \\
\hline C13 & $5275(6)$ & $8037(4)$ & $6132(5)$ & $65.1(11)$ \\
\hline C14 & $3407(7)$ & $833(5)$ & $9208(5)$ & $78.4(15)$ \\
\hline $\mathrm{C} 15$ & $-384(5)$ & $6440(5)$ & $5443(4)$ & $65.0(11)$ \\
\hline $\mathrm{C} 16$ & $4213(7)$ & $1868(6)$ & 9781(4) & 76.1(14) \\
\hline C17 & $3651(6)$ & $1597(4)$ & $7238(4)$ & $64.6(12)$ \\
\hline C18 & $6208(6)$ & $2443(5)$ & $8074(5)$ & $68.6(13)$ \\
\hline C19 & $-621(7)$ & $5868(7)$ & $7506(6)$ & $91.8(18)$ \\
\hline $\mathrm{C} 20$ & $5984(7)$ & $1690(6)$ & $10046(5)$ & $90.4(19)$ \\
\hline $\mathrm{C} 21$ & $5745(8)$ & $391(5)$ & $8327(6)$ & $91.7(19)$ \\
\hline $\mathrm{C} 22$ & $-23(8)$ & $7745(6)$ & $7157(6)$ & $87.8(16)$ \\
\hline $\mathrm{C} 23$ & $6552(6)$ & 1419(6) & $8905(5)$ & $83.2(16)$ \\
\hline $\mathrm{C} 24$ & $-331(9)$ & $7146(11)$ & $9092(7)$ & $119(3)$ \\
\hline $\mathrm{C} 25$ & $-615(10)$ & $6097(11)$ & $8659(8)$ & $120(3)$ \\
\hline $\mathrm{C} 26$ & $-7(10)$ & $8004(9)$ & $8338(8)$ & $118(3)$ \\
\hline
\end{tabular}

Table S7 Anisotropic Displacement Parameters $\left(\AA^{2} \times 10^{3}\right)$ for 3a. The Anisotropic displacement factor exponent takes the form: $-2 \pi^{2}\left[h^{2} a * 2 U_{11}+2 h k a * b * U_{12}+\ldots\right]$.

$\begin{array}{ccccccc}\text { Atom } & \mathbf{U}_{11} & \mathbf{U}_{\mathbf{2 2}} & \mathbf{U}_{\mathbf{3 3}} & \mathbf{U}_{\mathbf{2 3}} & \mathbf{U}_{13} & \mathbf{U}_{12} \\ \mathrm{~F} 1 & 108(2) & 44.2(15) & 112(2) & 9.6(14) & 38.0(18) & -10.2(15) \\ \text { O1 } & 76(2) & 42.3(16) & 58.2(16) & 3.6(12) & 0.6(14) & 5.0(15) \\ \text { O2 } & 84(2) & 61(2) & 74.5(19) & -1.3(16) & -9.7(18) & -5.5(18) \\ \text { O3 } & 56.4(18) & 49(2) & 139(3) & 7.7(19) & 8.6(18) & -12.4(16) \\ \text { O4 } & 116(4) & 213(8) & 85(3) & 5(4) & 9(3) & 50(5) \\ \text { O5 } & 170(5) & 99(4) & 119(3) & -20(3) & 62(4) & 22(4)\end{array}$




\begin{tabular}{|c|c|c|c|c|c|c|}
\hline N1 & $47.4(16)$ & $39.5(18)$ & $69(2)$ & $0.3(15)$ & $9.5(15)$ & $-1.1(14)$ \\
\hline $\mathrm{N} 2$ & $65(2)$ & $81(3)$ & $79(3)$ & $-15(2)$ & $5(2)$ & $1(2)$ \\
\hline $\mathrm{C} 1$ & $50(2)$ & $39(2)$ & $64(2)$ & $-4.2(17)$ & $16.0(17)$ & $-0.4(16)$ \\
\hline $\mathrm{C} 2$ & $62(2)$ & $43(2)$ & $57(2)$ & $-1.3(17)$ & $11(2)$ & $3.2(19)$ \\
\hline $\mathrm{C} 3$ & 49.7(19) & $43(2)$ & $67(2)$ & $-3.0(18)$ & $16.5(17)$ & $1.9(17)$ \\
\hline $\mathrm{C} 4$ & $48(2)$ & $49(2)$ & $69(2)$ & $-1.4(17)$ & $6.8(18)$ & $3.3(17)$ \\
\hline $\mathrm{C} 5$ & $50(2)$ & $44(2)$ & $84(3)$ & $-2(2)$ & $12(2)$ & $-2.3(19)$ \\
\hline C6 & $61(2)$ & $47(2)$ & $49(2)$ & $4.4(15)$ & $8.9(17)$ & $6.5(19)$ \\
\hline $\mathrm{C} 7$ & $53(2)$ & $53(3)$ & $77(3)$ & $-4(2)$ & $9.2(19)$ & $-5(2)$ \\
\hline $\mathrm{C} 8$ & $64(2)$ & $46(2)$ & $70(2)$ & 1.3(19) & $18(2)$ & $8(2)$ \\
\hline $\mathrm{C} 9$ & $77(3)$ & $41(2)$ & $78(3)$ & $-1(2)$ & $32(2)$ & $-7(2)$ \\
\hline C10 & $70(3)$ & $65(3)$ & $60(2)$ & $-9(2)$ & $17(2)$ & $0(2)$ \\
\hline C11 & $97(4)$ & $51(3)$ & $74(3)$ & $9(2)$ & $17(3)$ & $0(3)$ \\
\hline C12 & 43.2(19) & $73(3)$ & $85(3)$ & $6(2)$ & 15.7(19) & $9(2)$ \\
\hline C13 & $60(2)$ & $50(3)$ & $89(3)$ & $-6(2)$ & $21(2)$ & $-14(2)$ \\
\hline C14 & $81(3)$ & $80(4)$ & $75(3)$ & $19(3)$ & $17(3)$ & $-3(3)$ \\
\hline C15 & 45.1(19) & $65(3)$ & $83(3)$ & $6(2)$ & 8.6(19) & $7(2)$ \\
\hline C16 & $84(3)$ & $95(4)$ & $51(2)$ & $5(2)$ & $18(2)$ & $-5(3)$ \\
\hline C17 & $88(3)$ & $47(3)$ & $57(2)$ & $-0.8(18)$ & $10(2)$ & $2(2)$ \\
\hline C18 & $64(2)$ & $70(3)$ & $74(3)$ & $14(2)$ & $18(2)$ & $11(2)$ \\
\hline C19 & $78(3)$ & $104(5)$ & 101(4) & $18(4)$ & $36(3)$ & $-1(3)$ \\
\hline $\mathrm{C} 20$ & $90(4)$ & $103(5)$ & $70(3)$ & $30(3)$ & $-6(3)$ & $-3(3)$ \\
\hline $\mathrm{C} 21$ & $108(5)$ & $68(4)$ & $104(4)$ & $25(3)$ & $33(4)$ & $30(3)$ \\
\hline $\mathrm{C} 22$ & $92(4)$ & $83(4)$ & $93(4)$ & $-4(3)$ & $31(3)$ & $14(3)$ \\
\hline $\mathrm{C} 23$ & $64(3)$ & $93(4)$ & $92(3)$ & $34(3)$ & $11(2)$ & $20(3)$ \\
\hline $\mathrm{C} 24$ & $89(4)$ & 184(11) & $92(5)$ & $-4(6)$ & $33(4)$ & $25(5)$ \\
\hline $\mathrm{C} 25$ & $106(5)$ & $158(10)$ & $106(5)$ & $34(6)$ & $49(4)$ & $15(5)$ \\
\hline $\mathrm{C} 26$ & $120(6)$ & $126(7)$ & $116(5)$ & $-23(5)$ & $42(5)$ & $11(5)$ \\
\hline
\end{tabular}




\begin{tabular}{|c|c|c|c|c|c|}
\hline Atom & Atom & Length/Å & Atom & Atom & Length/Å \\
\hline $\mathrm{F} 1$ & C9 & $1.361(5)$ & $\mathrm{C} 7$ & C13 & $1.397(7)$ \\
\hline $\mathrm{O} 1$ & $\mathrm{C} 2$ & $1.317(6)$ & $\mathrm{C} 8$ & C9 & $1.374(7)$ \\
\hline $\mathrm{O} 1$ & C6 & $1.490(5)$ & C9 & C13 & $1.362(7)$ \\
\hline $\mathrm{O} 2$ & $\mathrm{C} 2$ & $1.195(5)$ & $\mathrm{C} 10$ & C16 & $1.527(8)$ \\
\hline $\mathrm{O} 3$ & $\mathrm{C} 5$ & $1.191(6)$ & $\mathrm{C} 11$ & C14 & $1.526(8)$ \\
\hline $\mathrm{O} 4$ & N2 & $1.217(8)$ & $\mathrm{C} 11$ & $\mathrm{C} 17$ & $1.537(7)$ \\
\hline O5 & N2 & $1.187(7)$ & $\mathrm{C} 11$ & $\mathrm{C} 21$ & $1.514(9)$ \\
\hline N1 & $\mathrm{C} 1$ & $1.422(5)$ & C12 & $\mathrm{C} 15$ & $1.505(8)$ \\
\hline N1 & $\mathrm{C} 2$ & $1.413(6)$ & $\mathrm{C} 12$ & C19 & $1.381(9)$ \\
\hline N1 & C5 & $1.407(5)$ & $\mathrm{C} 12$ & $\mathrm{C} 22$ & $1.371(9)$ \\
\hline N2 & $\mathrm{C} 4$ & $1.551(7)$ & C14 & C16 & $1.515(9)$ \\
\hline $\mathrm{C} 1$ & $\mathrm{C} 3$ & $1.382(6)$ & $\mathrm{C} 16$ & $\mathrm{C} 20$ & $1.530(8)$ \\
\hline $\mathrm{C} 1$ & $\mathrm{C} 7$ & $1.383(6)$ & $\mathrm{C} 18$ & $\mathrm{C} 23$ & $1.553(7)$ \\
\hline $\mathrm{C} 3$ & $\mathrm{C} 4$ & $1.491(6)$ & C19 & $\mathrm{C} 25$ & $1.355(12)$ \\
\hline C3 & $\mathrm{C} 8$ & $1.378(7)$ & $\mathrm{C} 20$ & $\mathrm{C} 23$ & $1.521(9)$ \\
\hline $\mathrm{C} 4$ & C5 & $1.543(6)$ & $\mathrm{C} 21$ & $\mathrm{C} 23$ & $1.512(10)$ \\
\hline $\mathrm{C} 4$ & C15 & $1.523(6)$ & $\mathrm{C} 22$ & $\mathrm{C} 26$ & $1.392(10)$ \\
\hline C6 & $\mathrm{C} 10$ & $1.518(6)$ & $\mathrm{C} 24$ & $\mathrm{C} 25$ & $1.360(14)$ \\
\hline C6 & $\mathrm{C} 17$ & $1.515(6)$ & $\mathrm{C} 24$ & $\mathrm{C} 26$ & $1.408(14)$ \\
\hline C6 & C18 & $1.511(6)$ & & & \\
\hline
\end{tabular}

Table S9 Bond Angles for 3a.

$\begin{array}{cccccccc}\text { Atom } & \text { Atom } & \text { Atom } & \text { Angle }^{\circ} & \text { Atom } & \text { Atom } & \text { Atom } & \text { Angle }^{\circ} \\ \text { C2 } & \text { O1 } & \text { C6 } & 120.9(3) & \text { C1 } & \text { C7 } & \text { C13 } & 117.2(4) \\ \text { C2 } & \text { N1 } & \text { C1 } & 123.0(3) & \text { C9 } & \text { C8 } & \text { C3 } & 116.8(4) \\ \text { C5 } & \text { N1 } & \text { C1 } & 110.5(3) & \text { F1 } & \text { C9 } & \text { C8 } & 118.3(5) \\ \text { C5 } & \text { N1 } & \text { C2 } & 126.3(4) & \text { F1 } & \text { C9 } & \text { C13 } & 118.8(4) \\ \text { O4 } & \text { N2 } & \text { C4 } & 116.9(5) & \text { C13 } & \text { C9 } & \text { C8 } & 122.9(4) \\ \text { O5 } & \text { N2 } & \text { O4 } & 122.6(5) & \text { C6 } & \text { C10 } & \text { C16 } & 108.2(4)\end{array}$




\begin{tabular}{|c|c|c|c|c|c|c|c|}
\hline $\mathrm{O} 5$ & $\mathrm{~N} 2$ & $\mathrm{C} 4$ & $119.3(5)$ & $\mathrm{C} 14$ & $\mathrm{C} 11$ & $\mathrm{C} 17$ & $108.8(5)$ \\
\hline $\mathrm{C} 3$ & $\mathrm{C} 1$ & N1 & $110.0(3)$ & $\mathrm{C} 21$ & $\mathrm{C} 11$ & $\mathrm{C} 14$ & $110.1(5)$ \\
\hline $\mathrm{C} 3$ & $\mathrm{C} 1$ & $\mathrm{C} 7$ & 121.1(4) & $\mathrm{C} 21$ & $\mathrm{C} 11$ & $\mathrm{C} 17$ & $108.7(5)$ \\
\hline $\mathrm{C} 7$ & $\mathrm{C} 1$ & N1 & $128.9(4)$ & $\mathrm{C} 19$ & $\mathrm{C} 12$ & $\mathrm{C} 15$ & $121.5(6)$ \\
\hline $\mathrm{O} 1$ & $\mathrm{C} 2$ & N1 & $109.9(3)$ & $\mathrm{C} 22$ & $\mathrm{C} 12$ & $\mathrm{C} 15$ & $120.2(5)$ \\
\hline $\mathrm{O} 2$ & $\mathrm{C} 2$ & $\mathrm{O} 1$ & $128.3(4)$ & $\mathrm{C} 22$ & $\mathrm{C} 12$ & C19 & $118.3(6)$ \\
\hline $\mathrm{O} 2$ & $\mathrm{C} 2$ & N1 & $121.8(4)$ & C9 & $\mathrm{C} 13$ & $\mathrm{C} 7$ & $120.5(4)$ \\
\hline $\mathrm{C} 1$ & $\mathrm{C} 3$ & $\mathrm{C} 4$ & $109.4(4)$ & $\mathrm{C} 16$ & $\mathrm{C} 14$ & $\mathrm{C} 11$ & 109.4(4) \\
\hline $\mathrm{C} 8$ & $\mathrm{C} 3$ & $\mathrm{C} 1$ & $121.5(4)$ & $\mathrm{C} 12$ & $\mathrm{C} 15$ & $\mathrm{C} 4$ & $112.7(4)$ \\
\hline $\mathrm{C} 8$ & $\mathrm{C} 3$ & $\mathrm{C} 4$ & $129.0(4)$ & $\mathrm{C} 10$ & C16 & $\mathrm{C} 20$ & $108.7(5)$ \\
\hline $\mathrm{C} 3$ & $\mathrm{C} 4$ & $\mathrm{~N} 2$ & $106.5(4)$ & $\mathrm{C} 14$ & $\mathrm{C} 16$ & $\mathrm{C} 10$ & $110.3(4)$ \\
\hline $\mathrm{C} 3$ & $\mathrm{C} 4$ & $\mathrm{C} 5$ & 103.7(3) & $\mathrm{C} 14$ & C16 & $\mathrm{C} 20$ & $110.0(6)$ \\
\hline $\mathrm{C} 3$ & $\mathrm{C} 4$ & $\mathrm{C} 15$ & $118.3(4)$ & C6 & $\mathrm{C} 17$ & $\mathrm{C} 11$ & $109.8(4)$ \\
\hline $\mathrm{C} 5$ & $\mathrm{C} 4$ & $\mathrm{~N} 2$ & $105.4(4)$ & C6 & $\mathrm{C} 18$ & $\mathrm{C} 23$ & $107.5(4)$ \\
\hline $\mathrm{C} 15$ & $\mathrm{C} 4$ & $\mathrm{~N} 2$ & $109.6(4)$ & $\mathrm{C} 25$ & C19 & $\mathrm{C} 12$ & $121.4(8)$ \\
\hline $\mathrm{C} 15$ & $\mathrm{C} 4$ & $\mathrm{C} 5$ & $112.4(4)$ & $\mathrm{C} 23$ & $\mathrm{C} 20$ & $\mathrm{C} 16$ & $109.4(4)$ \\
\hline $\mathrm{O} 3$ & $\mathrm{C} 5$ & N1 & $128.3(4)$ & $\mathrm{C} 23$ & $\mathrm{C} 21$ & C11 & $109.3(5)$ \\
\hline $\mathrm{O} 3$ & $\mathrm{C} 5$ & $\mathrm{C} 4$ & $125.1(4)$ & $\mathrm{C} 12$ & $\mathrm{C} 22$ & $\mathrm{C} 26$ & $121.6(8)$ \\
\hline N1 & $\mathrm{C} 5$ & $\mathrm{C} 4$ & $106.4(4)$ & $\mathrm{C} 20$ & $\mathrm{C} 23$ & $\mathrm{C} 18$ & $107.9(5)$ \\
\hline $\mathrm{O} 1$ & C6 & $\mathrm{C} 10$ & $110.0(4)$ & $\mathrm{C} 21$ & $\mathrm{C} 23$ & $\mathrm{C} 18$ & $110.7(5)$ \\
\hline $\mathrm{O} 1$ & C6 & $\mathrm{C} 17$ & $102.4(3)$ & $\mathrm{C} 21$ & $\mathrm{C} 23$ & $\mathrm{C} 20$ & $110.9(5)$ \\
\hline $\mathrm{O} 1$ & C6 & $\mathrm{C} 18$ & 113.1(4) & $\mathrm{C} 25$ & $\mathrm{C} 24$ & $\mathrm{C} 26$ & $119.6(8)$ \\
\hline $\mathrm{C} 17$ & C6 & $\mathrm{C} 10$ & $109.6(4)$ & C19 & $\mathrm{C} 25$ & $\mathrm{C} 24$ & $121.0(8)$ \\
\hline $\mathrm{C} 18$ & C6 & $\mathrm{C} 10$ & $111.6(4)$ & $\mathrm{C} 22$ & $\mathrm{C} 26$ & $\mathrm{C} 24$ & $118.2(9)$ \\
\hline C18 & C6 & $\mathrm{C} 17$ & 109.7(4) & & & & \\
\hline
\end{tabular}

\section{Table S10 Torsion Angles for 3a.}
$\begin{array}{llllllll}\text { A } & \text { B } & \text { C } & \text { D } & \text { Angle }^{\circ} & \text { A } & \text { B } & \text { C }\end{array}$
D
Angle $/^{\circ}$
$\begin{array}{llllllll}\mathrm{F} 1 & \mathrm{C} 9 & \mathrm{C} 13 & \mathrm{C} 7 & 179.6(4) & \mathrm{C} 6 & \mathrm{O} 1 & \mathrm{C} 2\end{array}$
$\mathrm{C} 2 \quad \mathrm{O} 2$
19.8(7)
$\mathrm{O} 1 \mathrm{C} 6 \quad \mathrm{C}$
$\begin{array}{lllll}\mathrm{C} 10 & \mathrm{C} 16 & 172.6(4) & \mathrm{C} 6 & \mathrm{O} 1\end{array}$
C2 N1
$-161.2(3)$ 
$\begin{array}{llllllllll}\mathrm{O} 1 & \mathrm{C} 6 & \mathrm{C} 17 & \mathrm{C} 11 & -177.7(4) & \mathrm{C} 6 & \mathrm{C} 10 & \mathrm{C} 16 & \mathrm{C} 14 & -60.9(5)\end{array}$

$\begin{array}{llllllllll}\mathrm{O} 1 & \mathrm{C} 6 & \mathrm{C} 18 & \mathrm{C} 23 & -173.9(4) & \mathrm{C} 6 & \mathrm{C} 10 & \mathrm{C} 16 & \mathrm{C} 20 & 59.7(5)\end{array}$

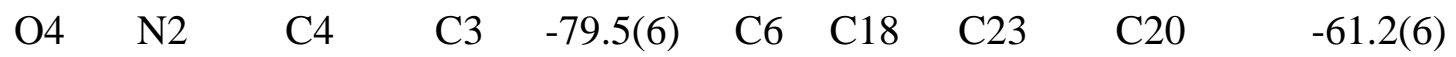

$\begin{array}{llllllllll}\mathrm{O} 4 & \mathrm{~N} 2 & \mathrm{C} 4 & \mathrm{C} 5 & 170.7(5) & \mathrm{C} 6 & \mathrm{C} 18 & \mathrm{C} 23 & \mathrm{C} 21 & 60.3(6)\end{array}$

$\begin{array}{llllllllll}\mathrm{O} 4 & \mathrm{~N} 2 & \mathrm{C} 4 & \mathrm{C} 15 & 49.6(7) & \mathrm{C} 7 & \mathrm{C} 1 & \mathrm{C} 3 & \mathrm{C} 4 & 177.2(4)\end{array}$

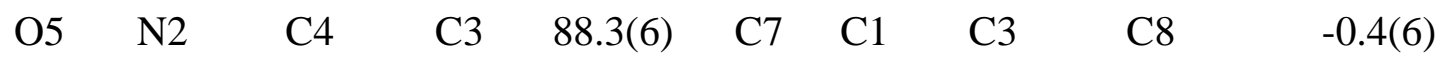

$\begin{array}{llllllllll}\mathrm{O} 5 & \mathrm{~N} 2 & \mathrm{C} 4 & \mathrm{C} 5 & -21.5(7) & \mathrm{C} 8 & \mathrm{C} 3 & \mathrm{C} 4 & \mathrm{~N} 2 & 67.1(6)\end{array}$

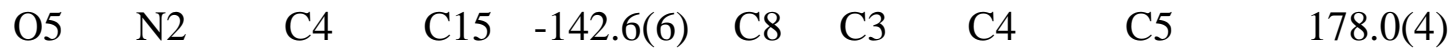

$\begin{array}{llllllllll}\mathrm{N} 1 & \mathrm{C} 1 & \mathrm{C} 3 & \mathrm{C} 4 & -0.7(5) & \mathrm{C} 8 & \mathrm{C} 3 & \mathrm{C} 4 & \mathrm{C} 15 & -56.8(6)\end{array}$

$\begin{array}{llllllllll}\mathrm{N} 1 & \mathrm{C} 1 & \mathrm{C} 3 & \mathrm{C} 8 & -178.3(4) & \mathrm{C} 8 & \mathrm{C} 9 & \mathrm{C} 13 & \mathrm{C} 7 & -0.7(7)\end{array}$

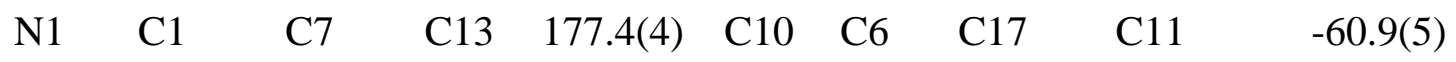

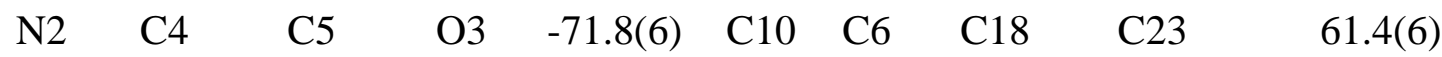

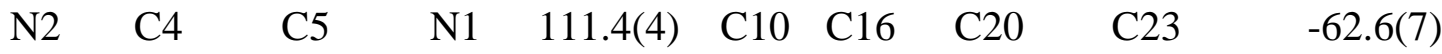

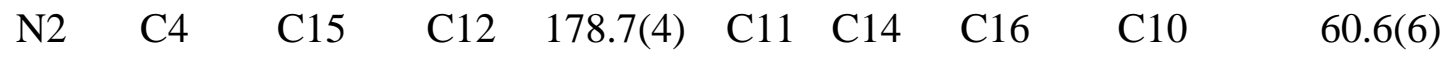

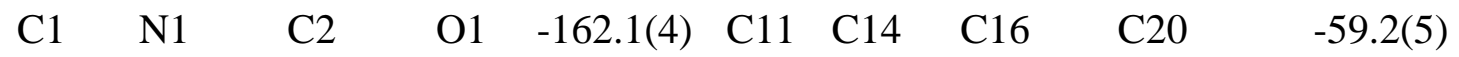

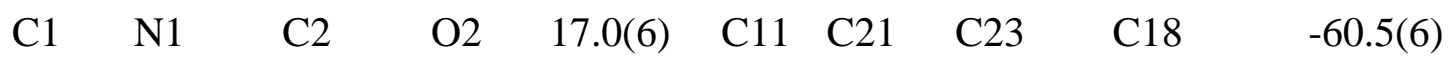

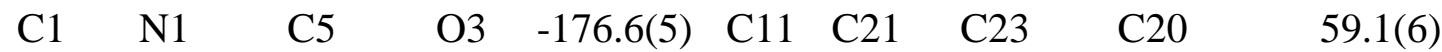

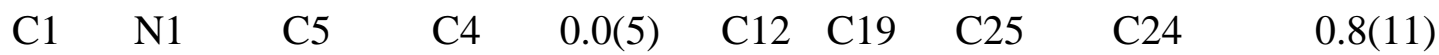

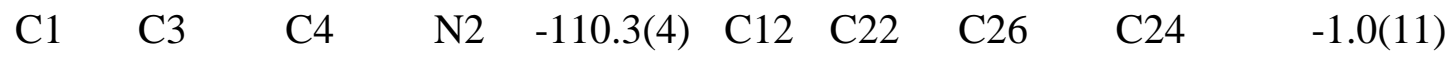

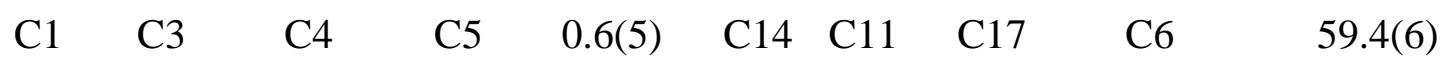

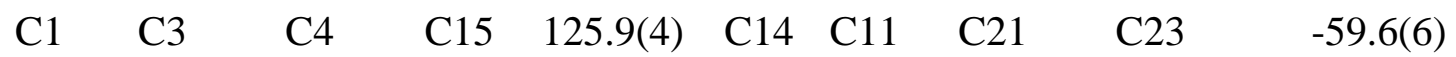

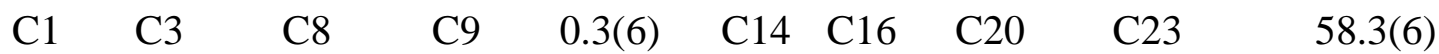

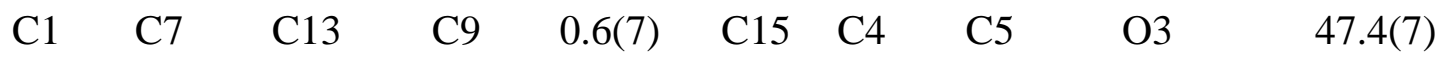

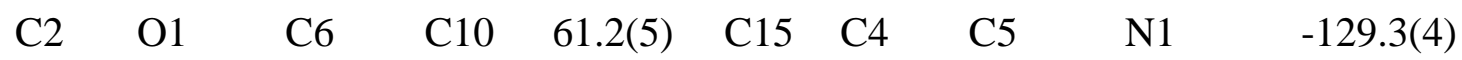

$\begin{array}{llllllllll}\mathrm{C} 2 & \mathrm{O} 1 & \mathrm{C} 6 & \mathrm{C} 17 & 177.7(4) & \mathrm{C} 15 & \mathrm{C} 12 & \mathrm{C} 19 & \mathrm{C} 25 & -177.8(6)\end{array}$

$\begin{array}{llllllllll}\mathrm{C} 2 & \mathrm{O} 1 & \mathrm{C} 6 & \mathrm{C} 18 & -64.3(5) & \mathrm{C} 15 & \mathrm{C} 12 & \mathrm{C} 22 & \mathrm{C} 26 & 177.9(6)\end{array}$

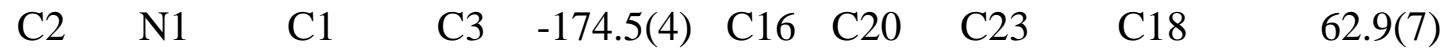

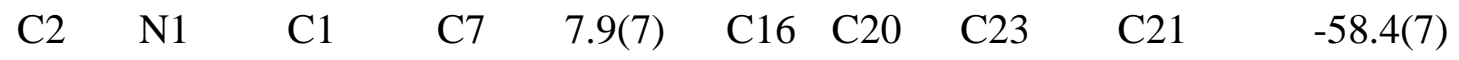

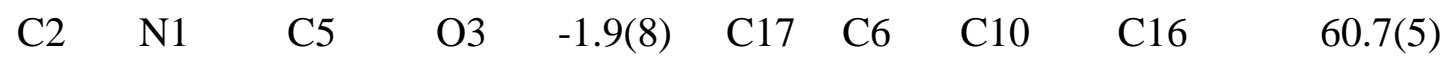




$\begin{array}{llllllllll}\mathrm{C} 2 & \mathrm{~N} 1 & \mathrm{C} 5 & \mathrm{C} 4 & 174.7(4) & \mathrm{C} 17 & \mathrm{C} 6 & \mathrm{C} 18 & \mathrm{C} 23 & -60.3(5) \\ \mathrm{C} 3 & \mathrm{C} 1 & \mathrm{C} 7 & \mathrm{C} 13 & 0.0(6) & \mathrm{C} 17 & \mathrm{C} 11 & \mathrm{C} 14 & \mathrm{C} 16 & -58.9(6) \\ \mathrm{C} 3 & \mathrm{C} 4 & \mathrm{C} 5 & \mathrm{O} 3 & 176.4(5) & \mathrm{C} 17 & \mathrm{C} 11 & \mathrm{C} 21 & \mathrm{C} 23 & 59.5(6) \\ \mathrm{C} 3 & \mathrm{C} 4 & \mathrm{C} 5 & \mathrm{~N} 1 & -0.4(5) & \mathrm{C} 18 & \mathrm{C} 6 & \mathrm{C} 10 & \mathrm{C} 16 & -61.0(5) \\ \mathrm{C} 3 & \mathrm{C} 4 & \mathrm{C} 15 & \mathrm{C} 12 & -59.0(6) & \mathrm{C} 18 & \mathrm{C} 6 & \mathrm{C} 17 & \mathrm{C} 11 & 61.9(5) \\ \mathrm{C} 3 & \mathrm{C} 8 & \mathrm{C} 9 & \mathrm{~F} 1 & 179.9(4) & \mathrm{C} 19 & \mathrm{C} 12 & \mathrm{C} 15 & \mathrm{C} 4 & -92.4(5) \\ \mathrm{C} 3 & \mathrm{C} 8 & \mathrm{C} 9 & \mathrm{C} 13 & 0.2(7) & \mathrm{C} 19 & \mathrm{C} 12 & \mathrm{C} 22 & \mathrm{C} 26 & 0.5(9) \\ \mathrm{C} 4 & \mathrm{C} 3 & \mathrm{C} 8 & \mathrm{C} 9 & -176.8(4) & \mathrm{C} 21 & \mathrm{C} 11 & \mathrm{C} 14 & \mathrm{C} 16 & 60.1(6) \\ \mathrm{C} 5 & \mathrm{~N} 1 & \mathrm{C} 1 & \mathrm{C} 3 & 0.4(5) & \mathrm{C} 21 & \mathrm{C} 11 & \mathrm{C} 17 & \mathrm{C} 6 & -60.5(6) \\ \mathrm{C} 5 & \mathrm{~N} 1 & \mathrm{C} 1 & \mathrm{C} 7 & -177.2(4) & \mathrm{C} 22 & \mathrm{C} 12 & \mathrm{C} 15 & \mathrm{C} 4 & 90.3(6) \\ \mathrm{C} 5 & \mathrm{~N} 1 & \mathrm{C} 2 & \mathrm{O} 1 & 23.8(6) & \mathrm{C} 22 & \mathrm{C} 12 & \mathrm{C} 19 & \mathrm{C} 25 & -0.4(9) \\ \mathrm{C} 5 & \mathrm{~N} 1 & \mathrm{C} 2 & \mathrm{O} 2 & -157.1(5) & \mathrm{C} 25 & \mathrm{C} 24 & \mathrm{C} 26 & \mathrm{C} 22 & 1.4(12) \\ \mathrm{C} 5 & \mathrm{C} 4 & \mathrm{C} 15 & \mathrm{C} 12 & 61.9(5) & \mathrm{C} 26 & \mathrm{C} 24 & \mathrm{C} 25 & \mathrm{C} 19 & -1.3(12)\end{array}$

Table S11 Hydrogen Atom Coordinates $\left(\AA \times 10^{4}\right)$ and Isotropic Displacement Parameters $\left(\AA^{2} \times 10^{3}\right)$ for $3 a$.

$\begin{array}{ccccc}\text { Atom } & \boldsymbol{x} & \boldsymbol{y} & \boldsymbol{z} & \mathbf{U}(\mathbf{e q}) \\ \text { H7 } & 6025 & 6591 & 6985 & 73 \\ \text { H8 } & 1772 & 8352 & 4623 & 71 \\ \text { H10A } & 2731 & 2997 & 8777 & 77 \\ \text { H10B } & 4349 & 3532 & 9333 & 77 \\ \text { H11 } & 3466 & -74 & 7685 & 89 \\ \text { H13 } & 6203 & 8432 & 6345 & 78 \\ \text { H14A } & 2285 & 950 & 9038 & 94 \\ \text { H14B } & 3626 & 205 & 9743 & 94 \\ \text { H15A } & -760 & 7096 & 4985 & 78 \\ \text { H15B } & -1124 & 5842 & 5204 & 78 \\ \text { H16 } & 3844 & 2019 & 10520 & 91 \\ \text { H17A } & 4019 & 1451 & 6508 & 78 \\ \text { H17B } & 2533 & 1725 & 7050 & 78\end{array}$




$\begin{array}{ccccc}\text { H18A } & 6735 & 3098 & 8448 & 82 \\ \text { H18B } & 6577 & 2307 & 7342 & 82 \\ \text { H19 } & -832 & 5145 & 7230 & 110 \\ \text { H20A } & 6498 & 2358 & 10398 & 108 \\ \text { H20B } & 6241 & 1082 & 10603 & 108 \\ \text { H21A } & 5985 & -245 & 8849 & 110 \\ \text { H21B } & 6115 & 233 & 7599 & 110 \\ \text { H22 } & 172 & 8304 & 6645 & 105 \\ \text { H23 } & 7682 & 1288 & 9082 & 100 \\ \text { H24 } & -351 & 7296 & 9881 & 143 \\ \text { H25 } & -808 & 5528 & 9162 & 143 \\ \text { H26 } & 212 & 8723 & 8620 & 141\end{array}$

\section{Experimental}

Single crystals of $\mathrm{C}_{26} \mathrm{H}_{25} \mathrm{FN}_{2} \mathrm{O}_{5} \mathbf{3 a}$ were obtained from diffusion of $n$-hexane into a sloution of the compound in isopropyl alcohol and methylene chloride (1:1) at room temperature. A suitable crystal was selected and measured on a XtaLAB Synergy, Dualflex, HyPix diffractometer. The crystal was kept at 293(2) K during data collection. Using Olex2 [1], the structure was solved with the Unknown [2] structure solution program using Unknown and refined with the Unknown [3] refinement package using Unknown minimisation.

1. Dolomanov, O.V., Bourhis, L.J., Gildea, R.J, Howard, J.A.K. \& Puschmann, H. (2009), J. Appl. Cryst. 42, 339-341.

2. Bourhis, L.J., Dolomanov, O.V., Gildea, R.J., Howard, J.A.K., Puschmann, H. (2015). Acta Cryst. A71, 59-75.

3. Bourhis, L.J., Dolomanov, O.V., Gildea, R.J., Howard, J.A.K., Puschmann, H. (2015). Acta Cryst. A71, 59-75.

\section{Crystal structure determination of [3a]}

Crystal Data for $\mathrm{C}_{26} \mathrm{H}_{25} \mathrm{FN}_{2} \mathrm{O}_{5}(M=464.48 \mathrm{~g} / \mathrm{mol})$ : monoclinic, space group $\mathrm{P} 2_{1}$ (no. 4), $a=$ 8.7046(3) $\AA, b=11.9983(4) \AA, c=11.5160(4) \AA, \beta=100.577(3)^{\circ}, V=1182.30(6) \AA^{3}, Z=2, T=$ 
293(2) $\mathrm{K}, \mu(\mathrm{CuK} \alpha)=0.798 \mathrm{~mm}^{-1}$, Dcalc $=1.305 \mathrm{~g} / \mathrm{cm}^{3}, 14443$ reflections measured $\left(7.81^{\circ} \leq 2 \Theta \leq\right.$ $\left.134.056^{\circ}\right), 4045$ unique $\left(R_{\text {int }}=0.0790, \mathrm{R}_{\text {sigma }}=0.0630\right)$ which were used in all calculations. The final $R_{1}$ was $0.0663(\mathrm{I}>2 \sigma(\mathrm{I}))$ and $w R_{2}$ was 0.1627 (all data). 


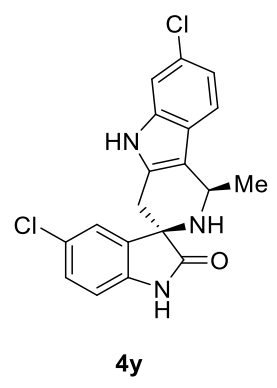

$4 y$

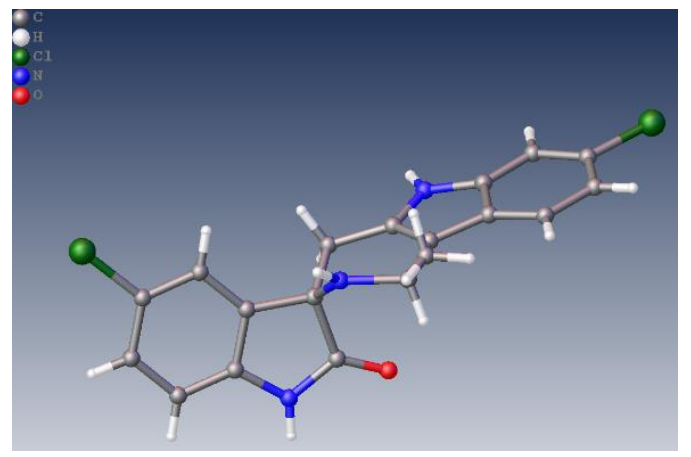

CCDC: 2097342

Table S12 Crystal data and structure refinement for $4 y$.

Identification code

Empirical formula

Formula weight

Temperature/K

Crystal system

Space group

$\mathrm{a} / \AA$

$\mathrm{b} / \AA$

$\mathrm{c} / \AA$

$\alpha^{\circ}$

$\beta /{ }^{\circ}$

$\gamma /{ }^{\circ}$

Volume $/ \AA^{3}$

Z

$\rho_{\text {calc }} \mathrm{g} / \mathrm{cm}^{3}$

$\mu / \mathrm{mm}^{-1}$

$\mathrm{F}(000)$

Crystal size $/ \mathrm{mm}^{3}$

Radiation
$4 y$

$\mathrm{C}_{22} \mathrm{H}_{23} \mathrm{Cl}_{2} \mathrm{~N}_{3} \mathrm{O}_{2}$

432.33

293(2)

monoclinic

$\mathrm{P} 21$

9.7747(3)

7.5306(3)

14.5872(4)

90

104.962(3)

90

1037.35(6)

2

1.384

3.008

452.0

$0.06 \times 0.06 \times 0.05$

$\operatorname{CuK} \alpha(\lambda=1.54184)$

$2 \Theta$ range for data collection/ ${ }^{\circ} 6.272$ to 134.16 
Index ranges

Reflections collected

Independent reflections

Data/restraints/parameters

Goodness-of-fit on $\mathrm{F}^{2}$

Final $\mathrm{R}$ indexes $[\mathrm{I}>=2 \sigma(\mathrm{I})]$

Final $\mathrm{R}$ indexes [all data]

Largest diff. peak/hole / e $\AA^{-3} 0.25 /-0.30$

Flack parameter

$-0.017(14)$
$-11 \leq \mathrm{h} \leq 11,-8 \leq \mathrm{k} \leq 8,-17 \leq 1 \leq 17$

10652

$3632\left[R_{\text {int }}=0.0596, R_{\text {sigma }}=0.0580\right]$

$3632 / 1 / 274$

1.084

$\mathrm{R}_{1}=0.0426, \mathrm{wR}_{2}=0.1110$

$\mathrm{R}_{1}=0.0492, \mathrm{wR}_{2}=0.1158$

Table S13 Fractional Atomic Coordinates $\left(\times 10^{4}\right)$ and Equivalent Isotropic Displacement Parameters $\left(\AA^{2} \times 10^{3}\right)$ for $4 y . U_{\text {eq }}$ is defined as $1 / 3$ of of the trace of the orthogonalised $U_{I J}$ tensor.

$\begin{array}{lcccc}\text { Atom } & \boldsymbol{x} & \boldsymbol{y} & \boldsymbol{z} & \mathbf{U}(\mathbf{e q}) \\ \mathrm{C} 11 & 3060.5(16) & 5090(2) & 228.0(7) & 76.6(4) \\ \mathrm{C} 12 & 7122.0(16) & 5236(2) & 9578.5(7) & 76.5(4) \\ \mathrm{O} 25 & 8741(3) & 2426(4) & 5020.0(17) & 42.0(6) \\ \mathrm{N} 1 & 3666(5) & 3835(2) & 39.1(7) \\ \mathrm{N} 2 & 4572(3) & 5995(4) & 5829(2) & 33.5(7) \\ \mathrm{N} 3 & 2139(5) & 6635(2) & 44.4(8) \\ \mathrm{C} 4 & 7773(3) & 6474(6) & 1562(3) & 52.6(11) \\ \mathrm{C} 5 & 9473(3) & 5328(7) & 1393(2) & 51.0(10) \\ \mathrm{C} 6 & 5232(5) & 4364(6) & 2096(3) & 46.6(9) \\ \mathrm{C} 7 & 4086(5) & 4546(5) & 3011(2) & 37.4(8) \\ \mathrm{C} 8 & 3733(4) & 5696(5) & 3212(2) & 35.1(8) \\ \mathrm{C} 9 & 4613(4) & 6667(6) & 2475(3) & 45.0(9) \\ \mathrm{C} 10 & 5803(4) & 5482(5) & 4212(2) & 32.7(7) \\ \mathrm{C} 11 & 6090(4) & 4254(5) & 4552(2) & 33.3(7) \\ \mathrm{C} 12 & 6470(3) & 3575(5) & 5548(2) & 36.4(8)\end{array}$




$\begin{array}{llllr}\mathrm{C} 13 & 7567(3) & 4128(5) & 6060(2) & 32.0(7) \\ \mathrm{C} 14 & 7847(4) & 8345(5) & 4702(3) & 38.5(8) \\ \mathrm{C} 15 & 7771(4) & 6356(5) & 4825(2) & 32.3(7) \\ \mathrm{C} 16 & 2805(5) & 5810(2) & 33.6(8) \\ \mathrm{C} 17 & 8645(4) & 3898(5) & 7126(2) & 35.2(8) \\ \mathrm{C} 18 & 7900(4) & 2767(6) & 7424(3) & 41.0(8) \\ \mathrm{C} 19 & 2445(7) & 8371(3) & 53.1(11) \\ \mathrm{C} 20 & 3050(4) & 9032(3) & 56.0(11) \\ \mathrm{C} 21 & 9665(5) & 4314(6) & 8733(3) & 50.8(10) \\ \mathrm{C} 22 & 9058(5) & 4657(5) & 7786(3) & 42.4(9) \\ \mathrm{O} 0 \mathrm{~A} & 7892(5) & 3544(5) & 3275(2) & 67.0(10) \\ \mathrm{C} 1 & 7281(4) & 3029(13) & 2364(6) & 43.8(18) \\ \mathrm{C} 1 \mathrm{~A} & 9210(4) & 2110(20) & 2622(9) & 52(3) \\ \mathrm{C} 2 & 8412(8) & 2523(8) & 1786(3) & 64.4(13) \\ \mathrm{C} 3 & 8658(13) & 1633(10) & 2419(5) & 90(2)\end{array}$

Table S14 Anisotropic Displacement Parameters $\left(\AA^{2} \times 10^{3}\right)$ for $4 y$. The Anisotropic displacement factor exponent takes the form: $-2 \pi^{2}\left[h^{2} a^{* 2} U_{11}+2 h k a * b * U_{12}+\ldots\right]$.

$\begin{array}{ccccccr}\text { Atom } & \mathbf{U}_{11} & \mathbf{U}_{22} & \mathbf{U}_{33} & \mathbf{U}_{23} & \mathbf{U}_{13} & \mathbf{U}_{12} \\ \text { C11 } & 106.1(10) & 71.0(8) & 35.8(5) & 1.1(6) & -12.3(5) & 11.8(8) \\ \text { C12 } & 115.7(10) & 75.7(9) & 43.7(5) & -6.5(6) & 30.8(6) & 10.3(8) \\ \text { O25 } & 46.5(14) & 39.2(16) & 41.2(14) & 0.3(11) & 12.6(11) & 4.7(12) \\ \text { N1 } & 34.4(15) & 45(2) & 34.2(15) & -0.2(13) & 2.0(11) & -9.4(13) \\ \text { N2 } & 36.8(15) & 28.9(16) & 32.8(15) & -1.1(11) & 5.3(12) & -1.3(12) \\ \text { N3 } & 43.6(17) & 45(2) & 42.4(17) & 7.4(14) & 7.5(13) & 14.5(15) \\ \text { C4 } & 71(3) & 50(3) & 36(2) & 10.7(18) & 12.2(18) & 8(2) \\ \text { C5 } & 64(3) & 46(2) & 35.0(17) & 0.8(19) & -1.8(16) & 14(2) \\ \text { C6 } & 45(2) & 48(2) & 39(2) & -1.4(17) & -3.7(15) & 0.7(17)\end{array}$




\begin{tabular}{|c|c|c|c|c|c|c|}
\hline $\mathrm{C} 7$ & $38.1(18)$ & $37(2)$ & $33.6(17)$ & $0.9(14)$ & $2.1(13)$ & $3.1(15)$ \\
\hline $\mathrm{C} 8$ & $36.0(18)$ & $35(2)$ & $33.6(17)$ & $0.9(14)$ & $8.2(13)$ & $4.4(15)$ \\
\hline $\mathrm{C} 9$ & $52(2)$ & $42(2)$ & $41.4(19)$ & 7.1(17) & $12.6(16)$ & $4.5(18)$ \\
\hline $\mathrm{C} 10$ & $30.2(16)$ & $33(2)$ & $33.3(15)$ & $1.4(14)$ & $6.3(12)$ & $0.0(14)$ \\
\hline $\mathrm{C} 11$ & $28.5(16)$ & $37(2)$ & $31.6(16)$ & $-1.3(14)$ & $2.7(12)$ & $-1.0(14)$ \\
\hline C12 & $32.9(18)$ & $39(2)$ & $35.2(18)$ & $2.5(15)$ & $6.1(13)$ & $-5.5(15)$ \\
\hline C13 & $32.2(17)$ & $29.4(18)$ & $31.4(16)$ & $1.8(14)$ & 3.1(13) & $0.8(14)$ \\
\hline C14 & $39.6(19)$ & $33(2)$ & 41.7(19) & $3.7(15)$ & $7.8(14)$ & $-2.6(15)$ \\
\hline $\mathrm{C} 15$ & $29.5(17)$ & $30.4(19)$ & $35.8(17)$ & $1.5(14)$ & $6.5(13)$ & $-2.2(13)$ \\
\hline C16 & $31.3(17)$ & $31.6(19)$ & $36.8(18)$ & $1.5(14)$ & $6.5(13)$ & $-0.9(14)$ \\
\hline C17 & $37.6(18)$ & $31(2)$ & $34.1(17)$ & $2.7(14)$ & 4.1(13) & $-2.3(15)$ \\
\hline C18 & $43(2)$ & $39(2)$ & $38.3(19)$ & $4.5(16)$ & $4.7(15)$ & $1.6(16)$ \\
\hline C19 & $56(2)$ & $57(3)$ & $40(2)$ & 11.6(19) & $0.8(17)$ & $9(2)$ \\
\hline $\mathrm{C} 20$ & $71(3)$ & $56(3)$ & $34(2)$ & $7.6(19)$ & $1.2(18)$ & $-3(2)$ \\
\hline $\mathrm{C} 21$ & $68(3)$ & $47(2)$ & $38(2)$ & $-2.6(17)$ & $13.8(17)$ & $-4(2)$ \\
\hline $\mathrm{C} 22$ & $46(2)$ & $41(2)$ & $39.6(18)$ & $-1.1(15)$ & $11.2(15)$ & $-1.8(16)$ \\
\hline $\mathrm{OOA}$ & $82(2)$ & $76(2)$ & $44.2(15)$ & $-13.4(16)$ & $19.6(14)$ & $-30.7(19)$ \\
\hline $\mathrm{C} 1$ & $46(4)$ & $37(5)$ & $43(4)$ & $5(3)$ & $1(3)$ & $4(3)$ \\
\hline $\mathrm{C} 1 \mathrm{~A}$ & $42(6)$ & $62(10)$ & $47(7)$ & $-9(6)$ & $2(5)$ & $2(6)$ \\
\hline $\mathrm{C} 2$ & $77(3)$ & $67(3)$ & $49(2)$ & $-7(2)$ & $15(2)$ & $5(3)$ \\
\hline $\mathrm{C} 3$ & $105(5)$ & $83(5)$ & $82(4)$ & $-22(3)$ & $25(3)$ & $-39(4)$ \\
\hline
\end{tabular}

Table S15 Bond Lengths for $4 y$.

\begin{tabular}{|c|c|c|c|c|c|}
\hline Atom & Atom & Length/Å & Atom & Atom & Length/Å \\
\hline $\mathrm{Cl1}$ & $\mathrm{C} 5$ & $1.745(4)$ & $\mathrm{C} 11$ & $\mathrm{C} 12$ & $1.494(5)$ \\
\hline $\mathrm{Cl} 2$ & $\mathrm{C} 21$ & $1.747(4)$ & $\mathrm{C} 12$ & $\mathrm{C} 13$ & $1.534(5)$ \\
\hline $\mathrm{O} 25$ & $\mathrm{C} 16$ & $1.214(4)$ & $\mathrm{C} 13$ & $\mathrm{C} 16$ & $1.560(5)$ \\
\hline N1 & $\mathrm{C} 7$ & $1.382(5)$ & $\mathrm{C} 13$ & $\mathrm{C} 17$ & $1.513(5)$ \\
\hline N1 & $\mathrm{C} 11$ & $1.390(4)$ & C14 & C15 & $1.512(5)$ \\
\hline
\end{tabular}




$\begin{array}{llllll}\mathrm{N} 2 & \mathrm{C} 13 & 1.472(5) & \mathrm{C} 17 & \mathrm{C} 18 & 1.387(5) \\ \mathrm{N} 2 & \mathrm{C} 15 & 1.489(5) & \mathrm{C} 17 & \mathrm{C} 22 & 1.386(5) \\ \mathrm{N} 3 & \mathrm{C} 16 & 1.361(5) & \mathrm{C} 18 & \mathrm{C} 19 & 1.378(5) \\ \mathrm{N} 3 & \mathrm{C} 18 & 1.401(5) & \mathrm{C} 19 & \mathrm{C} 20 & 1.389(7) \\ \mathrm{C} 4 & \mathrm{C} 5 & 1.385(7) & \mathrm{C} 20 & \mathrm{C} 21 & 1.377(7) \\ \mathrm{C} 4 & \mathrm{C} 9 & 1.386(6) & \mathrm{C} 21 & \mathrm{C} 22 & 1.380(6) \\ \mathrm{C} 5 & \mathrm{C} 6 & 1.371(6) & \mathrm{O} 0 \mathrm{~A} & \mathrm{C} 1 & 1.410(8) \\ \mathrm{C} 6 & \mathrm{C} 7 & 1.395(5) & \mathrm{O} 0 \mathrm{~A} & \mathrm{C} 1 \mathrm{~A} & 1.448(14) \\ \mathrm{C} 7 & \mathrm{C} 8 & 1.418(5) & \mathrm{C} 1 & \mathrm{C} 2 & 1.462(10) \\ \mathrm{C} 8 & \mathrm{C} 9 & 1.388(5) & \mathrm{C} 1 & \mathrm{C} 3 & 1.514(11) \\ \mathrm{C} 8 & \mathrm{C} 10 & 1.445(5) & \mathrm{C} 1 \mathrm{~A} & \mathrm{C} 2 & 1.582(14) \\ \mathrm{C} 10 & \mathrm{C} 11 & 1.357(5) & \mathrm{C} 1 \mathrm{~A} & \mathrm{C} 3 & 1.314(14) \\ \mathrm{C} 10 & \mathrm{C} 15 & 1.504(5) & & & \end{array}$

Table S16 Bond Angles for 4y.

\begin{tabular}{|c|c|c|c|c|c|c|c|}
\hline Atom & Atom & Atom & Angle ${ }^{\circ}$ & Atom & Atom & Atom & Angle ${ }^{\circ}$ \\
\hline $\mathrm{C} 7$ & N1 & $\mathrm{C} 11$ & $107.7(3)$ & $\mathrm{C} 17$ & C13 & $\mathrm{C} 12$ & $113.2(3)$ \\
\hline C13 & $\mathrm{N} 2$ & C15 & $115.8(3)$ & $\mathrm{C} 17$ & $\mathrm{C} 13$ & $\mathrm{C} 16$ & $100.8(3)$ \\
\hline $\mathrm{C} 16$ & N3 & $\mathrm{C} 18$ & 111.6(3) & $\mathrm{N} 2$ & $\mathrm{C} 15$ & $\mathrm{C} 10$ & $106.9(3)$ \\
\hline C5 & $\mathrm{C} 4$ & C9 & $119.9(4)$ & $\mathrm{N} 2$ & C15 & C14 & $108.1(3)$ \\
\hline $\mathrm{C} 4$ & C5 & $\mathrm{Cl1}$ & $118.2(3)$ & $\mathrm{C} 10$ & $\mathrm{C} 15$ & $\mathrm{C} 14$ & $115.0(3)$ \\
\hline C6 & $\mathrm{C} 5$ & $\mathrm{Cl1}$ & $118.8(4)$ & $\mathrm{O} 25$ & $\mathrm{C} 16$ & N3 & $125.4(3)$ \\
\hline C6 & $\mathrm{C} 5$ & $\mathrm{C} 4$ & $123.0(3)$ & $\mathrm{O} 25$ & $\mathrm{C} 16$ & $\mathrm{C} 13$ & $126.4(3)$ \\
\hline $\mathrm{C} 5$ & C6 & $\mathrm{C} 7$ & $116.8(4)$ & N3 & $\mathrm{C} 16$ & $\mathrm{C} 13$ & $108.2(3)$ \\
\hline $\mathrm{N} 1$ & $\mathrm{C} 7$ & C6 & $129.4(4)$ & $\mathrm{C} 18$ & $\mathrm{C} 17$ & $\mathrm{C} 13$ & $109.5(3)$ \\
\hline $\mathrm{N} 1$ & $\mathrm{C} 7$ & $\mathrm{C} 8$ & $108.6(3)$ & $\mathrm{C} 22$ & $\mathrm{C} 17$ & $\mathrm{C} 13$ & $130.5(3)$ \\
\hline C6 & $\mathrm{C} 7$ & $\mathrm{C} 8$ & $121.9(3)$ & $\mathrm{C} 22$ & $\mathrm{C} 17$ & $\mathrm{C} 18$ & $119.9(3)$ \\
\hline $\mathrm{C} 7$ & $\mathrm{C} 8$ & $\mathrm{C} 10$ & $105.9(3)$ & $\mathrm{C} 17$ & C18 & N3 & $109.7(3)$ \\
\hline C9 & $\mathrm{C} 8$ & $\mathrm{C} 7$ & $118.7(3)$ & C19 & C18 & N3 & $128.2(4)$ \\
\hline
\end{tabular}




$\begin{array}{cccccccc}\text { C9 } & \text { C8 } & \text { C10 } & 135.4(3) & \mathrm{C} 19 & \mathrm{C} 18 & \mathrm{C} 17 & 122.0(4) \\ \mathrm{C} 4 & \mathrm{C} 9 & \mathrm{C} 8 & 119.7(4) & \mathrm{C} 18 & \mathrm{C} 19 & \mathrm{C} 20 & 117.8(4) \\ \mathrm{C} 8 & \mathrm{C} 10 & \mathrm{C} 15 & 130.0(3) & \mathrm{C} 21 & \mathrm{C} 20 & \mathrm{C} 19 & 120.0(3) \\ \mathrm{C} 11 & \mathrm{C} 10 & \mathrm{C} 8 & 107.3(3) & \mathrm{C} 20 & \mathrm{C} 21 & \mathrm{C} 2 & 118.9(3) \\ \mathrm{C} 11 & \mathrm{C} 10 & \mathrm{C} 15 & 122.6(3) & \mathrm{C} 20 & \mathrm{C} 21 & \mathrm{C} 22 & 122.5(4) \\ \mathrm{N} 1 & \mathrm{C} 11 & \mathrm{C} 12 & 123.8(3) & \mathrm{C} 22 & \mathrm{C} 21 & \mathrm{C} 2 & 118.6(3) \\ \mathrm{C} 10 & \mathrm{C} 11 & \mathrm{~N} 1 & 110.5(3) & \mathrm{C} 21 & \mathrm{C} 22 & \mathrm{C} 17 & 117.7(4) \\ \mathrm{C} 10 & \mathrm{C} 11 & \mathrm{C} 12 & 125.7(3) & \mathrm{O} 0 \mathrm{~A} & \mathrm{C} 1 & \mathrm{C} 2 & 109.5(6) \\ \mathrm{C} 11 & \mathrm{C} 12 & \mathrm{C} 13 & 109.0(3) & \mathrm{O} 0 \mathrm{~A} & \mathrm{C} 1 & \mathrm{C} 3 & 111.6(6) \\ \mathrm{N} 2 & \mathrm{C} 13 & \mathrm{C} 12 & 108.6(3) & \mathrm{C} 2 & \mathrm{C} 1 & \mathrm{C} 3 & 114.4(7) \\ \mathrm{N} 2 & \mathrm{C} 13 & \mathrm{C} 16 & 114.6(3) & \mathrm{O} 0 \mathrm{~A} & \mathrm{C} 1 \mathrm{~A} & \mathrm{C} 2 & 101.4(10) \\ \mathrm{N} 2 & \mathrm{C} 13 & \mathrm{C} 17 & 109.8(3) & \mathrm{C} 3 & \mathrm{C} 1 \mathrm{~A} & \mathrm{O} 0 \mathrm{~A} & 122.2(10) \\ \mathrm{C} 12 & \mathrm{C} 13 & \mathrm{C} 16 & 109.8(3) & \mathrm{C} 3 & \mathrm{C} 1 \mathrm{~A} & \mathrm{C} 2 & 119.3(9)\end{array}$

\section{Table S17 Torsion Angles for $4 y$.}

$\begin{array}{lccccccccc}\text { A } & \mathbf{B} & \mathbf{C} & \mathbf{D} & \mathbf{A n g l e} /^{\circ} & \mathbf{A} & \mathbf{B} & \mathbf{C} & \mathbf{D} & \text { Angle }{ }^{\circ} \\ \mathrm{C} 11 & \mathrm{C} 5 & \mathrm{C} 6 & \mathrm{C} 7 & 177.7(3) & \mathrm{C} 12 & \mathrm{C} 13 & \mathrm{C} 17 & \mathrm{C} 18 & 121.8(3) \\ \mathrm{C} 12 & \mathrm{C} 21 & \mathrm{C} 22 & \mathrm{C} 17 & -179.3(3) & \mathrm{C} 12 & \mathrm{C} 13 & \mathrm{C} 17 & \mathrm{C} 22 & -60.5(5) \\ \mathrm{N} 1 & \mathrm{C} 7 & \mathrm{C} 8 & \mathrm{C} 9 & 177.4(3) & \mathrm{C} 13 & \mathrm{~N} 2 & \mathrm{C} 15 & \mathrm{C} 10 & 50.1(4) \\ \mathrm{N} 1 & \mathrm{C} 7 & \mathrm{C} 8 & \mathrm{C} 10 & -0.8(4) & \mathrm{C} 13 & \mathrm{~N} 2 & \mathrm{C} 15 & \mathrm{C} 14 & 174.4(3) \\ \mathrm{N} 1 & \mathrm{C} 11 & \mathrm{C} 12 & \mathrm{C} 13 & 164.8(3) & \mathrm{C} 13 & \mathrm{C} 17 & \mathrm{C} 18 & \mathrm{~N} 3 & -3.2(4) \\ \mathrm{N} 2 & \mathrm{C} 13 & \mathrm{C} 16 & \mathrm{O} 25 & -66.8(4) & \mathrm{C} 13 & \mathrm{C} 17 & \mathrm{C} 18 & \mathrm{C} 19 & 174.1(4) \\ \mathrm{N} 2 & \mathrm{C} 13 & \mathrm{C} 16 & \mathrm{~N} 3 & 113.2(3) & \mathrm{C} 13 & \mathrm{C} 17 & \mathrm{C} 22 & \mathrm{C} 21 & -174.5(4) \\ \mathrm{N} 2 & \mathrm{C} 13 & \mathrm{C} 17 & \mathrm{C} 18 & -116.6(3) & \mathrm{C} 15 & \mathrm{~N} 2 & \mathrm{C} 13 & \mathrm{C} 12 & -67.7(3) \\ \mathrm{N} 2 & \mathrm{C} 13 & \mathrm{C} 17 & \mathrm{C} 22 & 61.1(5) & \mathrm{C} 15 & \mathrm{~N} 2 & \mathrm{C} 13 & \mathrm{C} 16 & 55.4(4) \\ \mathrm{N} 3 & \mathrm{C} 18 & \mathrm{C} 19 & \mathrm{C} 20 & 179.4(4) & \mathrm{C} 15 & \mathrm{~N} 2 & \mathrm{C} 13 & \mathrm{C} 17 & 168.0(3) \\ \mathrm{C} 4 & \mathrm{C} 5 & \mathrm{C} 6 & \mathrm{C} 7 & -1.6(7) & \mathrm{C} 15 & \mathrm{C} 10 & \mathrm{C} 11 & \mathrm{~N} 1 & -179.8(3) \\ \mathrm{C} 5 & \mathrm{C} 4 & \mathrm{C} 9 & \mathrm{C} 8 & 0.6(6) & \mathrm{C} 15 & \mathrm{C} 10 & \mathrm{C} 11 & \mathrm{C} 12 & -1.2(6) \\ \mathrm{C} 5 & \mathrm{C} 6 & \mathrm{C} 7 & \mathrm{~N} 1 & -175.4(4) & \mathrm{C} 16 & \mathrm{~N} 3 & \mathrm{C} 18 & \mathrm{C} 17 & -0.1(5)\end{array}$




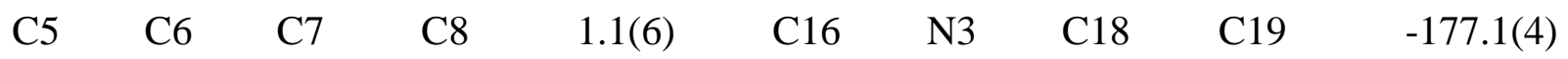

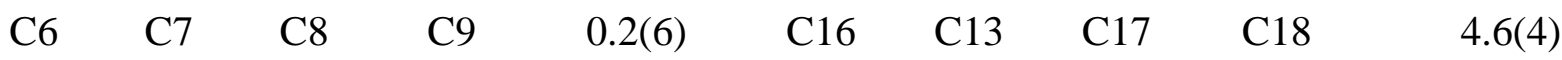

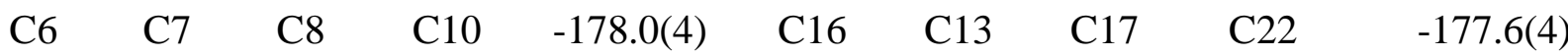

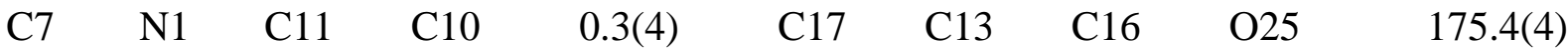

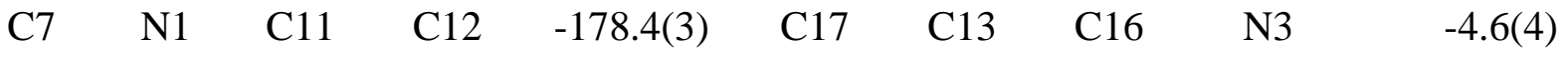

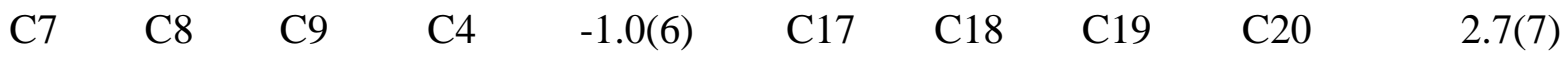

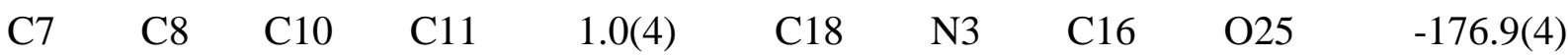

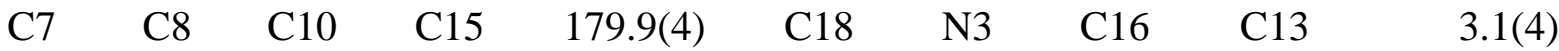

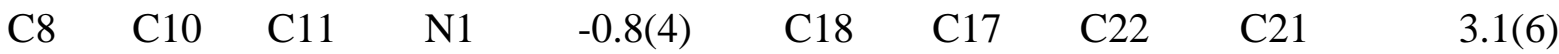

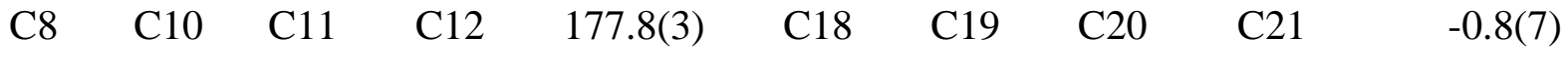

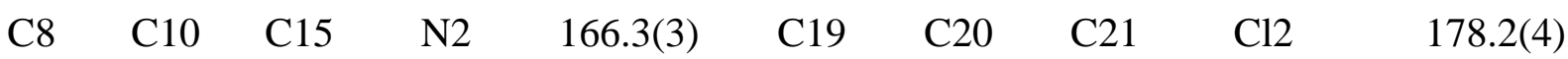

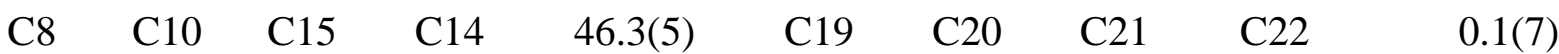

$\begin{array}{llllllllll}\text { C9 } & \text { C4 } & \text { C5 } & \text { C11 } & -178.5(3) & \text { C20 } & \text { C21 } & \text { C22 } & \text { C17 } & -1.2(7)\end{array}$

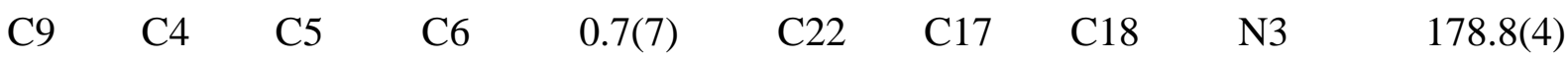

$\begin{array}{llllllllll}\text { C9 } & \mathrm{C} 8 & \mathrm{C} 10 & \mathrm{C} 11 & -176.7(4) & \mathrm{C} 22 & \mathrm{C} 17 & \mathrm{C} 18 & \mathrm{C} 19 & -4.0(6)\end{array}$

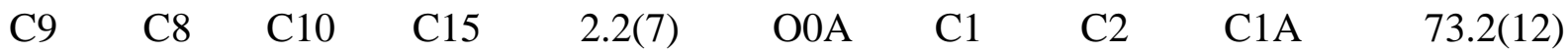

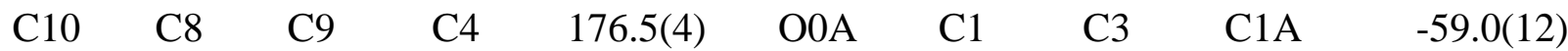

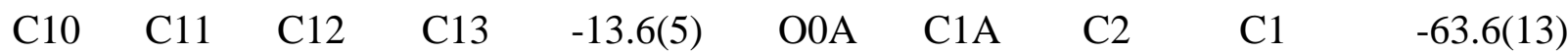

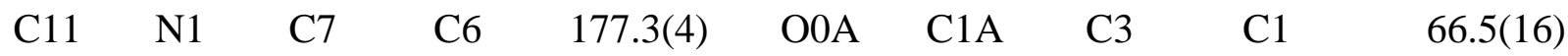

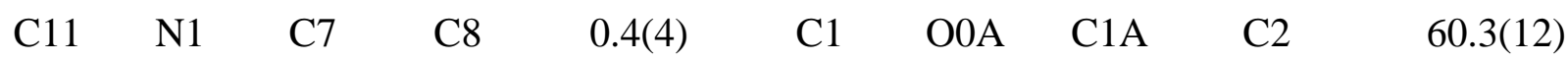

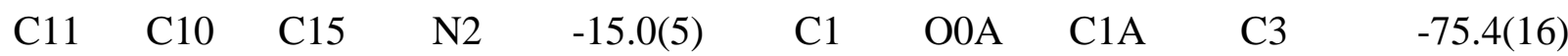

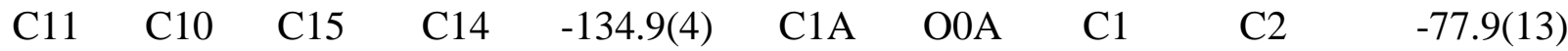

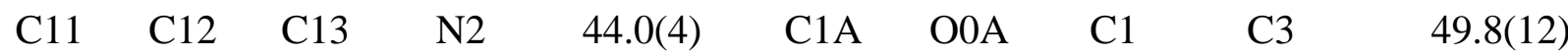

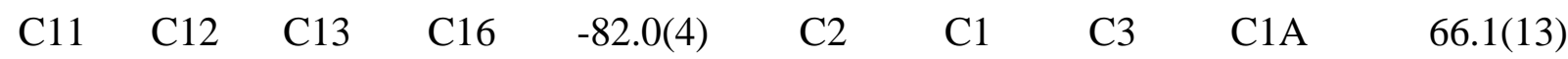

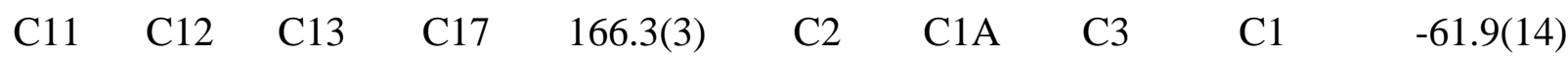

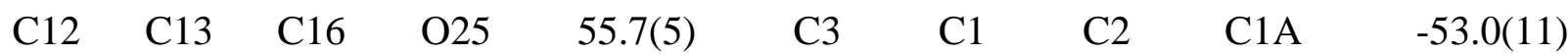

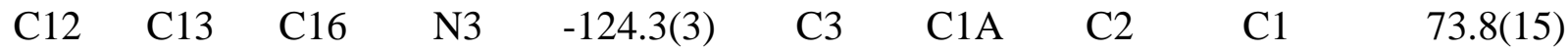


Table S18 Hydrogen Atom Coordinates $\left(\AA \times 10^{4}\right)$ and Isotropic Displacement Parameters $\left(\AA^{2} \times 10^{3}\right)$ for $4 y$.

\begin{tabular}{|c|c|c|c|c|}
\hline Atom & $x$ & $y$ & $z$ & $\mathrm{U}(\mathbf{e q})$ \\
\hline H1 & 3952 & 2893 & 3893 & 47 \\
\hline $\mathrm{H} 2$ & 7886 & 6824 & 6247 & 40 \\
\hline H3 & 10169 & 1421 & 6669 & 53 \\
\hline $\mathrm{H} 4$ & 5425 & 7112 & 1064 & 63 \\
\hline H6 & 2945 & 3624 & 1969 & 56 \\
\hline H9 & 6856 & 7444 & 2593 & 54 \\
\hline $\mathrm{H} 12 \mathrm{~A}$ & 5971 & 2291 & 5546 & 44 \\
\hline $\mathrm{H} 12 \mathrm{~B}$ & 5390 & 4061 & 5875 & 44 \\
\hline H14A & 6995 & 8883 & 4782 & 58 \\
\hline H14B & 7947 & 8603 & 4078 & 58 \\
\hline $\mathrm{H} 14 \mathrm{C}$ & 8647 & 8810 & 5167 & 58 \\
\hline H15 & 8612 & 5809 & 4696 & 39 \\
\hline H19 & 10460 & 1724 & 8561 & 64 \\
\hline $\mathrm{H} 20$ & 9440 & 3033 & 9676 & 67 \\
\hline $\mathrm{H} 22$ & 6482 & 5373 & 7598 & 51 \\
\hline $\mathrm{H} 0 \mathrm{~A}$ & 8888 & 3065 & 3678 & 100 \\
\hline H0AA & 9105 & 3302 & 3800 & 100 \\
\hline H1A & 7891 & 4081 & 2067 & 53 \\
\hline H1AA & 9167 & 1061 & 2930 & 63 \\
\hline $\mathrm{H} 2 \mathrm{AA}$ & 10095 & 3406 & 1847 & 97 \\
\hline $\mathrm{H} 2 \mathrm{AB}$ & 8844 & 2435 & 1133 & 97 \\
\hline $\mathrm{H} 2 \mathrm{AC}$ & 9793 & 1396 & 1997 & 97 \\
\hline $\mathrm{H} 2 \mathrm{BD}$ & 8903 & 3521 & 1427 & 97 \\
\hline $\mathrm{H} 2 \mathrm{BE}$ & 9280 & 1505 & 1379 & 97 \\
\hline $\mathrm{H} 2 \mathrm{BF}$ & 10352 & 2793 & 2045 & 97 \\
\hline H3AA & 7784 & 515 & 2597 & 135 \\
\hline
\end{tabular}




$\begin{array}{lllll}\text { H3AB } & 6653 & 1520 & 1810 & 135 \\ \text { H3AC } & 6832 & 1980 & 2883 & 135 \\ \text { H3BD } & 7039 & 1457 & 2996 & 135 \\ \text { H3BE } & 7198 & 547 & 2062 & 135 \\ \text { H3BF } & 6746 & 2547 & 2049 & 135\end{array}$

Table S19 Atomic Occupancy for $4 y$.

$\begin{array}{cccccc}\text { Atom } & \text { Occupancy } & \text { Atom } & \text { Occupancy } & \text { Atom } & \text { Occupancy } \\ \text { H0A } & 0.6 & \text { H0AA } & 0.4 & \text { C1 } & 0.6 \\ \text { H1A } & 0.6 & \text { C1A } & 0.4 & \text { H1AA } & 0.4 \\ \text { H2AA } & 0.6 & \text { H2AB } & 0.6 & \text { H2AC } & 0.6 \\ \text { H2BD } & 0.4 & \text { H2BE } & 0.4 & \text { H2BF } & 0.4 \\ \text { H3AA } & 0.6 & \text { H3AB } & 0.6 & \text { H3AC } & 0.6 \\ \text { H3BD } & 0.4 & \text { H3BE } & 0.4 & \text { H3BF } & 0.4\end{array}$

\section{Experimental}

Single crystals of $\mathrm{C}_{26} \mathrm{H}_{25} \mathrm{FN}_{2} \mathrm{O}_{5} 4 \mathrm{y}$ were obtained from diffusion of $n$-hexane into a sloution of the compound in isopropyl alcohol and methylene chloride (1:1) at room temperature. A suitable crystal was selected and measured on a XtaLAB Synergy, Dualflex, HyPix diffractometer. The crystal was kept at 293(2) K during data collection. Using Olex2 [1], the structure was solved with the Unknown [2] structure solution program using Unknown and refined with the Unknown [3] refinement package using Unknown minimisation.

1. Dolomanov, O.V., Bourhis, L.J., Gildea, R.J, Howard, J.A.K. \& Puschmann, H. (2009), J. Appl. Cryst. 42, 339-341.

2. Bourhis, L.J., Dolomanov, O.V., Gildea, R.J., Howard, J.A.K., Puschmann, H. (2015). Acta Cryst. A71, 59-75.

3. Bourhis, L.J., Dolomanov, O.V., Gildea, R.J., Howard, J.A.K., Puschmann, H. (2015). Acta Cryst. A71, 59-75. 
Crystal Data for $\mathrm{C}_{22} \mathrm{H}_{23} \mathrm{Cl}_{2} \mathrm{~N}_{3} \mathrm{O}_{2}(M=432.33 \mathrm{~g} / \mathrm{mol})$ : monoclinic, space group $\mathrm{P} 2{ }_{1}$ (no. 4), $a=$ 9.7747(3) $\AA, b=7.5306(3) \AA, c=14.5872(4) \AA, \beta=104.962(3)^{\circ}, V=1037.35(6) \AA^{3}, Z=2, T=293(2) \mathrm{K}$, $\mu(\mathrm{CuK \alpha})=3.008 \mathrm{~mm}^{-1}$, Dcalc $=1.384 \mathrm{~g} / \mathrm{cm}^{3}, 10652$ reflections measured $\left(6.272^{\circ} \leq 2 \Theta \leq 134.16^{\circ}\right), 3632$ unique $\left(R_{\text {int }}=0.0596, \mathrm{R}_{\text {sigma }}=0.0580\right)$ which were used in all calculations. The final $R_{1}$ was $0.0426(\mathrm{I}>$ $2 \sigma(\mathrm{I}))$ and $w R_{2}$ was 0.1158 (all data). 


\section{DFT calculations}

All the calculations were carried out in Gaussian 16 package ${ }^{[3]}$ by employing the density-functional theory (DFT) method ${ }^{[4]}$. For geometry optimization, all the structures including ground states [Int $1(R)$, Int $1(S)$, Int $2(R)$, Int $2(S)$, Int 3, Int $4(R)$, Int $4(S)$ ] were performed at B3LYP method ${ }^{[5]}$ with 6-31G basis set ${ }^{[6]}$ for $\mathrm{C}, \mathrm{H}, \mathrm{O}, \mathrm{N}, \mathrm{F}$ and $\mathrm{Cl}$ atoms, and Lanl2dz basis set ${ }^{[7]}$ for $\mathrm{Ni}$ atom. For vibration frequency calculation, same computational method with $6-31+\mathrm{G}(\mathrm{d})$ basis set ${ }^{[8]}$ for $\mathrm{C}, \mathrm{H}, \mathrm{O}, \mathrm{N}, \mathrm{F}$ and $\mathrm{Cl}$ atoms, and Lanl2dz basis set ${ }^{[7]}$ for $\mathrm{Ni}$ atom, was carried out to make sure that the optimized structures were true energy minima, and obtained the absolute free energies of the optimized molecules. All the calculations considered the solvation effect $\left(\mathrm{CH}_{2} \mathrm{Cl}_{2}\right.$ solvent) provided by the SMD solvent continuum models. ${ }^{[9]}$

Table S20. Free energies of Int $1(R, S)$, Int $2(R, S)$, Int 3 , Int $4(R, S), \mathrm{NO}_{2}{ }^{\circ},{ }^{t} \mathrm{BuO}$, and ${ }^{t} \mathrm{BuOH}^{[\mathrm{a}]}$

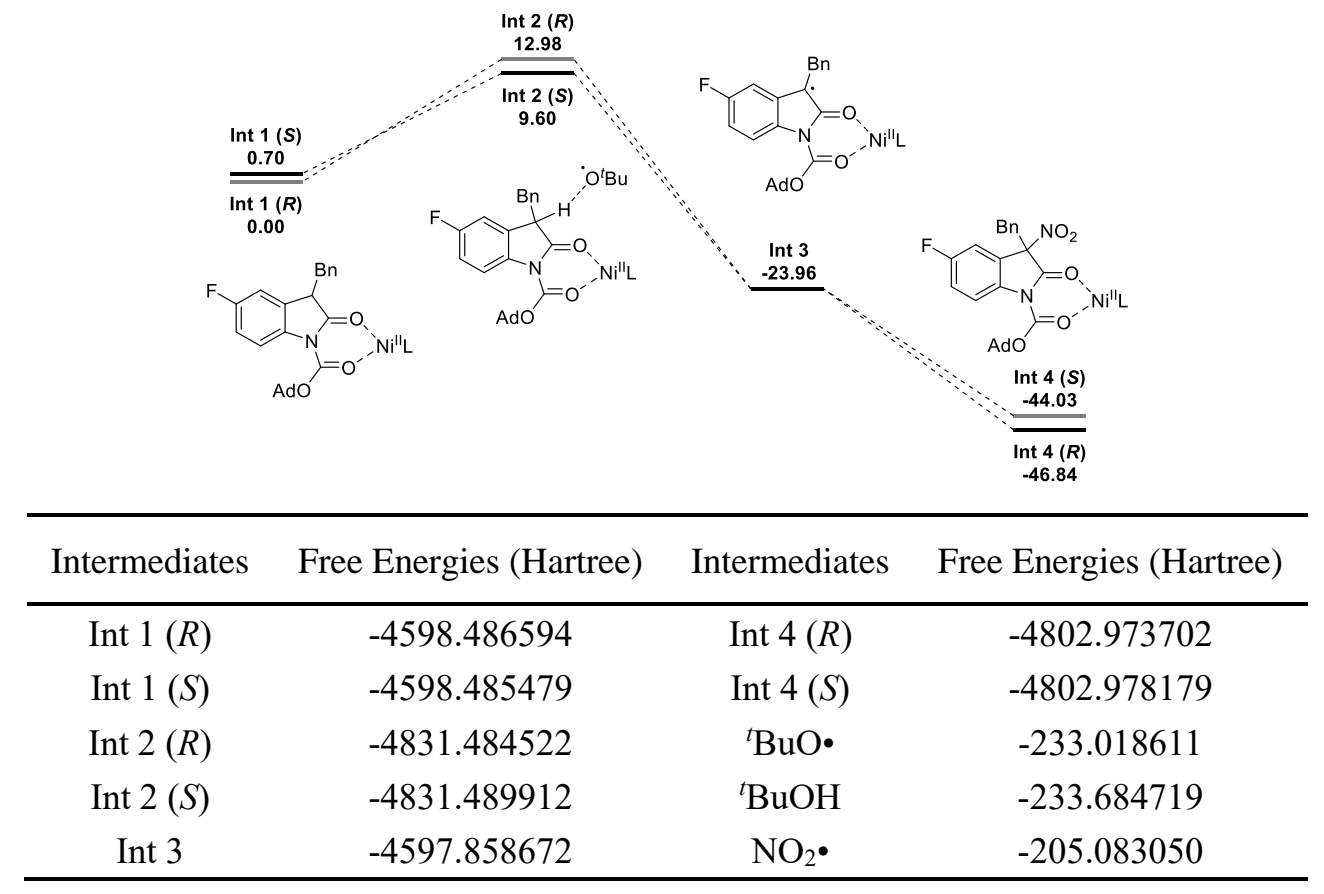

[a] Method: B3LYP; Basis Set: 6-31G(Lanl2dz)//6-31+G*(Lanl2dz); Solvent: $\mathrm{CH}_{2} \mathrm{Cl}_{2}$, SMD model. 1 Hartree $=627.5$ $\mathrm{kcal} / \mathrm{mol}$

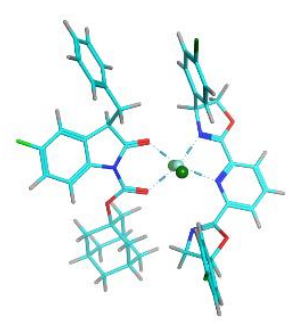

Int $1(R)$

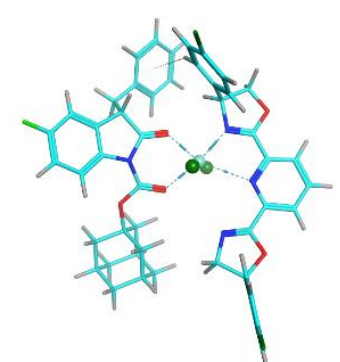

Int $1(S)$

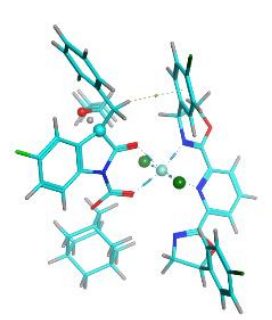

Int $2(R)$ 


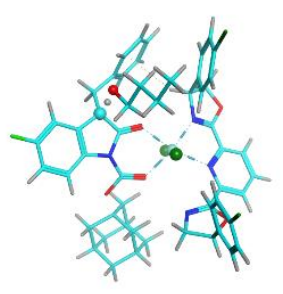

Int $2(S)$

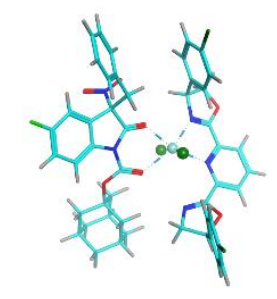

Int $4(S)$

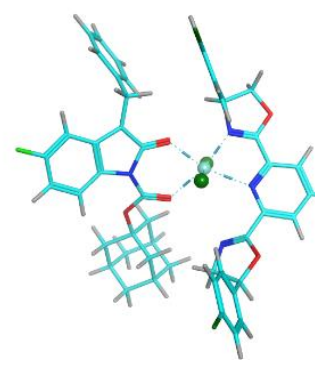

Int 3

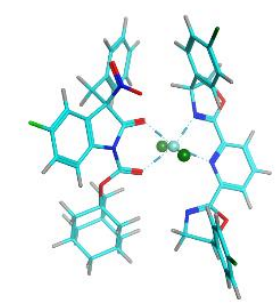

Int $4(R)$

Scheme S1. The calculation model of the intermediates

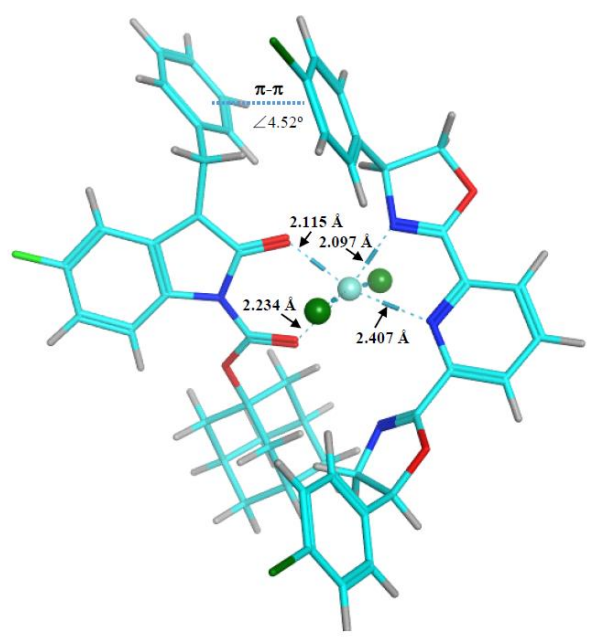

Scheme S2. The calculation model of TS

\section{Calculating data}

Int $1(S)$

$\Delta \mathbf{G}=\mathbf{- 4 5 9 8 . 4 8 5 4 7 9}$ hartree
C $\quad 4.744802$
$0.131233-2.425936$
$\begin{array}{llll}\text { C } & 3.675241 & -0.675335 & -2.794074\end{array}$
$\begin{array}{llll}\text { C } & 2.426657 & -0.477766 & -2.190861\end{array}$
$\begin{array}{llll}\text { C } & 2.316139 & 0.526833 & -1.232698\end{array}$
$\begin{array}{llll}\text { C } & 3.401783 & 1.339793 & -0.887569\end{array}$
$\begin{array}{llll}\text { C } & 4.645376 & 1.148139 & -1.481526\end{array}$
N $\quad \begin{array}{lll}1.154830 & 0.984792 & -0.519601\end{array}$
$\begin{array}{llll}\text { C } & 1.472595 & 2.141227 & 0.198151\end{array}$
C $2.980964 \quad 2.345922 \quad 0.150910$
$\begin{array}{llll}\text { C } & -0.061565 & 0.292288 & -0.334878\end{array}$
$\begin{array}{llll}\text { O } & 0.673826 & 2.861262 & 0.792534\end{array}$
$\begin{array}{llll}\text { C } & 3.659373 \quad 2.057629 & 1.536257\end{array}$
F $\quad 5.954601 \quad-0.076995 \quad-3.028340$
$\begin{array}{llll}\text { C } & 3.478990 & 3.114886 & 2.604402\end{array}$
$\begin{array}{llll}\text { C } & 4.301832 & 4.253971 & 2.616729\end{array}$ 


\begin{tabular}{|c|c|c|c|c|c|c|c|}
\hline $\mathrm{C}$ & 4.176548 & 5.219215 & 3.620386 & $\mathrm{C}$ & 0.395427 & 7.547426 & -1.897711 \\
\hline $\mathrm{C}$ & 3.223183 & 5.058175 & 4.633048 & $\mathrm{C}$ & -0.262324 & 6.903299 & -0.846875 \\
\hline $\mathrm{C}$ & 2.400300 & 3.927017 & 4.632898 & $\mathrm{C}$ & -8.298466 & 0.268745 & -3.54086 \\
\hline $\mathrm{C}$ & 2.530327 & 2.962979 & 3.626920 & $\mathrm{C}$ & -8.732886 & 0.332982 & -4.869017 \\
\hline $\mathrm{O}$ & 0.087219 & -0.983055 & -0.628186 & $\mathrm{C}$ & -8.030637 & 1.136687 & -5.765100 \\
\hline $\mathrm{O}$ & -1.076159 & 0.834415 & 0.081894 & $\mathrm{C}$ & -6.908894 & 1.867786 & -5.366182 \\
\hline $\mathrm{C}$ & -0.955080 & -2.029602 & -0.382267 & $\mathrm{C}$ & -6.485392 & 1.783398 & -4.0394 \\
\hline $\mathrm{C}$ & -2.197312 & -1.797367 & -1.248833 & $\mathrm{Cl}$ & 2.619788 & 8.528122 & -3.153152 \\
\hline $\mathrm{C}$ & -3.164498 & -2.988908 & -1.031255 & $\mathrm{Cl}$ & -8.571438 & 1.237512 & -7.44381 \\
\hline $\mathrm{C}$ & -2.475419 & -4.303639 & -1.452829 & $\mathrm{~N}$ & -4 & 1.393612 & 9117 \\
\hline $\mathrm{C}$ & -1.208663 & -4.516154 & -0.596996 & $\mathrm{O}$ & -6.684024 & 2.222785 & -1.069082 \\
\hline $\mathrm{C}$ & -0.248817 & -3.324202 & -0.807957 & $\mathrm{H}$ & 3.811078 & -1.446437 & -3.54584 \\
\hline $\mathrm{C}$ & -3.553926 & -3.066080 & 0.461178 & $\mathrm{H}$ & 1.585309 & -1.094123 & -2 \\
\hline $\mathrm{C}$ & -2.287567 & -3.284350 & 1.316532 & $\mathrm{H}$ & 5.507659 & 1.760517 & -1.235365 \\
\hline $\mathrm{C}$ & -1.597585 & -4.597697 & 0.894210 & $\mathrm{H}$ & 3.192085 & 3.377887 & -0.14767 \\
\hline $\mathrm{C}$ & -1.320131 & -2.093253 & 1.106718 & $\mathrm{H}$ & 3.304562 & 1.086294 & 1.89 \\
\hline $\mathrm{Ni}$ & -1.425903 & 2.965080 & 0.523399 & $\mathrm{H}$ & 4.727499 & 1.946251 & 1.321223 \\
\hline $\mathrm{Cl}$ & -1.278985 & 3.360190 & -1.833849 & $\mathrm{H}$ & 5.054584 & 4.379324 & 0232 \\
\hline $\mathrm{Cl}$ & -2.001032 & 2.259200 & 130 & $\mathrm{H}$ & 355 & 849 & 7836 \\
\hline $\mathrm{N}$ & -4.018439 & 4.004047 & 0.559152 & $\mathrm{H}$ & 3.128195 & 5.804319 & 5.418355 \\
\hline $\mathrm{C}$ & -5.270317 & 3.618255 & 0.270511 & $\mathrm{H}$ & 1.659455 & 3.789868 & 5.416857 \\
\hline $\mathrm{C}$ & -6.40 & 4.369182 & 0 & $\mathrm{H}$ & 4 & 2.0 & 3.638917 \\
\hline $\mathrm{C}$ & -6.229899 & 5.557983 & 1.331856 & $\mathrm{H}$ & -2.682796 & -0.858707 & -0.970658 \\
\hline $\mathrm{C}$ & -4.935152 & 5.972901 & 1.631980 & $\mathrm{H}$ & -1.903462 & -1.730333 & -2.304566 \\
\hline $\mathrm{C}$ & -3.8 & 5 & 1 & $\Pi$ & 9 & 86 & 2776 \\
\hline $\mathrm{C}$ & -2.482014 & 5.596919 & 1.470784 & $\mathrm{H}$ & -3.165091 & -5.148398 & -1.319393 \\
\hline $\mathrm{C}$ & -5.461593 & 2.347855 & -0.470001 & $\mathrm{H}$ & -2.210480 & -4.266655 & -2.518933 \\
\hline $\mathrm{O}$ & -2.319401 & 6.757415 & 2.130246 & $\mathrm{H}$ & -0.699180 & -5.438858 & -0.902819 \\
\hline $\mathrm{C}$ & -0.889731 & 6.865669 & 2.409429 & $\mathrm{H}$ & 0.664740 & -3.455815 & -0.214280 \\
\hline $\mathrm{C}$ & -0.251409 & 5.809947 & 1.468778 & $\mathrm{H}$ & 0.044316 & -3.252113 & -1.863075 \\
\hline $\mathrm{N}$ & -1.421113 & 4.973854 & 1.100506 & $\mathrm{H}$ & -4.059700 & -2.141185 & 0.769707 \\
\hline $\mathrm{C}$ & -6.717650 & 0.887955 & -1.677566 & $\mathrm{H}$ & -4.260128 & -3.892657 & 0.620617 \\
\hline $\mathrm{C}$ & -5.265123 & 0.379959 & -1.441724 & $\mathrm{H}$ & -2.559333 & -3.329100 & 2.378746 \\
\hline $\mathrm{C}$ & 0.446397 & 6.430134 & 0.265383 & $\mathrm{H}$ & -0.703464 & -4.770663 & 1.509717 \\
\hline $\mathrm{C}$ & -7.174877 & 0.985816 & -3.112372 & $\mathrm{H}$ & -2.273615 & -5.447952 & 1.060160 \\
\hline $\mathrm{C}$ & 1.834764 & 6.614917 & 0.306773 & $\mathrm{H}$ & -1.798565 & -1.162496 & 1.423907 \\
\hline $\mathrm{C}$ & 2.511388 & 7.257940 & -0.733520 & $\mathrm{H}$ & -0.408654 & -2.230688 & 1.703520 \\
\hline $\mathrm{C}$ & 1.778294 & 7.717249 & -1.827069 & $\mathrm{H}$ & -7.392315 & 4.014428 & 0.368559 \\
\hline
\end{tabular}




\begin{tabular}{|c|c|c|c|c|c|c|c|}
\hline & -7.086726 & 6.152985 & 1.633497 & $\mathrm{O}$ & -0.145320 & -1.133179 & 42581 \\
\hline$\Pi$ & -4.748613 & 6.896214 & 2.167914 & $\mathrm{O}$ & -1.328846 & 0.762369 & -0.122752 \\
\hline$\theta$ & -0.745410 & 6.627053 & 3.466504 & $\mathrm{C}$ & -1.125561 & -2.137146 & -0.018848 \\
\hline & -0.587974 & 7.893176 & 2.206036 & $\mathrm{C}$ & -2.468970 & -2.047123 & -0.748559 \\
\hline 11 & 0.463802 & 5.194548 & 2.016031 & $\mathrm{C}$ & -3.377592 & -3.188984 & -0.227567 \\
\hline $\mathrm{H}$ & -7.440500 & 0.304451 & -1.100587 & $\mathrm{C}$ & -2.719740 & -4.552866 & -0.519090 \\
\hline $\mathrm{H}$ & -5.256172 & -0.592634 & -0.942649 & $\mathrm{C}$ & -1.353274 & -4.625340 & 195496 \\
\hline H & -4.699075 & 0.283750 & -2.375380 & $\mathrm{C}$ & -0.449348 & -3.481494 & -0.319876 \\
\hline $\mathrm{H}$ & 2.400038 & 6.254583 & 1.162601 & $\mathrm{C}$ & -3.580258 & -3.025263 & 1.294556 \\
\hline $\mathrm{H}$ & 3.587545 & 7.394736 & -0.690665 & $\mathrm{C}$ & -2 & -3 & 2.009995 \\
\hline H & -0.162249 & 7.902725 & -2.758707 & $\mathrm{C}$ & -1.556058 & -4.468093 & 1.717491 \\
\hline $\mathrm{H}$ & -1.336322 & 6.760857 & -0.915283 & $\mathrm{C}$ & -1.302875 & -1.962934 & 1.494522 \\
\hline $\mathrm{H}$ & -8.848058 & -0.347499 & -2.833016 & $\mathrm{Ni}$ & -1.6 & 727 & 0.519380 \\
\hline $\mathrm{H}$ & -9.605142 & -0.227095 & -5.191346 & $\mathrm{Cl}$ & -1.942943 & 3.561095 & -1.780273 \\
\hline $\mathrm{H}$ & -6.374069 & 2.490256 & -6.076740 & $\mathrm{Cl}$ & -1.604124 & 1.928453 & 2.761918 \\
\hline $\mathrm{H}$ & -5.610727 & 2.349728 & -3.729498 & $\mathrm{~N}$ & -3.898642 & 3.567685 & 0.940051 \\
\hline \multicolumn{4}{|c|}{ Int $1(R)$} & E & -5.108559 & 3.131622 & 3565 \\
\hline \multirow{2}{*}{\multicolumn{4}{|c|}{$\Delta G=\mathbf{- 4 5 9 8 . 4 8 6 5 9 4}$ hartree }} & $\mathrm{C}$ & -6.304293 & 3.678712 & 1.053648 \\
\hline & & & & $\mathrm{C}$ & -6.249096 & 4.705593 & 1.987983 \\
\hline C & 4.358452 & -0.277683 & -2.813602 & $\mathrm{C}$ & -4.998940 & 5.195431 & 2.361792 \\
\hline C & 3.318029 & -1.193174 & -2.89 & $\mathrm{C}$ & -3.864835 & 4.609342 & 1.799687 \\
\hline C & 2.102736 & -0.904787 & -2.257757 & $\mathrm{C}$ & -2.525844 & 5.157704 & 2.06058 \\
\hline C & 1.991356 & 0.302216 & -1.571442 & $\mathrm{C}$ & -5.209585 & 2.063650 & -0.469656 \\
\hline C & 3.044325 & 1.227229 & -1.527447 & $\mathrm{O}$ & -2.393454 & 6.115361 & 2.988118 \\
\hline $\mathrm{C}$ & 4.257575 & 0.939270 & -2.146831 & $\mathrm{C}$ & -0.953392 & 6.346160 & 3.119961 \\
\hline $\mathrm{N}$ & 0.862597 & 0.848449 & -0.865381 & $\mathrm{C}$ & -0.335165 & 5.576013 & 1.913584 \\
\hline $\mathrm{C}$ & 1.176509 & 2.119958 & -0.388845 & $\mathrm{~N}$ & -1.477356 & 4.778284 & 1.427575 \\
\hline C & 2.611577 & 2.457724 & -0.774648 & $c$ & -6.181597 & 1.152626 & -2.304320 \\
\hline C & -0.320714 & 0.172258 & -0.477349 & $\mathrm{C}$ & -5.024167 & 0.279874 & -1.741365 \\
\hline $\mathrm{O}$ & 0.439337 & 2.85 & 0.259751 & $\mathrm{C}$ & 0.251037 & 6.500615 & 0.854914 \\
\hline C & 2.666689 & 3.805636 & -1.550216 & & -6.093781 & 1.528662 & -3.76 \\
\hline $\mathrm{F}$ & 5.542616 & -0.583229 & -3.426662 & $\mathrm{C}$ & 1.501228 & 7.091748 & 1.095273 \\
\hline $\mathrm{C}$ & 4.069507 & 4.321469 & -1.802748 & $\mathrm{C}$ & 2.056144 & 7.999503 & 0.191000 \\
\hline C & 4.908433 & 4.688294 & -0.736500 & $C$ & 1.347787 & 8.308140 & -0.97167 \\
\hline C & 6.195954 & 5.177964 & -0.972519 & $\mathrm{C}$ & 0.110529 & 7.727281 & -1.242737 \\
\hline $\mathrm{C}$ & 6.667868 & 5.313807 & -2.284203 & $\mathrm{C}$ & -0.432729 & 6.824804 & -0.322154 \\
\hline C & 5.840462 & 4.958016 & -3.353588 & $\mathrm{C}$ & -7.070516 & 1.079583 & -4.659548 \\
\hline C & 4.552450 & 4.465637 & -3.111774 & $\mathrm{C}$ & -6.996508 & 1.390653 & -6.021344 \\
\hline
\end{tabular}




\begin{tabular}{|c|c|c|c|}
\hline & 916 & & \\
\hline & 2 & 2 & \\
\hline & 7 & 517 & 42463 \\
\hline & 2.039853 & 9.454332 & 541 \\
\hline & 9 & & \\
\hline & & & \\
\hline & & 5 & \\
\hline & & & \\
\hline & 3 & & \\
\hline & & & \\
\hline & & & \\
\hline & 2 & 7 & -2 \\
\hline & 2.106574 & & \\
\hline & 4.5 & & \\
\hline $\mathrm{H}$ & 6.8 & 3 & \\
\hline & & & \\
\hline & & & \\
\hline & 3 & 18 & 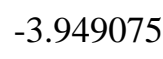 \\
\hline $\mathrm{H}$ & -2.93 & & \\
\hline & -2 & & \\
\hline $\mathrm{H}$ & -4.3 & -3 . & -0 \\
\hline $\mathrm{H}$ & -3.369441 & 7104 & \\
\hline $\mathrm{H}$ & -2 & & \\
\hline $\mathrm{H}$ & -0.8 & -5.5 & \\
\hline $\mathrm{H}$ & 0.532377 & & \\
\hline $\mathrm{H}$ & 36 & -3 . & -1 \\
\hline $\mathrm{H}$ & -4.0 & -2 & 1.5 \\
\hline $\mathrm{H}$ & & & \\
\hline$\pi$ & -2 & & \\
\hline $\mathrm{H}$ & -0.5 & -4 . & 2 \\
\hline & & & 2.09718 \\
\hline $\mathrm{H}$ & -1 & & \\
\hline $\mathrm{H}$ & -0 & -2 & 1.98513 \\
\hline$H$ & & & 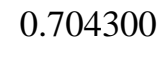 \\
\hline $\mathrm{H}$ & -7 & 869 & 2 \\
\hline $\mathrm{H}$ & -4 & 6 & 3 \\
\hline $\mathrm{H}$ & -0.04 & (2. & 4.05 \\
\hline & -0.788455 & .423318 & 3.09434 \\
\hline
\end{tabular}

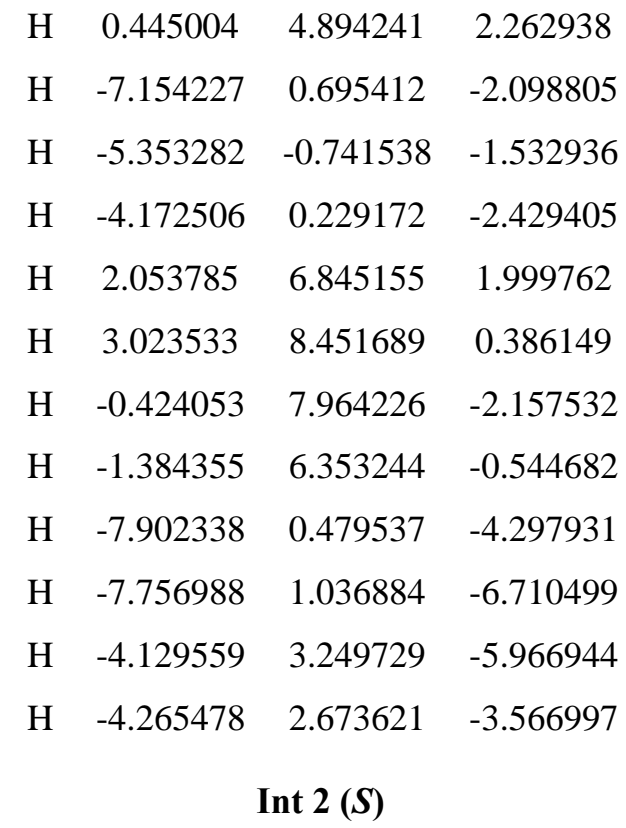
$\Delta G=\mathbf{- 4 8 3 1 . 4 8 9 9 1 2 ~ h a r t r e e ~}$

$\begin{array}{llll}\text { C } & 4.637712 & -1.108385 & -2.146785\end{array}$

$\begin{array}{llll}\text { C } & 3.498179 & -1.889066 & -2.327651\end{array}$

$\begin{array}{llll}\text { C } & 2.269802 & -1.462381 & -1.802639\end{array}$

$\begin{array}{llll}\text { C } & 2.241851 & -0.254538 & -1.109389\end{array}$

$\begin{array}{llll}\text { C } & 3.402427 & 0.529320 & -0.940356\end{array}$

$\begin{array}{llll}C & 4.624576 & 0.102787 & -1.462714\end{array}$

$\begin{array}{llll}\mathrm{N} & 1.143191 & 0.438200 & -0.499829\end{array}$

$\begin{array}{llll}\text { C } & 1.590826 & 1.674779 & 0.030503\end{array}$

$\begin{array}{llll}\text { C } & 3.074582 & 1.762507 & -0.186085\end{array}$

$\begin{array}{llll}\text { C } & -0.164405 & -0.042906 & -0.302939\end{array}$

$\begin{array}{llll}\mathrm{O} & 0.863751 & 2.525012 & 0.580060\end{array}$

$\begin{array}{llll}\text { C } & 3.990438 & 2.063898 & 1.024376\end{array}$

F $\quad 5.841637 \quad-1.557588 \quad-2.679005$

$\begin{array}{llll}\text { C } & 3.744871 & 3.306056 & 1.859717\end{array}$

$\begin{array}{llll}\text { C } & 4.109640 & 4.580396 & 1.388780\end{array}$

$\begin{array}{llll}C & 4.010417 & 5.697556 & 2.223602\end{array}$

$\begin{array}{llll}\text { C } & 3.541031 & 5.560531 & 3.537236\end{array}$

$\begin{array}{llll}\text { C } & 3.151629 & 4.300052 & 4.005761\end{array}$

$\begin{array}{llll}\text { C } & 3.254550 & 3.181232 & 3.170334\end{array}$

$\begin{array}{llll}\mathrm{O} & -0.216022 & -1.382248 & -0.415889\end{array}$

$\begin{array}{llll}\mathrm{O} & -1.125719 & 0.691535 & -0.030403\end{array}$

$\begin{array}{llll}\text { C } & -1.403401 & -2.205739 & 0.088724\end{array}$

$\begin{array}{llll}\text { C } & -2.658659 & -1.920489 & -0.745156\end{array}$ 


\begin{tabular}{|c|c|c|c|}
\hline $\mathrm{C}$ & -3.783499 & -2.875457 & -0.253706 \\
\hline $\mathrm{C}$ & -3.343220 & -4.347555 & 446598 \\
\hline $\mathrm{C}$ & -2.053628 & -4.609600 & 0.371143 \\
\hline $\mathrm{C}$ & -0.932272 & -3.652917 & -0.118066 \\
\hline $\mathrm{C}$ & -4.057867 & -2.605390 & 1.247316 \\
\hline $\mathrm{C}$ & -2.770787 & -2.875588 & 2.066672 \\
\hline $\mathrm{C}$ & -2.331474 & -4.347896 & 1.872682 \\
\hline $\mathrm{C}$ & -1.641263 & -1.924356 & 1.581294 \\
\hline $\mathrm{Ni}$ & -1.272240 & 2.733683 & 0.561587 \\
\hline $\mathrm{Cl}$ & -1.150576 & 3.387227 & -1.797630 \\
\hline $\mathrm{Cl}$ & -1.438036 & 1.784609 & 2.827654 \\
\hline $\mathrm{N}$ & -3.464002 & 3.421146 & 0.801519 \\
\hline $\mathrm{C}$ & -4.653607 & 3.042725 & 0.284944 \\
\hline $\mathrm{C}$ & -5.873075 & 3.516235 & 0.802701 \\
\hline $\mathrm{C}$ & -5.867883 & 4.388468 & 1.888020 \\
\hline $\mathrm{C}$ & -4.641542 & 4.830082 & 2.390185 \\
\hline $\mathrm{C}$ & -3.474234 & 4.343002 & 1.802703 \\
\hline $\mathrm{C}$ & -2.162141 & 4.882005 & 2.139043 \\
\hline $\mathrm{C}$ & -4.680795 & 2.201397 & -0.922185 \\
\hline $\mathrm{O}$ & -2.039917 & 5.771868 & 3.172021 \\
\hline $\mathrm{C}$ & -0.557767 & 5.979166 & 3.324591 \\
\hline $\mathrm{C}$ & 0.025575 & 5.451927 & 1.978810 \\
\hline $\mathrm{N}$ & -1.097281 & 4.603069 & 1.466665 \\
\hline $\mathrm{C}$ & -5.437357 & 1.751177 & -3.077356 \\
\hline $\mathrm{C}$ & -4.322085 & 0.764104 & -2.598278 \\
\hline $\mathrm{C}$ & 0.382930 & 6.562153 & 0.989385 \\
\hline $\mathrm{C}$ & -5.116175 & 2.572590 & -4.296576 \\
\hline $\mathrm{C}$ & 1.148834 & 7.659311 & 1.420446 \\
\hline $\mathrm{C}$ & 1.494087 & 8.690453 & 0.540655 \\
\hline $\mathrm{C}$ & 1.066580 & 8.608105 & -0.781912 \\
\hline $\mathrm{C}$ & 0.318944 & 7.530114 & -1.244355 \\
\hline $\mathrm{C}$ & -0.020820 & 6.503693 & -0.352785 \\
\hline $\mathrm{C}$ & -6.010600 & 2.600013 & -5.376674 \\
\hline $\mathrm{C}$ & -5.728767 & 3.347889 & -6.525849 \\
\hline $\mathrm{C}$ & -4.542263 & 4.073955 & -6.569152 \\
\hline $\mathrm{C}$ & -3.636079 & 4.074715 & -5.509722 \\
\hline $\mathrm{C}$ & -3.925715 & 3.318418 & -4.370356 \\
\hline $\mathrm{Cl}$ & 1.506863 & 9.956919 & -1.940189 \\
\hline
\end{tabular}

\begin{tabular}{|c|c|c|c|}
\hline & & & \\
\hline & 3 & 12 & 120065 \\
\hline & -5.598019 & & \\
\hline & & & \\
\hline & 77 & 7 & \\
\hline & 5.530629 & & \\
\hline & & & \\
\hline & 3.9 & & \\
\hline & & & \\
\hline & & & \\
\hline & & & \\
\hline & & & \\
\hline & & & \\
\hline & 2. & & \\
\hline & -2.96 & & \\
\hline & -2 & & \\
\hline & -4.692858 & -2.67 & \\
\hline & & & \\
\hline & & & \\
\hline & -1.72 & -5.64 & \\
\hline & & & \\
\hline & & & \\
\hline & & & \\
\hline & & & \\
\hline & -2.9 & & \\
\hline & 64 & & 2.4 \\
\hline & & & \\
\hline & 1. & & \\
\hline & 29 & 2 & 4 \\
\hline & & & \\
\hline & $-6.7 c$ & & \\
\hline & -4.5 & 5.5 & - \\
\hline & 00 & & \\
\hline & -0.4 & & \\
\hline & & & \\
\hline & & 1.249782 & \\
\hline & -4.6 & 6051 & -2 \\
\hline & & 0.84622 & $2 .-1$ \\
\hline
\end{tabular}




$\begin{array}{cccc}\mathrm{H} & 1.496278 & 7.716093 & 2.447224 \\ \mathrm{H} & 2.079851 & 9.536546 & 0.878849 \\ \mathrm{H} & -0.001066 & 7.481837 & -2.278085 \\ \mathrm{H} & -0.584698 & 5.651895 & -0.717032 \\ \mathrm{H} & -6.937428 & 2.035551 & -5.325730 \\ \mathrm{H} & -6.417751 & 3.366896 & -7.361219 \\ \mathrm{H} & -2.720699 & 4.650425 & -5.566752 \\ \mathrm{H} & -3.217402 & 3.320574 & -3.544876 \\ \mathrm{C} & 2.211533 & 6.953997 & -5.208539 \\ \mathrm{C} & 1.927412 & 5.585874 & -4.567153 \\ \mathrm{C} & 2.893703 & 5.272389 & -3.407895 \\ \mathrm{C} & 2.588680 & 3.900757 & -2.771861 \\ \mathrm{O} & 3.585039 & 3.686896 & -1.745878 \\ \mathrm{H} & 0.894413 & 5.554892 & -4.193853 \\ \mathrm{H} & 2.814317 & 6.042381 & -2.631306 \\ \mathrm{H} & 3.930177 & 5.277131 & -3.769510 \\ \mathrm{H} & 1.578656 & 3.916637 & -2.340690 \\ \mathrm{H} & 2.644393 & 3.109616 & -3.532699 \\ \mathrm{H} & 2.004368 & 4.797853 & -5.329850 \\ \mathrm{H} & 2.112784 & 7.764084 & -4.475722 \\ \mathrm{H} & 1.514150 & 7.156493 & -6.029672 \\ \mathrm{H} & 3.229551 & 6.995098 & -5.615716\end{array}$

Int $2(R)$

$\Delta G=\mathbf{- 4 8 3 1 . 4 8 4 5 2 2}$ hartree

$\begin{array}{llll}\mathrm{C} & 4.336566 & 0.039694 & -3.934223 \\ \mathrm{C} & 3.354675 & -0.934172 & -3.768226 \\ \mathrm{C} & 2.269571 & -0.688259 & -2.914968 \\ \mathrm{C} & 2.222846 & 0.537864 & -2.258609 \\ \mathrm{C} & 3.223866 & 1.521850 & -2.439122 \\ \mathrm{C} & 4.306606 & 1.269616 & -3.285721 \\ \mathrm{~N} & 1.208745 & 1.076381 & -1.393313 \\ \mathrm{C} & 1.538520 & 2.409691 & -1.062121 \\ \mathrm{C} & 2.911844 & 2.699158 & -1.593582 \\ \mathrm{C} & 0.110316 & 0.405238 & -0.831273 \\ \mathrm{O} & 0.848943 & 3.180225 & -0.367237 \\ \mathrm{C} & 3.151747 & 4.146376 & -2.042352 \\ \mathrm{~F} & 5.400364 & -0.233802 & -4.787747\end{array}$ $\begin{array}{llll}\text { C } & 4.576311 & 4.494366 & -2.444578\end{array}$

$\begin{array}{llll}\text { C } & 5.667057 & 4.252681 & -1.587168\end{array}$

$\begin{array}{llll}\text { C } & 6.962117 & 4.618832 & -1.969397\end{array}$

$\begin{array}{llll}\text { C } & 7.189702 & 5.236905 & -3.205436\end{array}$

$\begin{array}{llll}\text { C } & 6.111608 & 5.484709 & -4.061609\end{array}$

$\begin{array}{llll}\text { C } & 4.816304 & 5.113148 & -3.683193\end{array}$

$\begin{array}{lllll}\text { O } & 0.259750 & -0.927984 & -0.894817\end{array}$

$\begin{array}{llll}\mathrm{O} & -0.862678 & 0.990118 & -0.321925\end{array}$

$\begin{array}{lllll}\text { C } & -0.597628 & -1.896060 & -0.075278\end{array}$

$\begin{array}{llll}\text { C } & -2.052291 & -1.873414 & -0.561085\end{array}$

$\begin{array}{llll}\text { C } & -2.832327 & -2.962291 & 0.228998\end{array}$

$\begin{array}{llll}\text { C } & -2.205102 & -4.353781 & -0.031035\end{array}$

$\begin{array}{llll}\text { C } & -0.726847 & -4.346309 & 0.433522\end{array}$

$\begin{array}{llll}\text { C } & 0.050937 & -3.259080 & -0.357632\end{array}$

$\begin{array}{llll}\text { C } & -2.767356 & -2.631919 & 1.741191\end{array}$

$\begin{array}{llll}\text { C } & -1.289413 & -2.634371 & 2.207689\end{array}$

$\begin{array}{llll}\text { C } & -0.664024 & -4.026899 & 1.948190\end{array}$

$\begin{array}{llll}\mathrm{C} & -0.498122 & -1.552822 & 1.419590\end{array}$

$\begin{array}{llll}\mathrm{Ni} & -1.216620 & 2.948189 & 0.329279\end{array}$

$\begin{array}{lllll}\mathrm{Cl} & -2.034126 & 3.575045 & -1.841205\end{array}$

$\begin{array}{lllll}\mathrm{Cl} & -0.440965 & 2.161699 & 2.502019\end{array}$

$\begin{array}{llll}\mathrm{N} & -3.541307 & 3.421462 & 1.165799\end{array}$

$\begin{array}{llll}\text { C } & -4.776571 & 2.907454 & 0.985451\end{array}$

$\begin{array}{llll}\text { C } & -5.904367 & 3.419243 & 1.653025\end{array}$

$\begin{array}{llll}\text { C } & -5.750648 & 4.480999 & 2.542130\end{array}$

$\begin{array}{llll}\text { C } & -4.485226 & 5.048977 & 2.700490\end{array}$

$\begin{array}{llll}\text { C } & -3.421732 & 4.501698 & 1.976733\end{array}$

$\begin{array}{llll}\text { C } & -2.101830 & 5.120066 & 1.984068\end{array}$

$\begin{array}{llll}\text { C } & -4.964644 & 1.845352 & -0.013471\end{array}$

$\begin{array}{llll}\mathrm{O} & -1.855010 & 6.125876 & 2.882984\end{array}$

$\begin{array}{llll}\text { C } & -0.414814 & 6.494878 & 2.704893\end{array}$

$\begin{array}{llll}\mathrm{C} & 0.066946 & 5.607586 & 1.503257\end{array}$

$\begin{array}{llll}\mathrm{N} & -1.141222 & 4.790120 & 1.189703\end{array}$

$\begin{array}{llll}\mathrm{C} & -6.142956 & 0.907166 & -1.787967\end{array}$

$\begin{array}{llll}\text { C } & -4.804817 & 0.159916 & -1.476341\end{array}$

$\begin{array}{llll}\text { C } & 0.582026 & 6.423710 & 0.328750\end{array}$

$\begin{array}{llll}\text { C } & -6.226903 & 1.565476 & -3.139555\end{array}$

$\begin{array}{llll}\text { C } & 1.765305 & 7.165235 & 0.497330\end{array}$ 


\begin{tabular}{|c|c|c|c|}
\hline & & & \\
\hline & 0 & 7990783 & 7101 \\
\hline & .415525 & 7.257530 & \\
\hline & & & \\
\hline & & & \\
\hline & 390 & & \\
\hline & & & \\
\hline & & & \\
\hline & & & \\
\hline & & & \\
\hline & -6.5 & & \\
\hline & & & \\
\hline & & & \\
\hline$H$ & & & \\
\hline & & & \\
\hline & & & \\
\hline & & & \\
\hline & & & \\
\hline & & & \\
\hline & 5.5 & & \\
\hline & & & \\
\hline & & & \\
\hline & & & \\
\hline & & & \\
\hline & & & \\
\hline & & & \\
\hline & & & \\
\hline $\mathrm{H}$ & & & \\
\hline $\mathrm{H}$ & -2.2 & 5 & \\
\hline & & & \\
\hline & 1.10 & & \\
\hline$H$ & 0.01 & -3.47 & \\
\hline & 20 & -1.0496 & \\
\hline $\mathrm{H}$ & -3.3 & 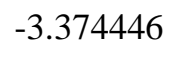 & \\
\hline $\mathrm{H}$ & & & \\
\hline & 0.378310 & -4.0431 & \\
\hline & 44 & -4.1 & 2. \\
\hline & -0.901046 & 0.0 & t \\
\hline
\end{tabular}

\begin{tabular}{cccc}
$\mathrm{H}$ & 0.555474 & -1.555873 & 1.726775 \\
$\mathrm{H}$ & -6.874697 & 2.984714 & 1.452975 \\
$\mathrm{H}$ & -6.603879 & 4.876120 & 3.081073 \\
$\mathrm{H}$ & -4.314291 & 5.903409 & 3.342346 \\
$\mathrm{H}$ & 0.085874 & 6.262964 & 3.645013 \\
$\mathrm{H}$ & -0.379333 & 7.563039 & 2.493730 \\
$\mathrm{H}$ & 0.837350 & 4.904652 & 1.835016 \\
$\mathrm{H}$ & -7.016508 & 0.275396 & -1.613096 \\
$\mathrm{H}$ & -4.972239 & -0.883989 & -1.196657 \\
$\mathrm{H}$ & -4.117144 & 0.184144 & -2.325407 \\
$\mathrm{H}$ & 2.305278 & 7.125673 & 1.440219 \\
$\mathrm{H}$ & 3.183995 & 8.526618 & -0.408723 \\
$\mathrm{H}$ & -0.097220 & 7.285450 & -2.902888 \\
$\mathrm{H}$ & -0.966741 & 5.862568 & -1.071285 \\
$\mathrm{H}$ & -8.105143 & 0.629858 & -3.651059 \\
$\mathrm{H}$ & -8.250995 & 1.688792 & -5.897024 \\
$\mathrm{H}$ & -4.531247 & 3.731189 & -5.165174 \\
$\mathrm{H}$ & -4.365982 & 2.677012 & -2.941206 \\
$\mathrm{C}$ & 3.831211 & 1.815966 & 5.556577 \\
$\mathrm{C}$ & 3.320825 & 2.558505 & 4.311010 \\
$\mathrm{C}$ & 3.992677 & 2.057536 & 3.017979 \\
$\mathrm{C}$ & 3.447988 & 2.795457 & 1.774893 \\
$\mathrm{O}$ & 4.147744 & 2.276243 & 0.627434 \\
$\mathrm{H}$ & 2.233461 & 2.434727 & 4.222125 \\
$\mathrm{H}$ & 3.809256 & 0.983994 & 2.888744 \\
$\mathrm{H}$ & 5.079872 & 2.199105 & 3.071640 \\
$\mathrm{H}$ & 2.370015 & 2.592939 & 1.700178 \\
$\mathrm{H}$ & 3.608088 & 3.878957 & 1.873932 \\
$\mathrm{H}$ & 3.509706 & 3.636394 & 4.425546 \\
$\mathrm{H}$ & 3.623960 & 0.741253 & 5.485209 \\
& 3.344349 & 2.189776 & 6.464685 \\
& 4.914497 & 1.940928 & 5.679592 \\
& & Int 3 & \\
\hline
\end{tabular}

\section{$\Delta G=\mathbf{- 4 5 9 7 . 8 5 8 6 7 2}$ hartree}

$\begin{array}{llll}\text { C } & 3.873876 & 0.181497 & -4.125365 \\ \text { C } & 2.914936 & -0.826414 & -3.965546 \\ \text { C } & 1.831838 & -0.635268 & -3.096291\end{array}$ 


\begin{tabular}{|c|c|c|c|}
\hline & & & \\
\hline & 6 & 1 & 04 \\
\hline & 3.820384 & 391904 & 041 \\
\hline & & & \\
\hline & .114605 & & \\
\hline & 2.367933 & & \\
\hline & & & \\
\hline & & & \\
\hline & & & \\
\hline & & & \\
\hline & 4 & & \\
\hline & & & \\
\hline & & & \\
\hline & & & \\
\hline & & & \\
\hline & & & \\
\hline & & & \\
\hline & & & \\
\hline & & & \\
\hline & -2. & & \\
\hline & & & \\
\hline & & & \\
\hline & & & \\
\hline & & & \\
\hline & -1 & & \\
\hline & & & \\
\hline & & & \\
\hline & & & \\
\hline & & 3.0 & 0 \\
\hline & & & \\
\hline & & & \\
\hline & -3 . & 3.7 & 0.781 \\
\hline & -5. & & \\
\hline & & & \\
\hline & -6 & 4. & \\
\hline & -4.777417 & 5. & 2.171015 \\
\hline & -3.6 & 4.8 & 1.5 \\
\hline & -2.344728 & אונ. & \\
\hline
\end{tabular}

$\begin{array}{llll}\text { C } & -5.241671 & 2.090659 & -0.346003\end{array}$

$\begin{array}{llll}\text { O } & -2.134532 & 6.398025 & 2.510335\end{array}$

$\begin{array}{llll}\text { C } & -0.694359 & 6.659071 & 2.483170\end{array}$

$\begin{array}{llll}\text { C } & -0.174735 & 5.797837 & 1.291000\end{array}$

$\begin{array}{llll}\mathrm{N} & -1.356603 & 4.989310 & 0.938288\end{array}$

$\begin{array}{llll}\text { C } & -6.454686 & 0.968769 & -1.899863\end{array}$

$\begin{array}{llll}\text { C } & -5.177056 & 0.197733 & -1.464476\end{array}$

$\begin{array}{llll}\text { C } & 0.359298 & 6.637255 & 0.138643\end{array}$

$\begin{array}{llll}\text { C } & -6.637622 & 1.196804 & -3.379472\end{array}$

$\begin{array}{llll}\text { C } & 1.603777 & 7.268580 & 0.291868\end{array}$

$\begin{array}{llll}\text { C } & 2.122024 & 8.094539 & -0.707234\end{array}$

$\begin{array}{llll}\text { C } & 1.382157 & 8.279430 & -1.876613\end{array}$

$\begin{array}{llll}\text { C } & 0.148243 & 7.658514 & -2.060249\end{array}$

$\begin{array}{llll}\text { C } & -0.358208 & 6.838572 & -1.045546\end{array}$

$\begin{array}{llll}\text { C } & -7.765237 & 0.684367 & -4.033138\end{array}$

$\begin{array}{llll}\text { C } & -7.945221 & 0.862434 & -5.408828\end{array}$

$\begin{array}{llll}\text { C } & -6.982940 & 1.573395 & -6.123322\end{array}$

$\begin{array}{llll}\text { C } & -5.851718 & 2.103058 & -5.497807\end{array}$

$\begin{array}{llll}\text { C } & -5.683598 & 1.906145 & -4.126682\end{array}$

$\begin{array}{llll}\mathrm{Cl} & 2.031192 & 9.316318 & -3.153460\end{array}$

$\begin{array}{llll}\mathrm{Cl} & -7.200503 & 1.815387 & -7.859829\end{array}$

$\begin{array}{llll}\mathrm{N} & -4.589572 & 0.999014 & -0.377499\end{array}$

$\begin{array}{llll}\mathrm{O} & -6.293861 & 2.251689 & -1.203317\end{array}$

$\begin{array}{llll}\mathrm{H} & 3.017355 & -1.752866 & -4.521696\end{array}$

H $\quad 1.089586 \quad-1.412462 \quad-2.979806$

$\mathrm{H} \quad 4.578896 \quad 2.151155 \quad-3.625750$

$\begin{array}{llll}\mathrm{H} & 3.409925 & 4.363509 & -2.638063\end{array}$

$\begin{array}{llll}\mathrm{H} & 2.334502 & 4.761738 & -1.298980\end{array}$

$\begin{array}{llll}\mathrm{H} & 3.148100 & 3.187427 & 0.973679\end{array}$

$\begin{array}{llll}\mathrm{H} & 5.078158 & 3.182770 & 2.516201\end{array}$

$\begin{array}{llll}\mathrm{H} & 7.306325 & 3.989000 & 1.742732\end{array}$

$\mathrm{H} \quad 7.575876 \quad 4.800030 \quad-0.599463$

$\begin{array}{llll}\mathrm{H} & 5.639452 & 4.798024 & -2.146054\end{array}$

$\begin{array}{llll}\mathrm{H} & -2.842901 & -1.026395 & -0.587132\end{array}$

$\begin{array}{llll}\mathrm{H} & -2.343476 & -2.144201 & -1.874012\end{array}$

$\begin{array}{llll}\mathrm{H} & -4.144153 & -3.149417 & -0.444665\end{array}$

$\begin{array}{llll}\mathrm{H} & -2.967187 & -5.295230 & 0.112370\end{array}$

$\begin{array}{llll}\mathrm{H} & -2.437606 & -4.676925 & -1.457748\end{array}$ 


$\begin{array}{cccc}\mathrm{H} & -0.459747 & -5.380187 & -0.093002 \\ \mathrm{H} & 0.828658 & -3.238345 & -0.233296 \\ \mathrm{H} & -0.204487 & -3.447555 & -1.660192 \\ \mathrm{H} & -3.605455 & -1.921726 & 1.671284 \\ \mathrm{H} & -3.663453 & -3.666580 & 1.962666 \\ \mathrm{H} & -1.639516 & -2.647010 & 3.041084 \\ \mathrm{H} & 0.072994 & -4.172815 & 2.034086 \\ \mathrm{H} & -1.483991 & -5.000925 & 2.172518 \\ \mathrm{H} & -1.343845 & -0.727153 & 1.483838 \\ \mathrm{H} & 0.158497 & -1.660463 & 1.595042 \\ \mathrm{H} & -7.163322 & 3.413517 & 0.908960 \\ \mathrm{H} & -6.921907 & 5.390289 & 2.427918 \\ \mathrm{H} & -4.616573 & 6.334087 & 2.785110 \\ \mathrm{H} & -0.290757 & 6.339176 & 3.446358 \\ \mathrm{H} & -0.556754 & 7.732341 & 2.349909 \\ \mathrm{H} & 0.613963 & 5.121896 & 1.633445 \\ \mathrm{H} & -7.353615 & 0.504488 & -1.482400 \\ \mathrm{H} & -5.414786 & -0.809613 & -1.112630 \\ \mathrm{H} & -4.448301 & 0.109233 & -2.278126 \\ \mathrm{H} & 2.182995 & 7.115097 & 1.200066 \\ \mathrm{H} & 3.085292 & 8.578323 & -0.578180 \\ \mathrm{H} & -0.412630 & 7.801961 & -2.978786 \\ \mathrm{H} & -1.307496 & 6.335057 & -1.197931 \\ \mathrm{H} & -8.517800 & 0.139850 & -3.467551 \\ \mathrm{H} & -8.821290 & 0.459829 & -5.907625 \\ \mathrm{H} & -5.112980 & 2.657701 & -6.067995 \\ & -4.802998 & 2.317871 & -3.640223\end{array}$

Int $4(S)$

\section{$\Delta G=\mathbf{- 4 8 0 2 . 9 7 3 7 0 2}$ hartree}

$\begin{array}{llll}\mathrm{C} & 4.421268 & -0.193790 & -2.690098 \\ \mathrm{C} & 3.385463 & -1.112607 & -2.799545 \\ \mathrm{C} & 2.162840 & -0.850718 & -2.170390 \\ \mathrm{C} & 2.038171 & 0.334962 & -1.451623 \\ \mathrm{C} & 3.085991 & 1.261435 & -1.377644 \\ \mathrm{C} & 4.306979 & 1.004097 & -1.994589 \\ \mathrm{~N} & 0.895531 & 0.847497 & -0.746297 \\ \mathrm{C} & 1.171824 & 2.101049 & -0.231717\end{array}$ $\begin{array}{llll}\text { C } & 2.629996 & 2.475006 & -0.624506\end{array}$

$\begin{array}{llll}\text { C } & -0.303147 & 0.155173 & -0.405533\end{array}$

$\begin{array}{llll}\text { O } & 0.431703 & 2.825016 & 0.413171\end{array}$

$\begin{array}{llll}\text { C } & 2.607727 & 3.828374 & -1.390238\end{array}$

$\begin{array}{llll}\text { F } & 5.609305 & -0.476363 & -3.299435\end{array}$

$\begin{array}{llll}\text { C } & 3.927667 & 4.301286 & -1.966167\end{array}$

$\begin{array}{llll}\text { C } & 4.899341 & 4.921361 & -1.162088\end{array}$

C $\quad 6.103376 \quad 5.368820 \quad-1.714290$

$\begin{array}{llll}\text { C } & 6.352493 & 5.211946 & -3.082134\end{array}$

$\begin{array}{llll}\text { C } & 5.386862 & 4.609052 & -3.894675\end{array}$

$\begin{array}{llll}C & 4.185985 & 4.157102 & -3.338732\end{array}$

$\begin{array}{llll}\mathrm{O} & -0.111616 & -1.144732 & -0.483310\end{array}$

$\begin{array}{llll}\text { O } & -1.317709 & 0.743078 & -0.079080\end{array}$

$\begin{array}{llll}\text { C } & -1.100828 & -2.161959 & 0.009069\end{array}$

$\begin{array}{llll}\text { C } & -2.430256 & -2.065258 & -0.744645\end{array}$

$\begin{array}{llll}\text { C } & -3.345145 & -3.214931 & -0.251738\end{array}$

$\begin{array}{llll}\text { C } & -2.678572 & -4.573605 & -0.547368\end{array}$

$\begin{array}{llll}\text { C } & -1.324818 & -4.651297 & 0.190606\end{array}$

$\begin{array}{llll}\text { C } & -0.414209 & -3.499484 & -0.295627\end{array}$

$\begin{array}{llll}\text { C } & -3.574508 & -3.068647 & 1.268308\end{array}$

$\begin{array}{llll}\text { C } & -2.221310 & -3.152573 & 2.007095\end{array}$

$\begin{array}{llll}\text { C } & -1.554176 & -4.511607 & 1.710524\end{array}$

$\begin{array}{llll}\text { C } & -1.304111 & -2.003544 & 1.520534\end{array}$

$\mathrm{Ni} \quad-1.733916 \quad 2.908129 \quad 0.603383$

$\begin{array}{lllll}\mathrm{Cl} & -1.874531 & 3.587592 & -1.694892\end{array}$

$\begin{array}{llll}\mathrm{Cl} & -1.766571 & 1.874830 & 2.797744\end{array}$

$\begin{array}{llll}\mathrm{N} & -3.911552 & 3.602913 & 0.946947\end{array}$

$\begin{array}{llll}\text { C } & -5.106934 & 3.182300 & 0.506871\end{array}$

$\begin{array}{llll}\text { C } & -6.317149 & 3.734076 & 0.958869\end{array}$

$\begin{array}{llll}\text { C } & -6.292297 & 4.745351 & 1.911545\end{array}$

$\begin{array}{llll}\text { C } & -5.055228 & 5.217083 & 2.348021\end{array}$

$\begin{array}{llll}\text { C } & -3.903698 & 4.630678 & 1.824319\end{array}$

$\begin{array}{llll}\text { C } & -2.569412 & 5.159294 & 2.142592\end{array}$

$\begin{array}{llll}\text { C } & -5.166185 & 2.122350 & -0.531283\end{array}$

$\begin{array}{llll}\mathrm{O} & -2.454170 & 6.110044 & 3.078121\end{array}$

$\begin{array}{llll}\text { C } & -1.013250 & 6.304264 & 3.266856\end{array}$

$\begin{array}{llll}\text { C } & -0.373112 & 5.564864 & 2.054616\end{array}$

$\begin{array}{llll}\mathrm{N} & -1.505675 & 4.768473 & 1.541582\end{array}$ 


\begin{tabular}{|c|c|c|c|}
\hline & & & \\
\hline & 1 & 349752 & \\
\hline & .209674 & .515468 & 01565 \\
\hline & -5.948008 & & \\
\hline & & & \\
\hline & 3 & 4 & 0.39585 \\
\hline & & & \\
\hline & & & \\
\hline & -0.46 & 00 & \\
\hline $\mathrm{C}$ & -( & & \\
\hline $\mathrm{C}$ & & & \\
\hline C & -5.7 & 2 & \\
\hline C & 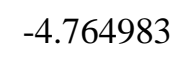 & & \\
\hline $\mathrm{C}$ & 66 & 2 & \\
\hline $\mathrm{Cl}$ & 1.98 & 9.5 & \\
\hline & $-5.5^{\prime}$ & & \\
\hline & & & \\
\hline $\mathrm{O}$ & -( & 2.396 & \\
\hline $\mathrm{N}$ & & & \\
\hline $\mathrm{O}$ & & & \\
\hline $\mathrm{O}$ & 3. & 7 & 1 \\
\hline $\mathrm{H}$ & & & \\
\hline $\mathrm{H}$ & & & \\
\hline $\mathrm{H}$ & 5.1 & 1.687519 & \\
\hline $\mathrm{H}$ & 1.882204 & & \\
\hline $\mathrm{H}$ & 2.1 & 7 & \\
\hline $\mathrm{H}$ & & 5.073012 & \\
\hline$H$ & & & \\
\hline $\mathrm{H}$ & 7.28 & .56 & \\
\hline $\mathrm{H}$ & 5.565332 & 4.492289 & -4.9608 \\
\hline $\mathrm{H}$ & & 54 & \\
\hline $\mathrm{H}$ & -2.90 & & \\
\hline $\mathrm{H}$ & 240 & 6 & -1.82443 \\
\hline $\mathrm{H}$ & -4.305294 & -3.147692 & . \\
\hline $\mathrm{H}$ & -3.3 & 85 & \\
\hline $\mathrm{H}$ & -2.527527 & 3 & -1.62947 \\
\hline $\mathrm{H}$ & -0.831964 & -5.607206 & -0 \\
\hline$U$ & 558079 & .537621 & .2 \\
\hline
\end{tabular}

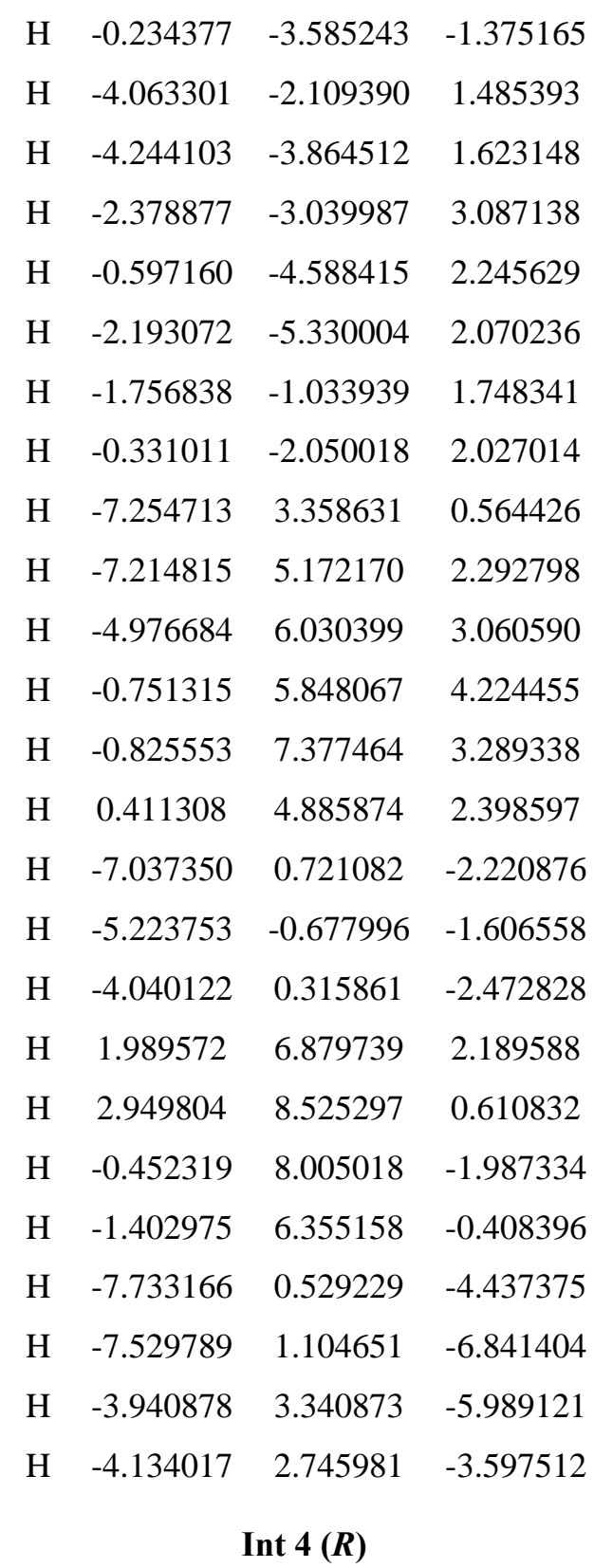

\section{$\Delta G=\mathbf{~ - 4 8 0 2 . 9 7 8 1 7 9}$ hartree}

$\begin{array}{llll}\text { C } & 4.104481 & -0.061399 & -3.187500\end{array}$

$\begin{array}{llll}\text { C } & 3.005757 & -0.901074 & -3.325909\end{array}$

$\begin{array}{llll}\text { C } & 1.849659 & -0.657901 & -2.574334\end{array}$

$\begin{array}{llll}\text { C } & 1.853102 & 0.427992 & -1.704049\end{array}$

$\begin{array}{llll}\text { C } & 2.966348 & 1.266557 & -1.595261\end{array}$

$\begin{array}{llll}\text { C } & 4.122966 & 1.034453 & -2.330650\end{array}$

$\begin{array}{llll}\mathrm{N} & 0.797670 & 0.930753 & -0.874919\end{array}$

$\begin{array}{llll}\text { C } & 1.169329 & 2.139940 & -0.297762\end{array}$

$\begin{array}{llll}\text { C } & 2.681477 & 2.339610 & -0.569820\end{array}$

$\begin{array}{llll}\text { C } & -0.367356 & 0.232177 & -0.452857\end{array}$ 


\begin{tabular}{|c|c|c|c|c|c|c|c|}
\hline $\mathrm{O}$ & 0.467482 & 2.884118 & 0.366557 & $\mathrm{C}$ & 0.124842 & 6.634168 & 31096 \\
\hline $\mathrm{C}$ & 3.565496 & 2.139469 & 0.697469 & $\mathrm{C}$ & -6.215488 & 1.424995 & 633657 \\
\hline $\mathrm{F}$ & 5.219142 & -0.319521 & -3.929065 & $\mathrm{C}$ & 1.398264 & 7.215347 & 0.528050 \\
\hline $\mathrm{C}$ & 3.362790 & 3.040058 & 1.900904 & $\mathrm{C}$ & 1.887298 & 8.054505 & -0.474782 \\
\hline $\mathrm{C}$ & 4.136307 & 4.199578 & 2.075061 & $\mathrm{C}$ & 1.086482 & 8.304756 & -1.589951 \\
\hline $\mathrm{C}$ & 4.007088 & 4.977373 & 3.230330 & $\mathrm{C}$ & -0.178802 & 7.735157 & -1.718060 \\
\hline $\mathrm{C}$ & 3.103799 & 4.604595 & 4.231626 & $\mathrm{C}$ & -0.653462 & 520 & -0.700995 \\
\hline $\mathrm{C}$ & 2.333116 & 3.447970 & 4.071017 & $\mathrm{C}$ & -7.210766 & 0.974063 & 09394 \\
\hline $\mathrm{C}$ & 2.466150 & 2.671879 & 2.916281 & $\mathrm{C}$ & -7.140760 & 1.242533 & -5.880494 \\
\hline $\mathrm{O}$ & -0.181506 & -1.066183 & -0.57 & $\mathrm{C}$ & -6 & 96 & 3591 \\
\hline $\mathrm{O}$ & -1.348341 & 0.805210 & -0.013101 & C & -5.058907 & 2.451259 & -5.513060 \\
\hline $\mathrm{C}$ & -1.122707 & -2.095928 & -0.022259 & $\mathrm{C}$ & -5.139564 & 2.164899 & -4.149687 \\
\hline $\mathrm{C}$ & -2.495640 & -2.014246 & -0.694416 & $\mathrm{Cl}$ & 1.694355 & 9.363494 & -2.868658 \\
\hline $\mathrm{C}$ & -3.366290 & -3.176889 & -0.154668 & $\mathrm{Cl}$ & -5.964652 & 2.338755 & -8.091607 \\
\hline $\mathrm{C}$ & -2.699914 & -4.526628 & -0.491877 & $\mathrm{~N}$ & -4.676862 & 1.003506 & -0.382785 \\
\hline $\mathrm{C}$ & -1.304879 & -4.587896 & 0.165531 & $\mathrm{O}$ & -6.228357 & 2.336529 & -1.370365 \\
\hline $\mathrm{C}$ & -0.438812 & -3.424170 & -0.370212 & $\mathrm{~N}$ & 2.909397 & 3.709860 & -1.222663 \\
\hline $\mathrm{C}$ & -3.509600 & -3.036370 & 1.376489 & $\mathrm{O}$ & 4.027148 & 4.205109 & -1.114648 \\
\hline $\mathrm{C}$ & -2.114991 & -3.1 & 2.0 & $\mathrm{O}$ & 1. & 374 & 660 \\
\hline $\mathrm{C}$ & -1.448900 & -4.454197 & 1.696634 & $\mathrm{H}$ & 3.048197 & -1.735215 & -4.019172 \\
\hline $\mathrm{C}$ & -1.240136 & -1.943529 & 1.499520 & $\mathrm{H}$ & 0.987519 & -1.302153 & -2.682161 \\
\hline $\mathrm{Ni}$ & -1.7 & 2 & 0.5 & $\mathrm{H}$ & 5.000549 & 425 & -2.260265 \\
\hline $\mathrm{Cl}$ & -1.939407 & 3.400442 & -1.831573 & + & 3.395292 & 1.097697 & 0.992239 \\
\hline $\mathrm{Cl}$ & -1.778120 & 2.148661 & 2.790480 & $\mathrm{H}$ & 4.600534 & 2.202031 & 0.349476 \\
\hline $\mathrm{N}$ & 4 & 3.708934 & 0.875612 & $\mathrm{H}$ & 4.852615 & 4.4 & 90 \\
\hline $\mathrm{C}$ & -5.177019 & 3.239179 & 0.565726 & $\mathrm{H}$ & 4.620067 & 5.867412 & 3.351143 \\
\hline $\mathrm{C}$ & -6.360167 & 3.803805 & 1.071731 & $\mathrm{H}$ & 3.007822 & 5.204422 & 5.133511 \\
\hline $\mathrm{C}$ & -6.282154 & 4.886680 & 1.938730 & $\mathbf{U}$ & 1.633603 & 3.143966 & 4.845589 \\
\hline $\mathrm{C}$ & -5.024939 & 5.411813 & 2.233411 & $\mathrm{H}$ & 1.875802 & 1.765461 & 2.809513 \\
\hline $\mathrm{C}$ & -3.905664 & 4.801715 & 1.667531 & $\mathrm{H}$ & -2.965958 & -1.055282 & -0.469305 \\
\hline $\mathrm{C}$ & -2.561965 & 5.369843 & 1.8 & $\mathrm{H}$ & -2.378 & 6029 & 783346 \\
\hline $\mathrm{C}$ & -5.300716 & 2.111416 & -0.393668 & $\mathrm{H}$ & -4.355470 & -3.120711 & -0.627568 \\
\hline $\mathrm{O}$ & -2.413445 & 6.420715 & 2.666187 & $\mathrm{H}$ & -3.322777 & -5.355275 & -0.127848 \\
\hline $\mathrm{C}$ & -0.968912 & 6.650436 & 2.755454 & $\mathrm{H}$ & -2.610170 & -4.643307 & -1.581208 \\
\hline $\mathrm{C}$ & -0.381458 & 5.792985 & 1.596409 & $\mathrm{H}$ & -0.813542 & -5.537241 & -0.083178 \\
\hline $\mathrm{N}$ & -1.523215 & 4.926630 & 1.240121 & $\mathrm{H}$ & 0.562546 & -3.452016 & 0.077935 \\
\hline $\mathrm{C}$ & -6.301683 & 1.102093 & -2.162740 & 11 & -0.321921 & -3.504881 & -1.458553 \\
\hline $\mathrm{C}$ & -5.137554 & 0.258613 & -1.567330 & $\mathrm{H}$ & -3.996955 & -2.083886 & 1.624136 \\
\hline
\end{tabular}




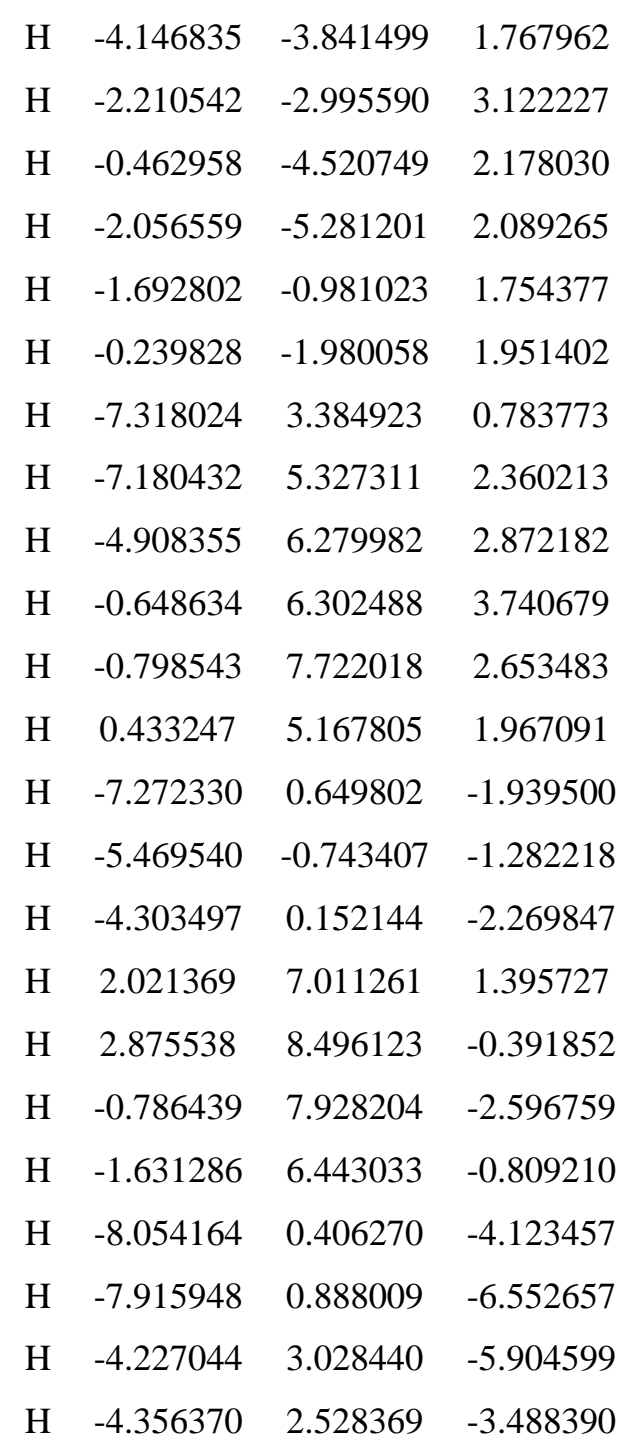

${ }^{t} \mathrm{BuO}^{\cdot}$

$$
\Delta \mathbf{G}=\mathbf{- 2 3 3 . 0 1 8 6 1 1} \text { hartree }
$$

$\begin{array}{cccc}\mathrm{C} & 2.466217 & -0.258327 & -0.001342 \\ \mathrm{C} & 1.148228 & 0.522798 & 0.004513 \\ \mathrm{C} & -0.086646 & -0.388236 & -0.001224 \\ \mathrm{C} & -1.396711 & 0.399643 & -0.008222 \\ \mathrm{O} & -2.547361 & -0.342081 & 0.000125 \\ \mathrm{H} & 1.114753 & 1.175685 & 0.889055\end{array}$
$\begin{array}{llll}\mathrm{H} & -0.075437 & -1.044644 & 0.880023\end{array}$

$\begin{array}{llll}\mathrm{H} & -0.066653 & -1.038359 & -0.887367\end{array}$

$\begin{array}{llll}\mathrm{H} & -1.470116 & 1.062994 & 0.885150\end{array}$

$\begin{array}{llll}\mathrm{H} & -1.448435 & 1.122451 & -0.849617\end{array}$

$\begin{array}{llll}\mathrm{H} & 1.114672 & 1.186825 & -0.871604\end{array}$

$\begin{array}{llll}\mathrm{H} & 2.547978 & -0.906667 & 0.881058\end{array}$

$\begin{array}{llll}\mathrm{H} & 3.329532 & 0.418963 & 0.001726\end{array}$

$\begin{array}{llll}\mathrm{H} & 2.546069 & -0.895870 & -0.891774\end{array}$

\section{${ }^{t} \mathrm{BuOH}$}

$\Delta \mathbf{G}=\mathbf{- 2 3 3 . 6 8 4 7 1 9}$ hartree

$\begin{array}{llll}\text { C } & -0.187612 & -0.097917 & 0.040080\end{array}$

$\begin{array}{llll}\text { C } & 1.344661 & -0.060580 & 0.041098\end{array}$

$\begin{array}{llll}\text { C } & 1.907617 & 1.311053 & -0.356177\end{array}$

$\begin{array}{llll}\text { C } & 3.429284 & 1.346934 & -0.354535\end{array}$

$\begin{array}{llll}\text { O } & 3.855812 & 2.664929 & -0.740740\end{array}$

$\begin{array}{llll}\mathrm{H} & 1.713896 & -0.331551 & 1.040880\end{array}$

$\begin{array}{llll}\mathrm{H} & 1.534300 & 2.079799 & 0.335574\end{array}$

$\begin{array}{llll}\mathrm{H} & 1.548836 & 1.582624 & -1.359241\end{array}$

$\begin{array}{llll}\mathrm{H} & 3.815316 & 1.106941 & 0.647488\end{array}$

$\begin{array}{llll}\mathrm{H} & 4.827719 & 2.686819 & -0.740236\end{array}$

$\begin{array}{llll}\mathrm{H} & 3.829548 & 0.602540 & -1.059141\end{array}$

H $\quad 1.727408 \quad-0.826343 \quad-0.649210$

$\begin{array}{llll}\mathrm{H} & -0.603643 & 0.632506 & 0.746638\end{array}$

$\begin{array}{llll}\mathrm{H} & -0.561513 & -1.088835 & 0.327359\end{array}$

$\begin{array}{llll}\mathrm{H} & -0.589627 & 0.135080 & -0.954837\end{array}$

$\cdot \mathrm{NO}_{2}$

\section{$\Delta G=\mathbf{- 2 0 5 . 0 8 3 0 5 0}$ hartree}

$\begin{array}{llll}\mathrm{N} & -0.346093 & 0.182699 & -0.214966\end{array}$

$\begin{array}{llll}\mathrm{O} & 0.794627 & 0.260823 & 0.153041\end{array}$

$\begin{array}{llll}\text { O } & -1.369876 & -0.164168 & 0.309464\end{array}$ 


\section{References}

1. Badiola, E.; Fiser, B.; Gómez-Bengoa, E.; Mielgo, A.; Olaizola, I.; Urruzuno, I.;

García, J. M.; Odriozola, J. M.; Razkin, J.; Oiarbide, M.; Palomo, C. Enantioselective Construction of Tetrasubstituted Stereogenic Carbons through Brønsted Base Catalyzed Michael Reactions: $\alpha^{\prime}$-Hydroxy Enones as Key Enoate Equivalent. J. Am. Chem. Soc. 2014, 136, 17869-17881.

2. Ishimaru, T.; Shibata, N.; Nagai, J.; Nakamura, S.; Toru, T.; Kanemasa, S. Lewis Acid-Catalyzed Enantioselective Hydroxylation Reactions of Oxindoles and $\beta$ Keto Esters Using DBFOX Ligand. J. Am. Chem. Soc. 2006, 128, 16488-16489.

3. Frisch, M. J.; Trucks, G. W.; Schlegel, H. B.; Scuseria, G. E.; Robb, M. A.; Cheeseman, J. R.; Scalmani, G.; Barone, V.; Petersson, G. A.; Nakatsuji, H.; Li, X.; Caricato, M.; Marenich, A. V.; Bloino, J.; Janesko, B. G.; Gomperts, R.; Mennucci, B.; Hratchian, H. P.; Ortiz, J. V.; Izmaylov, A. F.; Sonnenberg, J. L.; WilliamsYoung, D.; Ding, F.; Lipparini, F.; Egidi, F.; Goings, J.; Peng, B.; Petrone, A.; Henderson, T.; Ranasinghe, D.; Zakrzewski, V. G.; Gao, J.; Rega, N.; Zheng, G.; Liang, W.; Hada, M.; Ehara, M.; Toyota, K.; Fukuda, R.; Hasegawa, J.; Ishida, M.; Nakajima, T.; Honda, Y.; Kitao, O.; Nakai, H.; Vreven, T.; Throssell, K.; Montgomery, J. A.; Peralta, J. E.; Ogliaro, F.; Bearpark, M. J.; Heyd, J. J.; Brothers, E. N.; Kudin, K. N.; Staroverov, V. N.; Keith, T. A.; Kobayashi, R.; Normand, J.; Raghavachari, K.; Rendell, A. P.; Burant, J. C.; Iyengar, S. S.; Tomasi, J.; Cossi, M.; Millam, J. M.; Klene, M.; Adamo, C.; Cammi, R.; Ochterski, J. W.; Martin, R. L.; Morokuma, K.; Farkas, O.; Foresman, J. B.; Fox, D. J. Gaussian 16 Revision B.01, Gaussian Inc. Wallingford CT, 2016.

4. Kohn, W. Nobel Lecture: Electronic Structure of Matter-Wave Functions and Density Functionals. Rev. Mod. Phys., 1999, 71, 1253-1266.

5. Lee, C.; Yang, W.; Parr, R. G.; Development of the Colle-Salvetti CorrelationEnergy Formula into a Functional of the Electron Density. Phys. Rev. B, 1988, 37, 785-789.

6. Hariharan, P. C.; Pople, J. A. Influence of Polarization Functions on MolecularOrbital Hydrogenation Energies. Theory Chem. Acc., 1973, 28, 213-222.

7. Hay, P. J.; Wadt, W. R. Ab Initio Effective Core Potentials for Molecular Calculations-Potentials for $\mathrm{K}$ to $\mathrm{Au}$ Including the Outermost Core Orbitals. $J$. 
Chem. Phys., 1985, 82, 299-310.

8. McLean, A.; Chandler, G. Contracted Gaussian Basis Sets for Molecular Calculations. I. Second Row Atoms, $Z=11-18$. J. Chem. Phys, 1980, 72, 56395648 .

9. Marenich, A. V.; Cramer, C. J.; Truhlar, D. G. Universal Solvation Model Based on Solute Electron Density and A Continuum Model of the Solvent Defined by the Bulk Dielectric Constant and Atomic Surface Tensions. J. Phys. Chem. B, 2009, $113,6378-6396$. 